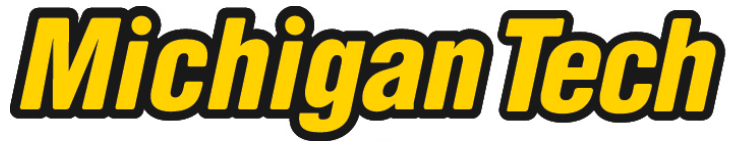 \\ Michigan Technological University Create the Future Digital Commons @ Michigan Tech
}

\section{Accounting for warping and differential drying shrinkage mechanisms in the design of jointed plain concrete payments}

Rita Elizabeth Lederle

Michigan Technological University

Follow this and additional works at: https://digitalcommons.mtu.edu/etds

Part of the Civil and Environmental Engineering Commons

Copyright 2011 Rita Elizabeth Lederle

\section{Recommended Citation}

Lederle, Rita Elizabeth, "Accounting for warping and differential drying shrinkage mechanisms in the design of jointed plain concrete payments", Master's Thesis, Michigan Technological University, 2011.

https://doi.org/10.37099/mtu.dc.etds/251

Follow this and additional works at: https://digitalcommons.mtu.edu/etds

3 Part of the Civil and Environmental Engineering Commons 
Rita Elizabeth Lederle

\author{
A THESIS \\ Submitted in partial fulfillment of the requirements for the degree of \\ MASTER OF SCIENCE \\ (Civil Engineering)
}

MICHIGAN TECHNOLOGICAL UNIVERSITY

2011

Copyright: Rita E. Lederle 2011 

This thesis, "Accounting for Warping and Differential Drying Shrinkage Mechanisms in the Design of Jointed Plain Concrete Pavements," is hereby approved in partial fulfillment of the requirements for the Degree of MASTER OF SCIENCE IN CIVIL ENGINEERING

Department of Civil and Environmental Engineering

Signatures:

Thesis Advisor

Dr. Jacob Hiller

Committee Member

Dr. Devin Harris

Committee Member

Dr. Gretchen Hein

Department Head

Dr. David Hand

Date 

As always,

for Andrew 



\section{Table of Contents}

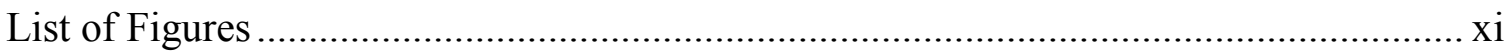

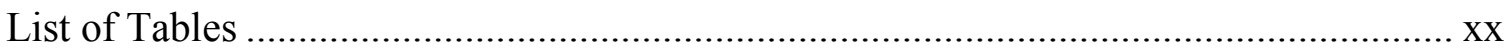

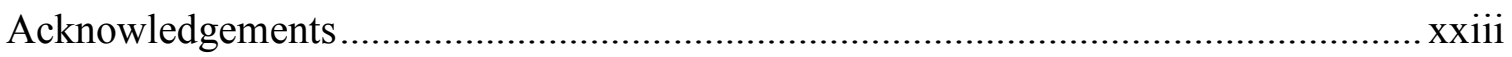

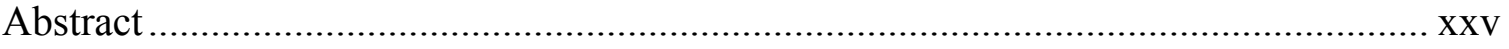

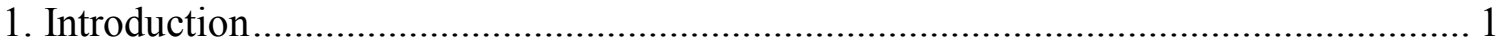

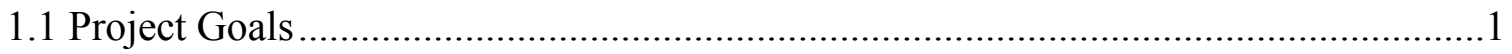

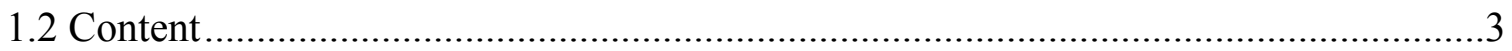

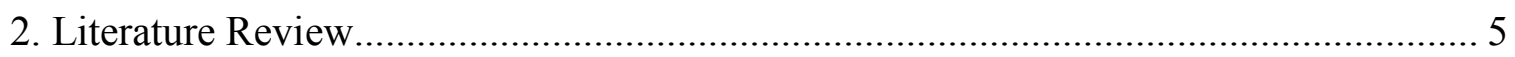

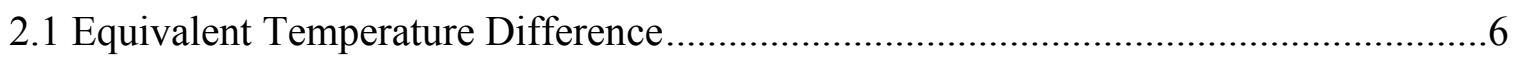

2.1.1 Actual Temperature Difference .................................................................. 7

2.1.2 Differential Drying Shrinkage ………………….................................... 9

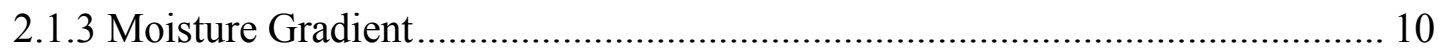

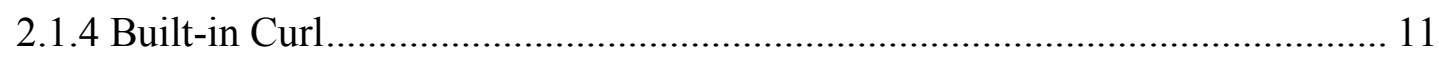

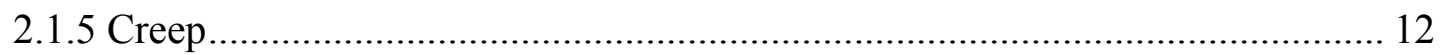

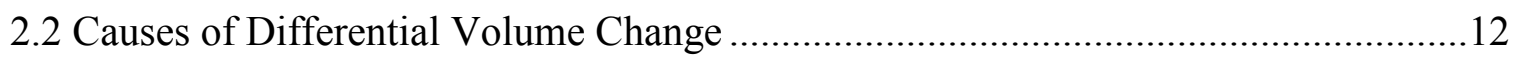

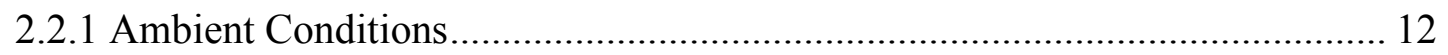

2.2.2 Concrete Material Properties …………………….................................... 14

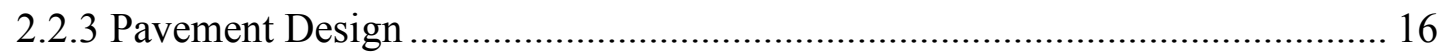

2.2.4 Curing Method/Drying History ………………......................................... 18

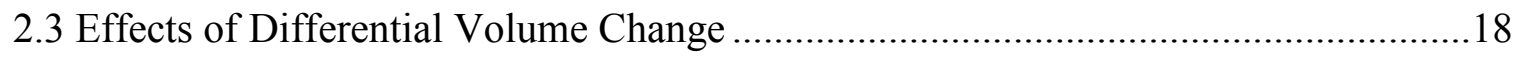

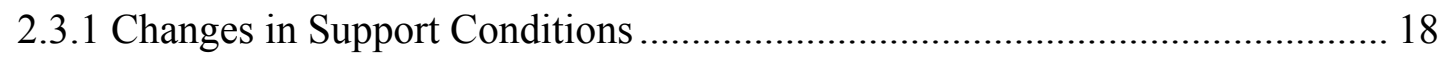

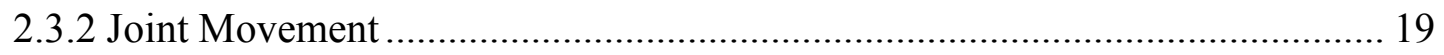

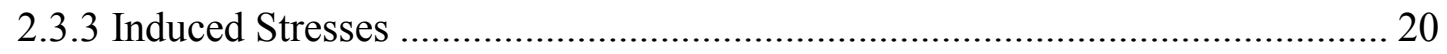

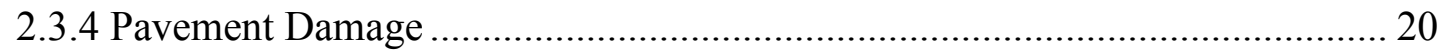

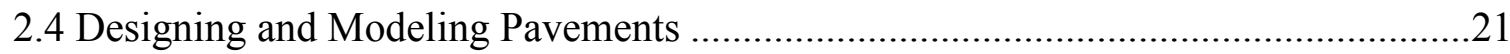

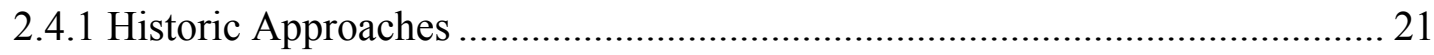

2.4.2 Modern Approaches ............................................................................ 22

2.4.3 Current Pavement Design Practices ............................................................. 22

2.4.4 Differential Volume Change in the MEDPG …………................................ 25

2.5 Measuring Differential Volume Change...............................................................26

2.5.1 Slab Measurement Techniques ................................................................... 26

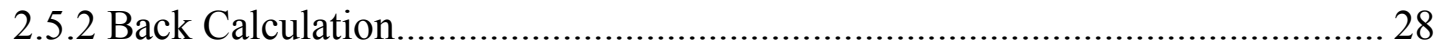

2.6 Summary of Literature Review............................................................................28 


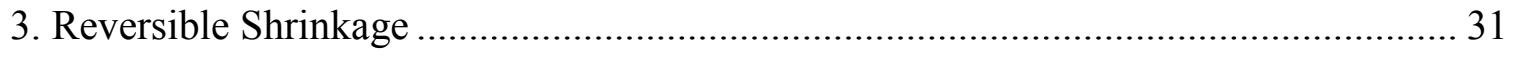

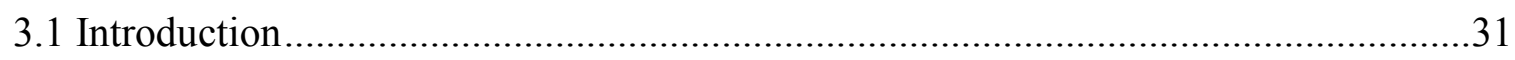

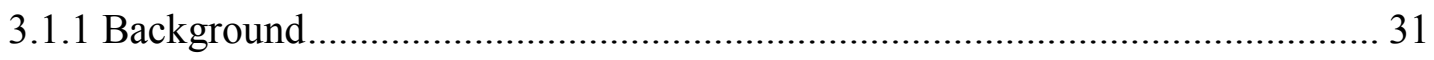

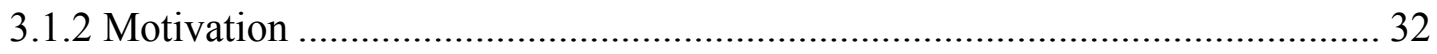

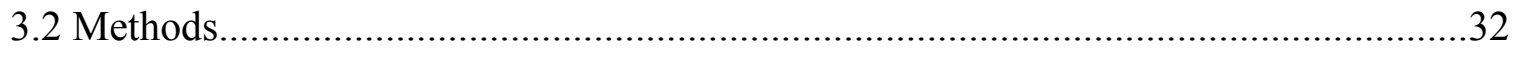

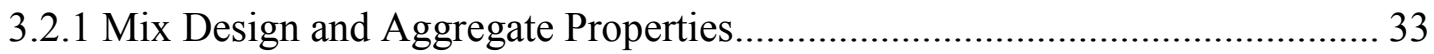

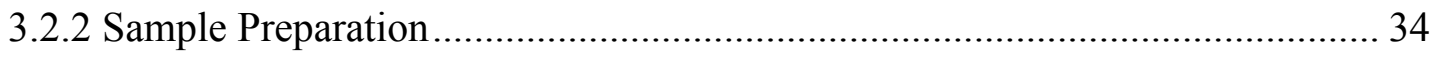

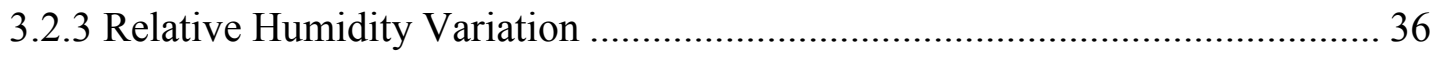

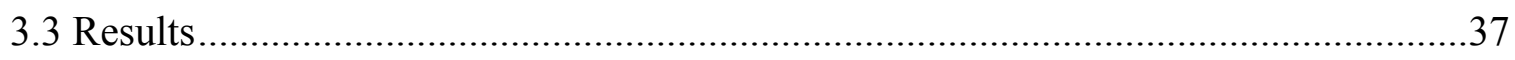

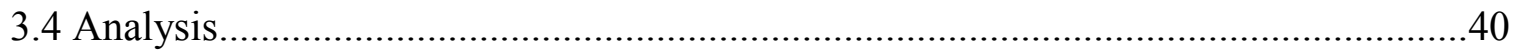

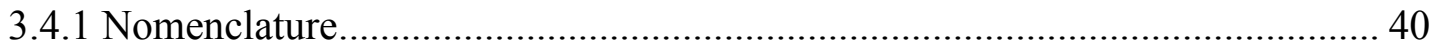

3.4.2 Magnitude of Ultimate and Drying Shrinkage ………....................................... 43

3.4.3 Reversible Shrinkage as a Function of Drying and Re-Wetting Cycles........... 45

3.4.4 Permanent Shrinkage as a Function of Drying and Re-wetting Cycles ........... 48

3.4.5 Reversible Shrinkage as a Function of Concrete Permeability and Aggregate

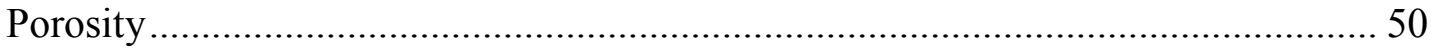

3.4.6 Shrinkage versus Weight Change ........................................................... 52

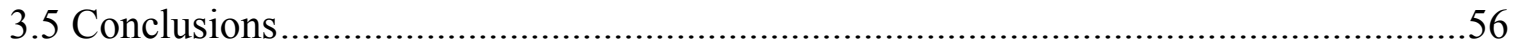

4. Warping and Differential Drying Shrinkage Models............................................... 59

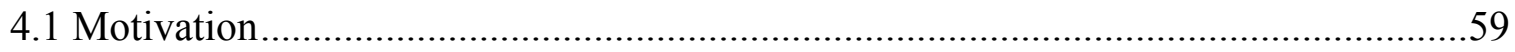

4.2 Current MEPDG Moisture Warping Model .............................................................60

4.3 Problems with the Current MEPDG Warping Model.....................................................63

4.3.1 Derivation of the Current MEPDG Warping Model .......................................... 64

4.3.2 Assumptions in the Current MEPDG Warping Model.......................................... 66

4.3.3 Amount of Warping Does Not Affect the Current MEPDG Cracking Model . 67

4.3.4 Relating Monthly Relative Humidity to the Annual Average ……..................... 69

4.4 Options for Improvement of the Warping Model ......................................................

4.4.1 Non-Linear Shrinkage Distribution with Plate Theory …………………….... 74

4.4.2 Remove Relation to Average Annual Relative Humidity …………………..... 81

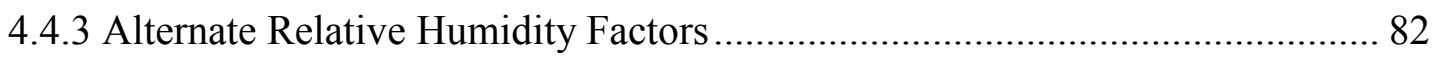

4.4.4 Account for Contribution of Autogenous Shrinkage....................................... 87

4.4.5 Alternate Reversible Shrinkage Factor ........................................................... 93

4.4.6 Alternate Combined Model to Account for Drying Shrinkage and Relative

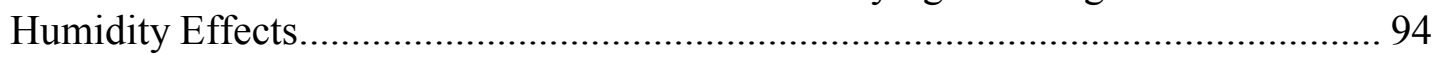


4.5 Final Proposed Models for Moisture Warping and Differential Drying Shrinkage ....95

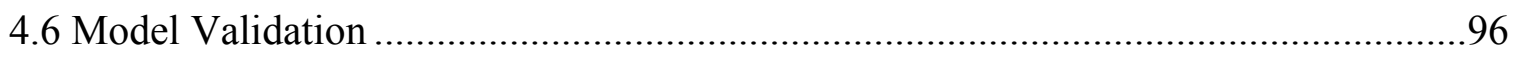

4.6.1 Warping Models Used for Comparison........................................................ 96

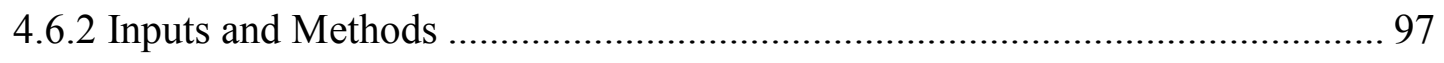

4.6.3 Results and Comparison of Warping Models ................................................. 98

4.7 Implications of Moisture Warping and Differential Drying Shrinkage on the Total Equivalent Temperature Difference.................................................................101

4.8 Design Aids for the Determination of Moisture Warping and Differential Drying

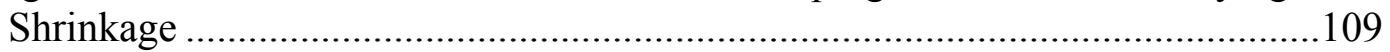

5. Quantifying Built-in Curl................................................................................. 113

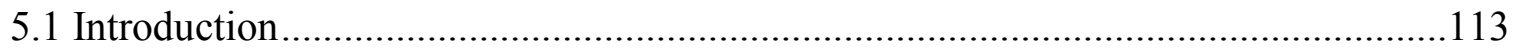

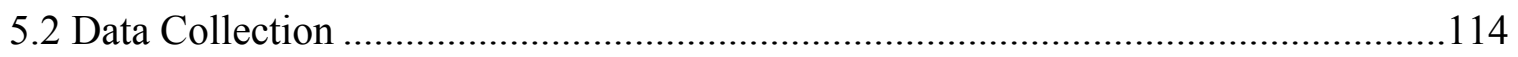

5.2.1 Test Cell Locations and Descriptions .......................................................... 114

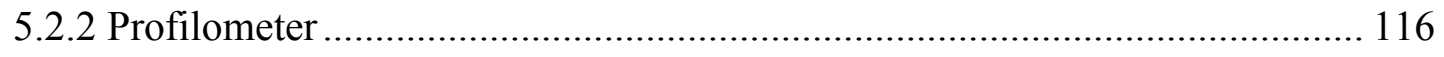

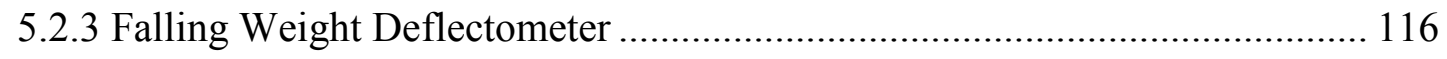

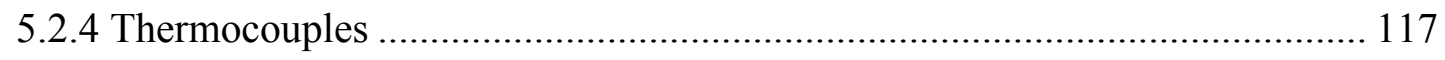

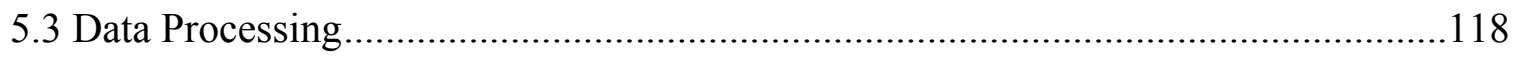

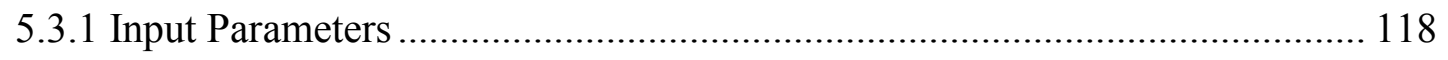

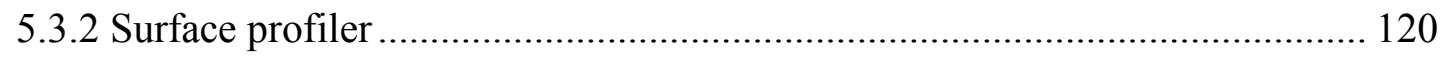

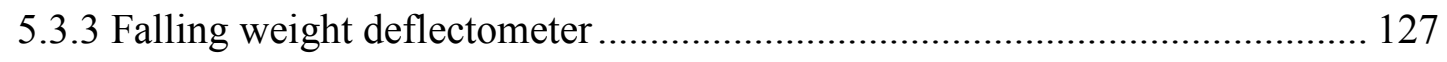

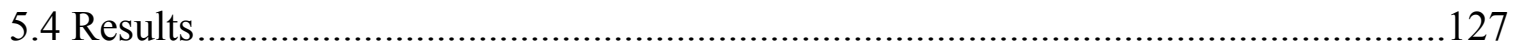

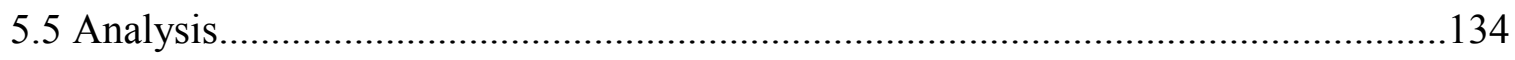

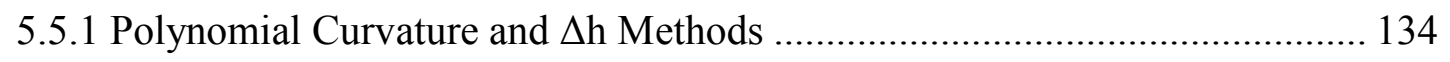

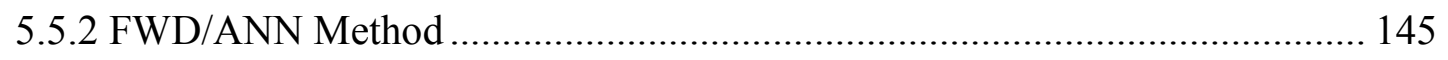

5.5.3 Minimum Error Method ............................................................................ 147

5.5.4 Comparison of Results from Different Methods ............................................. 153

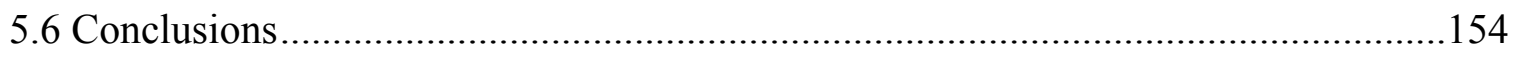

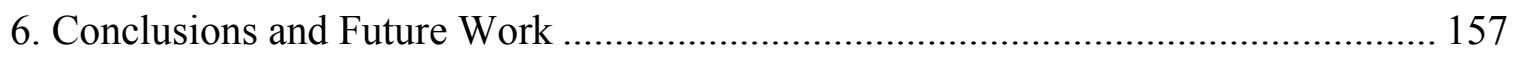

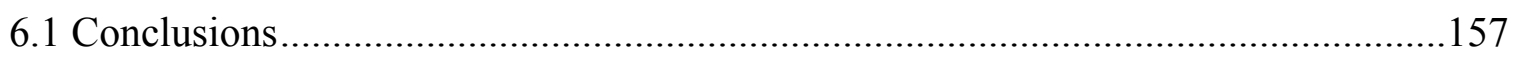

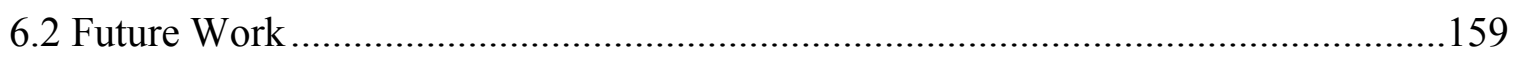

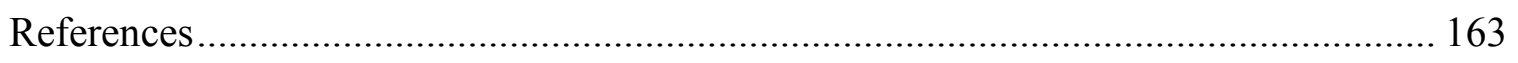

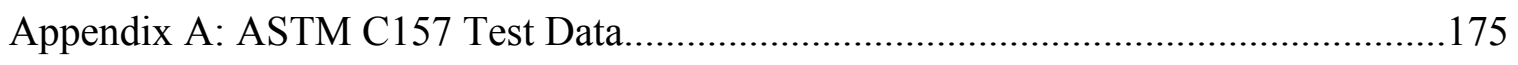

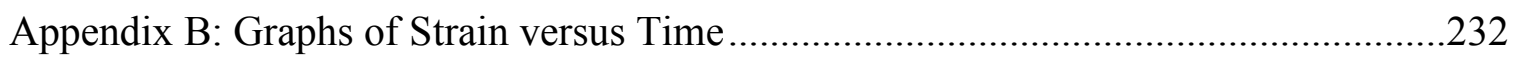

Appendix C: Graphs of Weight Change versus Time ………………….....................243 
Appendix D: Graphs of Changes in Strain versus Changes in Weight .........................254

Appendix E: Validation of Elliptical Approximation for All Locations .........................260

Appendix F: Design Aids for the Prediction of Moisture Warping and Differential

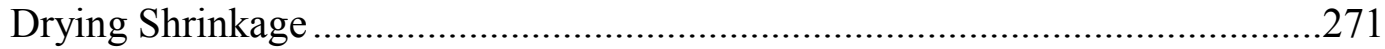

Appendix G: Input Parameters for ISLAB 2000 Runs ..............................................292 


\section{List of Figures}

Figure 2.1: A slab curled due to a temperature gradient a) upwards and b) downwards, and associated stress state.

Figure 2.2: A slab warped due to a moisture gradient a) upwards and b) downwards, and associated stress state.

Figure 2.3: Total temperature gradient and constituent components.............................. 8

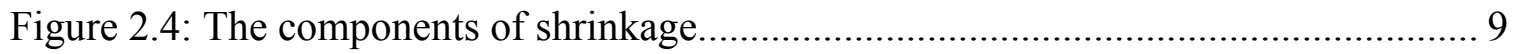

Figure 2.5: Top down cracking due to the combined effects of curling and traffic loading.

Figure 2.6: Accounting for equivalent temperature difference in the MEPDG.............. 25

Figure 3.1: Highly idealized curve of reversible shrinkage.................................... 32

Figure 3.2: Strain versus time for dry cured virgin aggregate concrete.......................... 37

Figure 3.3: Strain versus time for wet cured virgin aggregate concrete. ........................ 38

Figure 3.4: Strain versus time for dry cured gravel RCA concrete. ............................... 38

Figure 3.5: Strain versus time for wet cured gravel RCA concrete.............................. 39

Figure 3.6: Strain versus time for dry cured lightweight aggregate concrete................. 39

Figure 3.7: Strain versus time for wet cured lightweight aggregate ............................ 40

Figure 3.8: Highly idealized curve of shrinkage versus time for concrete initially exposed to drying followed by cyclic drying and re-wetting........................................ 41

Figure 3.9: Highly idealized curve of shrinkage versus time for concrete initially exposed to wetting followed by cyclic drying and re-wetting. ....................................... 41

Figure 3.10: Shrinkage versus concrete permeability.......................................... 45

Figure 3.11: Shrinkage versus aggregate porosity............................................... 45

Figure 3.12: Reversible shrinkage for different drying and re-wetting cycles. ............... 46

Figure 3.13: Reversible and permanent portions of ultimate shrinkage for samples which

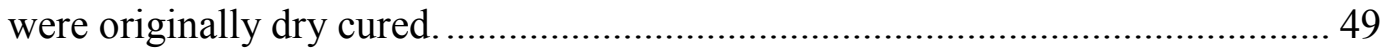


Figure 3.14: Reversible and permanent portions of ultimate shrinkage for samples which

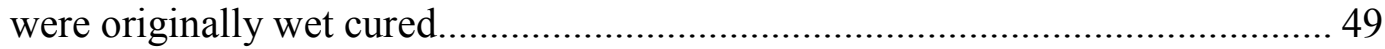

Figure 3.15: Reversible shrinkage versus concrete permeability as measured by the rapid chloride permeability test................................................................................... 51

Figure 3.16: Reversible shrinkage versus aggregate porosity. ......................................... 51

Figure 3.17: Change in strain as a function of change in weight for a virgin aggregate

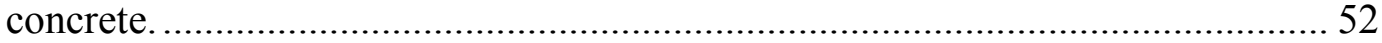

Figure 3.18: Change in strain as a function of change in weight for the gravel RCA

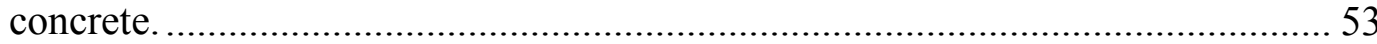

Figure 3.19: Change in strain as a function of change in weight for the lightweight

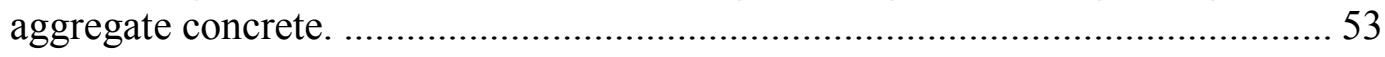

Figure 3.20: Length change as a function of weight change for dry cured samples......... 54

Figure 3.21: Length change as a function of weight change for wet cured samples........ 55

Figure 4.1: a) Eisenmann and Leykauf's rectangular shrinkage distribution and b)

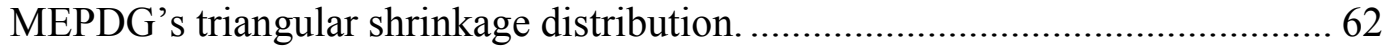

Figure 4.2: Equivalent linear temperature difference. ……………………………….... 65

Figure 4.3: Drying shrinkage through the thickness of (a) a concrete slab and (b) an ASTM C157 drying shrinkage prism, assuming a triangular shrinkage distribution with depth

Figure 4.4 Percent of slabs cracked at 20 years in Lincoln Nebraska. ............................. 69

Figure 4.5: Average monthly ambient relative humidity for Las Vegas and Seattle........ 71

Figure 4.6: Average monthly moisture warping for Las Vegas and Seattle..................... 71

Figure 4.7: Non-linear shrinkage gradient modeled as a quarter of an ellipse. ............... 74

Figure 4.8: Ultimate shrinkage through the depth of the pavement for Reno, Nevada five

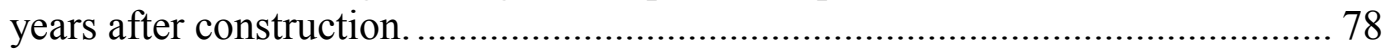

Figure 4.9: Area of the shrinkage distribution for Reno Nevada after five years of drying from a) the original data, b) the nonlinear elliptical approximation, and c) the

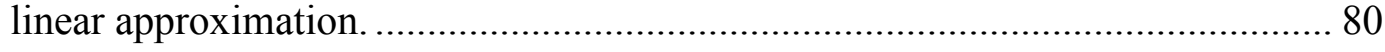

Figure 4.10: Comparison of shrinkage models. ............................................................ 86

Figure 4.11: $\omega$ vs. w/c, data and associated linear trend line........................................... 90 xii 
Figure 4.12: $\omega$ vs. $w / c$, data and associated bilinear trend line for a theoretical limit of hydration corresponding to a $\mathrm{w} / \mathrm{c}$ ratio of 0.36 .

Figure 4.13: $\omega$ vs. $w / c$, data and associated sigmoid trend line. 92

Figure 4.14: Equivalent temperature difference $\left({ }^{\circ} \mathrm{F}\right)$ from various models with the depth of the shrinkage zone determined from internal relative humidity data.

Figure 4.15: Equivalent temperature difference $\left({ }^{\circ} \mathrm{F}\right)$ from various models with the depth of the shrinkage zone equal to two inches in all locations

Figure 4.16: Predicted equivalent temperature difference in Las Vegas using a) the proposed warping and differential drying shrinkage models, and b) the MEPDG model warping model and $-10^{\circ} \mathrm{F}$ default value for BIC.

Figure 4.17: Predicted equivalent temperature difference in Seattle using a) the proposed warping and differential drying shrinkage models, and b) the MEPDG model warping model and $-10^{\circ} \mathrm{F}$ default value for BIC.

Figure 4.18: Design aid for the equivalent temperature difference due to warping for a 10 inch pavement with $h_{s}=3 \mathrm{in}, \mathrm{w} / \mathrm{c}=0.4, \varphi=.5$, and $\varepsilon_{\mathrm{su}}=600 \mu \varepsilon$.

Figure 5.1: MnROAD mainline cell locations with pertinant cells highlighted. 115

Figure 5.2: Mainline cell descriptions, (modified from Johnson et al. 2008)................. 115

Figure 5.3: MnROAD low volume road with pertinent cells highlighted. 116

Figure 5.4: Low volume road cell descriptions, (modified from Johnson et al. 2008)... 116

Figure 5.5: FWD configuration, with sensors numbered.

Figure 5.6: Deflection along the width of the slab for cell 36 , panel 19, early morning test unadjusted. 121

Figure 5.7: Deflection along the length of the slab after adjusting for extraneous data and subtracting given cross slope for cell 36 , panel 19 , early morning test.

Figure 5.8: Deflection along the length of the slab after adjusting for extraneous data and subtracting assumed cross slope for cell 36 , panel 19 , early morning test. 122

Figure 5.9: ISLAB2000 data and approximations. 123

Figure 5.10: 2nd order approximation of actual data for pass 2 of Cell 53 early test in October, and associated ISLAB2000 curves. 126

Figure 5.11: Best-fit polynomals for Cell 72, pass 3. 135 
Figure 5.12: Transverse profile of cracked slab without adjusting for cross slope - cell 37, panel 8, late morning test from October testing........................................... 144

Figure 5.13: Transverse profile of cracked slab after adjusting for cross slope - cell 37, panel 8, late morning test from October testing............................................. 144

Figure 5.14: 4th order polynomial approximation pass 2 of cell 53 early test vs. ISLAB2000......

Figure 5.15: 4th order polynomil approximation pass 2 of cell 53 early test vs. ISLAB2000, discarding first and last quarter of data. 150

Figure 5.16: 2nd order polynomial approximation pass 2 of cell 53 early test vs. ISLAB2000. 150

Figure 5.17: Actual data pass 1, cell 72 vs. ISLAB2000. 152

Figure B.1: Strain versus time for dry cured Virgin 1 232

Figure B.2: Strain versus time for wet cured Virgin 1. 232

Figure B.3: Strain versus time for dry cured Virgin 2 .......................................... 233

Figure B.4: Strain versus time for wet cured Virgin 2 ........................................ 233

Figure B.5: Strain versus time for dry cured Virgin 3.......................................... 234

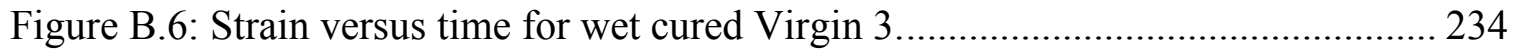

Figure B.7: Strain versus time for dry cured Slag RCA...................................... 235

Figure B.8: Strain versus time for wet cured Slag RCA.......................................... 235

Figure B.9: Strain versus time for dry cured Limestone RCA. ................................... 236

Figure B.10: Strain versus time for wet cured Limestone RCA................................ 236

Figure B.11: Strain versus time for dry cured Gravel RCA 1 ................................. 237

Figure B.12: Strain versus time for wet cured Gravel RCA 1 ................................. 237

Figure B.13: Strain versus time for dry cured Gravel RCA 2. ................................... 238

Figure B.14: Strain versus time for wet cured Gravel RCA 2 ................................ 238

Figure B.15: Strain versus time for dry cured Twice Recycled RCA. ........................ 239

Figure B.16: Strain versus time for wet cured Twice Recycled RCA.......................... 239 


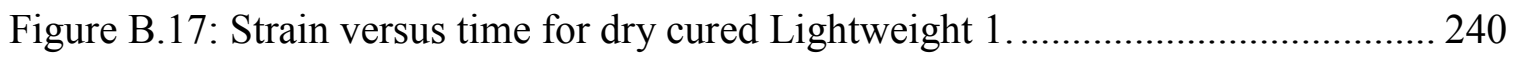

Figure B.18: Strain versus time for wet cured Lightweight 1 ................................... 240

Figure B.19: Strain versus time for dry cured Lightweight 2 ................................... 241

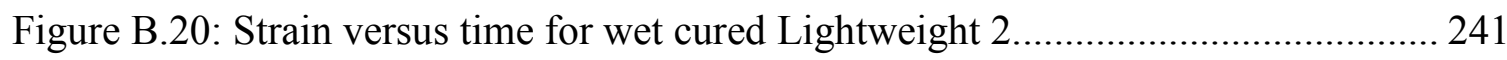

Figure B.21: Strain versus time for dry cured Lightweight 3 ................................ 242

Figure B.22: Strain versus time for wet cured Lightweight 3................................. 242

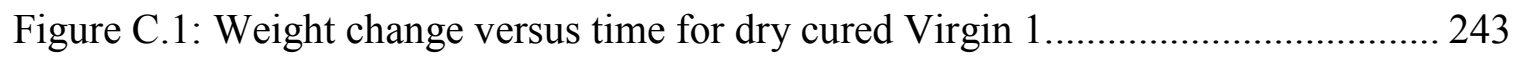

Figure C.2: Weight change versus time for wet cured Virgin 1................................. 243

Figure C.3: Weight change versus time for dry cured Virgin 2 .............................. 244

Figure C.4: Weight change versus time for wet cured Virgin 2 ............................... 244

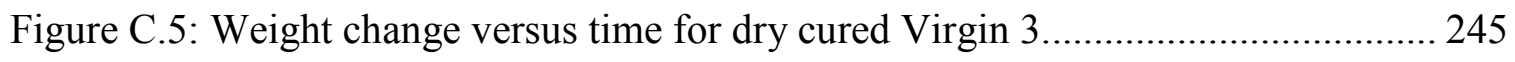

Figure C.6: Weight change versus time for wet cured Virgin 2 ............................... 245

Figure C.7: Weight change versus time for dry cured Slag RCA............................... 246

Figure C.8: Weight change versus time for wet cured Slag RCA. ............................. 246

Figure C.9: Weight change versus time for dry cured Limestone RCA...................... 247

Figure C.10: Weight change versus time for wet cured Limestone RCA. .................... 247

Figure C.11: Weight change versus time for dry cured Gravel RCA 1...................... 248

Figure C.12: Weight change versus time for wet cured Gravel RCA 1. ....................... 248

Figure C.13: Weight change versus time for dry cured Gravel RCA 2 ...................... 249

Figure C.14: Weight change versus time for wet cured Gravel RCA 1. ...................... 249

Figure C.15: Weight change versus time for dry cured Twice Recycled RCA............. 250

Figure C.16: Weight change versus time for wet cured Twice Recycled RCA. ........... 250

Figure C.17: Weight change versus time for dry cured Lightweight 1 . ....................... 251

Figure C.18: Weight change versus time for wet cured Lightweight 1...................... 251

Figure C.19: Weight change versus time for dry cured Lightweight 2. ...................... 252 
Figure C.20: Weight change versus time for wet cured Lightweight 2..................... 252

Figure C.21: Weight change versus time for dry cured Lightweight 3. ...................... 253

Figure C.22: Weight change versus time for wet cured Lightweight 3........................ 253

Figure D.1: Change in strain versus change in weight for Virgin 1........................... 254

Figure D.2: Change in strain versus change in weight for Virgin 2. .......................... 254

Figure D.3: Change in strain versus change in weight for Virgin 3.......................... 255

Figure D.4: Change in strain versus change in weight for Slag RCA. ......................... 255

Figure D.5: Change in strain versus change in weight for Limestone RCA.................. 256

Figure D.6: Change in strain versus change in weight for Gravel RCA 1 $\ldots \ldots \ldots \ldots \ldots \ldots \ldots . . . . . . . .256$

Figure D.7: Change in strain versus change in weight for Gravel RCA 2 ................... 257

Figure D.8: Change in strain versus change in weight for Twice Recycled.................. 257

Figure D.9: Change in strain versus change in weight for Lightweight 1 .................... 258

Figure D.10: Change in strain versus change in weight for Lightweight 2................. 258

Figure D.11: Change in strain versus change in weight for Lightweight 3................. 259

Figure E.1: Ultimate shrinkage through the depth of the pavement for Reno, Nevada 30

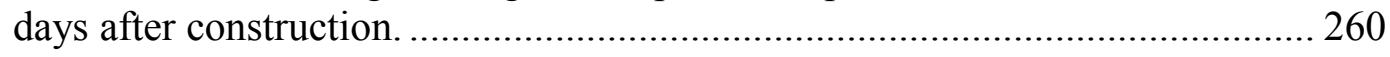

Figure E.2: Ultimate shrinkage through the depth of the pavement for Seattle, Washington 30 days after construction.................................................... 260

Figure E.3: Ultimate shrinkage through the depth of the pavement for Columbus, Ohio 30

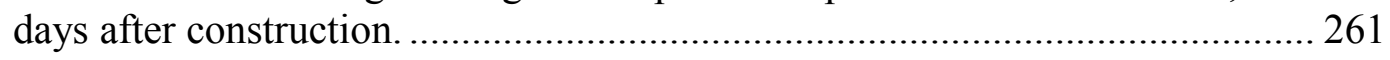

Figure E.4: Ultimate shrinkage through the depth of the pavement for New Orleans, Louisiana 30 days after construction. .......................................................... 261

Figure E.5: Ultimate shrinkage through the depth of the pavement for Los Angeles, California 30 days after construction. 262

Figure E.6: Ultimate shrinkage through the depth of the pavement for Las Vegas, Nevada, 30 days after construction. 262

Figure E.7: Ultimate shrinkage through the depth of the pavement for Astoria, Oregon 30 days after construction. 263 
Figure E.8: Ultimate shrinkage through the depth of the pavement for Reno, Nevada 1

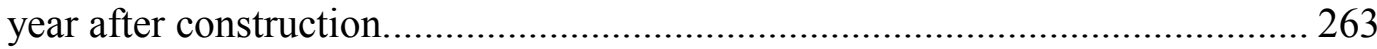

Figure E.9: Ultimate shrinkage through the depth of the pavement for Seattle, Washington 1 year after construction. 264

Figure E.10: Ultimate shrinkage through the depth of the pavement for Columbus, Ohio 1 year after construction............................................................................ 264

Figure E.11: Ultimate shrinkage through the depth of the pavement for New Orleans, Louisianal 1 year after construction. 265

Figure E.12: Ultimate shrinkage through the depth of the pavement for Los Angeles, California 1 year after construction. 265

Figure E.13: Ultimate shrinkage through the depth of the pavement for Las Vegas, Nevada 1 year after construction. 266

Figure E.14: Ultimate shrinkage through the depth of the pavement for Astoria, Oregon 1 year after construction. 266

Figure E.15: Ultimate shrinkage through the depth of the pavement for Reno, Nevada 5 years after construction. 267

Figure E.16: Ultimate shrinkage through the depth of the pavement for Seattle, Washington 5 years after construction. 267

Figure E.17: Ultimate shrinkage through the depth of the pavement for Columbus, Ohio 5 years after construction. 268

Figure E.18: Ultimate shrinkage through the depth of the pavement for New Orleans, Louisiana 5 years after construction. 268

Figure E.19: Ultimate shrinkage through the depth of the pavement for Los Angeles, California 5 years after construction. 269

Figure E.20: Ultimate shrinkage through the depth of the pavement for Los Angeles, California 5 years after construction.

Figure E.21: Ultimate shrinkage through the depth of the pavement for Astoria, Oregon 5 years after construction. 270

Figure F.1: Design Aid for a 10 inch thick slab with a w/c ratio of 0.4, ultimate shrinkage of $600 \mu \varepsilon$, a reversible shrinkage factor of 0.5 and a depth of shrinkage zone of 3 inches. 
Figure F.2: Design Aid for a 10 inch thick slab with a w/c ratio of 0.3, ultimate shrinkage of $600 \mu \varepsilon$, a reversible shrinkage factor of 0.5 and a depth of shrinkage zone of 3 inches.

Figure F.3: Design Aid for a 10 inch thick slab with a w/c ratio of 0.5, ultimate shrinkage of $600 \mu \varepsilon$, a reversible shrinkage factor of 0.5 and a depth of shrinkage zone of 3 inches.

Figure F.4: Design Aid for a 10 inch thick slab with a w/c ratio of 0.4, ultimate shrinkage of $600 \mu \varepsilon$, a reversible shrinkage factor of 0.5 and a depth of shrinkage zone of 1 inch.

Figure F.5: Design Aid for a 10 inch thick slab with a w/c ratio of 0.4, ultimate shrinkage of $600 \mu \varepsilon$, a reversible shrinkage factor of 0.5 and a depth of shrinkage zone of 2 inches.

Figure F.6: Design Aid for a 10 inch thick slab with a w/c ratio of 0.4, ultimate shrinkage of $600 \mu \varepsilon$, a reversible shrinkage factor of 0.5 and a depth of shrinkage zone of 4 inches. 276

Figure F.7: Design Aid for an 8 inch thick slab with a w/c ratio of 0.4 , ultimate shrinkage of $600 \mu \varepsilon$, a reversible shrinkage factor of 0.5 and a depth of shrinkage zone of 3 inches.

Figure F.8: Design Aid for a 12 inch thick slab with a w/c ratio of 0.4, ultimate shrinkage of $600 \mu \varepsilon$, a reversible shrinkage factor of 0.5 and a depth of shrinkage zone of 3 inches.

Figure F.9: Design Aid for a 10 inch thick slab with a w/c ratio of 0.4, ultimate shrinkage of $300 \mu \varepsilon$, a reversible shrinkage factor of 0.5 and a depth of shrinkage zone of 3 inches.

Figure F.10: Design Aid for a 10 inch thick slab with a w/c ratio of 0.4, ultimate shrinkage of $1000 \mu \varepsilon$, a reversible shrinkage factor of 0.5 and a depth of shrinkage zone of 3 inches.

Figure F.11: Design Aid for a 10 inch thick slab with a w/c ratio of 0.4, ultimate shrinkage of $600 \mu \varepsilon$, a reversible shrinkage factor of 0.6 and a depth of shrinkage zone of 3 inches.

Figure F.12: Design Aid for a 10 inch thick slab with a w/c ratio of 0.4, ultimate shrinkage of $600 \mu \varepsilon$, a reversible shrinkage factor of 0.3 and a depth of shrinkage zone of 3 inches. 
Figure F.13: Design Aid for a 10 inch thick slab with a w/c ratio of 0.4, ultimate shrinkage of $600 \mu \varepsilon$, a reversible shrinkage factor of 0.3 and a depth of shrinkage zone of 1 inch.

Figure F.14: Design Aid for a 10 inch thick slab with a w/c ratio of 0.4, ultimate shrinkage of $600 \mu \varepsilon$, a reversible shrinkage factor of 0.3 and a depth of shrinkage zone of 2 inches.

Figure F.15: Design Aid for a 10 inch thick slab with a w/c ratio of 0.4, ultimate shrinkage of $600 \mu \varepsilon$, a reversible shrinkage factor of 0.3 and a depth of shrinkage zone of 4 inches.

Figure F.16: Design Aid for a 10 inch thick slab with a w/c ratio of 0.3, ultimate shrinkage of $600 \mu \varepsilon$, a reversible shrinkage factor of 0.3 and a depth of shrinkage zone of 3 inches.

Figure F.17: Design Aid for a 10 inch thick slab with a w/c ratio of 0.5, ultimate shrinkage of $600 \mu \varepsilon$, a reversible shrinkage factor of 0.3 and a depth of shrinkage zone of 3 inches.

Figure F.18: Design Aid for an 8 inch thick slab with a w/c ratio of 0.4 , ultimate shrinkage of $600 \mu \varepsilon$, a reversible shrinkage factor of 0.3 and a depth of shrinkage zone of 3 inches.

Figure F.19: Design Aid for a 12 inch thick slab with a w/c ratio of 0.4, ultimate shrinkage of $600 \mu \varepsilon$, a reversible shrinkage factor of 0.3 and a depth of shrinkage zone of 3 inches.

Figure F.20: Design Aid for a 10 inch thick slab with a w/c ratio of 0.4, ultimate shrinkage of $300 \mu \varepsilon$, a reversible shrinkage factor of 0.3 and a depth of shrinkage zone of 3 inches.

Figure F.21: Design Aid for a 10 inch thick slab with a w/c ratio of 0.4, ultimate shrinkage of $1000 \mu \varepsilon$, a reversible shrinkage factor of 0.3 and a depth of shrinkage zone of 3 inches. 


\section{List of Tables}

Table 3.1 Aggregate properties, data from (Shorkey 2010) ………………………...... 34

Table 3.2 Mix proportions of concretes (in $\mathrm{lbs} / \mathrm{yd}^{3}$ of concrete) .................................... 35

Table 3.3 Plastic and hardened concrete test results (from Shorkey 2010) ..................... 36

Table 3.4 Ultimate and drying shrinkage for different aggregate types .......................... 43

Table 3.5 Calculated reversible shrinkage after 24 hours of re-wetting and percent of total reversible shrinkage which occurs in the first 24 hours of re-wetting.......... 48

Table 4.1 Percent of slabs cracked at 20 years for 10 inch pavements in various locations

Table 4.2 Ambient relative humidity for various locations in the United States.............. 72

Table 4.3 $\mathrm{ETG}_{\mathrm{SHi}}$ for various locations in the United States ........................................ 72

Table 4.4 Correlation coefficients for linear and nonlinear approximations .................... 79

Table 4.5 Area of shrinkage distribution for actual data, linear and nonlinear

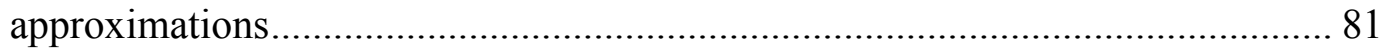

Table 4.6 Ratio of drying shrinkage to ultimate shrinkage for various w/c ratios........... 89

Table 4.7 Correlation coefficient for a bilinear relation between $\omega$ and $w / c$ for various theoretical limits of hydration ....................................................................... 90

Table 4.8 Alternate combined shrinkage and relative humidity model coefficients ........ 94

Table 4.9 Percent difference in equivalent temperature difference with a depth of shrinkage zone determined both empirically and equal to two inches ................ 101

Table 4.10 Variations in input variables used in the generation of design aids.............. 111

Table 5.1 Location of FWD sensors, measured to the right of the applied load ............ 117

Table 5.2 Correlation coefficients between different approximations and actual data... 124

Table 5.3 Built-in curl calculated via the polynomial curvature and $\Delta \mathrm{h}$ methods using full

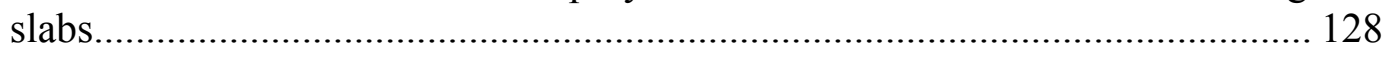

Table 5.4 Built-in curl calculated via the polynomial curvature and $\Delta \mathrm{h}$ methods using

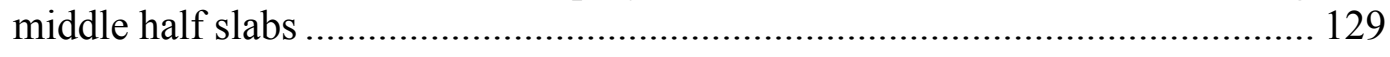

Table 5.5 Built-in curl from the FWD/ANN method - June test.................................. 130 
Table 5.6 Built-in curl from FWD/ANN method - October test ................................ 131

Table 5.7 Built-in curl determined via the minimum error method for full slabs .......... 132

Table 5.8 Built-in curl determined via the minimum error method for half slabs......... 133

Table 5.9 Built-in curl determined via the minimum error method using actual data and ISLAB2000 profiles............................................................................. 134

Table 5.10 Correlation coefficient $\left(\mathrm{R}^{2}\right)$ values for various order polynomials for Cell 72 ,

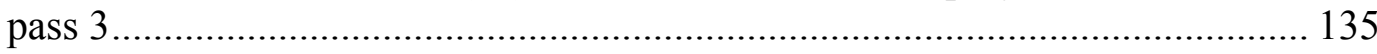

Table 5.11 Correlation coefficents $\left(\mathrm{R}^{2}\right)$ between actual data and polynomial approximations from whole slab analsysis ............................................. 136

Table 5.12 Correlation coefficients $\left(\mathrm{R}^{2}\right)$ between actual data and polynomial approximations from half slab analsysis.

Table 5.13 Sum of squares of errors between actual profile data and best fit polynomial for full slabs 138

Table 5.14 Sum of squares of errors between actual profile data and best fit polynomial for half slabs 139

Table 5.15 Polynomial order for which the sum of the squares of the error was minimized for full slabs 141

Table 5.16 Frequency of a polynomial being the best option 142

Table 5.17 Number of matches between full and half slab analsyes using the polynomial curvature method 143

Table 5.18 Lower bound value of built-in curl 147

Table 5.19 Polynomial order for which the sum of the squares of the error was minimized 148

Table 5.20 Number of matches for built-in curl between full and half slab analsyes using the minimum error method

Table 5.21 Built-in curl calculated using a second order polynomial approximation in both the polynomial curvature and minimum error methods.

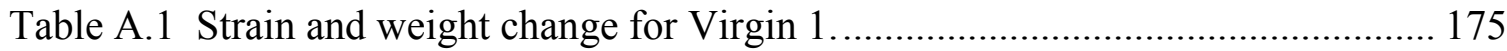

Table A.2 Strain and weight change for Virgin 2 ................................................ 180

Table A.3 Strain and weight change for Virgin 3 …........................................... 185 
Table A.4 Strain and weight change for Slag RCA....................................................... 190

Table A.5 Strain and weight change for Limestone RCA. ............................................. 196

Table A.6 Strain and weight change for Gravel RCA 1 ................................................ 202

Table A.7 Strain and weight change for Gravel RCA 2 .............................................. 207

Table A.8 Strain and weight change for Twice Recycled RCA. ……............................. 212

Table A.9 Strain and weight change for Lightweight 1............................................ 217

Table A.10 Strain and weight change for Lightweight 2............................................ 222

Table A.11 Strain and weight change for Lightweight 3 .............................................. 227

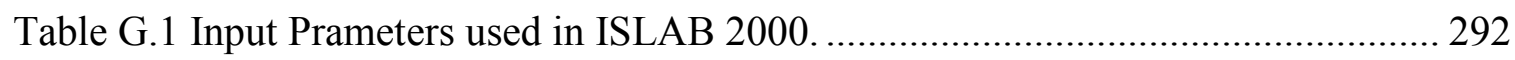




\section{Acknowledgements}

First and foremost, I would like to thank my adviser Jake Hiller for his guidance and support throughout my career at Michigan Tech. I appreciate all of the encouragement, time, and advice he gave me. This research could not have been conducted without the help of several colleagues and friends, who supplied not only ideas, but also time, effort and encouragement. Thanks go to my research mates Cory Shorkey and Yinghong Qin, Mike Yokie and his crew in the lab, Chris DeDene, Khatereh Vaghefi, Jason Flietstra, Kevin Mears, Morgan Hansen, Kyle Andrews, Luis Tomioka, and everyone else who helped measure samples and keep the humidifiers full. Ken Hansen and Chris DeDene generously donated time and expertise in the creation of figures and maps. Portions of the research presented in this thesis were funded by the Michigan and Minnesota Departments of Transportation. Their financial assistance is appreciated.

Finally, I would like to thank my friends and family for their love and support throughout my education. I am especially grateful to my parents and my brother. They have always encouraged me to pursue my interests by providing endless support, inspiration and love. 
xxiv 


\begin{abstract}
When a concrete slab experiences differential volume change due to temperature, moisture, and shrinkage gradients, it deforms. The stresses induced by these differential volume changes can reduce the pavement's fatigue life. Differential volume change is quantified by the equivalent temperature difference required to deform a comparable flat slab to the same shape as the actual slab. This thesis presents models to predict the equivalent temperature difference due to moisture warping and differential drying shrinkage. Moisture warping occurs because a portion of drying shrinkage is reversible, while differential drying shrinkage is due to the irreversible portion of drying shrinkage. The amount of reversible shrinkage was investigated for concretes made with different types of aggregate, including lightweight and recycled. Another source of differential volume change is built-in curl, which is caused by temperature gradients at the time of paving. This thesis also presents a comparison of methods used to quantify built-in curl.
\end{abstract}


xxvi 


\section{Introduction}

In 1963, Reddy et al. described how they would design a pavement, if only they had the tools necessary to conduct a proper analysis. The tool they proceeded to describe would mechanistically compute stresses induced by loading, and would accurately predict the distresses which would develop in the pavement. The Mechanistic-Empirical Pavement Design Guide (MEPDG) is an attempt to solve the problems in pavement design described years previously. The MEPDG mechanistically calculates stresses based on estimated traffic and environmental loading and correlates those stresses empirically to pavement damage. The accuracy of the MEPDG's predictions is dependent in part on the internal algorithms and models, and in part on the material property inputs. Improving either of these components will increase the overall accuracy of the MEPDG.

The MEPDG is an improvement over previous design methods because it attempts to account for the effects of differential volume change. Differential volume change occurs when a pavement is subjected to temperature, moisture, and shrinkage gradients, which induce deformations in a slab. These deformations can be large enough to cause the slab to separate from its underlying layers, which changes both support conditions of the slab, and how the slab responds to applied loads. If there is sufficient restraint to prevent the slab from deforming, stresses will be induced. Neglecting differential volume change can cause a slab to be under-designed or result in premature failure.

\subsection{Project Goals}

The goal of this research was to improve the ability of a design engineer to account for the effects of differential volume change when using the MEPDG in the design of jointed plain concrete pavements. The MEPDG uses two terms to account for differential volume change when computing stresses in a concrete pavement. The equivalent temperature difference (ETG) term uses climatic data and models to compute the effects of curling and warping, while the built-in curl (BIC) term is a user input which accounts for construction curl, differential drying shrinkage and creep. In order to improve the design of pavements which use the ETG and BIC terms, modifications to both terms were investigated. The MEPDG is set up as a compartmentalized system, where each model can be treated as a separate entity. Therefore, it is relatively simple to remove an existing model and replace it with an improved model.

Within the ETG term, there are two different models, one each for temperature and moisture gradients. These models compute equivalent temperature differences based on climatic data. One of the objectives of this research was to create a new model to predict the amount of moisture warping expected in a pavement based on the ambient relative humidity conditions. The goal of this new model was to predict warping more accurately than the current model, without requiring any new user inputs. To improve accuracy, the new model will be based on a non-linear moisture distribution through the thickness of the slab, and have assumptions which would accurately reflect the behavior of the slab in bending. 
Moisture warping occurs because a portion of the shrinkage in the concrete is reversible. One parameter required to compute moisture warping the amount of shrinkage expected to be reversible. The MEPDG recommends that $50 \%$ of the total shrinkage is assumed to be reversible in all instances. It is known that many different factors influence the amount of reversible shrinkage in concrete, and that reversible shrinkage is not always equal to half of the total shrinkage. Therefore, another objective of this study was to research the effects of material properties of the aggregate and concrete on reversible shrinkage and determine how much reversible shrinkage can be expected in concrete made with different types of aggregates, including lightweight and recycled concrete aggregates. By providing guidance to a pavement engineer about the amount of reversible shrinkage to be expected, especially with non-standard materials, the warping prediction will be improved. Design aids were developed to show typical values for warping and differential drying shrinkage throughout the United States.

Unlike the curling and warping terms in the MEPDG, which can be accounted for through the use of models and data, the MEPDG term which accounts for the effects of construction curl, differential drying shrinkage and creep, collectively known as built-in curl, is be defined entirely by the user. Currently, the default value that is recommended for the amount of built-in curl is $-10^{\circ} \mathrm{F}$. It has been shown that using $-10^{\circ} \mathrm{F}$ as the value of built-in curl is an unconservative assumption and will result in an under-designed slab. Rather than attempting to account for built-in curl as a single value, each of the components of built-in curl could be modeled separately and the effects summed. The built-in curl term in the MEPDG is meant to account for the effects of construction curl, differential drying shrinkage, and creep. Currently, there are not any models available to predict the amount of construction curl because it is highly dependent on the weather at the time of paving. There are also currently no models which will predict the differential drying shrinkage. Creep models do exist. One of the goals of this study was to develop a model for the amount of differential drying shrinkage to be expected. Because differential drying shrinkage and moisture warping are caused by the same mechanisms, the warping model developed for this study could be easily adapted to predict differential drying shrinkage.

Creating a new model to predict the amount of construction curl is beyond the scope of this investigation. Should a model be developed to predict construction curl, it would be necessary to devise a technique whereby the amount of built-in curl in a slab can be quantified. Subtracting the known values for differential drying shrinkage and creep components from the value of built-in curl would yield the construction curl component. This technique would make it possible to experimentally validate a construction curl model. Another objective of this study is to evaluate different techniques for quantifying built-in curl. Because no algorithms currently exist for the prediction of built-in curl, a design engineer must rely on information from previously cast slabs of similar geometry and materials in the same area. Until algorithms are developed to predict built-in curl, knowledge of how much built-in curl is typically found in a certain area could help a designer make a better assumption than the current value of $-10^{\circ} \mathrm{F}$ for the amount of overall built-in curl expected in a jointed plain concrete pavement. By determining 
which methods of quantifying built-in curl work best, an engineer will be able to better determine the amount of built-in curl in slabs which have already been cast.

\subsection{Content}

Chapter 2 is a literature review of pertinent background information. An experimental study of reversible shrinkage in concrete made from virgin, lightweight, and recycled concrete aggregates is contained in Chapter 3. This study investigates the behavior of concrete exposed to cyclic drying and wetting cycles, as well as the relationship between reversible shrinkage, concrete permeability and aggregate porosity. Chapter 4 presents new models to predict the amount of moisture warping and differential drying shrinkage expected based on ambient relative humidity. One input for these models is the amount of reversible shrinkage expected, as determined in Chapter 3. Chapter 5 describes and compares various techniques for quantifying the amount of built-in curl in a pavement. Finally, Chapter 6 offers concluding remarks and posits ideas for future research. 


\section{Literature Review}

A concrete slab subjected to a change in either temperature or moisture content experiences a change in volume. Increases in temperature or moisture are associated with expansion of the concrete, while decreases in either of these factors will result in contraction. If the specimen is restrained, these volumetric changes are limited, and stresses are induced. In a concrete pavement, these volumetric changes do not occur uniformly, but instead, are generally more prevalent on the surface of the pavement, which is exposed to the environment. The bottom of the pavement is protected from fluctuations in temperature and moisture by the insulation of the concrete layer, and therefore tends to experience less change in volume. Due to the lack of uniformity in the volume change of the concrete, there is differential expansion or contraction through the thickness of the slab.

When the top of the slab is warmer or wetter than the bottom (a positive gradient), the top of the slab expands, inducing a concave downward deflected shape. In some cases, this deflection is extreme enough to lift the middle of the slab off of the underlying layers, creating a gap under the middle of the slab (Shoukry 2000). Alternately, if the top of the slab is cooler or dryer than the bottom (a negative gradient), the slab will assume a concave upwards shape, sometimes lifting the corners off of the underlying layers (Hveem 1951). Figure 2.1 and Figure 2.2 show an exaggerated slab deformed due to temperature and moisture gradients, respectively, along with the induced stress states caused by the restraint of self-weight. If no temperature or moisture gradient exists, the slab, in theory, should be perfectly flat and in complete contact with the underlying layer.

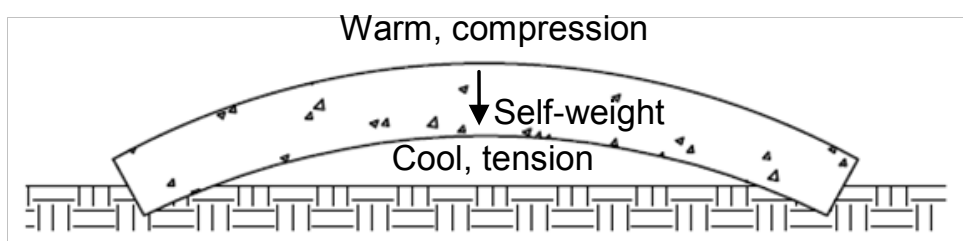

(b)

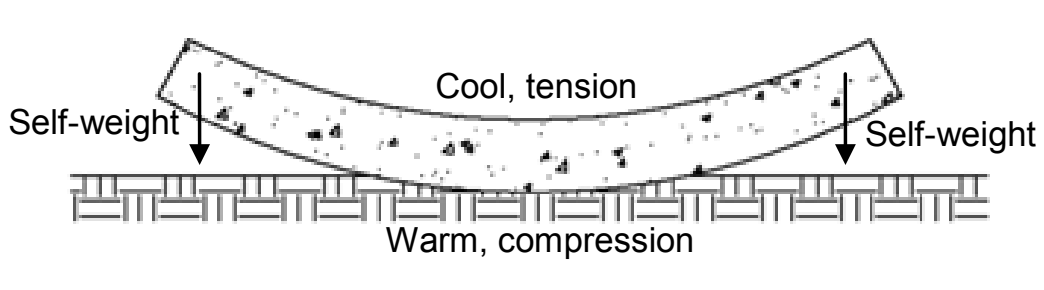

(a)

Figure 2.1: A slab curled due to a temperature gradient a) upwards and b) downwards, and associated stress state. 


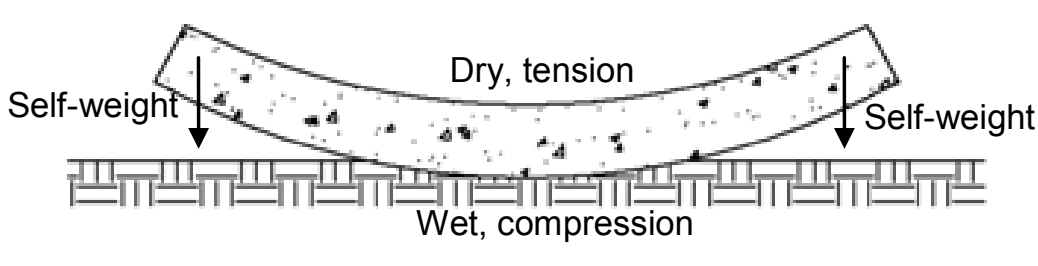

(a)

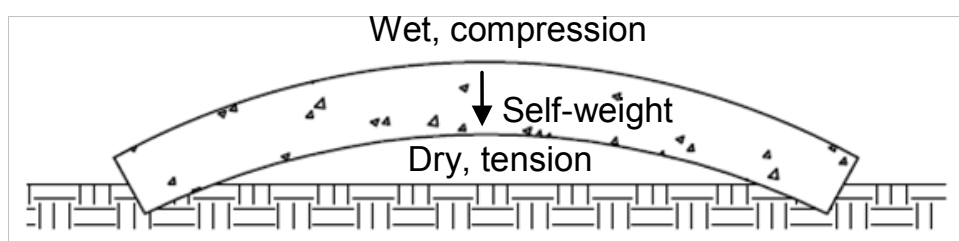

(b)

Figure 2.2: A slab warped due to a moisture gradient a) upwards and b) downwards, and associated stress state.

When differential expansion or contraction is due to a temperature gradient, it is called curling, while the same phenomenon due to a moisture gradient is called warping. Generally, a slab experiences both curling and warping simultaneously, and, as their effects cannot be measured separately in the field, these factors are grouped together.

The stresses induced in a slab by differential volume change have the opposite sign convention of what is generally intuitive. Applying a positive gradient causes the top of the slab to expand and the bottom to contract. The self-weight of the slab acts against this deformation, inducing compression in the top of the slab and tension in the bottom. Conversely, a negative gradient causes the top of the slab to be in tension while the bottom is in compression.

\subsection{Equivalent Temperature Difference}

The effects of differential volume change are quantified by the equivalent linear temperature difference that would be required to deform an identical, but theoretically flat slab to the same shape as the curled/warped slab. The equivalent temperature difference is often divided into five constituent components, which are considered separately in analysis, but often cannot be determined individually in field measurements. This equivalent temperature difference is also called the total effective linear temperature difference or $\Delta \mathrm{T}$, and is given by Equation 2.1 (Rao and Roesler 2005a).

$$
\Delta T=\Delta T_{t g}+\Delta T_{m g}+\Delta T_{b i}+\Delta T_{s h r}-\Delta T_{c r p}
$$

Equation 2.1

where:

$\Delta T_{t g}=$ the linear temperature difference between the top and bottom of the slab required to produce the same deformed shape as that induced 
by the actual, nonlinear temperature difference in the slab

$\Delta T_{m g}=$ the linear temperature difference between the top and bottom of the slab required to produce the same deformed shape as that induced by the actual, nonlinear moisture difference in the slab

$\Delta T_{b i}=$ the linear temperature difference between the top and bottom of the slab required to produce the same deformed shape as that induced by curl/warp which is built-in to the slab

$\Delta T_{s h r}=$ the linear temperature difference between the top and bottom of the slab required to produce the same deformed shape as that induced by differential drying shrinkage in the slab

$\Delta T_{c r p}=$ the linear temperature difference between the top and bottom of the slab required to produce the same deformed shape as that induced by the creep of the slab.

While an attempt can be made to separate the behavior of the slab into these components, and indeed, this is quite necessary for calculations, in reality, all of these effects overlap and influence each other.

\subsubsection{Actual Temperature Difference}

The top of the slab is exposed to the environment, and consequently, it experiences more frequent changes in temperature than the bottom of the slab. This causes a temperature gradient to develop through the thickness of the slab. During the day, the slab is often warmer on the surface, and cooler on the bottom, while at night, the reverse is true, leading to a slab profile which is curled downwards during the day and upwards at night. Many studies have found that the temperature gradient of a slab does not vary linearly with depth, but is instead non-linear (Teller and Sutherland 1935; Armaghani et al. 1987; Poblete et al. 1988; Choubane and Tia 1995; Beckemeyer et al. 2002). Additionally, it has been proven on numerous occasions that the assumption of a linear temperature gradient in design is unconservative (Reddy et al. 1963; Choubane and Tia 1995; Mohamed and Hansen 1997; Rao et al. 2001; Siddique et al. 2006).

Generally, the quadratic equation is used to model variation of temperature gradients through the depth of the slab because it matches the actual profile fairly well and is computationally simple (Richardson and Armaghani 1987; Choubane and Tia 1992; 1995). It has been shown that a non-linear temperature gradient will cause stresses to develop to achieve equilibrium in the slab, even if it is unrestrained, (Timoshenko and Goodier 1951). Stresses induced by temperature gradients are generally divided into three components: uniform, linear, and nonlinear self-equilibrating (Bradbury 1938; Thomlinson 1940), which are shown in Figure 2.3 


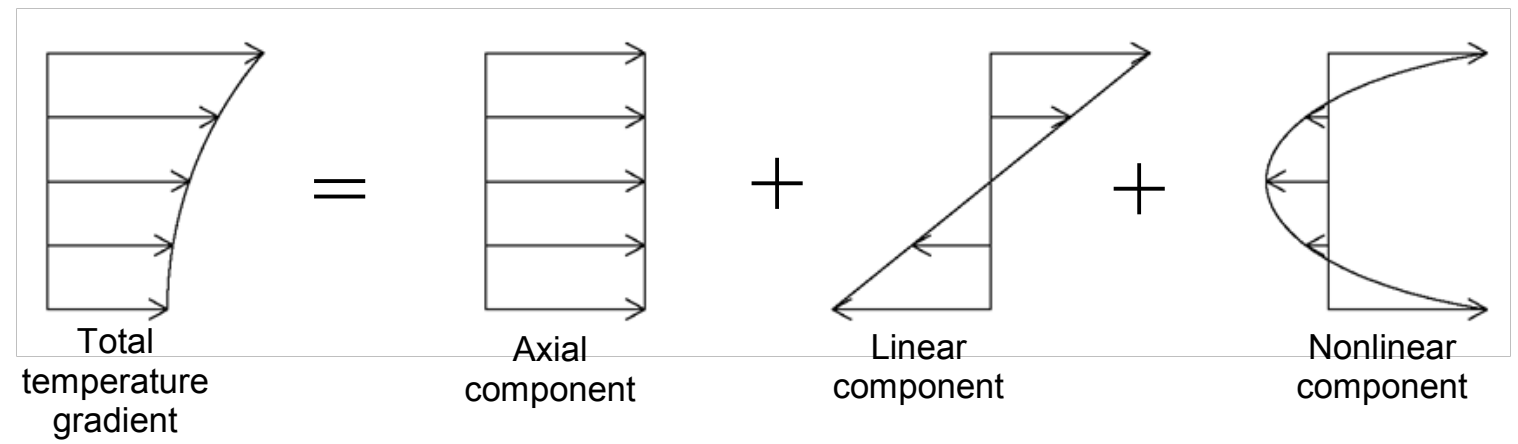

Figure 2.3: Total temperature gradient and constituent components.

The axial component is due to the uniform temperature distribution and causes a uniform stress distribution through the slab. In classic plate theory, the assumption that plane sections remain plane means that a uniform stress distribution will not cause any bending moment in the slab at all, though axial stresses will still be induced. It has been shown that the assumption of plane sections remaining plane is true for most pavements (Pane et al. 1998). The axial component is generally small and is typically ignored in computation for pavements with fully developed strength. For freshly cast slabs which have not yet gained the majority of their strength, the stresses induced by axial deformation can be of the same magnitude as the tensile strength of the young concrete, and therefore are of greater concern.

The linear component of the temperature gradient is responsible for all bending deformation in the slab, as well as the majority of the induced stresses. When the slab is exposed to a temperature gradient, the warmer side expands, while the cooler side contracts. Since all of the bending in the slab is due to the linear component, this component must produce the same bending moment as the equivalent linear temperature difference.

Summing the effects of the uniform and linear components of the actual temperature gradient yields a linear gradient; however, the actual gradient is nonlinear. The difference between the two gradients is the nonlinear or self-equilibrating stress. This stress balances out, or equilibrates, the thermal stress profile in the slab such that there is zero total force acting on any single part of the slab. No deformations are induced by the nonlinear component because of the assumption that plane sections remain plane; however, it does introduce additional stresses (Choubane and Tia 1995; Ioannides and Khazanovich 1998).

If there are no major deformations present in the slab due to other effects, such as built-in curl or differential drying shrinkage, then neglecting the self-equilibrating stresses will result in under-predicting the fatigue life of the pavement and overdesigning the slab. If, however, there are substantial deformations due to other sources, neglecting the selfequilibrating stresses will over-predict the fatigue life of the pavement, which is unconservative (Hiller and Roesler 2010). 


\subsubsection{Differential Drying Shrinkage}

Drying shrinkage is caused by loss of water from the concrete matrix, particularly from the calcium silicate hydrate (C-S-H) gel pore and smaller capillary pores in the concrete's void system. Water loss is generally due to the evaporation of water from the concrete when the ambient relative humidity $(\mathrm{RH})$ is less than the moisture content of the concrete. This can be caused by several factors, and the associated shrinkage may or may not be reversible. The reversible portion of drying shrinkage causes moisture warping. The irreversible portion is considered as differential drying shrinkage, and is due to permanent drying shrinkage (Neville 1997). The components of shrinkage are shown in Figure 2.4.

Because most of the moisture movement in the slab occurs within the top few inches (Janssen 1987), the vast majority of drying shrinkage also occurs in this region, leading to differential volume changes in the slab (Wells et al. 2006b), which, in turn, causes a warped shape. One study found that the rate at which drying shrinkage occurs is proportional to the rate at which the slab warps (Bissonnette et al. 2007). The $\Delta T_{s h r}$ term in Equation 2.1 only accounts for the permanent component of this differential drying shrinkage. The reversible component of drying shrinkage is accounted for in the moisture warping $\Delta T_{m g}$ term.

There is an additional portion of permanent shrinkage called autogenous shrinkage, which does not contribute to differential volume change. Autogenous shrinkage is the self-desiccating effect of the hydration process due to the withdrawal of water from the capillary pores in the paste matrix. Essentially, the volume of the hydrated paste is less than the sum of the volume of the water and the cement. The majority of autogenous shrinkage is generally fairly uniform throughout the slab and occurs during the initial hydration process (Neville 1997). In fact, most of the initial shrinkage a sample will experience occurs in the first 24 hours (L'Hermite 1947; Helmuth and Turk 1967). Due to its uniformity, autogenous does not contribute to deformation in the slab.

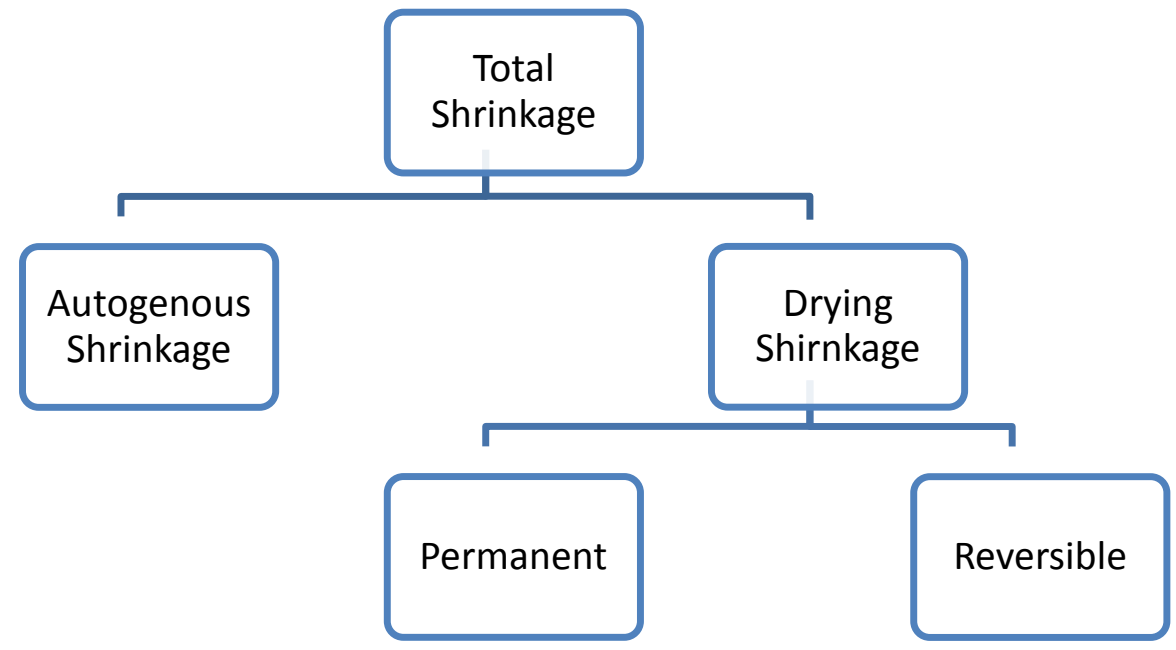

Figure 2.4: The components of shrinkage. 


\subsubsection{Moisture Gradient}

Moisture variation through the depth of the slab causes warping because a portion of the shrinkage in the concrete is reversible. Janssen (1987) found that the majority of moisture loss in concrete pavements occurs in the top two inches of the pavement, while the remaining thickness of the slab is at least $80 \%$ saturated, regardless of ambient conditions, and the bottom of the slab is generally fully saturated. Within the top few inches, the moisture gradient is non-linear. Using a coupled temperature and moisture model to compute the moisture gradients through the thickness of the pavement around the country however, shows that the depth of the shrinkage zone is more likely to be between one and four inches, depending on the location (Qin 2011).

Unlike temperature gradients, which generally follow a set pattern of diurnal variation, moisture gradients are quite variable, depending on the ambient relative humidity and rain events. For this reason, moisture gradients are generally considered on a monthly basis (NCHRP 2003). When the top surface of the pavement is dry, the concrete shrinks, causing the slab to warp upwards. When the top surface becomes saturated, the shrinkage is at least partially reversed, which reverses the warping.

The amount of shrinkage which is reversible depends on the properties of the concrete and the degree and duration of wetting. This phenomenon has been noted by some researchers (L'Hermite 1947; L'Hermite et al. 1949; Shacklock and Keene 1957; Helmuth and Turk 1967; Granger et al. 1994), but it is still not well known or understood, and no prediction equation for the amount of shrinkage which will be reversible currently exists. The majority of the studies on reversible shrinkage were conducted in Europe between 1940 and 1975, and did not necessarily use conventional or repeatable experimental practices. For example, L'Hermite prevented moisture loss in his samples by storing them in vats of mercury. Through this and other more conventional experiments, he found that $40-70 \%$ of the total shrinkage in a concrete is reversible (L'Hermite 1960); however, for concrete stored in water for an extended period of time before being exposed to cyclic drying and re-wetting, shrinkage was found to be fully reversible (L'Hermite et al. 1949).

Neville (1997) hypothesizes that reversible shrinkage exists because C-S-H gels form bonds when they are in close proximity during a drying phase. When the concrete is again exposed to moisture, it swells, but these bonds hold it together, preventing shrinkage from being fully reversible. This theory fits with the behavior observed by others (L'Hermite 1947; Helmuth and Turk 1967), but has not been conclusively proven.

It is worth noting that very little information is readily available on the topic of reversible shrinkage in concrete. Within the pavements community, the most widely used information on reversible shrinkage is a highly idealized curve taken from Mindness and Young, or the later edition, by Mindness, Young, and Darwin (2003), which shows that $50 \%$ of all shrinkage is reversible, but does not actually state a numeric value for the amount of reversible shrinkage. This is significant because a value of $50 \%$ reversible shrinkage is often assumed in calculations (NCHRP 2003), and may not be correct in all 
cases (L'Hermite et al. 1949). However, due to a lack of information, most designers are likely unaware that such considerations even exist.

Proper estimation of the amount of moisture warping in a pavement is important because the equivalent temperature difference due to moisture warping is often of the same magnitude as that due to temperature curling (Hveem and Bailey 1957), and it has been found that neglecting the effects of moisture warping is unconservative (Rao et al. 2001). No work has yet examined the reversible shrinkage behavior of concrete made with light weight aggregates or recycled concrete aggregates. As quality virgin aggregates become more expensive and less available, recycled concrete aggregates are likely to become more prevalent in pavements.

\subsubsection{Built-in Curl}

Built-in curl is also called construction curl, and is due to the presence of a temperature gradient at the time of initial set of the concrete. When a slab is cast with a temperature gradient, it cannot deform as it usually would because the concrete is plastic. Instead, it sets as a flat slab. If the temperature gradient differs from that at the time of casting, the slab reacts by deforming. Therefore, when the slab is of uniform temperature, it behaves as though there is a temperature gradient present of the same magnitude as the gradient during the final set of the concrete. Similarly; the slab will only be flat when the temperature gradient is the same as it was when the slab was cast (disregarding all other differential volume change factors) (Yu and Khazanovich 2001). Because most slabs are cast during the day (in the presence of a positive temperature gradient), at a zero temperature gradient condition, they behave as though the positive gradient has been removed (i.e. as though a negative gradient has been applied). This positive temperature gradient at the time of set is exacerbated by heat produced internally in the concrete as part of the chemical reactions associated with hydration.

The concept of built-in curl due to construction conditions was first presented by Eisenmann and Leykauf, when they observed that slabs were not flat when there was no temperature gradient present, but instead were curled upwards (1990a). Poblete et al. (1988) actually noted the phenomenon of built-in curl earlier, but attributed the permanent deformations in their slabs to moisture warping and differential drying shrinkage. However, they accurately described the effects built-in curl can have on early age cracking and reduced fatigue life.

Many researchers have found pavements with negative built-in curl (for example Armaghani et al. 1987; Poblete et al. 1988; Guo 2001; Rao and Roesler 2005b). For a slab with a negative built-in curl, a very high positive temperature would be needed to not only counter the negative built-in curl, but to then further curl the slab downwards (Poblete et al. 1991). Because this does not happen frequently, slabs are generally curled upwards, regardless of the ambient temperature.

There is some confusion regarding the use of the term built-in curl to characterize the initial shape of the slab after the concrete is set. The equivalent temperature gradient required to deform a theoretical slab to the same shape as the actual slab at the time of set 
is called the built-in curl. However, there are other mechanisms causing the slab to have a deformed shape at the time of set, such as differential drying shrinkage. Because these mechanisms cannot be separated, their effects are often counted as built-in curl. For the sake of simplicity, in this document, construction curl will refer to the curl set into the slab at the time of set of the concrete due solely to a temperature differential at the time of set. Built-in curl will refer to the total permanent curl in the slab at the time of set.

\subsubsection{Creep}

Creep is defined as deformation due to sustained loading; in the case of a concrete slab, the load is the weight of the slab itself. Curl, warp, shrinkage and built-in curl all induce deformations, which are countered by the self-weight of the slab. Regardless of how the other components cause the slab to deform, creep will always work against those deformations, which is why it is subtracted from all of the other components of the equivalent temperature difference. Studies on the influence of creep on the other factors of differential volume change have found that creep counteracts shrinkage (Altoubat and Lange 2001), curling (Teller and Sutherland 1935; Rao et al. 2001; Sondag and Snyder 2003), and warping (Teller and Sutherland 1935; Bissonnette et al. 2007). Reduced deformations due to creep also cause stress relaxation, which can increase the strain capacity of the concrete (Altoubat and Lange 2001). One study found that if creep was neglected in estimating the total deformations induced by curl, warp, shrinkage and builtin curl, then edge displacements would be almost double those expected if creep were considered. Likewise, stresses computed without considering the stress relaxation effects of creep were three times higher than when creep was included in calculations (Lee et al. 2011).

One important component to creep that must be considered is time. Creep does not occur instantaneously, but is instead due to sustained loading over time. When considering curl due to daily fluctuations in temperature, there is generally not sufficient time for creep to take effect, and therefore, it can generally be neglected. However, effects due to moisture vary seasonally, which allows for creep to counteract moisture induced deformations. In this case, creep must be considered in computations (Lee et al. 2011). The effects of creep are much more prominent early in the life of the pavement.

\subsection{Causes of Differential Volume Change}

Differential volume change in concrete slabs is influenced by many different factors, some of which can be controlled and some of which cannot. The single largest influence on the amount of differential volume change in a slab is the ambient conditions to which the slab surface is exposed. The pavement material properties, slab geometry, weather conditions during paving, and curing methods all play a role in determining how a slab will react to temperature and moisture gradients, as well as the differential drying shrinkage and built-in curl with which the slab will set.

\subsubsection{Ambient Conditions}

Ambient conditions not only play the largest role in determining the amount of differential volume change experienced by a pavement, but they are also the factor which can be least controlled. 


\subsubsection{Ambient Conditions During Service}

Ambient conditions during service affect the amount of temperature curling and moisture warping in a pavement. The obvious example of this is that the ambient temperature and relative humidity cause temperature and moisture gradients, respectively, through the thickness of the pavement. However, other aspects of the weather also play a role in determining temperature and moisture gradients.

The temperature gradient through the thickness of the slab depends on weather factors such as solar radiation, wind, and rain, in addition to the actual temperature. When there is a large amount of solar radiation, the pavement absorbs that energy and gets much warmer at the surface than the actual air temperature. One study found that the temperature of the top of the pavement on a sunny day can actually be $15-25^{\circ} \mathrm{F}$ hotter than the ambient air temperature. However, this affect is concentrated at the surface, as the sun's rays cannot penetrate the concrete easily, which induces a gradient. Additionally, wind and rain help to cool the slab, reducing the effects of ambient temperature. In fact, these factors can contribute much more to the temperature gradient in the slab than the actual ambient temperature (Armaghani et al. 1987).

Moisture gradients are also affected by factors other than just the ambient relative humidity. The top surface of a pavement exposed to solar radiation will lose water faster to evaporation than a slab which is shaded. Likewise, more evaporation will occur in a slab on a windy day than on a calm one.

One interesting case which illustrated the effects of wind and solar radiation as well as site conditions is presented by Hveem (1951). He observed a pavement in California which had a substantial upward curl and warp for most of its length. The warp was due to the fact that the soil under the pavement was saturated, while the curl was due to temperature gradients. There was one section however, where the slabs were much flatter than the rest. This section was adjacent to a grove of trees which were planted close together to serve as a windbreak. The trees took up much of the soil moisture, such that the moisture content of the base beneath the pavement adjacent to the trees was only $53 \%$, compared to $98 \%$ away from the trees. Additionally, the trees also blocked the wind and provided some amount of shade for the pavement. Not only was this section much smoother, but it also had markedly less cracking, even though it was exposed to the same ambient temperature and relative humidity as the other sections of the roadway.

\subsubsection{Paving Conditions}

Ambient conditions during paving influence differential volume change differently than those during service because young concrete has different properties than fully cured concrete. During paving, concrete is plastic and cannot deform in response to environmental loading. Instead, conditions during paving factor into the built-in curl which sets into the slab, and into the amount of differential drying shrinkage which causes permanent warp.

Paving on a warm, sunny day causes more construction curl because both warm temperatures and solar radiation cause the top of the slab to heat up much more than it 
would on a cool or cloudy day, causing a larger temperature gradient at the time of set (Eisenmann and Leykauf 1990a; Rao et al. 2001; Bendana et al. 2003). Because pavements are generally cast in the morning, the base tends to be cool and moist. As the slab sets, the ambient temperature increases and the sun rises higher in the sky, increasing the effects of solar radiation. This effect is more pronounced in the summer because the days are hotter and the sun shines brighter. Paving in the fall instead of in the summer decreases the amount of construction curl in the slab because these effects are lessened (Hansen et al. 2006). Hot, windy days also cause surface moisture to evaporate faster, leading to larger drying shrinkage gradients (Eisenmann and Leykauf 1990a). This is exacerbated by paving in areas with a low ambient humidity because the rate of evaporation of surface moisture is increased (Heath et al. 2001).

\subsubsection{Concrete Material Properties}

Properties of the concrete itself, as well as those of its constituent ingredients affect how a slab will respond to differential volume change. These factors are some of the few parameters over which the design engineer is able to exercise some measure of control. Generally, the constituent materials in the concrete affect the shrinkage behavior of the mix, which in turn affects the differential drying shrinkage and moisture warping. However, certain aggregate properties affect the coefficient of thermal expansion, which is directly proportional to the volume change of the concrete in response to a temperature change.

\subsubsection{Cement Paste}

Paste is the source of shrinkage in the concrete, as aggregates generally do not undergo volume change. Concrete mixes with a higher ratio of paste to aggregates will experience more shrinkage because more of the material is able to shrink (Wei et al. 2011). The permanent portion of drying shrinkage contributes to the differential drying shrinkage, while the reversible portion causes moisture warping.

The cement content and the water to cement (w/c) ratio of the paste also affect the amount of shrinkage. Higher cement content (like that associated with high strength concrete) and lower water to cement ratio leads to more self-desiccation/autogenous shrinkage because more cement granules require hydration. However, this selfdesiccation occurs uniformly, and does not contribute to warping. Therefore, though lower water to cement ratios cause more overall shrinkage, less of that shrinkage causes warping (Kim and Lee 1999). Concrete with a higher water to cement ratio has more evaporable water and therefore is more prone to drying shrinkage. For mixes with the same water to cement ratio, a higher overall cement content will result in increased shrinkage because there is more paste in the same volume of concrete (Neville 1997).

The type of cementitious materials used in the mix also affects the amount of shrinkage experienced by the concrete. Use of pozzolans, such as fly ash and slag, reduce the swelling of the concrete when it is exposed to high relative humidity or to wetting (Wei et al. 2011). However, expansion and contraction due to wetting and drying cycles is not affected by the pozzolan mix proportions (Shacklock and Keene 1957). For an ordinary Portland cement, both tricalcium aluminate $\left(\mathrm{C}_{3} \mathrm{~A}\right)$ and gypsum have been found to 
influence the amount of shrinkage. Higher levels of $\mathrm{C}_{3} \mathrm{~A}$, which are correlated to higher levels of sulfoaluminates, lead to increased shrinkage, while either an excess or a dearth of gypsum will also cause higher shrinkage (Mindness et al. 2003).

The degree of hydration of the paste matrix affects the amount of shrinkage, and the behavior of the concrete upon exposure to cyclic wetting and drying. Pastes which are more fully hydrated initially are less susceptible to volume change with subsequent changes in relative humidity (Neville 1997).

\subsubsection{Aggregates}

The aggregates in a concrete mix do not shrink; instead they influence shrinkage in by acting as a restraint mechanism (Pickett 1956). The exceptions to this are certain shrinking and argillaceous aggregates. Clay undergoes drying shrinkage, and inclusion of aggregates containing clay will cause more drying shrinkage in the concrete (Neville 1997). Porous aggregates, such as light weight aggregates or sandstone, do not themselves shrink, but they increase the diffusivity of the concrete, which leads to increased overall shrinkage (Kosmatka et al. 2002; Shorkey 2010).

The amount of restraint against shrinkage an aggregate can provide is dependent on its strength and compressibility. Harder aggregates, such as granite and quartz, provide more restraint and produce concrete with less shrinkage (Kosmatka et al. 2002). The strength and hardness of the aggregates also influences the modulus of elasticity and the strength of the concrete. Higher strength concrete generally experiences more curling than lower strength concrete (Bissonnette et al. 2007). Concrete with a lower modulus of elasticity, due to either lower strength or lightweight aggregates, tends to also have higher shrinkage, due to less restraint (Mindness et al. 2003).

Recycled concrete aggregate (RCA) is made by crushing old concrete, and has different properties than virgin aggregates. RCA has two constituent materials: the original aggregate and any adhered mortar from the original concrete. Concrete made with RCA has a higher porosity than concrete made with virgin aggregates, due to the fact that RCA is more porous than virgin aggregate and that concrete made with RCA has a higher natural air content. The porosity of new paste has also been found to increase with the use of RCA. Examining the components of RCA, one study found that both the adhered mortar and the original aggregate were more porous than virgin aggregates. The increased porosity in the original aggregate was attributed to stress in the aggregate during both use and crushing (Etxeberria et al. 2006). In addition to increased porosity, concrete made with RCA also has increased creep and shrinkage compared with virgin concrete. One study found that concrete made with RCA experiences up to $70 \%$ more shrinkage and 50\% more creep than concrete made with virgin aggregates. Of that additional shrinkage, almost all is drying shrinkage, which is attributed to the increased porosity of the RCA concrete (Domingo et al. 2010). It was found in one study that concrete containing RCA has 1.3-2.5 times more drying shrinkage than concrete made with virgin aggregate. The use of RCA also influences the rate at which shrinkage strain develops in the concrete, again, due to the increased porosity (Sato et al. 2007). 
The quantity and gradation of aggregate in the concrete affect the amount of shrinkage. Concrete mixes with a higher ratio of aggregate to paste tend to have less shrinkage because of the lower amount of cement in the mix (Neville 1997). Concrete made of aggregate without a good gradation, such as a mix lacking mid-sized aggregates or with high fines content, has higher shrinkage than concrete made from well graded aggregates (Kosmatka et al. 2002).

Aggregates play a role in determining the coefficient of thermal expansion of the concrete. The coefficient of thermal expansion of a material determines how much it will expand or contract when exposed to temperature changes, and is a direct input in calculating the stresses induced in a slab due to a thermal gradient. The coefficient of thermal expansion is also used to determine the equivalent temperature difference due to moisture warping. Cement type plays a fairly small role in determining the coefficient of thermal expansion of the concrete (Kosmatka et al. 2002). The type of aggregate, however, greatly affects the value of the coefficient of thermal expansion. For example, dolomite and gravel produce a concrete with a much larger coefficient of thermal expansion than limestone aggregates (Jahangirnejad et al. 2009). This is important because aggregate type varies significantly by location.

\subsubsection{Pavement Design}

The geometry of the pavement, the constituent material of the underlying layers and the restraint mechanisms affect the amount of differential volume change in the pavement, as well as the magnitude of the stresses induced.

\subsubsection{Slab Geometry}

Deformations induced in a slab by differential volume change are countered by the weight of the slab, which is directly proportional to its size. If the self-weight provides sufficient restraint to prevent deformation, stresses are induced. Thicker and wider slabs experience less deformation due to differential volume change because they have more self-weight to act as a restraint. However, they also have higher induced stresses than a thinner slab would, due to the additional restraint (Bradbury 1938; Sondag and Snyder 2003; Jeong and Zollinger 2004). Thinner slabs are more sensitive to variations in air temperature, but thicker slabs have larger actual temperature differences (Teller and Sutherland 1935). Thinner slabs are also more sensitive to variations in built-in curl. For example, an eight inch pavement with a built-in curl of $-10^{\circ} \mathrm{F}$ was found to have $20 \%$ cracked panels at 20 years, and increasing the built-in curl to $-20^{\circ} \mathrm{F}$ resulted in over $90 \%$ cracked panels at 20 years. Increasing the slab thickness to 12 inches resulted in almost no panel cracked at 20 years for either value of built-in curl (Lothschutz 2009).

Slab length affects curl in much the same way as slab width, longer slabs have less deformation due to curling, but more induced stress (Beckemeyer et al. 2002). The main difference is that slab width is fairly standard, both on an individual stretch of road, and between pavements in general, while slab length is quite variable. Not only do some pavements have variable joint spacing, but cracks act as de facto joints. A slab cracked in half will curl and warp as two independent, adjacent slabs, each with an effective length 
equal to half the length of the original, uncracked slab. This will decrease the restraint from self-weight, and therefore the induced stresses in each slab.

One parameter not affected by the slab geometry is the total amount of shrinkage experienced by a slab. The ultimate shrinkage is dependent upon the constituent materials of the concrete and the drying/curing regime. Slab geometry does affect the time required for the concrete to reach its ultimate shrinkage (Muller and Hilsdorf 1990) because the rate of water loss in concrete is nonlinear (Bazant and Najjar 1972). Due to this, thicker slabs have a larger differential drying shrinkage gradient, and therefore are more prone to shrinkage cracking (L'Hermite and Grieu 1952).

\subsubsection{Base Stiffness}

The stiffness of the underlying layers is very important to the design of a successful pavement. Generally, stiffer subgrades are preferred because they will better support the slab during traffic loading. However, the exact opposite is true for environmental loading. Stresses due to differential volume change are induced when the slab cannot freely deform. Having a softer base allows the slab to "push in" to the base and deform, reducing the amount of stress induced and ultimately the amount of cracking (Reddy et al. 1963; Eisenmann and Leykauf 1990a). For the case of traffic loading on a curled or warped slab, it is best to have a stiffer base, which will reduce the stress due to traffic loading, even though the stresses due to the curl will increase (Dere et al. 2006). A stiffened base, such as an asphalt treated base, will prevent deflections, but also induce more stress in the slab than an ordinary granular base (Beckemeyer et al. 2002)

\subsubsection{Restraints}

When a slab is restrained in any manner, deformations are limited or prevented completely, which causes stress in the slab. It is generally desirable to prevent deformations in pavements because they increase faulting and decrease ride quality. Restraints also prevent the slab from lifting off of the underlying layers and loosing subgrade support. However, if the slab cannot deform freely, stresses will be induced, which can lead to damage. Slab restraint originates from slab self-weight, aggregate interlock between adjacent slabs, and dowel/tie bars.

The slab self-weight is essentially a function of the slab geometry, as concrete used in paving applications is generally normal weight concrete. Joint width dictates the amount of friction between, and support provided by, adjacent slabs and shoulders, if no dowel or tie bars are present. Wider joint spacing means there is less aggregate interlock between slabs, and therefore less restraint, which allows more deformation due to differential volume change (Wells et al. 2006a). The presence of shoulders and adjacent slabs have been found to reduce the amount of curling (Teller and Sutherland 1935), while unrestrained edges have more deformations (Bendana et al. 2003).

One way to increase the amount of restraint between adjacent slabs and shoulders is through the use of dowel and tie bars. Dowel bars have been found effective at reducing deformations due to curling (Armaghani et al. 1987; Rao et al. 2001; Sondag and Snyder 2003) and warping (Bissonnette et al. 2007). One study found that doweled slabs deflect 
half as much as unrestrained slabs (Wells et al. 2006a). Additionally, slabs with dowel and tie bars curl more symmetrically when the provided restraint is insufficient to prevent deformation (Rao and Roesler 2005b).

\subsubsection{Curing Method/Drying History}

The curing method and drying history of the concrete affect the construction curl, differential drying shrinkage and moisture warping a slab will experience. Wet curing decreases the temperature gradient at the time of set, and therefore the amount of construction curl in the pavement (Eisenmann and Leykauf 1990a; Hiller et al. 2004). Wet curing will also decrease the amount of drying shrinkage experienced by the pavement, as there is less evaporation. Mat curing leads to less shrinkage and decreased built-in curl, but more moisture warping, while the reverse is true for membrane curing (use of a curing compound). Essentially the differences between the two cancel out, and both techniques result in the same amount of deformation (Jeong and Zollinger 2004). Sealed concrete will still experience shrinkage due to self-desiccation, but this shrinkage is uniform and does not contribute to moisture warping or differential drying shrinkage (Altoubat and Lange 2001). Using some form of curing to prevent the evaporation of water has the added benefit of reducing or eliminating shrinkage cracking (L'Hermite and Grieu 1952).

The drying history affects the rate of development of differential drying shrinkage and the amount of reversible shrinkage, which affects the amount of moisture warping in a slab. Drying history affects which portion of ultimate shrinkage is due to autogenous shrinkage versus which portion is due to drying shrinkage. Concrete which is soaked in water for an extended period of time before drying will have essentially no permanent shrinkage (L'Hermite et al. 1949), while concrete which is only moist cured for a few days will have 40-70\% reversible shrinkage (L'Hermite 1960). While it seems that concrete stored in water and then dried would have less overall shrinkage, this is not the case. Long term exposure to wet conditions does not diminish the potential for shrinkage, it merely increases the proportion of shrinkage which is reversible (L'Hermite 1947). Storing concrete initially in water results a more fully hydrated system, and therefore the restraining effect of unhydrated cement granules is diminished (Neville 1997). It has been shown that the amount of ultimate shrinkage a specific mix experiences will be the same, regardless of whether the concrete was stored in water and then let dry, or dried and then soaked in water (L'Hermite 1947).

\subsection{Effects of Differential Volume Change}

Deformations and stresses induced by differential volume changes in concrete are important because of their effects. If a slab deforms enough, it can separate from the underlying layers and become partially unsupported, which changes how loads are distributed. If the slab is sufficiently restrained, these deformations do not occur, but instead, stresses are induced.

\subsubsection{Changes in Support Conditions}

Differential volume change can induce sufficient deformation to cause the slab to separate from the underlying layers. The base cannot exert a tensile force on the slab, 
thus the only restraint against uplift comes from the slab's self-weight, dowel or tie bars, and friction from adjacent slabs. If these restraints are insufficient to resist the forces caused by differential volume change, then a portion of the slab will lift off of the underlying layers and become unsupported. When any portion of the pavement becomes unsupported, the stiffness of the entire pavement system is degraded (Armaghani et al. 1987).

If the slab curls downwards, then the corners will push into the base and the middle will lift off. If the slab curls upwards, then the corners will be unsupported (Teller and Sutherland 1935; Armaghani et al. 1987; Shoukry 2000; Wells et al. 2006a). Slabs very rarely experience uplift due to positive curl simply because a very large positive temperature gradient is required to overcome the downward deflections due to construction curl, moisture warping and differential drying shrinkage (Poblete et al. 1991). Instead, most slabs have a negative curl, which causes corners to be unsupported; this loss of support has been found to extend up to several feet at each corner (Hveem 1951; Rao and Roesler 2005b). In fact, most slabs experience sufficient curl and warp so as to be partially unsupported quite frequently (Harr and Leonards 1959; Poblete et al. 1991).

Loss of support from underlying layers at the corners of a slab essentially creates unreinforced concrete cantilevers, which are apt to fail when traffic loading is applied. In a standard jointed concrete pavement, edge loading is the worst loading scenario and produces the highest stresses. However, if the corners become unsupported, they will have higher stresses than the slab edge when loaded (Teller and Sutherland 1936). Additionally, slabs which have separated from the base at the corners are more prone to pumping and erosion of the underlying layers (Hveem and Bailey 1957).

\subsubsection{Joint Movement}

Uniform temperature changes cause slabs to expand and contract axially. Slabs incorporate joints to relieve the stress and strain induced by these uniform volume changes, and prevent thermal cracking. Temperature increases cause the pavement to expand, and occupy some of the space in the joint. If the expansion is of sufficient magnitude, the joint can even close or lock up, at which point it ceases to be functional. This increases the load transfer efficiency (LTE), because the slabs now act essentially as one, but also greatly increases the amount of stress in the pavement. A uniform decrease in temperature will cause the concrete to contract, and the joint to widen. This decreases the LTE between slabs, but also decreases the amount of axial stress in the pavement. Loss of LTE, however, will increases stresses due to traffic loading, as the load cannot be shared with adjacent slabs (Vandenbossche 2007).

Differential volume change can also cause joints to open and close, which changes the effective geometry of the slab, as well as the restraint conditions (Armaghani et al. 1987). When a slab is curled upwards, joints open up. This causes a decrease in the LTE from friction and aggregate interlock between adjacent slabs (Rao et al. 2001). Restraint provided by dowels and ties will reduce the loss in LTE, but will also induce more internal bending stress as deformation is prevented (Vandenbossche 2007). Downward 
curl closes joints, which increases LTE between slabs. However, if the joint closes fully, then adjacent slabs essentially act as one, which increases the amount of bending stress induced in the pavement (Poblete et al. 1988).

\subsubsection{Induced Stresses}

The main source of loading and induced stress in pavement is generally assumed to be vehicular. However, the stresses induced by curling can be larger than those caused by traffic (Bradbury 1938). Stresses are induced by differential volume change when the slab is sufficiently restrained to prevent deformations. Additional stresses in the pavement due to traffic loading compound the stresses due to differential volume change, but, due to nonlinearity, the two sets of stresses cannot be simply superimposed (Teller and Sutherland 1935; Ioannides and Salsilli-Murua 1989). Stresses due to positive and negative equivalent temperature gradients of the same magnitude are not equivalent; otherwise put, equal but opposite loads do not have equal but opposite reactions. This is due to the effects of gravity and slab self-weight (Siddique et al. 2006; Shoukry et al. 2007).

The sign convention of stresses induced by differential volume change is opposite that which is generally intuitive. This is because stresses are not induced by the deformations themselves, but by the resistance provided by the self-weight of the concrete and any other restraint mechanisms. For a slab with a concave downwards shape, such as a slab with very little built-in curl and moisture warping, and a positive thermal gradient, the top of the slab is in compression while the bottom is in tension. This decreases the load carrying capacity of the slab, because traffic loads place the top of the slab in compression and the bottom in tension, even though the deflections produced by these loads are opposite. For a slab with a concave upwards shape, the top of the slab is in tension while the bottom is in compression. Therefore the stresses induced by differential volume change negate those from traffic loading, allowing the slab to carry more load (Ioannides and Salsilli-Murua 1989).

A built-in upward curl (negative gradient), will reduce the stresses of a positive temperature gradient (generally during the day), but will exacerbate the stress at night, when a negative temperature gradient is present ( $\mathrm{Yu}$ and Khazanovich 2001; Bendana et al. 2003). Stresses due to moisture warping will behave in a similar manner, as slabs are almost always warped upwards.

\subsubsection{Pavement Damage}

In a flat slab, traffic loading causes tension at the bottom of the slab and compression at the top. A load placed at the edge of the slab will cause higher stresses than a load placed at the corners or in the middle of the slab (Westergaard 1926). Because most slabs are permanently curled upwards, the downward curl and associated bottom up cracking, which are normally considered to be the critical case in a pavement, become less concerning (Poblete et al. 1991). Instead, top down cracking at the corners of the pavement slab becomes the critical failure mechanism (Yu and Khazanovich 2001), see Figure 2.5. 


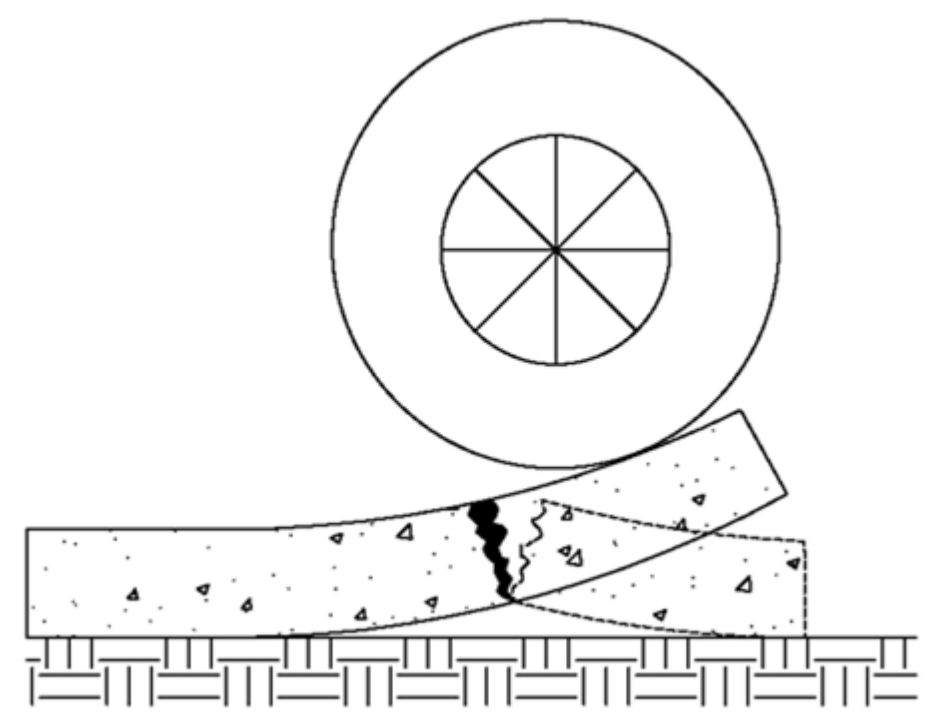

Figure 2.5: Top down cracking due to the combined effects of curling and traffic loading.

Even without traffic loading, the effects of temperature, moisture, shrinkage and built-in curl can create sufficient stresses in a slab to cause cracking (Teller and Sutherland 1935). This is particularly true when the concrete is young, and has not fully developed its strength. Many researchers have observed slabs which cracked within days of casting due to the effects of built-in curl, differential drying shrinkage, and thermal gradients (Eisenmann and Leykauf 1990a; Springenschmid and Hiller 1998). When stresses due to traffic loading are intensified by differential volume change, pavements fail sooner than intended in fatigue (Poblete et al. 1988).

\subsection{Designing and Modeling Pavements}

The complex, nonlinear relation between deformations and stresses induced by traffic and ambient conditions make modeling the behavior of concrete pavements very difficult. Computers have drastically increased analysis capabilities and allowed for a semimechanistic approach to be taken in design.

\subsubsection{Historic Approaches}

Westergaard first presented a closed-form solution to determine the stresses induced by temperature loading. His solutions were based on several simplifying assumptions, and allowed stresses at the corner, edge and middle of the slab to be determined. Westergaard assumed that the temperature gradient was linear, the slab behaved as a beam on a Winkler foundation, concrete is a linear elastic material, and the slab and the underlying layers were constantly in contact (Westergaard 1927). Additionally, Westergaard used superposition to combine the stresses induced by thermal loading with the stresses induced by traffic loading, found through his earlier closed-form solutions (Westergaard 1926).

It has since been shown that temperature gradients are nonlinear and that the slab does indeed separate from the subgrade (Teller and Sutherland 1935). Due to the nonlinearity 
of temperature gradients and the separation of the slab from the underlying layers, stresses induced by thermal and traffic loads cannot be superimposed (Teller and Sutherland 1935; Ioannides and Salsilli-Murua 1989). Despite this, Westergaard's solutions are still generally capable of finding a maximum induced stresses in a slab which is in the same range as those found with modern methods, though they cannot be used for estimating the stress distribution (Tang et al. 1993; Ioannides et al. 1999).

Other closed-form solutions which attempted to correct some of the simplifying assumptions made by Westergaard have since been proposed. Harr and Leonards (1959) developed a two-part stress prediction equation which accounted for the lack of support in slabs, but still assumed a linear temperature gradient and a Winkler foundation. Reddy et al. (1963) improved on this equation by assuming a non-linear temperature gradient, and using a subgrade model which accounted for time dependent deformations in the underlying layers.

\subsubsection{Modern Approaches}

Despite the derivation of various closed-form solutions, it was not until the invention of computers that more sophisticated analysis became feasible. Initially, these computer analyses were limited due to their computational intensity and the capability of computers. Ioannides and Salsilli-Murua (1989) developed a factor which can be applied to Westergaard's original solution to solve for nonlinear stresses induced by both traffic and thermal loading. Though the factor is based on finite element results, the end user needs only simple math to implement the solution.

As computational power has become increasingly available, the use of finite element methods (FEM) to solve for the stresses in pavements has become much more practical. FEM software programs specifically for concrete pavements, such as ISLAB2000 (Khazanovich et al. 2000b) or EverFE (Davids et al. 1998), now allow a user to solve for stresses and strains at any point on the pavement due to various combined thermal and traffic loads using a personal computer. FEM can also be used to generate theoretical data for a specific pavement for use in comparisons or back-estimation.

Another relatively new analysis tool is the artificial neural network (ANN). An ANN is an algorithm which is capable of "learning" from data and is used to correlate inputs and outputs. To the end user, the ANN is a proverbial black box, where known inputs are fed in, and output values are produced. Creating an ANN requires having a large amount of data with known inputs and end results. The ANN is trained with the known dataset so it can predict an output based on new user input values. ANNs have been found to be a practical tool for solving problems with several independent variables, and are becoming more prevalent in the field of concrete pavements (Ioannides et al. 1996; NCHRP 2004a; Lothschutz 2009).

\subsubsection{Current Pavement Design Practices}

Despite the existence of sophisticated, computerized tools for calculating stresses in concrete pavements, many states still use the design guidelines from the American Association of State Highway and Transportation Officials (AASHTO). The design 
guidelines, commonly called AASHTO-93, are a set of nomographs based purely on empirical test data obtained from a large scale test in Ottawa, Illinois, conducted between 1958 and 1960. The basic design philosophy in AASHTO-93 is that the user defines the acceptable loss of service in terms of ride quality, and then determines the required slab thickness necessary to achieve that loss of service. The inputs considered are the effective modulus of subgrade reaction, the modulus of rupture of the concrete, the estimated LTE between slabs, a coefficient to account for drainage, the number of equivalent single axle loads (ESALs) expected, the acceptable loss in design serviceability, and reliability factors (AASHTO 1993). The user connects the dots between input values to solve the nomograph and obtain a pavement thickness. This type of procedure does not indicate how the pavement will fail, nor does it allow the engineer any control over how to change the design other than to alter the thickness.

There are several problems commonly recognized with this method, mostly stemming from the limited scope of the AASHO road test. One major concern is that there is a lack of variables, for example, there is no direct way to account for the effects of climate on design. The empirical data is only for one site, and was extrapolated for the entire country; other countries have also adopted these nomographs despite having drastically different climates. As was previously discussed, the effects of temperature gradients, moisture gradients and built-in curl play a large role in determining the damage to a concrete pavement. These factors are largely determined from the ambient conditions to which a pavement is exposed. While they are implicitly incorporated in the performance of test sections in the AASHO Road Test, the effects of specific locations on these factors cannot be separated using the empirical AASHTO-93 design procedure. The AASHTO93 design procedure also does not account for the effects of differential volume change directly (NCHRP 2004a).

In an effort to remedy the deficiencies of the AASHTO-93 design method, AASHTO, the Federal Highway Administration (FHWA), and the National Cooperative Highway Research Program (NCHRP), partnered to sponsor the creation of the MechanisticEmpirical Pavement Design Guide (MEPDG). The MEPDG is widely seen as an improvement over the AASHTO-93 design standards because the MEPDG accounts for local weather and traffic conditions, different material properties and geometry, and factors such as built-in curl, which were originally neglected by the AASHTO-93 standards. Accurate estimates for the input parameters in the MEPDG are important because pavements designed with the MEPDG are generally thinner than those designed with AASHTO-93 (Timm 2006).

The MEPDG is a computer program which mechanistically calculates stresses induced by traffic and environmental loading. These stresses are then empirically correlated to damage based on a national database of observed pavement distresses. The amount of various types of damage can be predicted at different points in a pavement's life. Based on the predicted damage, the design engineer can decide if the pavement is acceptable or not. If the predicted damage is above an allowable threshold, parameters can be varied and the analysis repeated until a satisfactory design is achieved. This represents a drastic 
change from the AASHTO-93 procedures, where the engineer specified an acceptable loss in service as an input and was given the required concrete thickness.

In contrast, the MEPDG procedure provides the engineer with a predicted value of ride quality using the international roughness index (IRI), the amount of faulting, and the percent of slabs which will be cracked. Additionally, the predicted damage is calculated over the life of the pavement, so it can be determined at which age the pavement will fail for each distress type. If the damage level is found to be unacceptable, the engineer can vary different parameters to find an optimal design. This allows the engineer to incorporate other factors, such as cost, in their decision. For example, if a pavement design is analyzed in the MEPDG and found to have an unacceptable level of damage during its design life, the engineer may decide that changing the pavement thickness or using a different base material are not good options for that specific design. Instead, they may change the joint spacing, or add dowels to improve the performance of the pavement. By repeating the analysis for multiple designs, it can be determined which combinations of materials and geometry achieve an acceptable level of performance. The engineer can then select the most cost-effective design. This was not possible with the AASHTO-93 design procedure (NCHRP 2004a).

To achieve the increased capabilities in prediction afforded by the MEPDG, the user must input much more information than was originally required by AASHTO-93 procedures. For example, the original AASHTO inputs did not include any information about the geometry of the pavement. MEPDG requires slab thickness, joint spacing, dowel size and spacing, and edge support conditions (such as if there is a shoulder and if it is tied). The MEPDG has three levels of input, based on the amount of information available to the design engineer. In cases where the information may not be known, such as the amount of ultimate shrinkage anticipated in the concrete, guidance is generally given as to how values should be estimated; occasionally, default values are provided (NCHRP 2006).

Climate is determined through use of the enhanced integrated climatic model (EICM) (Larson and Dempsey 1997) based on historic data from the nearest weather station. The EICM can also interpolate between data from several nearby weather stations. This is a marked improvement over the AASHTO-93 procedure, which did not have any inputs to account for climate. The equivalent temperature difference due to temperature and moisture effects is computed in the MEPDG based on the EICM data.

In AASHTO-93, the sole factor accounting for traffic was the total number of ESALs. In MEPDG, the designer must specify the anticipated annual average daily truck traffic (AADTT), and can make adjustments for vehicle class distribution, axle configurations, monthly and hourly traffic distributions, and traffic growth factors, among other things. Default values are provided for these factors if the engineer is lacking information.

The stresses induced by the combined effects of differential volume change and traffic loading are calculated for every month in the pavement life using a neural network based 
on thousands of cases simulated using the finite element software ISLAB2000 (Khazanovich et al. 2000b). This stress is then correlated to damage through a correlation algorithm. Damage is accumulated with time according to Palmgren-Miner partial damage hypothesis (Tepfers et al. 1977), which allows a design engineer to view the amount of damage on a monthly basis and determine when exactly the pavement fails. The MEPDG correlation algorithm between stress and damage was calibrated based on data from the Long Term Pavement Performance (LTPP) database (FHWA 2009), and is therefore applicable to pavements located throughout the United States. Local calibration can refine this algorithm for improved accuracy (NCHRP 2004a).

\subsubsection{Differential Volume Change in the MEDPG}

One of the main differences between the MEPDG and AASHTO-93 is that the MEPDG is able to account for the effects of differential volume change. The MEPDG uses two terms to account for differential volume change when calculating stresses: equivalent temperature gradient (ETG) and built-in curl (BIC). The ETG term is used to quantify the effects of curl due to actual temperature gradients and warp due to moisture gradients, while the BIC term is the equivalent temperature gradient due to construction curl, the permanent component of warp due to differential drying shrinkage, and creep, collectively called built-in curl (NCHRP 2003). The relation of these terms is given in Figure 2.6. To avoid confusing, in this document, built-in curl and equivalent temperature gradient will refer to the phenomena, while BIC and ETG will refer to the MEPDG input parameters.

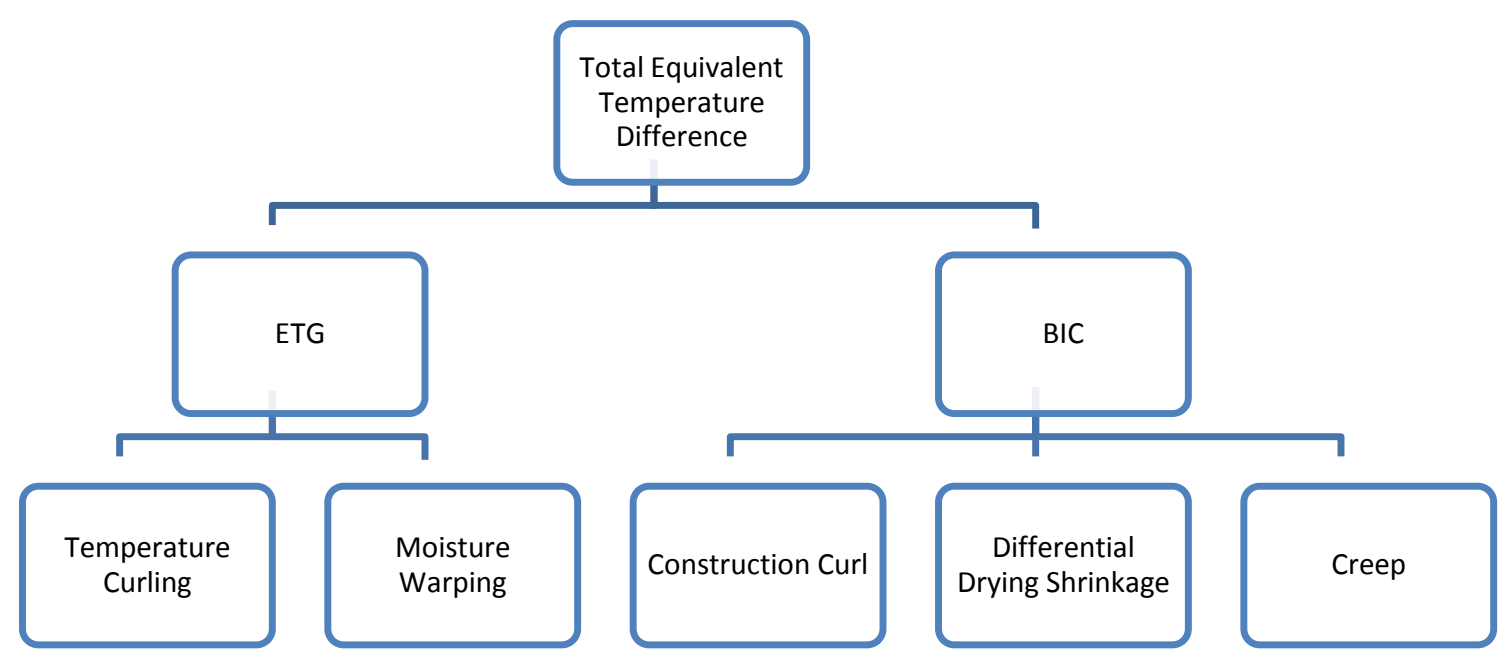

Figure 2.6: Accounting for equivalent temperature difference in the MEPDG.

To calculate the total ETG, the actual temperature gradient from climatic data is summed with the equivalent temperature gradient due to moisture warping. The moisture warping is predicted based on an assumed linear shrinkage distribution through the top few inches of the pavement due to variations in relative humidity, also taken from climatic data. 
This value is then adjusted to account for the age of the slab. Temperature curling is computed hourly, while moisture warping is computed monthly (NCHRP 2003).

The value of BIC is a user input, with a default setting of $-10^{\circ} \mathrm{F}$. BIC is also a calibration factor used in the creation of MEPDG to calibrate predicted distress levels (NCHRP 2003). It has been shown that assuming $\mathrm{BIC}$ is equal to $-10^{\circ} \mathrm{F}$ results in an unconservative design in almost all cases, because $-10^{\circ} \mathrm{F}$ corresponds well to the value of BIC for which damage is minimized (Lothschutz 2009; Vandenbossche et al. 2010). This is due to the use of BIC as a calibration factor and not expressly as a factor which is influenced by paving conditions, slab geometry, material properties, etc. During the MEPDG calibration process, factors such as the BIC factor were used to minimize the error between the predicted and observed distresses from LTPP test sections (Vandenbossche et al. 2010). Therefore, it would make sense that the BIC calibration factor corresponds to the value of BIC for which damage would be minimized in most cases. However, using this factor as a calibration factor and a user input results in an under prediction of the fatigue life of the pavement. This is compounded by the fact that no guidance is given for estimating the value of BIC which should be used (Lothschutz 2009; Lothschutz et al. 2011).

\subsection{Measuring Differential Volume Change}

Differential volume change is quantified by the linear temperature gradient required to deform a similar, but theoretically flat slab to the same shape as the actual slab; this temperature gradient is known as the equivalent temperature difference. As this quantity cannot be measured directly, any technique used to characterize the amount of differential volume change in a slab must include both actual measurements from the slab and a theoretical model or algorithm used to correlate the actual measurements to an equivalent temperature difference. The actual measurements generally are slab profile measurements or falling weight deflectometer (FWD) drops, while the correlation is typically made with either a model, such as a finite element model, or an ANN.

\subsubsection{Slab Measurement Techniques}

Measurements of the slab profile are obtained from a profiler device, which measures the elevation of various points on the slab relative to a datum. From this data, a profile of the top surface of a cross section of the slab is obtained. By profiling at several locations across the slab, a general three-dimensional image of the slab can be obtained. One advantage of profiling is that the data can be visualized, and is presented in a format that intuitively shows the shape of the slab.

The FWD measures the deflection of a pavement at various locations in response to an applied load. This data obtained from this test in its own right does not present a clear picture of the shape of the slab, as profiler data does. However, once the data from and FWD test is processed, it can give information which a profile cannot. The FWD response incorporates the entire slab, as well as the underlying layers, and therefore can be used to characterize the entire slab and its relationship with the base layer. In contrast, a profile can only show the shape of the top surface of the slab. 


\subsubsection{Profile Measurement Devices}

There are several different types of device used to obtain a profile of the slab, including dipsticks, on-site profilometers, and high-speed profilometers, each of which present their own benefits and drawbacks. The least sophisticated form of profiling is to use a survey rod and level, but it is difficult to obtain measurements of the precision required for profiling, which is often on the order of a few hundredths of an inch. A dipstick profiler is a proprietary device which consist of a computer and a measuring device on two "feet". The dipstick profiler is "walked" across the surface of the pavement and automatically records data. Though this device is an improvement over the rod and level system, it is still slow and cumbersome (Sayers and Karamihas 1998).

An onsite profilometer consist of a laser bar mounted to a small personal utility vehicle such as a lawn tractor. This vehicle can drive between locations, but is stationary as readings are taken. The laser bar takes readings across the slab to determine the profile. This device presents an advantage over the dipstick or the rod and level methods because it can measure the entire profile at once, allowing for faster data collection. However, this device still moves relatively slowly, and must be stopped to take a reading; therefore the pavement which it is measuring must be closed to traffic during the data collection process.

Similar to an onsite profilometer, a high speed profiler is a vehicle mounted mobile device for measuring elevation differences from a reference to establish the profile of the slab. The differences are that the high speed profile is housed in a much larger vehicle, such as a van, and it travels at highway speeds. Therefore, the road which is being measured can be open to traffic, and many profiles can be obtained quickly. High speed profilers are also called inertial profilers, because, unlike the onsite profilometer, they must be moving to function. Due to this, the data obtained from high speed profilers is not of the same form as that obtained with other profiler devices, and requires processing before it can be used (Sayers and Karamihas 1998).

The main drawback of profilers is that they only measure the top surface of the slab, which is not representative of the entire slab. For example, the surface of a slab contains many irregularities due to tining, and surface defects, such as cracks or spalls. Some types of profilers are also affected by the slope or grade of the road. A surface profile will include the effects of all of these irregularities, which add error when the data is processed (Byrum 2001; Sondag and Snyder 2003). More importantly, the profile cannot indicate whether or not the slab is fully supported on the underlying layers. This is important because loss of support causes pavements to fail prematurely in fatigue ( $\mathrm{Yu}$ and Khazanovich 2001; Beckemeyer et al. 2002).

\subsubsection{Falling Weight Deflectometer}

An FWD generally consists of a load plate and a series of deflection sensors mounted on a trailer. The sensors are placed on the pavement extending away from the load plate in a line; often additional deflection sensors are placed under the load and adjacent to it in one or more directions. When the load is dropped on to the pavement, which simulates a vehicle load, the sensors measure how the induced deflections dissipate with distance 
from the load. Based on this, information about the slab, such as the modulus of elasticity of the concrete or the modulus of subgrade reaction, can be back-calculated using various algorithms (Khazanovich et al. 2000a). When using FWD back-calculation techniques, it is important that the back-calculation algorithm does not assume linearly varying deflections because any pavement which is not fully supported will have nonlinear deflections (Choubane and Tia 1995).

FWD data is particularly useful in determining the support conditions of a slab because it can be used to detect voids under the pavement which would not otherwise be visible or observable by only considering the surface of the slab. However, it cannot be determined from the data whether the void is due to slab deformation as a result of curl or warp, or due to erosion (Rao and Roesler 2005a). Temperature gradients at the time of testing can also affect slab shape determined from FWD data (Vandenbossche 2003).

\subsubsection{Back Calculation}

Either type of data collection, profiler or FWD can be coupled with either an FEM model or an ANN. In general, profilers are only coupled with finite element models because training an ANN to work with profiler data would require thousands of cases of profiler data, which is more time consuming to collect and process than FWD data. For that reason, it is much more common to pair FWD with an ANN than profiler data (Lothschutz 2009). FWD measurements can also be compared with finite element software to back calculate the amount of curl (Vandenbossche 2003; Rao and Roesler 2005b; 2005a).

When using FEM, the surface profile obtained from either the profiler or the FWD can then be plotted and an equation fitted to the shape of the surface. This equation is compared to curves generated using a finite element program representing the deflected shape of a theoretical flat slab of the same geometry and material properties which is exposed to various temperature gradients. The temperature gradient required to produce the same deflected shape as that of the observed slab less the actual temperature gradient at the time of testing is the built-in temperature gradient. This brute force procedure is time consuming and error prone, but does not require specialized techniques or equipment.

Alternately, the data can be run through an ANN. This method is much more automated and therefore, the potential to introduce human error is greatly reduced. However, it does require that the user have an appropriately trained ANN at their disposal, as well as access to data which is suited for use in the ANN. In general, it is desirable to have FWD data obtained when there is not a large positive overall equivalent temperature difference. As such, FWD testing should be conducted at night or in the early morning (Shoukry 2000; Lothschutz 2009).

\subsection{Summary of Literature Review}

Differential volume change in slabs is due to the combined effects of temperature curling, moisture warping, differential drying shrinkage, construction curl, and creep, all of which act to induce deformations. These differential volume changes are all quantified by the 
equivalent temperature difference required to deform a theoretically flat slab to the same shape as the actual slab. If a slab is not free to deform, stresses will be induced. Factors affecting both the amount of and response to differential volume change include the slab geometry, material properties of the both the concrete and the underlying layers, ambient conditions during paving and service life, and restraint mechanisms.

It is important to consider the effects of differential volume change because induced deformations can be sufficient to cause damage to the pavement, even before considering the effects of traffic loading. Differential volume change can also cause the slab to separate from the underlying layers, which drastically changes how the pavement responds to loading. The critical load case can change from bottom-up cracking at the edge of the slab to top-down cracking at the corners. Differential volume change can cause early age cracking and shorten the fatigue life of the pavement.

Though differential volume change is an important factor in the design of concrete pavements, it is neglected in the current AASHTO-93 design procedure. The new MEPDG design method accounts for the effects of differential volume changes in the computation of stresses, which are then correlated to pavement damage. Within the MEPDG, the terms ETG and BIC are used to account for differential volume change. The ETG factor is based on equations and historic climatic data, and accounts for the effects of temperature curling and moisture warping. The BIC factor is a user input which accounts for the effects of construction curl, differential drying shrinkage, and creep. Differential volume change in pavements in the field cannot be measured directly. Instead data such as the slab profile or response to FWD testing is processed with FEM models or an ANN to back-calculate the equivalent temperature difference. 


\section{Reversible Shrinkage}

\subsection{Introduction}

Reversible shrinkage is that portion of shrinkage in concrete which can be recovered upon soaking the concrete in water. Very little is currently known about reversible shrinkage beyond the fact that it exists. However, reversible shrinkage plays an important role in determining the amount of moisture warping in a pavement, and therefore is of great interest. This study investigated the amount of reversible shrinkage for concrete made of different types of aggregate, including recycled and lightweight aggregates.

\subsubsection{Background}

The amount of growth and shrinkage is measured as strain, $\varepsilon$, which is calculated as the change in length over the initial length of the specimen, see Equation 3.1. Strain is given in units of length/length, or as a dimensionless quantity. Strains are often very small for volume change in concrete, and therefore are generally expressed in terms of microstrain or $\mu \varepsilon$, which is strain* $10^{-6}$. Strain due to shrinkage is negative because the sample contracts, yielding a negative $\Delta \mathrm{L}$; strain due to swelling has a positive sign convention.

$$
\varepsilon=\frac{\Delta L}{L}
$$

Equation 3.1

where: $\Delta \mathrm{L}$ is the change in length

$\mathrm{L}$ is the initial length

Much of what is generally known about reversible shrinkage can be represented by a highly idealized curve shown in Figure 3.1(Mindness et al. 2003; NCHRP 2004b). This curve is sometimes shown mirrored about the abscissa such that negative strain is increasing on the ordinate. Regardless of the orientation, this figure only shows the behavior for one initial storage condition (dry cured) and one re-wetting cycle.

Work by L'Hermite et al. (1949) showed that repeated wetting and drying will cause this curve to repeat itself, though the amount of reversible and permanent shrinkage is not constant, but changes with each cycle. Their work eventually quantified the amount of shrinkage which was initially reversible as between 40 and $70 \%$, but did not calculate the amount of additional permanent or reversible shrinkage from subsequent cycles. (L'Hermite 1960). The curve in Figure 3.1 does not suggest that there is any further behavior after the first rewetting cycle, nor does it imply that there is a range of values for the amount of reversible shrinkage. Based on this curve, it is often assumed that half of all shrinkage is reversible and half is not. 


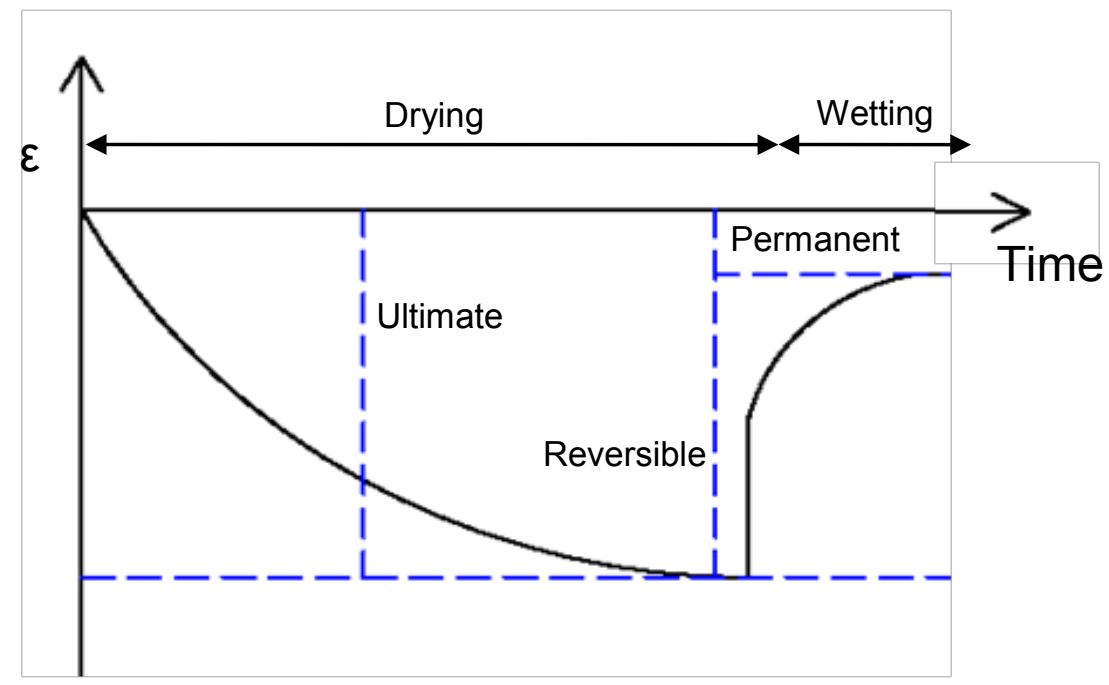

Figure 3.1: Highly idealized curve of reversible shrinkage.

\subsubsection{Motivation}

The goal of this study was to characterize the amount of reversible shrinkage in concrete made with virgin, recycled concrete and lightweight aggregates. This information is of particular use to pavement engineers because reversible shrinkage is an input in the MEPDG, but is also of general interest to the broader concrete community. There has been essentially no research on the topic of reversible shrinkage for the last several decades, and there has never been any research on the amount of reversible shrinkage for concrete made with lightweight or recycled concrete aggregates.

One important input parameter in the MEPDG for the design of jointed plain concrete pavements is the reversible shrinkage factor, $\phi$. Moisture warping is due to changes in volume of the reversible portion of drying shrinkage when the slab is exposed to changes in relative humidity. The reversible shrinkage factor is multiplied by the ultimate shrinkage in the warping model to determine the amount of shrinkage which is expected to be reversible. The default value recommended for the reversible shrinkage factor in the MEPDG is 0.5 (NCHRP 2003), indicating that half of all shrinkage is reversible. Studies have shown that this is not necessarily the case, and also that the amount of reversible shrinkage is highly dependent on both the material properties and curing regime of the concrete (L'Hermite et al. 1949; Shacklock and Keene 1957; Helmuth and Turk 1967).

\subsection{Methods}

To determine the relative shrinkage factor for concrete mixes with various aggregates, the ASTM C157 Standard Test Method for Length Change of Hardened Hydraulic-Cement Mortar and Concrete (ASTM 2008e) was performed for concretes made of virgin, lightweight and recycled concrete aggregates. Concrete prisms were stored in both 50\% and $100 \%$ relative humidity chambers with constant temperature. The length change was recorded and used to calculate the shrinkage strain that the samples experienced as the concrete hydrated and as the relative humidity to which they were exposed was varied. 
The specimens used in this experiment were originally used by Shorkey (2010) to determine the unrestrained shrinkage behavior of concrete made with various aggregates as part of a project for the Michigan Department of Transportation (MDOT). The aggregate testing, mix design, sample preparation and initial sample measurements were part of that study. The samples in Shorkey's study were stored in a constant relative humidity environment, which was maintained after the study was concluded. It was determined that these samples were well suited for a study on the reversible shrinkage behavior of concrete with various aggregates because they had been stored in constant relative humidity for at least 300 days. This was the time required to achieve ultimate shrinkage, as determined by previous research (Dutron 1934; L'Hermite et al. 1949). Additionally, use of these samples was advantageous because a continuous record of their shrinkage strain had been maintained since the beginning of Shorkey's study.

\subsubsection{Mix Design and Aggregate Properties}

Six different aggregate types were tested in this experiment, a virgin aggregate, four different recycled concrete aggregates and a lightweight/virgin aggregate blend. The virgin aggregate was a crushed gravel obtained from a local ready-mix contractor and is typical of an aggregate used in standard concrete for structural and paving applications. This aggregate was used as the control in this study, and was designated as "Virgin". While the reversible shrinkage of concrete made with such aggregate has been studied, albeit on a limited scale, no research has yet been conducted on the reversible shrinkage of concretes made with lightweight or recycled concrete aggregates.

In standard practice, lightweight concrete is not made completely of lightweight aggregates because they lack strength. Therefore, to represent typical industry practice, $30 \%$ lightweight aggregate and $70 \%$ of the same virgin aggregate from the control samples was used to create a lightweight aggregate blend which was designated as "Lightweight". A lightweight aggregate was selected because they are commonly used in construction applications where decreased self-weight of the concrete is desirable. One of the main parameters which influences the shrinkage of the concrete is the ability of water to diffuse through the matrix (Bazant and Najjar 1972), which is related to the permeability of the concrete. Lightweight aggregates are very porous, which produces more permeable concrete and promotes internal long-term curing (Kosmatka et al. 2002).

As the emphasis on using sustainable materials continues to grow, and sources of viable virgin aggregate become depleted or increasingly costly, it is expected that the use of recycled concrete aggregates will increase. Previous research in the use of recycled concrete aggregates (RCA) has shown that they cause more shrinkage than virgin aggregates, and the magnitude of that shrinkage is difficult to predict (Kosmatka et al. 2002). The recycled concrete aggregates in this study came from four different sites in Michigan. The recycled slag was from I-69 in Shiawassee County (designated as "Slag RCA"), the recycled limestone was from I-75 in Saginaw (designated as "Limestone RCA"), and the recycled gravel was from US-41 in Houghton (designated as "Gravel RCA"). The fourth recycled aggregate in this study was a bit of an oddity among recycled concrete aggregates. This aggregate was from US-131 in Kalamazoo County, and had already been used as a recycled aggregate previously, which led to the name 
"Twice Recycled". The pavement in which this aggregate was a virgin aggregate was ground up and used in another pavement, which was subsequently ground up and used again. When this pavement reached the end of its service life, it was ground up and used to make the sample for this study. At this time, there is no documented research on the use of twice recycled concrete aggregates, making this sample particularly interesting.

Depending on the quantity of each aggregate available, several batches of concrete were made of some aggregates, while there was only enough aggregate for one batch of concrete from other aggregates. When there were several batches, they were given the same name, and numbered sequentially. For each batch of concrete made, the properties of the aggregate were tested separately. Table 3.1 shows the absorption capacity, bulk specific gravity and effective porosity of each aggregate.

Table 3.1

Aggregate properties, (data from Shorkey 2010).

\begin{tabular}{|c|c|c|c|}
\hline Aggregate & Absorption capacity & Bulk specific gravity & Effective porosity \\
\hline Slag RCA & $2.8 \%$ & 2.2 & $6.2 \%$ \\
\hline Limestone RCA & $4.2 \%$ & 2.4 & $10.1 \%$ \\
\hline Virgin 1 & $0.7 \%$ & 2.7 & $1.9 \%$ \\
\hline Virgin 2 & $0.9 \%$ & 2.7 & $2.4 \%$ \\
\hline Virgin 3 & $0.7 \%$ & 2.7 & $1.9 \%$ \\
\hline Gravel RCA 1 & $2.8 \%$ & 2.5 & $7.0 \%$ \\
\hline Gravel RCA 2 & $4.5 \%$ & 2.4 & $10.8 \%$ \\
\hline Twice Recycled & $2.5 \%$ & 3.1 & $7.8 \%$ \\
\hline LWA 1 & $4.8 \%$ & 2.4 & $11.5 \%$ \\
\hline LWA 2 & $4.8 \%$ & 2.4 & $11.5 \%$ \\
\hline LWA 3 & $5.5 \%$ & 2.3 & $12.7 \%$ \\
\hline
\end{tabular}

The porosity of the aggregate is particularly important because it will influence the moisture diffusivity through the concrete, which is hypothesized to affect the reversible shrinkage. From Table 3.1, it can be seen that all of the recycled aggregates have much higher porosity than the virgin aggregates, as does the lightweight aggregate. The twice recycled aggregate has a lower porosity than most of the other aggregates; this is due to delayed ettringite formation in the adhered paste, which filled many of the voids (Hiller et al. 2011). Several studies have found that concrete made with RCA have increased drying shrinkage (ex: Sato et al. 2007; Domingo et al. 2010). Given that the amount of shrinkage which is reversible is a function of drying shrinkage, samples made with the more porous recycled and lightweight aggregates are anticipated to have higher reversible shrinkage.

\subsubsection{Sample Preparation}

The concrete made from each of these different aggregates varied only in the aggregate type used. As the original use of these samples was a research project funded by the 
MDOT, the mixes were designed follow MDOT P1 mix specifications (MDOT 2003), which is the mix used for slip-form paver applications. All mixes had a water to cement ratio of 0.42 and cement content of $610 \mathrm{lb} / \mathrm{yd}^{3}$. The dry-rodded course aggregate content by volume was $72 \%$, and all mixes contained the same MDOT 6AAA coarse aggregate gradation. A local virgin sand source conforming to MDOT Standard Specification Series 2NS was used as the fine aggregate (MDOT 2003). The mix proportions of the concrete are shown in Table 3.2. Sieve-specific grading of the course and fine aggregates was maintained to assure a particle size distribution representing the middle of the upper and lower specification. The concrete was air entrained using vinsol resin at a dosage of $1.247 \mathrm{~g} \mathrm{resin} / \mathrm{kg}$ cement, and all mixes had a target air content of $6.5 \%$ (Shorkey 2010 ).

Table 3.2

Mix proportions of concretes (in $\mathrm{lbs} / \mathrm{yd}^{3}$ of concrete).

\begin{tabular}{|c|c|c|c|c|c|}
\hline $\begin{array}{c}\text { Material } \\
\text { (lb/yd }{ }^{3} \text { of } \\
\text { concrete) }\end{array}$ & $\begin{array}{c}\text { Crushed } \\
\text { Gravel }\end{array}$ & Slag RCA & $\begin{array}{c}\text { Limestone } \\
\text { RCA }\end{array}$ & $\begin{array}{c}\text { Crushed } \\
\text { Gravel } \\
\text { RCA }\end{array}$ & $\begin{array}{c}\text { Twice } \\
\text { Recycled } \\
\text { RCA }\end{array}$ \\
\hline $\begin{array}{c}\text { Type I } \\
\text { Cement }\end{array}$ & 610 & 610 & 610 & 610 & 610 \\
\hline Water & 260 & 260 & 260 & 260 & 260 \\
\hline $\begin{array}{c}\text { Coarse } \\
\text { Aggregate }\end{array}$ & 1965 & 1807 & 1836 & 1930 & 1793 \\
\hline $\begin{array}{c}\text { Fine } \\
\text { Aggregate }\end{array}$ & 1160 & 834 & 957 & 981 & 1048 \\
\hline
\end{tabular}

The concrete was batched in accordance with standard MDOT procedures (MDOT 2003). The concrete was placed in standard ASTM C157 (2008e) prism molds to create 3 "x3"x12" specimens; each mix produced six prisms for use in this study, as well as samples for other research, such as compression test cylinders. These specimens were demolded after 18 hours. Four of the samples from each mix were placed in a 50\% relative humidity chamber, two in a sealed condition and two unsealed, and the remaining two samples were placed in a 100\% relative humidity chamber. After several months, one of the $100 \%$ relative humidity samples for some of the mixes was used for another research project and was no longer included in this study. The sealed samples stored in the $50 \%$ relative humidity chamber were used to calculate the autogenous shrinkage, but as they were sealed, they could not be used for the relative humidity variation portion of the study. The samples originally stored in the $50 \%$ relative humidity chamber were designated as "dry cured" while those initially placed in the $100 \%$ relative humidity chamber were designated as "wet cured".

Standard testing was conducted on the plastic hardened concrete. The air content of the plastic concrete was measured with a pressure meter (ASTM 2008d). Initial testing conducted after 28-days on the concrete included compressive strength (ASTM 2008c), 
elastic modulus (ASTM 2008b), modulus of rupture (ASTM 2008a) and concrete permeability (ASTM 2008f). Not all testing could be conducted on the twice recycled aggregate concrete because there was an insufficient amount of aggregate to make the required samples. Test results are given in Table 3.3.

Table 3.3

Plastic and hardened concrete test results (data from Shorkey 2010).

\begin{tabular}{|c|c|c|c|c|c|c|}
\hline Mix & $\begin{array}{c}\text { Aggregate } \\
\text { Type }\end{array}$ & $\begin{array}{c}\text { Air } \\
\text { content } \\
(\%)\end{array}$ & $\begin{array}{c}\text { Compressive } \\
\text { Strength } \\
\text { (psi) }\end{array}$ & $\begin{array}{c}\text { Elastic } \\
\text { Modulus } \\
\text { (psi) }\end{array}$ & $\begin{array}{c}\text { Modulus of } \\
\text { Rupture } \\
\text { (psi) }\end{array}$ & $\begin{array}{c}\text { Concrete } \\
\text { Permeability } \\
\text { (Coulombs) }\end{array}$ \\
\hline Virgin & $\begin{array}{c}\text { Virgin } \\
\text { gravel }\end{array}$ & 4.9 & 5542 & 3336431 & 779 & 1405 \\
\hline Slag RCA & $\begin{array}{c}\text { Recycled } \\
\text { slag }\end{array}$ & 3.5 & 2648 & 2706245 & 627 & 2557 \\
\hline $\begin{array}{c}\text { Limeston } \\
\text { e RCA }\end{array}$ & $\begin{array}{c}\text { Recycled } \\
\text { limestone }\end{array}$ & 5.8 & 4302 & 2634307 & 568 & 3069 \\
\hline $\begin{array}{c}\text { Gravel } \\
\text { RCA }\end{array}$ & $\begin{array}{c}\text { Recycled } \\
\text { gravel }\end{array}$ & 5.8 & 4144 & 2771585 & 621.5 & 2047 \\
\hline $\begin{array}{c}\text { Twice } \\
\text { Recycled }\end{array}$ & $\begin{array}{c}\text { Twice } \\
\text { recycled }\end{array}$ & 5.5 & 5490 & - & - & 1925 \\
\hline $\begin{array}{c}\text { Light- } \\
\text { weight }\end{array}$ & $\begin{array}{c}30 \% \text { LWA, } \\
70 \% \text { virgin }\end{array}$ & 9.0 & 3925 & 2319287 & 514 & 1528 \\
\hline
\end{tabular}

\subsubsection{Relative Humidity Variation}

Previous studies have found that concrete stored under constant temperature and relative humidity conditions will take between 100 (Helmuth and Turk 1967) and 300 days (L'Hermite et al. 1949) to stop growing or shrinking appreciably, although volume change will continue on an infinitesimal scale indefinitely (Neville 1997). To ensure that the volume change had essentially stabilized before the storage conditions were changed, all samples were aged a minimum of 300 days under their initial storage conditions. Then, the samples from the $50 \%$ relative humidity chamber were placed in the $100 \%$ relative humidity chamber and the samples from the $100 \%$ relative humidity chamber were placed in the $50 \%$ relative humidity chamber. After the volume change had stabilized, the samples were placed back in their original conditions; this is the same procedure used by L'Hermite et al. (1949). This process was repeated for several cycles of drying and re-wetting to determine how the amount of reversible shrinkage changed with repeated drying and re-wetting.

As not all of the samples were cast on the same day, they reached the 300 day mark at different times. For the sake of simplicity, the samples were divided into two groups based on age, and conditions were changed for the entire group of samples on the same 
day. Group 1 consisted of sample sets Virgin 1 and 2, Slab RCA, Limestone RCA, and Gravel RCA 1 and 2, while Group 2 consisted of sample sets Virgin 3, Twice Recycled, and Lightweight 1,2 and 3. Due to the difference in ages, Group 1 had an additional cycle of drying and re-wetting. The samples which were wet cured had to be dried before reversible shrinkage testing could begin; therefore, these samples also have one less drying and re-wetting cycle.

\subsection{Results}

The change in length and the weight of each sample were measured in accordance with ASTM C157 (ASTM 2008e). By this standard, the frequency of the measurements decreases with the age of the sample. Every time the relative humidity conditions were varied, measurements recommenced with the frequency required for freshly cast samples. From these measurements, both the strain and weight change were calculated.

Full results for all samples are given in tabular form in Appendix A and graphically in Appendix B and Appendix C, while selected results are shown below. The two unsealed dry cured prisms from each mix are designated as "dry cure 1" and "dry cure 2", while the wet cured prisms are designated as "wet cure 1", and "wet cure 2", where applicable. Again, a negative strain value indicates shrinkage, while a positive value represents swelling from the initial measurement after demolding. The strain versus time for a virgin aggregate set is given in Figure 3.2 for samples which were dry cured and in Figure 3.3 for samples which were wet cured.

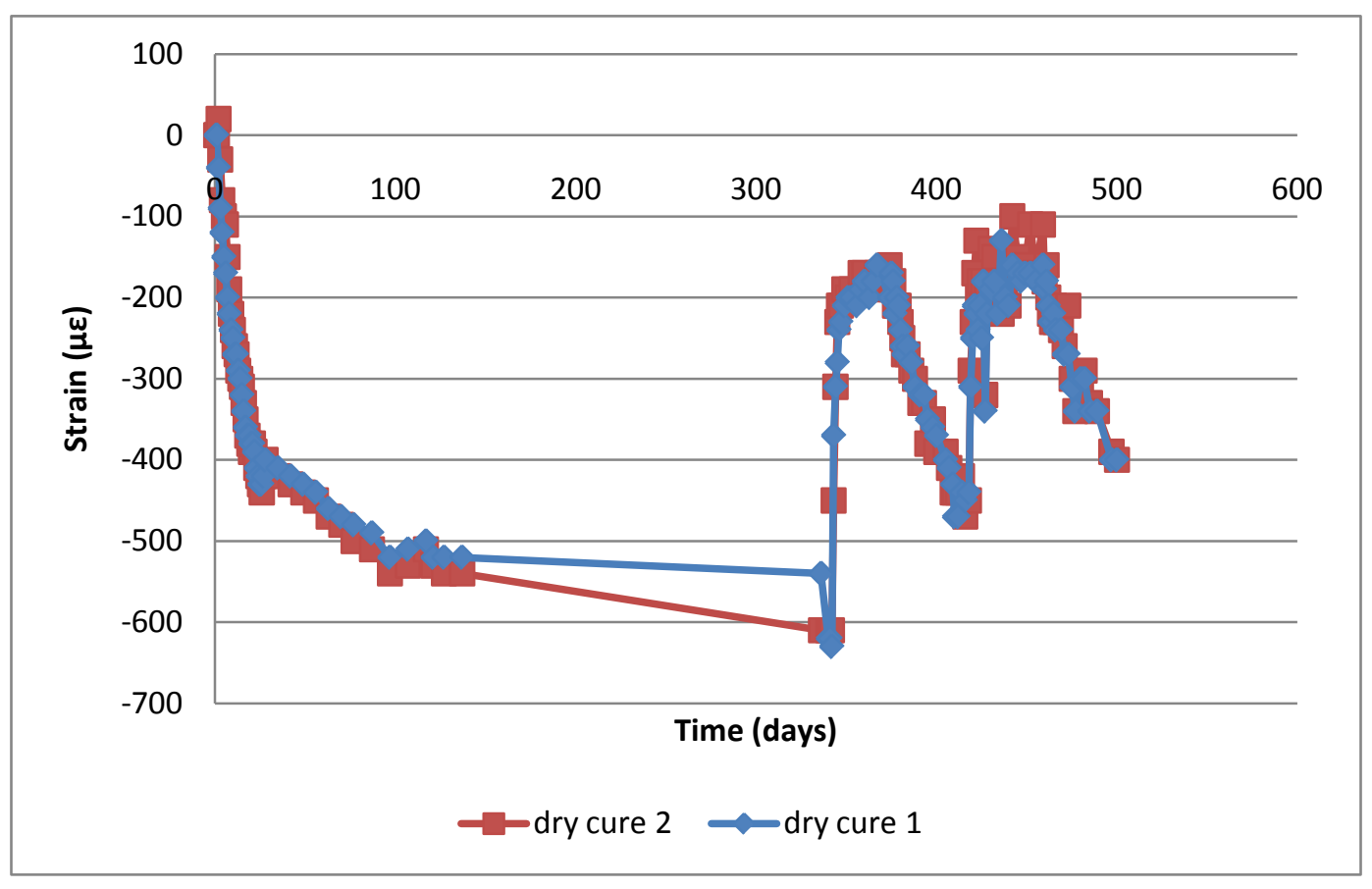

Figure 3.2: Strain versus time for dry cured virgin aggregate concrete. 


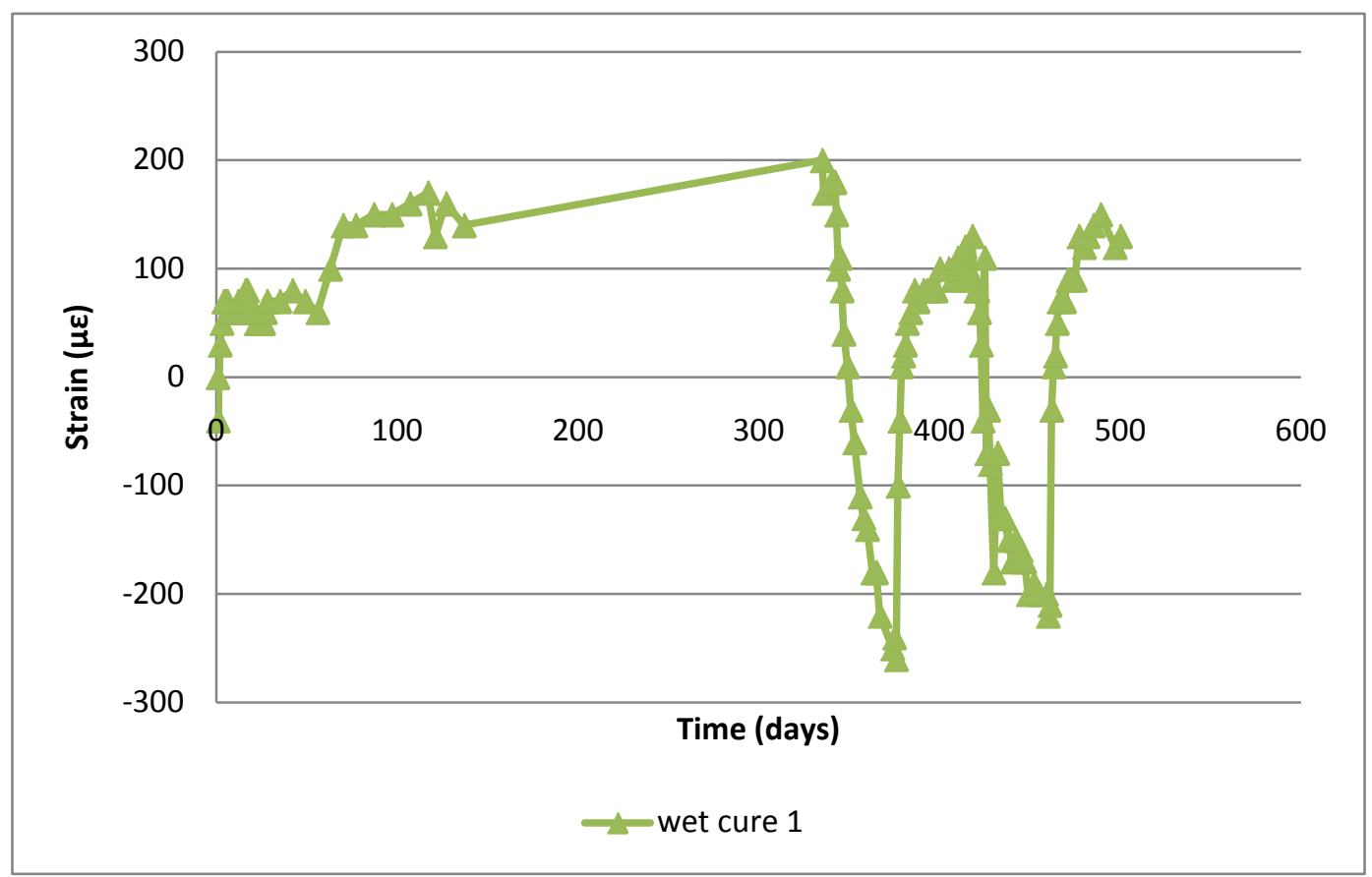

Figure 3.3: Strain versus time for wet cured virgin aggregate concrete.

The strain versus time for a recycled aggregate set is given in Figure 3.4 for samples which were dry cured and in Figure 3.5 for samples which were wet cured.

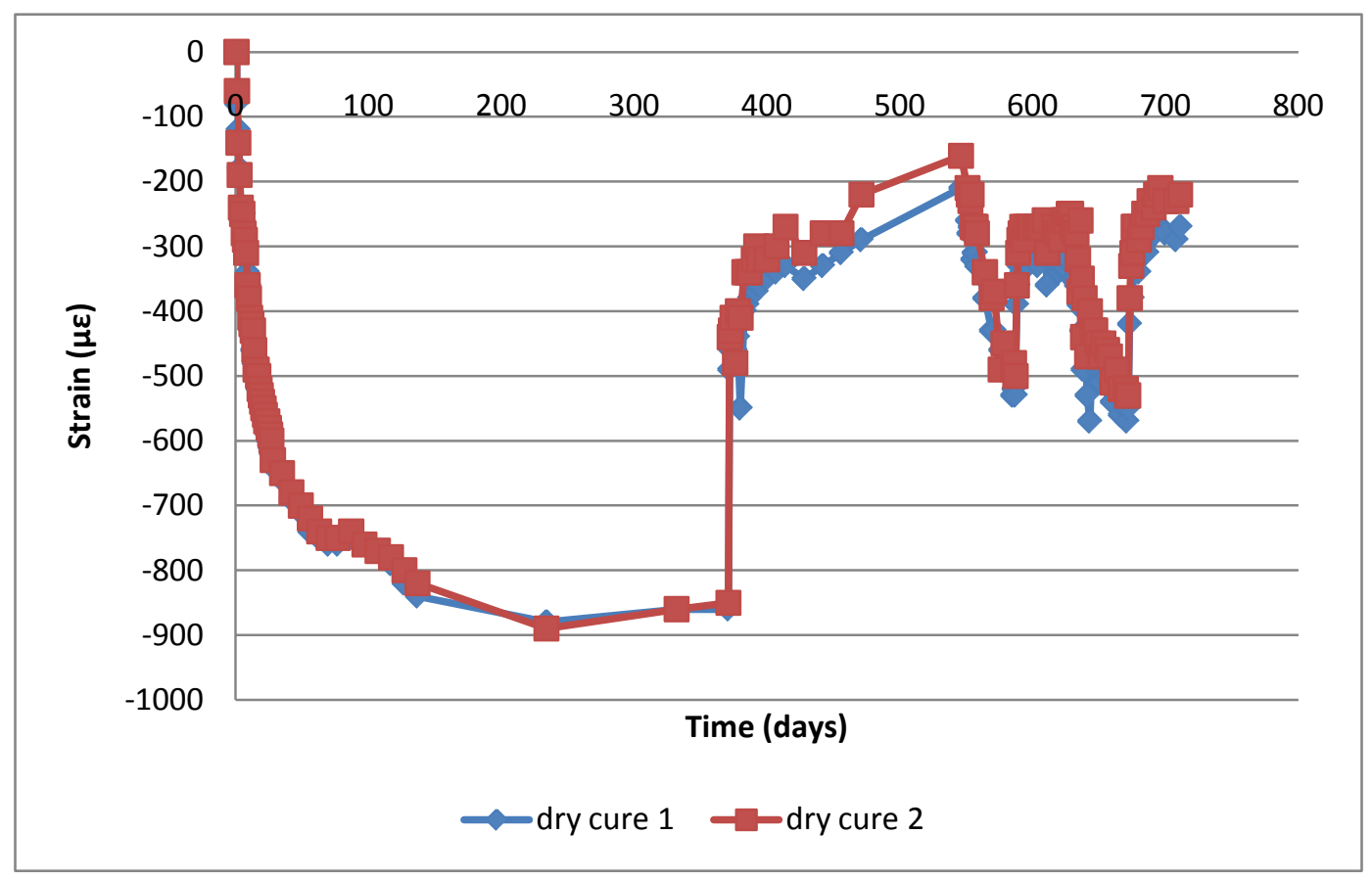

Figure 3.4: Strain versus time for dry cured gravel RCA concrete. 


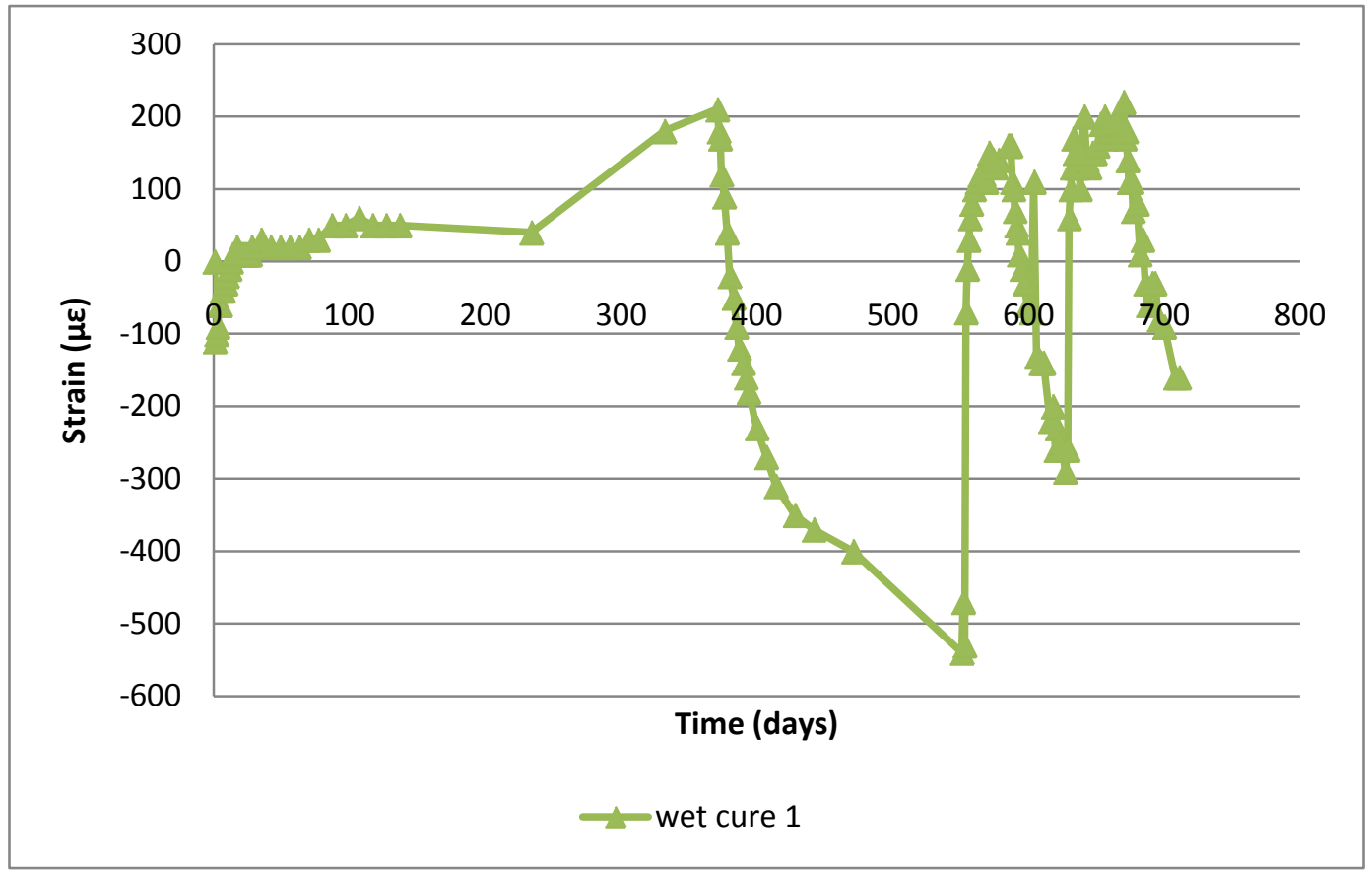

Figure 3.5: Strain versus time for wet cured gravel RCA concrete.

The strain versus time for a lightweight aggregate set is given in Figure 3.6 for samples which were dry cured and in Figure 3.7 for samples which were wet cured.

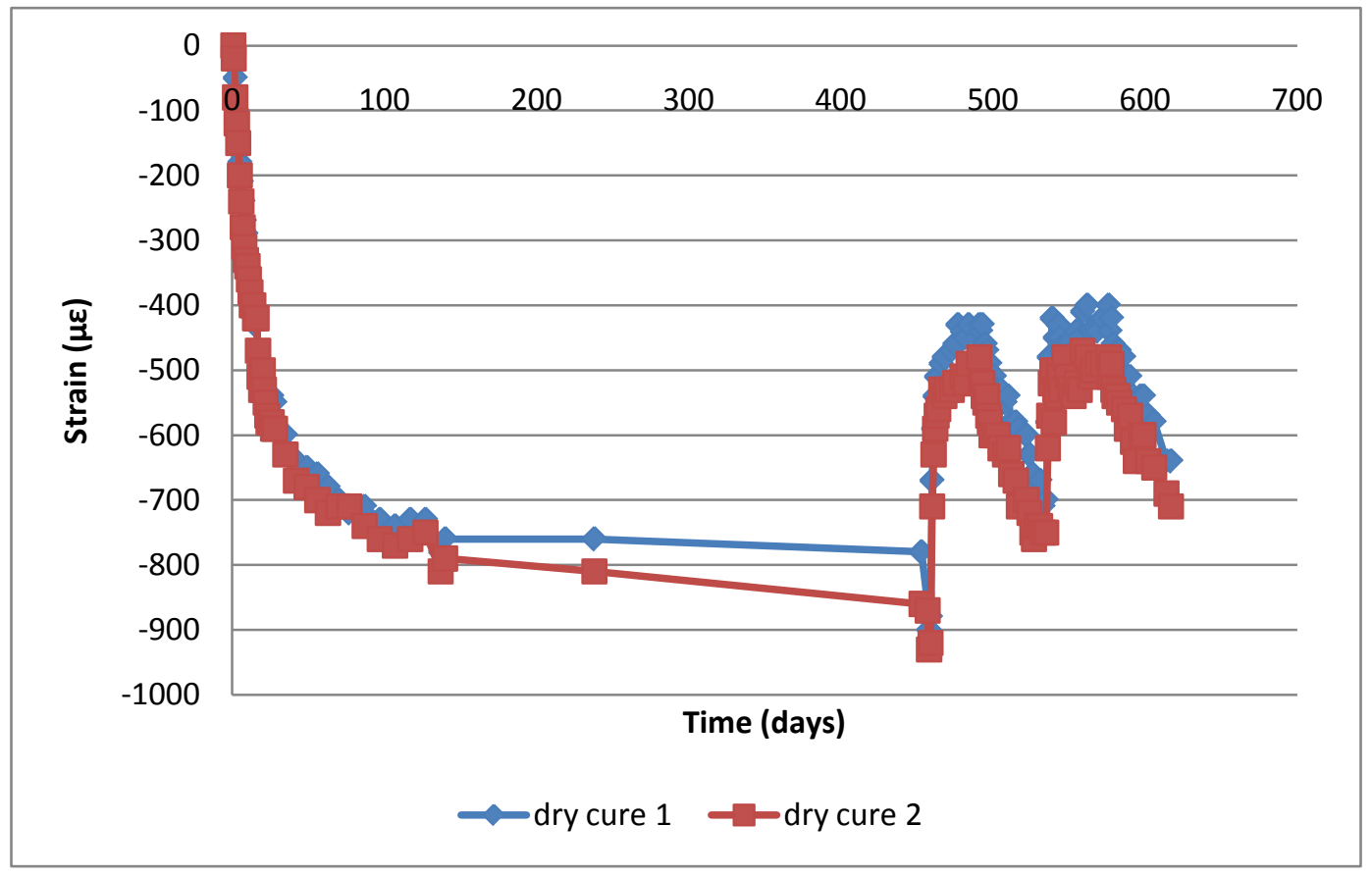

Figure 3.6: Strain versus time for dry cured lightweight aggregate concrete. 


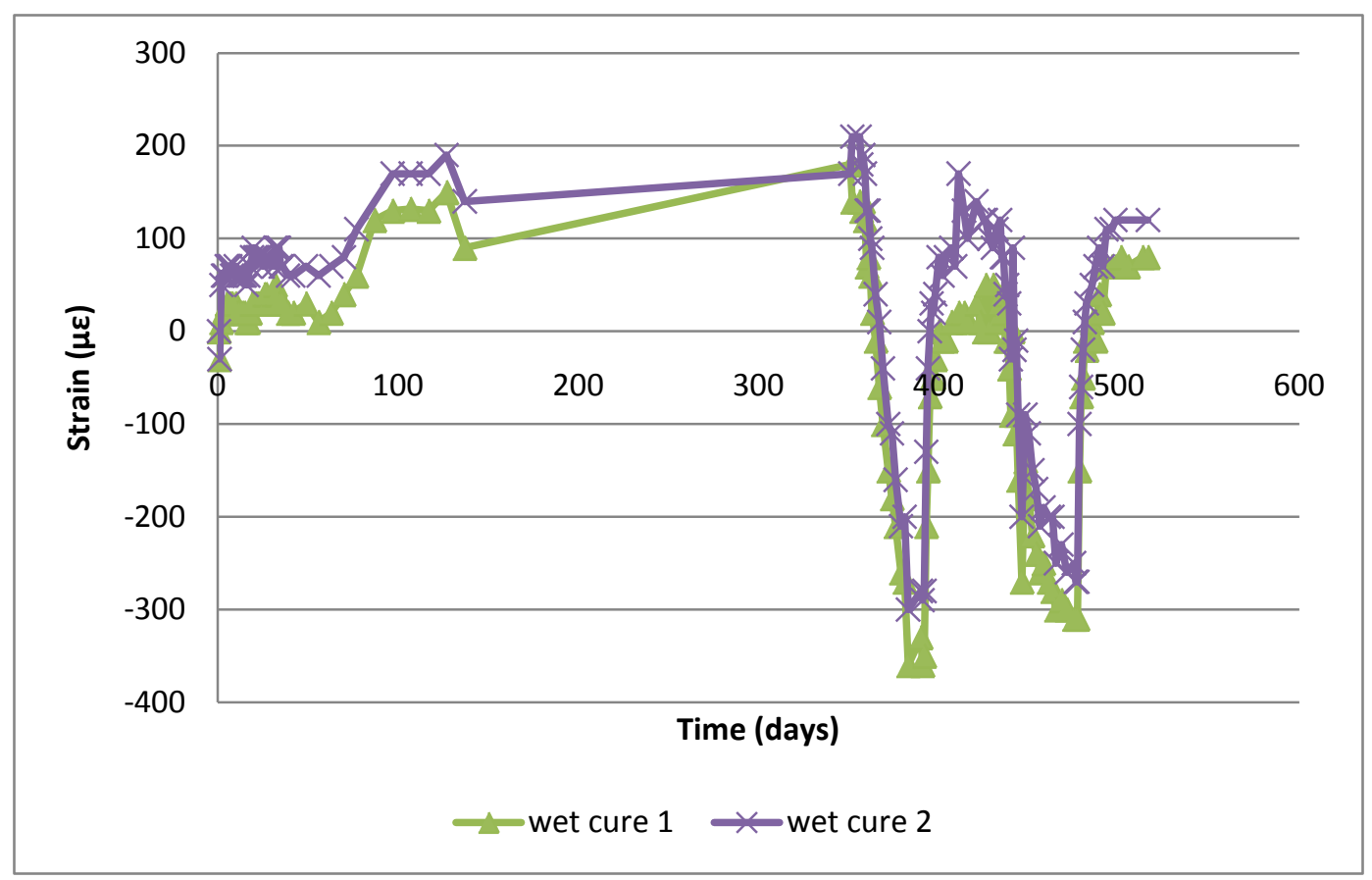

Figure 3.7: Strain versus time for wet cured lightweight aggregate.

\subsection{Analysis}

The behavior of the concrete subjected to cyclic drying and re-wetting was analyzed and the amount of reversible shrinkage was calculated for each cycle. Additionally, the effect of wet and dry curing on the amount of reversible shrinkage was examined. The classic assumption that weight change and shrinkage/growth are proportional was also evaluated. To compare the behavior of concrete made with virgin aggregates to concrete made with lightweight and recycled concrete aggregates, the amount of reversible shrinkage was compared with the permeability of the concrete and the porosity of the aggregates.

\subsubsection{Nomenclature}

Relatively few studies have investigated the behavior of concrete exposed to repeated drying and re-wetting. Often, the focus of previous studies has not been simply the behavior of the concrete, but rather the influence of different cementitious materials (Helmuth and Turk 1967) or on the effects of admixtures (Feldman and Swenson 1975). The few studies which focused purely on the behavior of plain concrete concerned themselves mainly with discussing broad trends, rather than defining specific terms. For these reasons, there is no standard terminology to describe the amount of shrinkage which is reversible or permanent for each cycle of drying and re-wetting. Therefore, it is necessary to first define the different parameters which will be discussed.

The behavior of the concrete subjected to cyclic drying and re-wetting is highly dependent upon the initial storage conditions (L'Hermite et al. 1949). Figure 3.8 shows a highly idealized curve of shrinkage over time for concrete subjected to cyclic drying and 
re-wetting when the concrete is initially stored in a dry environment until ultimate shrinkage has been reached. In contrast, Figure 3.9 shows the shrinkage with time for concrete subjected to cyclic drying and re-wetting when the concrete is initially stored in a dry environment until it ceases to swell.

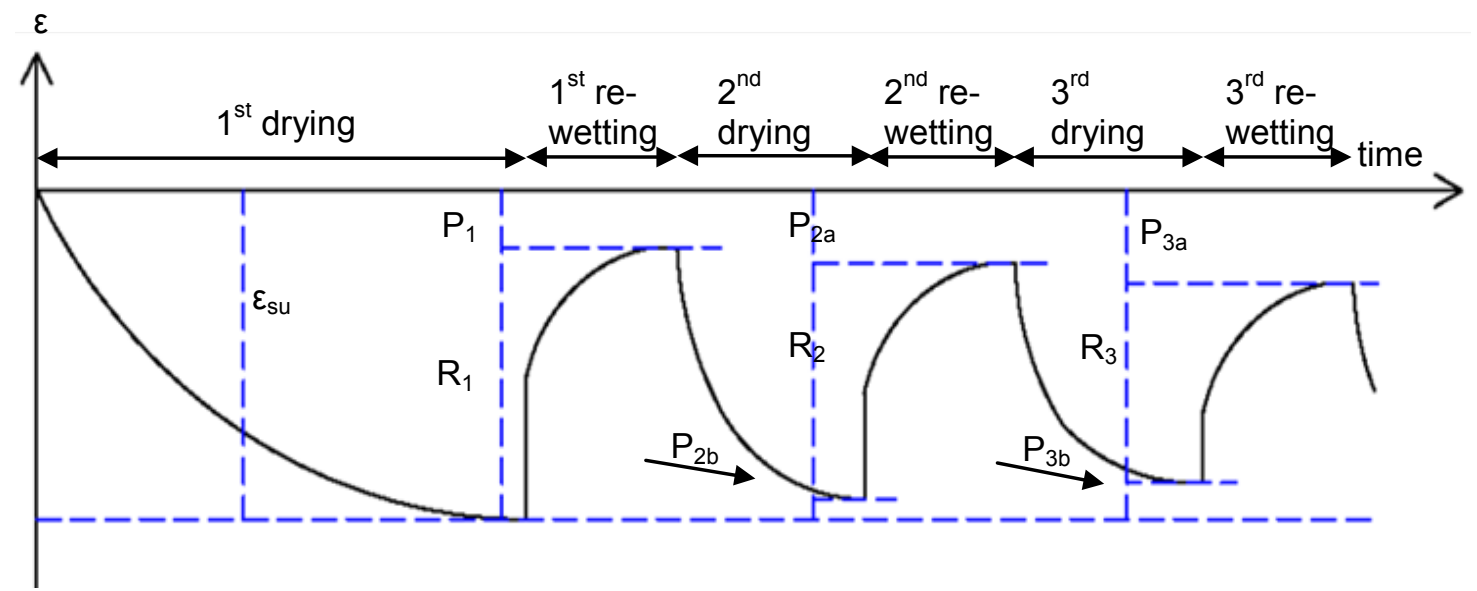

Figure 3.8: Highly idealized curve of shrinkage versus time for concrete initially exposed to drying followed by cyclic drying and re-wetting.

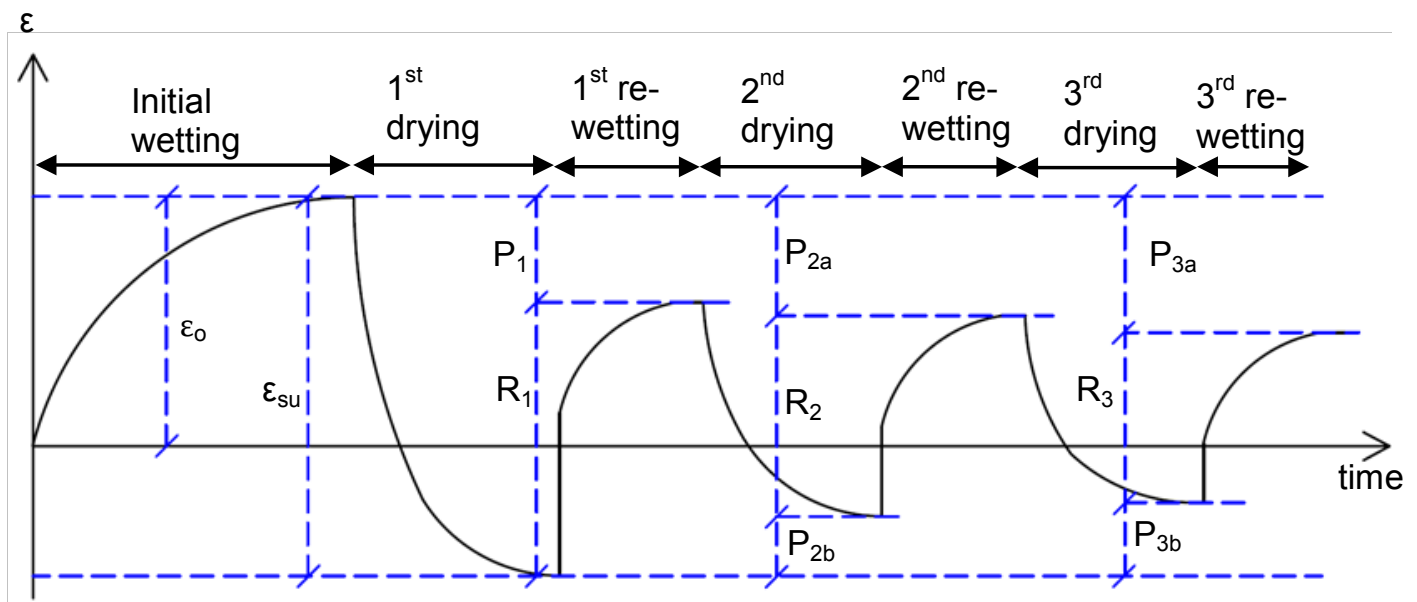

Figure 3.9: Highly idealized curve of shrinkage versus time for concrete initially exposed to wetting followed by cyclic drying and re-wetting.

From comparing these figures, it can be seen that concrete initially stored in water swells, but after that swelling, the curves are essentially identical. This initial swelling induces a strain in the concrete, which is not seen in the concrete preliminarily stored in air. In both cases, the first cycle of drying and rewetting results in a portion of the shrinkage being reversible $(R)$ and a portion of the shrinkage being permanent $(P)$. For subsequent cycles, the permanent portion is split into a primary portion (denoted by an $a$ ) and a secondary portion (denoted by a $b$ ). The total permanent shrinkage is the sum of these two portions. 
A cycle is defined as a drying phase followed by a re-wetting phase. For the dry cured samples, the cycle starts right away with a drying phase. For the wet cured samples, there is an initial wetting phase, followed by the first cycle of drying and re-wetting.

From Figure 3.8 and Figure 3.9, the following terms can be defined.

$\varepsilon_{\mathrm{o}}=$ The initial strain in the concrete at the end of the preliminary swelling if the concrete is wet cured. For concrete cured in air, this is equal to zero

$\varepsilon_{\mathrm{su}}=$ The ultimate shrinkage of the concrete, which is equal to the shrinkage at the end of the first drying cycle less the initial strain.

$$
\varepsilon_{\text {su }}=\text { strain at end of first drying cycle }-\varepsilon_{o}
$$

Equation 3.2

The amount of shrinkage which is reversed in the first re-wetting cycle,

$\mathrm{R}_{1}=$ which is the difference between the strain at the end of the first rewetting cycle and the strain at the end of the first drying cycle

$$
\begin{aligned}
R_{1}= & \text { strain at end of first rewetting cycle } \\
& - \text { strain at the end of the first dyring cycle }
\end{aligned}
$$

Equation 3.3

The portion of shrinkage which is not reversed by the first re-wetting $\mathrm{P}_{1}=$ cycle, which is equal to the difference between the initial strain and the strain at the end of the first re-wetting cycle

$$
P_{1}=\varepsilon_{o}-\text { strain at end of first rewetting cycle }
$$

Equation 3.4

After the first drying and re-wetting cycle, $\mathrm{R}$ and $\mathrm{P}$ of all subsequent cycles are computed in the same manner. Let $i$ be the cycle number.

The amount of shrinkage which is reversed in the second re-wetting

$\mathrm{R}_{i}=$ cycle, which is the difference between the strain at the end of the second re-wetting cycle and the strain at the end of the second drying cycle

$$
\begin{aligned}
& R_{i}=\text { strain at end of } i^{\text {th }} \text { rewetting cycle } \\
& \quad-\text { strain at end of } i^{\text {th }}
\end{aligned}
$$

Equation 3.5

The primary permanent shrinkage is part of shrinkage which is not $\mathrm{P}_{i \mathrm{a}}=$ reversed by the $i^{\text {th }}$ re-wetting cycle, and is equal to the difference between the initial strain and the strain at the end of the $i^{\text {th }}$ re-wetting cycle

$$
P_{i a}=\varepsilon_{o}-\text { strain at end of } i^{\text {th }} \text { rewetting cycle }
$$

$\mathrm{P}_{i \mathrm{~b}}=$ The secondary permanent shrinkage is a part of shrinkage which is not reversed by the $i^{\text {th }}$ re-wetting cycle and is equal to the difference between 
the ultimate shrinkage and the sum of the reversible shrinkage and the primary permanent shrinkage

$$
P_{i b}=\varepsilon_{o}-\varepsilon_{s u}-R_{i}-P_{i a}
$$

Equation 3.7

$\mathrm{P}_{i}=\begin{aligned} & \text { The total permanent shrinkage which is the sum of the primary and } \\ & \text { secondary permanent shrinkage from the } i^{\text {th }} \text { rewetting cycle. }\end{aligned}$

$$
\mathrm{P}_{\mathrm{i}}=\mathrm{P}_{\mathrm{ia}}+\mathrm{P}_{\mathrm{ib}}
$$

Equation 3.8

Reversible and permanent shrinkage values from subsequent drying and re-wetting cycles are calculated in the same manner. Percentages of $R_{1}, P_{l}$, etc. are found by dividing by the ultimate shrinkage $\varepsilon_{s u}$. Thus, the percent of reversible shrinkage from the second rewetting cycle, $\% R_{2}$, will be $R_{2} / \varepsilon_{s u}$.

\subsubsection{Magnitude of Ultimate and Drying Shrinkage}

Use of recycled concrete and lightweight aggregates generally increases both the ultimate and drying shrinkage of the concrete (Kosmatka et al. 2002). This is concerning for designers because increases in both ultimate shrinkage and drying shrinkage can lead to increased moisture warping and differential drying shrinkage, as well as increased cracking in the concrete.

In this study, the ultimate shrinkage, $\varepsilon_{s u}$, was measured as the shrinkage after at least 300 days of dry curing, or as the difference between the initial growth during wet curing and the strain at the end of the first drying cycle. As part of Shorkey's study (2010), the autogenous shrinkage was also measured using sealed samples. The drying shrinkage was calculated by subtracting the autogenous shrinkage from the ultimate shrinkage measured from the dry cured samples. Table 3.4 shows the amount of ultimate and drying shrinkage for each aggregate type. Because it is very rare for concrete in a construction application to be wet cured for a year, dry cured values for ultimate shrinkage better reflect concrete that is placed in the field.

Table 3.4

Ultimate and drying shrinkage for different aggregate types.

\begin{tabular}{|c|c|c|c|c|}
\hline Aggregate Type & $\begin{array}{c}\varepsilon_{\mathrm{su}}, \text { Dry } \\
\text { Cure }(\mu \varepsilon)\end{array}$ & $\begin{array}{c}\varepsilon_{\mathrm{su}}, \text { Wet } \\
\text { Cure }(\mu \varepsilon)\end{array}$ & $\begin{array}{c}\text { Drying } \\
\text { Shrinkage }(\mu \varepsilon)\end{array}$ & $\begin{array}{c}\text { Drying Shrinkage as a } \\
\text { Percent of Ultimate }\end{array}$ \\
\hline Virgin & -830 & -555 & -208 & $25 \%$ \\
\hline Slag RCA & -615 & -810 & -305 & $50 \%$ \\
\hline Limestone RCA & -650 & -680 & -155 & $24 \%$ \\
\hline Gravel RCA & -983 & -580 & -430 & $44 \%$ \\
\hline Twice Recycled & -630 & -390 & -375 & $60 \%$ \\
\hline Lightweight & -873 & -508 & -348 & $40 \%$ \\
\hline
\end{tabular}


From Table 3.4, it can be seen that the dry cured virgin aggregate concrete experienced slightly more ultimate shrinkage than is typically expected, but is still in the normal range. The dry cured slag, limestone, and twice recycled RCA mixes all experienced ultimate shrinkage of the magnitude expected for normal concrete, while the dry cured gravel RCA and lightweight concrete experienced more shrinkage. The wet cured mixes all experienced less ultimate shrinkage than their dry cured counterparts, with the exception of the slag and limestone RCA concretes. It is expected that wet cured concrete undergoes less shrinkage because there is initially no drying shrinkage due to water loss. The limestone RCA concrete did not have a significant difference in the ultimate shrinkage between the wet and dry cured samples, while the slag RCA concrete had much higher ultimate shrinkage for the wet cured sample. This may be because the concrete made from both the slag and limestone RCA was very permeable, which would allow more water both into and out of the concrete's pore system.

The amount of drying shrinkage for all concretes, except for the limestone RCA, was greater than that of the virgin aggregate concrete. This fits with established literature that drying shrinkage is more prevalent in concrete made with lightweight and RCA (Sato et al. 2007; Domingo et al. 2010). The percentage of shrinkage due to drying was also much higher for the recycled concrete and lightweight mixes than for the virgin mix, again with the exception of the limestone RCA. However, this can be attributed to the low drying shrinkage of that mix. The comparatively low drying shrinkage of the limestone RCA concrete may be due to the fact that the limestone RCA was crushed in such a way that it had much less residual mortar than the other RCA samples. This caused the concrete made from the limestone RCA to behave more like concrete made from virgin aggregates.

Comparing the ultimate and drying shrinkage to both the rapid chloride permeability of the concrete (obtained from ASTM 2008f) and the porosity of the aggregates, no direct trends were found; see Figure 3.10 and Figure 3.11, respectively. It may be that there are simply too few samples included in this study for a discernable trend to be determined. 


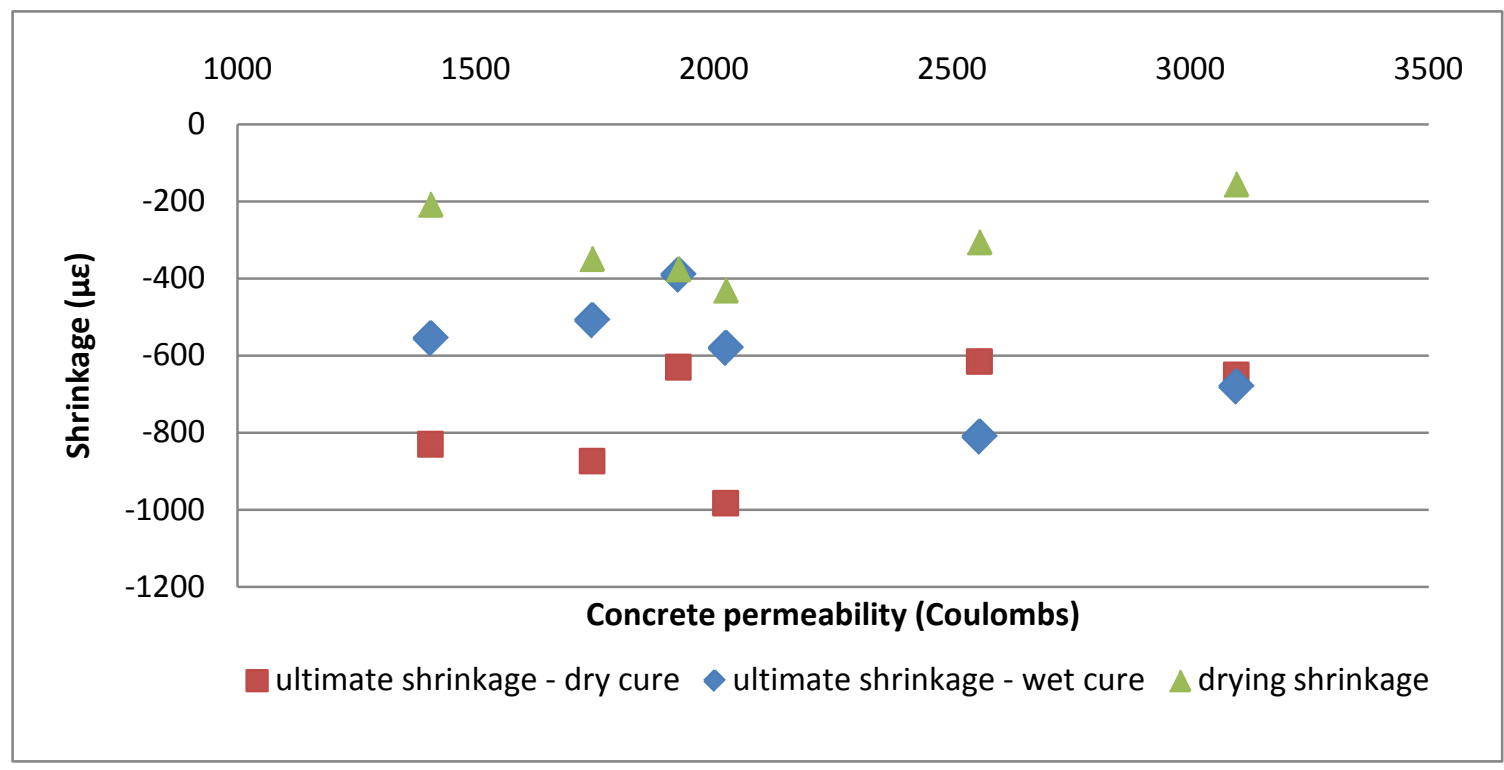

Figure 3.10: Shrinkage versus concrete permeability.

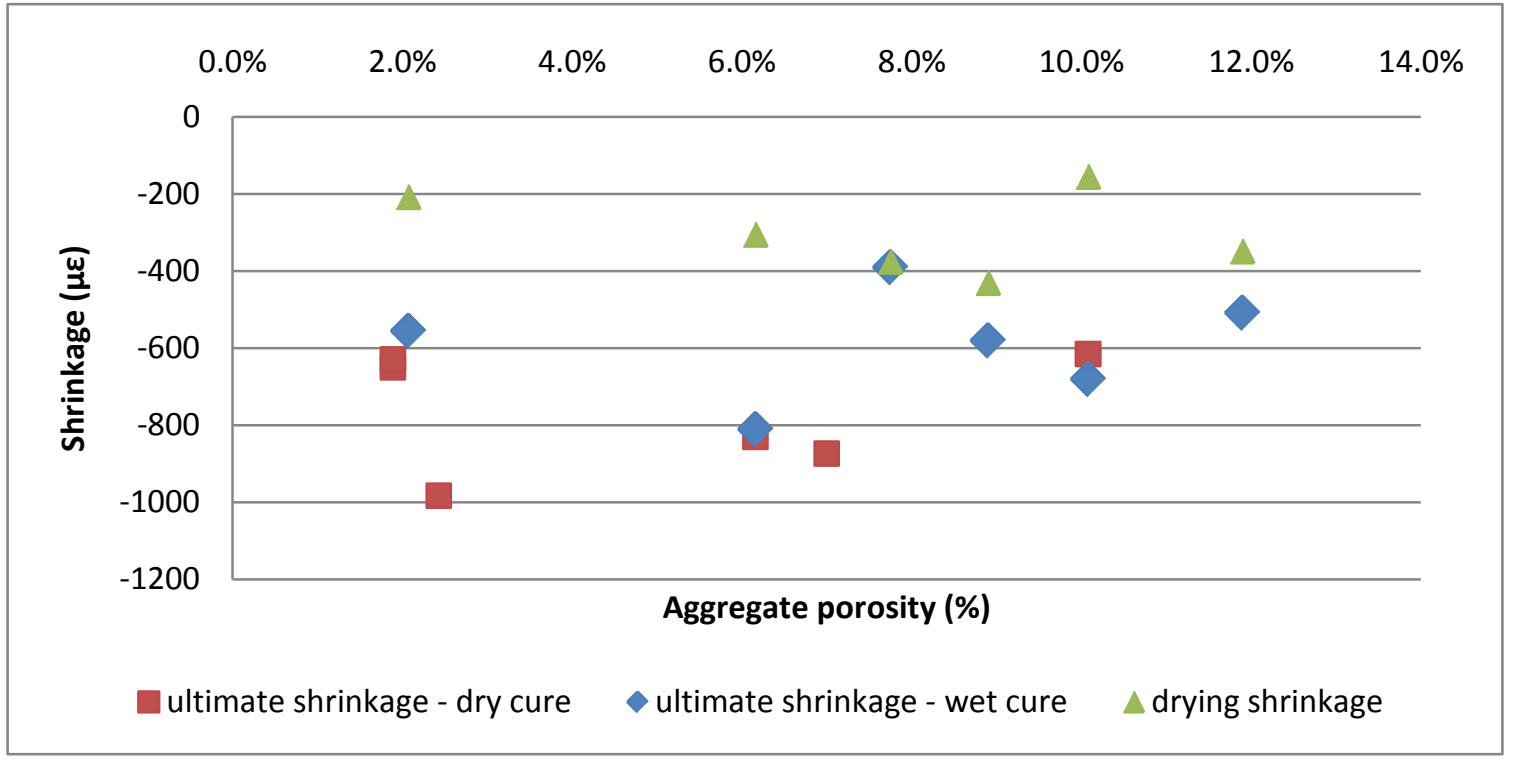

Figure 3.11: Shrinkage versus aggregate porosity.

\subsubsection{Reversible Shrinkage as a Function of Drying and Re-Wetting Cycles}

Previous studies have found that the curing regime plays a role in determining the reversible shrinkage of the concrete (L'Hermite 1947). A specimen stored in a wet environment will be more hydrated than one stored in a dry environment after this initial period. More importantly, the hydration process for the specimen in the wet environment will take place when the specimen is swelled from excess relative humidity. This growth causes the cement particles to be further apart when the C-S-H gels form (Neville 1997). The resulting microstructure is locked in due to the almost fully hydrated state of the 
paste, but may allow for more reversible shrinkage due to this increased C-S-H gel pore spacing.

In contrast, a sample stored initially in a dry environment will form C-S-H gels when the cement particles are closer together, and bonds will form between these gels holding the particles closer together. However, dry curing results in a less fully hydrated system, and the microstructure is less locked in. When the samples are re-wetted, they will further hydrate, but will do so with the concrete in a swelled state, which puts the cement particles and their surrounding gels further apart. Reversible shrinkage was calculated using Equation 3.3 and Equation 3.5. The effects of wet and dry curing on the reversible shrinkage can be seen in Figure 3.12, which shows the percent of reversible shrinkage for concrete of each aggregate type for drying and wetting cycle. In almost all cases, the wet cured samples have higher amounts of reversible shrinkage than their dry cured counterparts. The magnitude of the reversible shrinkage for the virgin aggregate concrete fit well with that of the established literature for both wet and dry cured samples (L'Hermite et al. 1949; Helmuth and Turk 1967).

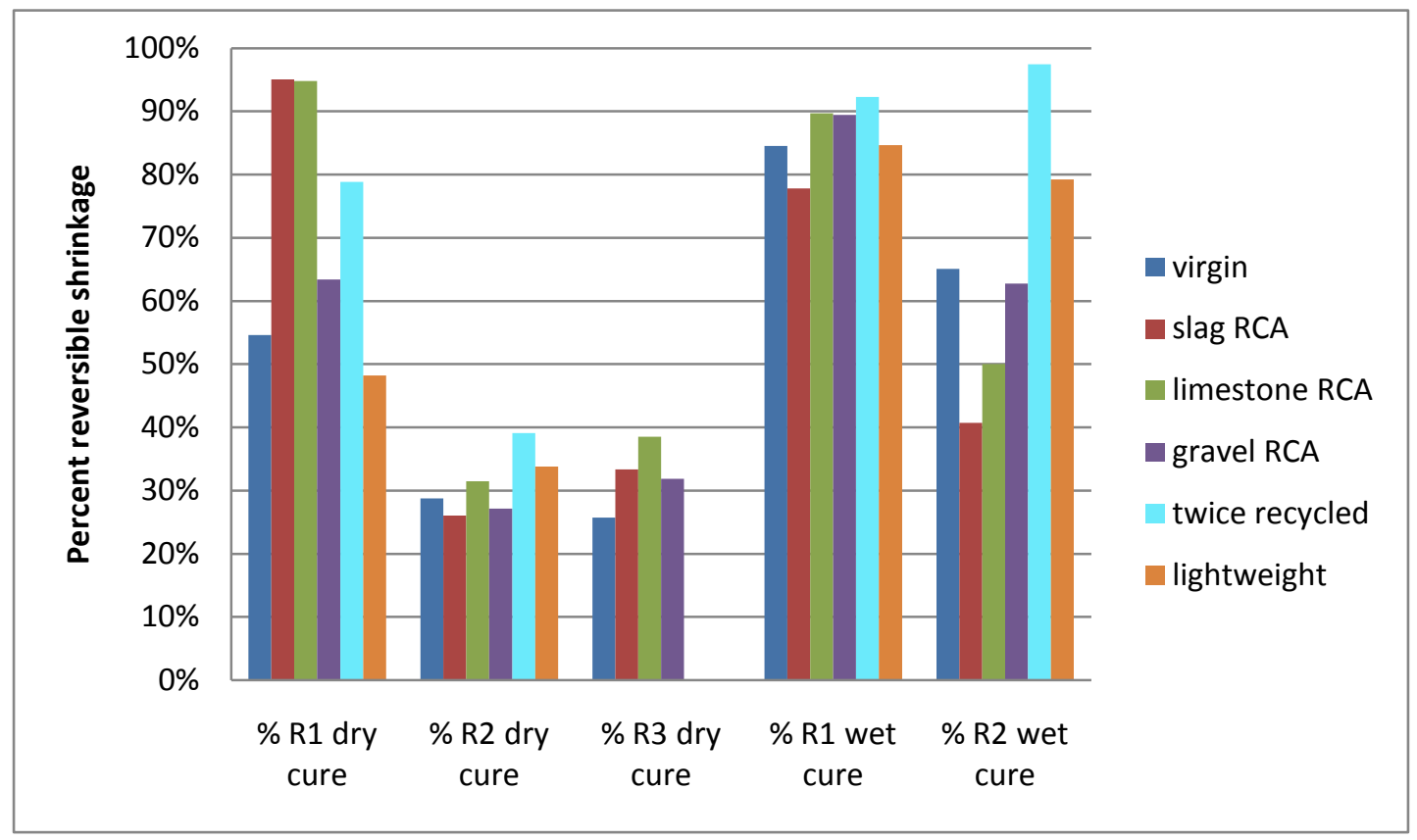

Figure 3.12: Reversible shrinkage for different drying and re-wetting cycles.

From Figure 3.12, it can be seen that the reversible shrinkage decreases between drying and re-wetting cycles 1 and 2 for all dry cured samples, but this decrease is much more drastically for the concretes made from slag, limestone and twice recycled aggregates. The reversible shrinkage showed no appreciable change between cycles 2 and 3 for all dry cured samples. For the wet cured samples, the reversible shrinkage decreased between cycles 1 and 2 for all samples except the twice recycled and lightweight, which did not change significantly. 
The lack of a change between $R_{1}$ and $R_{2}$ for the wet cured lightweight and twice recycled samples may be due to a lower modulus of elasticity of the aggregate in conjunction with the high coarse aggregate content used in this paving mix. Lightweight aggregate is known to have a lower modulus of elasticity than regular aggregate (Neville 1997), and the concrete made from lightweight aggregates in this study also had a lower modulus of elasticity than the concrete made from virgin, or recycled aggregates (Shorkey 2010). Modulus of elasticity testing was not conducted on the concrete made from the twice recycled aggregates, due to a lack of aggregate material. However, the twice recycled aggregates had significantly more adhered paste than other aggregates, likely a result of both its previous use and the crushing process. Given that paste has a lower modulus of elasticity than high quality original aggregate, increased paste content would lower the modulus of the twice recycled aggregate.

A lower elastic modulus could mean that the aggregates are excessively deforming elastically, in addition to restraining the shrinkage/growth in the twice recycled and lightweight aggregate samples. Lower strength and lightweight aggregates have been found to experience increased shrinkage due to less restraint caused by lower elastic moduli (Mindness et al. 2003). Alternately, internal curing due to increased porosity could be increasing the hydration of the lightweight aggregate concrete, though this is unlikely as all specimens were wet cured and therefore well hydrated. This also fails to explain the behavior of the twice recycled aggregate. More testing will be necessary to determine if the lower modulus of elasticity does indeed play a role in the lack of reduction in reversible shrinkage. Further testing is also needed to determine the value of $R_{3}$ for the wet cured samples, and how it compares to $R_{2}$.

The consistency between $R_{2}$ and $R_{3}$ indicates that, after the first rewetting cycle, the amount of reversible shrinkage stabilizes. This is likely due to increased hydration achieved during the first re-wetting cycle, which locked in the microstructure of the concrete. This microstructure then did not change appreciably between the second and third drying and re-wetting cycles, resulting in the same amount of reversible shrinkage for both. Further testing will be necessary to determine the value of $R_{4}$, and how it compares to $R_{2}$ and $R_{3}$.

The amount of reversible shrinkage attained after prolonged drying and re-wetting is of interest from a general materials perspective. From a design perspective, most structures or pavements exposed to cyclic drying and re-wetting due to ambient conditions are not going to be exposed to wetting at $100 \%$ relative humidity for 30 days. During a rain event, however, it is very reasonable to expect 24 hours of wetting at $100 \%$ relative humidity. Wetting due to high ambient relative humidity would also cause shrinkage to reverse, but not as much as rain event of the same duration. The amount of reversible shrinkage which occurs after 24 hours was computed from Equation 3.5, using the strain after 24 hours of re-wetting rather than after 30 days. The ratio of this 24 hour reversible shrinkage to the total reversible shrinkage for that drying and re-wetting cycle was also computed. The results of these computations are given in Table 3.5 
Table 3.5

Calculated reversible shrinkage after 24 hours of re-wetting and percent of total reversible shrinkage which occurs in the first 24 hours of re-wetting.

\begin{tabular}{|c|c|c|c|c|c|c|c|}
\hline & $\begin{array}{l}\text { Cure } \\
\text { type }\end{array}$ & Virgin & $\begin{array}{l}\text { Slag } \\
\text { RCA }\end{array}$ & $\begin{array}{l}\text { Limestone } \\
\text { RCA }\end{array}$ & $\begin{array}{c}\text { Gravel } \\
\text { RCA }\end{array}$ & $\begin{array}{c}\text { Twice } \\
\text { Recycled }\end{array}$ & $\begin{array}{l}\text { Light- } \\
\text { weight }\end{array}$ \\
\hline$\% \mathrm{R}_{1,24 \mathrm{hr}}$ & \multirow{3}{*}{$\begin{array}{l}\text { Dry } \\
\text { cure }\end{array}$} & $33 \%$ & $54 \%$ & $55 \%$ & $40 \%$ & $35 \%$ & $25 \%$ \\
\hline$\% \mathrm{R}_{2,24 \mathrm{hr}}$ & & $17 \%$ & $12 \%$ & $19 \%$ & $16 \%$ & $17 \%$ & $17 \%$ \\
\hline$\% \mathrm{R}_{3,24 \mathrm{hr}}$ & & $13 \%$ & $15 \%$ & $18 \%$ & $16 \%$ & - & - \\
\hline$\% \mathrm{R}_{1,24 \mathrm{hr}}$ & \multirow{2}{*}{$\begin{array}{l}\text { Wet } \\
\text { cure }\end{array}$} & $47 \%$ & $52 \%$ & $60 \%$ & $43 \%$ & $36 \%$ & $33 \%$ \\
\hline$\% \mathrm{R}_{2,24 \mathrm{hr}}$ & & $39 \%$ & $28 \%$ & $35 \%$ & $40 \%$ & $41 \%$ & $37 \%$ \\
\hline $\mathrm{R}_{1,24 \mathrm{hr}} / \mathrm{R}_{1}$ & \multirow{3}{*}{$\begin{array}{l}\text { Dry } \\
\text { cure }\end{array}$} & $63 \%$ & $57 \%$ & $59 \%$ & $63 \%$ & $43 \%$ & $53 \%$ \\
\hline $\mathrm{R}_{2,24 \mathrm{hr}} / \mathrm{R}_{2}$ & & $61 \%$ & $47 \%$ & $61 \%$ & $61 \%$ & $43 \%$ & $49 \%$ \\
\hline $\mathrm{R}_{3,24 \mathrm{hr}} / \mathrm{R}_{3}$ & & $51 \%$ & $44 \%$ & $48 \%$ & $49 \%$ & - & - \\
\hline $\mathrm{R}_{1,24 \mathrm{hr}} / \mathrm{R}_{1}$ & \multirow{2}{*}{$\begin{array}{l}\text { Wet } \\
\text { cure }\end{array}$} & $56 \%$ & $67 \%$ & $67 \%$ & $56 \%$ & $39 \%$ & $39 \%$ \\
\hline $\mathrm{R}_{2,24 \mathrm{hr}} / \mathrm{R}_{2}$ & & $61 \%$ & $70 \%$ & $71 \%$ & $64 \%$ & $42 \%$ & $47 \%$ \\
\hline
\end{tabular}

From Table 3.5, it can be seen that $43-63 \%$ of total reversible shrinkage occurs within the first 24 hours of re-wetting for dry cured specimens. For wet cured concrete, this range is slightly larger, $39-71 \%$. This is important because most concrete in use, particularly in pavements, is not exposed to 30 day periods of wetting. The percentage of total reversible shrinkage which occurs in 24 hours does decrease with subsequent drying and re-wetting cycles, but only slightly. The finding that a large portion of the reversible shrinkage occurs within the first day of re-wetting agrees with the results of L'Hermite (1947), and Helmuth and Turk (1967), though that study included only cement paste without the influence of coarse aggregates.

\subsubsection{Permanent Shrinkage as a Function of Drying and Re-wetting Cycles}

The permanent component of shrinkage can be divided into primary and secondary components, as seen in Figure 3.8 and Figure 3.9. Previous studies have only examined the primary component, which is the permanent shrinkage after one drying and re-wetting cycle. Subsequent cycles of drying and re-wetting result in a secondary component of shrinkage. The secondary portion of permanent shrinkage is caused by the same drying shrinkage mechanisms as the primary component, it just occurs during later drying and re-wetting cycles.

The permanent portion of the ultimate shrinkage is the sum of the autogenous shrinkage, and the irreversible portion of drying shrinkage. In the first drying and re-wetting cycle, the permanent shrinkage $P_{1}$ will include both these components. Autogenous shrinkage occurs at early ages due to the hydration process, and can be considered as complete before the alternate relative humidity storage began. For subsequent drying and rewetting cycles, any additional permanent shrinkage will be secondary permanent shrinkage, which is only due to additional, irreversible drying shrinkage. This is 
illustrated in Figure 3.13 and Figure 3.14, which show the reversible and permanent portions of shrinkage for dry and wet cured samples, respectively. The primary permanent shrinkage was calculated using Equation 3.6 while the secondary shrinkage was calculated using Equation 3.7.

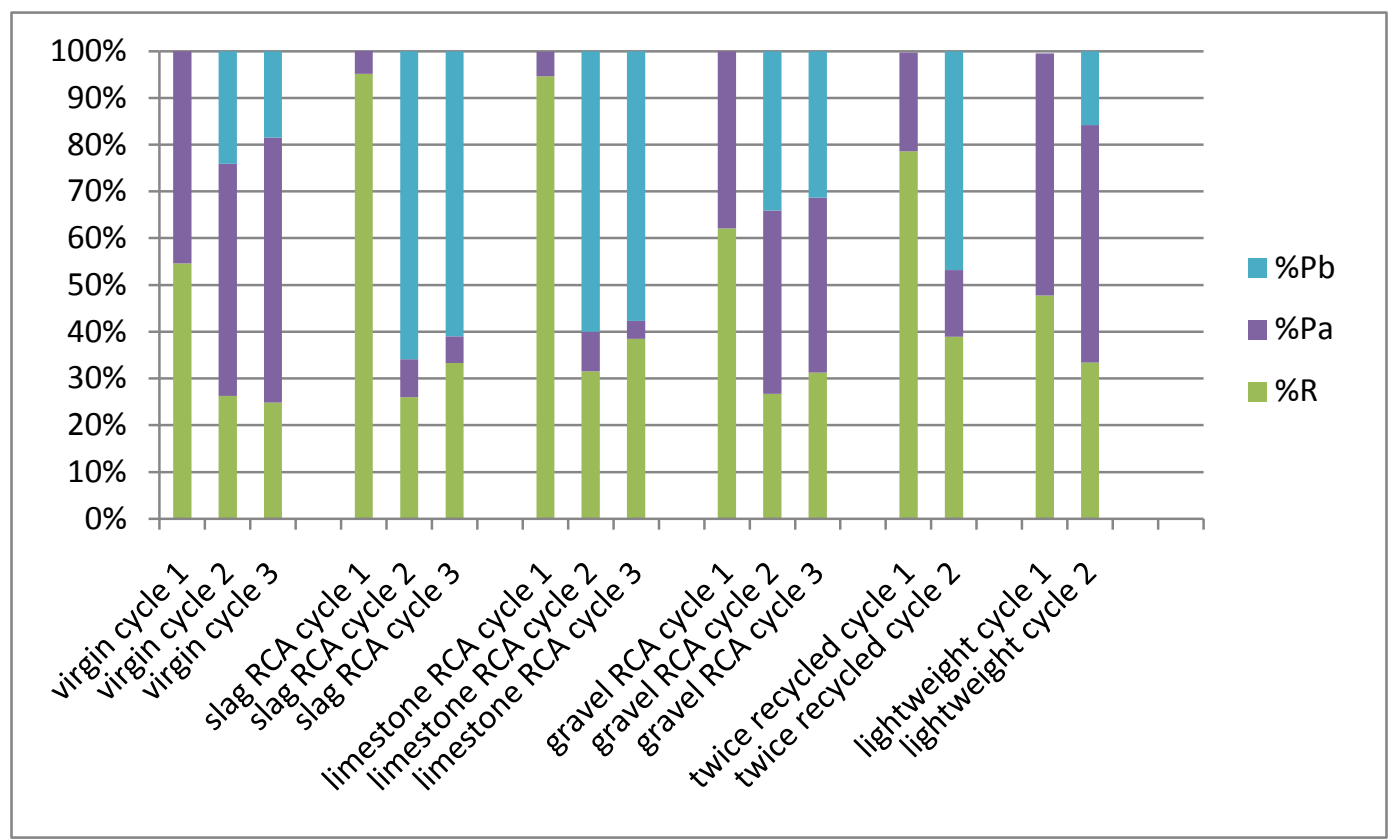

Figure 3.13: Reversible and permanent portions of ultimate shrinkage for samples which were originally dry cured.

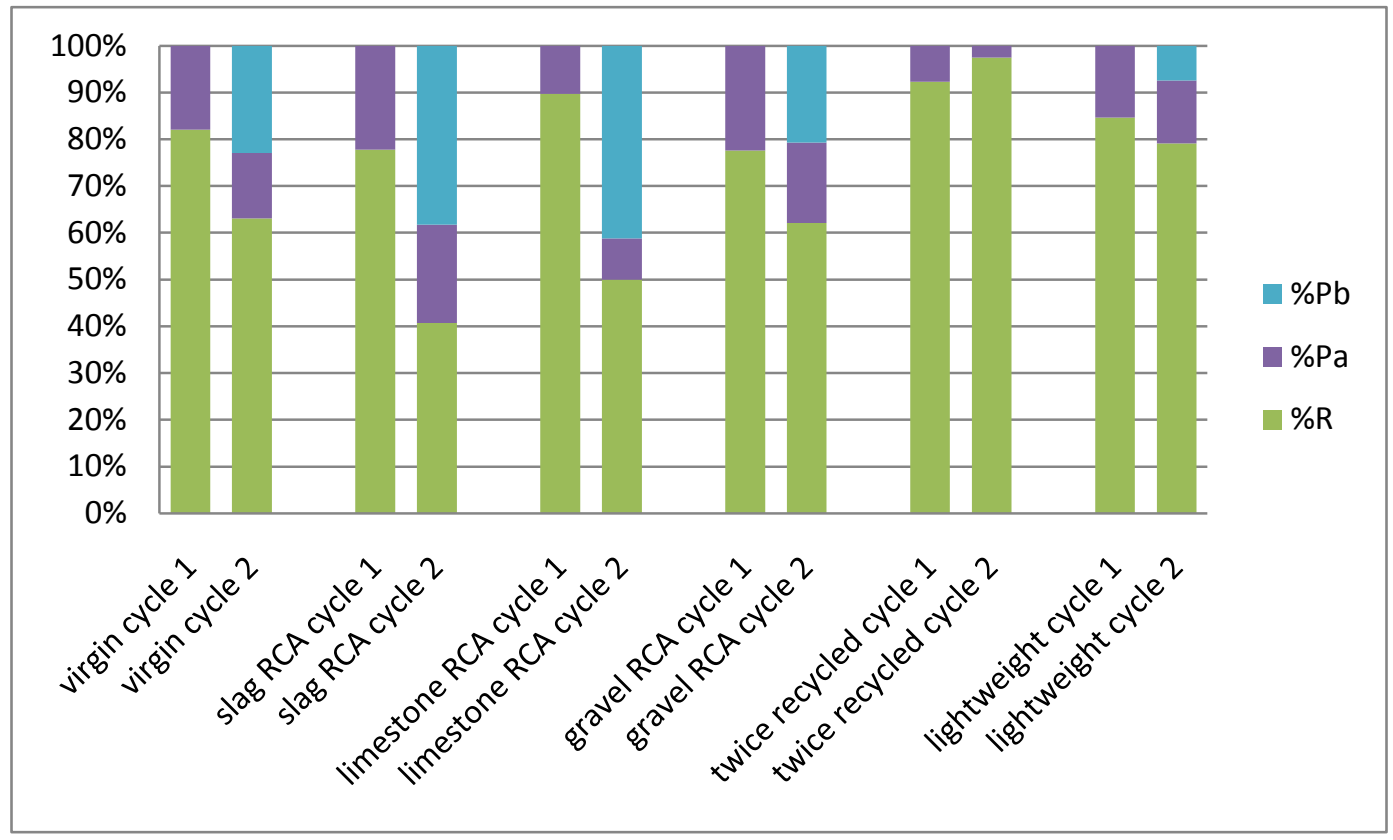

Figure 3.14: Reversible and permanent portions of ultimate shrinkage for samples which were originally wet cured. 
From these figures, it can be seen that the amount of primary permanent shrinkage, $P_{a}$, does not change significantly with subsequent drying and re-wetting cycles. Instead, all additional permanent shrinkage is secondary permanent shrinkage, $P_{b}$. This is true for both wet and dry cured samples. For some of the wet cured samples, there was relatively little change in the amount of reversible shrinkage between the first and second drying and re-wetting cycles. In these cases, there was no increase in the amount of permanent shrinkage, and therefore, all permanent shrinkage was still attributed to primary permanent shrinkage. This supports the theory that secondary permanent shrinkage is only due to additional drying shrinkage from subsequent drying and re-wetting cycles.

\subsubsection{Reversible Shrinkage as a Function of Concrete Permeability and Aggregate Porosity}

The permeability of concrete is affected by the internal pore structure. For pores to transport water, they must be sufficiently large to allow water molecules to pass, and be connected to the exterior of the sample (Neville 1997). The permeability of concrete is a function mainly of the water to cement ratio of the paste and the degree of hydration (FIB 2008), as well as the permeability of the aggregates and the air entrainment (Kosmatka et al. 2002). The porosity of aggregates can contribute to the permeability of concrete because the pores in the aggregate are part of the pore structure of the concrete matrix. If the pores in the aggregate of are sufficient size and are connected to the pores in the cement paste, then they would increase the permeability of the concrete (Neville 1997).

Shorkey (2010) found no correlation between concrete permeability and aggregate porosity for the materials used in this study. This may be due to the fact that, for water to cement ratios of 0.42 and above, the porosity of the paste, and therefore the permeability of the concrete increases drastically. At this water to cement ratio, the porosity of the paste becomes the dominant factor in determining the permeability of the paste, eclipsing all other factors (Mindness et al. 2003). All of the mixes used in this study had a water to cement ratio of 0.42 . Therefore, the amount of reversible shrinkage must be compared to both concrete permeability and aggregate porosity to determine if a trend exists, though it is unlikely that a correlation with aggregate porosity could be found.

Figure 3.15 shows the amount of reversible shrinkage for the different drying and rewetting cycles versus the permeability of the concrete. From this figure, it can be seen that the percent of shrinkage which is reversible is only dependent on the permeability of the concrete for the first drying and re-wetting cycle of the dry cured samples. For the wet cure samples and all drying and re-wetting cycles other than the first cycle of the dry cure samples, the permeability of the concrete is not correlated with reversible shrinkage. The initially dry cured samples are not fully hydrated before the first re-wetting cycle; while after the first re-wetting cycle, the hydration process is much more complete, as it is for the wet cured samples. This hydration process locks in the microstructure of the concrete matrix, preventing further changes. 


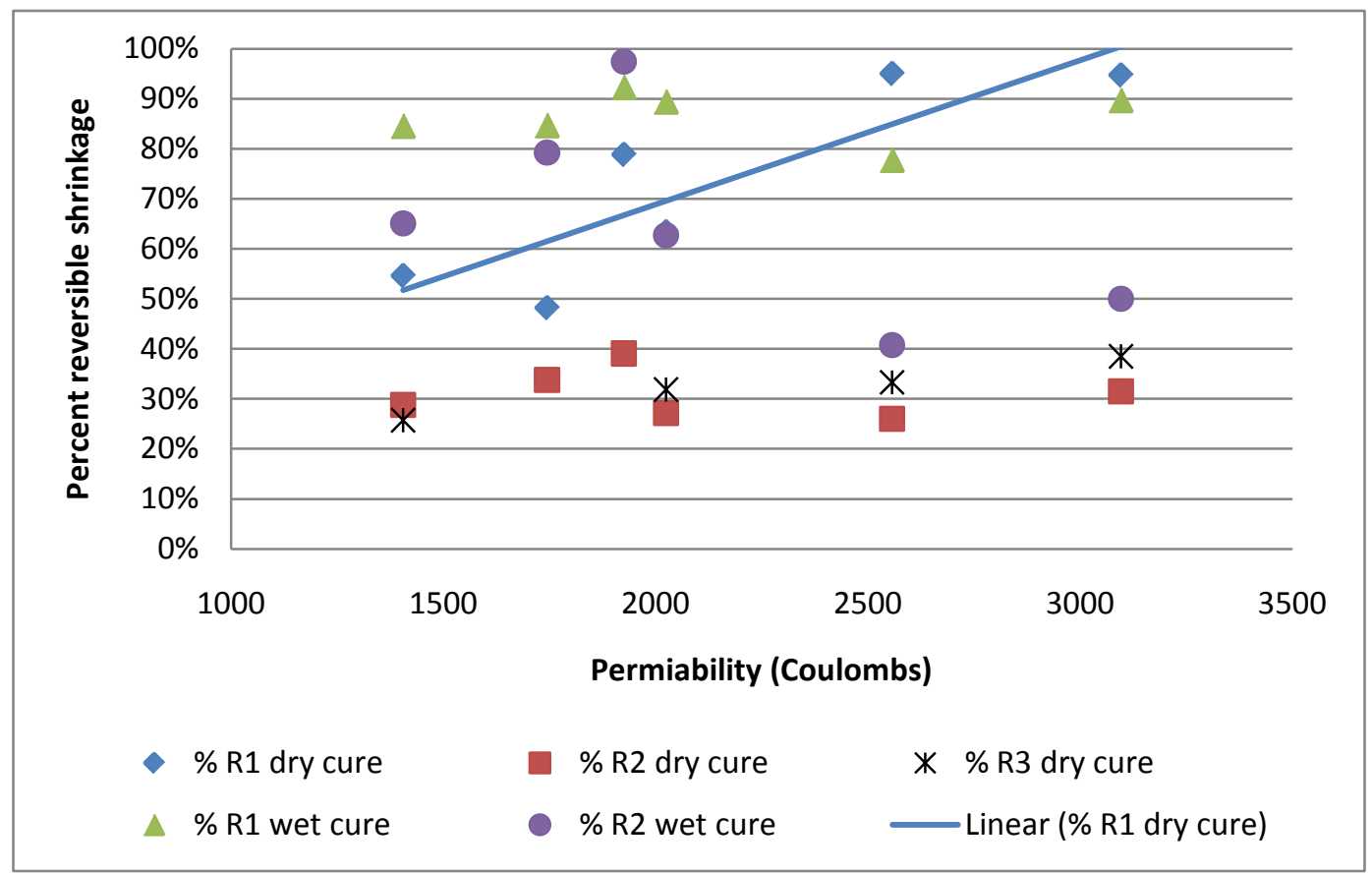

Figure 3.15: Reversible shrinkage versus concrete permeability as measured by the rapid chloride permeability test.

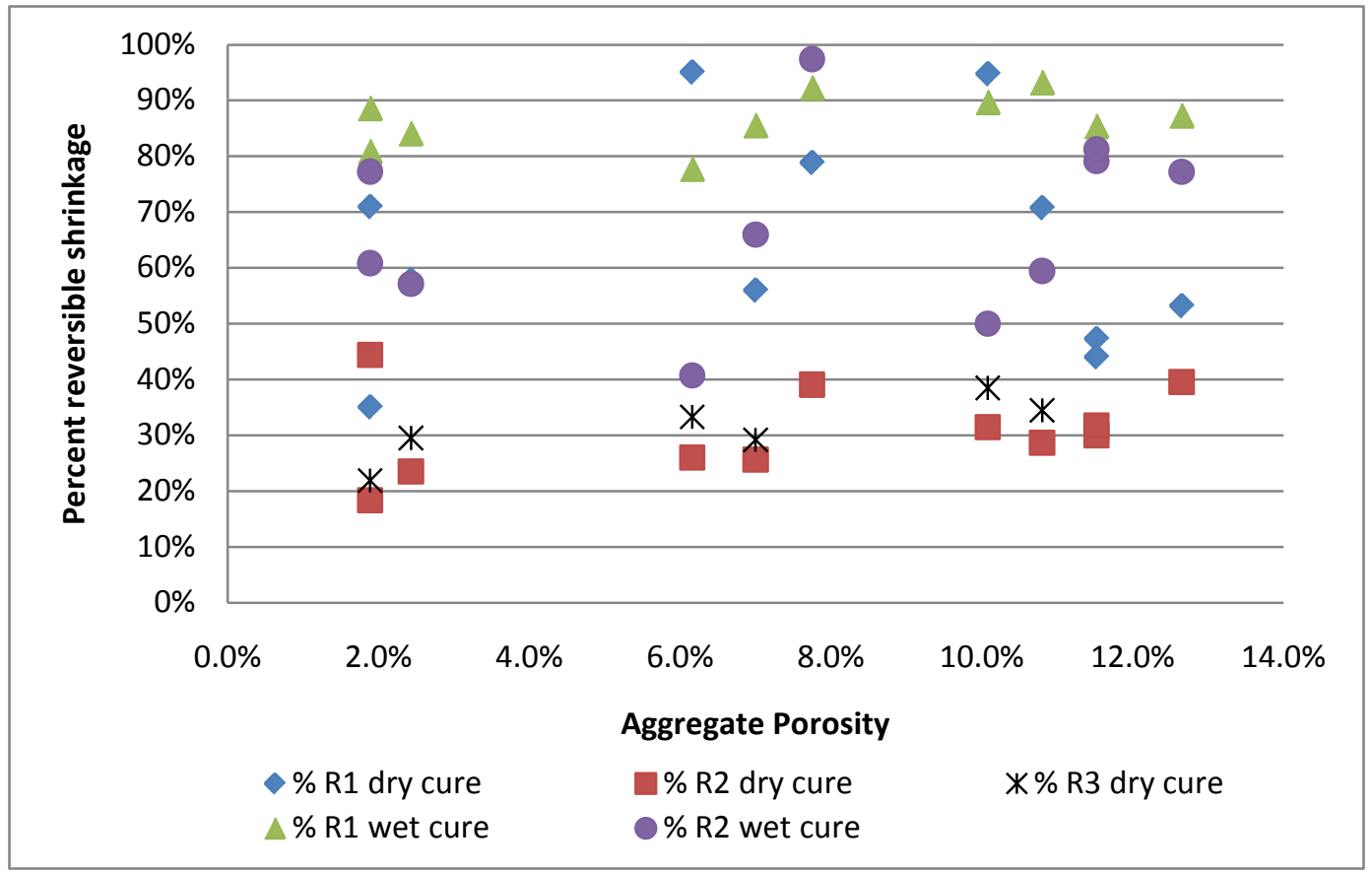

Figure 3.16: Reversible shrinkage versus aggregate porosity. 
Figure 3.16 shows the amount of reversible shrinkage versus the porosity of the aggregates. From this figure, it can be seen that no trend exists in the data. This is expected as there was no correlation between the aggregate porosity and the concrete permeability, likely due to the water to cement ratio being above the 0.42 threshold. Further research using concretes made with lower water to cement ratios could yield a correlation between aggregate porosity and reversible shrinkage.

\subsubsection{Shrinkage versus Weight Change}

One classic assumption made in the study of volume change in concrete is that growth and shrinkage are proportional to changes in weight (Dutron 1934; Granger et al. 1994; Torrenti et al. 1999); however, nowhere in the literature is there any indication that this assumption has been tested. Therefore, the length change as a function of weight change was plotted for each sample. Full results for all samples are given graphically in Appendix D, while selected results are shown below in Figure 3.17, Figure 3.18, and Figure 3.19 for a virgin, recycled concrete, and lightweight aggregate, respectively.

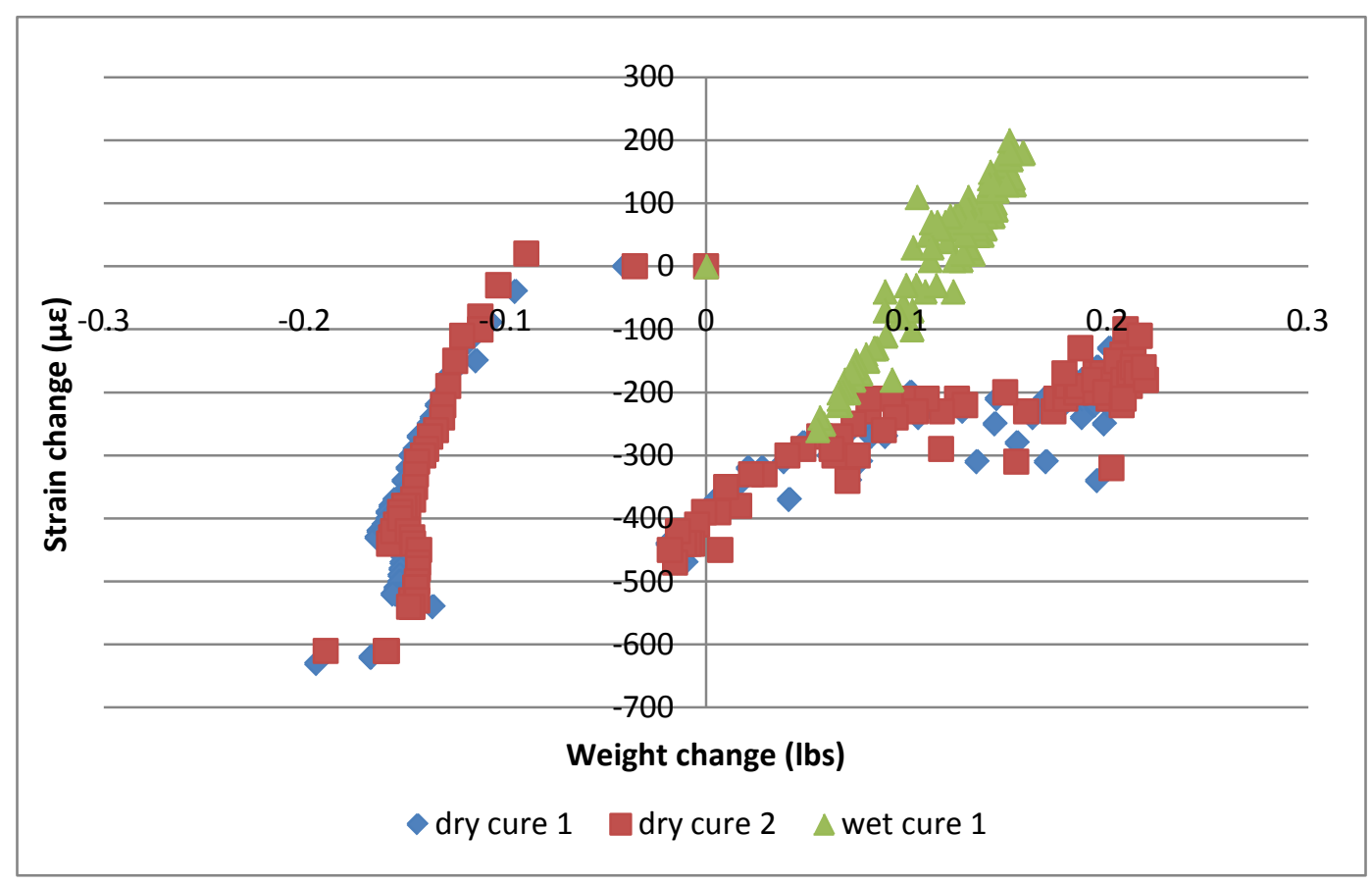

Figure 3.17: Change in strain as a function of change in weight for a virgin aggregate concrete. 


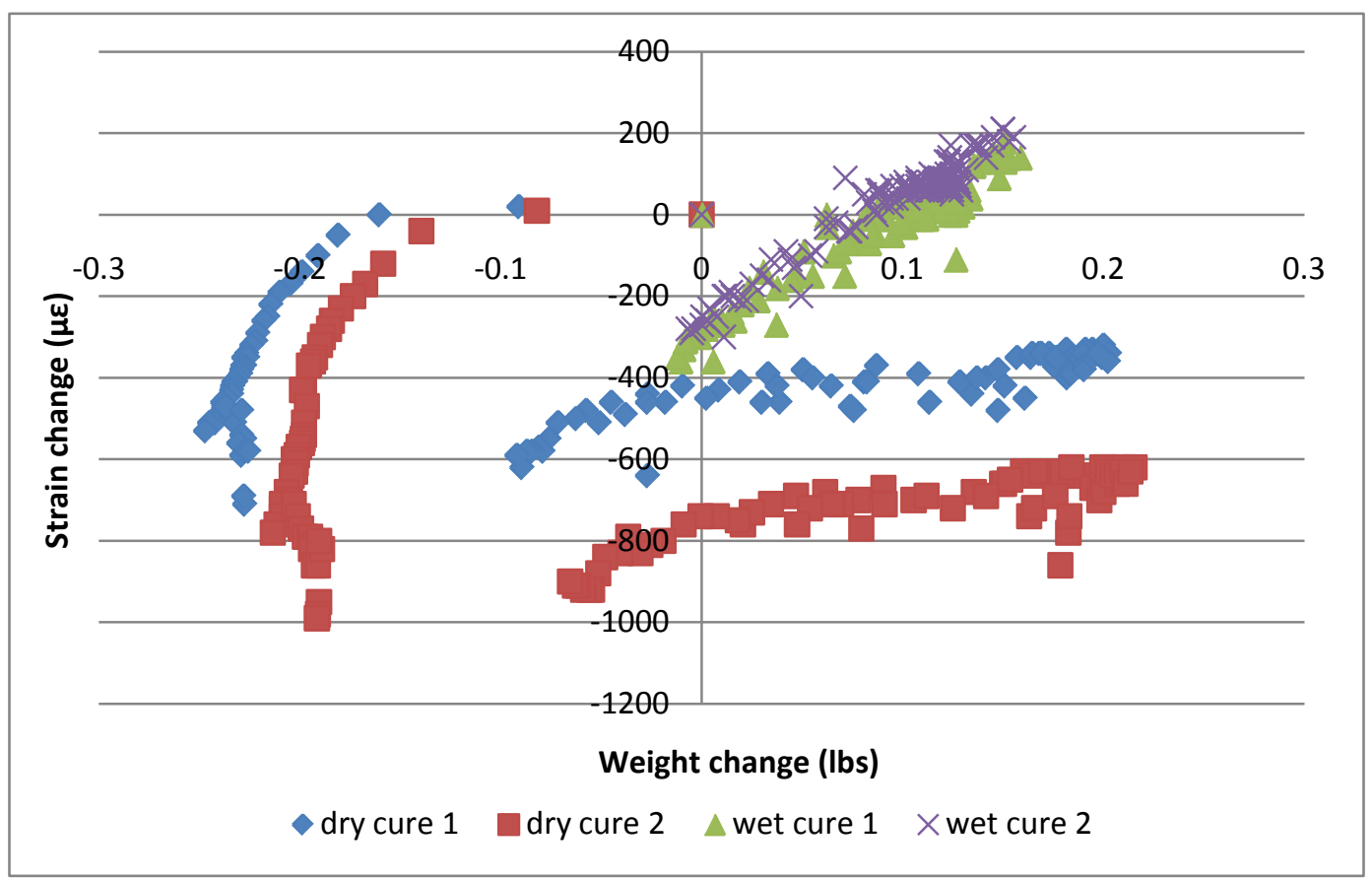

Figure 3.18: Change in strain as a function of change in weight for the gravel RCA concrete.

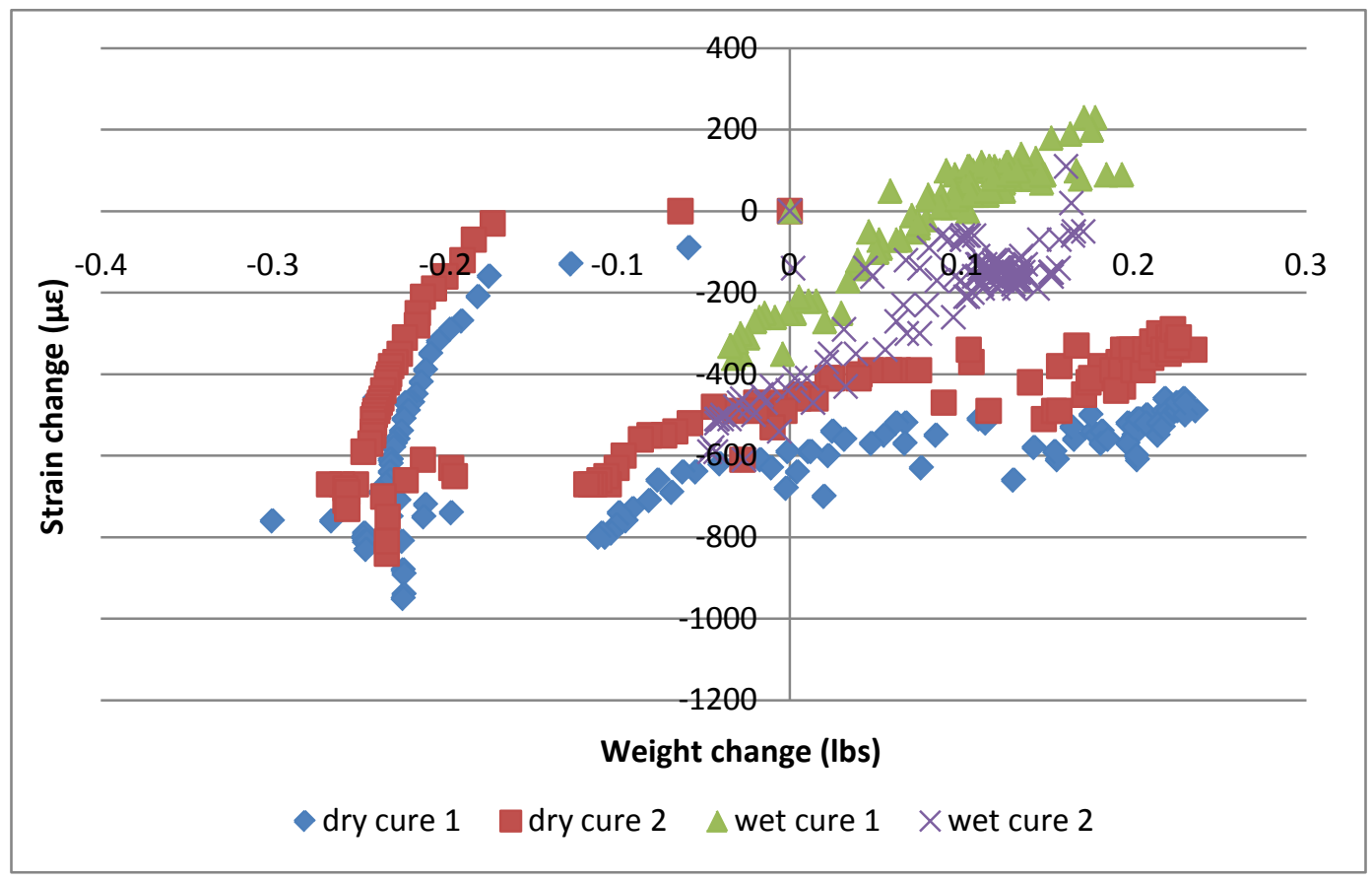

Figure 3.19: Change in strain as a function of change in weight for the lightweight aggregate concrete.

From these figures, it can be seen that change in length as a function of change in weight occurs along a fairly straight line, and the two are indeed proportional. In each figure, 
there is one line of points for each of the dry cured specimens that is separate from the main cluster of points. These points represent the first drying cycle of the dry cured specimens. The data for all subsequent drying and re-wetting cycles is clustered together elsewhere on the graph. The slope of the lines created by the data points from the initial drying is much steeper than the slopes of the lines created by the points for the subsequent drying and re-wetting cycles. This indicates that, for initial drying, changes in weight are associated with larger changes in volume than for subsequent cycles of drying and re-wetting.

The data cluster from subsequent cycles of drying and re-wetting of both the wet and dry cured samples can be broken down in to further cycles, which reveals that, for each instance of wetting or drying, the data forms a nearly straight line. The slope of that line changes slightly for each case. The data from the wet cured samples is clustered above the data from the dry cured samples because the wet cured samples have much higher levels of swelling.

The slope of the line formed by the data for length change versus weight change was calculated for each instance of drying and re-wetting and averaged for each sample set. These slopes are compared in Figure 3.20 for samples which were dry cured and in Figure 3.21 for samples which were wet cured. Note that a decrease in weight was associated with shrinkage (negative strain) while an increase in weight was associated with growth (positive strain). Therefore, the length change over the weight change will always be positive. A low or shallow slope means a change in water content does not cause much change in length, while a high or steep slope means that a change in water content results in a large change in length.

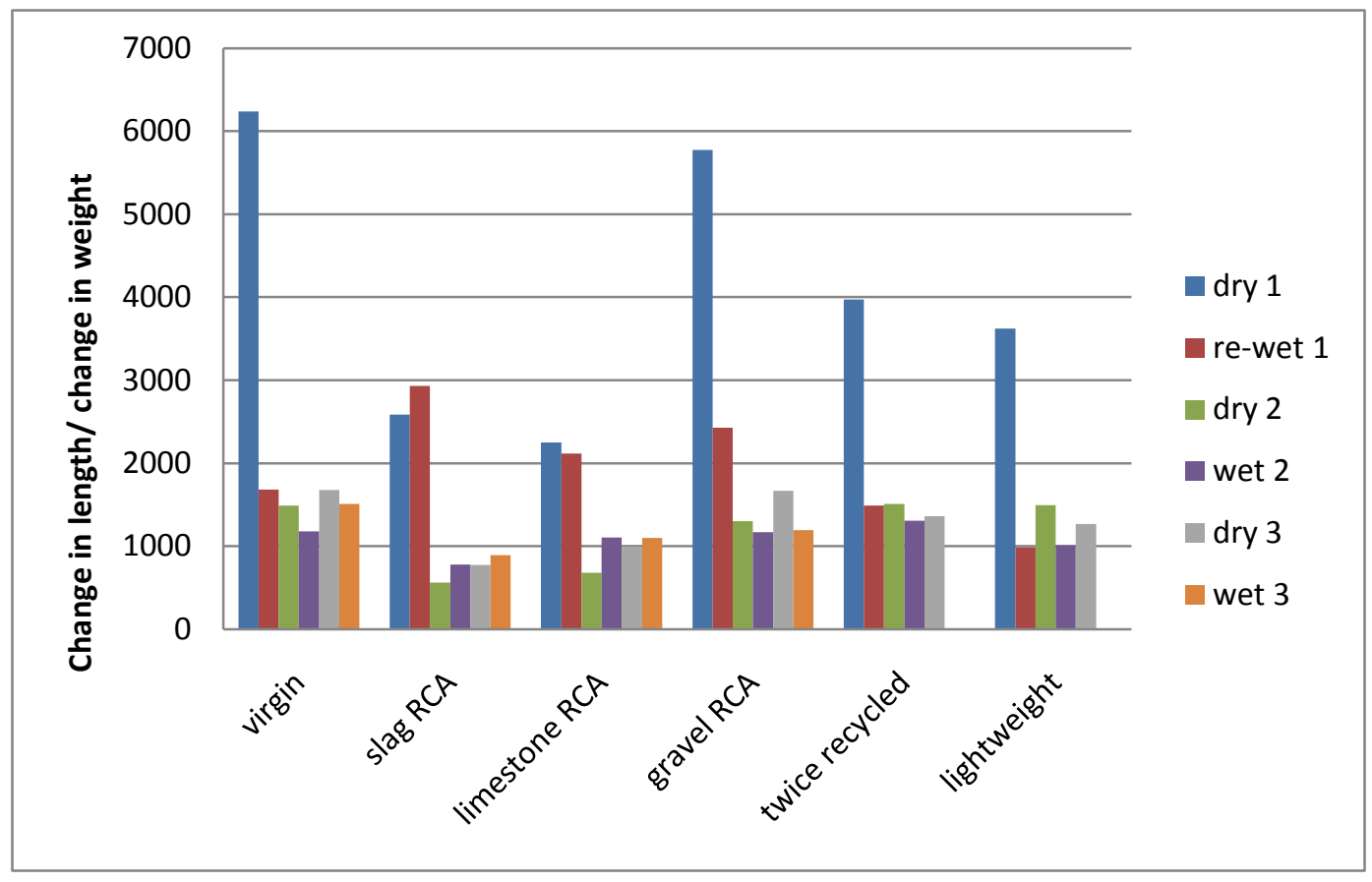

Figure 3.20: Length change as a function of weight change for dry cured samples. 


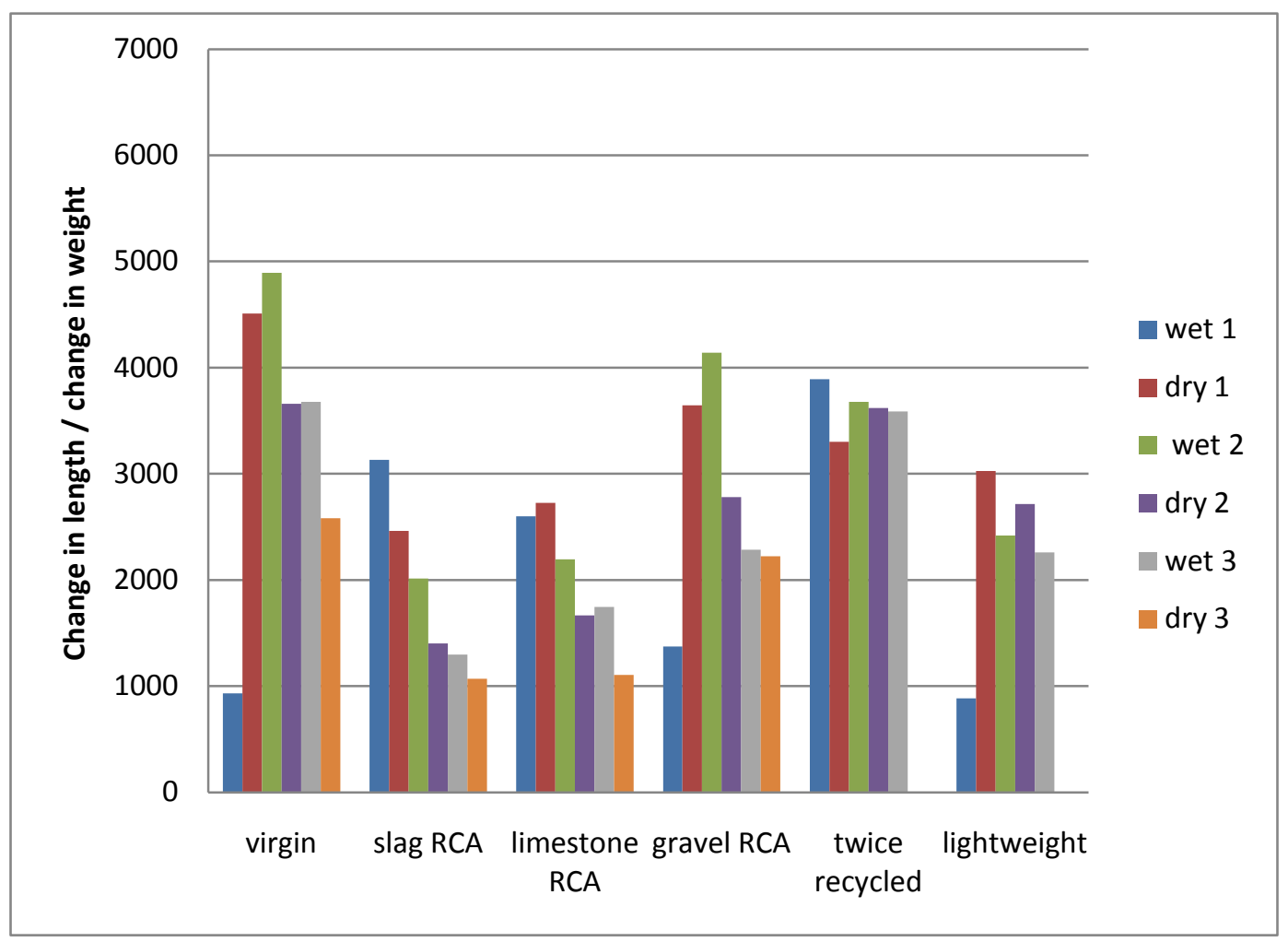

Figure 3.21: Length change as a function of weight change for wet cured samples.

From Figure 3.20, it can be seen that the slope of the first drying is much higher than the slopes of all subsequent drying and re-wetting conditions for all of the concretes, except those made with slag and limestone RCA. After the first drying cycle, the slopes do not change appreciable. For the case of the slag and limestone RCA mixes, the slopes are higher for the first drying and re-wetting cycle than for the subsequent cycles, but are lower relative to all other mixes. After the first cycle of drying and re-wetting, the slopes were very similar for all mixes except the slag and limestone, which were lower, but matched each other. The slope of the initial drying is much higher than for subsequent cycles because the initial evaporation and initial drying are occurring simultaneously. During the initial drying phase, concrete will shrink, even without changing weight due to autogenous shrinkage. This is evident when shrinkage occurs in sealed samples, which have no moisture movement between the sample and the environment and therefore no weight change. This shrinkage coupled with the drying shrinkage due to evaporation, which also causes weight loss results in the high slopes.

From Figure 3.21, it can be seen that the slopes of length change as a function of weight change were generally lower for the initial wetting, and then higher for subsequent drying and re-wetting cycles. As the drying and re-wetting proceed, the slopes begin to gradually decrease slightly with each cycle. Again, the slopes for all mixes were similar except for the slag and limestone RCA concretes, which were lower, but similar to each other. For the wet cured samples, the slope of the first sample is lower compared to all 
other slopes because the concrete was initially close to saturated when the wetting cycle began. The concrete was demolded after 18 hours in sealed conditions and placed in the $100 \%$ relative humidity chamber. Eighteen hours after casting, the concrete was no longer plastic, but the system was still very close to saturated, and therefore could not absorb much water, resulting in a low weight gain. The swelling associated with the high relative humidity was also counteracted by chemical shrinkage, and was therefore lower than would be expected for stable concrete soaked in water. The decreased swelling coupled with the low weight gain caused a low slope for the initial wetting. In the cases of both the wet and dry cured samples, it is unknown why the slag and limestone RCA mixes behaved differently. It is possible that this is related to their high permeability, but further testing would be required to confirm this.

\subsection{Conclusions}

In this study, the ASTM C157 length change test was conducted on concrete prisms containing different types of aggregates. Aggregates tested included virgin, lightweight, and four different recycled concrete aggregates. Samples from each mix were placed in either wet ( $100 \%$ relative humidity) or dry (50\% relative humidity) conditions for a minimum of one year before the samples were exposed to cyclic drying and re-wetting. The percentage of total shrinkage which was reversed upon rewetting was calculated for each drying and re-wetting cycle.

It was found that the initial storage conditions greatly affected the amount of reversible shrinkage, likely because samples stored in a wet environment were more hydrated than samples stored in a dry environment. Dry cured samples had less reversible shrinkage than wet cured samples. Subsequent cycles of drying and re-wetting caused secondary permanent shrinkage to develop, which further decreased the amount of reversible shrinkage in all samples compared to the first cycle of drying and re-wetting. It appears that after the second cycle, the amount of reversible shrinkage stabilizes to a constant value. Further testing is needed to determine how additional cycles contribute to the behavior of the concrete. The amount of reversible shrinkage determined from the second and third cycles of drying and re-wetting was not dependent on the aggregate type for dry cure samples. Further testing is required to determine if this is the case for wet cured samples, though preliminary results suggest that a dependence exists. This study also confirmed the classic assumption that length change is proportional to weight change.

One of the goals of this study was to determine if there was a relation between aggregate type and reversible shrinkage. This would be particularly useful to design engineers to predict the amount of reversible shrinkage based on material properties. The specific properties examined in this study were the porosity of the aggregates and the permeability of the concrete. No correlation was found between the reversible shrinkage and the aggregate porosity, likely because the water to cement ratio of the mixes studied was such that aggregate porosity was not a factor in determining the permeability of the concrete. A correlation was found between the permeability of the concrete and the reversible shrinkage due to the first re-wetting cycle for the dry cured samples. No correlation was found for reversible shrinkage from subsequent drying and re-wetting 
cycles or for the wet cured samples. It is worth noting, however, that the two most permeable mixes often exhibited different behavior than the other mixes. Further research with a greater number of samples and different water to cement ratios may find a relation between reversible shrinkage and either aggregate porosity or concrete permeability. 


\section{Warping and Differential Drying Shrinkage Models}

Differential volume change caused by drying shrinkage can be divided into two categories: permanent and reversible. Both components occur due to removal of water from the capillary pore structure of the paste matrix. The permanent component of drying shrinkage occurs as the concrete sets and cures. It is theorized that, as the C-S-H gel bonds form during the hydration process, they interlock in such a way that they cannot be fully separated when re-wetting causes the material to expand. Thus, the paste cannot achieve unlimited expansion. However, because some expansion does occur when the ambient relative humidity is higher than the moisture content of the concrete, a portion of the shrinkage is reversible (Neville 1997).

\subsection{Motivation}

When computing the differential volume change due to drying shrinkage, the permanent and reversible components are considered separately. Within the MEPDG, permanent differential drying shrinkage is combined with construction curl and creep effects in the built-in curl (BIC) ${ }^{1}$ term. There is no equation to estimate BIC, but rather, it is a user defined input with a default value of $-10^{\circ} \mathrm{F}$. Additionally, no guidance is given in this program as to how much of BIC is attributable to differential drying shrinkage, construction curl or creep, though it is acknowledged that $-10^{\circ} \mathrm{F}$ is not an accurate estimate of BIC in all cases (NCHRP 2003). Users are advised not to change this term without great caution, should they use an alternate estimate. It has been shown that this default setting corresponds to the value of BIC which is required to minimize the expected pavement damage in most pavements (Lothschutz 2009; Vandenbossche et al. 2010), and therefore this default results in unconservative designs (Lothschutz et al.

2011). Given that there currently is no way to predict the amount of built-in curl which will set in a pavement, a value for BIC could be estimated based on the amount of built-in curl seen in similar pavements in the same climatic region. Methods for quantifying the amount of built-in curl in slabs will be discussed in Chapter 5. The ability to predict the expected differential drying shrinkage would help improve estimation of the overall value of BIC because differential drying shrinkage is included in the BIC term.

Differential volume change due to the reversible portion of drying shrinkage is computed in the MEPDG by a moisture warping model as part of the equivalent temperature difference (ETG) factor. This ETG factor is the sum of two temperature gradients, the actual temperature gradient and the equivalent temperature gradient due to the moisture gradient. In MEPDG, moisture warping is considered on a monthly basis (NCHRP 2003). A previous study found that concrete exposed to ambient conditions experiences changes in moisture warping on a seasonal basis (Granger et al. 1994). Given the variation in seasons throughout the country, calculating moisture warping on a monthly basis accurately captures changes in the amount of warping, but is not overly computationally intensive.

\footnotetext{
${ }^{1}$ Recall that in this document, the term built-in curl refers to the actual phenomenon while BIC refers to the MEPDG user input which attempts to characterize the sum of construction curl, differential drying shrinkage and creep.
} 
The current MEPDG warping model uses a triangular shrinkage distribution within the shrinkage zone, and modification factors to account for the amount of total shrinkage which is expected to be reversible, and the ambient relative humidity. This model predicts values for the equivalent temperature gradient which are improbable and/or representative of physically impossible phenomena. Improvements to the current model were made by using a non-linear shrinkage distribution and plate theory to re-derive the basic equation, creating a new model. The basic equation for this new model is based on the ultimate shrinkage expected in the concrete, as this is an MEPDG input. Modification factors are used to account for the effects of reversible shrinkage and to remove the contributions of autogenous shrinkage and permanent drying shrinkage, so that only the reversible portion of drying shrinkage is considered when computing moisture warping. The new model does not require inputs beyond those already used in the MEPDG, and predicts moisture warping monthly using geometric and material properties of the slab, and the monthly average ambient relative humidity.

The new model can also be used to model differential drying shrinkage, provided the modification factors are changed such that only the irreversible portion of drying shrinkage is considered. Additionally, the effects of relative humidity would need to be computed using an annual average relative humidity or the monthly average relative humidity for the month in which the pavement is expected to be built. It is possible to use the same base model for both differential drying shrinkage and moisture warping because they are caused by essentially the same phenomenon and are only separated for modeling purposes. In the development of the base model, the focus of the derivation is on moisture warping, because that was the use of the original MEPDG warping model, which one of the new models replaces. However, disregarding the reversible shrinkage factor and the computation on a monthly basis, all other discussion on the warping model is also applicable to modeling differential drying shrinkage.

\subsection{Current MEPDG Moisture Warping Model}

The current MEPDG equation for the monthly equivalent temperature difference ETG $_{\mathrm{SHi}}$ is given in Equation 4.1 (NCHRP 2003). This equation is based on an equation from Eisenmann and Leykauf (1990b), with heavy modification based on the ACI 209 shrinkage prediction model (ACI 2008).

$$
E T G_{S H i}=\frac{3\left(\phi \varepsilon_{s u}\right)\left(S_{h i}-S_{\text {have }}\right) h_{s}\left(\frac{h}{2}-\frac{h_{s}}{3}\right)}{\alpha h^{2} 100}
$$

Equation 4.1

Where:

$$
\begin{aligned}
E T G_{S H i}= & \text { Equivalent temperature difference due to the deviation of } \\
& \text { moisture warping in month i from the annual average, in }{ }^{\circ} \mathrm{F} \\
\phi= & \text { Reversible shrinkage factor }=\text { fraction of total shrinkage } \\
& \text { which is reversible. A default value of } 0.5 \text { is used unless } \\
& \text { more accurate information is available. }
\end{aligned}
$$




$$
\begin{aligned}
& \varepsilon_{s u}=\text { The ultimate shrinkage strain (which may be estimated } \\
& \text { based on concrete mix properties if it is not known), * } 10^{-6} \\
& S_{h i}=\text { The relative humidity factor for month } \mathrm{i} \\
& \mathrm{S}_{\mathrm{hi}}=1.1 \quad \text { for } \mathrm{RH}_{\mathrm{a}}<30 \% \\
& \mathrm{~S}_{\mathrm{hi}}=1.4-0.01 \mathrm{RH}_{\mathrm{a}} \quad \text { for } 30 \%<\mathrm{RH}_{\mathrm{a}}<80 \% \\
& \mathrm{~S}_{\mathrm{hi}}=3.0-0.03 \mathrm{RH}_{\mathrm{a}} \quad \text { for } \mathrm{RH}_{\mathrm{a}}>80 \%
\end{aligned}
$$

ETG at any time $t$ days after placement is given by Equation 4.2 (NCHRP 2003), which is taken from ACI 209 (ACI 2008).

$$
E T G_{S H t}=S_{t} * E T G_{S h i}
$$

Where:

$$
\begin{aligned}
E T G_{S H t}= & \begin{array}{l}
\text { ETG } \\
\text { placement, in }{ }^{\circ} \mathrm{F}
\end{array} \\
E T G_{S H i}= & \begin{array}{l}
\text { Equivalent temperature difference due to the } \\
\text { deviation of moisture warping in month i from } \\
\text { the annual average, in }{ }^{\circ} \mathrm{F}
\end{array} \\
S_{t}= & \begin{array}{l}
\text { a time factor to account for the time required to } \\
\text { develop ultimate shrinkage }
\end{array}
\end{aligned}
$$

$$
S_{t}=\frac{A g e}{n+A g e}
$$

$$
\begin{aligned}
\text { Age }= & \text { Age in days since concrete placement } \\
n= & \text { The time in days required to develop } 50 \% \text { of } \\
& \text { the ultimate shrinkage. A value of } 35 \text { is } \\
& \text { recommended unless more accurate } \\
& \text { information is available. }
\end{aligned}
$$

It should be noted that in the original ACI equation, Age is the age in days after the curing regime of the concrete is complete, not the days after placement (ACI 2008). However, given that pavements are rarely cured in a water bath or steam room, these are 
likely to be the same. The original ACI approach in determining shrinkage does not separate the effects of autogenous and drying shrinkage (Mindness et al. 2003); the inclusion of the reversible shrinkage factor $\phi$ in Equation 4.1 is to ensure that only the reversible portion of the shrinkage is used in the ETG term, as this is the only portion which contributes to moisture warping (NCHRP 2003). The amount of reversible shrinkage which can be expected in normal Portland cement concrete (PCC), as well as PCC made with recycled concrete and lightweight aggregates, was explored in Chapter 3.

Equation 4.1 is based on an assumed triangular distribution of a shrinkage gradient through the depth of the shrinkage zone, with modifications to account for the cyclic nature of relative humidity. Tracing the origins of this equation back, it can be seen that it is a modification of the equation developed by Eisenmann and Leykauf (1990b) (see Equation 4.3), which used a rectangular distribution to model the shrinkage and was derived under the assumption of beam bending theory.

$$
E T G_{E \& L}=\frac{6 h_{s} \varepsilon_{s u}\left(h-h_{s}\right)}{\alpha h^{3}}
$$

Equation 4.3

Figure 4.1 shows the rectangular and triangular stress distributions. For the purposes of discussion and in order to compare models, the term $a$ will be used to represent the product of all variables which account for shrinkage. In the original MEPDG equation, $a$ will be equal to $\phi \varepsilon_{s u}\left(S_{h i}-S_{\text {have }}\right)$, while in the Eisenmann and Leykauf model, $a$ is simply $\varepsilon_{s u}$.

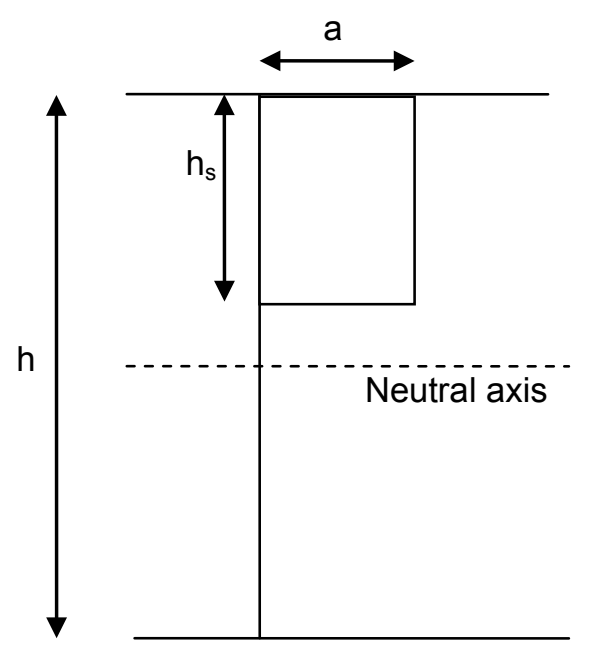

(a)

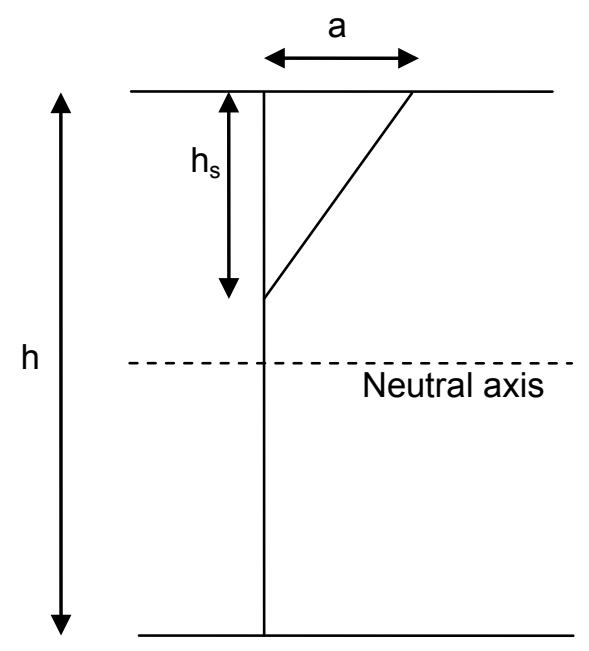

(b)

Figure 4.1: a) Eisenmann and Leykauf's rectangular shrinkage distribution and b) MEPDG's triangular shrinkage distribution. 
Equation 4.3 gives the temperature gradient in units of degrees/inch of thickness; multiplying this equation by the thickness of the pavement $h$ will give the overall gradient, as in the MEPDG model.

Equation 4.1 also differs from Eisenmann and Leykauf's equation in that it incorporates a term, $\left(S_{h i}-S_{\text {have }}\right)$, to account for the variation in relative humidity throughout the year. This term is loosely based on a correction factor included in ACI 209 to account for the effects of higher than expected ambient relative humidity for the duration of the life of the slab. To develop the term $\left(S_{h i}-S_{\text {have }}\right)$, the original ACI correction factor $\gamma_{r h}$ was multiplied by the ultimate shrinkage, where $\gamma_{r h}$ is given by Equation 4.4 (ACI 2008).

$$
\begin{aligned}
\text { for } 40 \%<\mathrm{RH}<80 \%: & \gamma_{\mathrm{rh}}=1.4-0.01 \mathrm{RH} \\
\text { for } \mathrm{RH}>80 \%: & \gamma_{\mathrm{rh}}=3.0-0.03 \mathrm{RH}
\end{aligned}
$$

Equation 4.4

In Equation 4.1, $\gamma_{r h}$ is modified slightly and renamed $S_{h i}$. This equation is not intended to account for cyclic changes in ambient relative humidity, but is instead intended for structural concrete permanently exposed to higher relative humidity, such as for a dam or holding tank (Mindness et al. 2003). However, the argument can be made that, in computing ETG $_{\mathrm{SHi}}$ on a monthly basis with the $S_{h i}$ factor, cyclic relative humidity is not being considered. Because the relative humidity over the month is assumed to be constant, and $\mathrm{ETG}_{\mathrm{SHi}}$ is computed on a monthly basis, only the case of constant relative humidity is being considered in each computation, and therefore the $S_{h i}$ factor is applicable.

In an attempt to modify the ACI correction factor and apply it to the case of cyclic loading, Equation 4.4 was modified, and included in Equation 4.1, through the term $\left(S_{h i}-\right.$ $S_{\text {have }}$ ), which accounts for the difference in relative humidity in a given month from the average relative humidity seen throughout the year. Relating the relative humidity in a given month to the annual average presents several problems, which will be explored later.

With this modification, and the inclusion of the reversible shrinkage factor, the ultimate shrinkage term in Eisenmann and Leykauf's original equation (Equation 4.3) becomes $\phi \varepsilon_{s u}\left(S_{h i}-S_{\text {have }}\right)$ in Equation 4.1. There are still some additional terms in the MEPDG equation, however, that cannot be accounted for.

\subsection{Problems with the Current MEPDG Warping Model}

The MEPDG equation for warping is not a published equation, but rather is a conglomeration of several modified models. A closer examination of this equation reveals several shortcomings, including terms of unknown origin, and a propensity to predict physically impossible behavior. Identifying the problems with the current MEPDG warping model is important because any new model should address all known issues with the current model 


\subsubsection{Derivation of the Current MEPDG Warping Model}

The MEPDG model is based on the Eisenmann and Leykauf model, with extensive modifications. Below, the current MEPDG model is re-derived using the process from Eisenmann and Leykauf (1990b), and incorporating the changes used in the new MEPDG model. From this re-derivation, it can be seen that there are terms in the MEPDG which are not found in a re-derivation.

Again, $a$ is the term which accounts for shrinkage. Assuming a triangular strain distribution which extends from the top of the slab to the edge of the shrinkage zone, the force in caused by this shrinkage $F_{S}$ is given by:

$$
F_{s}=\frac{1}{2} a h_{s} E
$$

Equation 4.5

where:

$\mathrm{E}$ is the elastic modulus of the concrete.

The distance between the centroid of this triangular strain distribution and the neutral axis of the slab is $e$ :

$$
e=\frac{h}{2}-\frac{h_{s}}{3}
$$

Equation 4.6

The moment about the neutral axis of the slab induced by the shrinkage is $M$ :

$$
M=F_{s} e=\frac{1}{2} a h_{s} E\left(\frac{h}{2}-\frac{h_{s}}{3}\right)
$$

Equation 4.7

The stress in the slab induced by this moment is $\sigma_{\mathrm{s}}$ :

$$
\sigma_{s}=\frac{M y}{I}
$$

Equation 4.8

where:

$y$ is the depth to the neutral axis, $y=\frac{h}{2}$

$I$ is the moment of inertia of the slab. Assuming a unit slab width, $I=\frac{h}{12}^{3}$

Therefore, $\sigma_{\mathrm{s}}$ is given by:

$$
\sigma_{s}=\frac{\frac{1}{2} a h_{s} E\left(\frac{h}{2}-\frac{h_{s}}{3}\right) \frac{h}{2}}{h^{3} / 12}=\frac{3 a h_{s} E\left(\frac{h}{2}-\frac{h_{s}}{3}\right)}{h^{2}}
$$

Equation 4.9

The edge stress $\sigma_{\mathrm{t}}$ due to an equivalent linear temperature distribution $\Delta T$ is:

$$
\sigma_{t}=E \alpha h \frac{\Delta T}{2}
$$

Equation 4.10 
This equivalent linear temperature distribution is shown in Figure 4.2.

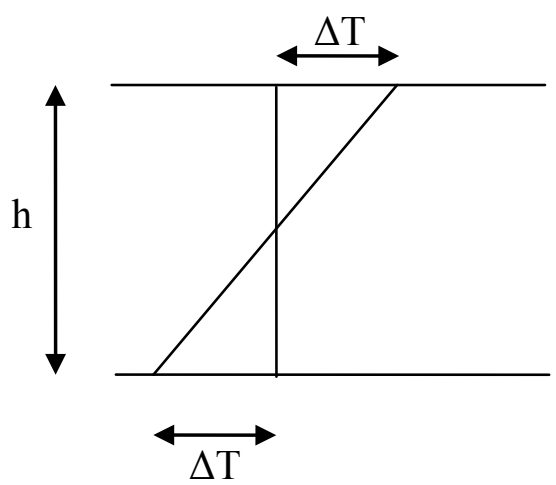

Figure 4.2: Equivalent linear temperature difference.

Equating these two stresses and solving for $\Delta T$ :

$$
\Delta T=\frac{6 a h_{s}\left(\frac{h}{2}-\frac{h_{s}}{3}\right)}{\alpha h^{3}}
$$

This equation is for the temperature gradient per unit thickness of the slab. In order to compare this equation to the MEPDG model, it must be multiplied by the total height of the slab to get the total temperature gradient for the entire slab ETG:

$$
\mathrm{ETG}=\frac{6 a h_{s}\left(\frac{h}{2}-\frac{h_{s}}{3}\right)}{\alpha h^{2}}
$$

Equation 4.12

When this equation is compared to the actual equation used in the MEPDG (Equation 4.1 ), there is a difference of $1 / 200$, which will substantially change any numerical values obtained using this equation. No indication is given in the MEPDG as to the source of this term. To better hypothesize where this factor comes from, it can be split into a factor of $1 / 2$ and a factor of $1 / 100$.

There are two potential sources for the factor of $1 / 2$, both of which demonstrate shortcomings of the MEPDG model. One option is that it accounts for the fact that the shrinkage term used in the model is that of ultimate shrinkage, not drying shrinkage. However, only the reversible portion of the drying shrinkage is responsible for moisture warping. The ultimate shrinkage term includes not only the drying shrinkage, but also the autogenous shrinkage, which is both permanent and uniform, and therefore not associated with moisture warping. The factor of $1 / 2$ could be from an assumption that half of all shrinkage is drying shrinkage and the other half is autogenous shrinkage. Multiplying the ultimate shrinkage by half would then eliminate the effects of autogenous shrinkage. It has been found that the autogenous shrinkage accounts for approximately half of the overall shrinkage in concretes with a water to cement ratio of 0.3 or less (Kosmatka et al. 2002). However, this assumption is not valid for concrete mixes with higher water to cement ratios, as is typical in paving mixes. 
Alternately, the factor of $1 / 2$ could account for the fact that the ASTM C157 length change test, from which ultimate shrinkage value is generally obtained, is for a sample where shrinkage occurs on both sides. In a slab, drying shrinkage occurs only on the top of the slab, as the bottom is protected from evaporation by the insulative nature of the slab itself (see Figure 4.3). In this case, dividing the stress due to shrinkage by $1 / 2$ could eliminate the effects of drying shrinkage on the bottom of the slab. One concern associated with this assumption is that, when considering long term drying, the depth of the shrinkage zone will likely extend beyond the centerline of the prism, as the prisms used in the ASTM C157 test are only three inches wide (ASTM 2008e).

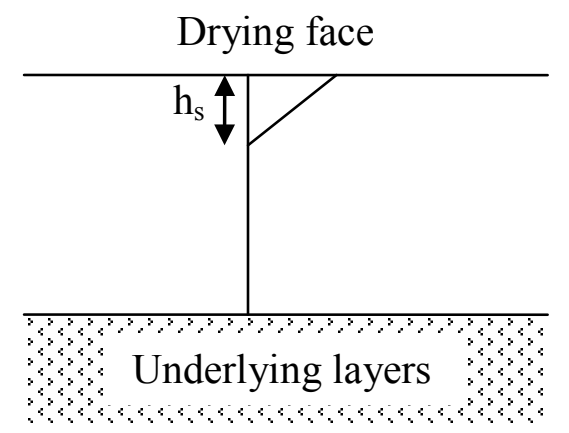

(a)

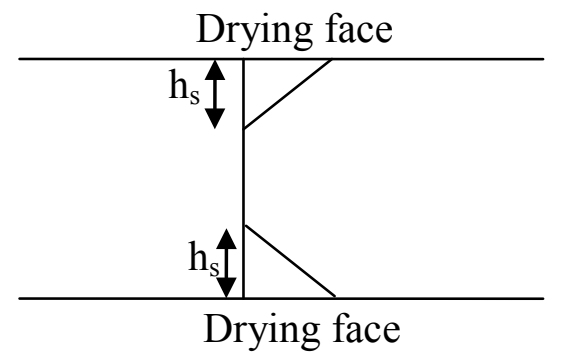

(b)

Figure 4.3: Drying shrinkage through the thickness of (a) a concrete slab and (b) an ASTM C157 drying shrinkage prism, assuming a triangular shrinkage distribution with depth.

If the first hypothesis is true, and the $1 / 2$ is to remove the effects of autogenous shrinkage, then the effects of drying shrinkage occurring on the top of the slab have been neglected. If the reverse is true, and the $1 / 2$ factor is to account for the drying shrinkage occurring on the top of the slab, then the autogenous shrinkage has not been removed from the shrinkage term. Another possibility is that the $1 / 2$ accounts for another effect altogether and the effects of both the autogenous shrinkage and drying on the top half of the slab have been ignored. All of these cases must be considered in any new warping model.

The $1 / 100$ factor is harder to rationalize. One possibility is that it is to unnecessarily convert the relative humidity in the $S_{h i}$ and $S_{\text {have }}$ factors from a percent to a decimal. Other standard shrinkage models use relative humidity in decimal form; however the MEPDG input is in percent. In this case, the factor of $1 / 100$ is unnecessary because the relative humidity is already assumed to be a percent in the calculation of the relative humidity adjustment factors. The 1/100 factor could also be a calibration factor, or a unit conversion for some other quantity. In any case, it is both unnecessary, and unaccounted for in the current warping model.

\subsubsection{Assumptions in the Current MEPDG Warping Model}

One assumption made during the derivation of the current MEPDG warping model was that shrinkage varies linearly through the depth of the shrinkage zone. While this approximation is an improvement over the rectangular approximation used by Eisenmann 
and Leykauf, a non-linear approximation would be more representative of actual drying (Janssen 1987).

Eisenmann and Leykauf's equation is also derived using the temperature stress associated with a beam, which experiences one way bending. A slab acts as a two way system due to its dimensions, and is therefore best characterized using plate theory, as opposed to beam theory (Timoshenko and Goodier 1951).

\subsubsection{Amount of Warping Does Not Affect the Current MEPDG Cracking Model}

When the current MEPDG warping model is used to compute the equivalent temperature difference which would be expected for a standard pavement using actual relative

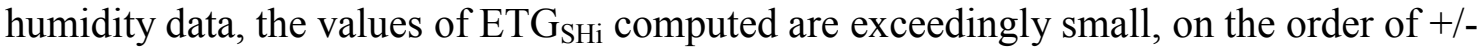
.001 to $.01^{\circ} \mathrm{F}$. If the equivalent temperature difference caused by warping were really this small, it would not be worth the effort to model it, especially considering that other contributions to the total equivalent temperature difference are several orders of magnitude larger.

Additionally, these values of warping do not match those found in the literature. There have been very few studies on the magnitude of moisture warping, but it is generally agreed that warp exists and is of a large enough magnitude that it must be considered in design (Teller and Sutherland 1935). One study by Wei et al. (2008) back-calculated the equivalent temperature gradient in an eight inch thick slab being held at a constant temperature with a relative humidity of $50 \%$ at the top, and fully saturated at the bottom. Through back-calculation with profiler data and an FEM model, this slab was found to have an equivalent temperature difference of -20 to $-35^{\circ} \mathrm{F}$. Another study found that the deflections due to warping in concrete pavements in the arid climate of California were of similar magnitude as curl due to temperature gradients (Hveem and Bailey 1957). A predictive model for warp should be finding values on this order of magnitude.

The equivalent temperature difference predicted for a standard pavement using the current MEPDG warping model is so small compared to the other terms that it is actually insignificant in the overall MEPDG JPCP cracking model. A factorial was run for a standard PCC pavement in different locations to examine the effects of varying the amount of total shrinkage and the reversible shrinkage factor. For several locations around the country, analysis was conducted on a 10 inch thick PCC pavement with a 12 inch A-1-a base, A-7-6 subgrade, 1 inch diameter dowels with 12 inch spacing, and 15 foot joint spacing. The annual average daily truck traffic (AADTT) was 5000, and MEPDG defaults were used for all other values. For different trials, an ultimate shrinkage of 600, 900 and $1200 \mu \varepsilon$ was used with a reversible shrinkage factor of 0,25 , 50,75 , and $100 \%$ for each of the values of ultimate shrinkage. Climatic models were used from the EICM at strategic locations around the country. Because warping is dependent on ambient relative humidity, it was desirable to include locations with a variety of humidity regimes. Seattle, Washington and Las Vegas, Nevada were selected as wet and dry locations, respectively. Additional locations were selected in the middle of the country because they experience both wet and dry months. Several sites located in close proximity to each other with both wet and dry seasons were selected to eliminate 
the potential from error in climatic files after preliminary results yielded strange trends. These sites were Omaha, Nebraska; Lincoln Nebraska; Kansas City, Missouri; Des Moines, Iowa; and Topeka, Kansas. Results for the percent of slabs cracked at 20 years from this factorial are given in Table 4.1

Table 4.1

Percent of slabs cracked at 20 years for 10 inch pavements in various locations.

\begin{tabular}{|c|c|c|c|c|c|c|c|c|}
\hline $\begin{array}{c}\text { Total } \\
\text { strain } \\
(\mu \varepsilon)\end{array}$ & $\begin{array}{c}\% \\
\text { reversible }\end{array}$ & $\begin{array}{c}\text { Omaha } \\
\text { NE }\end{array}$ & $\begin{array}{c}\text { Lincoln } \\
\text { NE }\end{array}$ & $\begin{array}{c}\text { Topeka } \\
\text { KS }\end{array}$ & $\begin{array}{c}\text { Kansas } \\
\text { City } \\
\text { MO }\end{array}$ & $\begin{array}{c}\text { Des } \\
\text { Moines } \\
\text { IA }\end{array}$ & $\begin{array}{c}\text { Las } \\
\text { Vegas } \\
\text { NV }\end{array}$ & $\begin{array}{c}\text { Seattle } \\
\text { WA }\end{array}$ \\
\hline 600 & 0 & 2.3 & 2.7 & 4 & 0.6 & 0.5 & 1.7 & 0.1 \\
\hline 600 & 25 & 1.7 & 2.7 & 4 & 0.6 & 0.5 & 1.7 & 0.1 \\
\hline 600 & 50 & 1.8 & 2.7 & 4 & 0.6 & 0.5 & 1.8 & 0.1 \\
\hline 600 & 75 & 1.8 & 2.6 & 4 & 0.6 & 0.4 & 1.8 & 0.1 \\
\hline 600 & 100 & 1.9 & 2.6 & 4 & 0.6 & 0.4 & 1.9 & 0.1 \\
\hline 900 & 0 & 1.7 & 2.7 & 4 & 0.6 & 0.5 & 1.7 & 0.1 \\
\hline 900 & 25 & 1.7 & 2.7 & 4 & 0.6 & 0.5 & 1.7 & 0.1 \\
\hline 900 & 50 & 1.8 & 2.6 & 4 & 0.6 & 0.4 & 1.8 & 0.1 \\
\hline 900 & 75 & 2 & 2.6 & 4 & 0.6 & 0.4 & 2 & 0.2 \\
\hline 900 & 100 & 2.2 & 2.7 & 4 & 0.6 & 0.4 & 2.2 & 0.2 \\
\hline 1200 & 0 & 1.7 & 2.7 & 4 & 0.6 & 0.5 & 1.7 & 0.1 \\
\hline 1200 & 25 & 1.8 & 2.7 & 4 & 0.6 & 0.5 & 1.8 & 0.1 \\
\hline 1200 & 50 & 1.9 & 2.6 & 4 & 0.6 & 0.4 & 1.9 & 0.1 \\
\hline 1200 & 75 & 2.2 & 2.7 & 4 & 0.6 & 0.4 & 2.2 & 0.2 \\
\hline 1200 & 100 & 2.7 & 2.7 & 4 & 0.6 & 0.5 & 2.7 & 0.4 \\
\hline
\end{tabular}

From Table 4.1, it can be seen that there is little or no variation in the percent of cracked slabs with changes in total strain or the amount of reversible shrinkage for any location. When there is any amount of variation, it does not follow any logical trend, as seen for example, in Figure 4.4. It should be noted that when variation in the predicted percentage of cracked slabs was present, it was in such small amounts (less than 1\%) as to be insignificant in design. Additional analysis on six and eight inch thick concrete pavements, and at higher traffic levels revealed the same insensitive results.

This lack of variation indicates that the warping model does not contribute to the calculation of the percent of slabs cracked. In the current MEPDG warping model, the value of equivalent temperature difference is directly proportional to the ultimate shrinkage. Therefore, doubling the ultimate shrinkage will lead to an equivalent temperature difference that is twice as large. As can be seen from Table 4.1, doubling the equivalent temperature difference due to warping had essentially zero effect on the predicted cracking in the slab. If the contribution to slab damage from moisture warping is this insignificant, it is not worth the effort to consider it in design. However, as 
previous studies have shown that warping is of importance, then the current model is not an accurate representation of slab behavior and requires modification.

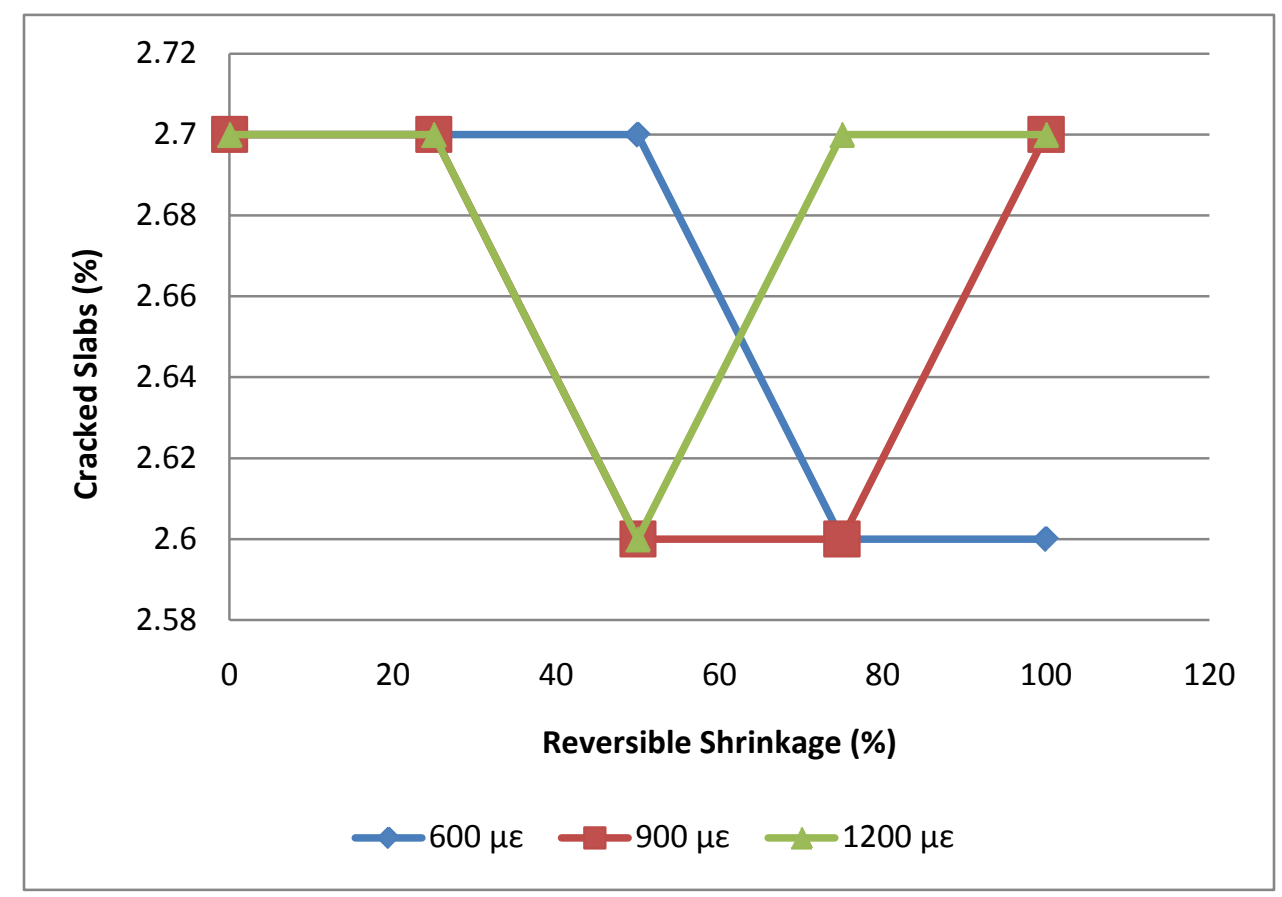

Figure 4.4 Percent of slabs cracked at 20 years in Lincoln Nebraska.

One potential reason that the current warping model predicts such low values is the $1 / 100$ factor which cannot be explained when the model is re-derived. However, simply multiplying the results of the current equation by 100 does not yield realistic values, though it is an improvement. This indicates that the factor of $1 / 100$ is not the only problem with the equation.

\subsubsection{Relating Monthly Relative Humidity to the Annual Average}

The use of the $\left(S_{h i}-S_{\text {have }}\right)$ term to account for cyclic changes in relative humidity presents a fundamental problem. This term relates the relative humidity in any given month to the annual average, essentially saying that any dry months will cancel out any wet months, and therefore, the net annual warp in a slab is zero. An analysis of the implications of this assumption quickly reveals that this is not the case.

A slab in a desert region, such as Las Vegas, will generally be dry on top because the ambient relative humidity in that climate is low. Figure 4.5 shows the relative humidity data from the EICM in the MEPDG for Las Vegas (NCHRP 2006). Even in the wettest month, the relative humidity is never above $45 \%$. In the dry climate of Las Vegas, Qin (2011) has shown that after five years of drying, the relative humidity at the bottom of a ten inch thick slab is $78 \%$. From this, it is obvious that the relative humidity of the top of the slab is never greater than the relative humidity at the bottom of the slab. Note that this analysis is on a monthly basis, which is how the MEPDG computes moisture warping. During a rain event, it is likely that the relative humidity at the top of the slab is 
greater than at the bottom; however, a rain event is an isolated incident, and is not representative of the average monthly relative humidity.

If the top of the slab is never wetter than the bottom on a long term basis, then the warping due to moisture gradients would never be downwards. However, if Equation 4.1 is used to calculate the expected ETG $_{\mathrm{SHi}}$ for a slab in Las Vegas exposed to the relative humidity values given in Figure 4.5, the equation predicts that, for 4 months of the year, the slab will be have a positive equivalent temperature difference, causing it to be warped downwards. Figure 4.6 shows the values of ETG $_{\mathrm{SHi}}$ calculated using Equation 4.1 for a 10 inch thick PCC slab with an ultimate shrinkage of $600 \mu \varepsilon$ and MEPDG default values for all other parameters. Note that, as previously discussed, the values of equivalent temperature gradient predicted by the current warping model are much smaller than would be considered reasonable. Therefore, the actual value of the numbers being compared should not be the focus of an analysis; rather the relative magnitude of the values of the equivalent temperature difference, as well as trends in these values and whether they are positive or negative, are what is important.

Though this analytical exercise clearly demonstrates that a slab in the desert will never experience downward moisture warping, MEPDG predicts that it will from February through May, because of the assumption that average warping will be zero for the year. It is also interesting to note that the values of positive warp are much larger than those of negative warp, in most cases almost double. This means that not only will the slab warp downwards, but the downwards warping will be more severe than the upward warp. Again, simply thinking through the logistics shows that this is not possible.

Another case to consider is that on the opposite extreme, a slab in a very moist environment. Figure 4.5 shows the EICM values for relative humidity in Seattle Washington used in the MEPDG (NCHRP 2006). Seattle has a very wet climate, and even assuming that the base of any pavement is always saturated, the moisture gradient in a slab would not be very large compared with a pavement in Las Vegas. Knowing that the base of a pavement is generally close to saturated (Janssen 1987), and given the ambient relative humidity in Seattle, it is expected that there would be either no gradient, or a very small negative gradient. However, computing the values of $\mathrm{ETG}_{\mathrm{SHi}}$ calculated using Equation 4.1 for a 10 inch thick PCC slab with an ultimate shrinkage of $600 \mu \varepsilon$ and MEPDG default values for all other parameters in Seattle, the results are again improbable and defy logic, see Figure 4.6. 


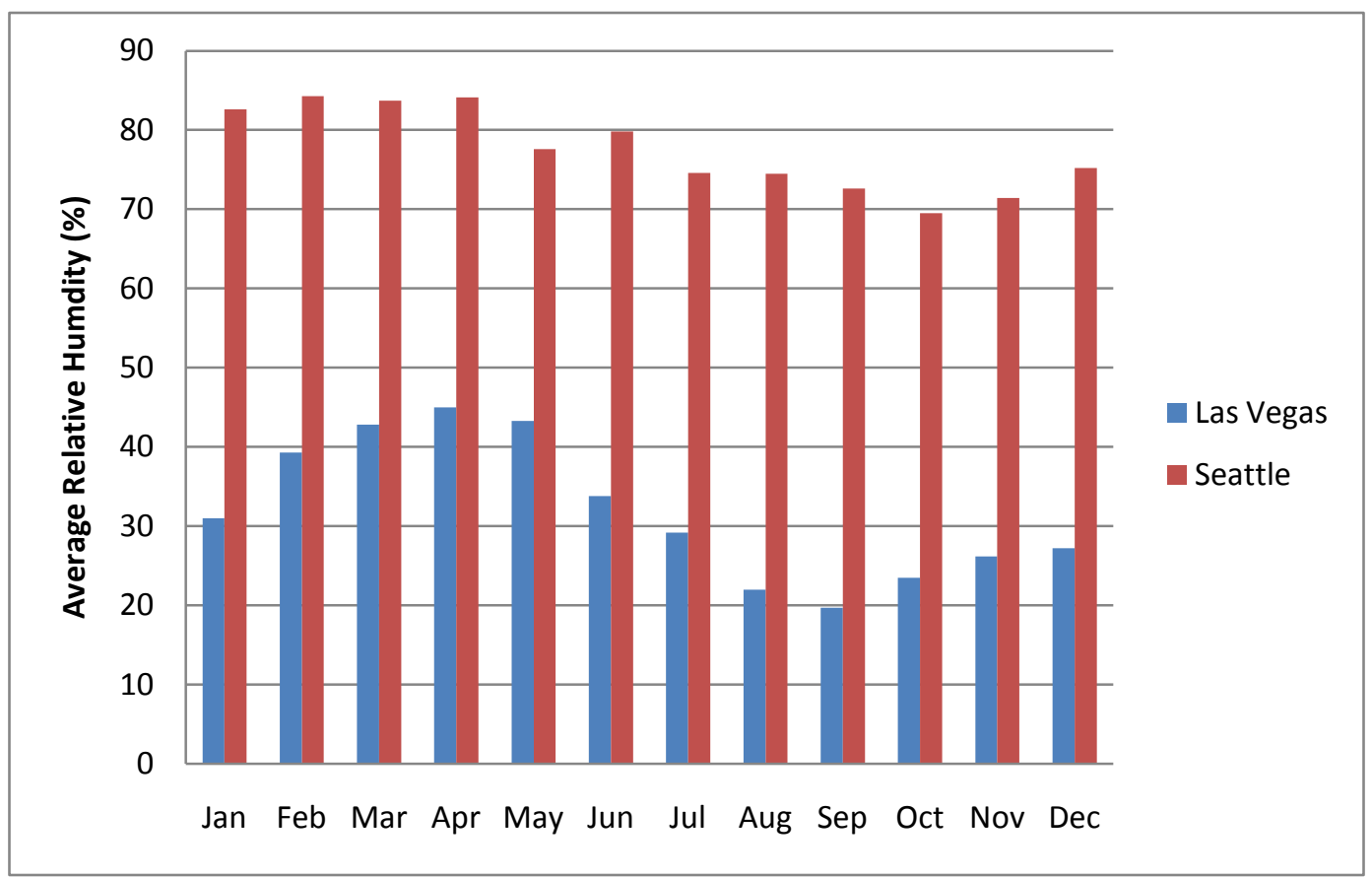

Figure 4.5: Average monthly ambient relative humidity for Las Vegas and Seattle.

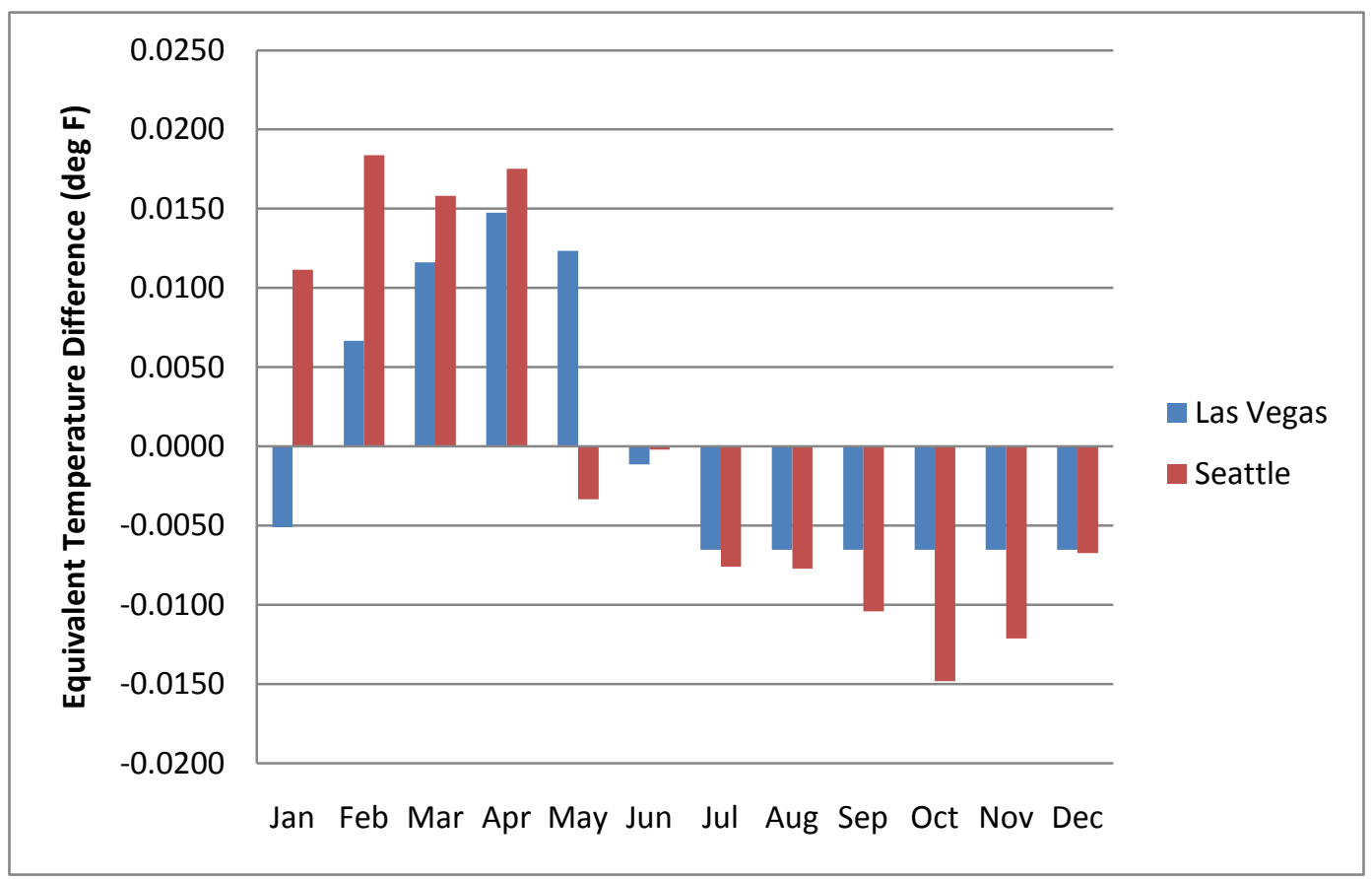

Figure 4.6: Average monthly moisture warping for Las Vegas and Seattle.

In comparing the results for $\mathrm{ETG}_{\mathrm{SHi}}$ in Seattle and Las Vegas, it can be seen that they follow similar trends in that there are large positive gradients expected for four months of the year, and smaller negative gradients are predicted the remainder of the time. Similar 
computations performed for less extreme sites in the middle of the country also show this trend, see Table 4.2 and Table 4.3 for values of relative humidity and computed ETG $_{\mathrm{SHi}}$ respectively. All calculations were for a 10 inch slab with an ultimate shrinkage of 600 $\mu \varepsilon$ and MEPDG default values for all other parameters. From Table 4.3 , it can be seen that in locations without extremely wet or dry climates, the current MEPDG model predicts that slabs will be warped downwards or flat for three to six months a year.

Table 4.2

Ambient relative humidity for various locations in the United States.

\begin{tabular}{|c|c|c|c|c|c|c|c|}
\hline Month & $\begin{array}{c}\text { Omaha } \\
\text { NE }\end{array}$ & $\begin{array}{c}\text { Minneapolis } \\
\text { MN }\end{array}$ & $\begin{array}{c}\text { Houston } \\
\text { TX }\end{array}$ & $\begin{array}{c}\text { Miami } \\
\text { FL }\end{array}$ & $\begin{array}{c}\text { Lexington } \\
\text { KY }\end{array}$ & $\begin{array}{c}\text { Portland } \\
\text { OR }\end{array}$ & $\begin{array}{c}\text { Los } \\
\text { Angeles } \\
\text { CA }\end{array}$ \\
\hline January & 68.8 & 75.6 & 74.3 & 74.0 & 74.5 & 68.2 & 69.9 \\
\hline February & 72.1 & 73.6 & 74.9 & 74.6 & 72.5 & 64.8 & 72.1 \\
\hline March & 75.6 & 68.3 & 76 & 72.3 & 68 & 67.7 & 76.6 \\
\hline April & 74.0 & 59.8 & 75.5 & 68.5 & 68 & 64.7 & 75.3 \\
\hline May & 74.2 & 62.9 & 76.7 & 71.8 & 72.2 & 72.4 & 79.3 \\
\hline June & 68.5 & 66.5 & 77.5 & 77.4 & 75.1 & 74.9 & 78.3 \\
\hline July & 65.4 & 67.8 & 76.9 & 74.6 & 74.4 & 75.2 & 78.9 \\
\hline August & 67.6 & 70.1 & 75.9 & 75.7 & 73.7 & 76.1 & 78.7 \\
\hline September & 69.2 & 68.4 & 74.1 & 78.8 & 71.4 & 77 & 77.7 \\
\hline October & 72.7 & 67.7 & 76.6 & 75.1 & 73.1 & 74.2 & 76.5 \\
\hline November & 75.2 & 72.8 & 76.3 & 74.0 & 72.4 & 72.1 & 69.5 \\
\hline December & 68.5 & 75.7 & 73.1 & 75.1 & 76.9 & 69.7 & 64.1 \\
\hline
\end{tabular}

Table 4.3

ETG $_{\mathrm{SHi}}$ for various locations in the United States.

\begin{tabular}{|c|c|c|c|c|c|c|c|}
\hline Month & $\begin{array}{c}\text { Omaha } \\
\text { NE }\end{array}$ & $\begin{array}{c}\text { Minneapolis } \\
\text { MN }\end{array}$ & $\begin{array}{c}\text { Houston } \\
\text { TX }\end{array}$ & $\begin{array}{c}\text { Miami } \\
\text { FL }\end{array}$ & $\begin{array}{c}\text { Lexington } \\
\text { KY }\end{array}$ & $\begin{array}{c}\text { Portland } \\
\text { OR }\end{array}$ & $\begin{array}{c}\text { Los } \\
\text { Angeles } \\
\text { CA }\end{array}$ \\
\hline January & -0.003 & 0.009 & -0.002 & 0.000 & 0.003 & -0.005 & -0.007 \\
\hline February & 0.002 & 0.006 & -0.001 & 0.000 & 0.000 & -0.009 & -0.004 \\
\hline March & 0.007 & -0.001 & 0.000 & -0.003 & -0.007 & -0.005 & 0.003 \\
\hline April & 0.004 & -0.013 & 0.000 & -0.008 & -0.007 & -0.010 & 0.001 \\
\hline May & 0.005 & -0.009 & 0.001 & -0.004 & -0.001 & 0.001 & 0.006 \\
\hline June & -0.004 & 0.004 & 0.003 & 0.004 & 0.003 & 0.005 & 0.005 \\
\hline July & -0.008 & -0.002 & 0.002 & 0.000 & 0.002 & 0.005 & 0.006 \\
\hline August & -0.005 & 0.001 & 0.000 & 0.002 & 0.001 & 0.007 & 0.006 \\
\hline September & -0.003 & -0.001 & -0.002 & 0.006 & -0.002 & 0.008 & 0.004 \\
\hline October & 0.002 & -0.002 & 0.001 & 0.001 & 0.001 & 0.004 & 0.002 \\
\hline November & 0.006 & 0.005 & 0.001 & 0.000 & 0.000 & 0.001 & -0.007 \\
\hline December & -0.004 & 0.009 & -0.004 & 0.001 & 0.006 & -0.002 & -0.015 \\
\hline
\end{tabular}


From these case studies, it is obvious that tying the relative humidity in any given month to the annual average is unreasonable, because it mathematically forces the equation to predict impossible behavior.

Another important observation from these studies is that the value of ETG SHi $_{\text {Shedicted is }}$ much lower than would be expected. Taking, for example, the case of Las Vegas in September, where the ambient relative humidity is $19.7 \%$, the estimated equivalent temperature gradient is $-0.0065^{\circ} \mathrm{F}$. After five years of drying, the relative humidity at the base of the slab is 78\% (Qin 2011), resulting in a 58\% difference in relative humidity through the thickness of the slab. For a 10 inch pavement, one would expect to find much larger values for warp. In reality, most of this gradient is concentrated in the shrinkage zone, which for Las Vegas has been shown to be four inches (Qin 2011). In this case, that $58 \%$ difference in relative humidity is mostly across four inches of concrete. The warping produced by this should be much larger than that of a $-0.0065^{\circ} \mathrm{F}$ gradient. Two studies found that the equivalent temperature gradient induced by moisture warping was generally on the order of -20 to $-35^{\circ} \mathrm{F}$ (Kosmatka et al. 2002; Wei et al. 2011); warping of this order of magnitude would be expected.

\subsection{Options for Improvement of the Warping Model}

There are several options for improving the current MEPDG warping model to correct for the deficiencies discussed above. The current equation has terms which cannot be accounted for when the equation is re-derived; these terms should be removed unless they can be satisfactorily explained. Alternately, and preferably, the equation should be rederived using a non-linear shrinkage distribution, in which case the unexplained factors become irrelevant. Plate theory should be considered in this derivation, as opposed to beam theory, as it more accurately represents slab behavior. Relative humidity factors from a different model could be used if they would provide a more accurate representation of the actual material behavior, and the term relating monthly relative humidity to the annual average should be removed. Additionally, only the drying shrinkage should be considered in computations, and the contribution of autogenous shrinkage should be removed, because only drying shrinkage contributes to warping.

Given that the permanent and transient components of drying shrinkage are caused by the same mechanisms, improvements to the warping model could also be used in a differential drying shrinkage model. The base equation for both models will be the same, while only some of the modification factors and the time factor (monthly versus yearly averages) would need to be different.

One large constraint in the development of a new warping or differential drying shrinkage model is that no new inputs beyond those already in the MEPDG must be required. The current MEPDG has three levels of input, based on the amount of information available to the design engineer. A good warping model will be able to accurately predict the moisture warping while only requiring the inputs currently included in the lowest level of MEPDG inputs, because this is often the only information a designer has. This eliminates the possibility of modifying the current warping models available because they require additional inputs or knowledge. For example, the warping model recently developed by Wei and Hansen (2011) is dependent upon the variation of 
relative humidity with depth, information which is generally not available to a pavement designer. Lee et al. (2011) also have a predictive model, but it requires finite element simulations using a proprietary program, which would be difficult to introduce into the current MEPDG program, would make the design process much more computationally intensive, and increase overall run time.

\subsubsection{Non-Linear Shrinkage Distribution with Plate Theory}

A derivation of the MEPDG equation using the linear shrinkage variation was discussed previously, and the final result of this is given in Equation 4.12. The triangular distribution of the shrinkage gradient used in the current MEPDG is more representative of the actual conditions within a slab than the original rectangular distribution; however, a non-linear distribution would be more accurate (Janssen 1987). The current MEPDG warping model was derived under the same assumption of beam behavior used by Eisenmann and Leykauf (1990b). In reality, a slab behaves as a plate, not a beam, and therefore plate theory should be used to compute the bending stress in the derivation of a new equation (Timoshenko and Goodier 1951).

\subsubsection{Derivation of a New Moisture Warping Model}

A major challenge in characterizing a non-linear gradient through the shrinkage zone is that only two points on the curve are known: the top and bottom of the shrinkage zone. To best fit any type of curve would require a minimum of three points. Additionally, it is not known if this curve should be a parabolic, an exponential, or some other type of curve. To eliminate this issue, it was assumed that the shrinkage gradient follows the arc of a quarter of an ellipse, as shown in Figure 4.7. In this figure, $a$ is the shrinkage term (which will be discussed in detail later) and the minor radius of the ellipse; $h_{s}$ is the depth of the shrinkage zone and the major radius of the ellipse; and $h$ is the thickness of the concrete slab.

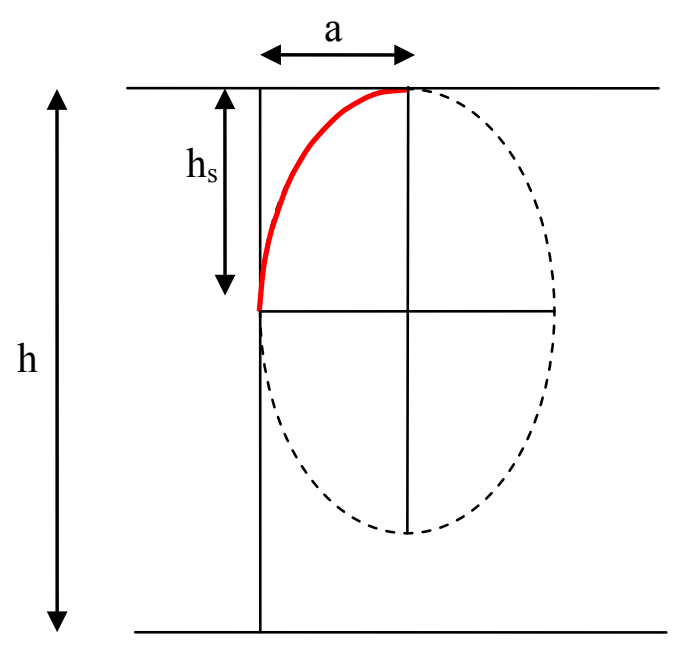

Figure 4.7: Non-linear shrinkage gradient modeled as a quarter of an ellipse.

Using a circle for this approximation does not work, because this implicitly assumes that $h_{s}$ and $a$ are equal, which is not the case. An ellipse, however, eliminates this problem 
because it has both major and minor radii, which do not need to be equal. The arc used to describe the non-linear shrinkage variation must be equal to a quarter of the ellipse; otherwise it would be necessary to locate points along the circumference of the ellipse, which would require more knowledge about the shape of the non-linear gradient than is available. Additionally, the geometric properties of the quarter ellipse of unequal radii are well documented, which facilitates calculations.

The current MEPDG warping model was derived using beam theory; however plate theory is more representative of the actual behavior of the slab in two-way bending. In the derivation of the non-linear model, plate theory is used to compute the bending stress induced in the slab by the moisture gradient. This derivation is the same for bending stresses induced by a differential drying shrinkage gradient.

The force caused by the non-linear shrinkage gradient $F_{s}$ is given by:

$$
F_{s}=a h_{s} E\left(1-\frac{\pi}{4}\right)
$$

Equation 4.13

The distance between the centroid of this distribution and the neutral axis of the slab is $e$ :

$$
e=\frac{h}{2}-h_{s}+\frac{2 h_{s}}{12-3 \pi}
$$

The moment about the neutral axis of the slab induced by the shrinkage is $M$ :

$$
M=F_{S} e=\frac{1}{24} a h_{s} E\left[-3 h(-4+\pi)-20 h_{s}+6 \pi h_{s}\right]
$$

The stress in the slab induced by this moment is $\sigma_{\mathrm{s}}$ :

$$
\sigma_{s}=\frac{M y}{I}
$$

Where $y=$ distance to the neutral axis, in this case $y=h / 2$

$I=$ second moment of inertia, in this case, for a unit width, $I=h^{3} / 12$

Therefore, $\sigma_{\mathrm{s}}$ is given by:

$$
\begin{array}{r}
\sigma_{s}=\frac{\frac{1}{24} a h_{s} E\left[-3 h(-4+\pi)-20 h_{s}+6 \pi h_{s}\right] * \frac{h}{2}}{h^{3} / 12} \\
=\frac{a h_{s} E\left[-3 h(-4+\pi)-20 h_{s}+6 \pi h_{s}\right]}{4 h^{2}}
\end{array}
$$

The stress $\sigma_{\mathrm{t}}$ due to an equivalent linear temperature distribution from plate theory is (Yoder and Witczak 1975): 


$$
\sigma_{t}=\frac{E \alpha h \Delta T}{2(1-\mu)}
$$

where $\mu$ is Poisson's ratio of the concrete

Equating these two stresses and solving for $\Delta T$ :

$$
\Delta T=\frac{a h_{s}\left[-3 h(-4+\pi)-20 h_{s}+6 \pi h_{s}\right](1-\mu)}{2 h^{3} \alpha}
$$

Equation 4.19

This equation is for the temperature gradient per unit thickness of the slab. To compare to the MEPDG model, it must be multiplied by the total height of the slab to get the total temperature gradient for the entire slab ETG:

$$
\mathrm{ETG}=\frac{a h_{s}\left[-3 h(-4+\pi)-20 h_{s}+6 \pi h_{s}\right](1-\mu)}{2 h^{2} \alpha}
$$

Equation 4.20

This equation does not require any inputs beyond those which are already required in the MEPDG, so it can be easily implemented. The only new term in the warping model is Poisson's ratio, $\mu$, but this term is already a required input in the MEPDG. It also improves on the triangular strain distribution currently used by assuming a non-linear distribution, and by using plate theory to better model the stresses in the slab. Further improvements will be made by changing the shrinkage term $a$. Note that the term $a$ will be different for the warping and differential drying shrinkage models, but the base equation will be the same.

\subsubsection{Validation of Elliptical Approximation}

To validate the proposed model, the non-linear elliptical approximation was compared to shrinkage variation with depth through a slab, as well as to the current linear approximation. Data on the variation of shrinkage through the depth of a pavement is very difficult to obtain. Instead, a model developed by Qin (2011) was used to predict the internal relative humidity with depth for a slab in several different locations around the country. The shrinkage model developed by Wei et al. (2011) was then used to convert the internal relative humidity to an ultimate shrinkage at intervals through the depth of a pavement. The shape of this shrinkage curve was then compared with that predicted by the non-linear elliptical approximation.

Qin's model is a coupled temperature and moisture model for the prediction of curling and warping which treats concrete as a visco-elastic material and accounts for the effects of creep and relaxation. One of the outputs of this model is the internal relative humidity (or pore relative humidity) through the depth of the slab. This program was run for several locations around the country for a 10 inch pavement with a water to cement ratio of 0.45. The locations were New Orleans, Louisiana; Columbus, Ohio; Los Angeles, California; Seattle, Washington; Reno, Nevada; Astoria, Oregon; and Las Vegas, Nevada. These sites were selected because some are very wet, others are very dry and 
some have relative humidity which varies seasonally; therefore a broad spectrum of climates is represented.

The output of these runs was a value of internal relative humidity at 0.125 inch intervals through the depth of the pavement every hour for five years. Data was taken at 30 days, one year and five years to give a representative picture over the life of the concrete. The value of internal relative humidity at each depth was converted to a value of shrinkage in the paste using an empirical relation from Wei et al. (2011), which is given in Equation 4.21 .

$$
\varepsilon_{\text {paste }}=\left[6150 *\left(1-\frac{R H}{100}\right)\right] * 10^{6}
$$

Equation 4.21

where $\mathrm{RH}$ is the internal relative humidity as a percent.

The shrinkage in the paste is then converted to an overall shrinkage in the concrete through Pickett's model (1956) to account for the confining effects of aggregate, as seen in Equation 4.22 (Wei et al. 2011).

$$
\varepsilon_{\text {concrete }}=\varepsilon_{\text {paste }} *\left(1-V_{a}\right)^{n}
$$

Equation 4.22

Where $\mathrm{V}_{\mathrm{a}}=$ the volume fraction of the aggregates

$\mathrm{n}=$ a correlation parameter

This method was used by Wei et al. to compute the autogenous shrinkage in the concrete in one study (2011), and by Wei and Hansen to compute the total shrinkage (drying and autogenous) in another (2011). No explanation is given as to why the same equation can be used to predict both autogenous and drying shrinkage, but it can be surmised that the authors' of those studies assumed that both drying and autogenous shrinkage have the same relation to relative humidity, even though they are caused by very different mechanisms. In both cases, a volume fraction of aggregate of $40 \%$ and an $n$ value of 1.68 were used. It should be noted that mixes intended for paving applications, especially when a slip-form paver is to be used, often have a higher volume fraction of aggregate, such as $72 \%$, which is used in Michigan (MDOT 2003). However, given that no other data was available, values of $\mathrm{V}_{\mathrm{a}}=40$ and $\mathrm{n}=1.68$ were used here as well.

The profiles of shrinkage versus depth were plotted for each location. The depth of the shrinkage zone was determined visually to the nearest inch based on the inflection point where the shrinkage profile changed from being mostly linear to being a distinct curve. Though in practice the depth of the shrinkage zone is generally assumed in design to be two inches, this is not always the case. To accurately assess the validity of the non-linear elliptical approximation given the depth of the shrinkage zone and the value of ultimate shrinkage at the top of the pavement, those actual values were used as $h_{s}$ and $a$, respectively. The linear approximation was also determined for comparison purposes using those values. The actual data curve, as well as the linear and non-linear 
approximations, for Reno at five years is given in Figure 4.8. The same curves for all other locations and ages are given in Appendix E.

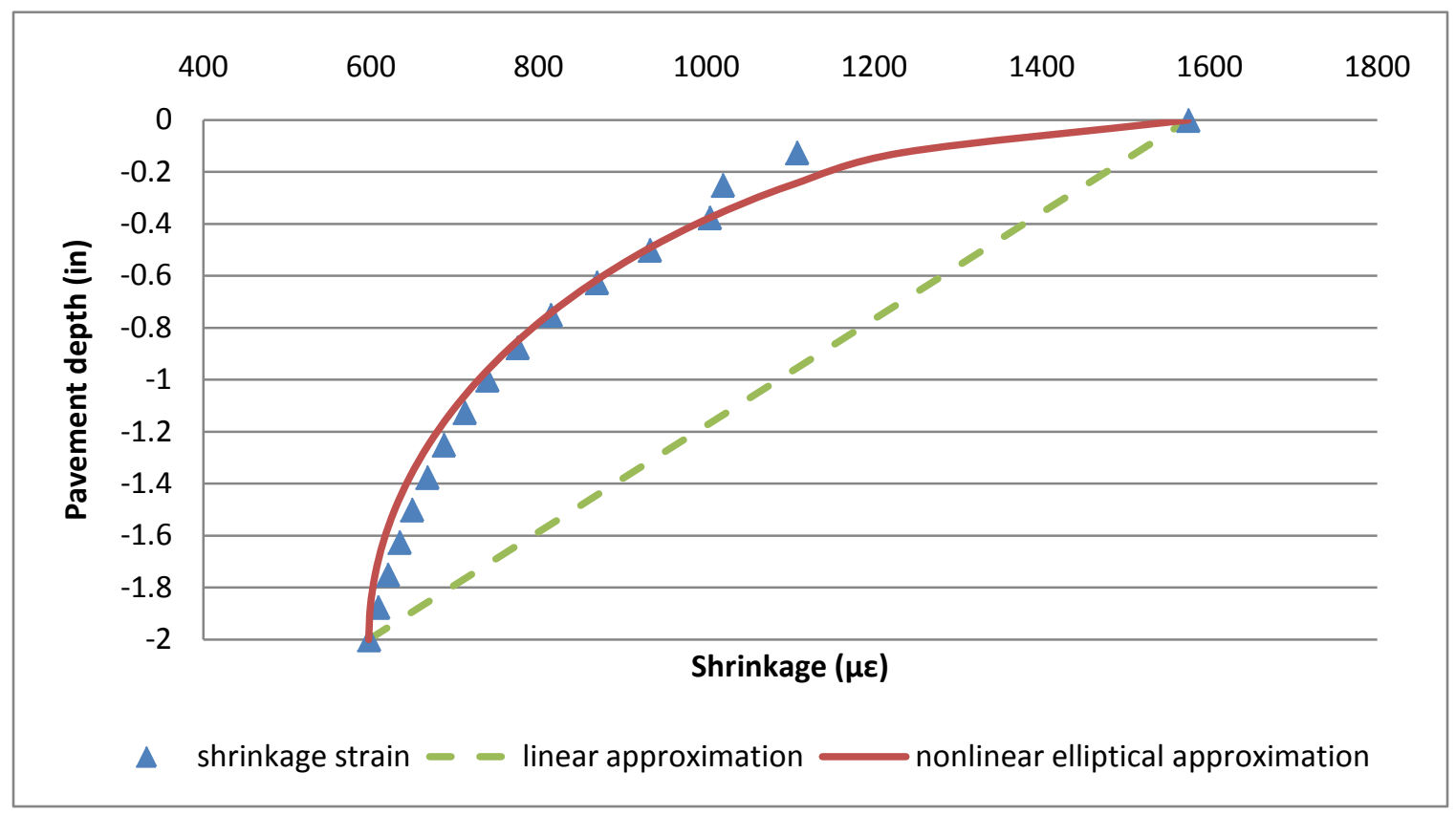

Figure 4.8: Ultimate shrinkage through the depth of the pavement for Reno, Nevada five years after construction.

As can be seen in Figure 4.8, the non-linear approximation is much closer to the actual data than the linear approximation. The correlation coefficients $\mathrm{R}^{2}$ for both the linear and non-linear approximations are given in Table 4.4. From this table, it can be seen that the non-linear approximation has a better correlation in every location, and at every pavement age. 
Table 4.4

Correlation coefficients for linear and nonlinear approximations.

\begin{tabular}{|c|c|c|c|}
\hline & & \multicolumn{2}{|c|}{$R^{\wedge} 2$} \\
\hline & & Linear & Non-linear \\
\hline \multirow{3}{*}{$\begin{array}{c}\text { New } \\
\text { Orleans }\end{array}$} & 30 days & 0.87 & 0.98 \\
\hline & 1 year & 0.82 & 0.86 \\
\hline & 5 years & 0.67 & 0.89 \\
\hline \multirow{3}{*}{ Columbus } & 30 days & 0.82 & 0.98 \\
\hline & 1 year & 0.90 & 0.93 \\
\hline & 5 years & 0.83 & 0.82 \\
\hline \multirow{3}{*}{ Los Angeles } & 30 days & 0.84 & 0.97 \\
\hline & 1 year & 0.91 & 0.98 \\
\hline & 5 years & 0.57 & 0.93 \\
\hline \multirow{3}{*}{ Seattle } & 30 days & 0.80 & 0.99 \\
\hline & 1 year & 0.82 & 0.99 \\
\hline & 5 years & 0.69 & 0.95 \\
\hline \multirow{3}{*}{ Reno } & 30 days & 0.74 & 0.98 \\
\hline & 1 year & 0.66 & 0.94 \\
\hline & 5 years & 0.76 & 0.98 \\
\hline \multirow{3}{*}{ Astoria } & 30 days & 0.90 & 0.95 \\
\hline & 1 year & 0.87 & 0.99 \\
\hline & 5 years & 0.69 & 0.92 \\
\hline \multirow{3}{*}{ Las Vegas } & 30 days & 0.65 & 0.95 \\
\hline & 1 year & 0.69 & 0.97 \\
\hline & 5 years & 0.87 & 0.99 \\
\hline
\end{tabular}

Though the fit of the approximation to the actual data is pertinent, it is more important that the area of the shrinkage distribution used in computing the bending moment be as accurate as possible.

Figure 4.9 show this area for the actual data, and the linear and nonlinear approximations. From this figure, it can be seen that the area of the shrinkage as defined by the nonlinear approximation is very close to that of the actual data, while the area of the shrinkage ad defined by the linear approximation is much larger. This area was computed for the actual data and the non-linear approximation using numerical integration via the trapezoidal rule, and for the linear approximation using basic geometry. The value of this area is given in Table 4.5, as is the percent difference between the actual data and each of the approximations. From this table, it can be seen that the non-linear approximation is better in every location and at every pavement age. 


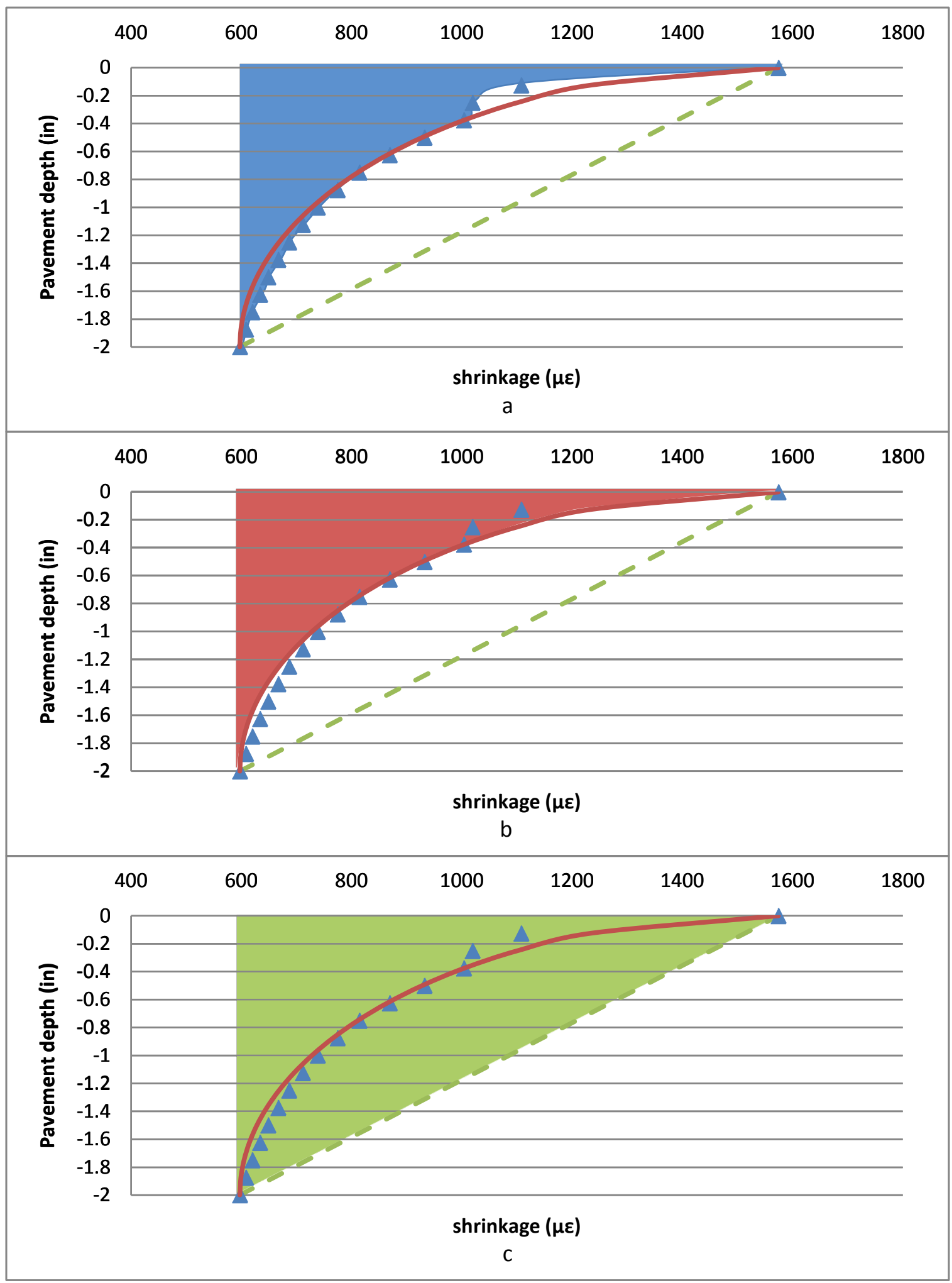

Figure 4.9: Area of the shrinkage distribution for Reno Nevada after five years of drying from a) the original data, b) the nonlinear elliptical approximation, and c) the linear approximation. 
Table 4.5

Area of shrinkage distribution for actual data, linear and nonlinear approximations.

\begin{tabular}{|c|c|c|c|c|c|c|}
\hline & & \multicolumn{3}{|c|}{ Area (inch* $\mu \varepsilon$ ) } & \multicolumn{2}{|c|}{$\begin{array}{c}\% \text { Difference from } \\
\text { original area }\end{array}$} \\
\hline & & Actual & Linear & Non-linear & Linear & Non-linear \\
\hline \multirow{3}{*}{$\begin{array}{c}\text { New } \\
\text { Orleans }\end{array}$} & 30 days & -2082 & -815 & -1278 & $61 \%$ & $39 \%$ \\
\hline & 1 year & -362 & -131 & -205 & $64 \%$ & $43 \%$ \\
\hline & 5 years & -321 & -132 & -206 & $59 \%$ & $36 \%$ \\
\hline \multirow{3}{*}{ Columbus } & 30 days & -3646 & -1477 & -2316 & $59 \%$ & $36 \%$ \\
\hline & 1 year & -720 & -269 & -421 & $63 \%$ & $42 \%$ \\
\hline & 5 years & -230 & -95 & -139 & $59 \%$ & $40 \%$ \\
\hline \multirow{3}{*}{ Los Angeles } & 30 days & -3974 & -1551 & -2403 & $61 \%$ & $40 \%$ \\
\hline & 1 year & -4879 & -180 & -1282 & $96 \%$ & $74 \%$ \\
\hline & 5 years & -124 & -53 & -80 & $57 \%$ & $36 \%$ \\
\hline \multirow{3}{*}{ Seattle } & 30 days & -6203 & -2515 & -3911 & $59 \%$ & $37 \%$ \\
\hline & 1 year & -7196 & -2247 & -3953 & $69 \%$ & $45 \%$ \\
\hline & 5 years & -2226 & -951 & -1490 & $57 \%$ & $33 \%$ \\
\hline \multirow{3}{*}{ Reno } & 30 days & -7294 & -3068 & -4809 & $58 \%$ & $34 \%$ \\
\hline & 1 year & -9816 & -4249 & -6666 & $57 \%$ & $32 \%$ \\
\hline & 5 years & -2374 & -977 & -1526 & $59 \%$ & $36 \%$ \\
\hline \multirow{3}{*}{ Astoria } & 30 days & -3439 & -1282 & -2011 & $63 \%$ & $42 \%$ \\
\hline & 1 year & -5174 & -2108 & -3309 & $59 \%$ & $36 \%$ \\
\hline & 5 years & -1149 & -488 & -766 & $58 \%$ & $33 \%$ \\
\hline \multirow{3}{*}{ Las Vegas } & 30 days & -8164 & -3492 & -5474 & $57 \%$ & $33 \%$ \\
\hline & 1 year & -8984 & -3784 & -5931 & $58 \%$ & $34 \%$ \\
\hline & 5 years & -7477 & -2933 & -4597 & $61 \%$ & $39 \%$ \\
\hline
\end{tabular}

Based on the findings of this validation study, the non-linear approximation described by a quarter of an ellipse is a better representation of the actual variation of shrinkage with depth than the linear approximation currently used by the MEPDG. The non-linear approximation could be improved by best-fitting a curve to actual data; however, this would require more information than is currently available to the design engineer. The elliptical approximation requires the same inputs as the linear model, but offers a better fit and more accurate results. Because this approximation is a model for drying shrinkage in general, it can be used for both warping and differential drying shrinkage.

\subsubsection{Remove Relation to Average Annual Relative Humidity}

In the current MEPDG model, the effects of shrinkage in each month are related to the annual average. One way to improve the current model is to eliminate the average relative humidity term $S_{\text {have }}$. Subtracting the annual average relative humidity factor from the monthly relative humidity factor forces unrealistic behavior, as previously discussed. The use of the $S_{\text {have }}$ factor causes the average warp for the year to be zero; whether this 
was an intentional assumption or the unintended consequence of another assumption is unclear.

An average annual warp of zero implies that any upward warp due to the top of the slab being dryer than the bottom (as is generally the case) will be counteracted by downward warp. This means that the slab must spend some months every a year with the top of the slab on average wetter than the bottom. As the moisture content at the base of a slab is generally around 100\% (Janssen 1987), this is highly unlikely in even the wettest of climates.

This improvement is also applicable to the differential drying shrinkage model. In fact, failure to remove this term would cause the model to predict that the differential drying shrinkage is equal to zero in all cases. Differential drying shrinkage should be predicted based on either the average monthly relative humidity for the month in which the pavement will be built, or the annual average relative humidity. If the annual average is used, then $S_{h i}$ will be equal to $S_{h a v e}$, and multiplying the entire equation by the difference of the two would yield an unrealistic value of zero for differential drying shrinkage.

\subsubsection{Alternate Relative Humidity Factors}

The current MEPDG model uses a relative humidity factor which is modified from the ACI 209 shrinkage model. One way to improve the current MEPDG model is to explore whether or not other shrinkage models have better relative humidity factors which could be used in place of the current $S_{h i}$ factor. To this end, five alternate relative humidity factors from different shrinkage models were investigated, including the original ACI model (Equation 4.4). Note that in the discussion of the models, the original notation used in each model is preserved.

\subsubsection{Relative Humidity Factor from the B3 Model}

The B3 model was developed by Bazant and Baweja (1995c), and builds on previous shrinkage models, including the BP and BP-KK (Bazant and Baweja 1995b). The B3 model is simpler than its predecessors while still yielding accurate results. RILEM's extensive data bank was used to calibrate the model, which has a 95\% confidence limit (Bazant and Baweja 1995a). In this model, the ultimate shrinkage is multiplied by a humidity dependence factor $k_{h}$, which is given in Equation 4.23 (Bazant and Baweja 1995c).

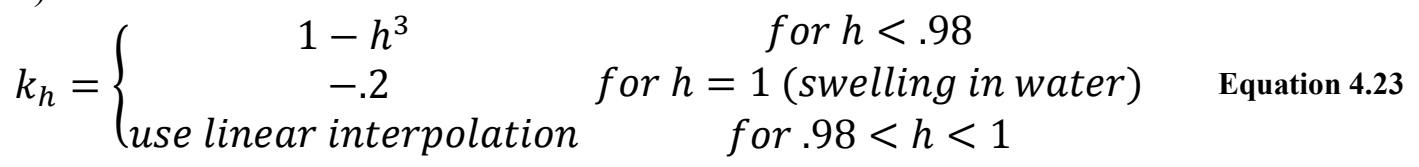

where: $h$ is the relative humidity as a decimal.

\subsubsection{Relative Humidity Factor from the CEB90 Model}

The CEB90 model was developed by the Comité Euro-International du Béton for the EuroCode (European structural building code). This model is semi-empirical, and gives the overall or average shrinkage across a cross section. The mechanistic portion of this model is based on diffusion theory; however, the model is not fully mechanistic because it was developed as a general code equation which only incorporates parameters which 
would be known at the time of design. The model was empirically optimized using a databank of laboratory test results for structural concrete. In this model, the drying shrinkage is multiplied by the coefficient of relative humidity, $\beta_{R H}$, which is given in Equation 4.24 (Muller and Hilsdorf 1990).

$$
\begin{aligned}
& \beta_{R H}=-1.55 \beta_{A R H} \\
& \beta_{A R H}=1-\left(\frac{R H}{100}\right)^{3}
\end{aligned}
$$

where: $R H$ is relative humidity as a percentage

\subsubsection{Relative Humidity Factor from the FIB Model}

The Fédération Internationale du Béton (FIB) shrinkage model was developed to predict shrinkage for both normal and high performance concretes. This model is based on the CEB90 model, but is modified to incorporate different factors at higher and lower humidity. In this model, the drying shrinkage is multiplied by the relative humidity coefficient $\beta_{R H}$, as seen in Equation 4.25 (FIB 2008).

$$
\beta_{R H}=\left\{\begin{array}{cc}
1 & \text { for } R H<40 \% \\
-1.55 *\left[1-\left(\frac{R H}{R H_{0}}\right)^{3}\right] & \text { for } 40<R H<99 \% \\
0.25 & \text { for } R H>99 \%
\end{array}\right.
$$

Equation 4.25

where: $R H$ is humidity as a percent and $R H_{0}=100 \%$

\subsubsection{Relative Humidity Factor from the GL2000 Model}

The GL2000 model was designed by Gardner and Lockman as a tool for designers, and, as such, the only required inputs for the model are those likely to be known at the time of design. Due to this, it does not account for the restraining effects of aggregates and is not intended to be highly accurate. This model assumes that $96 \%$ relative humidity is the hygroequilibric point, and only accounts for shrinkage due to self-desiccation; additionally it is only valid for concrete with a compressive strength less than 12000 psi. In this model, the ultimate shrinkage is multiplied by a humidity correction term $\beta_{h}$, which is given in Equation 4.26 (Gardner and Lockman 2001).

$$
\beta_{h}=1-1.18 h^{4}
$$

Equation 4.26

where: $h$ is humidity as a decimal.

\subsubsection{Comparison of Shrinkage Models}

To determine the best model from which to pull relative humidity adjustment factors, several criteria must be considered. First, any new relative humidity factor must only be dependent on variables already known in the MEPDG. It is important to note that the entire shrinkage model is not being used in the development of the new working model. Only the relative humidity adjustment factors from the various models are being considered. This means that the shrinkage model itself can incorporate factors not 
considered by MEPDG, as long as the relative humidity factors do not incorporate terms outside the scope of MEPDG inputs.

Also, it is important to note whether the relative humidity factor is applied to the ultimate shrinkage or only to the drying shrinkage. The current MEPDG model uses the ultimate shrinkage to calculate warping, however, only the reversible portion of drying shrinkage is actually responsible for slab deformations due to moisture. Ideally, a relative humidity factor from a model using the same shrinkage quantity (whether ultimate or drying) as that used in the MEPDG model would be selected.

Though several studies have been conducted to compare these models to each other, the accuracy of the relative humidity adjustment factors is not something which is generally compared in the literature. Instead, the predicted shrinkage is compared to actual values taken from data bases with thousands of data points. Therefore, an assumption must be made that the best relative humidity adjustment factor will come from the most accurate shrinkage model.

Results of the ACI 209, CEB90, B3 and GL2000 models have been compared with each other in several different studies. The ACI 209 model was found to be the worst model at predicting shrinkage in multiple studies when compared with shrinkage in standard state department of transportation mixes (Mokarem et al. 2003) and the RILEM data bank (AlManaseer and Lam 2005; Bazant and Li 2008). The ACI model predictions have a large amount of scatter when compared with actual data, and produce illogical trends (Gardner and Lockman 2001). The model was also found to overestimate shrinkage (Bazant and Li 2008).

The CEB model was found to be the best predictor of shrinkage when compared to the ACI, B3, and GL2000 models in one study, which compared the shrinkage model with data from shrinkage tests conducted on standard mix designs used by a state department of transportation for paving concrete (Mokarem et al. 2003). Another study, however, found that CEB ranked third out of four when compared to the same models in predicting shrinkage for a very large number of test cases from the RILEM data bank. The CEB90 model was found to both under-predict (Gardner and Lockman 2001; Al-Manaseer and Lam 2005), and overestimate shrinkage (Al-Manaseer and Lam 2005) depending on the case.

The FIB model was not compared to any other models except the CEB 90 model. These two models use the same adjustment factor in the standard humidity range (40-99\%), and are very similar. The FIB model was found to have almost identical accuracy for normal strength concrete, and is only an improvement over the CEB 90 model in the prediction of shrinkage in high-strength concretes (FIB 2008), which are not generally used for paving applications.

The GL2000 model was found by most studies to be one of the best models when compared with the B3, CEB90 and ACI models. Though the model tends to 
overestimate, it was found to be the best at predicting shrinkage overall in one study (AlManaseer and Lam 2005). Another study found that the GL2000 model was the best model in the design stage, when mix properties were assumed in calculation, but second best to the B3 model when actual mix properties were used instead (Gardner and Lockman 2001). Two other studies found that the GL2000 model was the second best model overall to predict shrinkage (Al-Manaseer and Lam 2005; Bazant and Li 2008), and that the results obtained were similar to the B3 model, though not quite as accurate (Bazant and Li 2008). It is important to note, however, that the GL2000 model is only intended to predict autogenous shrinkage, which does not contribute to warping.

The B3 model is considered to be one of the best models. The model slightly underestimates shrinkage generally (Al-Manaseer and Lam 2005), and requires more inputs than the rest, though this is not the case for the computation of relative humidity adjustment factors. One study found the GL2000 model to be slightly superior overall to the B3 model. However, in that study, the GL2000 model ranked very well in some categories, and very low in others, while the B3 model was consistently ranked as one of the top two models (Al-Manaseer and Lam 2005). Almost all of the other studies considered the B3 model to be the best at predicting shrinkage (Gardner and Lockman 2001; Mokarem et al. 2003; Bazant and Li 2008).

Overall, the general consensus was that the ACI 209 model was the worst model, and either the B3 or the GL2000 model was the best. As the current MEPDG relative humidity correction factor is very closely related to the ACI 209 relative humidity correction factor, it is recommended that the current relative humidity adjustment factor be replaced with one taken from the B3 or GL2000 models. The B3 model would be the best option because it is consistently found to be the most accurate representation of shrinkage behavior. The GL2000 model is slightly less accurate, but still a very good option, especially considering that it is specifically intended to be used as a design aid. The main drawback of this model is that it is only intended to predict autogenous shrinkage.

To further compare the relative humidity adjustment factors from the various models, the relative humidity adjustment factor was calculated for relative humidity ranging from 0 to $100 \%$, as shown in Figure 4.10. From this figure, it can be seen that, though the models differ greatly, many of the relative humidity adjustment factors are quite similar. The current $S_{h i}$ factor is fairly comparable to the factors used in the ACI, B3, and GL2000 models. The CEB90 and FIB models have much larger adjustment factors than the rest. Again, comparisons of the models in the literature were for the entire model, not solely the relative humidity adjustment factor. As such, it is not necessarily surprising that models whose results differ greatly may have closely aligned relative humidity adjustment factors; other portions of the model also affect their accuracy.

It is interesting to note that the only two models which account for swelling upon immersion in water through the relative humidity correction factor are the B3 and GL2000 models, which are generally considered the best models. All other models have 
a positive relative humidity correction factor when the ambient relative humidity is $100 \%$. In the GL2000 model and the B3 model, the relative humidity factor is negative; when this factor is multiplied by the ultimate shrinkage in the model, the result will be negative shrinkage, or swelling. This swelling is consistent with the expected behavior when the concrete is soaked in water. Therefore, at very large values of relative humidity, the B3 and GL2000 relative humidity adjustment factors are better at predicting behavior than those of the other models. Though it is unlikely that the ambient relative humidity would be so high for an entire month (which is the time interval for which warping is calculated in the MEPDG), this is nonetheless a metric by which the relative humidity adjustment factors, and not just the models, can be compared.

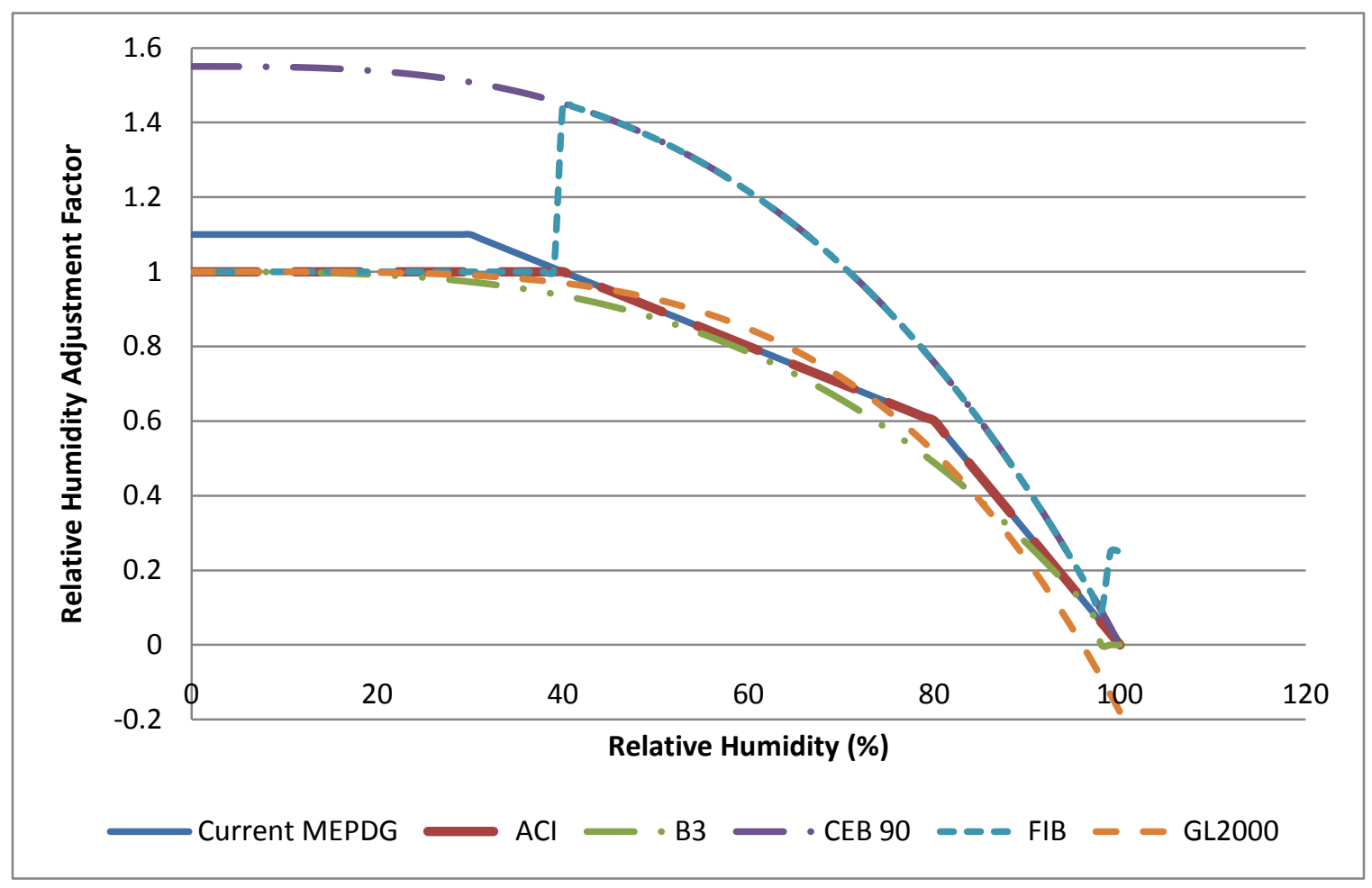

Figure 4.10: Comparison of shrinkage models.

\subsubsection{Selection of Relative Humidity Adjustment Factor}

The new relative humidity adjustment factor will be called $Я$ (pronounced yah), so as to distinguish it from the original $S_{h i}$ factor; $S_{h i}$ will not appear in the proposed model. The relative humidity adjustment factor from the B3 model (Bazant and Baweja 1995c) was selected due to its ability to consistently and accurately predict shrinkage. The B3 model is a step function which uses different equations to predict the relative humidity adjustment factor for ranges of internal relative humidity of $0-98 \%, 98 \%-100 \%$ and at $100 \%$. In practice, the relative humidity of a pavement is never going to be above $98 \%$ for an entire month. Therefore, it is possible to pare down the equation to only the relevant term, for the sake of simplicity. Thus, the new relative humidity adjustment factor $Я$ will be given by Equation 4.27 


$$
\text { Я }=1-(R H / 100)^{3}
$$

Equation 4.27

where: $R H$ is the relative humidity as a percentage.

This relative humidity adjustment factor is also applicable to the proposed differential drying shrinkage model. The only difference is that the differential drying shrinkage is only computed once for the life of the pavement while moisture warping is computed on a monthly basis. When computing $Я$ for the differential drying shrinkage model, it will be computed using the annual average relative humidity or the monthly average humidity for the month in which the pavement is built. In the moisture warping model, $Я$ will be computed on a monthly basis using the monthly average relative humidity.

\subsubsection{Account for Contribution of Autogenous Shrinkage}

Warping occurs because a portion of the drying shrinkage is reversible when the concrete is exposed to high ambient relative humidity. Differential drying shrinkage is that portion of shrinkage which is permanent, but does not occur uniformly in the slab. Summed together, the permanent and reversible portions of shrinkage equal the total drying shrinkage in the slab. The ultimate shrinkage is comprised of this drying shrinkage and autogenous shrinkage due to self-desiccation. Autogenous shrinkage occurs uniformly through the slab, and therefore does not cause any slab bending deformations, though it does induce a uniform reduction in both the length and width of the slab. Therefore, autogenous shrinkage cannot contribute to moisture warping or differential drying shrinkage. The current MEPDG warping model uses the reversible shrinkage factor $\phi$ to account for the fact that only a portion of shrinkage is reversible and only that portion causes warping. However, the $\phi$ factor is multiplied by the total shrinkage, which includes autogenous shrinkage. This would cause the model to overestimate the amount of warping expected. Inclusion of autogenous shrinkage in the proposed differential drying shrinkage model would have the same effects.

It is possible that the current MEPDG warping model removes the contribution of autogenous shrinkage by assuming that half of all shrinkage is autogenous and half is due to drying. This possibility was discussed above as a potential source of the mysterious $1 / 2$ factor in the current equation which cannot be accounted for. However, the assumption that the half of the total shrinkage is autogenous only applies to concrete with a water to cement ratio of 0.3 or less (Kosmatka et al. 2002), which is generally not the case for paving mixes.

To remedy this issue, a factor, $\omega$, is proposed as the ratio between drying shrinkage and ultimate shrinkage. Multiplying the ultimate shrinkage by the drying shrinkage factor $\omega$ will ensure that only the drying shrinkage is considered in computing warping and differential drying shrinkage. However, there currently is no model which will predict the amount of total shrinkage which is autogenous. It is widely held that the amount of autogenous shrinkage is dependent on the water to cement ratio of the paste (Neville 1997; Wei et al. 2011). Generally, it is assumed that autogenous shrinkage occurs only in 
concrete with a water to cement ratio less than the theoretical limit required for hydration (Holt 2001), which is generally given as 0.36 (Mindness et al. 2003) to 0.38 (Neville 1997) but is some times estimated to be as high as 0.42 (Holt 2001), depending on the source. Neville recommends that self-desiccation be considered in any concrete with a water to cement less than 0.5 , even though this is above his lower bound on hydration (1997). However, the only direct relation found in the literature states that half of all shrinkage is autogenous for a water to cement ratio of 0.3 , though no study is listed to support this claim (Kosmatka et al. 2002).

Therefore, a relation between the ratio of drying shrinkage to ultimate shrinkage, $\omega$, and water to cement ratio was developed. Data on ultimate and autogenous shrinkage for ordinary concrete of various water to cement ratios was taken from a variety of studies. All of the studies measured the shrinkage of sealed (autogenous) and unsealed (ultimate) concrete specimens. All of the data selected was for concrete which could be used in a typical paving application; samples may have contained slag or flyash as pozzolans, or superplasticizer, and may or may not have been air entrained. Any samples containing silica fume, fibers, shrinkage reducing admixture, and lightweight or recycled concrete aggregates were excluded because they are not representative of typical paving mixes. Though much data was available on the shrinkage of mortar, it was excluded because coarse aggregates provide a confining effect and have a large influence on the amount of shrinkage experienced by the concrete (Pickett 1956).

For each mix, the percentage of ultimate shrinkage due to drying shrinkage was calculated using Equation 4.28. Table 4.6 shows the calculated value of $\omega$ and the water to cement ratio for each mix, as well as the data source.

$$
\omega=\frac{\text { ultimate shrinkage }- \text { autogenous shrinkage }}{\text { ultimate shrinkage }}
$$


Table 4.6

Ratio of drying shrinkage to ultimate shrinkage for various w/c ratios.

\begin{tabular}{|c|c|c|}
\hline$w / c$ & $\omega$ & Source \\
\hline 0.4 & 0.6 & \multirow{4}{*}{$\begin{array}{l}\text { (Tazawa } \\
\text { and } \\
\text { Miyazawa } \\
\text { 1995) }\end{array}$} \\
\hline 0.3 & 0.5 & \\
\hline 0.23 & 0.2 & \\
\hline 0.17 & 0 & \\
\hline 0.20 & 0.24 & \multirow{5}{*}{$\begin{array}{l}\text { (Tazawa } \\
\text { et al. } \\
2000 \text { ) }\end{array}$} \\
\hline 0.30 & 0.51 & \\
\hline 0.40 & 0.64 & \\
\hline 0.50 & 0.88 & \\
\hline 0.60 & 0.84 & \\
\hline 0.43 & 0.72 & \multirow{5}{*}{$\begin{array}{l}\text { (Baroghel- } \\
\text { Bouny } \\
\text { and Godin } \\
2000 \text { ) }\end{array}$} \\
\hline 0.44 & 0.78 & \\
\hline 0.34 & 0.42 & \\
\hline 0.3 & 0.54 & \\
\hline 0.26 & 0.51 & \\
\hline 0.37 & 0.70 & \multirow{6}{*}{$\begin{array}{c}\text { (Persson } \\
1999)\end{array}$} \\
\hline 0.38 & 0.78 & \\
\hline 0.41 & 0.77 & \\
\hline 0.44 & 0.80 & \\
\hline 0.55 & 0.80 & \\
\hline 0.30 & 0.67 & \\
\hline 0.3 & 0.41 & \multirow{3}{*}{$\begin{array}{l}\text { (Zhang et } \\
\text { al. 2003) }\end{array}$} \\
\hline 0.26 & 0.29 & \\
\hline 0.35 & 0.90 & \\
\hline 0.43 & 0.56 & \multirow{2}{*}{$\begin{array}{l}\text { (Saliba et } \\
\text { al. 2011) }\end{array}$} \\
\hline 0.65 & 0.78 & \\
\hline
\end{tabular}

To determine a function for $\omega$ in terms of water to cement, three different relations were investigated: linear, bilinear, and sigmoid. The linear relation was determined through linear regression and is given in Equation 4.29.

$$
\omega=1.5492 *\left(\frac{w}{c}\right)+0.0173
$$


The correlation coefficient, $\mathrm{R}^{2}$, for the linear model was 0.6238 . Figure 4.11 shows the drying shrinkage as a fraction of ultimate shrinkage and the linear trend line. From this figure it can be seen that the data has a definite trend, which is highlighted by the linear model, but the low correlation coefficient suggests that another approximation may be a better fit.

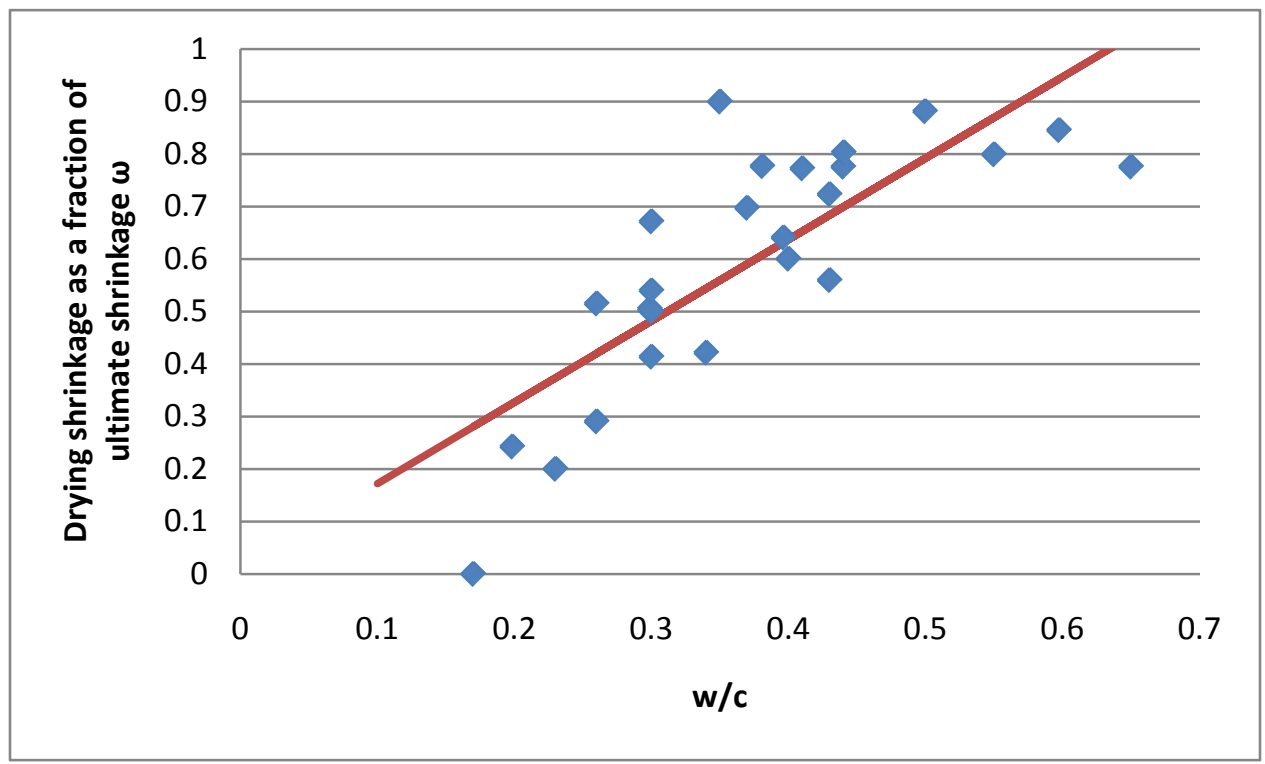

Figure 4.11: $\omega$ vs. $w / c$, data and associated linear trend line.

Based on the premise that the amount of autogenous shrinkage changes at the water to cement ratio required to fully hydrate the concrete system (Holt 2001), a bilinear model could potentially better capture the dependence of $\omega$ on the water to cement ratio. In such a model, the first line would end and the second begin at the value of water to cement equal to the theoretical limit of hydration (Baroghel-Bouny and Mounanga 2005). As there is some contention about which water to cement is the lower limit of hydration, break points at a water to cement ratio of $0.36,0.38,0.40$, and 0.42 were all tested. Linear regression was used to best fit both lines, and the overall correlation coefficient was calculated for each break point, see Table 4.7.

Table 4.7

Correlation coefficient for a bilinear relation between $\omega$ and $w / c$ for various theoretical limits for hydration.

\begin{tabular}{|c|c|}
\hline $\begin{array}{c}\text { w/c corresponding } \\
\text { to the limit for } \\
\text { hydration }\end{array}$ & $\mathrm{R}^{2}$ \\
\hline 0.36 & 0.8045 \\
\hline 0.38 & 0.7886 \\
\hline 0.4 & 0.7682 \\
\hline 0.42 & 0.7698 \\
\hline
\end{tabular}


The model with a water to cement ratio of 0.36 for the theoretical limit of hydration had the highest correlation coefficient, and was therefore considered to be the best fitting bilinear model. This model is given in Equation 4.30, and shown in Figure 4.12.

$$
\omega= \begin{cases}3.6308(w / c)-0.5675, & w / c \leq 0.36 \\ 0.5463(w / c)+0.4901, & w / c>0.36\end{cases}
$$

Equation 4.30

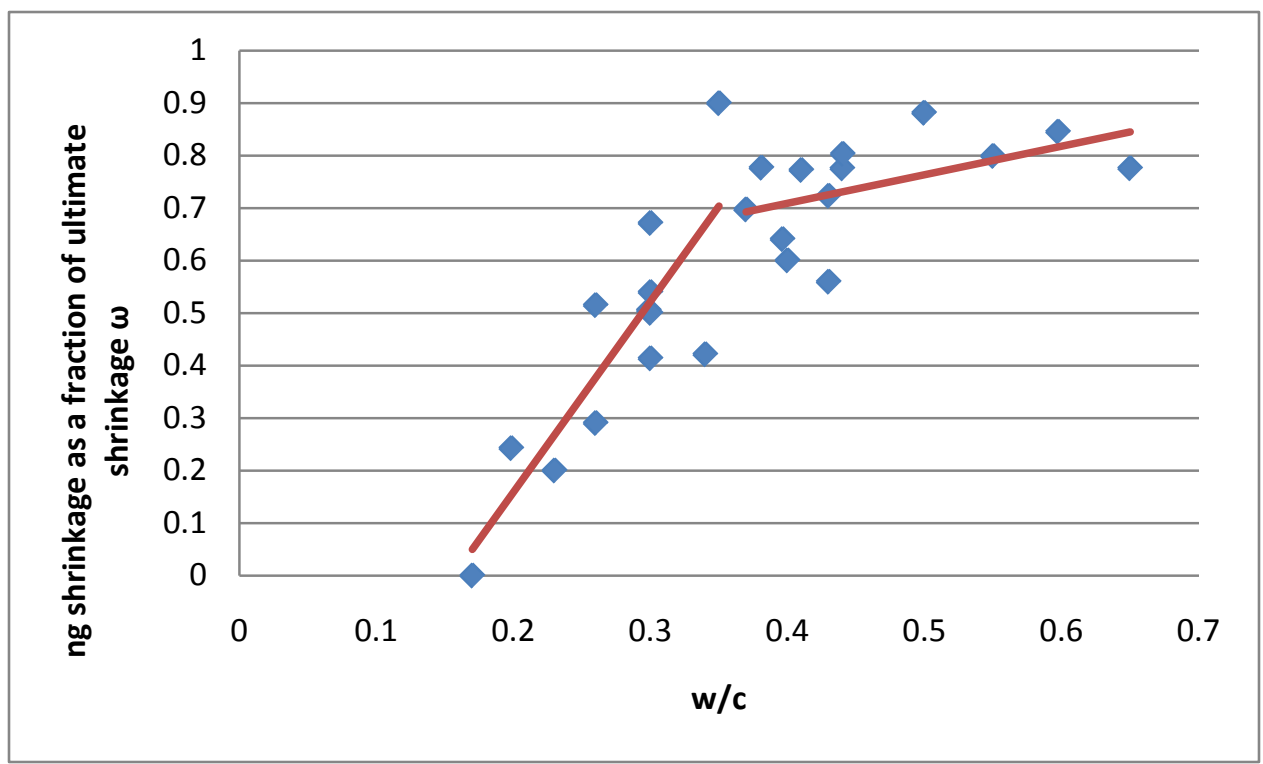

Figure 4.12: $\omega$ vs. w/c, data and associated bilinear trend line for a theoretical limit of hydration corresponding to a w/c ratio of 0.36 .

From Figure 4.12, it can be seen that the slope of the trend line for a water to cement ratio above 0.36 is much smaller than that of the trend line for a water to cement below 0.36 . The bilinear model fits well with the conventional notion that above a water to cement ratio corresponding to the minimum water to cement ratio required for full hydration, shrinkage is primarily due to drying, and autogenous shrinkage is small. Many researchers have opined that the effects of autogenous shrinkage can be neglected at large water to cement ratios (Neville 1997; Holt 2001; Kosmatka et al. 2002). However, ignoring the contribution of autogenous shrinkage would tend to over-predict the amount of moisture warping; therefore it should not be disregarded.

The final model considered to relate $\omega$ and water to cement was a sigmoid function. This function was selected because it is often used to graph a trend that has a low and a high plateau connected by a rise or decent in the middle. This function is particularly useful in mathematical applications and computer programming because it is continuous. The basic sigmoid function is centered at zero, but this can be shifted over by a value of $q$, see Equation 4.31. 


$$
y=\frac{1}{1+\exp [-n *(x-q)]}
$$

Even though the sigmoid is centered at the point $q$, this point is merely an inflection point, and not necessarily equivalent to the water to cement ratio corresponding to the theoretical limit for hydration. An optimization algorithm was used to find the values of $n$ and $q$ which would minimize the sum of the squares of the error between the actual data and the sigmoid. This yielded a relation given in Equation 4.32, which is plotted along with the data in Figure 4.13. The sigmoid relation had a correlation coefficient of 0.7115.

$$
\omega=\frac{1}{1+\exp [-9.2376 *((w / c)-0.309)]}
$$

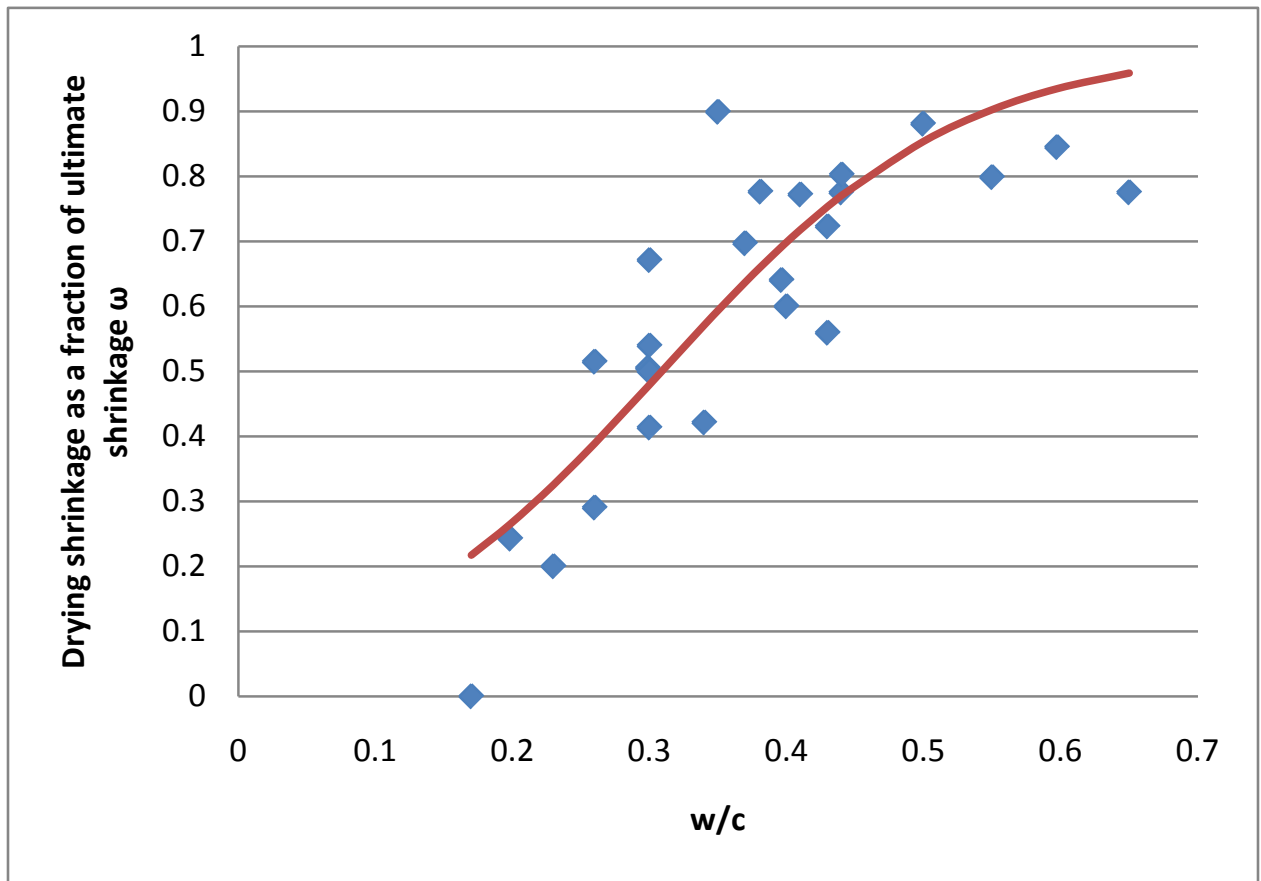

Figure 4.13: $\omega$ vs. $w / c$, data and associated sigmoid trend line.

Between the three models, the bilinear model (Equation 4.30) fit the data the best because it had the highest correlation coefficient. Therefore the bilinear model was selected as the equation for $\omega$. Multiplying $\omega$ by the ultimate shrinkage will give the amount of shrinkage which is due to drying mechanisms only. Introducing this term in the proposed warping and differential drying shrinkage models will eliminate the contribution of autogenous shrinkage, which the current warping model erroneously includes in the calculation for slab bending. 


\subsubsection{Alternate Reversible Shrinkage Factor}

The fraction of shrinkage which is reversible, given by the factor $\phi$, is dependent on the type of aggregate used, as well as the permeability of the concrete, and is further influenced by the curing regime and drying history of the pavement. This was explored in great detail in Chapter 3. Currently, the MEPDG assumes that half of all shrinkage is reversible, or $\phi=0.5$ (NCHRP 2004b). However, this value does not match those found in the reversible shrinkage study in Chapter 3.

It is important to note that the MEPDG assumes that concrete is dried in a $40 \%$ relative humidity environment for measuring total shrinkage and predicting reversible shrinkage. The MEPDG recommends using the AASHTO T160 test to measure shrinkage at after drying at 40\% humidity (NCHRP 2004b). Both the AASHTO T160 length change test and its equivalent ASTM C157 counterpart recommend drying at 50\% relative humidity (Lawler 2007; ASTM 2008e). No guidance is given in the MEPDG as to why drying at $40 \%$ relative humidity is used. The testing conducted in Chapter 3 was conducted in accordance with ASTM standards so that the results could be compared with those of future researchers, and to lend credence to the results. Further study will be required to determine how drying the concrete at $40 \%$ relative humidity instead of $50 \%$ will change the results.

The reversible shrinkage study found that the amount of shrinkage which is reversible depends mainly on the curing regime. For pavements, a value of $\phi=0.3$ is recommended, indicating that $30 \%$ of shrinkage is reversible. This corresponds to the average value of the percentage of shrinkage which was reversible for the second and third cycles of drying and re-wetting of dry cured specimens. The dry cured case was selected because it was considered extremely unlikely that a pavement would be wet cured in the field for an entire year. The amount of reversible shrinkage for the first rewetting cycle for the dry cured samples was considerably higher than $30 \%$. This value from the first re-wetting cycle was not selected because pavements are exposed to wetting during every precipitation event. This model is designed to predict long term behavior, and the pavement will experience many wetting events throughout the course of its life. The reversible shrinkage for the second and third cycles of drying and rewetting appears to be relatively constant, and therefore is a good assumption for the amount of reversible shrinkage expected. Future work will be necessary to determine if this is the case for further drying and re-wetting cycles.

A reversible shrinkage factor $\phi=0.3$ means that the irreversible shrinkage factor $(1-\phi)$ is equal to $70 \%$ for the differential drying shrinkage model. The fact that the reversible and irreversible shrinkage factors sum to one is helpful in that, if an unconservative assumption is made regarding the amount of reversible shrinkage anticipated, the additional differential volume change still will be considered in design in the form of differential drying shrinkage. 


\subsubsection{Alternate Combined Model to Account for Drying Shrinkage and Relative Humidity Effects}

In the current MEPDG model, the shrinkage term $a$ is equal to $\phi \varepsilon_{s u}\left(S_{h i}-S_{\text {have }}\right.$ ) (NCHRP 2003). The proposed warping model would redefine $a$ to be equal to $\phi \varepsilon_{s u} \omega Я$, where $\omega$ accounts for the drying shrinkage and $Я$ accounts for the effects of ambient relative humidity. In the proposed differential drying shrinkage model, $a$ would be redefined as $(1-\phi) \varepsilon_{s u} \omega Я$. An alternate option for both the differential drying shrinkage and warping models would be to use a model proposed by Miyazawa and Tazawa (2000), which predicts the drying shrinkage with variations in time and relative humidity and is given in Equation 4.33.

$$
\begin{aligned}
\varepsilon_{d}\left(t, t_{d}\right)=e * & {\left[1-\exp \left(\frac{R H-R H_{0}}{100}\right)\right] } \\
* & {\left[1-\exp \left(1-c\left(t-t_{d}\right)^{d}\right)\right] }
\end{aligned}
$$

Equation 4.33

where:

$R H$ is the ambient relative humidity as a percent

$t$ is the age of the concrete in days

$t_{d}$ is the age at initial set in days

$R H_{0}, c, d$, and $e$. are coefficients dependent upon the water to cement ratio of the concrete shown in Table 4.8

Table 4.8

Alternate combined shrinkage and relative humidity model coefficients.

\begin{tabular}{|c|c|c|c|c|}
\hline $\mathrm{w} / \mathrm{c}$ & $\mathrm{RH}_{0}$ & $\mathrm{c}$ & $\mathrm{d}$ & $\mathrm{e}$ \\
\hline 0.20 & 74.9 & 0.135 & 0.56 & 2200 \\
\hline 0.30 & 84.7 & 0.100 & 0.65 & 1800 \\
\hline 0.40 & 90.0 & 0.080 & 0.75 & 1600 \\
\hline 0.50 & 95.0 & 0.035 & 0.90 & 2000 \\
\hline 0.60 & 97.0 & 0.025 & 0.95 & 2000 \\
\hline
\end{tabular}

Using this model would eliminate the need for both the $\omega$ and $Я$ factors, and the need to compute warping as a function of time (Equation 4.2). However, this would require more modification to the MEPDG than was intended when a new warping model was

proposed. Additionally, this model is only valid for concrete which is described as having "a usual aggregate fraction", though no guidance is give as to what a usual amount would be. Concrete used in paving applications generally has an aggregate fraction that is much higher than that of concrete used in structural applications. No information is available for how the model should be applied to concretes with a water to cement ratio for which coefficients are not explicitly given. For example, it is not apparent if linear 
interpolation is valid for computing the required coefficients at a water to cement ratio of 0.42 .

The Miyazawa and Tazawa model is not a practical choice for inclusion in the MEPDG at this time. However, the possibility of a model which predicts drying shrinkage based on ambient relative humidity and water to cement is particularly intriguing, and more research should be conducted in the future to determine how such a model could be implemented. Also, this model could be used as a comparison tool for current or proposed models, once it was determined that it is valid for paving mixes.

\subsection{Final Proposed Models for Moisture Warping and Differential Drying Shrinkage}

To improve the current MEPDG warping prediction, changes were made to nearly every aspect of the model. First, the basic equation for the equivalent temperature difference was re-derived based on an assumed shrinkage distribution through the depth of the slab which followed the curve of a quarter of an ellipse. This eliminated problems associated with both the linear distribution assumption and unexplained factors in the original equation. Additionally, stresses were calculated based on plate theory, rather than beam theory, because slabs experience two way bending. The new basic equation is the basis for both the warping and differential drying shrinkage models.

The drying shrinkage factor, $\omega$, was introduced to remove the contribution of autogenous shrinkage, so that only the drying shrinkage is included in the models. This is more accurate than the ultimate shrinkage used in the current model because autogenous shrinkage is uniform and therefore does not contribute to slab bending for either moisture warping or differential drying shrinkage.

Monthly relative humidity is no longer related to the average, as the $S_{\text {have }}$ term was removed. Without this term, the proposed model predicts actual slab bending, as opposed to physically impossible behavior. Additionally, the monthly relative humidity modification factor $S_{h i}$ was changed to a new factor $Я$, based on the B3 model (Bazant and Baweja 1995c), to more accurately account for the effects of relative humidity.

Finally, changes to the reversible shrinkage factor $\phi$ were proposed to more accurately reflect the behavior of concrete exposed to cyclic drying and re-wetting. Additionally, recommendations were made for the modification of this factor to account for the use of recycled and lightweight aggregates. In the differential drying shrinkage factor, this becomes the irreversible shrinkage factor $(1-\phi)$, but the same recommendations still apply.

The proposed moisture warping model is given in Equation 4.34. The proposed differential drying shrinkage model is given in Equation 4.35. 


$$
\begin{aligned}
& E T G_{\text {warp }}=\frac{\phi Я \omega \varepsilon_{u} h_{s}\left[-3 \mathrm{~h}(-4+\pi)-20 \mathrm{~h}_{\mathrm{s}}+6 \pi \mathrm{h}_{\mathrm{s}}\right](1-\mu)}{2 h^{2} \alpha} \quad \text { Equation 4.34 } \\
& E T G_{D D S} \\
& =\frac{(1-\phi) Я \omega \varepsilon_{u} h_{s}\left[-3 h(-4+\pi)-20 \mathrm{~h}_{\mathrm{s}}+6 \pi \mathrm{h}_{\mathrm{s}}\right](1-\mu)}{2 h^{2} \alpha} \quad \text { Equation } 4.35
\end{aligned}
$$

where:

$$
\begin{aligned}
& \omega=\left\{\begin{array}{ll}
3.6308(w / c)-0.5675, & w / c \leq 0.36 \\
0.5463(w / c)+0.4901, & w / c>0.36
\end{array} \quad\right. \text { Equation 4.30 } \\
& \text { Я }=1-(R H / 100)^{3}
\end{aligned}
$$

\subsection{Model Validation}

The proposed warping model was validated through comparison with other models since empirical validation was not possible. There are currently no models for predicting the amount of differential drying shrinkage in a slab. However, given that the base equation for both models is the same, validation of the proposed warping model alone is sufficient to show that the base equation effectively predicts the bending behavior of a slab due to drying shrinkage. The only difference between the models is whether or not the drying shrinkage is permanent.

\subsubsection{Warping Models Used for Comparison}

A review of the literature shows that there has been very limited study of the amount of warping in concrete slabs due to moisture. This is due in part to the fact that, until recently, moisture warping was very poorly understood and often ignored, and partly due to the difficultly in measuring the amount of deformation a slab experiences and correlating it to a moisture gradient. This process would be similar to quantifying the amount of built-in curl, which will be discussed in Chapter 5, only the effects of moisture would have to be isolated.

Due to the lack of data on moisture warping, it is not possible to validate the proposed model with actual testing. Additionally, designing and conducting a test to obtain such data is far beyond the scope of this project. Therefore, the new model (Equation 4.34) was compared with the existing model (Equation 4.1), and the re-derived existing model (Equation 4.3). These models are repeated below for the purpose of comparison.

Current MEPDG model:

$$
E T G_{S H i}=\frac{3\left(\phi \varepsilon_{s u}\right)\left(S_{h i}-S_{h a v e}\right) h_{s}\left(\frac{h}{2}-\frac{h_{s}}{3}\right)}{\alpha h^{2} 100}
$$


Re-derived MEPDG model:

$$
\mathrm{ETG}=\frac{6 a h_{s}\left(\frac{h}{2}-\frac{h_{s}}{3}\right)}{\alpha h^{2}}
$$

In the re-derived model, the shrinkage term $a$ was taken as $\phi \varepsilon_{s u} S_{h i}$

New model:

$$
E T G_{\text {warp }}=\frac{\phi Я \omega \varepsilon_{u} h_{s}\left[-3 \mathrm{~h}(-4+\pi)-20 \mathrm{~h}_{\mathrm{s}}+6 \pi \mathrm{h}_{\mathrm{s}}\right](1-\mu)}{2 h^{2} \alpha} \quad \text { Equation } 4.34
$$

Additionally, warping was computed using a model proposed by Wei and Hansen (2011). Wei and Hansen's model relates moisture deformation to shrinkage caused by changes in relative pore humidity for slabs on grade. It is based on a linear regression equation relating autogenous shrinkage to internal relative humidity which was originally developed by Wei et al. (2011). Coupling this model with a Pickett model (1956) allowed Wei et al. to predict the autogenous shrinkage in concrete. Wei and Hansen use this same relation in their warping model, except it is used to predict total shrinkage, not just autogenous shrinkage, which implicitly assumes that drying and autogenous shrinkage have the same relation to variations in pore relative humidity with depth. This model for the equivalent temperature difference in a slab due to warping is given in Equation 4.36 (Wei and Hansen 2011).

$$
\Delta T_{e}=\frac{12}{\alpha h^{2}} \int_{-\frac{h}{2}}^{\frac{h}{2}}[6150(1-R H(z))]\left(1-V_{A}\right)^{n} * 10^{-6} * Z * d z \quad \text { Equation } 4.36
$$

where:

$\Delta \mathrm{T}_{\mathrm{e}}=$ equivalent temperature difference due to warp

$\mathrm{RH}(\mathrm{z})=$ variation in pore relative humidity with depth

$\mathrm{h}=$ slab thickness

$\mathrm{z}=$ distance from the neutral axis

$\alpha=$ coefficient of thermal expansion of the concrete

$\mathrm{V}_{\mathrm{A}}=$ volume fraction of the aggregate

$\mathrm{n}=$ constant from Pickett's model, here use 1.68

Qin's coupled temperature and moisture model (2011) was used to generate the data on internal relative humidity variation with depth at several locations around the country for the model comparisons.

\subsubsection{Inputs and Methods}

Each model was run for a typical concrete pavement in seven different cities located around the country: New Orleans, Louisiana; Columbus, Ohio; Seattle, Washington; Los Angeles, California; Reno Nevada; Las Vegas, Nevada; and Astoria, Oregon. These sites were selected because they represent regions with different humidity regimes and because data on the relative humidity variation through the depth of the slab could be 
readily obtained for use in the Wei and Hansen model. For the other three models, climatic data from the EICM was generated for the same cities (NCHRP 2006).

The slab used was a 10 inch thick PCC pavement with a water to cement of 0.45 , Poisson's ratio of 0.15 , a coefficient of thermal expansion of $5.5^{*} 10^{-6}$ per ${ }^{\circ} \mathrm{F}$, and a volume fraction of aggregate of 0.75 . The ultimate shrinkage of the concrete as assumed to be $600 \mu \varepsilon$. These values are all representative of a standard pavement. The value of $n$ for Wei and Hansen's model was taken as 1.68, per their recommendation (2011). The reversible shrinkage was assumed to be $30 \%$ of the total shrinkage for a pavement with virgin aggregates, as was found in Chapter 3. Warping was computed at an age of five years, as the proposed model is intended to predict long term behavior of the pavement.

As was previously discussed in the validation of the elliptical approximation, the depth of the shrinkage zone $h_{s}$ is generally assumed to be two inches, but varies depending on climate to which the pavement is exposed. In order to accurately compare the proposed model with Wei and Hansen's model, the value of $h_{s}$ used in all models was that actually found in the input data from Qin's model. However, in design, the depth of the shrinkage zone is typically not known in advance. Therefore, the expected equivalent temperature difference due to warping was also computed for all models with an assumed value of $h_{s}$ equal to two inches, which is the value recommended by the MEPDG (NCHRP 2004c).

Wei and Hansen's model does not directly require ambient relative humidity as an input, as this information is contained in the relative humidity profile with depth. However, the current and re-derived MEPDG models and the proposed warping model rely on ambient humidity from the EICM. In order to make comparisons based on the same data, the equivalent temperature difference due to warping was computed twice with the proposed model using two different ambient relative humidity adjustment factors. $Я$ was computed using the EICM for comparison with the MEPDG models, and with the value of internal relative humidity at the top surface of the slab from Qin's data for comparison with the Wei and Hansen model. Ambient relative humidity for the month of July was taken from the EICM for each location, as Qin's data at an age of five years was based on conditions in July.

\subsubsection{Results and Comparison of Warping Models}

The equivalent temperature difference due to warping (in ${ }^{\circ} \mathrm{F}$ ) computed for each location, with the depth of the shrinkage zone determined empirically, is shown in Figure 4.14. The equivalent temperature difference due to warping computed for each location, with the depth of the shrinkage zone equal to two inches for all locations, is given in Figure 4.15 Note that the current MEPDG model appears to be missing from the graph. This is because the current MEPDG warping model always predicts a value which is approximately zero, and therefore is not visible on the graph. Due to differences in required inputs, the results from the current and re-derived MEPDG models should be compared with those from the new model which uses a value of $\boldsymbol{A}$ based on EICM data, while Wei and Hansen's model should be compared with the new model which uses a value of $A$ based on Qin's data. 


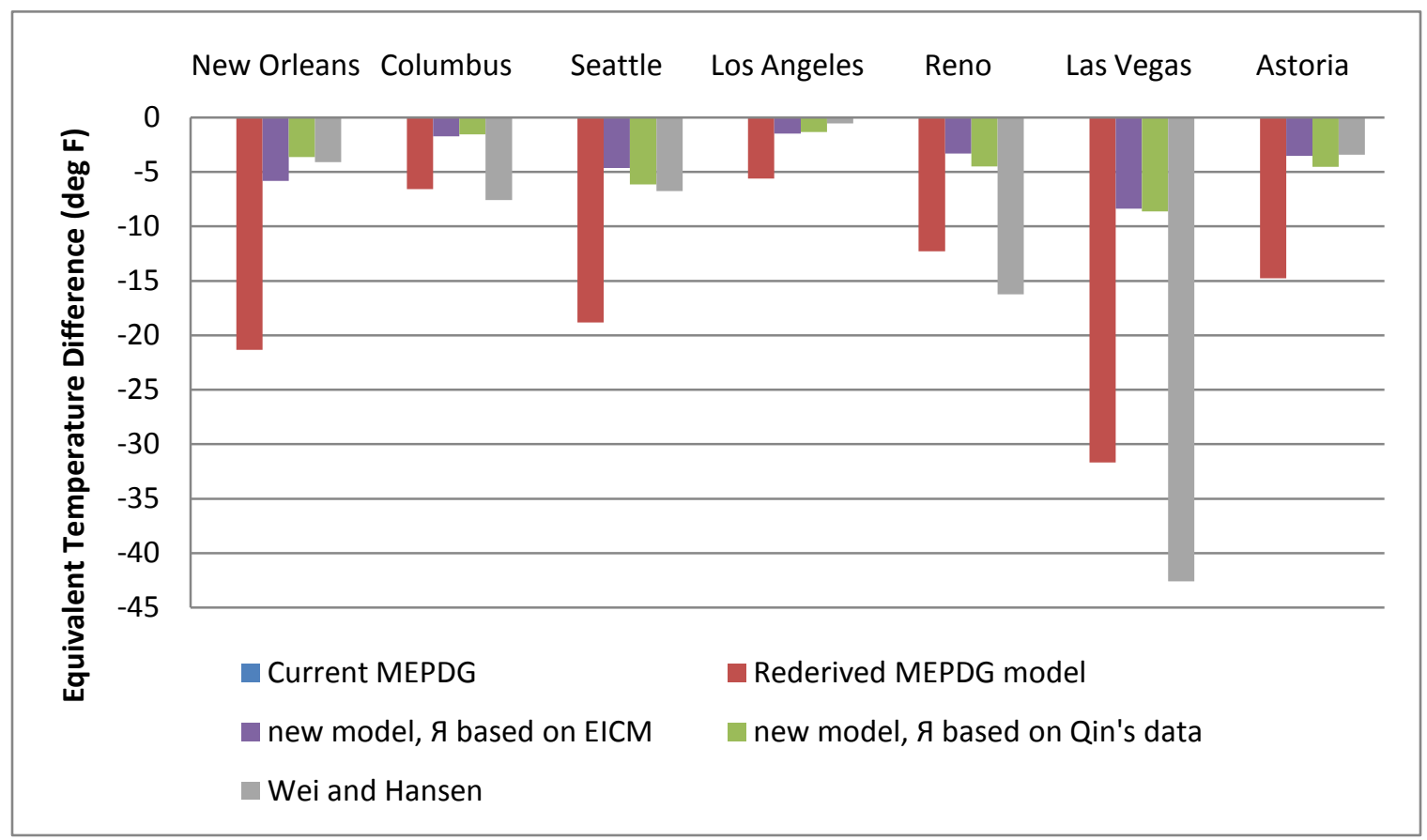

Figure 4.14: Equivalent temperature difference $\left({ }^{\circ} \mathrm{F}\right)$ from various models with the depth of the shrinkage zone determined from internal relative humidity data.

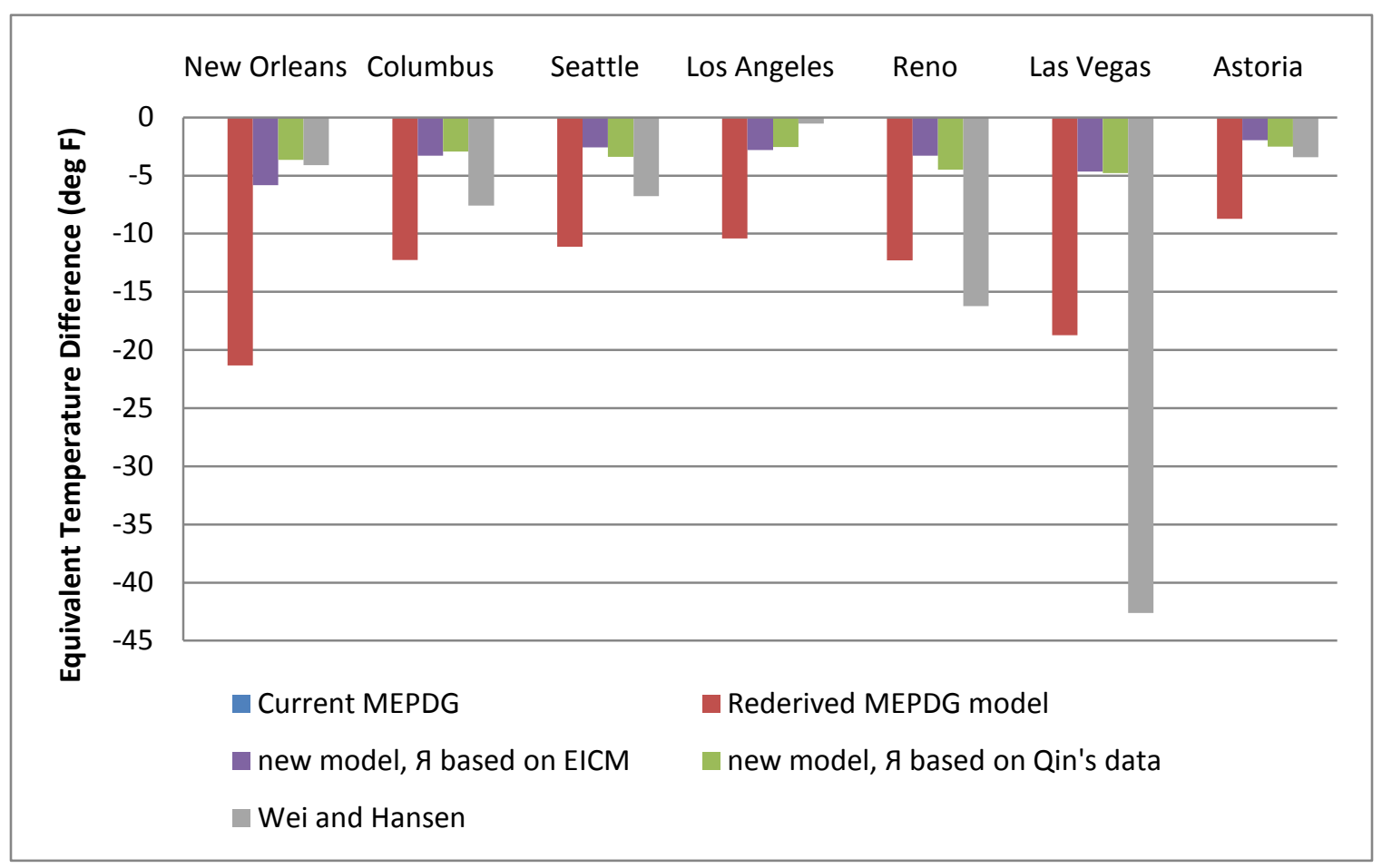

Figure 4.15: Equivalent temperature difference $\left({ }^{\circ} \mathrm{F}\right)$ from various models with the depth of the shrinkage zone equal to two inches in all locations. 
From these figures, it can be seen that, regardless of the depth of the shrinkage zone, certain trends exist in all locations studied. First, the current MEPDG model predicts essentially no warping in any location, for reasons previously discussed. The re-derived MEPDG model predicts much higher warping than the proposed model. This is to be expected, as the area of the shrinkage "force" distribution which causes the deformation is 40 to $45 \%$ larger for a linear shrinkage distribution than a more accurate non-linear, elliptical distribution. Additionally, the shrinkage considered to cause warping in the proposed model is much smaller than that considered in the re-derived MEPDG model due to the $Я$ and $\omega$ factors.

Wei and Hansen's model predicted an equivalent temperature difference due to warping which was either smaller or larger than that predicted by the proposed model, depending on the location of the pavement. This is likely because Wei and Hansen's model is dependent on the variation of relative humidity with depth through the entire slab, while the proposed model depends only on the depth of the shrinkage zone and the relative humidity at the surface of the slab. For the purpose of validating the new model, the fact that Wei and Hansen's model tends to bound the proposed model shows that the proposed model predicts reasonable values of equivalent temperature difference.

One interesting observation is that on occasion, the Wei and Hansen model predicts warping in amounts close to or larger than the re-derived MEPDG model. The MEPDG warping model is based on a linear shrinkage distribution, which would tend to over predict the deformations induced by warping. This is particularly true in dry climates, such as Las Vegas and Reno, Nevada, and happens even when the depth of the shrinkage zone used to compute the warping in the re-derived MEPDG model is determined empirically. Based on this limited study, this data suggests that Wei and Hansen's model potentially over predicts warping in dry climates.

The value given for the depth of the shrinkage zone has a large impact on the value of the equivalent temperature gradient determined using either the current or re-derived MEPDG models, or the proposed new model. This is to be expected, since the moment due to the moisture gradient which causes warping is directly related to the area of the shrinkage "force" distribution. Choosing a value of $h_{s}$ which is close to the actual depth of the shrinkage zone will make the model more accurate.

Table 4.9 shows the percent difference in equivalent temperature difference due to moisture warping for a shrinkage zone assumed to be two inches deep and one found empirically. Note that the depth of the shrinkage zone in New Orleans and Reno was found empirically to be two inches, and therefore the percent difference for all models was zero. The Wei and Hansen model is unaffected by the value of $h_{s}$ because it is not an input parameter in that model. The proposed model is affected by a change in $h_{s}$ slightly more than the current and re-derived MEPDG models, though all models are greatly affected. Given this sensitivity, it is important to properly estimate the depth of the shrinkage zone. 
Table 4.9

Percent difference in equivalent temperature difference with a depth of shrinkage zone determined both empirically and equal to two inches.

\begin{tabular}{|c|c|c|c|c|c|}
\hline Site & $\begin{array}{c}\text { Current } \\
\text { MEPDG }\end{array}$ & $\begin{array}{c}\text { Re-derived } \\
\text { MEPDG } \\
\text { model }\end{array}$ & $\begin{array}{c}\text { new model, } Я \\
\text { based on } \\
\text { Qin's data }\end{array}$ & $\begin{array}{c}\text { new model, } Я \\
\text { based on } \\
\text { EICM data }\end{array}$ & $\begin{array}{c}\text { Wei and } \\
\text { Hansen }\end{array}$ \\
\hline Columbus & $60 \%$ & $60 \%$ & $62 \%$ & $62 \%$ & $0 \%$ \\
\hline Seattle & $51 \%$ & $51 \%$ & $57 \%$ & $57 \%$ & $0 \%$ \\
\hline Los Angeles & $60 \%$ & $60 \%$ & $62 \%$ & $62 \%$ & $0 \%$ \\
\hline Las Vegas & $51 \%$ & $51 \%$ & $57 \%$ & $57 \%$ & $0 \%$ \\
\hline Astoria & $51 \%$ & $51 \%$ & $57 \%$ & $57 \%$ & $0 \%$ \\
\hline
\end{tabular}

The depth of the shrinkage zone is generally assumed to be two inches for all pavements, regardless of thickness or location. This assumption is based on a study by Janssen which included relatively few sites and pavement thicknesses (1987). The coupled temperature and moisture model from Qin showed that the depth of the shrinkage zone is indeed two inches for some pavements, but this is not always the case. For the seven sites examined in this study, the depth of the shrinkage zone in the wet climates was found to be between two and four inches, while in the dry climates it was between one and four inches, and was one inch in a moderate climate (Qin 2011). Kim and Lee (1999) also studied the moisture movement in concrete with several different water to cement ratios. Their study corrected for the effects of autogenous shrinkage, and found the depth of the drying shrinkage zone to be closer to three inches. Janssen's data fits nicely within this spectrum, but does not give the impression that there is a range of values for $h_{s}$.

From the elliptical shape validation (Section 4.4.1.2 ), and the overall model validation shown above, it is obvious that correct selection of the depth of the shrinkage zone is crucial in the estimation of warping. More work will be needed in the future to determine how the depth of the shrinkage zone can be predicted for design purposes.

\subsection{Implications of Moisture Warping and Differential Drying Shrinkage on the Total Equivalent Temperature Difference}

To examine the effects of using the proposed moisture warping and differential drying shrinkage models in the MEPDG, the total equivalent temperature difference was calculated using both the new models and the current MEPDG methods. Las Vegas, Nevada and Seattle, Washington, were selected for this study because they are representative of a very dry and very wet climate, respectively, thus bounding the problem. For both sites, a typical 10 inch thick concrete pavement was considered and evaluated in the MEPDG using default inputs for all parameters. The temperature gradient at every hour for the $20^{\text {th }}$ year of the pavement's life was obtained from the MEPDG thermal model (NCHRP 2006). This temperature gradient was used to compute the total equivalent temperature difference using both the current MEPDG methods and the new proposed models. 
For the case of the current MEPDG methods, relative humidity data from the EICM was used to calculate the predicted monthly moisture warping for the $20^{\text {th }}$ year of the pavement's life with the current MEPDG warping model (Equation 4.1). The default value for $\mathrm{BIC}$ of $-10^{\circ} \mathrm{F}$ was used. The total temperature gradient was calculated for each hour for one year as the sum of the temperature gradient, the equivalent temperature gradient due to moisture warping and the BIC.

For the case of the new model, relative humidity data from the EICM was used to calculate the predicted monthly moisture warping for the $20^{\text {th }}$ year of the pavement's life with the proposed warping model (Equation 4.34). The BIC term is comprised of the differential drying shrinkage, construction curl and creep effects. A value of differential drying shrinkage was calculated using the proposed differential drying shrinkage model (Equation 4.35) using an average annual relative humidity based on data from the EICM. A simplifying assumption was made that the construction curl would be negated by the creep. Though this assumption is unlikely to be completely representative of real behavior, and is not recommended for generally practice, it was necessary for the purposes of comparison. The total temperature gradient was calculated for each hour for one year as the sum of the temperature gradient, and the equivalent temperature gradients due to moisture warping and differential drying shrinkage. Figure $4.16 \mathrm{a}$ and $\mathrm{b}$ show the total equivalent temperature gradient and its constituent components for Las Vegas, calculated with the proposed models and the current MEPDG, respectively. Figure $4.17 \mathrm{a}$ and $b$ show the same comparison for Seattle. In these figures, the horizontal axis has been highlighted for clarity and comparison purposes.

From these figures, it can be seen that the current MEPDG models predict that slabs are concave upwards (an overall negative gradient) much less frequently than the proposed model does for Las Vegas. The two models predict roughly the same frequency of upwardly concave deformations in Seattle. Warping plays essentially no role in determining the deformed shape of the slab in the current MEPDG model, while slabs are always warped upwards with the new model. The effects of using a differential drying shrinkage model to predict the amount of built-in curl rather than the default $-10^{\circ} \mathrm{F}$ BIC value are much more pronounced in Seattle than in Las Vegas. This is because Las Vegas has a much drier climate than Seattle, and therefore experiences much more differential drying shrinkage. This increase in differential drying shrinkage, as well as an increase in the expected moisture warping, means that the proposed models predict that in Las Vegas, a slab would be deformed upwards much more frequently than in Seattle.

Using the MEPDG models, it was found that slabs in Las Vegas are curled upwards $74 \%$ of the time; this increased to $91 \%$ using the proposed models. In Seattle, it was found that slabs are curled upwards $87 \%$ of the time, regardless of which model was used. This is because the equivalent temperature differences predicted by the new warping and differential drying shrinkage models sum to approximately $-10^{\circ} \mathrm{F}$, which is the value of $\mathrm{BIC}$ assumed by the current MEPDG. It is important to note that the models matched well only for this location, slab thickness, and material properties, and that this is not always the case. Upwardly curled slabs can separate from underlying layers, leading to 
top-down cracking and premature fatigue failure. Therefore, it is imperative that the deformed shape of the pavement be accurately estimated in design. 


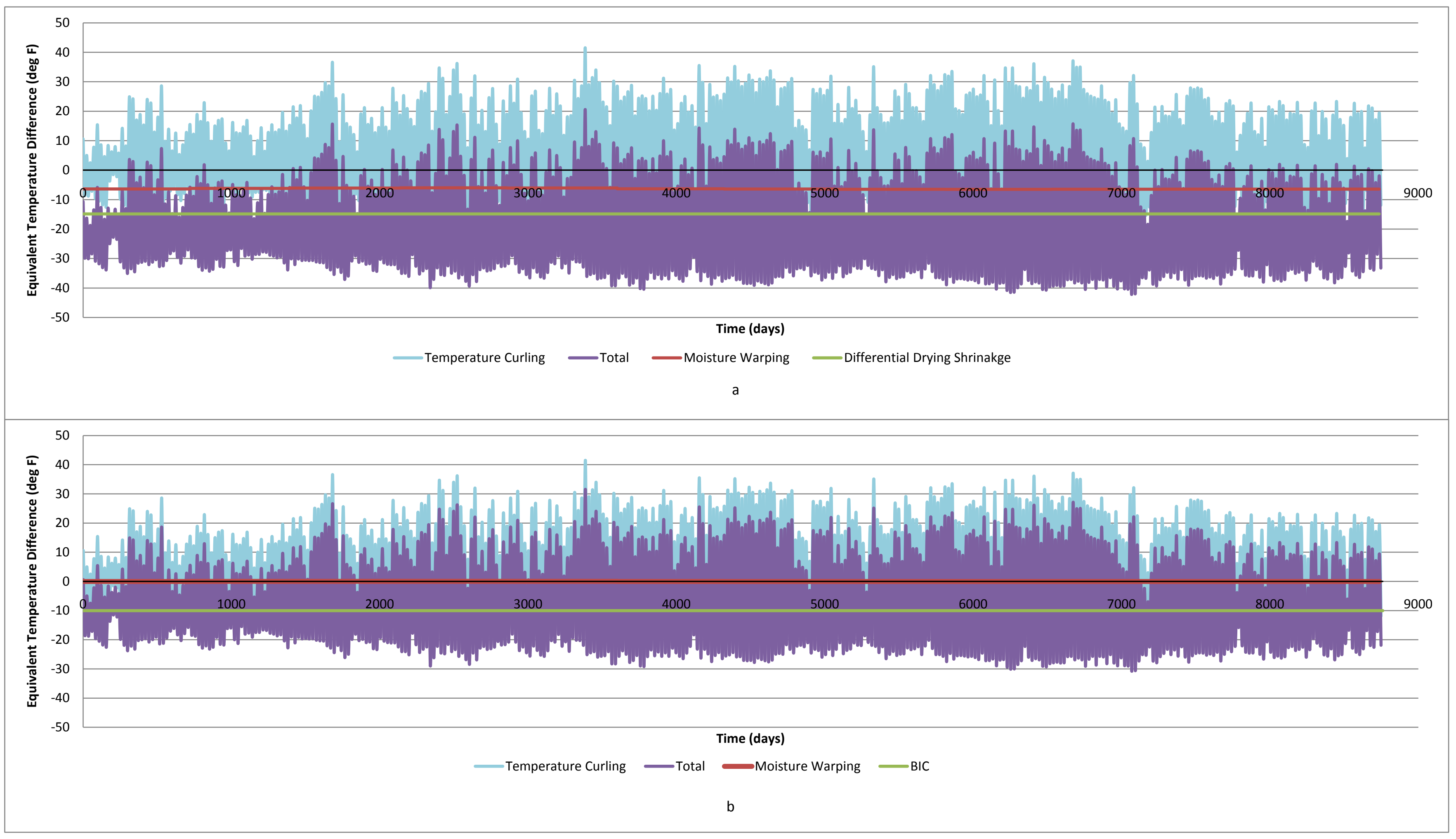

Figure 4.1: Predicted equivalent temperature difference in Las Vegas using a) the proposed warping and differential drying shrinkage models, and b) the MEPDG model warping model and -10 ${ }^{\circ} \mathrm{F}$ default value for BIC 


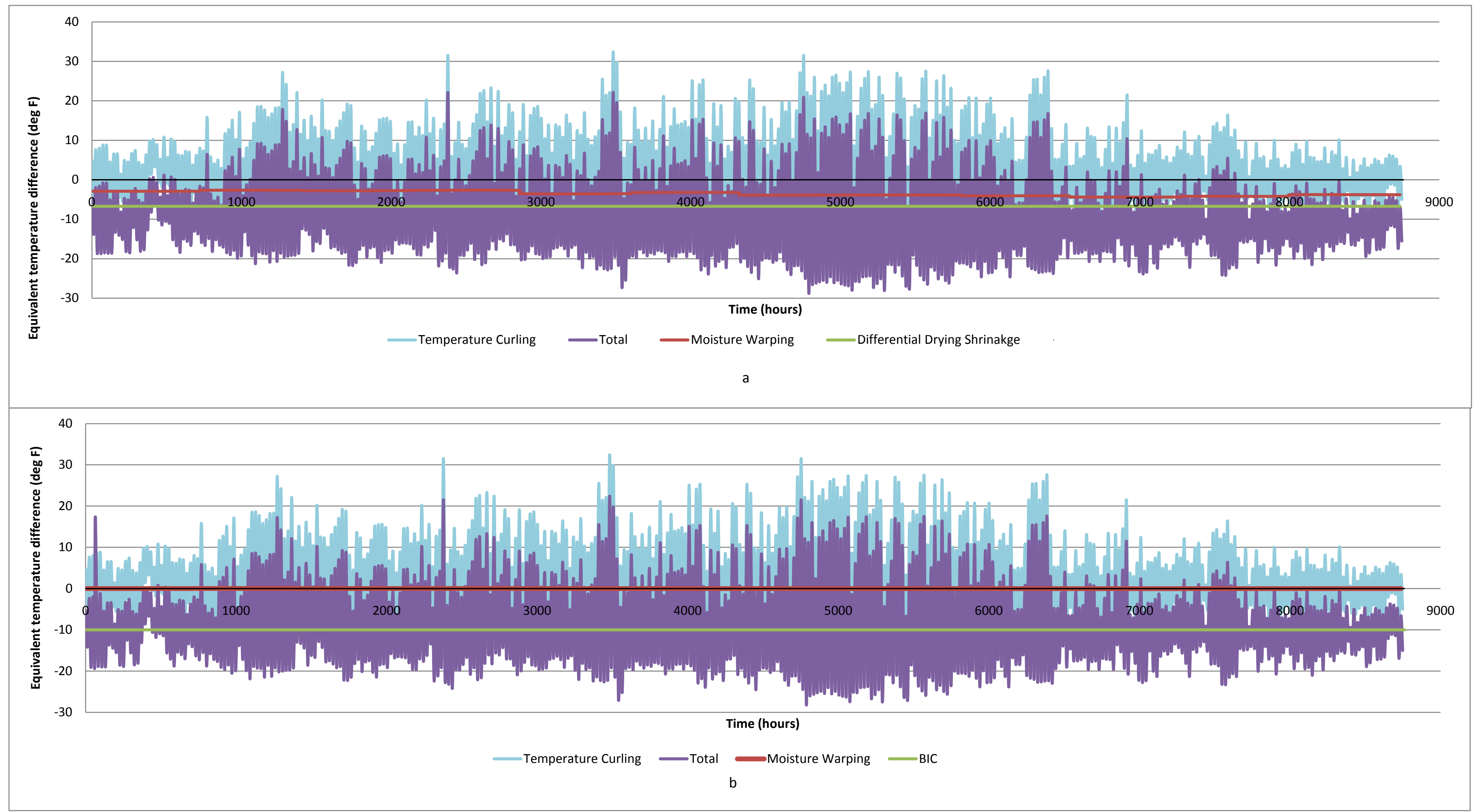

Figure 4.16: Predicted equivalent temperature difference in Seattle using a) the proposed warping and differential drying shrinkage models, and b) the MEPDG model warping model and -10 ${ }^{\circ} \mathrm{F}$ default value for BIC 
The sum of the reversible and irreversible components of drying shrinkage is the total shrinkage, and therefore, it may be appear that the separate equivalent temperature differences due to moisture warping and differential drying shrinkage could simply be removed and one equation could be used to predict the overall equivalent temperature gradient due to differential drying shrinkage and an annual average moisture warping. However, differential drying shrinkage does not change seasonally and moisture warping varies on a monthly basis. These two terms should remain separate because moisture warping should be computed on a seasonal or monthly basis. Failure to consider variations in the amount of moisture warping could both under- and over-predict the stresses in the pavement at different times of year. This is compounded by the fact that moisture and temperature in the slab are coupled in reality (Qin 2011), even though they are generally considered separately for the sake of simplicity.

Moisture warping varies seasonally, and changes in moisture gradients may coincide with expected temperature gradients. For example, a region might have dry summers, and temperatures which are cool at night and hot during the day, leading to large thermal gradients. In the same region, winters may lack such drastic temperature changes and be wetter in general. In this case, the larger moisture gradients in the summer coincide with larger temperature gradients, while the smaller moisture gradients in the winter are coincident with smaller thermal gradients. This may be compounded by other weather factors, such as the fact that winters tend to be cloudier and have less solar radiation. In this case, failure to compute moisture warping on a monthly basis would over-predict stresses in the winter, but under-predict them in the summer.

\subsection{Design Aids for the Determination of Moisture Warping and Differential Drying Shrinkage}

The amount of both warping and differential drying shrinkage expected in a pavement varies with the ambient relative humidity, which in turn varies by location. To aid design engineers in determining how much warping or differential drying shrinkage is to be expected, the proposed models were used to create several design aids. These design aids use contour lines to show the variation in warping or differential drying shrinkage for a pavement of given thickness and water to cement ratio throughout the country.

Data for the ambient relative humidity for each month of the year was obtained from the National Oceanic and Aeronautics Administration (NOAA) climatic database for 256 sites scattered around the United States. At each location, the mean value of average morning (AM) and afternoon (PM) relative humidity in each month was given based on historic data. Depending on the location, the historical climatic record stretched back between 6 and 96 years (NOAA 2008). To obtain an average daily value of relative humidity from morning and afternoon values, a weighted average was used, as given in Equation 4.37 (Daly and Taylor 2001).

$$
\text { Daily average } R H=0.65 * R H_{A M}+0.3 * R H_{P M}
$$

Equation 4.37

The annual average of the average daily relative humidity was used as an input in the proposed warping model to compute the expected equivalent temperature difference due 
to moisture warping in a pavement of given thickness and water to cement ratio. Calculations were also made on a monthly basis, as in the MEPDG, but there was insufficient variation to justify monthly maps. Maps based on the annual average are also usable to predict the amount of differential drying shrinkage, so long as proper attention is given to map selection based on the values of $\phi$ and (1- $\phi)$. For the case of $\phi=0.5$, the map is applicable to both moisture warping and differential drying shrinkage because they are equal.

As was previously discussed, correct determination of the depth of shrinkage zone is critical to accurately predicting warping. Given that the actual depth of the shrinkage zone varies by location, and is not the conventionally assumed two inches in all areas, design aids were created for various values of $h_{\mathrm{s}}, \mathrm{w} / \mathrm{c}, \varphi$ and $\varepsilon_{\mathrm{su}}$ as well. Figure 4.18 shows the design for what was considered a reference case, that is a $10 \mathrm{inch}$ pavement with $h_{s}=3$ in, w/c $=0.4, \phi=.3$, and $\varepsilon_{\mathrm{su}}=600 \mu \varepsilon$.

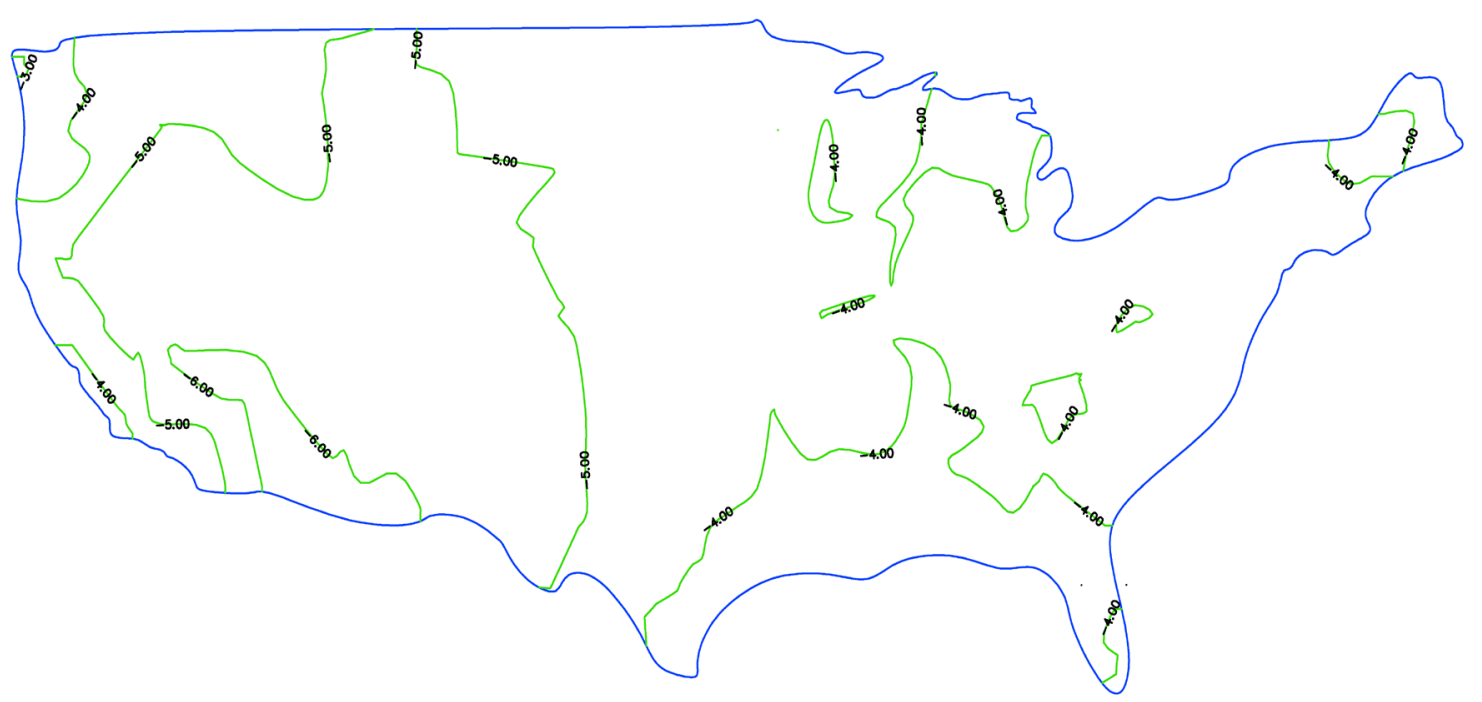

Figure 4.18: Design aid for the equivalent temperature difference due to warping for a 10 inch pavement with $h_{s}=3 \mathrm{in}, \mathrm{w} / \mathrm{c}=0.4, \varphi=.3$, and $\varepsilon_{\mathrm{su}}=600 \mu \varepsilon$.

The input parameters for the design aids were varied to give designers an understanding of how changing each variable will affect the final value of equivalent temperature difference due to warping. These input variables are shown in Table 4.10, and the associated design aids can be found in Appendix F. 
Table 4.10

Variations in input variables used in the generation of design aids.

\begin{tabular}{|c|c|c|c|c|c|c|}
\hline $\begin{array}{l}\text { Slab } \\
\text { Depth }\end{array}$ & $\begin{array}{c}\text { Total } \\
\text { Shrinkage }\end{array}$ & $w / c$ & $\begin{array}{l}\text { Depth of } \\
\text { Shrinkage } \\
\text { Zone }\end{array}$ & $\begin{array}{c}\text { Reversible } \\
\text { Shrinkage } \\
\text { Factor }\end{array}$ & $\begin{array}{c}\text { To Compare } \\
\text { Effects of }\end{array}$ & $\begin{array}{c}\text { Figure } \\
\text { Number in } \\
\text { Appendix } F\end{array}$ \\
\hline h (in) & $\varepsilon_{\mathrm{su}}(\mu \varepsilon)$ & - & $\mathrm{h}_{\mathrm{s}}$ (in) & $\phi$ & & \\
\hline 10 & 600 & 0.4 & 3 & 0.3 & $\begin{array}{c}\text { standard } \\
\text { case } 1\end{array}$ & F12 \\
\hline 10 & 600 & 0.3 & 3 & 0.3 & \multirow{2}{*}{$w / c$} & F16 \\
\hline 10 & 600 & 0.5 & 3 & 0.3 & & F17 \\
\hline 10 & 600 & 0.4 & 1 & 0.3 & \multirow{3}{*}{$h_{s}$} & F13 \\
\hline 10 & 600 & 0.4 & 2 & 0.3 & & F14 \\
\hline 10 & 600 & 0.4 & 4 & 0.3 & & F15 \\
\hline 8 & 600 & 0.4 & 3 & 0.3 & \multirow{2}{*}{$\mathrm{h}$} & F18 \\
\hline 12 & 600 & 0.4 & 3 & 0.3 & & F19 \\
\hline 10 & 300 & 0.4 & 3 & 0.3 & \multirow{2}{*}{$\varepsilon_{\mathrm{su}}$} & $\mathrm{F} 20$ \\
\hline 10 & 1000 & 0.4 & 3 & 0.3 & & F21 \\
\hline 10 & 600 & 0.4 & 3 & 0.5 & $\begin{array}{c}\text { standard } \\
\text { case } 2 \\
\end{array}$ & F1 \\
\hline 10 & 600 & 0.5 & 3 & 0.5 & \multirow{2}{*}{$w / c$} & $\mathrm{~F} 2$ \\
\hline 10 & 600 & 0.3 & 3 & 0.5 & & F3 \\
\hline 10 & 600 & 0.4 & 1 & 0.5 & \multirow{3}{*}{$h_{s}$} & F4 \\
\hline 10 & 600 & 0.4 & 2 & 0.5 & & F5 \\
\hline 10 & 600 & 0.4 & 4 & 0.5 & & F6 \\
\hline 8 & 600 & 0.4 & 3 & 0.5 & \multirow{2}{*}{$\mathrm{h}$} & F7 \\
\hline 12 & 600 & 0.4 & 3 & 0.5 & & F8 \\
\hline 10 & 600 & 0.4 & 3 & 0.6 & $\phi$ & F11 \\
\hline 10 & 300 & 0.4 & 3 & 0.5 & \multirow{2}{*}{$\varepsilon_{\mathrm{su}}$} & F9 \\
\hline 10 & 1000 & 0.4 & 3 & 0.5 & & F10 \\
\hline
\end{tabular}

From the design aids generated, it can be seen that the majority of the variation in the amount of predicted warping occurs on the west coast, in the southwest and in New England. This is because those locations have areas of very different climate close together due to the effects of topography. The largest amount of both moisture warping and differential drying shrinkage occurs in the southwest because it has the driest climate. New England and the northwest have less warping because they are wetter.

Changing the input parameters greatly affects both the value of warping and the spacing of the contour lines. Increasing the water to cement ratio causes more warping overall, and leads to more tightly spaced contours. Thinner pavements have more warping, and closer spaced contour lines. Pavements with a larger amount of reversible shrinkage will 
have more warping and more tightly spaced contours, as will pavements with higher values of ultimate shrinkage.

Changing the depth of the shrinkage zone had the most drastic effect on both the magnitude of the warping, and its spatial distribution. The map with a depth of the shrinkage zone equal to one inch has almost no variation, the entire country has an equivalent temperature gradient due to warping of -2 to $-3^{\circ} \mathrm{F}$. In contrast, the map with a depth of the shrinkage zone equal to four inches shows significant variation in the expected equivalent temperature distribution around the country. Additionally, the values are much larger. This is especially important because $h_{s}$ is one input which is often left at the default setting of two inches, despite evidence that this is often not the case.

These design aids are intended for use by design engineers to predict the amounts of moisture warping and differential drying shrinkage expected in a pavement, since the current MEPDG does not account for moisture warping on an appropriate scale and neglects differential drying shrinkage completely. Though implementing a new model will take time, these design aids can be used immediately. To account for the expected amount of warp, the equivalent temperature difference from the design aid can be directly added to the amount of built-in curl anticipated. This will neglect any time dependent effects of warping, but at least the overall deformed shape and any associated damage will be accounted for. 


\section{Quantifying Built-in Curl}

The amount of built-in curl in a slab contributes to changes in its support conditions, and therefore factors into the estimate for the fatigue life of the pavement. This is of particular importance in the MEPDG, because built-in curl is an input factor for the design of rigid pavements. Proper estimation of the amount of built-in curl expected in a slab is critical to determining the amount of fatigue damage which can be expected to occur in the pavement. Small changes in the amount of built-in curl can significantly change the predicted pavement performance. Because no algorithm currently exists for the prediction of built-in curl, a design engineer must rely on information from previously cast slabs of similar geometry and material in the area. Additionally, if a model to predict built-in curl were developed, it would be necessary to measure actual slabs to validate that model. The goal of this study was to compare methods of quantifying built in curl and make a recommendation as to which method is best.

\subsection{Introduction}

Built-in curl is often quantified by the equivalent temperature gradient needed to deform a flat slab to the same shape as the curled slab. There are two main schools of thought regarding the ideal way to measure the amount of curl built into a concrete slab. A surface profiler can be used to measure the deflections along the length of the slab. This can be accomplished through a variety of different methods including dipsticking, on-site profilometers, high-speed profilometers, etc., which each present their own benefits and drawbacks. These surface profiles can then be plotted and an equation fitted to the shape of the surface. This equation is compared to curves generated using a FEM program representing the deflected shape of a theoretical flat slab of the same geometry and properties that is exposed to various temperature gradients. The temperature gradient used to produce the same deflected shape as that of the observed slab is the built-in temperature gradient. This brute force procedure is time consuming and error prone, but does not require specialized techniques or equipment.

Alternately, an FWD can be used to measure the response of a pavement to various applied loads. The data obtained from this test can be run through an ANN, which has been designed and trained to back estimate the built-in curl. This method is much more automated and therefore, the potential to introduce human error is greatly reduced. However, it does require that the user have an appropriately trained ANN at their disposal, as well as access to FWD data at specific times of the day.

In this study, several instrumented test slabs at the Minnesota Road Research Facility (MnROAD) were measured with an on-site profilometer and tested with an FWD. Temperature data for these slabs was obtained from thermal couples embedded in the slabs. Both methods discussed above were used to estimate the built-in curl of the slabs. The deflection curves obtained from the profilometer were compared to curves for 21 different temperature gradients (from $-70^{\circ} \mathrm{F}$ to $30^{\circ} \mathrm{F}$ in $5^{\circ} \mathrm{F}$ increments) generated using ISLAB2000. The FWD data was run through an ANN developed by (Lothschutz 2009). A third method was also used where the deflected shape measured by the surface profiler was matched with the deflected shapes generated in ISLAB2000 for slabs exposed to 
various temperature gradients. In this case, the actual profile was matched with the ISLAB2000 profile for which the sum of the squares of the errors between the actual profile and the ISLAB2000 profile was minimized.

\subsection{Data Collection}

Data was collected at the MnROAD facility, from both the mainline and low volume loops. For each cell, five transverse profiles were collected, along with FWD deflection data at a variety of locations on the slab. Thermocouple data provided by Mn/DOT was used to determine the temperature profile through the depth of the slab at the time of both profilometer and FWD testing.

Testing was conducted in June and October 2010. Initial results from the June tests showed that the actual temperature gradients at the time of testing were not negative enough to produce sufficient curl that was measurable with the ANN. The ANN used in this study was only trained to predict total curl which is more negative than $-9^{\circ} \mathrm{F}$. Therefore, the curl due to the actual temperature gradient and the built-in curl must sum to a value more negative than $-9^{\circ} \mathrm{F}$. The June tests were conducted in the late morning and in the afternoon when positive temperature gradients were present. This caused the total curl to be less negative than $-9^{\circ} \mathrm{F}$, meaning that the ANN outputs were not usable. The tests were therefore repeated in the early morning in October, in an attempt to capture larger negative total curls. The full analysis was conducted using the October 2010 data set.

\subsubsection{Test Cell Locations and Descriptions}

Several concrete test slabs were selected for this study. They are identified by their cell and panel number; when only one panel in a cell was tested, that panel is referred to by its cell number only. Cells 7, 71, 72, 12, 213, 305, 513, and 614 are located on the mainline portion of the MnROAD facility; see Figure 5.1 for cell locations and Figure 5.2 for cell descriptions. Cell 305 is an unbounded PCC overlay section, and a subsection in cell 5. Cell 70 is an asphalt section, and therefore, was not included in testing regime, as all the test methods used are only applicable to concrete pavements. Cells 213 and 513 are thin concrete sections which are subsections of cell 13. Restraint is provided by flat plate dowels. Cell 614 is a whitetopping section that is a subsection in cell 14 (Johnson et al. 2008). Profilometer, FWD and thermocouple data was collected from these cells in the early morning (4-7AM) on October 26, 2010. 


\begin{tabular}{|l|l|l|l|l|l|l|l|l|l|l|l|l|l|l|l|l|l|l|l}
\hline 5 & 6 & 7 & 8 & 9 & 60 & 61 & 62 & 63 & 96 & 97 & 92 & 10 & 11 & 12 & 13 & 14 & 15 & 16 & \\
\hline
\end{tabular}

Figure 5.1: MnROAD mainline cell locations with pertinant cells highlighted.

\begin{tabular}{|c|c|c|c|c|c|c|c|}
\hline 305 & 7 & 71 & 72 & 12 & 513 & 213 & 614 \\
\hline $5 "$ & \multirow{2}{*}{$\begin{array}{l}7.5 " \\
\text { Trans } \\
\text { Tined }\end{array}$} & $\begin{array}{c}\text { 3" PCC } \\
\text { EAC }\end{array}$ & 3" PCC & \multirow{3}{*}{$\begin{array}{l}9.5 " \\
\text { Trans } \\
\text { Tined }\end{array}$} & 5.5" PCC & 5.5" PCC & \multirow{2}{*}{$\begin{array}{c}\text { 6" PCC } \\
\text { Long } \\
\text { Broom }\end{array}$} \\
\hline 1" PASB & & \multirow{2}{*}{$\begin{array}{l}\text { 6" PCC } \\
\text { Recycle }\end{array}$} & \multirow{2}{*}{$\begin{array}{l}\text { 6" PCC } \\
\text { Low Cost }\end{array}$} & & & \multirow[b]{2}{*}{$\begin{array}{c}\text { 5" CL1 } \\
\text { Stab Agg }\end{array}$} & \\
\hline $\begin{array}{c}7.5 " \\
03\end{array}$ & 4" PSAB & & & & $\begin{array}{c}\text { 5" CL1 } \\
\text { Stab Agg }\end{array}$ & & \multirow{2}{*}{$\begin{array}{c}7 " \\
58-28 \\
\text { '93 HMA }\end{array}$} \\
\hline 935 r & 3" CL4 & \multirow{4}{*}{$\begin{array}{c}8 " \\
\text { Class } 7\end{array}$} & \multirow{4}{*}{$\begin{array}{c}8 " \\
\text { Class } 7\end{array}$} & \multirow{3}{*}{$\begin{array}{c}5^{\prime \prime} \\
\text { Class } 5\end{array}$} & & \multirow{3}{*}{$\begin{array}{c}4.5 " \\
\text { Class } 5\end{array}$} & \\
\hline 3. CL4 & \multirow[t]{3}{*}{ Clay } & & & & $\begin{array}{l}5 " \\
\text { Class } 5\end{array}$ & & \multirow{3}{*}{ Clay } \\
\hline $27 "$ & & & & & & & \\
\hline Class & & & & \multirow[t]{2}{*}{ Clay } & Clay & \multirow[t]{2}{*}{ Clay } & \\
\hline 3 & \multirow{3}{*}{$\begin{array}{c}20 \times 14 \\
20 \times 13 \\
1 " \\
\text { dowel }\end{array}$} & \multirow{5}{*}{$\begin{array}{c}\text { Clay } \\
\text { Innovative } \\
\text { DG(driving) } \\
\text { Convent. } \\
\text { DG } \\
\text { (passing) }\end{array}$} & Clay & & & & \multirow{7}{*}{$\begin{array}{c}\text { 6'x12' } \\
\text { Flat } \\
\text { Dowels } \\
\text { Driving } \\
\\
\text { No } \\
\text { Dowels } \\
\text { Passing }\end{array}$} \\
\hline $15 \times 14$ & & & & 15'x12' & Heavy & Heavy & \\
\hline & & & $\begin{array}{c}\text { EAC } \\
\text { Surface }\end{array}$ & $\begin{array}{c}1.25 " \\
\text { dowel }\end{array}$ & & Turf & \\
\hline dowels & \multirow{4}{*}{$\begin{array}{l}2007 \\
\text { Innov } \\
\text { Grind }\end{array}$} & & $\begin{array}{c}15^{\prime} \times 12^{\prime} \\
1.25^{\prime \prime}\end{array}$ & & $\begin{array}{l}12^{\prime} \times 12^{\prime} \\
15^{\prime} \times 12^{\prime}\end{array}$ & $15^{\prime} \times 12^{\prime}$ & \\
\hline $\begin{array}{l}\text { Trad } \\
\text { Grind }\end{array}$ & & & dowel & & Flat & & \\
\hline & & $1.25 "$ & & & dowels & & \\
\hline clay & & dowels & & & & & \\
\hline
\end{tabular}

Figure 5.2: Mainline cell descriptions, (modified from Johnson et al. 2008).

Cells 36, 37, and 53 are located on the low volume road portion of the MnROAD facility; see Figure 5.3 for cell locations and Figure 5.4 for cell descriptions. Cell 36 was subdivided into panels 19 and 20, while cell 37 was subdivided into panels 8 and 9 . Profilometer, FWD and thermocouple data was collected from these cells in the early morning (4-7 AM) and late morning (10:30-11:30 AM) on October 26, 2010. 


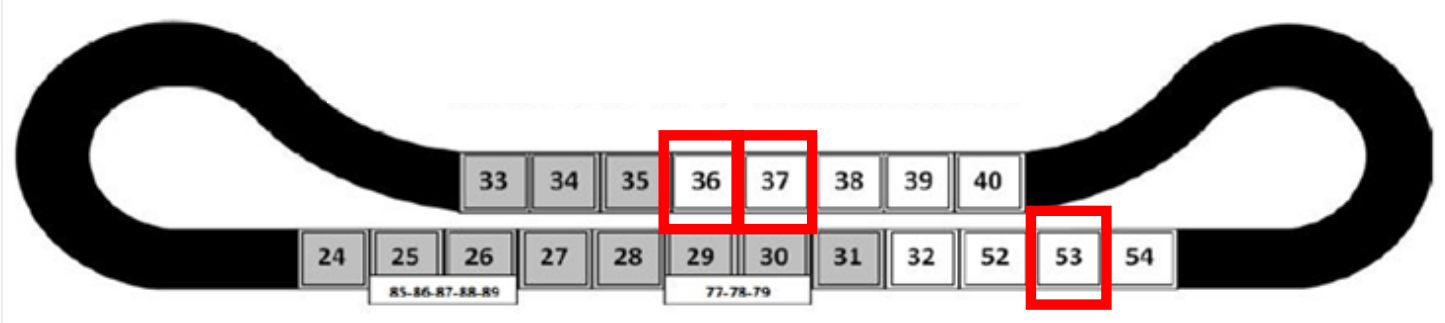

Figure 5.3: MnROAD low volume road with pertinent cells highlighted.

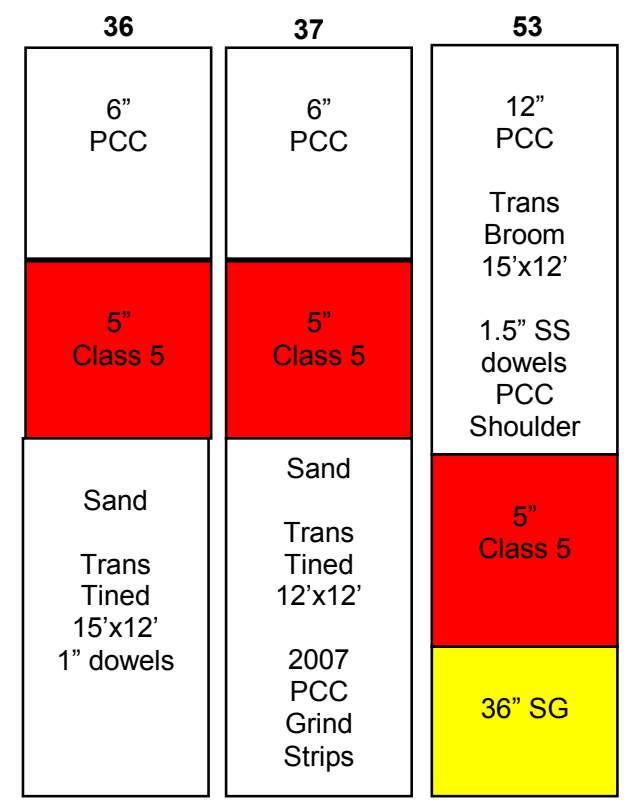

Figure 5.4: Low volume road cell descriptions, (modified from Johnson et al. 2008).

\subsubsection{Profilometer}

The Automated Laser Profile System 2 (ALPS2) is an automated system developed by MnROAD to collect measurements of the profile of the slab in both the longitudinal and transverse directions. The ALPS2 profilometer was used to obtain longitudinal and transverse surface profiles for each cell. In the June tests, 10-15 transverse profiles were taken per slab. In the October tests, five transverse profiles were taken for each slab.

\subsubsection{Falling Weight Deflectometer}

The FWD tested the slab in the corners, the middle and at the longitudinal and transverse edges. The transverse edge of the adjacent slab was also tested so that the load transfer efficiency could be calculated. Data from the FWD tests was used to back-calculate the modulus of subgrade reaction in addition to the load transfer efficiency to be used in the ISLAB2000 models. See Figure 5.5 for the configuration of the FWD sensors, and Table 5.1 for the sensor locations in relation to the applied load. 


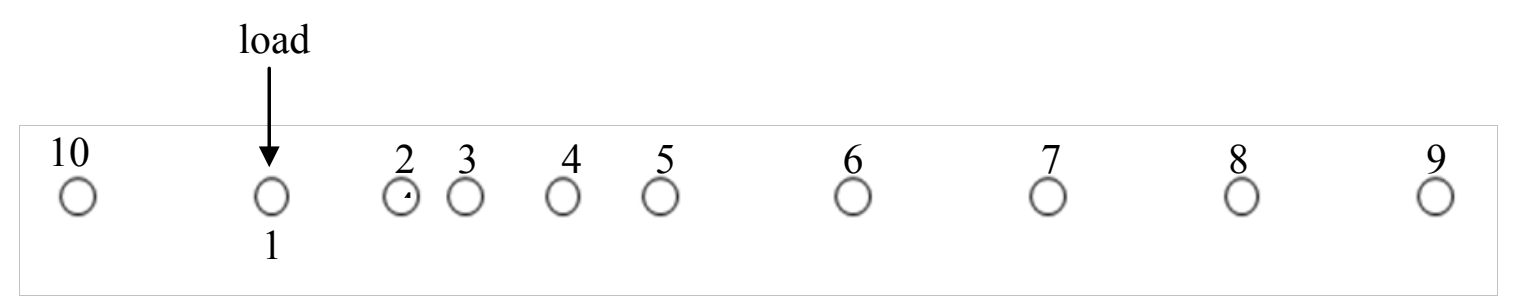

Figure 5.5: FWD configuration, with sensors numbered.

Table 5.1

Location of FWD sensors, measured to the right of the applied load.

\begin{tabular}{|r|r|}
\hline $\begin{array}{r}\text { sensor } \\
\text { number }\end{array}$ & $\begin{array}{c}\text { distance } \\
\text { from } \\
\text { load (in) }\end{array}$ \\
\hline 1 & 0 \\
\hline 2 & 8 \\
\hline 3 & 12 \\
\hline 4 & 18 \\
\hline 5 & 24 \\
\hline 6 & 36 \\
\hline 7 & 48 \\
\hline 8 & 60 \\
\hline 9 & 72 \\
\hline 10 & -12 \\
\hline
\end{tabular}

\subsubsection{Thermocouples}

Thermocouple data was available for the majority of the slabs in this study. In general, thermocouple sensors were embedded in the pavement at a variety of depths. Though it is well known that temperature gradients are nonlinear in concrete pavements, in this case it was appropriate to assume a linear temperature gradient. This is because equivalent temperature difference is calculated as a linear temperature gradient, and the actual temperature gradient must be subtracted from the total calculated curl, which is also linear. The linear temperature difference is also highly related to the level of bending experienced in a concrete pavement slab.

The sensors in the concrete layer were not located at exactly the top and bottom of the slab, because they had to be embedded in the concrete. To obtain a linear profile for the entire depth of the concrete slab, the thermocouple data from the top- and bottom- most sensors in the concrete layer was linearly extrapolated for the entire pavement thickness. When no thermocouple data was available for a slab, data from a nearby slab with similar structural characteristics was used. In the event that the similar slab had a different 
thickness than the actual slab, the thermocouple data was extrapolated for a slab of the same thickness as the actual slab. No thermocouple data was available for cells 71,72 , $213,513,614$. In all cases, cell 12 was the closest cell with a similar structure and available thermocouple data. The actual temperature difference was interpolated for these cases. Again, in this case it was acceptable to use a linear gradient because the total temperature gradient was subtracted from the linear total curl to get the linear equivalent temperature difference.

\subsection{Data Processing}

Before the ALPS2 data could be used in the profiler and minimum error methods, it had to be processed to remove extraneous points and the cross slope of the pavement. Data from the FWD sensors was used to calculate the modulus of subgrade reaction and the load transfer efficiency. These parameters, along with the slab geometry and material properties were used to calculate the total curl using the polynomial curvature method, the $\Delta \mathrm{h}$ method, the FWD/ANN method, and the minimum error method. For the polynomial curvature and minimum error methods, $2^{\text {nd }}, 3^{\text {rd }}, 4^{\text {th }}, 5^{\text {th }}$, and $6^{\text {th }}$, order polynomial approximations were used for the data, and the tests were conducted for both the entire slab and only the middle half of the slab. All in all, 22 different methods were used to calculate the built-in curl for each pass of the ALPS2 on every slab evaluated.

\subsubsection{Input Parameters}

The same input parameters for the pavement geometry and materials were used for both the surface profiler and the FWD/ANN methods. The parameters used for each cell are given in Appendix G. The values of static coefficient of subgrade reaction $\mathrm{k}$, were calculated based on the FWD test results using the AREA method shown in Equation 5.1 though Equation 5.5 (Fabrizzio 1998).

$$
\begin{gathered}
\boldsymbol{k}_{\text {static }}=\frac{\boldsymbol{k}_{\text {dynamic }}}{2} \\
\boldsymbol{k}_{\text {dynamic }}=\frac{\operatorname{Load} * D_{i}^{*}}{\frac{D_{i}}{1000}} \ell^{2} \\
D_{i}^{*}=a_{i} e^{-b_{i} e^{-c_{i} \ell}}
\end{gathered}
$$

Equation 5.3

where: $\mathrm{a}, \mathrm{b}$, and c are known constants for each load location,

\begin{tabular}{|c|c|c|c|c|c|c|c|}
\hline & $\mathbf{D}^{*} \mathbf{0}$ & $\mathbf{D}^{*} \mathbf{8}$ & $\mathbf{D}^{*} \mathbf{1 2}$ & $\mathbf{D}^{*} \mathbf{1 8}$ & $\mathbf{D}^{*} \mathbf{2 4}$ & $\mathbf{D}^{*} \mathbf{3 6}$ & $\mathbf{D}^{*} \mathbf{6 0}$ \\
\hline $\mathrm{a}$ & 0.1245 & 0.12323 & 0.12188 & 0.11933 & 0.11634 & 0.1096 & 0.09521 \\
\hline $\mathrm{b}$ & 0.14707 & 0.46911 & 0.79432 & 1.38363 & 2.06115 & 3.62187 & 7.41241 \\
\hline $\mathrm{c}$ & 0.07565 & 0.07209 & 0.07074 & 0.06909 & 0.06775 & 0.06568 & 0.06255 \\
\hline
\end{tabular}




$$
\begin{gathered}
\ell=\left[\frac{\ln \left(\frac{60-\text { Area }}{289.708}\right)}{-.698}\right]^{2.566} \\
\text { Area }=4+6\left(\frac{D_{8}}{D_{0}}\right)+5\left(\frac{D_{12}}{D_{0}}\right)+6\left(\frac{D_{18}}{D_{0}}\right)+9\left(\frac{D_{24}}{D_{0}}\right) \\
+18\left(\frac{D_{36}}{D_{0}}\right)+12\left(\frac{D_{60}}{D_{0}}\right)
\end{gathered}
$$

Equation 5.5

The LTE for longitudinal joints was assumed to be $60 \%$ for all cases. The LTE for transverse joints was taken as an average of the LTE calculated from the tests before and after the joint, which were found using Equation 5.6 and Equation 5.7.

$$
\begin{gathered}
L T E_{\text {joint before }}=\frac{D_{10}}{D_{1}} \\
L T E_{j o i n t ~ a f t e r}=\frac{D_{3}}{D_{1}}
\end{gathered}
$$

Equation 5.6

Equation 5.7

The thickness of the slab was taken as the given thickness for all cases except those noted below. In all cases, the contribution from unbound base layers was ignored. For the thin concrete section (cell $213 \& 513$ ), which had slab thicknesses of less than 6 inches, a PCC thickness of 6 inches was used in the FWD/ANN computations since the ANN is not configured for slabs less than 6 inches thick. For the unbonded overlay section (cell 305), an equivalent thickness (Khazanovich 1994) was computed using various configurations to account for the multiple layers. The configuration which gave an equivalent thickness which could be used to compute the most realistic values of the static modulus of subgrade reaction was selected. For PCC layers bonded to a stabilized base (cell 305), the transformed section thickness was calculated based on Equation 5.8 and Equation 5.9.

$$
\begin{aligned}
h_{\text {equiv }}=\left[\left(\boldsymbol{h}_{\text {pcc }}{ }^{3}+\frac{E_{\text {base }}}{E_{\text {pcc }}} \boldsymbol{h}_{\text {base }}{ }^{3}\right)\right. \\
+12\left(\left(x-\frac{h_{p c c}}{2}\right)^{2} h_{p c c}\right. \\
\left.\left.+\frac{E_{\text {base }}}{E_{p c c}}\left(h_{p c c}-x+\frac{h_{\text {base }}}{2}\right)^{2} h_{\text {base }}\right)\right]^{\frac{1}{3}}
\end{aligned}
$$




$$
x=\frac{E_{p c c} h_{p c c} \frac{h_{p c c}}{2}+E_{p c c}\left(h_{p c c}+\frac{h_{b a s e}}{2}\right)}{E_{p c c} h_{p c c}+E_{b a s e} h_{b a s e}}
$$

\subsubsection{Surface profiler}

The surface profiler data was plotted using Excel to make graphs of deflection versus point number, see Figure 5.6. Note that the distance along the transverse dimension of the slab is in "points", which the unit was given by the profiler device. Since the profiler was wider than the slab, there was a certain amount of data on either side of the slab which needed to be deleted. By manually examining the surface profile graphs, the points which required deletion were determined to be those at the beginning of the curve which were quite variable (indicating the shoulder) and those at the end of the curve where the graph peaked and began to descend (indicating the crown in the road and the longitudinal joint/adjacent slab). The first point on the graph after the data from the profiler overhang was subtracted was called the zero point. After the extraneous points were removed, the difference in elevation due to a constant cross slope (regardless of time of day tested) was subtracted from the deflection, see Figure 5.7. This was accomplished by applying the following formula to all of the data remaining after the data from the profiler overhangs were eliminated.

$$
d_{a d j}=d-c\left(p-p_{z}\right)
$$

Equation 5.10

Where:

$\mathrm{d}_{\text {adj }}=$ adjusted deflection

$\mathrm{d}=$ original deflection

$\mathrm{c}=$ cross slope

$\mathrm{p}=$ point number corresponding to deflection

$\mathrm{p}_{\mathrm{z}}=$ point number of the zero point 


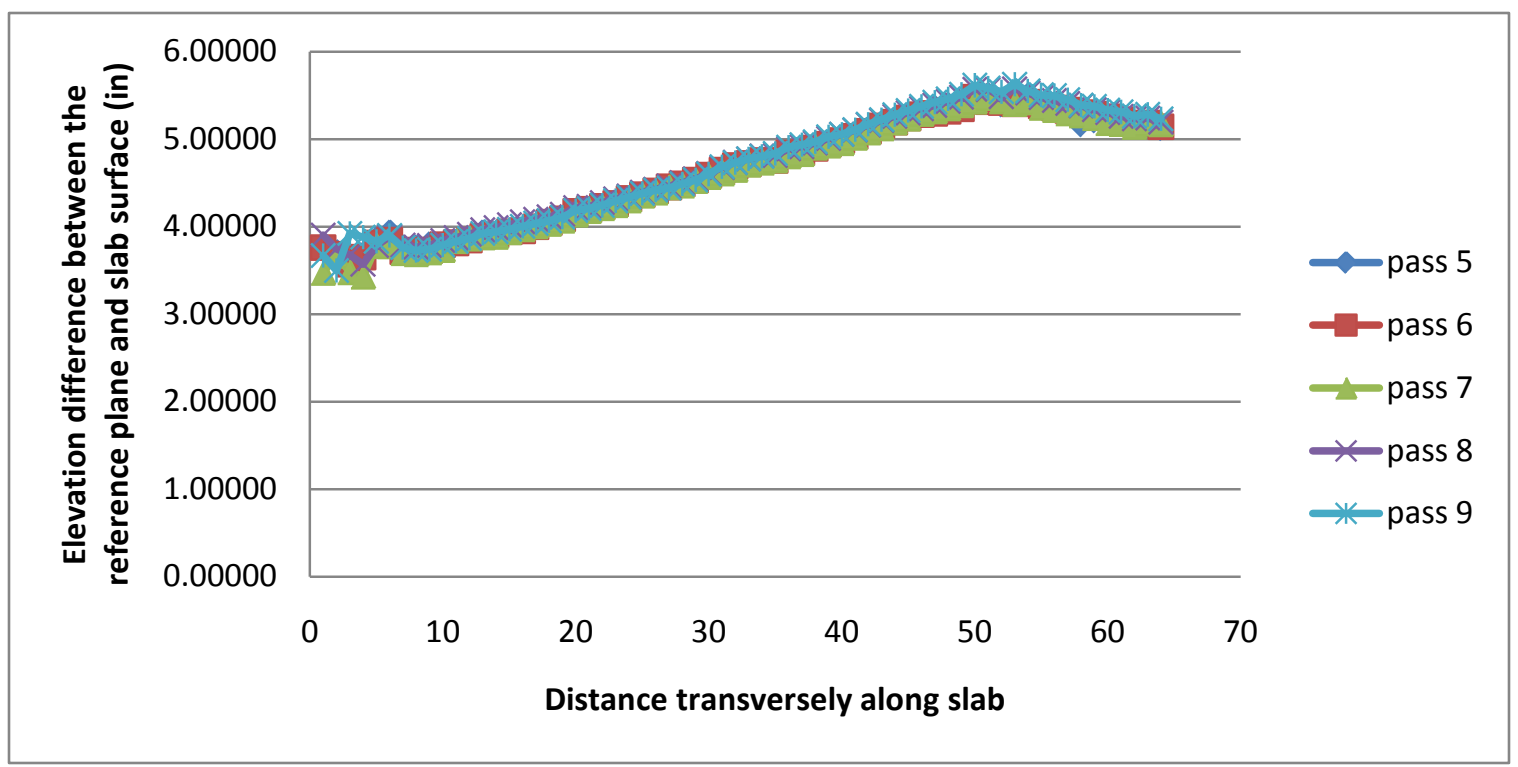

Figure 5.6: Deflection along the width of the slab for cell 36, panel 19, early morning test unadjusted.

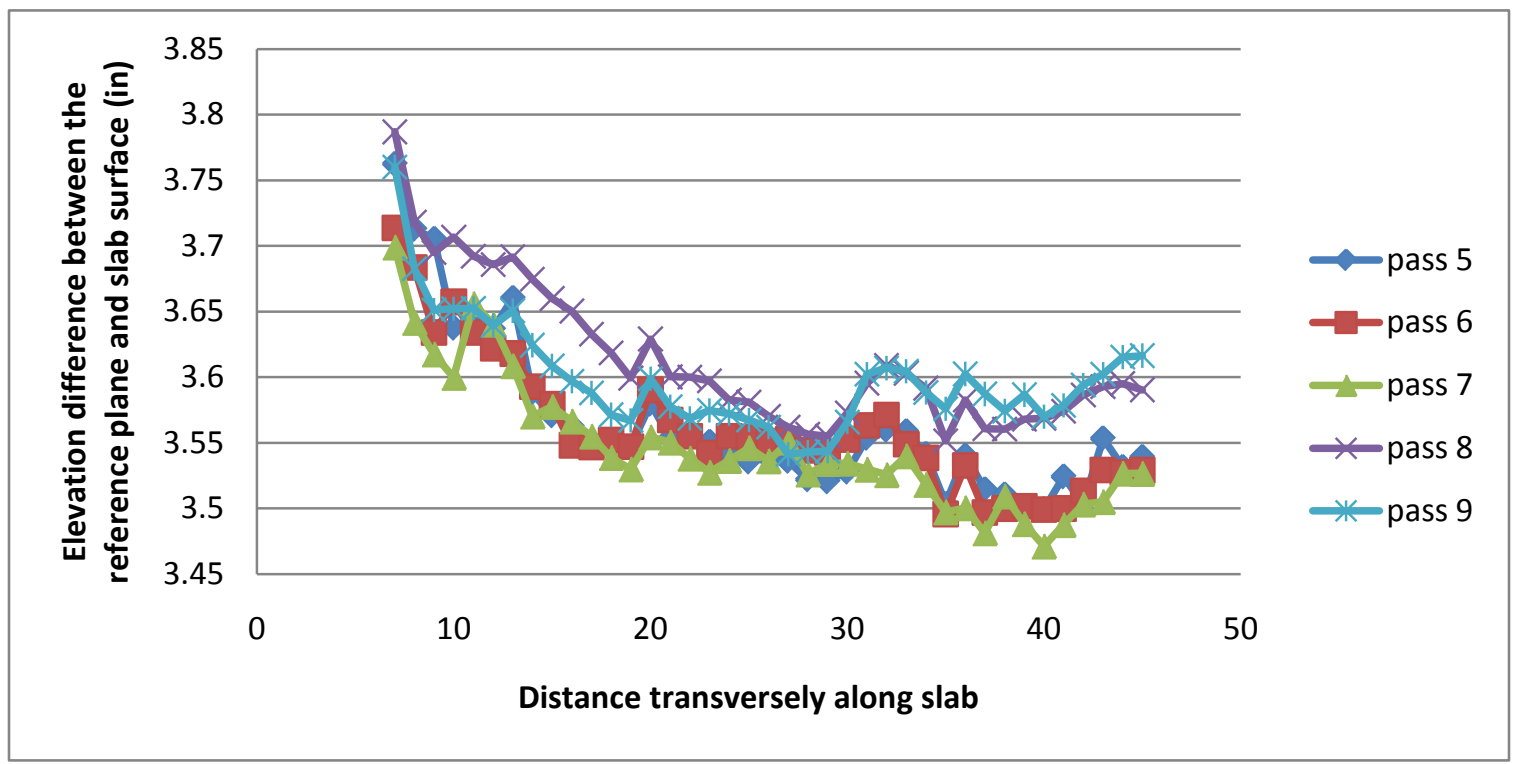

Figure 5.7: Deflection along the length of the slab after adjusting for extraneous data and subtracting given cross slope for cell 36 , panel 19, early morning test.

Subtracting out the given cross slope did not always produce a graph which reflected the shape of the slab in reality. From Figure 5.7, it can be assumed that the slab does not have the shape of a linearly decreasing line. Adjusting the cross slope very slightly through trial and error, it is possible to obtain a bowl shaped curve, indicative of a slightly curled up slab, which is much more likely to be the actual shape of the slab. Given that it is difficult to achieve the exact cross-slope called for in design, it is likely that the actual cross slope of the slabs does not match the given values of cross slope perfectly. To standardize the process for determining the value of built-in curl to subtract 
from the profile, it was assumed that the slab would deform symmetrically in the transverse direction. Therefore, the value of cross slope which produced the most symmetric shaped slab was used; see Figure 5.8 for an example.

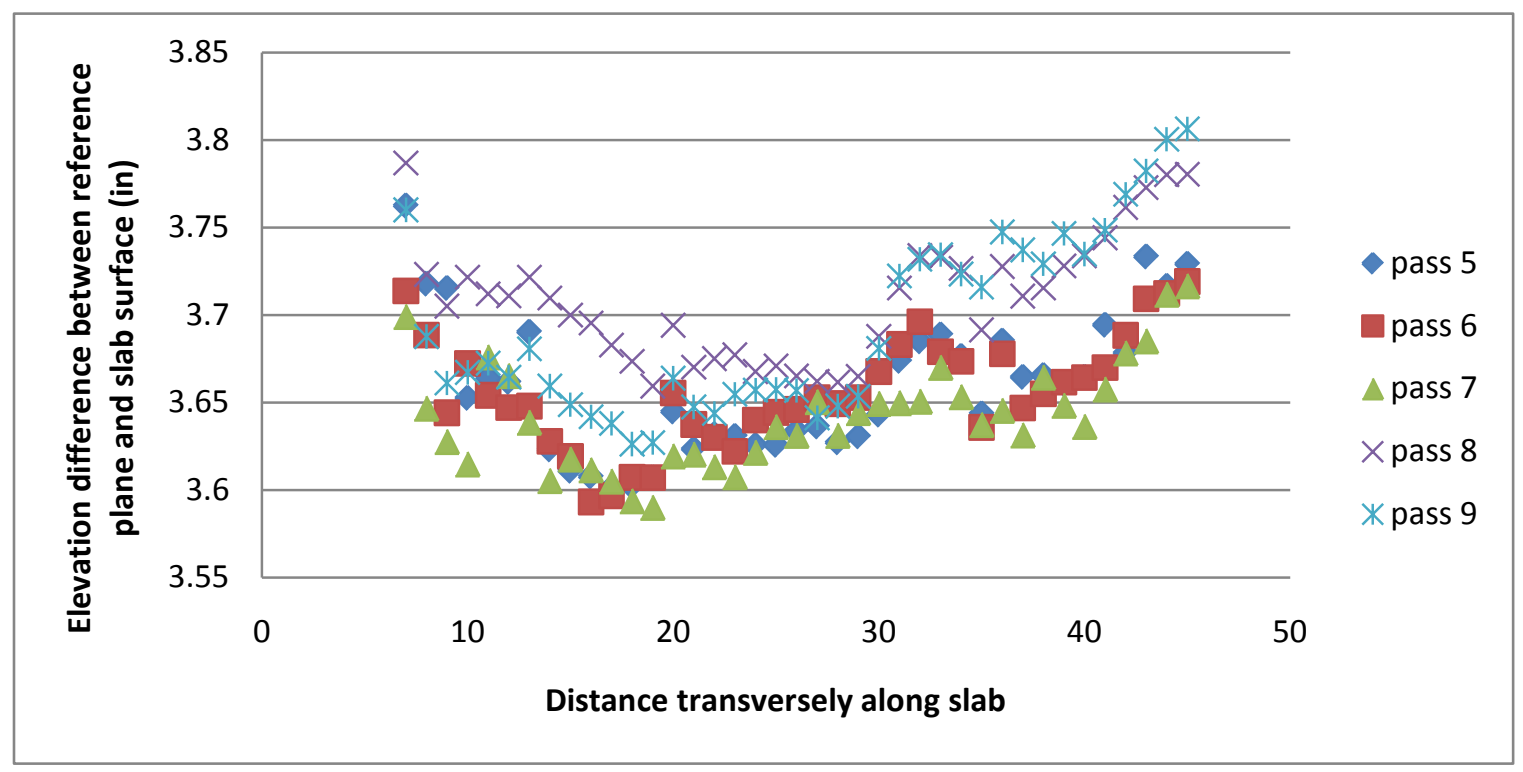

Figure 5.8: Deflection along the length of the slab after adjusting for extraneous data and subtracting assumed cross slope for cell 36 , panel 19 , early morning test.

This procedure did not affect the calculated values of built-in curl for any of the polynomial curvature methods used except the $\Delta \mathrm{h}$ method, which was dependant on the slope of the profile. It should be noted that the scales on the abscissa and the ordinate of the deflection curve are not in the same units, and therefore the curvature of the slab is greatly exaggerated.

\subsubsection{Polynomial Curvature Methods}

The adjusted data was plotted against point number and best fit with polynomial curves of order two through six were determined using linear algebra. The forms of these polynomials are given in Equation 5.11 through Equation 5.15.

$$
\begin{array}{cr}
\text { Second order: } \alpha x^{2}+\beta x+\gamma & \text { Equation 5.11 } \\
\text { Third order: } \alpha x^{3}+\beta x^{2}+\gamma x+\delta & \text { Equation 5.12 } \\
\text { Fourth order: } \alpha x^{4}+\beta x^{3}+\gamma x^{2}+\delta x+\epsilon & \text { Equation 5.13 } \\
\text { Fifth order: } \alpha x^{5}+\beta x^{4}+\gamma x^{3}+\delta x^{2}+\epsilon x+\zeta & \text { Equation 5.14 } \\
\text { Sixth order: } \alpha x^{6}+\beta x^{5}+\gamma x^{4}+\delta x^{3}+\epsilon x^{2}+\zeta x+\eta & \text { Equation 5.15 }
\end{array}
$$


A constant curvature $(\kappa)$ of the best fit polynomial was calculated for each curve by taking the second derivative. For example, the curvature of the second order polynomial was constant at $\kappa=2 \alpha$. For larger order polynomials where the curvature is dependent on distance along the slab, the curvature was calculated for each point along the slab, and then averaged. This average value of curvature was to determine the corresponding builtin curl.

The finite element modeling program ISLAB2000 (Khazanovich et al. 2000b) was used to generate deflection profiles at the same locations where profiler data was taken for a slab of the same geometry, support conditions, PCC properties, etc., but with a temperature difference ranging from -30 to $+70^{0} \mathrm{~F}$, in increments of $5^{\circ} \mathrm{F}$. For each pass of the surface profiler, 21 theoretical deflection curves were generated (one for each temperature difference). The theoretical deflections were plotted in Excel and best fit with polynomial curves of order two through six were determined using linear algebra. The forms of these polynomials are given in Equation 5.11 through Equation 5.15. The ISLAB2000 model produced very smooth curves, see

Figure 5.9, therefore, all of the lines of best correlated almost perfectly with the ISLAB2000 data; see Table 5.2 for the correlation coefficients.

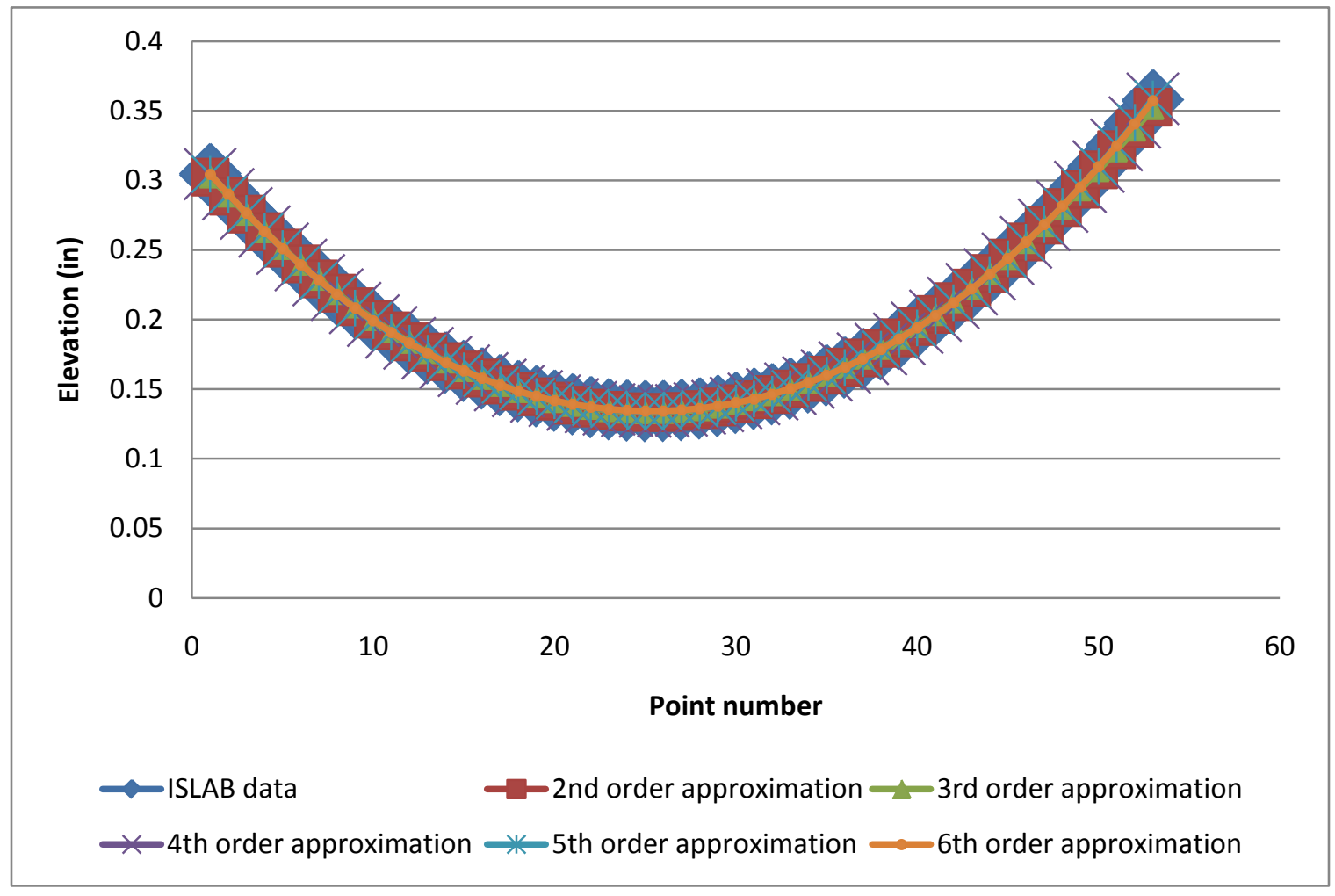

Figure 5.9: ISLAB2000 data and approximations. 
Table 5.2

Correlation coefficients between different approximations and actual data.

\begin{tabular}{|c|c|}
\hline Approximation & $\mathrm{R}^{2}$ \\
\hline 2nd order & 0.9997 \\
\hline 3rd order & 0.9998 \\
\hline 4th order & 1 \\
\hline 5th order & 1 \\
\hline 6th order & 1 \\
\hline
\end{tabular}

The curvature of each best fit polynomial was determined by taking the second derivative of the polynomial. The curvature of each deflection curve on the actual slab was matched up with the 21 theoretical curves for each polynomial order to determine the temperature difference required to produce the observed defections. This process was repeated for each profiler pass on the slab. To automate this process in order to reduce error and human bias, a built-in feature in Excel was used to return the temperature gradient associated with the slab whose curvature most closely matched that of the actual slab. The values of temperature required to produce the observed shape for each pass on the slab were then averaged. It should be noted that the values of curvature obtained for each method were the same, regardless of whether the given or assumed cross slope was used. This is because adjusting the cross slope essentially shifts the data, but does not change the curvature of the curve.

For the higher order polynomials (mainly fifth and sixth order), there were occasions when none of the theoretical curvatures closely matched the actual curvature. In these instances, the program returned a value of "\#N/A". Because no value could be determined, the built-in curl was not calculated using the polynomial which produced the error. Manually determining the temperature gradient for which the curvature of the theoretical slab most closely matched that of the actual slab, it was found that generally the temperature gradient was $-70^{\circ} \mathrm{F}$ for these higher order polynomials.

The actual temperature difference at the time of profiler testing was obtained from thermocouple sensors imbedded in the slab. Because no sensors were available at the very top and bottom of the slab, linear extrapolation was used to determine the temperature difference across the entire slab thickness. For slabs where no thermocouple data was available, data from a nearby cell with a similar structure was used.

The value of built-in curl is equal to the total curl less the actual temperature difference, as given in Equation 5.16.

$$
T_{B I C}=T_{r e q}-T_{a c t}
$$

Where:

$T_{B I C}=$ amount of curl built-in to the slab 


$$
\begin{aligned}
T_{\text {req }}= & \text { average temperature difference required to deform theoretical slab } \\
& \text { to observed profile } \\
T_{a c t}= & \text { actual temperature difference across the entire thickness of the field } \\
& \text { slab at the time of testing }
\end{aligned}
$$

This entire procedure was then repeated using only the middle half of the actual slab, and the middle half of the ISLAB2000 model slabs. Many of the slabs used in this study were restrained at the edges with dowels and ties, and all experienced some restraint due to friction at the joints with other slabs and/or shoulders. The motivation behind discarding a quarter of the length of a pass on either end was the thought that the slabs may deform more symmetrically in the middle, where the edge restraint would have less of an effect.

\subsubsection{Ah Method}

In addition to determining the total curl based on polynomial lines of best fit, another method was used whereby the height differential between the midpoint of the slab and the edges along each pass were compared between the profiled slab and the 21 theoretical slabs subjected to temperature gradients. The profiles generated by ISLAB2000 for the polynomial curvature methods were used in the $\Delta \mathrm{h}$ method. The same Excel program used in the polynomial curvature method was used to find the temperature gradient required to produce the same amount of differential deflection between the middle and the corners of the theoretical slab as in the actual slab. The temperature gradient required to achieve the same differential deflections in the theoretically flat slab as in the actual slab is computed for each pass, and these values are averaged to find the total curl in the slab. It should be noted that using the given cross slopes to create the profiles from which the height differential between the midpoint and the edge of the slab was determined caused unrealistic values of $\Delta \mathrm{h}$ because the resulting profile is linear (see Figure 5.7). Using the assumed cross-slope to generate the profile (see Figure 5.8) yields meaningful results for $\Delta \mathrm{h}$.

Once the total curl in the slab was determined, Equation 21 was used to find the amount of curl built in to the slab, just as in the polynomial curvature method. In this case, $\mathrm{T}_{\mathrm{req}}$ is the temperature difference required to obtain the same differential deflections in the theoretically flat slab as in the actual slab.

\subsubsection{Minimum Error Method}

The minimum error method matched the deflected shape measured by the surface profiler with the deflected shapes generated in ISLAB2000 for slabs exposed to various temperature gradients. The same ISLAB2000 profiles and the line of best fit for the actual profiles used in the polynomial curvature method were used in the minimum error method. The actual profile was matched with the ISLAB2000 profile for which the sum of the squares of the errors between the line of best fit for the actual profile and the ISLAB2000 profile was minimized. The ISLAB2000 profiles were superimposed on the line of best fit such that midpoints had the same elevation. 
This method removed any inherent error in the polynomial curvature method due to curvature calculations by simply matching shapes, not curvatures. Figure 5.10 shows the second order approximation of the actual data (blue dotted line), and the associated ISLAB2000 profiles (smooth curves) for the same slab exposed to 21 different temperature gradients.

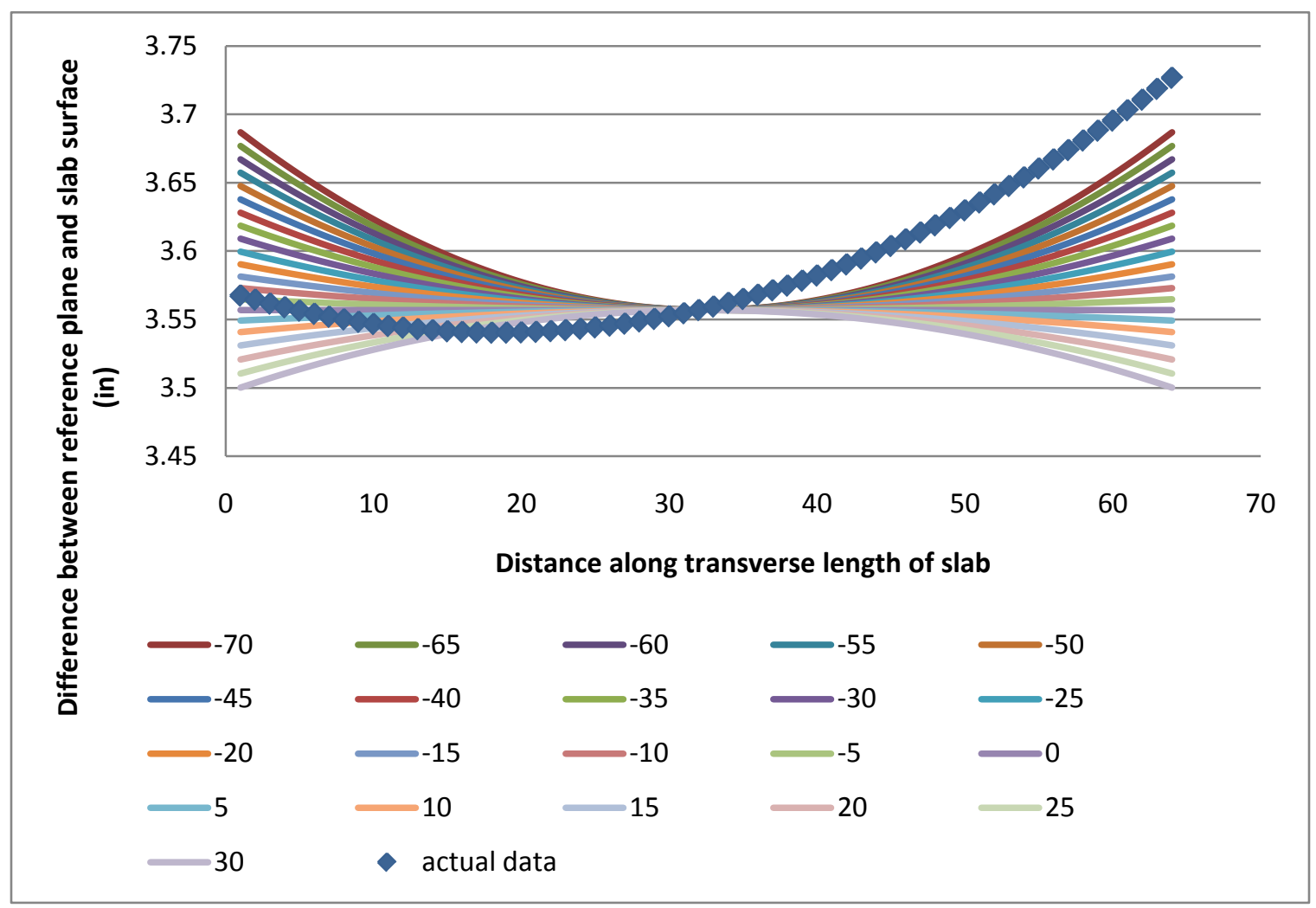

Figure 5.10: 2nd order approximation of actual data for pass 2 of Cell 53 early test in October, and associated ISLAB2000 curves.

As in the other methods, the output of this method was the total curl. The value of builtin curl is equal to the total curl less the actual temperature difference. Once the total curl in the slab was determined, Equation 21 was used to find the amount of curl built in to the slab, just as in the polynomial curvature method.

This entire procedure was then repeated using only the middle half of the actual slab, and the middle half of the ISLAB2000 model slabs. Many of the slabs used in this study were restrained at the edges with dowels and ties, and all experienced some restraint due to friction at the joints with other slabs and/or shoulders. The motivation behind discarding a quarter of the length of a pass on either end was the thought that the slabs may deform more symmetrically in the middle, where the edge restraint would have less of an effect. 


\subsubsection{Falling weight deflectometer}

The deflection data obtained from the FWD test was used in conjunction with an ANN to back-calculate the total curl of the slab. The ANN is a computer program, where the user inputs the slab geometry and concrete properties in addition to the FWD test loads and associated deflections. The program then calculates the total curl of the slab. The ANN used in this study was developed specifically for use at the MnROAD facility by Lothschutz (2009). It should be noted that the ANN was trained only for certain geometries typical of jointed plain concrete pavements. Therefore, built-in curl for cells 614 could not be calculated (short slab width spacing) and built-in curl for cell 213 was calculated assuming a pavement thickness of 6 inches.

The actual temperature difference at the time of FWD testing was obtained from thermocouple sensors embedded in the slab. Just as with the surface profile testing regime, since no sensors were available at the very top and bottom of the slab, linear extrapolation was used to determine the temperature difference across the entire slab thickness.

The value of built-in curl is equal to the total curl less the actual temperature difference.

$$
T_{B I C}=T_{A N N}-T_{a c t}
$$

Equation 5.17

Where:

$T_{B I C}=$ amount of curl built-in to the slab

$T_{A N N}=$ total equivalent temperature difference calculated by the ANN

$T_{a c t}=$ actual temperature difference across the entire thickness of the slab at the time of testing

The ANN only provides meaningful results for slabs with a total curl more extreme than $9^{\circ} \mathrm{F}$. If the ANN yields a total curl of less than $-9^{\circ} \mathrm{F}$ (i.e. the total curl is a small negative number or a positive number), then the curl is either extremely small, positive, or nonexistent.

\subsection{Results}

The amount of built-in curl for each test slab was back estimated using the surface profiler/finite element method for whole and half slabs, the FWD/ANN method, and the minimum error method for whole and half slabs. The results for these tests, after accounting for the actual temperature difference in the slabs, are presented below. A full analysis of the surface profile data using the polynomial curvature, $\Delta \mathrm{h}$, and minimum error methods was only conducted on the October test data. The FWD/ANN method was run for both the October and June test data. 
Table 5.3 shows the built-in curl calculated via the various polynomial curvature methods for the full slab profiles.

Table 5.3

Built-in curl calculated via the polynomial curvature and $\Delta \mathrm{h}$ methods using full slabs.

\begin{tabular}{|c|c|c|c|c|c|c|c|c|}
\hline \multirow[b]{2}{*}{ cell } & \multirow[b]{2}{*}{ panel } & \multirow[b]{2}{*}{ time } & \multicolumn{6}{|c|}{ Built-in Curl $\left({ }^{\circ} \mathrm{F}\right)$} \\
\hline & & & $\begin{array}{c}\Delta \mathrm{h} \\
\text { method }\end{array}$ & $\begin{array}{c}2^{\text {nd }} \\
\text { order }\end{array}$ & $\begin{array}{c}3^{\text {rd }} \\
\text { order }\end{array}$ & $\begin{array}{c}4^{\text {th }} \\
\text { order }\end{array}$ & $\begin{array}{c}5^{\text {th }} \\
\text { order }\end{array}$ & $\begin{array}{c}6^{\text {th }} \\
\text { order }\end{array}$ \\
\hline 7 & 14 & early & -41.21 & -33.21 & 6.79 & $\# N / A$ & -63.21 & $\# \mathrm{~N} / \mathrm{A}$ \\
\hline 12 & 19 & early & 13.9 & -56.1 & 11.9 & $\# N / A$ & -59.1 & $\# N / A$ \\
\hline 36 & 19 & early & -45.8 & -50.8 & 15.2 & -45.8 & -59.8 & -59.8 \\
\hline 36 & 19 & late & -76.1 & -54.1 & -27.1 & -76.1 & $\# N / A$ & $\mathrm{~N} / \mathrm{A}$ \\
\hline 36 & 20 & early & -47.8 & -43.8 & 15.2 & $\# \mathrm{~N} / \mathrm{A}$ & $\# N / A$ & $\# \mathrm{~N} / \mathrm{A}$ \\
\hline 36 & 20 & late & -76.1 & -76.1 & -31.1 & -76.1 & $\# N / A$ & -76.1 \\
\hline 37 & 8 & early & -59.6 & -58.6 & 21.4 & $\# N / A$ & $\# N / A$ & $\# \mathrm{~N} / \mathrm{A}$ \\
\hline 37 & 8 & late & -66.8 & -79.8 & -14.8 & -79.8 & $\# \mathrm{~N} / \mathrm{A}$ & $\# \mathrm{~N} / \mathrm{A}$ \\
\hline 37 & 9 & early & -59.6 & -54.6 & 20.4 & $\# N / A$ & $\# N / A$ & $\# \mathrm{~N} / \mathrm{A}$ \\
\hline 37 & 9 & late & -79.8 & -79.8 & -15.8 & -79.8 & $\# \mathrm{~N} / \mathrm{A}$ & $\# \mathrm{~N} / \mathrm{A}$ \\
\hline 53 & 3 & early & -48.6 & $\# \mathrm{~N} / \mathrm{A}$ & 18.4 & $\# \mathrm{~N} / \mathrm{A}$ & $\# N / A$ & $\# N / A$ \\
\hline 53 & 3 & late & -77.0 & $\# N / A$ & -3.0 & -77.0 & $\# \mathrm{~N} / \mathrm{A}$ & -72.0 \\
\hline $71^{*}$ & 11 & early & -50.7 & -59.7 & 15.3 & $\# N / A$ & -59.7 & 3.3 \\
\hline $72 *$ & 27 & early & -60.0 & -60.0 & 10.0 & -30.0 & $\# N / A$ & $\# \mathrm{~N} / \mathrm{A}$ \\
\hline $213^{*}$ & 15 & early & -60.2 & -66.2 & 7.8 & $\# \mathrm{~N} / \mathrm{A}$ & $\# \mathrm{~N} / \mathrm{A}$ & -66.2 \\
\hline 305 & 23 & early & -63.7 & -63.7 & 6.3 & $\# \mathrm{~N} / \mathrm{A}$ & $\# N / A$ & -31.7 \\
\hline $513 *$ & 5 & early & -66.2 & -66.2 & 3.8 & -3.2 & $\# N / A$ & -66.2 \\
\hline $614^{*}$ & 57 & early & -65.2 & -60.2 & -5.2 & $\# \mathrm{~N} / \mathrm{A}$ & $\# \mathrm{~N} / \mathrm{A}$ & -66.2 \\
\hline
\end{tabular}

Thermo-couple data was not available for this cell; instead, data from cell 12 was used. 
Table 5.4 shows the built-in curl calculated with the same polynomial curvature methods, though only considering the middle half of the slab, and discarding the profile measurements taken from the first and last quarter of the transverse width of the slab. Note that the $\Delta \mathrm{h}$ method was not applicable to the analysis of the middle half of the slabs.

Table 5.4

Built-in curl calculated via the polynomial curvature and $\Delta \mathrm{h}$ methods using middle half slabs.

\begin{tabular}{|c|c|c|c|c|c|c|c|}
\hline \multirow[b]{2}{*}{ cell } & \multirow[b]{2}{*}{ panel } & \multirow[b]{2}{*}{ time } & \multicolumn{5}{|c|}{ Built-in Curl ( $\left.{ }^{\circ} \mathrm{F}\right)$} \\
\hline & & & $\begin{array}{c}2^{\text {nd }} \\
\text { order }\end{array}$ & $\begin{array}{c}3^{\text {rd }} \\
\text { order }\end{array}$ & $\begin{array}{c}4^{\text {th }} \\
\text { order }\end{array}$ & $\begin{array}{c}5^{\text {th }} \\
\text { order }\end{array}$ & $\begin{array}{c}6^{\text {th }} \\
\text { order }\end{array}$ \\
\hline 7 & 14 & early & -63.21 & -63.21 & 10.79 & $\# N / A$ & $\# N / A$ \\
\hline 12 & 19 & early & -59.07 & 16.93 & $\# N / A$ & -59.07 & $\# N / A$ \\
\hline 36 & 19 & early & -15.8 & 8.2 & -34.8 & $\# N / A$ & $\# N / A$ \\
\hline 36 & 19 & late & -76.12 & -30.12 & -75.12 & $\# N / A$ & $\mathrm{~N} / \mathrm{A}$ \\
\hline 36 & 20 & early & -25.8 & -10.2 & $\# N / A$ & $\# N / A$ & $\# N / A$ \\
\hline 36 & 20 & late & -76.12 & -40.12 & -76.12 & -76.12 & -76.12 \\
\hline 37 & 8 & early & $\# \mathrm{~N} / \mathrm{A}$ & 15.43 & -6.57 & $\# N / A$ & -45.57 \\
\hline 37 & 8 & late & -59.75 & -28.75 & $\# \mathrm{~N} / \mathrm{A}$ & $\# N / A$ & $\# \mathrm{~N} / \mathrm{A}$ \\
\hline 37 & 9 & early & 3.43 & 17.43 & $\# N / A$ & $\# N / A$ & $\# N / A$ \\
\hline 37 & 9 & late & -79.75 & -27.75 & $\# N / A$ & -79.75 & $\# N / A$ \\
\hline 53 & 3 & early & $\# N / A$ & 16.37 & $\# N / A$ & $\# N / A$ & $\# N / A$ \\
\hline 53 & 3 & late & $\# N / A$ & -7.03 & $\# N / A$ & $\# N / A$ & $\# N / A$ \\
\hline $71 *$ & 11 & early & -49.71 & 22.29 & $\# N / A$ & $\# N / A$ & -49.71 \\
\hline $72 *$ & 27 & early & -60.01 & 9.99 & $\# N / A$ & $\# N / A$ & $\# N / A$ \\
\hline 213* & 15 & early & -62.22 & 0.78 & -66.22 & $\# N / A$ & -64.22 \\
\hline 305 & 23 & early & -63.71 & 5.29 & -33.71 & -63.71 & -63.71 \\
\hline 513* & 5 & early & -60.22 & -4.22 & -66.22 & $\# N / A$ & -66.22 \\
\hline 614* & 57 & early & -49.22 & -20.22 & -66.22 & 3.782519 & -66.22 \\
\hline
\end{tabular}

* Thermo-couple data was not available for this cell; instead, data from cell 12 was used. 
Table 5.5 and Table 5.6 show the built-in curl calculated using the FWD/ANN method based on the June and October test data respectively. It should be noted that, as the ANN can only reliably calculate the curl in slabs with a total curl more negative than $-9^{\circ} \mathrm{F}$, none of the results from the June test can be considered valid, and only the results for cell 36 panel 20 early and cell 305 from the October test should be considered valid. The cells for which the ANN results are invalid are shown in gray in Table 5.5 and Table 5.6.

Table 5.5

Built-in curl from the FWD/ANN method - June test.

\begin{tabular}{|c|c|c|c|c|c|}
\hline cell & panel & time & $\begin{array}{c}\text { average } \\
\text { total curl } \\
\quad\left({ }^{\circ} \mathrm{F}\right)\end{array}$ & $\begin{array}{c}\Delta \mathrm{T} \text { at } \\
\text { time of } \\
\text { testing } \\
\left({ }^{\circ} \mathrm{F}\right)\end{array}$ & $\begin{array}{c}\text { Built- } \\
\text { in curl } \\
\left({ }^{\circ} \mathrm{F}\right)\end{array}$ \\
\hline 7 & 12 & AM & -1.024 & 4.7 & -5.7 \\
\hline 7 & 12 & PM & 4.437 & 10.2 & -5.8 \\
\hline 7 & 14 & AM & 3.078 & 0.6 & 2.5 \\
\hline 7 & 14 & PM & 3.352 & 6.82 & -3.5 \\
\hline 12 & 19 & AM & 4.302 & -6.28 & 10.6 \\
\hline 12 & 19 & PM & 4.387 & 17.04 & -12.7 \\
\hline 12 & 24 & AM & 2.630 & -6.76 & 9.4 \\
\hline 12 & 24 & PM & 4.306 & 17.32 & -13.0 \\
\hline 36 & 19 & AM & 4.332 & -10.12 & 14.5 \\
\hline 36 & 19 & PM & 4.422 & 13.05 & -8.6 \\
\hline 36 & 20 & AM & 4.307 & -10.12 & 14.4 \\
\hline 36 & 20 & PM & 4.399 & 13.05 & -8.7 \\
\hline 37 & 8 & AM & 4.342 & -3.13 & 7.5 \\
\hline 37 & 8 & PM & 4.397 & 8.77 & -4.4 \\
\hline 37 & 9 & AM & 4.347 & -3.13 & 7.5 \\
\hline 37 & 9 & PM & 4.383 & 8.77 & -4.4 \\
\hline 53 & 13 & AM & 4.386 & -1.53 & 5.9 \\
\hline 53 & 13 & PM & 4.369 & 11.49 & -7.1 \\
\hline 213 & 15 & AM & 4.414 & -7.27 & 11.7 \\
\hline $213^{a}$ & 15 & PM & 4.418 & 19.31 & -14.9 \\
\hline $305^{b}$ & 23 & AM & 2.747 & -8.55 & 11.3 \\
\hline 313 & 26 & AM & 4.406 & -7.53 & 11.9 \\
\hline 313 & 26 & PM & 4.422 & 17.04 & -12.6 \\
\hline $513^{a}$ & 5 & AM & 4.370 & -6.25 & 10.6 \\
\hline $513^{a}$ & 5 & PM & 4.420 & 16.47 & -12.0 \\
\hline
\end{tabular}


Table 5.6

Built-in curl from FWD/ANN method - October test.

\begin{tabular}{|c|c|c|c|c|c|}
\hline cell & panel & time & $\begin{array}{c}\text { average } \\
\text { total curl } \\
\left({ }^{\circ} \mathrm{F}\right)\end{array}$ & $\begin{array}{c}\Delta \mathrm{T} \text { at time } \\
\text { of testing } \\
\left({ }^{\circ} \mathrm{F}\right)\end{array}$ & $\begin{array}{c}\text { built in } \\
\text { curl } \\
\left({ }^{\circ} \mathrm{F}\right)\end{array}$ \\
\hline 7 & 14 & early & 2.8 & -7.1 & 9.9 \\
\hline 12 & 19 & early & 2.6 & -9.7 & 12.2 \\
\hline 36 & 19 & early & 2.8 & -10.2 & 13.0 \\
\hline 36 & 19 & late & 4.4 & 5.7 & -1.3 \\
\hline 36 & 20 & early & -10.7 & -10.2 & -0.5 \\
\hline 36 & 20 & late & 4.1 & 5.7 & -1.6 \\
\hline 37 & 8 & early & 4.2 & -9.1 & 13.3 \\
\hline 37 & 8 & late & 4.3 & 12.0 & -7.7 \\
\hline 37 & 9 & early & -0.8 & -9.1 & 8.2 \\
\hline 37 & 9 & late & 4.4 & 12.0 & -7.7 \\
\hline 53 & 3 & early & 2.3 & -15.1 & 17.4 \\
\hline 53 & 3 & late & 2.9 & 10.3 & -7.4 \\
\hline $71^{*}$ & 11 & early & 3.4 & -10.0 & 13.4 \\
\hline $72^{*}$ & 27 & early & 3.4 & -9.4 & 12.7 \\
\hline $213^{*}$ & 15 & early & 0.2 & -3.5 & 3.8 \\
\hline 305 & 23 & early & -14.9 & -4.2 & -10.8 \\
\hline $513^{*}$ & 5 & early & -5.0 & -3.5 & -1.5 \\
\hline$*$
\end{tabular}

* Thermocouple data was not available for this cell; data from cell 12 was used instead. 
Table 5.7 shows the results of the minimum error method. The final value for built-in curl was based on the polynomial approximation which gave the least amount of error between the line of best fit of the actual data and the ISLAB2000.

Table 5.7

Built-in curl determined via the minimum error method for full slabs.

\begin{tabular}{|c|c|c|c|c|c|c|c|}
\hline \multirow[b]{2}{*}{ cell } & \multirow[b]{2}{*}{ panel } & \multirow[b]{2}{*}{ time } & \multicolumn{5}{|c|}{ Built-in Curl $\left({ }^{\circ} \mathrm{F}\right)$} \\
\hline & & & $\begin{array}{c}2^{\text {nd }} \\
\text { order }\end{array}$ & $\begin{array}{c}3^{\text {rd }} \\
\text { order }\end{array}$ & $\begin{array}{c}4^{\text {th }} \\
\text { order }\end{array}$ & $\begin{array}{c}5^{\text {th }} \\
\text { order }\end{array}$ & $\begin{array}{c}6^{\text {th }} \\
\text { order }\end{array}$ \\
\hline 7 & 14 & early & -48.21 & -43.21 & -33.21 & -3.21 & 4.29 \\
\hline 12 & 19 & early & -56.57 & -55.32 & 24.68 & 10.93 & 5.93 \\
\hline 36 & 19 & early & -51.05 & -17.3 & -56.05 & -59.8 & -59.8 \\
\hline 36 & 19 & late & -56.12 & -76.12 & -76.12 & 23.88 & -1.12 \\
\hline 36 & 20 & early & -47.3 & -11.05 & -58.55 & -59.8 & -59.8 \\
\hline 36 & 20 & late & -76.12 & -76.12 & -76.12 & -63.62 & -76.12 \\
\hline 37 & 8 & early & -59.57 & 15.43 & -44.57 & -13.32 & -34.57 \\
\hline 37 & 8 & late & -79.75 & -79.75 & -79.75 & 20.25 & -29.75 \\
\hline 37 & 9 & early & -59.57 & 24.18 & -47.07 & 6.68 & -59.57 \\
\hline 37 & 9 & late & -79.75 & -79.75 & -79.75 & 20.25 & -79.75 \\
\hline 53 & 3 & early & -4.88 & -2.38 & -27.38 & -3.63 & -28.63 \\
\hline 53 & 3 & late & -34.53 & -24.53 & -77.03 & -64.53 & -77.03 \\
\hline $71^{*}$ & 11 & early & -59.71 & -54.71 & -59.71 & -59.71 & 40.29 \\
\hline $72 *$ & 27 & early & -60.01 & -60.01 & -60.01 & -60.01 & -32.51 \\
\hline $213^{*}$ & 15 & early & -66.22 & -66.22 & -66.22 & -63.72 & 33.78 \\
\hline 305 & 23 & early & -65.57 & -65.57 & -65.57 & -65.57 & -65.57 \\
\hline $513 *$ & 5 & early & -61.22 & -63.72 & -66.22 & -66.22 & -16.22 \\
\hline $614^{*}$ & 57 & early & -37.47 & -37.47 & -37.47 & -37.47 & 31.28 \\
\hline
\end{tabular}

* Thermo-couple data was not available for this cell; instead, data from cell 12 was used. 
Table 5.8 shows the built-in curl calculated with the same method, though only considering the middle half of the slab, and discarding the profile measurements taken from the first and last quarter of the transverse width of the slab. Note that the $\Delta \mathrm{h}$ method was not applicable to the analysis of the middle half of the slabs.

Table 5.8

Built-in curl determined via the minimum error method for half slabs.

\begin{tabular}{|c|c|c|c|c|c|c|c|}
\hline \multirow[b]{2}{*}{ cell } & \multirow[b]{2}{*}{ panel } & \multirow[b]{2}{*}{ time } & \multicolumn{5}{|c|}{ Built-in Curl $\left({ }^{\circ} \mathrm{F}\right)$} \\
\hline & & & $\begin{array}{c}2^{\text {nd }} \\
\text { order }\end{array}$ & $\begin{array}{c}3^{\text {rd }} \\
\text { order }\end{array}$ & $\begin{array}{c}4^{\text {th }} \\
\text { order }\end{array}$ & $\begin{array}{c}5^{\text {th }} \\
\text { order }\end{array}$ & $\begin{array}{c}6^{\text {th }} \\
\text { order }\end{array}$ \\
\hline 7 & 14 & early & -63.21 & -63.21 & -63.21 & -63.21 & -63.21 \\
\hline 12 & 19 & early & -59.07 & -59.07 & -59.07 & -59.07 & -59.07 \\
\hline 36 & 19 & early & -9.8 & -9.8 & -7.3 & -7.3 & 6.45 \\
\hline 36 & 19 & late & -76.12 & -76.12 & -76.12 & -76.12 & -76.12 \\
\hline 36 & 20 & early & -27.3 & -27.3 & -17.3 & -17.3 & -9.8 \\
\hline 36 & 20 & late & -76.12 & -76.12 & -76.12 & -76.12 & -76.12 \\
\hline 37 & 8 & early & 22.93 & 22.93 & 31.68 & 31.68 & 37.93 \\
\hline 37 & 8 & late & -79.75 & -79.75 & -79.75 & -79.75 & -79.75 \\
\hline 37 & 9 & early & 12.93 & 12.93 & 15.43 & 15.43 & 27.93 \\
\hline 37 & 9 & late & -79.75 & -79.75 & -79.75 & -79.75 & -79.75 \\
\hline 53 & 3 & early & 2.62 & 2.62 & -3.63 & -3.63 & -3.63 \\
\hline 53 & 3 & late & 4.22 & 4.22 & 0.47 & 0.47 & -2.03 \\
\hline $71 *$ & 11 & early & -40.96 & -40.96 & -10.96 & -10.96 & -2.21 \\
\hline $72 *$ & 27 & early & -60.01 & -60.01 & -60.01 & -60.01 & -58.76 \\
\hline $213 *$ & 15 & early & -61.22 & -61.22 & -39.97 & -39.97 & -27.47 \\
\hline 305 & 23 & early & -65.57 & -65.57 & -65.57 & -65.57 & -65.57 \\
\hline $513^{*}$ & 5 & early & -53.72 & -53.72 & -32.47 & -32.47 & -32.47 \\
\hline $614^{*}$ & 57 & early & -32.47 & -32.47 & -23.72 & -23.72 & -16.22 \\
\hline
\end{tabular}

${ }^{*}$ Thermo-couple data was not available for this cell; instead, data from cell 12 was used. 
Table 5.9 shows the built-in curl determined using the minimum error method to compare the actual data and the ISLAB2000 profiles, rather than an approximation.

Table 5.9

Built-in curl determined via the minimum error method using actual data and ISLAB2000 profiles.

\begin{tabular}{|c|c|c|r|}
\hline cell & panel & time & \multicolumn{1}{c|}{$\begin{array}{r}\text { Built-in } \\
\left.\text { Curl ( }{ }^{\circ} \mathrm{F}\right)\end{array}$} \\
\hline 7 & 14 & early & -63.21 \\
\hline 12 & 19 & early & -59.07 \\
\hline 36 & 19 & early & 31.2 \\
\hline 36 & 19 & late & -76.12 \\
\hline 36 & 20 & early & -5.8 \\
\hline 36 & 20 & late & -63.88 \\
\hline 37 & 8 & early & 26.43 \\
\hline 37 & 8 & late & -79.75 \\
\hline 37 & 9 & early & 26.43 \\
\hline 37 & 9 & late & -70.75 \\
\hline 53 & 3 & early & 7.37 \\
\hline 53 & 3 & late & -1.03 \\
\hline $71 *$ & 11 & early & -43.71 \\
\hline $72^{*}$ & 27 & early & -59.01 \\
\hline $213^{*}$ & 15 & early & -53.22 \\
\hline 305 & 23 & early & -65.57 \\
\hline $513^{*}$ & 5 & early & -64.22 \\
\hline $614^{*}$ & 57 & early & -61.22 \\
\hline
\end{tabular}

${ }^{*}$ Thermo-couple data was not available for this cell; instead, data from cell 12 was used.

\subsection{Analysis}

The surface profiler/finite element results were evaluated using the polynomial curvature method, $\Delta \mathrm{h}$ method, and minimum error method. None of these methods consistently gave realistic results; however, as the built-in curl for the slabs investigated is not known, there is no way of determining which method gave the correct value of built-in curl most frequently. Given that these methods did not provide consistent results, they could not be used to verify the FWD/ANN results. The FWD/ANN method is only valid for cells with a total curl more negative than $-9^{\circ} \mathrm{F}$; in this test, only two cells met this requirement. The values of built-in curl determined by the FWD/ANN for these two cells were both plausible, however, they could not be confirmed.

\subsubsection{Polynomial Curvature and $\Delta \mathrm{h}$ Methods}

The polynomial curvature method calculated values for built-in curl ranging from large positive to large negative values. Multiple methods of calculating the built-in curl were 
used in an effort to find a method that would work best. Fitting the data with a quadratic equation is, by far, the most common method for two reasons. First, a curled up slab naturally has a parabolic shape, so the best-fit curve actually has a physical meaning: it is the actual shape of the slab. Second, the derivative of a quadratic is a constant, which makes comparisons between the curvatures of the actual and the theoretical slabs very straightforward .

Characterizing the profile with higher order polynomials may lead to slight increases in the correlation coefficient between the actual profile and the best fit curve. For example, Table 5.10 shows the correlation coefficient $\left(\mathrm{R}^{2}\right)$ value for each order polynomial used to best fit the data for Cell 72, pass 3, which is shown in Figure 5.11.

Table 5.10

Correlation coefficient $\left(R^{2}\right)$ values for various order polynomials for Cell 72 , pass 3 .

\begin{tabular}{|c|c|}
\hline order & $\mathrm{R}^{2}$ \\
\hline 2nd & 0.8029 \\
\hline 3rd & 0.8166 \\
\hline 4th & 0.8213 \\
\hline 5th & 0.8224 \\
\hline 6th & 0.8502 \\
\hline
\end{tabular}

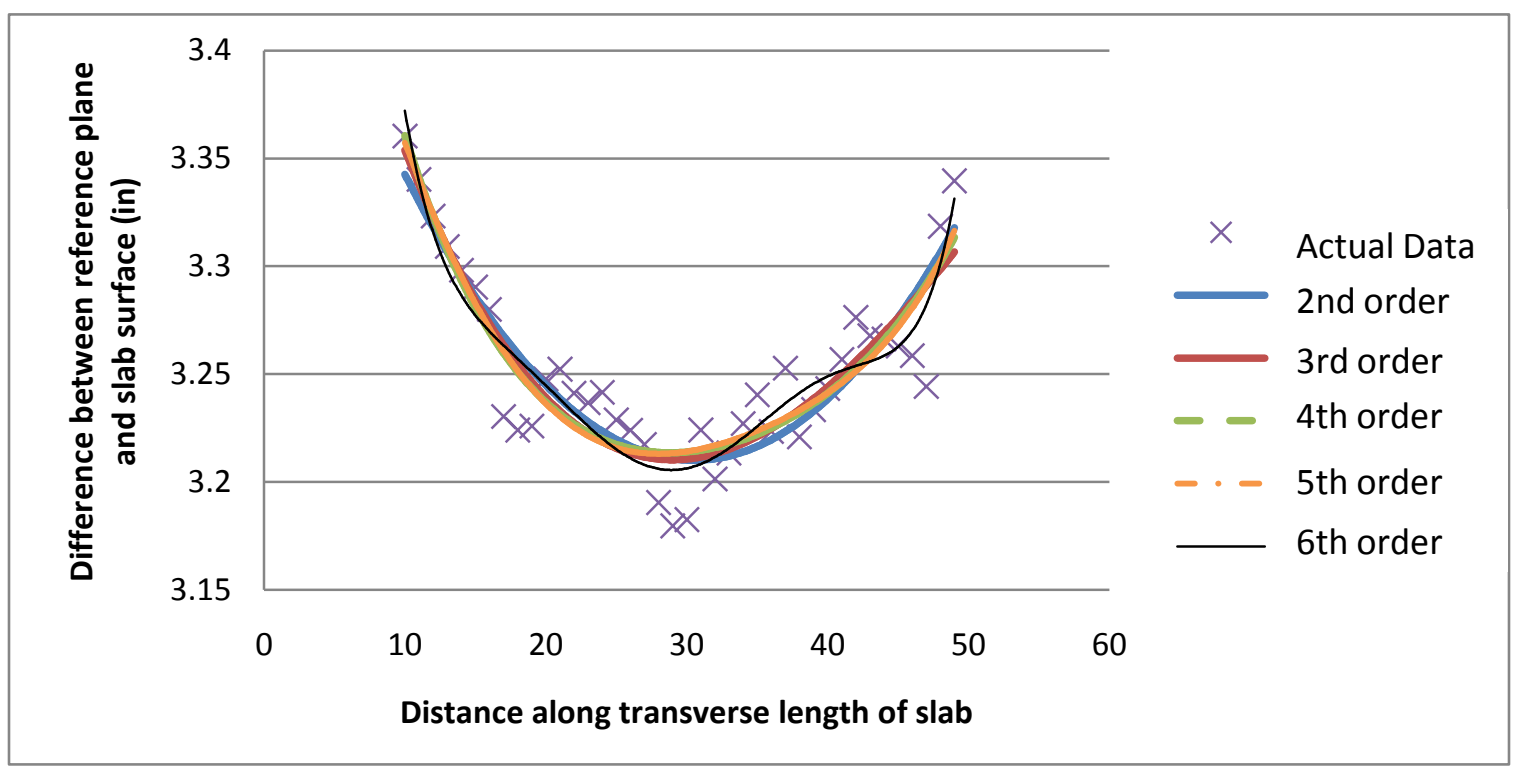

Figure 5.11: Best-fit polynomals for Cell 72, pass 3 .

As can be seen in this example, increasing the polynomial order does lead to a slightly better correlation, but the best-fit curve then loses its physical meaning and becomes simply a mathematical model. The one exception to this is that the curvature of the fourth order polynomial is a quadratic and therefore does represent the deflected shape of 
the slab (Byrum 2000). However, the curvature of a higher order polynomial is dependent on the location along the slab and therefore more computationally intensive. The correlation coefficient was computed between the actual data and each order best fit polynomial for both the transverse pass across the whole slab, and only the middle portion of the slab, see Table 5.11 and Table 5.12 respectively. In these tables, the highest correlation coefficient for a particular slab is shaded.

Table 5.11

Correlation coefficents $\left(R^{2}\right)$ between actual data and polynomial approximations from whole slab analsysis.

\begin{tabular}{|c|c|c|r|r|r|r|r|}
\cline { 4 - 8 } \multicolumn{2}{c}{} & \multicolumn{6}{c|}{ Correlation coefficients } \\
\hline cell & panel & time & $\begin{array}{l}\text { 2nd } \\
\text { order }\end{array}$ & \multicolumn{1}{l}{$\begin{array}{l}\text { 3rd } \\
\text { order }\end{array}$} & $\begin{array}{l}\text { 4th } \\
\text { order }\end{array}$ & $\begin{array}{l}\text { 5th } \\
\text { order }\end{array}$ & $\begin{array}{l}\text { 6th } \\
\text { order }\end{array}$ \\
\hline 7 & 14 & early & 0.53 & 0.56 & 0.70 & 0.74 & 0.74 \\
\hline 12 & 19 & early & 0.63 & 0.71 & 0.82 & 0.91 & 0.92 \\
\hline 36 & 19 & early & 0.67 & 0.73 & 0.79 & 0.81 & 0.82 \\
\hline 36 & 19 & late & 0.30 & 0.76 & 0.74 & 0.05 & 0.23 \\
\hline 36 & 20 & early & 0.90 & 0.96 & 0.96 & 0.96 & 0.97 \\
\hline 36 & 20 & late & 0.90 & 0.96 & 0.96 & 0.96 & 0.97 \\
\hline 37 & 8 & early & 0.63 & 0.71 & 0.82 & 0.91 & 0.92 \\
\hline 37 & 8 & late & 0.63 & 0.71 & 0.82 & 0.91 & 0.92 \\
\hline 37 & 9 & early & 0.44 & 0.76 & 0.80 & 0.84 & 0.86 \\
\hline 37 & 9 & late & 0.41 & 0.43 & 0.49 & 0.74 & 0.80 \\
\hline 53 & 3 & early & 0.34 & 0.36 & 0.46 & 0.54 & 0.60 \\
\hline 53 & 3 & late & 0.63 & 0.71 & 0.82 & 0.91 & 0.92 \\
\hline 71 & 11 & early & 0.67 & 0.90 & 0.91 & 0.93 & 0.94 \\
\hline 72 & 27 & early & 0.79 & 0.86 & 0.86 & 0.87 & 0.85 \\
\hline 213 & 15 & early & 0.93 & 0.97 & 0.97 & 0.98 & 0.99 \\
\hline 305 & 23 & early & 0.81 & 0.95 & 0.97 & 0.98 & 0.98 \\
\hline 513 & 5 & early & 0.80 & 0.91 & 0.92 & 0.88 & 0.94 \\
\hline 614 & 57 & early & 0.63 & 0.71 & 0.69 & 0.38 & 0.74 \\
\hline
\end{tabular}


Table 5.12

Correlation coefficients $\left(\mathrm{R}^{2}\right)$ between actual data and polynomial approximations from half slab analsysis.

\begin{tabular}{|c|c|c|c|c|c|c|c|}
\hline \multirow[b]{2}{*}{ cell } & \multirow[b]{2}{*}{ panel } & \multirow[b]{2}{*}{ time } & \multicolumn{5}{|c|}{ Half slabs correlation coefficient } \\
\hline & & & $\begin{array}{l}\text { 2nd } \\
\text { order }\end{array}$ & $\begin{array}{l}\text { 3rd } \\
\text { order }\end{array}$ & $\begin{array}{l}\text { 4th } \\
\text { order }\end{array}$ & $\begin{array}{l}\text { 5th } \\
\text { order }\end{array}$ & $\begin{array}{l}\text { 6th } \\
\text { order }\end{array}$ \\
\hline 7 & 14 & early & 0.68 & 0.76 & 0.76 & 0.77 & 0.78 \\
\hline 12 & 19 & early & 0.71 & 0.87 & 0.90 & 0.94 & 0.96 \\
\hline 36 & 19 & early & 0.84 & 0.86 & 0.87 & 0.88 & 0.91 \\
\hline 36 & 19 & late & 0.62 & 0.69 & 0.78 & 0.84 & 0.86 \\
\hline 36 & 20 & early & 0.48 & 0.52 & 0.58 & 0.61 & 0.64 \\
\hline 36 & 20 & late & 0.90 & 0.93 & 0.94 & 0.96 & 0.96 \\
\hline 37 & 8 & early & 0.84 & 0.86 & 0.88 & 0.89 & 0.93 \\
\hline 37 & 8 & late & 0.49 & 0.58 & 0.72 & 0.73 & 0.76 \\
\hline 37 & 9 & early & 0.80 & 0.83 & 0.85 & 0.86 & 0.92 \\
\hline 37 & 9 & late & 0.55 & 0.59 & 0.76 & 0.76 & 0.77 \\
\hline 53 & 3 & early & 0.35 & 0.40 & 0.65 & 0.70 & 0.73 \\
\hline 53 & 3 & late & 0.61 & 0.67 & 0.79 & 0.81 & 0.82 \\
\hline 71 & 11 & early & 0.78 & 0.92 & 0.96 & 0.96 & 0.97 \\
\hline 72 & 27 & early & 0.60 & 0.63 & 0.65 & 0.66 & 0.73 \\
\hline 213 & 15 & early & 0.84 & 0.84 & 0.90 & 0.91 & 0.92 \\
\hline 305 & 23 & early & 0.94 & 0.96 & 0.96 & 0.97 & 0.98 \\
\hline 513 & 5 & early & 0.91 & 0.92 & 0.96 & 0.96 & 0.96 \\
\hline 614 & 57 & early & 0.88 & 0.89 & 0.91 & 0.92 & 0.92 \\
\hline
\end{tabular}

While the general trend was for the correlation coefficient to increase with higher order polynomials, this was not always the case for the full slab analysis. This is to be expected, as linear algebra dictates that a better fit of data points will be made with a higher order polynomial. When the correlation coefficient did increase with the use of higher order polynomials, this increase was generally not substantial.

Another method to determine which polynomial best fit the data was to compute the sum of the squares of the difference (or error) between the actual data and the line of best fit. This was determined for both the full and half slab analyses; see

Table 5.13 and Table 5.14 respectively, again, the best polynomial approximation for each case is shaded. 
Table 5.13

Sum of squares of errors between actual profile data and best fit polynomial for full slabs.

\begin{tabular}{|c|c|c|c|c|c|c|c|}
\hline \multirow[b]{2}{*}{ cell } & \multirow[b]{2}{*}{ panel } & \multirow[b]{2}{*}{ time } & \multicolumn{5}{|c|}{ sum of squares of error } \\
\hline & & & $\begin{array}{l}\text { 2nd } \\
\text { order }\end{array}$ & $\begin{array}{l}\text { 3rd } \\
\text { order }\end{array}$ & $\begin{array}{l}\text { 4th } \\
\text { order }\end{array}$ & $\begin{array}{l}5 \text { th } \\
\text { order }\end{array}$ & $\begin{array}{l}\text { 6th } \\
\text { order }\end{array}$ \\
\hline 7 & 14 & early & 0.068455 & 0.065575 & 0.046325 & 0.039782 & 0.039467 \\
\hline 12 & 19 & early & 0.159266 & 0.123332 & 0.075014 & 0.037239 & 0.037745 \\
\hline 36 & 19 & early & 0.082388 & 0.065131 & 0.051983 & 0.048055 & 0.043695 \\
\hline 36 & 19 & late & 1.414262 & 0.448095 & 0.522003 & 3.949544 & 3.000933 \\
\hline 36 & 20 & early & 0.108859 & 0.076159 & 0.075048 & 0.082185 & 0.081695 \\
\hline 36 & 20 & late & 0.996398 & 0.403063 & 0.395741 & 0.381508 & 0.295709 \\
\hline 37 & 8 & early & 0.547331 & 0.175613 & 0.124412 & 0.114777 & 0.109796 \\
\hline 37 & 8 & late & 0.794096 & 0.767706 & 0.642451 & 0.30033 & 0.264691 \\
\hline 37 & 9 & early & 0.55283 & 0.225641 & 0.186442 & 0.14536 & 0.132411 \\
\hline 37 & 9 & late & 0.913603 & 0.888148 & 0.782845 & 0.401994 & 0.310879 \\
\hline 53 & 3 & early & 0.347501 & 0.330325 & 0.268344 & 0.234935 & 0.215294 \\
\hline 53 & 3 & late & 0.469503 & 0.452533 & 0.286458 & 0.260481 & 0.218923 \\
\hline 71 & 11 & early & 0.184218 & 0.054421 & 0.050044 & 0.037609 & 0.031481 \\
\hline 72 & 27 & early & 0.238296 & 0.160075 & 0.16062 & 0.162877 & 0.187812 \\
\hline 213 & 15 & early & 0.47396 & 0.22224 & 0.218355 & 0.179817 & 0.085203 \\
\hline 305 & 23 & early & 0.821379 & 0.205393 & 0.109287 & 0.093565 & 0.092965 \\
\hline 513 & 5 & early & 0.329148 & 0.146821 & 0.139454 & 0.265197 & 0.119431 \\
\hline 614 & 57 & early & 1.715912 & 3.296648 & 8.649725 & 15.92525 & 46.848 \\
\hline
\end{tabular}


Table 5.14

Sum of squares of errors between actual profile data and best fit polynomial for half slabs.

\begin{tabular}{|c|c|c|l|l|l|l|l|}
\cline { 4 - 8 } \multicolumn{2}{c}{} & \multicolumn{5}{c|}{ half slabs sum of squares of error } \\
\hline cell & panel & time & $\begin{array}{l}\text { 2nd } \\
\text { order }\end{array}$ & $\begin{array}{l}\text { 3rd } \\
\text { order }\end{array}$ & $\begin{array}{l}\text { 4th } \\
\text { order }\end{array}$ & $\begin{array}{l}\text { 5th } \\
\text { order }\end{array}$ & $\begin{array}{l}\text { 6th } \\
\text { order }\end{array}$ \\
\hline 7 & 14 & early & 0.156558 & 0.111082 & 0.10849 & 0.106295 & 0.101262 \\
\hline 12 & 19 & early & 0.078638 & 0.036671 & 0.02866 & 0.017956 & 0.011851 \\
\hline 36 & 19 & early & 0.041507 & 0.03715 & 0.035996 & 0.032446 & 0.025465 \\
\hline 36 & 19 & late & 0.386065 & 0.312982 & 0.230026 & 0.168537 & 0.144081 \\
\hline 36 & 20 & early & 0.035924 & 0.034369 & 0.029796 & 0.026386 & 0.023186 \\
\hline 36 & 20 & late & 0.432074 & 0.305998 & 0.263802 & 0.184969 & 0.159737 \\
\hline 37 & 8 & early & 0.049813 & 0.044889 & 0.037559 & 0.032768 & 0.023063 \\
\hline 37 & 8 & late & 0.348778 & 0.288121 & 0.190448 & 0.185198 & 0.16384 \\
\hline 37 & 9 & early & 0.065147 & 0.054551 & 0.049422 & 0.044936 & 0.029145 \\
\hline 37 & 9 & late & 0.322533 & 0.291988 & 0.169372 & 0.168452 & 0.163544 \\
\hline 53 & 3 & early & 0.117137 & 0.107803 & 0.063558 & 0.056061 & 0.050159 \\
\hline 53 & 3 & late & 0.100166 & 0.085788 & 0.052473 & 0.046461 & 0.043113 \\
\hline 71 & 11 & early & 0.083743 & 0.030311 & 0.013885 & 0.013689 & 0.011555 \\
\hline 72 & 27 & early & 0.100464 & 0.094239 & 0.086344 & 0.083164 & 0.067067 \\
\hline 213 & 15 & early & 0.109408 & 0.108077 & 0.067547 & 0.062264 & 0.052665 \\
\hline 305 & 23 & early & 0.045792 & 0.031741 & 0.030239 & 0.021815 & 0.017822 \\
\hline 513 & 5 & early & 0.054447 & 0.047851 & 0.025062 & 0.024568 & 0.023392 \\
\hline 614 & 57 & early & 0.088741 & 0.074746 & 0.061634 & 0.055318 & 0.049337 \\
\hline
\end{tabular}

The sixth order polynomial was the best approximation of the actual data in most cases for the full slab analysis, and in all cases for the half slab analysis. Again, this is to be expected, based on simple linear algebra. However, it is important to note that even though a higher order approximation fits the data better, it is not necessarily the best approximation to use in calculations. The data obtained by a profilometer is inherently erratic, due to cracks, surface texture, etc, and is only representative of the surface of the slab. To determine the amount of built-in curl in a slab, it is necessary to determine the shape of the neutral axis of the slab, not the top surface. Lower order approximations do not show all of the elevation changes in the top of the slab, but do capture the basic behavior of the entire slab. Therefore, it should not be concluded that the order polynomial which bests fits the actual data will be the best option to be used to determine the built-in curl in the slab.

The various polynomials used to best-fit the profile and determine the built-in curl in the slab produced widely varied results. None of the different order polynomials matched with each other, and therefore cannot be used to validate one model versus another. The graphical representations of the profiles (see Figure 5.8 as an example) all have a slight bowl shape or upward curvature (exaggerated due to the scale of the graph), which is indicative of a slab with a negative total gradient. The profiles all had this concave 
shape, regardless of the temperature difference at the time of profiling. Cells 36, 37, and 53 were all tested in both the early and the late morning, while all other cells were only tested in the early morning. The early morning temperature gradients were all negative (i.e. the top of the slab is cooler than the bottom), while in the late morning tests, all of the temperature gradients were positive (i.e. the top is warmer than the bottom). Given that all of the measured profiles had a negative total curl (based on their shape), regardless of the temperature gradient present, it can be concluded that the expected builtin gradient was negative.

The only polynomial which consistently predicted negative built-in curl was the fifth order polynomial. However, the fifth order polynomial was also the most unreliable method which returned a value of "N/A" in 14 cases out of 18 . This is likely due to the fact that the fifth order polynomial has no physical meaning, but is merely a line which is closest to the most points in the profile. However, profile data has a large amount of small variations due surface imperfections and therefore matching the largest amount of points does not guarantee a more accurate representation of the shape of the slab. When the fifth order polynomial did produce results, it predicted large negative values of builtin curl, from -23 to $-60^{\circ} \mathrm{F}$, which are unlikely to be accurate. The sixth order polynomial also returned a value of "N/A" on 10 occasions, and predicted large negative values of built-in curl, as did the second and fourth order polynomials, and the $\Delta \mathrm{h}$ method. The third order polynomial curvature methods gave generally low negative or even positive values of built-in curl. Given that the exact values of curl are not known, it is difficult to states that one method can be declared more accurate than the others.

In an effort to reduce the error caused by edge restraints, the data for the first and last quarter of each pass was discarded, and the remaining data was reanalyzed via the polynomial curvature method. In this analysis, the third order polynomial was the only approximation that consistently produced results; the $4^{\text {th }}, 5^{\text {th }}$, and $6^{\text {th }}$ order approximations all yielded a value of "N/A" for many of the cases and predicted very large values of built-in curl when results were produced. The third order polynomial predicted values of built-in curl that were either positive or less negative than those given by the other methods. Though the values of built-in curl determined from the half slab analysis differed from those calculated in the full slab analysis, the trends in the data were similar.

One way to compare the validity of the various methods is to examine the results given for the same cell tested at different times. In the October round of testing, cells 36, 37, and 53 were all tested in both the early morning (5-7am) and late morning (10-11am). The $2^{\text {nd }}$ order polynomial predicted values of built-in curl which were very similar for the early and late tests for cell 36 using both the polynomial curvature and minimum error methods.

In an effort to determine which order polynomial was most likely to produce accurate results, a statistical analysis was conducted. In this analysis, the sum of the square of the errors between the actual data, and the ISLAB2000 profile which best matched the actual 
data based on curvature was computed for each order polynomial assumed in the polynomial curvature method. Additionally, the sum of the square of the errors between the line of best fit for the actual data and the ISLAB2000 profile which best matched the actual data based on curvature was computed for each order polynomial assumed in the polynomial curvature method.

Table 5.15 shows which order polynomial minimized the sum of the squares of the error. The best option is based on value which minimized the sum of the squares of the errors between the actual data and the ISLAB2000 profile selected based on the profilometer method.

Table 5.15

Polynomial order for which the sum of the squares of the error was minimized for full slabs.

\begin{tabular}{|c|c|c|c|c|}
\hline cell & panel & time & $\begin{array}{l}\text { best option } \\
\text { from } \\
\text { actual data and } \\
\text { ISLAB2000 }\end{array}$ & $\begin{array}{c}\text { best option } \\
\text { from } \\
\text { best fit curve of } \\
\text { actual data and } \\
\text { ISLAB2000 }\end{array}$ \\
\hline 7 & 14 & early & 2nd order & 2nd order \\
\hline 12 & 19 & early & 5th order & 2nd order \\
\hline 36 & 19 & early & $3 r d$ order & 3rd order \\
\hline 36 & 19 & late & 4th order & 2nd order \\
\hline 36 & 20 & early & 3rd order & 2nd order \\
\hline 36 & 20 & late & 2nd order & 2nd order \\
\hline 37 & 8 & early & 3rd order & 2nd order \\
\hline 37 & 8 & late & 2nd order & 2nd order \\
\hline 37 & 9 & early & 3rd order & 2nd order \\
\hline 37 & 9 & late & 2nd order & 2nd order \\
\hline 53 & 3 & early & 3 rd order & 3rd order \\
\hline 53 & 3 & late & 4th order & 3rd order \\
\hline 71 & 11 & early & 5th order & 3rd order \\
\hline 72 & 27 & early & 2nd order & 2nd order \\
\hline 213 & 15 & early & 6 th order & 2nd order \\
\hline 305 & 23 & early & 2nd order & 2nd order \\
\hline 513 & 5 & early & 2nd order & 2nd order \\
\hline 614 & 57 & early & 6 th order & 3rd order \\
\hline
\end{tabular}

As can be seen in Table 5.15, the lower order polynomials were better at minimizing the sum of the square of the error between the actual data and the ISLAB2000 profile selected via the polynomial curvature method. The same was true for minimizing the sum of the square of the error between the best fit curve of the actual data and the ISLAB2000 profile selected via the polynomial curvature method a majority of the time. 
This is summarized in Table 5.16, which shows the number of times, out of the 18 total cases considered, that using a certain order polynomial to match the actual data to the ISLAB2000 data via the polynomial curvature method minimized the sum of the squares of the errors between either the actual data and the ISLAB2000 curve, or the line of the best fit of the actual data and the ISLAB2000 curve.

Table 5.16

Frequency of a polynomial being the best option.

\begin{tabular}{|c|c|c|}
\hline polynomial & $\begin{array}{c}\text { actual data } \\
\text { and ISLAB2000 }\end{array}$ & $\begin{array}{c}\text { best fit curve of } \\
\text { actual data and } \\
\text { ISLAB2000 }\end{array}$ \\
\hline 2nd order & 7 & 13 \\
\hline 3rd order & 5 & 5 \\
\hline 4th order & 2 & 0 \\
\hline 5th order & 2 & 0 \\
\hline 6th order & 2 & 0 \\
\hline
\end{tabular}

As can be seen from Table 5.16, lower order polynomials, in general, minimized the sum of the square of the error between the ISLAB2000 profile selected via the polynomial curvature method and either the actual data or the best fit curve of the actual data. This is likely because lower order polynomials "smooth out" the imperfections in the surface profile due to tining, cracks, spalls, etc., and are more representative of the behavior of the entire slab. The ISLAB2000 model does not include distresses or discrepancies in the slab surface; therefore, approximations which produce smoother curves will match the ISLAB2000 models more accurately.

Another way the accuracy of various polynomial approximations used in the polynomial curvature method was investigated was through the analysis using on the profilometer data from the middle half of the slabs. Consistency between the results of the whole and half slab analyses shows that a particular approximation is a good fit for the data. Table 5.17 shows the number of times that the built-in curl calculated by the polynomial curvature method for the full slabs was within $4^{\circ} \mathrm{F}$ of that calculated using only data from the middle half of the slab for each order polynomial approximation used. It can be seen that the $2^{\text {nd }}, 3^{\text {rd }}$, and $6^{\text {th }}$ order approximations yielded matches most frequently. However, none of the order polynomial approximations produced at match more than five times out of 18 . One reason the $2^{\text {nd }}$ order approximation may have had the most matches between the full and half slab analyses is that the curvature is not dependent on the location along the slab for a quadratic. 
Table 5.17

Number of matches between full and half slab analsyes using the polynomial curvature method.

\begin{tabular}{|c|c|}
\hline $\begin{array}{c}\text { polynomial } \\
\text { order }\end{array}$ & $\begin{array}{c}\text { number of } \\
\text { matches }\end{array}$ \\
\hline $2^{\text {nd }}$ & 5 \\
\hline $3^{\text {rd }}$ & 4 \\
\hline $4^{\text {th }}$ & 2 \\
\hline $5^{\text {th }}$ & 1 \\
\hline $6^{\text {th }}$ & 4 \\
\hline
\end{tabular}

The lack of consistency between the full and half slab analyses shows that the polynomial curvature method is very sensitive to the data profile. The motivation behind the half slab analysis was that restraints and edge effects could distort the ends of the profile. Removing a quarter of the data on either end of the profile would remove these edge effects. However, any gross deviations from the general trend of the profile would have been removed in the data processing phase. Therefore, the half slab analysis removed extra data points which could have been used to better fit a curve, but likely did not increase accuracy from the removal of edge effects.

Since the profiler method is so sensitive to the data profile, it is important to ensure that the data is as accurate as possible. The actual profiler device used to make the measurements, the ALPS2, has a margin of error of approximately $+/-0.015 \mathrm{in}$, increasing to $+/-.025 \mathrm{in}$ at the ends of the device. This is a fairly large margin of error, given that the profiler obtains data out to the hundred thousandth of an inch. Adjacent data points often vary by only hundredths or thousandths of an inch, which within the margin of error of the ALPS2.

Another source of error was that several of the slabs in this study had surface defects or discontinuities, such as cracks, spalls, tining and diamond grinding. Cracks function as joints, and change how the pavement slab deforms. The polynomial curvature and $\Delta \mathrm{h}$ methods assume that the slab deforms symmetrically, which is not the case for cracked slabs. Additionally, the profiler measurements are for the slab surface, so any variation in the surface will be shown in the profile.

Figure 5.12 shows the transverse profile of a slab with some form of discrepancy, cell 37 , panel 8, before subtracting the cross slope. Approximately half way across the slab, the profile for each pass has a significant jump. The damage report for this cell indicates that there is not a crack in this location; however, the cell does have strips of diamond grinding, which could account for the discontinuity. 


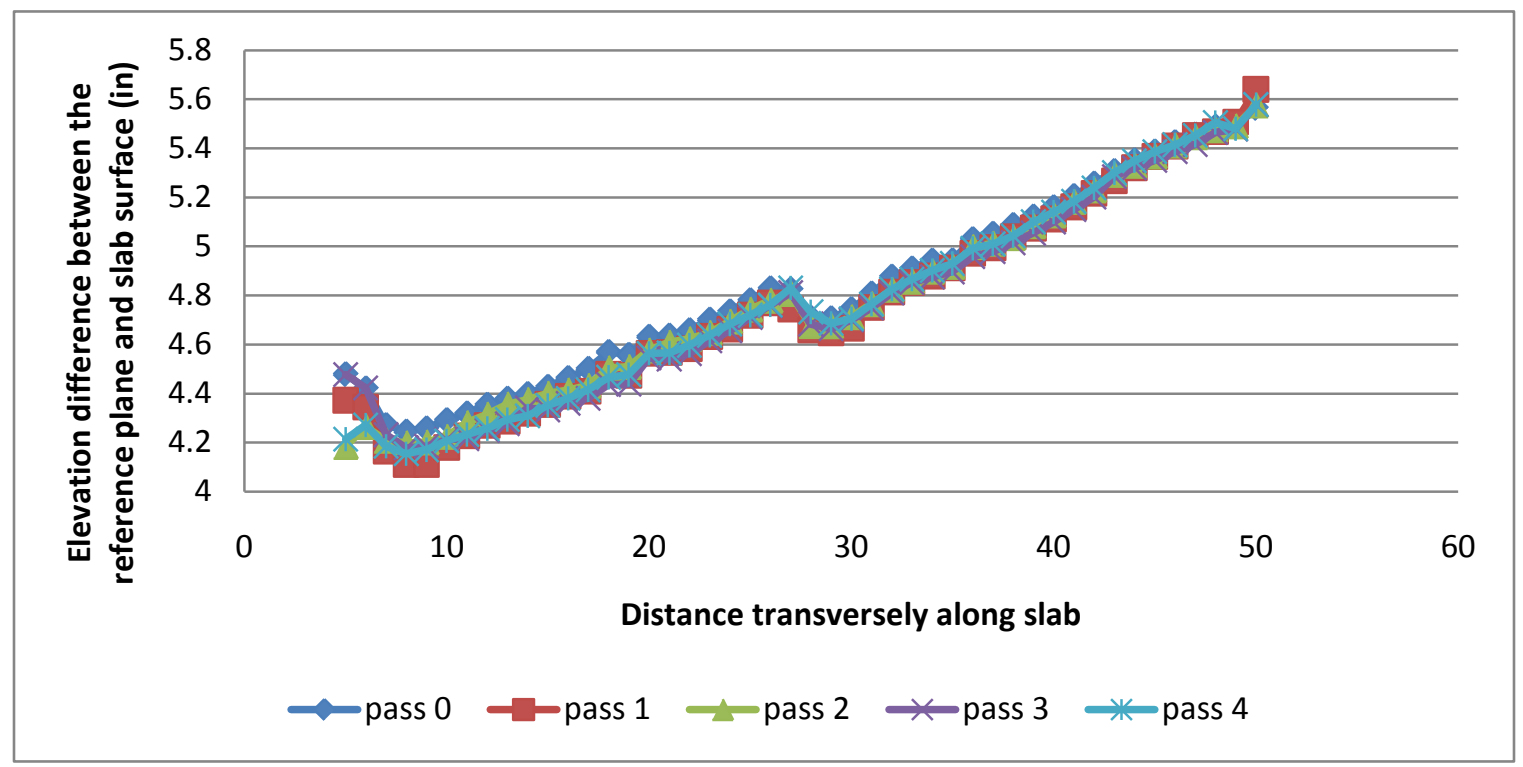

Figure 5.12: Transverse profile of cracked slab without adjusting for cross slope - cell 37, panel 8, late morning test from October testing.

Figure 5.13 shows the same set of data, cell 37 panel 8, after adjusting for the cross slope. It can be seen that this is not a smooth, bowl shaped curve, and the deformations are certainly not symmetrical. This lack of symmetry is important because the ISLAB2000 program used to generate the model slabs to which the actual data was compared does not account for cracks or other discontinuities in the slab.

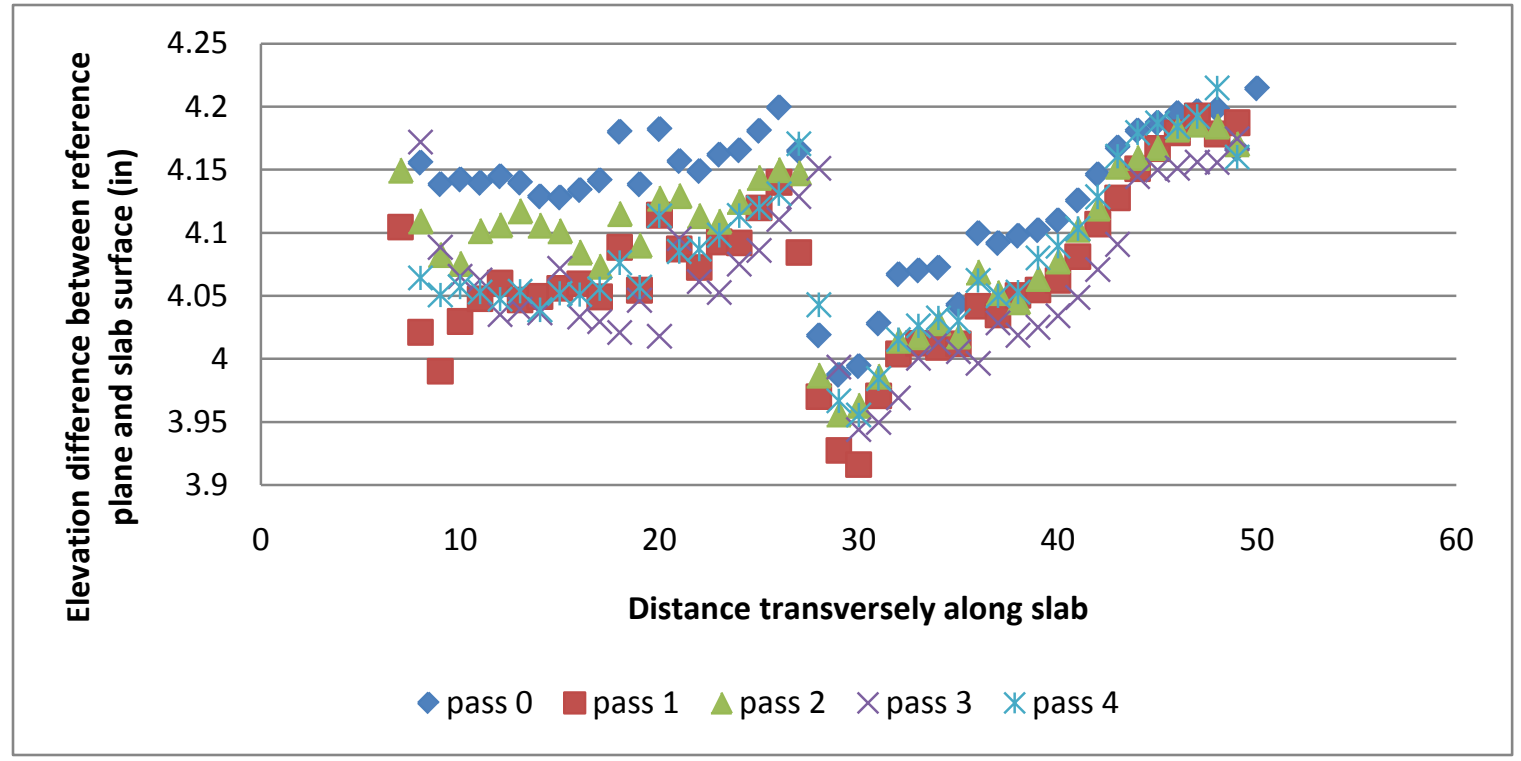

Figure 5.13: Transverse profile of cracked slab after adjusting for cross slope - cell 37, panel 8, late morning test from October testing. 
In addition to error in the data collection process, the polynomial curvature method has several sources of inherent error and ample opportunity to introduce inaccuracy through human variability. The transverse profiles from the June test varied in terms of the number of points in a pass and the number of transverse passes per slab; this made it more difficult to automate the data processing procedure. In the October tests, five transverse profiles were taken for each slab. Each profile was the average of three scans in the same location, and contained the exact same number of data points. Receiving the data in a slightly processed format, where every run had the same number of data points was very helpful to automate the processing procedure, and it is recommended that the data always be given in this format. The zero-point is selected quite subjectively by the data processor, as are the extraneous points. The choice of which points to include or exclude greatly influenced the curvature of the best-fit curve, which is used to estimate the total curl. In an effort to reduce this source of error, the same data processor selected which points should be deleted each time. Another source of error is that the FEM model curves were only run in increments of $5^{\circ} \mathrm{F}$. Using theoretical deflection curves in one or two degree increments would have increased accuracy, but was computationally too intensive to be feasible. To reduce error in the process of matching up the actual and theoretical deflection curves, a computer program was used, so that the selection criteria were consistent.

Another source of error was that the ISLAB2000 program used to create finite element models of the slabs and generate the deflected shapes for slabs subjected to various temperature gradients assumes a rectangular geometry and cannot account for skewed joints. Therefore, the ISLAB2000 profiles do not reflect the actual geometry of any slabs with skewed joints (cells 7, 36, and 37). Slabs with skewed joints will also not deform symmetrically in the transverse direction; when processing the profilometer data to find the actual profiles, an assumption of symmetrical deformation in the transverse direction was made.

\subsubsection{FWD/ANN Method}

The FWD/ANN method did not produce usable values for built-in curl for most cells tested because the total curl calculated was less negative than $-9^{\circ} \mathrm{F}$. The ANN used in this study was only trained to predict total curl which is more negative than $-9^{\circ} \mathrm{F}$. In an effort to obtain usable results, the test data from the FWD tests conducted in the morning was used because it is likely that the temperature gradient of a slab would be negative. This negative gradient coupled with a negative built-in curl gradient (which is typically found in pavement slabs) was more likely to be a large enough negative value that the ANN would be able to back estimate total curl. The tests conducted in the afternoon, when the actual temperature gradient is positive, would require a very large value of built-in curl before the ANN would be able to accurately estimate the total curl. Indeed, when analysis was conducted on the FWD test data from the afternoon, all results were found to be unusable.

Since many of the values of total curl calculated by the ANN were more positive than $9^{\circ} \mathrm{F}$, it may be concluded that those of the slabs tested have either very low negative, or no built in curl. It could also indicate that the slabs have a positive built-in curl; however, 
this is highly unlikely, as this condition is very rare for pavements paved under conditions similar to those at MnROAD (wet climate, restraint from initial construction, high negative temperature difference in concrete at final set, etc.). Values of very low or no built-in curl for other MnROAD test slabs are also consistent to those found by Vandenbossche (2003). The ANN could be used to calculate such low values for built-in curl if the actual temperature gradient were great enough such that the total value of built in curl were more negative than $-9^{\circ} \mathrm{F}$. This is likely to occur in the morning in the fall in Minnesota, and based on this recommendation additional FWD testing was conducted in the fall of 2010 .

Previous testing, which occurred in June, was not conducted in the presence of a temperature gradient sufficiently negative to produce usable data for the ANN. Cell 513 and cell 36 panel 20 were tested in June, and the ANN was not able to calculate usable results; for both cells, the ANN returned a total curl of $4^{\circ} \mathrm{F}$. The same slabs were tested in October, and found to have a total curl large enough that the ANN results can be considered valid. Subtracting out the actual temperature difference from the total curl gives very small negative values of built-in curl for both cells, which is consistent with the built-in curl generally found in MnROAD PCC pavements (Vandenbossche 2003).

Though many of the cells did not have a sufficiently negative gradient at the time of testing to obtain an accurate value of built-in curl, the theoretical lower bound on the amount of built-in curl present can be calculated. As discussed previously, the ANN will not return usable values of built-in curl if the total temperature gradient at the time of testing is less negative that $-9^{\circ} \mathrm{F}$. Recall that the total temperature gradient is the sum of the actual temperature gradient at the time of testing and the built-in gradient. By assuming that the total gradient is the largest possible value it could be, in this case $-9^{\circ} \mathrm{F}$, and subtracting the actual gradient, a theoretical lower limit on amount of built-in curl in the slab was calculated. These values are given in Table 5.18. For these calculations, only the early morning October tests were used, as these tests had temperature gradients more similar to those with which the ANN was trained. 
Table 5.18

Lower bound value of built-in curl.

\begin{tabular}{|c|c|c|c|}
\hline cell & panel & $\begin{array}{l}\Delta T \text { at time of } \\
\text { testing (deg } F \text { ) }\end{array}$ & $\begin{array}{l}\text { Lower limit of } \\
\text { built in curl } \\
\text { (deg F) }\end{array}$ \\
\hline 7 & 14 & -7.1 & -1.9 \\
\hline 12 & 19 & -9.7 & 0.7 \\
\hline 36 & 19 & -10.2 & 1.2 \\
\hline 37 & 8 & -9.1 & 0.1 \\
\hline 37 & 9 & -9.1 & 0.1 \\
\hline 53 & 3 & -15.1 & 6.1 \\
\hline 71* & 11 & -10.0 & 1.0 \\
\hline $72 *$ & 27 & -9.4 & 0.4 \\
\hline $213 *$ & 15 & -3.5 & -5.5 \\
\hline 305 & 23 & -4.2 & -4.8 \\
\hline 513* & 5 & -3.5 & -5.5 \\
\hline 614* & 57 & -3.5 & -5.5 \\
\hline
\end{tabular}

The lower bound calculated in this manner was generally a small negative or small positive value. This is consistent with the amount of built-in curl determined for other cells in the MnROAD facility (Vandenbossche 2003).

\subsubsection{Minimum Error Method}

Similar to the polynomial curvature method, the minimum error method produced quite varied results. The $5^{\text {th }}$ and $6^{\text {th }}$ order approximations predicted a mix of positive and negative values for built-in curl, while the $2^{\text {nd }}, 3^{\text {rd }}$, and $4^{\text {th }}$ order approximations were generally just negative. For all approximations, if a built-in curl was predicted to be negative, it tended to be a very large negative number.

To determine which polynomial approximation was likely to be the most accurate, the sums of the squares of the errors between the line of best fit of the data and the ISLAB2000 profile were compared. The $2^{\text {nd }}$ order polynomial was by far the best approximation in both the whole and half slab analyses, as can be seen in Table 5.19. In this table, the best option is the order polynomial of the line of best fit of the actual data for which the sum of the squares of the errors between that line of best fit and the ISLAB2000 approximation was minimized. 
Table 5.19

Polynomial order for which the sum of the squares of the error was minimized.

\begin{tabular}{|c|c|c|c|c|}
\hline cell & panel & $\begin{array}{c}\text { Time of } \\
\text { morning }\end{array}$ & $\begin{array}{c}\text { best option for } \\
\text { whole slabs }\end{array}$ & $\begin{array}{c}\text { best option for } \\
\text { half slabs }\end{array}$ \\
\hline 7 & 14 & early & 2nd order & 2nd order \\
\hline 12 & 19 & early & 2nd order & 2nd order \\
\hline 36 & 19 & early & 3rd order & 2nd order \\
\hline 36 & 19 & late & 2nd order & 2nd order \\
\hline 36 & 20 & early & 2nd order & 2nd order \\
\hline 36 & 20 & late & 2nd order & 3rd order \\
\hline 37 & 8 & early & 2nd order & 3rd order \\
\hline 37 & 8 & late & 2nd order & 2nd order \\
\hline 37 & 9 & early & 2nd order & 3rd order \\
\hline 37 & 9 & late & 2nd order & 2nd order \\
\hline 53 & 3 & early & 2nd order & 2nd order \\
\hline 53 & 3 & late & 2nd order & 2nd order \\
\hline 71 & 11 & early & 2nd order & 2nd order \\
\hline 72 & 27 & early & 2nd order & 2nd order \\
\hline 213 & 15 & early & 2nd order & 2nd order \\
\hline 305 & 23 & early & 2nd order & 3rd order \\
\hline 513 & 5 & early & 2nd order & 3rd order \\
\hline 614 & 57 & early & 2nd order & 2nd order \\
\hline
\end{tabular}

One way the accuracy of various polynomial approximations used in the minimum error was investigated was through the analysis using only the profilometer data from the middle half of the slabs. For higher order polynomial approximations, there was a general trend for the polynomial to fit the profile poorly at the edges of the slab, as seen in Figure 5.14. In this figure, the blue dots are the $4^{\text {th }}$ order approximation of the actual data for pass 2 of cell 53, early test in October. The smooth lines are the various ISLAB2000 models for the same slab exposed to various temperature gradients. As can be seen in, the approximation is very far from the ISLAB2000 model curves at the beginning and end of the slab. The impetus behind the half slab study was to eliminate these effects at the edges. 


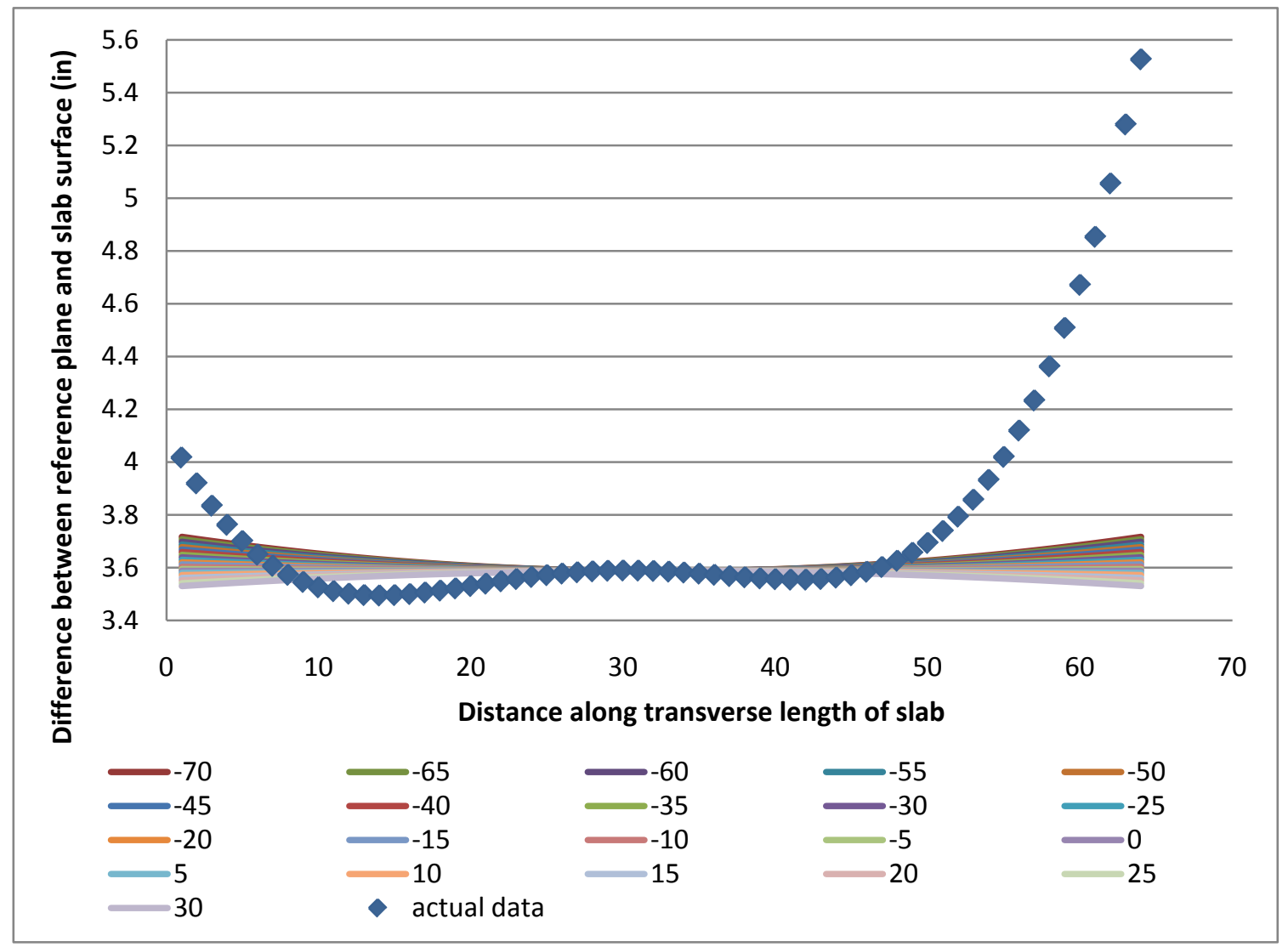

Figure 5.14: 4th order polynomial approximation pass 2 of cell 53 early test vs. ISLAB2000.

For consistency between all cases, the first and last quarter of the actual data was discarded (points 0-15, and 49-64). This effectively eliminated the edge effects, as can be seen in Figure 5.15, which shows the same $4^{\text {th }}$ order approximation for the pass in the same cell as Figure 5.14, only with the first and last quarter of the approximation discarded. It should be noted that the scale on these figures is different because the outliers were eliminated.

Though the edge effects were removed, for this case, the line of best fit is does not match the ISLAB2000 profiles well. This is eliminated by using a lower order polynomial, such as the quadratic. Figure 5.16 shows the second order approximation of the same pass in the same cell as Figure 5.14 and Figure 5.15. It can be seen in this figure that the second order approximation will match the ISLAB2000 profiles better than the $4^{\text {th }}$ order. 


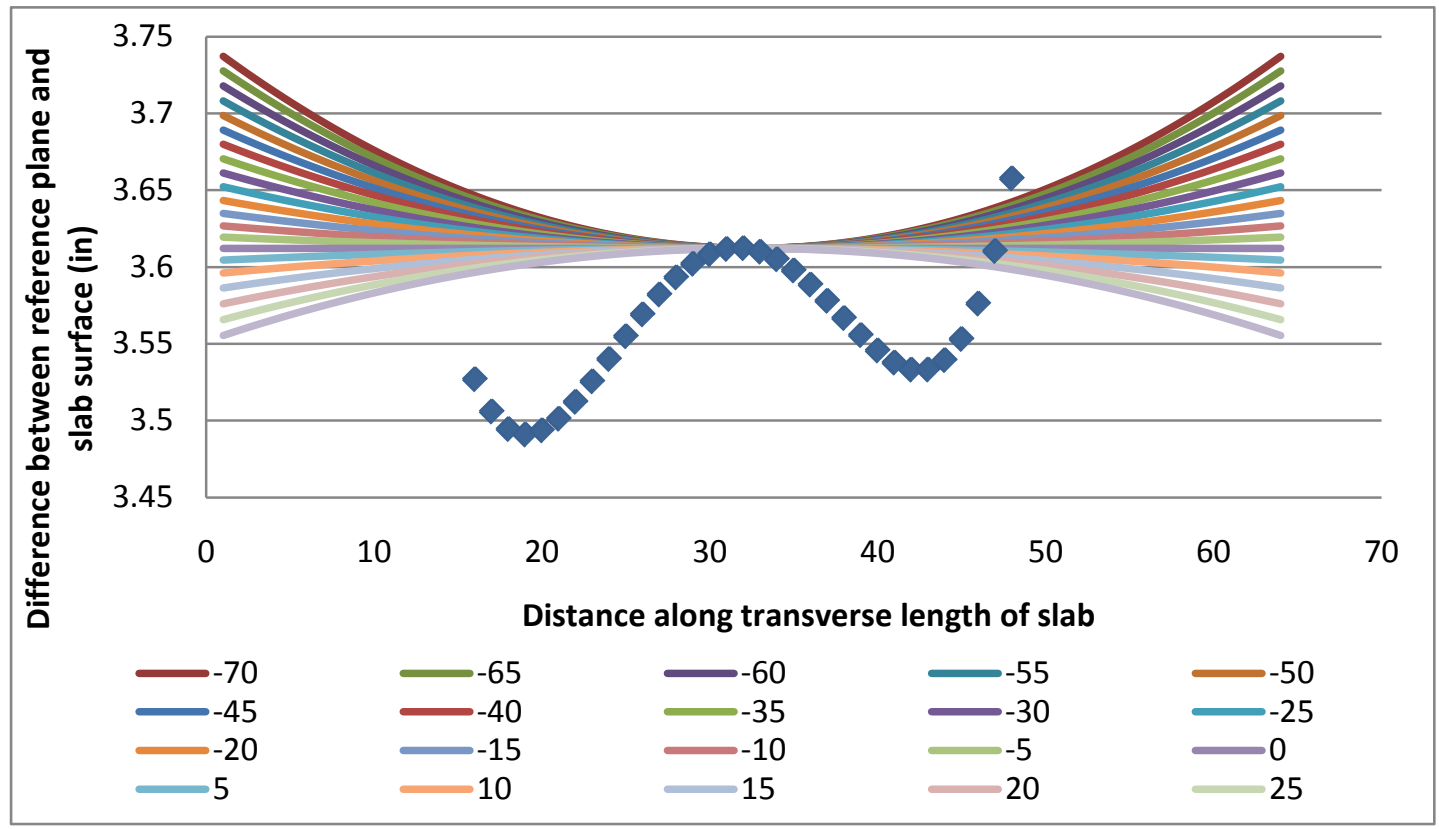

Figure 5.15: 4th order polynomil approximation pass 2 of cell 53 early test vs. ISLAB2000, discarding first and last quarter of data.

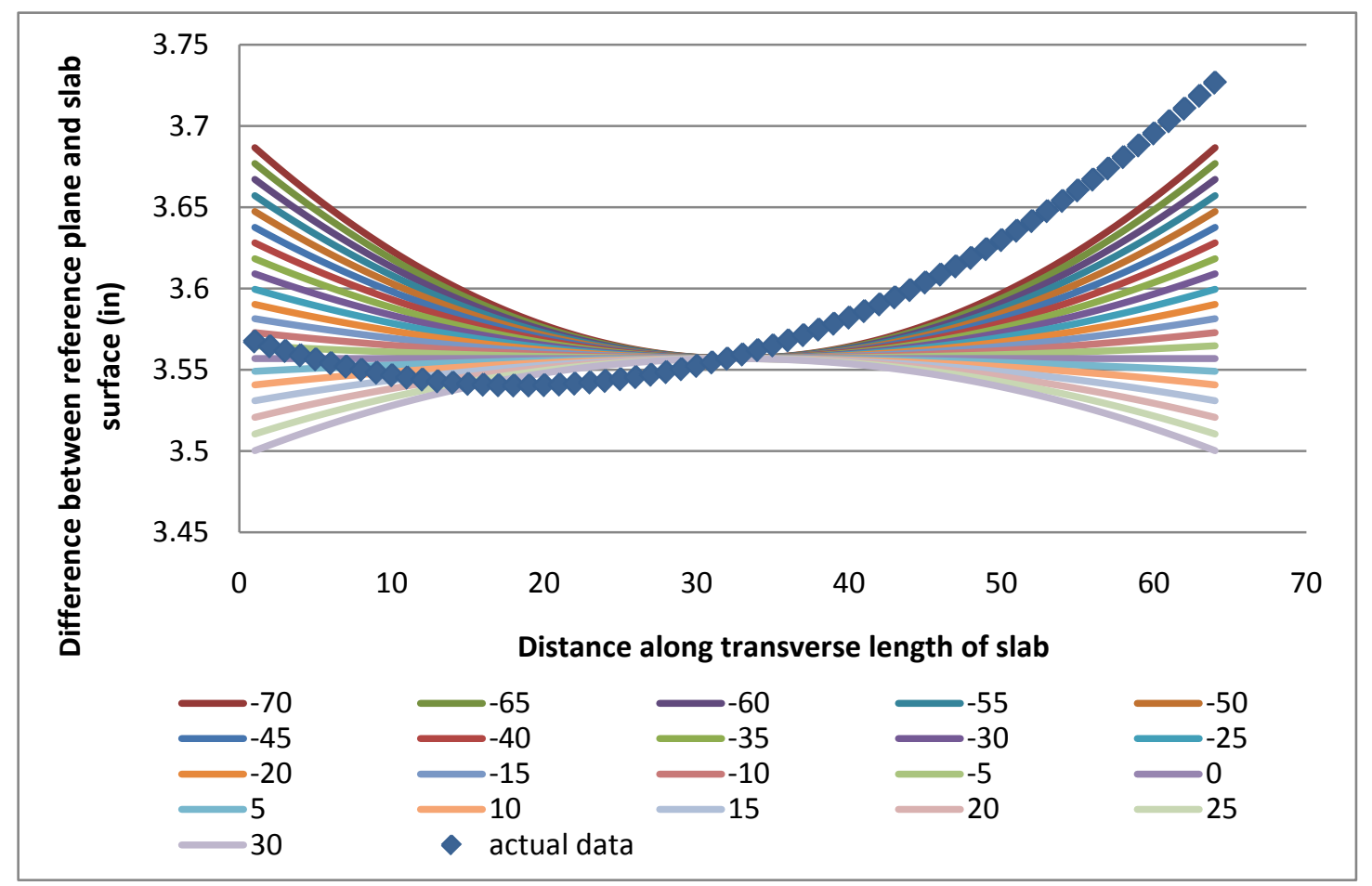

Figure 5.16: 2nd order polynomial approximation pass 2 of cell 53 early test vs. ISLAB2000.

Consistency between the results of the whole and half slab analyses shows that a particular approximation fits all of the data well, rather than just the middle portion. It 
does not mean that a particular approximation is best for calculating the built-in curl. Table 5.20 shows the number of times that the built-in curl calculated by the minimum error method for the full slabs was within $4^{\circ} \mathrm{F}$ of that calculated using only data from the middle half of the slab for each order polynomial approximation used. No one order polynomial stood out as a better option when compared to the rest, however, the $6^{\text {th }}$ order approximation had the least number of matches between the whole and half slabs.

Table 5.20

Number of matches for built-in curl between full and half slab analsyes using the minimum error method.

\begin{tabular}{|c|c|}
\hline $\begin{array}{c}\text { polynomial } \\
\text { order }\end{array}$ & $\begin{array}{c}\text { number of } \\
\text { matches }\end{array}$ \\
\hline $2^{\text {nd }}$ & 6 \\
\hline $3^{\text {rd }}$ & 8 \\
\hline $4^{\text {th }}$ & 6 \\
\hline $5^{\text {th }}$ & 8 \\
\hline $6^{\text {th }}$ & 3 \\
\hline
\end{tabular}

Compared with the number of matches between the whole and half slab analyses for the polynomial curvature method (Table 5.17), the minimum error method shows more consistency. This is likely due to the fact that the minimum error method compared the ISLAB2000 profiles to the line of best fit for the actual data, so many of the imperfections in the measured slab profile did not affect the error calculation as significantly as in the polynomial curvature method.

This was further shown by the additional test performed to determine the built-in curl based on the ISLAB2000 profile which minimized the sum of the square of the errors between the actual data and the ISLAB2000 profiles. Figure 5.17 shows the actual data (blue dots) superimposed on the 21 different ISLAB2000 models (smooth lines). 


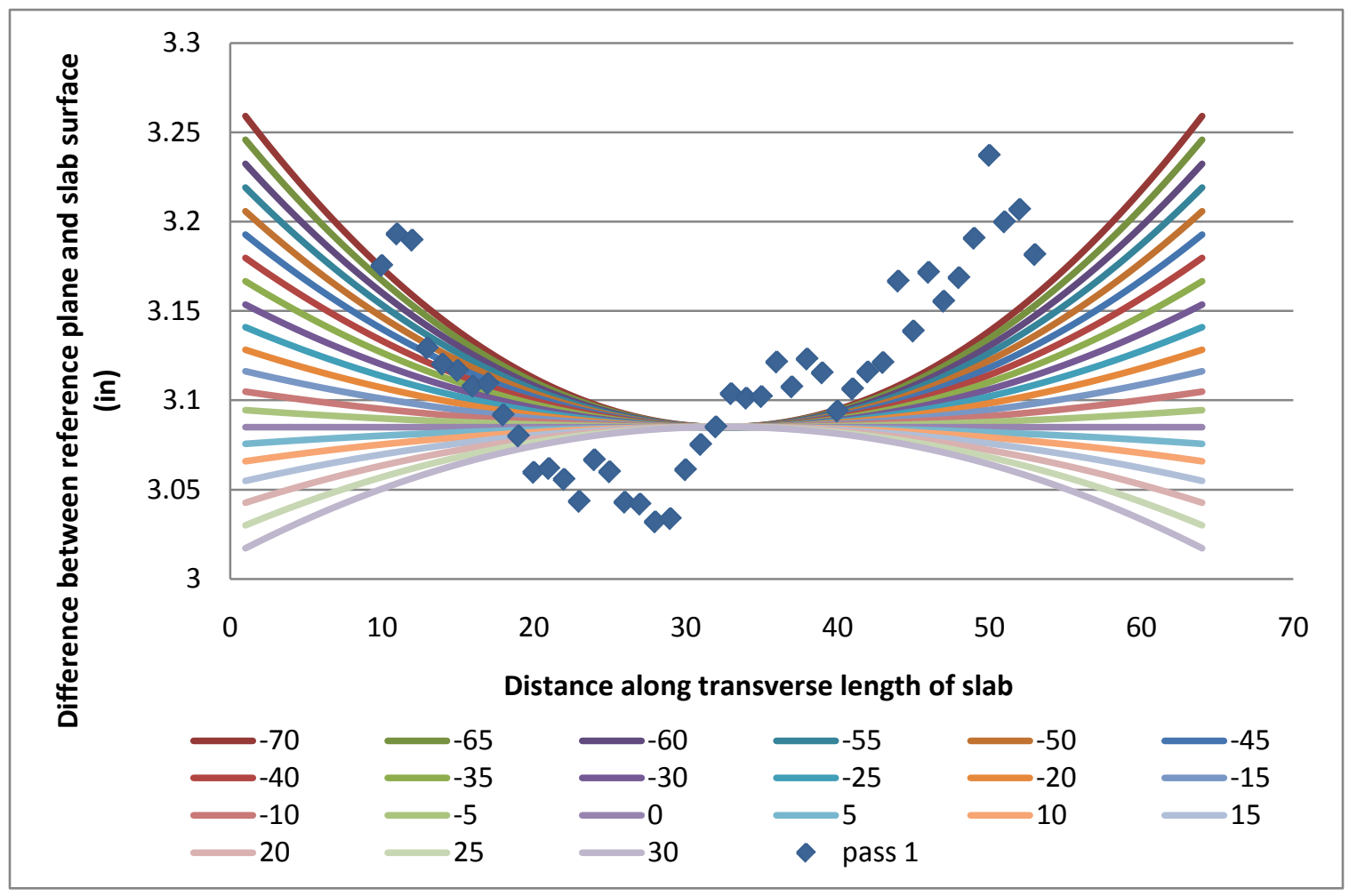

Figure 5.17: Actual data pass 1, cell 72 vs. ISLAB2000.

As can be seen from this figure, the actual data is inherently very erratic. Additionally this data incorporates surface discrepancies, such as tining and distresses, which do not affect the entire thickness of the slab. The error between the actual data and ISLAB2000 profiles, and the error between polynomial approximations and ISLAB2000 profiles were not compared directly because the data sets contained different numbers of points, which means one dataset would have inherently more error than the other. However, it was generally observed that the error per point was much lower the comparison between ISLAB2000 and the polynomial approximations than for the comparison between ISLAB2000 and the actual data. This reinforces the concept that the surface profile only captures the profile of the surface, which is not representative of the entire shape, especially if the slab is tined, or has surface defects.

The sources of error in the minimum error method were the same as with polynomial curvature method because they use same profile data, processing techniques and ISLAB2000 models. The only difference in error sources was that the polynomial curvature method depended on the curvature of the line of best fit, and the assumption that the average curvature was representative of the curvature of the slab. The minimum error method eliminated this source of error by directly comparing deformed shapes, rather than curvatures. 


\subsubsection{Comparison of Results from Different Methods}

Due to limitations in the ANN discussed above, results were only obtained for cell 305, and the cell 36 panel 20 early test. The value of built-in curl obtained for these cells by any of the profilometer methods does not match with the built-in curl obtained by the ANN. It should be noted that, as none of the profilometer methods were verified, this does not mean that the ANN results are incorrect, just that they cannot be validated. As the expected built-in curl values for this section are quite low (or near zero), this is not completely surprising.

The biggest difference between the polynomial curvature method and the minimum error method was that the polynomial curvature method matched the actual data to an ISLAB2000 model based on curvature, while the minimum error method matched the profiles based on their actual shape. For any polynomial of higher order than a quadratic, curvature is simply a mathematical parameter with no physical meaning in terms of the overall slab shape. For all order polynomials, the minimum error method retains physical meaning.

One major advantage the minimum error method had over the polynomial curvature method was that the minimum error method always produced results. Recall that for the polynomial curvature method, anytime the difference between the curvature of the line of best fit of the actual data and the curvature of the ISLAB2000 profiles was outside of the tolerance of the automated matching program, a value of "N/A" was returned. This was not an issue in the minimum error method because profiles were matched based on the minimum amount of error, rather than on a similarity between two sets of data. However, it should be noted that even though the minimum error method can consistently produce results, this does not mean those results are any more accurate than those from the polynomial curvature method.

A comparison of the results of the polynomial curvature method and the minimum error method showed that the value of built-in curl predicted using the $2^{\text {nd }}$ order polynomial approximation are very consistent, particularly for the full slab analysis, as can be seen in Table 5.21. 
Table 5.21

Built-in curl calculated using a second order polynomial approximation in both the polynomial curvature and minimum error methods.

\begin{tabular}{|c|c|c|c|c|c|}
\hline cell & panel & time & $\begin{array}{c}\text { Built-in Curl }\left({ }^{\circ} \mathrm{F}\right) \\
\text { from the } \\
\text { minimum error } \\
\text { method }\end{array}$ & $\begin{array}{c}\text { Built-in Curl } \\
\left({ }^{\circ} \mathrm{F}\right) \text { from the } \\
\text { polynomial } \\
\text { curvature } \\
\text { method }\end{array}$ & $\begin{array}{c}\text { percent } \\
\text { difference }\end{array}$ \\
\hline 7 & 14 & early & -6.96 & -9.21 & $28 \%$ \\
\hline 12 & 19 & early & -56.57 & -56.07 & $-1 \%$ \\
\hline 36 & 19 & early & -51.05 & -50.8 & $0 \%$ \\
\hline 36 & 19 & late & -56.12 & -54.12 & $-4 \%$ \\
\hline 36 & 20 & early & -47.3 & -43.8 & $-8 \%$ \\
\hline 36 & 20 & late & -76.12 & -76.12 & $0 \%$ \\
\hline 37 & 8 & early & -59.57 & -58.57 & $-2 \%$ \\
\hline 37 & 8 & late & -79.75 & -79.75 & $0 \%$ \\
\hline 37 & 9 & early & -59.57 & -54.57 & $-9 \%$ \\
\hline 37 & 9 & late & -79.75 & -79.75 & $0 \%$ \\
\hline 53 & 3 & early & -4.88 & \#N/A & $\mathrm{N} / \mathrm{A}$ \\
\hline 53 & 3 & late & -34.53 & \#N/A & $\mathrm{N} / \mathrm{A}$ \\
\hline 71 & 11 & early & -59.71 & -59.71 & $0 \%$ \\
\hline 72 & 27 & early & -60.01 & -60.01 & $0 \%$ \\
\hline 213 & 15 & early & -66.22 & -66.22 & $0 \%$ \\
\hline 305 & 23 & early & -65.57 & -63.71 & $-3 \%$ \\
\hline 513 & 5 & early & -61.22 & -66.22 & $8 \%$ \\
\hline 614 & 57 & early & -37.47 & -60.22 & $47 \%$ \\
\hline & & & & & \\
\hline
\end{tabular}

\subsection{Conclusions}

In this study, cells of PCC pavements at MnROAD were profiled using an ALPS2 profilometer and tested with an FWD. The profile data was plotted and best-fitted with various order polynomial curves in Excel. ISLAB2000 was used to generate similar profiles for a theoretically flat slab of the same composition, subjected to different temperature gradients, and similar equations of best-fit were found. The differential deflection between the middle and edge of the slab profiles were also found for both the actual and theoretical slabs. The curvature and differential deflections of the profiles were compared to find the temperature gradient required to produce the same shape in the theoretical profile as that of the actual slab; this temperature gradient is called the total temperature gradient. The total temperature gradient required to deform the theoretical slab to the actual slab was also determined by finding the theoretical slab profile required to minimize the sum of the squares of the errors between the actual the theoretical slabs. The actual temperature gradient at the time of testing was subtracted from the 
temperature gradient to find the built-in curl. Data obtained from the FWD tests were run through an ANN to backcalculate the built-in curl of the same slabs.

The results obtained from all of the methods tested were compared and the following conclusions were drawn:

- FWD testing must be performed when a large negative total gradient is present to use the current ANN as the sensitivity to corner deflections is minimal at built-in curl level greater than $-9^{\circ} \mathrm{F}$ (close to zero).

- Based on the graphs of the profiles, all cells tested have negative built-in temperature gradients.

- A second order polynomial is the easiest to work with when fitting profiles because the curvature is a constant value. The quadratic equation also gives a physical representation of the shape of the slab.

- A higher order polynomial has no physical meaning, but is merely a line which is closest to the most points in the profile. However, profile data has a large amount of small variation due to cracks and spalls, surface texture, etc, and therefore matching the largest amount of points does not guarantee a more accurate representation of the shape of the slab.

- The second and third order polynomials produced the most realistic values of built-in curl.

- The results from both the polynomial curvature and minimum error methods for the second order polynomial approximation matched fairly well.

- A fifth order polynomial gave the least realistic values of built-in curl and was the most likely to not return a value of built-in curl.

Due to the fact that exact values of curl in the slabs tested are not known, no one method can be declared more accurate than the others. Further testing on slabs of known built-in curl will be required before such a determination can be made. 


\section{Conclusions and Future Work}

The goal of this thesis was to improve the ability of a pavement designer to account for the effects of differential volume change in the design of jointed plain concrete pavements. This was accomplished through better characterization of the amount of reversible shrinkage, the development of new warping and differential drying shrinkage models, and design aids to predict warping throughout the United States, and an evaluation of different methods for quantifying the amount of built-in curl in standard pavements. While these do allow an engineer to better account for the effects of differential volume change, there are still many improvements which can be made.

\subsection{Conclusions}

From the research conducted for this thesis, the following conclusions were reached:

- Reversible shrinkage occurs when concrete is subjected to drying and re-wetting cycles. The amount of ultimate shrinkage which is reversible is highly dependent on the initial curing regime and the number of drying and re-wetting cycles to which the concrete has been subjected. These finding matched those of L'Hermite et al. (1949) for concrete made from virgin aggregates. This study found that concrete made from lightweight and recycled concrete aggregates had similar behavior to concrete made from virgin aggregate when exposed to cyclic drying and re-wetting. However, the magnitude of the reversible shrinkage was different between concrete made with virgin aggregates and lightweight or recycled aggregates.

- Changes in length were found to be proportional to changes in weight, confirming a classic assumption about volume change. The slope of the line for the change in length over change in weight was found to be dependent on the initial curing regime. A decrease in weight is associated with shrinkage (negative strain), while an increase in weight is associate with growth (positive strain). The ratio of growth over weight gain was not found to be equal to the ratio of shrinkage over weight loss.

- Forty to $70 \%$ of the total reversible shrinkage was recovered within the first 24 hours of a re-wetting cycle. This is important in the realm of pavement design because pavements are generally not exposed to the 30 days of continuous wetting used as an experimental condition. A rain event, however, could reasonably cause 24 hours of wetting, which this study shows is enough for a significant amount of shrinkage to reverse.

- The amount of reversible shrinkage from the first re-wetting cycle for dry cured samples was dependent on the permeability of the concrete. More permeable concrete had higher reversible shrinkage. If there were a relationship between the reversible shrinkage and a parameter which can be easily measured, such as permeability, then a program like the MEPDG would be able to better predict the amount of reversible shrinkage expected. 
- For dry cured samples, the amount of reversible shrinkage due to subsequent drying and re-wetting cycles was approximately 30\%, regardless of the type of aggregate used, or the permeability of the concrete. The behavior of the dry cured samples after several drying and re-wetting cycles is most representative of how a pavement would act when exposed to long-term ambient conditions.

- The amount of reversible shrinkage assumed to contribute to warping should be based on the amount of reversible shrinkage from cycles after the first cycle, because warping is a long-term behavior. Therefore, the reversible shrinkage factor, $\phi$, should be equal to 0.3 . This means that $30 \%$ of drying shrinkage will cause warping, and the rest will cause differential drying shrinkage.

- The amounts of warping and differential drying shrinkage in a pavement slab vary by location around the country due to climatic differences. Failure to properly account for moisture warping and differential drying shrinkage can cause the total equivalent temperature difference to be under-predicted, especially in dry climates. The design aids developed as part of this research will assist design engineers in determining the amount of warping and differential drying shrinkage which can be assumed for different regions of the United States.

- The current MEPDG warping model does not properly compute the amount of moisture warping due to errors in the derivation and poor assumptions. The proposed warping model (Equation 4.34) corrects these deficiencies. With slight modification, this model can also be used to predict differential drying shrinkage (Equation 4.35), which is currently not modeled in the MEPDG.

- Using a non-linear approximation based on the arc formed by a quarter of an ellipse to model drying shrinkage provides a better fit to model shrinkage through a slab than a linear approximation. Proper determination of the depth of the shrinkage was found to be critical for obtaining a good fit between shrinkage and the non-linear elliptical approximation.

- Quantifying built-in curl with FWD testing requires an appropriately trained ANN. If such a tool is available, using FWD data pared with an ANN drastically reduces computational time and effort when compared with methods involving profilometer data and finite element models. The FWD/ANN method is also much more automated, which greatly reduces the potential for human error.

- When using a profilometer pared with finite element models to determine built-in curl, a second order polynomial approximation produces the most realistic results, is the simplest approximation to work with, and is the only approximation which is representative of physical phenomena. 


\subsection{Future Work}

Throughout the research performed in this thesis, areas were identified where future study would be warranted. These areas were outside the scope of the current research, and would require extensive study and/or testing to fully investigate. Suggestions for future work include the following:

- The reversible shrinkage study should be expanded to include more aggregate types and water to cement ratios. Using different water to cement ratios could potentially circumvent the issue of paste properties dictating the permeability of the concrete, and could allow a correlation to be found between the porosity of the aggregate and reversible shrinkage. Testing with more aggregate types would expand knowledge of the relationship between reversible shrinkage and aggregate type. Additionally, the number of samples in the study should be expanded such that statistically significant conclusions can be drawn.

- It was theorized that the reason there was such a decrease in the amount of reversible shrinkage from the first re-wetting of the dry cured samples compared to than that of subsequent re-wetting cycles was due to the increase in hydration upon re-wetting. A study should be conducted on the degree of hydration of the concrete at each phase in the reversible shrinkage experiment to test this theory.

- The reversible shrinkage in this study was investigated under laboratory conditions, where the relative humidity was held constant for 30-45 days for each instance of drying and re-wetting. While this was necessary for investigation of behavior from a materials perspective, it is not representative of the conditions to which an in-service pavement would be exposed. To obtain a better estimate of the reversible shrinkage factor which should be used in design, the reversible shrinkage testing should be repeated with drying and re-wetting cycles whose duration is representative of field conditions. The need for such a study is bolstered by the finding that a large percentage of reversible shrinkage occurs within the first 24 hours of re-wetting.

- The MEPDG defines ultimate shrinkage as the ultimate shrinkage achieved when the concrete is dried at $40 \%$ relative humidity. Both AASHTO and ASTM procedures involve drying the concrete at $50 \%$ relative humidity, and this condition was used in the determination of $\phi$. Further testing should be conducted at both 40 and $50 \%$ relative humidity to determine how $\phi$ changes. If the change is significant, it should be determined which value of relative humidity should be used when measuring the ultimate shrinkage for use in pavement design.

- For the phenomena of both differential drying shrinkage and moisture warping, drying shrinkage, not ultimate shrinkage, is responsible for differential volume change. When the reversible shrinkage factor is calculated to distribute the drying shrinkage between warping and differential drying shrinkage, it should be 
calculated based only on the amount of drying shrinkage which is reversible. However, in the laboratory, it is only possible to measure the amount of ultimate shrinkage which is reversible. A future study should devise a method whereby only the reversible portion of drying shrinkage, not reversible shrinkage, can be measured.

- The ultimate shrinkage, as determined by the ASTM C157 test, is an average shrinkage value for the prism at the middle of the cross section of the sample. In reality, the edges of the prism will experience more shrinkage than the middle. When determining the shrinkage gradient in a pavement, the topmost fiber of the slab will experience the most shrinkage. The shrinkage in the topmost fiber will be akin to the shrinkage at the edges of the prism. The value of this shrinkage is the true ultimate shrinkage of the concrete, and should be used for computing the drying shrinkage which causes moisture warping and differential drying shrinkage. A method should be devised to measure this value, as the current ASTM C157 test cannot do this.

- The factor $\omega$ was developed to remove the contribution of autogenous shrinkage from the ultimate shrinkage, so that only drying shrinkage would be considered in the warping and differential drying shrinkage models. The empirical determination of $\omega$ was based solely on tests of normal concrete. Given that other terms in the warping and differential drying shrinkage models are applicable to concrete made with standard, lightweight and recycled aggregates, $\omega$ should be determined for those cases as well. It is possible that $\omega$ could be dependent on both the water to cement ratio of the concrete and aggregate properties, such as porosity. Because there is a lack of available data on the amount of autogenous versus drying shrinkage for these types of aggregates, this would likely involve more testing.

- The depth of the shrinkage zone is not always two inches (Kim and Lee 1999; Qin 2011), as is often assumed. Further research should be conducted to determine the mechanisms which dictate the depth of the shrinkage zone and whether or not an equation can be developed to predict it. Also, if the expected depth of the shrinkage zone varies with ambient conditions, the possibility of design aids showing expected regional variation should be explored.

- The proposed warping and differential drying shrinkage models assume that shrinkage is constant from the bottom of the shrinkage zone to the bottom of the pavement, and therefore do not contribute to slab bending. In reality, the majority of the shrinkage is confined to the shrinkage zone, but there is still a small amount of shrinkage variation with depth between the bottom of the shrinkage zone and the bottom of the slab. The proposed models could be expanded to account for this variation. To do so, however, would involve predicting the amount of shrinkage at the bottom of the shrinkage zone and at the bottom of the slab. 
- Differential drying shrinkage is considered constant throughout the life of the pavement in the proposed model. This is not the case, particularly at early ages. A future study could investigate the variation of differential drying shrinkage with time, in order to better account for its contribution to differential volume change.

- Currently, the MEPDG does not compute differential drying shrinkage, instead, it is lumped into the BIC term, along with construction curl and creep effects. If a model were developed to predict the construction curl, it could be pared with the proposed differential drying shrinkage models, and one of several previously developed creep prediction model to determine the estimated built-in curl. 


\section{References}

AASHTO. 1993. Guide for Design of Pavement Structures. Washington DC: American Association of State Highway and Transportation Officials.

ACI. 2008. ACI 209R-92: Prediction of Creep, Shrinkage, and Temperature Effects in Concrete Structures. Farmington Hills: American Concrete Institute.

Al-Manaseer A, Lam J-P. 2005. Statistical Evaluation of Shrinkage and Creep Models. ACI Materials Journal 102(3):170-176.

Altoubat SA, Lange DA. 2001. Creep, Shrinkage, and Cracking of Restrained Concrete at Early Age. ACI Materials Journal 98(4):323-331.

Armaghani JM, Larsen TJ, Smith LL. 1987. Temperature Response of Concrete Pavements. Transportation Research Record(1121):23-33.

ASTM. 2008a. ASTM C78 Standard Test Method for Flexural Strength of Concrete (Using Simple Beam with Third-Point Loading). West Conshohocken, PA: American Society for Testing and Materials.

ASTM. 2008b. ASTM C469 Standard Test Method for Static Modulus of Elasticity and Poisson's Ratio of Concrete in Compression. West Conshohocken, PA: American Society for Testing and Materials.

ASTM. 2008c. ASTM C39 Test Method for Compressive Strength of Cylindrical Concrete Specimens. West Conshohocken, PA: American Society for Testing and Materials.

ASTM. 2008d. ASTM C231 Test Method for Air Content of Freshly Mixed Concrete by the Pressure Method. West Conshohocken, PA: American Society for Testing and Materials.

ASTM. 2008e. ASTM C157 Standard Test Method for Length Change of Hardened Hydaurlic-Cement Mortar and Concrete. West Conshohocken, PA: American Society for Testing and Materials.

ASTM. 2008f. ASTM C1202 Standard Test Method for Electrical Indication of Concrete's Ability to Resist Chloride Ion Penetration. West Conshohocken, PA: American Society for Testing and Materials.

Baroghel-Bouny V, Godin J. 2000. Experimental Study on Drying Shrinkage of Ordinary and High-Performance Cementitious Materials. In: Baroghel-Bouny V, Aitcin P$\mathrm{C}$, editors. International RILEM Workshop on Shrinkage of Concrete. Bagneux, France.: RILEM. 
Baroghel-Bouny V, Mounanga P. 2005. Effects of Self-Desiccation on Autogenous Deformations, Microstructure and Long-term Hygral Behaviour. In: Persson BSM, Bentz DP, Nilsson L-O, editors. Self-Desiccation and its Importance in Concrete Technology. . Lund, Sweden: Lund Institute of Technology.

Bazant ZP, Najjar LJ. 1972. Nonlinear Water Diffusion in Nonsaturated Concrete. Materials and Structurs/Materiaux et Constructions 5(1):3-20.

Bazant ZP, Baweja S. 1995a. Justification and Refinements of Model B3 for Concrete Creep and Shrinkage: 2. Updating and Theoretical Basis. Materials and Structurs/Materiaux et Constructions 28(8):488-495.

Bazant ZP, Baweja S. 1995b. Justification and Refinements of Model B3 for Concrete Creep and Shrinkage: 1. Statistics and Sensitivity. Materials and Structurs/Materiaux et Constructions 28(7):415-430.

Bazant ZP, Baweja S. 1995c. Creep and Shrinkage Prediction Model for Analysis and Design of Concrete Structures - Model B3. Materials and Structurs/Materiaux et Constructions 28(6):357-365.

Bazant ZP, Li G-H. 2008. Unbiased Statistical Comparison of Creep and Shrinkage Prediction Models. ACI Materials Journal 105(6):610-621.

Beckemeyer CA, Khazanovich L, Yu HT. 2002. Determining the Amount of Built-in Curling in JPCP: A Case Study of Pennsylvania I-80. Transportation Research Board Annual Meeting. Washington, D.C.

Bendana LJ, Sargand SM, Khoury I, Selle R. 2003. Enviornmental Influence of Early Age Response of PCC Pavement. Transportation Research Record.

Bissonnette B, Attiogbe EK, Miltenberger MA, Fortin C. 2007. Drying Shrinkage, Curling, and Joint Opening of Slabs-on-Ground. ACI Materials Journal 104(3):259-267.

Bradbury RD. 1938. Reinforced Concrete Pavements. Washington D.C.: Wire Reinforcement Institute.

Byrum CR. 2000. Analysis by High Speed Profile of Jointed Concrete Pavement Slab Curvatures. Transportation Research Record 1730:1-9.

Byrum CR. 2001. A High Speed Profiler Based Slab Curvature Index for Jointed Concrete Pavement Curling and Warping Analysis. [Ann Arbor]: University of Michigan.

Choubane B, Tia M. 1992. Nonlinear Temperature Gradient Effects on Maximum Warping Stresses in Rigid Pavements. Transportation Research Record 1370:1119. 
Choubane B, Tia M. 1995. Analysis and Verification of Thermal-Gradient Effects on Concrete Pavement. Journal of Transportation Engineering 121(1):75-81.

Daly C, Taylor G. United States Average Monthly or Annual Relative Humidity, 1961-90 [Internet]. 2001. Corvallis, OR: Spatial Climate Analysis Service at Oregon State University. Available from: http://www.climatesource.com/us/fact_sheets/meta_rh_us.html

Davids WG, Turkiyyah GM, Mahoney J. 1998. EverFE - Rigid Pavement Finite Element Analysis Tool. Transportation Research Record(1629):41-49.

Dere Y, Asgari A, Sotelino ED, Archer GC. 2006. Failure Prediction of Skewed Jointed Plain Concrete Pavements Using 3D FE Analysis. Engineering Failure Analysis 13(6):898-913.

Domingo A, Lazaro C, Gayarre FL, Serrano MA, Lopez-Colina C. 2010. Long Term Deformations by Creep and Shrinkage in Recycled Aggregate Concrete. Materials and Structurs/Materiaux et Constructions 43:1147-1160.

Dutron R. 1934. Le Retrait des Ciments, Mortiers, et Betons. Annales des Travaux Publics de Belgique 35(3):347-429.

Eisenmann J, Leykauf G. 1990a. Effect of Paving Temperature on Pavement Performance. 2nd International Workshop on the Theoretical Design of Concrete Pavements. Siguenza, Spain. p. 419-428.

Eisenmann J, Leykauf G. 1990b. Simplified Calculation Method of Slab Curling Caused by Surface Shrinkage. Second International Workshop on the Theoretical Design of Concrete Pavements. Madrid: 6th International Symposium on Concrete Roads.

Etxeberria M, Vaquez E, Mari A. 2006. Microstructure Analysis of Hardened Recycled Aggregate Concrete. Magazine of Concrete Research 58(10):683-690.

Fabrizzio MA. 1998. Field Investigation of Transverse Cracking in Jointed Concrete Pavements [Thesis]. [East Lansing]: Michigan State University.

Feldman RF, Swenson EG. 1975. Volume Change on First Drying of Hydrated Portland Cement with and without Admixtures. Cement and Concrete Research 5:23-35.

FHWA. 2009. Long Term Pavement Performance Database. Federal Highway Administration.

FIB. 2008. Constitutive Modelling of High Strength/High Performance Concrete. Lausanne: Federation International du Beton. 
Gardner NJ, Lockman MJ. 2001. Design Provisions for Drying Shrinkage and Creep of Normal Strength Concrete. ACI Materials Journal 98(2):159-167.

Granger L, Torrenti J-M, Diruy M. 1994. Simulation Numerique du Retrait du Beton Sous Hygrometrie Variable. Bulletin de Liaison de Laboratoires des Ponts et Chaussees 190(3881):57-64.

Guo EH. 2001. Back-Estimation of Slab Curling and Joint Stiffness. 7th International Conference on Concrete Pavements. Orlando, FL.

Hansen W, Wei Y, Smiley DL, Peng Y, Jensen EA. 2006. Effects of Paving Conditions on Built-in Curling and Pavement Performance. International Journal of Pavement Engineering 7(4):291-296.

Harr ME, Leonards GA. 1959. Warping Stresses and Deflections in Concrete Pavements. Transportation Research Record 38:286-320.

Heath AC, Roesler JR, Harvey JT. 2001. Quantifying Longitudinal, Corner and Transverse Cracking in Jointed Concrete Pavements. Transportation Research Board Annual Meeting. Washington, D.C.

Helmuth RA, Turk DH. 1967. The Reversible and Irreversible Drying Shrinkage of Hardened Portland Cement and Tricalcium Silicate Pastes. Research and Development Laboratories of the Portland Cement Association.

Hiller E, Springenschmid R, Fleischer W. The influence of the Curing Method on Early Cracking Risk During Hot Weather Paving. 9th International Symposium on Concrete Roads; 2004; Istanbul, Turkey.

Hiller JE, Roesler JR. 2010. Simplifying Non-linear Temperature Distribution for Concrete Pavement Analysis. Journal of Transportation Engineering 131(8):654663.

Hiller JE, Deshpande YS, Qin YH, Shorkey CJ. 2011. Efficient Use of Recycled Concrete in Transportation Infrastructure. Lansing: Michigan Department of Transportation.

Holt EE. 2001. Early Age Autogenous Shrinkage. Technical Research Center of Finland.

Hveem FN. 1951. Slab Warping Affects Pavement Joint Performance. Journal of the American Concrete Institute 22(10):797-808.

Hveem FN, Bailey T. 1957. Some Factors Influencing Shrinkage of Concrete Pavements. Journal of the American Concrete Institute 53(2):781-789. 
Ioannides AM, Salsilli-Murua RA. 1989. Temperature Curling in Rigid Pavements: An Application of Dimensional Analysis. Transportation Research Record(1227):111.

Ioannides AM, Alexander DR, Hammons MI, Davis CM. 1996. Application of Artificial Neural Networks to Concrete Pavement Joint Evaluation. Transportation Research Record(1540):56-64.

Ioannides AM, Khazanovich L. 1998. General Formulation for Multilayered Pavement Systems. Journal of Transportation Engineering 124(1):82-90.

Ioannides AM, Davis CM, Weber CM. 1999. Westergaard Curling Solution Reconsidered. Transportation Research Record(1684):61-70.

Jahangirnejad S, Bush N, Kravchenko A. 2009. Evaluation of Coefficient of Thermal Expansion Test Protocol and Its Impact on Jointed Concrete Pavement Performance. ACI Materials Journal 106(1):64-71.

Janssen DJ. 1987. Moisture in Portland Cement Concrete. Transportation Research Record(1121):40-44.

Jeong J-H, Zollinger DG. 2004. Insights on Early-Age Curling and Warping Behavior from a Fully Instrumented Test Slab System. Transportation Research Board Annual Meeting. Washington, D.C.

Johnson A, Clyne TR, Worel BJ. 2008. 2008 MnROAD Phase II Construction Report.

Khazanovich L. 1994. Structural Analysis of Multi-Layered Concrete Pavement Systems. [Urbana]: University of Illinois Urbana-Champaign.

Khazanovich L, McPeak TJ, Tayabji SD. 2000a. LTPP Rigid Pavement FWD Deflection Analysis and Backcalculation Procedure. In: Tayabji SD, Lukanen EO, editors. Nondestructive Testing of Pavements and Backcalculation of Moduli: Third Volume, ASTM STP 1375. West Conshohocken, PA: American Society for Testing and Materials.

Khazanovich L, Yu HT, Rao S, Galasova K, Shats E, Jones R. 2000b. ISLAB2000 Finite Element Analysis Program for Rigid and Composite Pavements. User's Guide. Champaign, Ill.: ERES Consultants.

Kim J-K, Lee C-S. 1999. Moisture Diffusion of Concrete Considering Self-Desiccation at Early Ages. Cement and Concrete Research 29:1921-1927.

Kosmatka SH, Kerkhoff B, Panarese WC. 2002. Design and Control of Concrete Mixtures. Skokie: Portland Cement Association. 
L'Hermite R. 1947. Le Retrait des Ciments, Mortiers et Beton. Laboratoires du Batiment de des Travaux Publics.

L'Hermite R, Chefdeville J, Grieu JJ. 1949. Memoires sur la Mechanique-Physique du Beton: Nouvelle Contribution a L'Etude du Retrait des Ciments. Liants Hydrauliques, Annales de L'Institut Technique du Batiment et des Travaux Publics 106(5):2-28.

L'Hermite R, Grieu JJ. 1952. Memoires sur la Mecanique-Physique du Beton: Etude Experimentales Recentes sur le Retrait des Ciments et des Betons. Liants Hydrauliques, Annales de L'Institut Technique du Batiment et des Travaux Publics 6(52):492-514.

L'Hermite R. Volume Changes of Concrete. 4th International Symposium on the Chemistry of Cement; 1960; Washington DC. p. 659-694.

Larson G, Dempsey BJ. 1997. Enhanced Integrated Climatic Model 2.0 ed. Urbana University of Illinois - Urbana Champaign.

Lawler J. 2007. Guildelines for Concrete Mixtures Containing Supplemenatry Cementitious Materials to Enhance Durability of Bridge Decks. Washington DC: Transportation Research Borad.

Lee CJ, Lange DA, Liu Y-S. 2011. Prediction of Moisture Curling of Concrete Slabs. Materials and Structurs/Materiaux et Constructions 44(4):787-803.

Lothschutz RW. 2009. Back-Calculation of Effective Built-In Temperature Difference in Jointed Plain Concrete Pavement. [Houghton]: Michigan Technological University.

Lothschutz RW, Lederle RE, Hiller JE. 2011. Sensitivity of Built-in Curl on Predicted Fatigue Cracking Performance in Jointed Plain Concrete Pavements. Presented at Transportation Research Board. Washington DC: Transportation Research Board.

MDOT. 2003. Michigan Department of Transportation Standard Specification for Construction. Lansing: MDOT.

Mindness S, Young JF, Darwin D. 2003. Concrete. Upper Saddle River: Prentice Hall Pearson Education

Miyazawa S, Tazawa E. 2000. Influence of Specimen Size and Relative Humidity on Shrinkage of High-Strength Concrete. In: Baroghel-Bouny V, Aitcin P-C, editors. International RILEM Workshop on Shrinkage of Concrete. Bagneux, France: RILEM.

Mohamed AR, Hansen W. 1997. Effect of Nonlinear Temperature Gradient on Curling Stress in Concrete Pavement. Transportation Research Record(1568):65-71. 
Mokarem DW, Weyers RE, Lane DS. 2003. Development of Performance Specifications for Shrinkage of Portland Cement Concrete. Transportation Research Record 2420:40-47.

Muller HS, Hilsdorf HK. 1990. Evaluation of the Time Dependent Behavior of Concrete. Lausanne, Switzerland: Comite Euro-International du Beton.

NCHRP. 2003. Guide for Mechanistic-Empirical Design for New and Rehabilitated Pavement Structures: Appendix KK - Transverse Cracking of JPCP. Washington DC: Transportation Research Board.

NCHRP. 2004a. Guide for Mechanistic-Empirical Design of New and Rehabilitated Pavement Structures: Part 1: Introduction: Chapter 1: Background, Scope and Overview. Washington DC: Transportation Reserach Board.

NCHRP. 2004b. Guide for Mechanistic-Empirical Design of New and Rehabilitated Pavement Structures: Part 2: Design Inputs: Chapter 2: Material Characterization. Washington DC: Transportation Research Board.

NCHRP. 2004c. Guide for Mechanistic-Empirical Design of New and Rehabilitated Pavement Structures: Part 3 Design Analysis: Chapter 4: Design of New and Reconstructed Rigid Pavements. Washington DC: Transportation Research Board. Final Report.

NCHRP. 2006. Mechanistic Empirical Pavement Design Guide. 1.1 ed. Washington DC: Transportation Research Board.

Neville AM. 1997. Properties of Concrete. New York: Wiley \& Sons, Inc.

NOAA. Average Relative Humidity (\%) [Internet]. 2008. 2008. National Oceanic and Aeronautics Administration. Available from: http://wf.ncdc.noaa.gov/oa/climate/online/ccd/avgrh.html

Pane I, Hansen W, Mohamed AR. 1998. Three-Dimensional Finite Element Study on Effects of Nonlinear Temperature Gradients in Concrete Pavements. Transportation Research Record(1629):58-66.

Persson BSM. 1999. Shrinkage of High-Performance Concrete. In: Tazawa E, editor. Autogenous Shrinkage of Concrete: Proceedings of the International Workshop Organized by the JCI. New York: Routledge.

Pickett G. 1956. Effect of Aggregate on Shrinkage of Concrete and a Hypothesis Concerning Shrinkage. Journal of the American Concrete Institute 22(5):581-590.

Poblete M, Salsilli R, Valenzuela R, Bull A, Spratz P. 1988. Field Evaluation of Thermal Deformations in Undoweled PCC Pavement Slabs. Transportation Research Record(1207):217-227(+?). 
Poblete M, Ceza P, David J, Espinosa R, Garcia A, Gonzalez J. 1991. Model of Slab Cracking for Portland Cement Concrete Pavements. Transportation Research Record(1307):154-161.

Qin YH. 2011. Numerical Study on the Curling and Warping of Hardened Rigid Pavement Slabs [Dissertation]. [Houghton, MI]: Michigan Technological University.

Rao C, Barenberg EJ, Snyder MB, Schmidt S. 2001. Effects of Temperature and Moisture on the Response of Jointed Concrete Pavements. 7th International Conference on Concrete Pavements. Orlando, FL.

Rao S, Roesler JR. 2005a. Nondestructive Testing of Concrete Pavements for Characterization of Effective Built-In Curling. Journal of Testing and Evaluation 33(5):1-8.

Rao S, Roesler JR. 2005b. Characterizing Effective Built-In Curling from Concrete Pavement Field Measurements. Journal of Transportation Engineering 131(4):320-327.

Reddy AS, Leonards GA, Harr ME. Warping Stresses and Deflections in Concrete Pavements: Part III. Highway Research Board Proceedings; 1963. p. 1-24.

Richardson JM, Armaghani JM. 1987. Stress Caused by Temperature Gradients in Portland Cement Concrete Pavements. Transportation Research Record(1121):713.

Saliba J, Roziere E, Grondin F, Loukili A. 2011. Influence of shrinkage-reducing admixtures on plastic and long-term shrinkage. Cement and Concrete Composites 33:209-217.

Sato R, Maruyama I, Sogabe T, Sogo M. 2007. Flexural Behavior of Reinforced Recycled Concrete Beams. Journal of Advanced Concrete Technology 5(1):4361.

Sayers MW, Karamihas SM. 1998. The Little Book of Profiling: Basic Information about Measuring and Interpreting Road Profiles. Ann Arbor: University of Michigan.

Shacklock BW, Keene PW. 1957. The Effect of Mix Proportions and Testing Conditions on Drying Shrinkage and Moisture Movement of Concrete. Cement and Concrete Association.

Shorkey CJ. 2010. Mechanical Properties of Concrete Products Utilizing Recycled Concrete Aggregates: Studying the Effects of Recycled Materials on Concrete Mixtures [Master's Report]. [Houghton]: Michigan Technological University. 
Shoukry SN. 2000. Backcalculation of Thermally Deformed Concrete Pavements. Transportation Research Record(1716):64-72.

Shoukry SN, Fahmy M, Prucz J, William G. 2007. Validation of 3DFE Analysis of Rigid Pavement Dynamic Response to Moving Traffic and Nonlinear Temperature Gradient Effects. International Journal of Geomechanics 7(1):16-24.

Siddique Z, Hossain M, Meggers D. Curling and Curling Stresses of New Concrete. Airfield and Highway Pavements Specialty Conference; 2006: American Society of Civil Engineers. p. 671-682.

Sondag SK, Snyder MB. 2003. Analysis of "Built-in" Curling and Warping of PCC Pavements.

Springenschmid R, Hiller E. Influence of Temperature During Curing on Stresses in Concrete Pavements. 8th International Symposium on Concrete Roads; 1998; Lisbon p. 259-263.

Tang T, Zollinger DG, Senadheera S. 1993. Analysis of Concave Curling in Concrete Slabs. Journal of Transportation Engineering 119(4):618-633.

Tazawa E, Miyazawa S. 1995. Experimental Study on Mechanism of Autogenous Shrinkage of Concrete. Cement and Concrete Research 25(8):1633-1638.

Tazawa E, Sato R, Sakai E, Miyazawa S. 2000. Work of JCI Committee on Autogenous Shrinkage. In: Baroghel-Bouny V, Aitcin P-C, editors. International RILEM Workshop on Shrinkage of Concrete. Bagneux, France: RILEM.

Teller LW, Sutherland EC. 1935. The Structural Design of Concrete Pavements: Observed Effects of Variations in Temperature and Moisture on the Size, Shape, and Stress Resistance of Concrete Pavement Slabs. Public Roads 16(9):169-200.

Teller LW, Sutherland EC. 1936. The Sturctural Design of Concrete Pavements: A Study of the Structural Action of Several Types of Transverse and Longitudinal Joint Designs. Public Roads 17(7):143-71.

Tepfers R, Friden C, Georgsson L. 1977. Study of the Applicability to the Fatigue of Concrete of teh Palmgren-Miner Partial Damage Hypothesis. Magazine of Concrete Research 29(100):123-130.

Thomlinson J. 1940. Temperature Variations and Consequent Stresses Produced by Daily and Seasonal Temperature Cycles in Concrete Slabs. Concrete and Constructional Engineering 35(7):352-360.

Timm DH. Design Comparisons Using the New Mechanistic-Empirical Rigid Pavement Design Guide. In: Imad LA-Q, editor. 2006: ASCE. p. 20. 
Timoshenko S, Goodier JN. 1951. Theory of Elasticity. New York: McGraw Hill Book Company.

Torrenti J-M, Granger L, Diruy M, Genin P. 1999. Modeling Concrete Shrinkage under Variable Ambient Conditions. ACI Materials Journal 96(1):35-39.

Vandenbossche JM. 2003. Interpreting Falling Weight Deflectometer Results for Curled and Warped Portland Cement Concrete Pavements [Thesis]. [Minneapolis, MN]: University of Minnesota. p. 337.

Vandenbossche JM. 2007. Effects of Slab Temperature Profiles on Use of Falling Weight Deflectometer Data to Monitor Joint Performance and Detect Voids. Transportation Research Record(2005):75-85.

Vandenbossche JM, Mu F, Gutierrez JJ, Sherwood J. 2010. An Evaluation of the Built-In Temperature Difference Input Parameter in the Jointed Plain Concrete Pavement Cracking Model of the Mechanistic-Empirical Pavement Design Guide. International Journal of Pavement Engineering.

Wei Y, Hansen W, Schlangen E. 2008. Moisture Warping in Slabs on Grade. In: Al-Qadi, Scarpas, Loizos, editors. Pavement Cracking. London: Taylor and Francis Group.

Wei Y, Hansen W. 2011. Characterization of Moisture Transport and Its Effects on Deformations in Jointed Concrete Pavement. Transportation Research Record Accepted for Publication.

Wei Y, Hansen W, Biernacki JJ, Schlangen E. 2011. Unified Shrinkage Model for Concrete from Autogenous Shrinkage Test on Paste with and without GroundGranulated Blast-Furnace Slag. ACI Materials Journal 108(1):13-20.

Wells SA, Phillips BM, Vandenbossche JM. 2006a. Quantifying Built-in Construction Gradients and Early-age Slab Deformation Caused by Environmental Loads in a Jointed Plain Concrete Pavement. International Journal of Pavement Engineering 7(4):275-289.

Wells SA, Phillips BM, Vandenbossche JM. 2006b. Characterizing Strain Induced by Environmental Loads in Jointed Plain Concrete Pavements. Transportation Research Record(1947):36-48.

Westergaard HM. 1926. Stresses in Concrete Pavements Computed by Theoretical Analysis. Public Roads 7(2):25-35.

Westergaard HM. 1927. Analysis of Stresses in Concrete Roads Caused by Variations of Temperature. Public Roads 8(3):54-60.

Yoder EJ, Witczak MW. 1975. Principles of Pavement Design. New York: John Wiley and Sons, Inc. 
Yu HT, Khazanovich L. 2001. Effects of Construction Curling on Concrete Pavement Behavior. 7th International Conference on Concrete Pavements. Orlando, FL.

Zhang MH, Tam CT, Leow MP. 2003. Effect of water-to-cementitious materials ratio and silica fume on the autogenous shrinkage of concrete Cement and Concrete Research 33(10):1687-1694. 


\section{Appendix A: ASTM C157 Test Data}

Table A.1

Strain and weight change for Virgin 1.

\begin{tabular}{|c|c|c|c|c|c|c|c|c|}
\hline \multirow[b]{3}{*}{$\begin{array}{c}\text { age } \\
\text { (days) }\end{array}$} & \multicolumn{8}{|c|}{ Virgin 1} \\
\hline & \multicolumn{2}{|c|}{ dry cure 1} & \multicolumn{2}{|c|}{ dry cure 2} & \multicolumn{2}{|c|}{ wet cure 1} & \multicolumn{2}{|c|}{ wet cure 2} \\
\hline & $\begin{array}{l}\text { strain } \\
(\mu \varepsilon)\end{array}$ & $\begin{array}{c}\text { weight } \\
\text { change } \\
\text { (Ibs) }\end{array}$ & $\begin{array}{l}\text { strain } \\
(\mu \varepsilon)\end{array}$ & $\begin{array}{c}\text { weight } \\
\text { change } \\
\text { (Ibs) }\end{array}$ & $\begin{array}{c}\text { strain } \\
(\mu \varepsilon)\end{array}$ & $\begin{array}{c}\text { weight } \\
\text { change } \\
\text { (lbs) }\end{array}$ & $\begin{array}{c}\text { strain } \\
(\mu \varepsilon)\end{array}$ & $\begin{array}{l}\text { weight } \\
\text { change } \\
\text { (Ibs) }\end{array}$ \\
\hline 0.75 & 0 & 0 & 0 & 0 & 0 & 0 & 0 & 0 \\
\hline 1 & -40 & -0.0425 & -50 & -0.04 & -60 & 0.04 & -40 & 0.03 \\
\hline 2 & -80 & -0.077 & -100 & -0.0735 & -200 & 0.0685 & -50 & 0.0585 \\
\hline 3 & -110 & -0.093 & -170 & -0.0895 & -210 & 0.073 & -90 & 0.062 \\
\hline 4 & -180 & -0.1045 & -200 & -0.1005 & -210 & 0.066 & -80 & 0.056 \\
\hline 5 & -220 & -0.11 & -240 & -0.1065 & -220 & 0.079 & -80 & 0.066 \\
\hline 6 & -220 & -0.109 & -250 & -0.1055 & -220 & 0.081 & -70 & 0.0695 \\
\hline 7 & -220 & -0.111 & -280 & -0.105 & -230 & 0.0815 & -110 & 0.0685 \\
\hline 8 & -260 & -0.1125 & -300 & -0.109 & -200 & 0.08 & -120 & 0.0675 \\
\hline 9 & -400 & -0.117 & -460 & -0.1115 & -100 & 0.0855 & 100 & 0.0725 \\
\hline 10 & -300 & -0.1155 & -460 & -0.112 & -310 & 0.0825 & -30 & 0.0715 \\
\hline 11 & -360 & -0.117 & -450 & -0.1135 & -200 & 0.09 & -10 & 0.078 \\
\hline 12 & -380 & -0.1175 & -400 & -0.1135 & -180 & 0.089 & -70 & 0.0775 \\
\hline 13 & -370 & -0.118 & -440 & -0.1145 & -210 & 0.09 & -30 & 0.078 \\
\hline 14 & -380 & -0.1185 & -530 & -0.115 & -220 & 0.0885 & -160 & 0.0765 \\
\hline 15 & -360 & -0.1195 & -520 & -0.116 & -240 & 0.089 & -190 & 0.077 \\
\hline 16 & -280 & -0.1195 & -430 & -0.116 & -230 & 0.0875 & -90 & 0.0755 \\
\hline 17 & -400 & -0.121 & -620 & -0.117 & -210 & 0.0885 & -90 & 0.077 \\
\hline 18 & -420 & -0.121 & -680 & -0.1175 & -220 & 0.089 & -70 & 0.0775 \\
\hline 19 & -410 & -0.1225 & -660 & -0.119 & -210 & 0.092 & -50 & 0.0805 \\
\hline 20 & -420 & -0.123 & -690 & -0.119 & -220 & 0.0925 & -60 & 0.08 \\
\hline 21 & -440 & -0.1235 & -710 & -0.1195 & -220 & 0.0915 & -60 & 0.078 \\
\hline 22 & -440 & -0.1235 & -710 & -0.1195 & -220 & 0.0915 & -60 & 0.078 \\
\hline 23 & -450 & -0.1265 & -740 & -0.123 & -200 & 0.094 & -30 & 0.0835 \\
\hline 24 & -460 & -0.1265 & -780 & -0.1225 & -210 & 0.0915 & -10 & 0.0805 \\
\hline 25 & -460 & -0.1275 & -770 & -0.1235 & -210 & 0.0935 & -20 & 0.0825 \\
\hline 26 & -470 & -0.1285 & -770 & -0.1255 & -220 & 0.097 & -40 & 0.0855 \\
\hline 27 & -470 & -0.1295 & -790 & -0.1255 & -220 & 0.095 & -50 & 0.0825 \\
\hline 28 & -750 & -0.1305 & -810 & -0.1265 & -210 & 0.0985 & -30 & 0.0875 \\
\hline
\end{tabular}


Table A.1, continued.

\begin{tabular}{|c|c|c|c|c|c|c|c|c|}
\hline \multirow[b]{3}{*}{$\begin{array}{c}\text { age } \\
\text { (days) }\end{array}$} & \multicolumn{8}{|c|}{ Virgin 1} \\
\hline & \multicolumn{2}{|c|}{ dry cure 1} & \multicolumn{2}{|c|}{ dry cure 2} & \multicolumn{2}{|c|}{ wet cure 1} & \multicolumn{2}{|c|}{ wet cure 2} \\
\hline & $\begin{array}{c}\text { strain } \\
(\mu \varepsilon)\end{array}$ & $\begin{array}{c}\text { weight } \\
\text { change } \\
\text { (Ibs) }\end{array}$ & $\begin{array}{c}\text { strain } \\
(\mu \varepsilon)\end{array}$ & $\begin{array}{c}\text { weight } \\
\text { change } \\
\text { (lbs) }\end{array}$ & $\begin{array}{c}\text { strain } \\
(\mu \varepsilon)\end{array}$ & $\begin{array}{c}\text { weight } \\
\text { change } \\
\text { (lbs) }\end{array}$ & $\begin{array}{l}\text { strain } \\
(\mu \varepsilon)\end{array}$ & $\begin{array}{c}\text { weight } \\
\text { change } \\
\text { (Ibs) }\end{array}$ \\
\hline 34 & -750 & -0.1305 & -810 & -0.1265 & -210 & 0.0985 & -30 & 0.0875 \\
\hline 41 & -780 & -0.139 & -890 & -0.1345 & -200 & 0.101 & 10 & 0.09 \\
\hline 48 & -810 & -0.1395 & -980 & -0.13 & -180 & 0.106 & 20 & 0.092 \\
\hline 55 & -850 & -0.152 & -1110 & -0.148 & -170 & 0.114 & 10 & 0.105 \\
\hline 62 & -830 & -0.15 & -1090 & -0.1455 & -170 & 0.1215 & 10 & 0.1115 \\
\hline 69 & -840 & -0.151 & -1130 & -0.146 & -160 & 0.124 & 30 & 0.1145 \\
\hline 76 & -870 & -0.1505 & -1150 & -0.146 & -160 & 0.1275 & 30 & 0.1165 \\
\hline 86 & -860 & -0.1485 & -1140 & -0.1435 & -150 & 0.129 & 30 & 0.12 \\
\hline 96 & -860 & -0.146 & -1140 & -0.142 & -140 & 0.1305 & 40 & 0.1215 \\
\hline 106 & -860 & -0.1435 & -1130 & -0.14 & -130 & 0.132 & 40 & \\
\hline 116 & -880 & -0.148 & -1160 & -0.1425 & -130 & 0.1265 & 50 & 0.119 \\
\hline 126 & -890 & -0.148 & -1170 & -0.143 & -130 & 0.1375 & 50 & 0.128 \\
\hline 135 & -890 & -0.148 & -1170 & -0.1415 & -120 & 0.139 & 60 & 0.13 \\
\hline 374 & -980 & -0.1565 & -1260 & -0.1505 & -140 & 0.155 & 30 & 0.143 \\
\hline 472 & -960 & -0.148 & -1240 & -0.142 & -30 & 0.1595 & 130 & 0.147 \\
\hline 511 & -970 & & -1240 & -0.1435 & -10 & 0.165 & 160 & 0.1545 \\
\hline 512 & -680 & 0.132 & -950 & 0.146 & -50 & 0.142 & 120 & 0.1335 \\
\hline 513 & -650 & 0.138 & -930 & 0.147 & -70 & 0.1355 & 120 & 0.128 \\
\hline 514 & -640 & 0.1355 & -920 & 0.151 & -120 & 0.1295 & 70 & 0.123 \\
\hline 516 & -780 & 0.141 & -990 & 0.1535 & -160 & 0.1215 & 40 & 0.1145 \\
\hline 518 & -720 & 0.1375 & -930 & 0.1495 & -210 & 0.115 & 10 & 0.109 \\
\hline 520 & -700 & 0.15 & -940 & 0.1625 & -250 & 0.1105 & -30 & 0.1045 \\
\hline 523 & -670 & 0.153 & -900 & 0.163 & -300 & 0.1055 & -70 & 0.0995 \\
\hline 525 & -670 & 0.155 & -890 & 0.169 & -330 & 0.1025 & -110 & 0.0965 \\
\hline 527 & -670 & 0.1575 & -890 & 0.1625 & -360 & 0.1 & -140 & 0.093 \\
\hline 530 & -660 & 0.158 & -870 & 0.17 & -370 & 0.0955 & -140 & 0.0895 \\
\hline 532 & -650 & 0.1585 & -870 & 0.1695 & -380 & 0.093 & -150 & 0.086 \\
\hline 534 & -660 & 0.1545 & -870 & 0.168 & & 0.091 & -190 & 0.0835 \\
\hline 540 & -640 & 0.165 & -870 & 0.174 & -460 & 0.085 & -210 & 0.078 \\
\hline 547 & -670 & 0.161 & -870 & 0.1765 & -470 & 0.0795 & -210 & 0.0725 \\
\hline
\end{tabular}


Table A.1, continued.

\begin{tabular}{|c|c|c|c|c|c|c|c|c|}
\hline \multirow[b]{3}{*}{$\begin{array}{l}\text { age } \\
\text { (days) }\end{array}$} & \multicolumn{8}{|c|}{ Virgin 1} \\
\hline & \multicolumn{2}{|c|}{ dry cure 1} & \multicolumn{2}{|c|}{ dry cure 2} & \multicolumn{2}{|c|}{ wet cure 1} & \multicolumn{2}{|c|}{ wet cure 2} \\
\hline & $\begin{array}{c}\text { strain } \\
(\mu \varepsilon)\end{array}$ & $\begin{array}{c}\text { weight } \\
\text { change } \\
\text { (Ibs) }\end{array}$ & $\begin{array}{c}\text { strain } \\
(\mu \varepsilon)\end{array}$ & $\begin{array}{c}\text { weight } \\
\text { change } \\
\text { (lbs) }\end{array}$ & $\begin{array}{c}\text { strain } \\
(\mu \varepsilon)\end{array}$ & $\begin{array}{c}\text { weight } \\
\text { change } \\
\text { (Ibs) }\end{array}$ & $\begin{array}{c}\text { strain } \\
(\mu \varepsilon)\end{array}$ & $\begin{array}{c}\text { weight } \\
\text { change } \\
\text { (lbs) }\end{array}$ \\
\hline 554 & -640 & 0.16 & -880 & 0.1735 & -510 & 0.0715 & -210 & 0.067 \\
\hline 568 & -650 & 0.17 & -860 & 0.184 & -520 & 0.065 & -300 & 0.0575 \\
\hline 582 & -650 & 0.1735 & -850 & 0.186 & -530 & 0.0565 & -310 & 0.0495 \\
\hline 596 & -670 & 0.175 & -880 & 0.1875 & & 0.0525 & -200 & 0.0455 \\
\hline 611 & -620 & 0.1795 & -790 & 0.192 & -560 & 0.0485 & -310 & 0.0405 \\
\hline 686 & & 0.1815 & & 0.197 & & 0.026 & -410 & 0.0185 \\
\hline 691 & & 0.1805 & & 0.1955 & & 0.0245 & & 0.016 \\
\hline 692 & -620 & 0.1805 & -820 & 0.198 & -590 & 0.025 & -420 & 0.016 \\
\hline 693 & -610 & 0.1815 & -830 & 0.1925 & & 0.0245 & -410 & 0.0155 \\
\hline 694 & -620 & 0.1315 & -830 & 0.143 & -340 & 0.114 & -140 & 0.093 \\
\hline 695 & -650 & 0.116 & -870 & 0.127 & -280 & 0.122 & -110 & 0.105 \\
\hline 696 & -640 & 0.103 & -870 & 0.114 & -240 & 0.124 & -50 & 0.112 \\
\hline 697 & -650 & 0.0935 & -870 & 0.1045 & -220 & 0.1275 & -30 & 0.1135 \\
\hline 698 & -660 & 0.086 & -870 & 0.097 & -200 & 0.1285 & -10 & 0.114 \\
\hline 700 & & & & & -180 & 0.1375 & 10 & 0.123 \\
\hline 702 & & & & & -170 & 0.1405 & 20 & 0.1265 \\
\hline 704 & -700 & 0.0615 & -910 & 0.0725 & -160 & 0.1385 & 30 & 0.1225 \\
\hline 707 & & & & & -170 & 0.147 & 30 & 0.1315 \\
\hline 709 & -730 & 0.047 & -930 & & -150 & 0.143 & 50 & 0.1295 \\
\hline 711 & -740 & 0.041 & -930 & 0.052 & -130 & 0.143 & 60 & 0.128 \\
\hline 714 & & & & & -140 & 0.147 & 60 & 0.132 \\
\hline 716 & -750 & 0.03 & -960 & 0.041 & -150 & 0.1475 & 60 & 0.13 \\
\hline 718 & -790 & 0.027 & -970 & 0.0375 & -160 & 0.149 & 60 & 0.135 \\
\hline 725 & -810 & 0.0155 & -1030 & 0.0265 & -110 & 0.1515 & 70 & 0.136 \\
\hline 726 & -830 & & -1030 & 0.0255 & -120 & 0.148 & 70 & 0.132 \\
\hline 727 & -820 & 0.013 & -1030 & 0.024 & -140 & 0.159 & 70 & 0.1415 \\
\hline 728 & -720 & 0.119 & -910 & 0.137 & -190 & 0.1315 & 40 & 0.1185 \\
\hline 729 & -690 & 0.1335 & -890 & 0.1485 & -170 & 0.123 & 30 & 0.1115 \\
\hline 730 & -650 & 0.1375 & -860 & 0.151 & -190 & & 20 & 0.107 \\
\hline 731 & -660 & 0.1425 & & 0.1615 & -220 & 0.1125 & -10 & 0.102 \\
\hline
\end{tabular}


Table A.1, continued.

\begin{tabular}{|c|c|c|c|c|c|c|c|c|}
\hline \multirow[b]{3}{*}{$\begin{array}{c}\text { age } \\
\text { (days) }\end{array}$} & \multicolumn{8}{|c|}{ Virgin 1} \\
\hline & \multicolumn{2}{|c|}{ dry cure 1} & \multicolumn{2}{|c|}{ dry cure 2} & \multicolumn{2}{|c|}{ wet cure 1} & \multicolumn{2}{|c|}{ wet cure 2} \\
\hline & $\begin{array}{c}\text { strain } \\
(\mu \varepsilon)\end{array}$ & $\begin{array}{c}\text { weight } \\
\text { change } \\
\text { (Ibs) }\end{array}$ & $\begin{array}{c}\text { strain } \\
(\mu \varepsilon)\end{array}$ & $\begin{array}{c}\text { weight } \\
\text { change } \\
\text { (lbs) }\end{array}$ & $\begin{array}{c}\text { strain } \\
(\mu \varepsilon)\end{array}$ & $\begin{array}{c}\text { weight } \\
\text { change } \\
\text { (Ibs) }\end{array}$ & $\begin{array}{c}\text { strain } \\
(\mu \varepsilon)\end{array}$ & $\begin{array}{c}\text { weight } \\
\text { change } \\
\text { (lbs) }\end{array}$ \\
\hline 732 & -670 & 0.151 & -870 & 0.1665 & -250 & 0.139 & -20 & 0.0985 \\
\hline 733 & -650 & 0.1545 & -840 & 0.17 & -260 & 0.105 & -50 & 0.095 \\
\hline 735 & -640 & 0.154 & -830 & 0.171 & -290 & 0.096 & -50 & 0.09 \\
\hline 737 & -630 & 0.1585 & -850 & 0.173 & -310 & 0.095 & -70 & 0.086 \\
\hline 739 & -660 & 0.1575 & -840 & 0.1755 & -330 & 0.09 & -100 & 0.0815 \\
\hline 742 & -630 & 0.162 & -830 & 0.182 & -370 & 0.084 & -130 & 0.0755 \\
\hline 744 & -630 & 0.163 & -840 & 0.181 & -360 & 0.0805 & -120 & 0.072 \\
\hline 746 & -620 & 0.163 & -840 & 0.178 & -390 & 0.078 & -160 & 0.069 \\
\hline 749 & -630 & 0.163 & -830 & 0.1775 & -370 & 0.0745 & -150 & 0.0655 \\
\hline 751 & -630 & 0.161 & -840 & 0.1775 & -430 & 0.0725 & -190 & 0.063 \\
\hline 756 & -670 & 0.1655 & -830 & 0.1795 & -460 & 0.0675 & -230 & 0.0589 \\
\hline 758 & -660 & 0.161 & -840 & 0.1785 & -460 & 0.066 & -210 & 0.0565 \\
\hline 760 & -680 & 0.162 & -850 & 0.1705 & -490 & 0.065 & -230 & 0.056 \\
\hline 761 & -650 & 0.165 & -870 & 0.181 & -490 & 0.0635 & -240 & 0.0545 \\
\hline 763 & -640 & 0.1625 & -860 & 0.18 & -460 & 0.062 & -250 & 0.053 \\
\hline 765 & -630 & 0.1635 & -840 & 0.1815 & -460 & 0.0595 & -240 & 0.051 \\
\hline 767 & -620 & 0.1685 & -820 & 0.178 & -490 & 0.0585 & -260 & 0.0495 \\
\hline 769 & -620 & 0.166 & -830 & 0.1805 & -470 & 0.0575 & -250 & 0.048 \\
\hline 770 & -660 & 0.1245 & -880 & 0.138 & -240 & 0.1255 & -60 & 0.1105 \\
\hline 771 & -660 & 0.1125 & -880 & 0.126 & -200 & 0.1355 & -20 & 0.123 \\
\hline 772 & -670 & 0.103 & -890 & 0.032 & -190 & 0.1375 & 10 & 0.125 \\
\hline 773 & -660 & 0.0955 & -870 & 0.108 & -170 & 0.1405 & 80 & 0.1285 \\
\hline 774 & -690 & 0.089 & -890 & 0.102 & -180 & 0.145 & 40 & 0.131 \\
\hline 775 & -710 & 0.0845 & -980 & 0.097 & -140 & 0.1455 & 30 & 0.1315 \\
\hline 776 & -700 & 0.08 & -920 & 0.092 & -160 & 0.149 & 30 & 0.136 \\
\hline 777 & -720 & 0.0755 & -940 & 0.0885 & -120 & 0.1495 & -240 & 0.138 \\
\hline 778 & -720 & 0.0725 & -980 & 0.0845 & -220 & 0.1495 & 10 & 0.1385 \\
\hline 779 & -740 & 0.0695 & -930 & 0.0815 & -150 & 0.1495 & 10 & 0.138 \\
\hline 781 & -830 & 0.065 & -1050 & 0.077 & -120 & 0.153 & -30 & 0.1355 \\
\hline 783 & -800 & 0.0605 & -1050 & 0.0715 & -230 & 0.1535 & 50 & 0.1435 \\
\hline
\end{tabular}


Table A.1, continued.

\begin{tabular}{|c|c|c|c|c|c|c|c|c|}
\hline \multirow[b]{3}{*}{$\begin{array}{l}\text { age } \\
\text { (days) }\end{array}$} & \multicolumn{8}{|c|}{ Virgin 1} \\
\hline & \multicolumn{2}{|c|}{ dry cure 1} & \multicolumn{2}{|c|}{ dry cure 2} & \multicolumn{2}{|c|}{ wet cure 1} & \multicolumn{2}{|c|}{ wet cure 2} \\
\hline & $\begin{array}{c}\text { strain } \\
(\mu \varepsilon)\end{array}$ & $\begin{array}{c}\text { weight } \\
\text { change } \\
\text { (Ibs) }\end{array}$ & $\begin{array}{c}\text { strain } \\
(\mu \varepsilon)\end{array}$ & $\begin{array}{c}\text { weight } \\
\text { change } \\
\text { (lbs) }\end{array}$ & $\begin{array}{c}\text { strain } \\
(\mu \varepsilon)\end{array}$ & $\begin{array}{c}\text { weight } \\
\text { change } \\
\text { (lbs) }\end{array}$ & $\begin{array}{c}\text { strain } \\
(\mu \varepsilon)\end{array}$ & $\begin{array}{c}\text { weight } \\
\text { change } \\
\text { (lbs) }\end{array}$ \\
\hline 785 & -760 & 0.056 & -980 & 0.046 & -150 & 0.1515 & 0 & 0.141 \\
\hline 787 & -770 & 0.053 & -970 & 0.0645 & -140 & 0.1535 & 40 & 0.143 \\
\hline 789 & -780 & 0.049 & -1010 & 0.0615 & -130 & 0.1565 & 50 & 0.1475 \\
\hline 791 & -810 & 0.0455 & -1010 & 0.056 & -140 & 0.154 & 50 & 0.146 \\
\hline 793 & -780 & 0.043 & -990 & 0.054 & -100 & 0.157 & 90 & 0.1445 \\
\hline 796 & -790 & 0.041 & -990 & 0.0525 & -100 & 0.158 & 90 & 0.1505 \\
\hline 798 & -800 & 0.0385 & -1010 & 0.0505 & -130 & 0.159 & 70 & 0.1485 \\
\hline 800 & -830 & 0.036 & -1030 & 0.0485 & -130 & 0.156 & 60 & 0.1475 \\
\hline 803 & -810 & 0.0335 & -1020 & 0.0455 & -90 & 0.16 & 80 & 0.1505 \\
\hline 806 & -840 & 0.032 & -1050 & 0.044 & -110 & 0.157 & 70 & 0.149 \\
\hline 810 & -800 & 0.03 & -1040 & 0.0415 & -100 & 0.1615 & 80 & 0.1495 \\
\hline 811 & -860 & 0.029 & -1060 & 0.041 & -110 & 0.159 & 70 & 0.1515 \\
\hline 812 & -860 & 0.0295 & -1070 & 0.041 & -110 & 0.159 & 90 & 0.152 \\
\hline 813 & -750 & 0.104 & -950 & 0.124 & -150 & 0.1405 & 40 & 0.0962 \\
\hline 814 & -710 & 0.12 & -920 & 0.132 & -160 & 0.1325 & 30 & 0.123 \\
\hline 815 & -710 & 0.1215 & -900 & 0.139 & -180 & 0.1265 & 10 & 0.1175 \\
\hline 816 & -660 & 0.1255 & -880 & 0.143 & -180 & 0.122 & 20 & 0.1135 \\
\hline 817 & -670 & 0.1325 & -880 & 0.1485 & -190 & 0.1145 & 0 & 0.1105 \\
\hline 820 & -660 & 0.141 & -880 & 0.1595 & -210 & 0.112 & -10 & 0.1045 \\
\hline 822 & & 0.144 & -900 & 0.1565 & -260 & 0.108 & -50 & 0.1005 \\
\hline 824 & -650 & 0.146 & -860 & 0.1595 & -250 & 0.1035 & -50 & 0.096 \\
\hline 826 & -660 & 0.153 & -870 & 0.1695 & -280 & 0.1005 & -80 & 0.093 \\
\hline 828 & -630 & 0.1555 & -820 & 0.1735 & -300 & 0.097 & -120 & 0.09 \\
\hline 833 & -630 & 0.159 & -830 & 0.176 & -300 & 0.0935 & -90 & 0.0865 \\
\hline 833 & -640 & 0.164 & -830 & 0.1775 & -310 & 0.0915 & -90 & 0.084 \\
\hline 836 & -620 & 0.1625 & -810 & 0.1795 & -340 & 0.0885 & -120 & 0.0815 \\
\hline 840 & -630 & 0.164 & -830 & 0.1805 & -350 & 0.0845 & -140 & \\
\hline 848 & -640 & 0.1675 & -840 & 0.1845 & & 0.078 & -170 & 0.0705 \\
\hline 851 & -630 & 0.168 & -820 & 0.1855 & -400 & 0.077 & -190 & 0.069 \\
\hline
\end{tabular}


Table A.2

Strain and weight change for Virgin 2.

\begin{tabular}{|c|c|c|c|c|c|c|}
\hline \multirow[b]{3}{*}{$\begin{array}{c}\text { age } \\
\text { (days) }\end{array}$} & \multicolumn{6}{|c|}{ Virgin 2} \\
\hline & \multicolumn{2}{|c|}{ dry cure 1} & \multicolumn{2}{|c|}{ dry cure 2} & \multicolumn{2}{|c|}{ wet cure 1} \\
\hline & $\begin{array}{l}\text { strain } \\
(\mu \varepsilon)\end{array}$ & $\begin{array}{l}\text { weight change } \\
\text { (lbs) }\end{array}$ & $\begin{array}{l}\text { strain } \\
(\mu \varepsilon)\end{array}$ & $\begin{array}{c}\text { weight change } \\
\text { (Ibs) }\end{array}$ & $\begin{array}{c}\text { strain } \\
(\mu \varepsilon)\end{array}$ & $\begin{array}{c}\text { weight change } \\
\text { (Ibs) }\end{array}$ \\
\hline 0.75 & 0 & 0 & 0 & 0 & 0 & 0 \\
\hline 1 & -70 & -0.0525 & -50 & -0.0495 & -50 & 0.066 \\
\hline 2 & -120 & -0.072 & -100 & -0.0915 & -10 & 0.072 \\
\hline 3 & -160 & -0.127 & -150 & -0.121 & 0 & 0.078 \\
\hline 4 & -190 & -0.137 & -190 & -0.131 & 0 & 0.088 \\
\hline 5 & -230 & -0.1425 & -230 & -0.1355 & 0 & 0.0925 \\
\hline 6 & -260 & -0.1465 & -270 & -0.1405 & 10 & 0.097 \\
\hline 7 & -280 & -0.149 & -280 & -0.1415 & 0 & 0.101 \\
\hline 8 & -300 & -0.152 & -310 & -0.1445 & 20 & 0.1025 \\
\hline 9 & -330 & -0.154 & -340 & -0.146 & 10 & 0.1035 \\
\hline 10 & -350 & -0.156 & -350 & -0.148 & 20 & 0.1015 \\
\hline 11 & -370 & -0.158 & -380 & -0.1495 & 10 & 0.108 \\
\hline 12 & -360 & -0.1575 & -390 & -0.1485 & 30 & 0.11 \\
\hline 13 & -370 & -0.1545 & -390 & -0.146 & 10 & 0.1065 \\
\hline 14 & -390 & -0.1565 & -420 & -0.1475 & 20 & 0.1075 \\
\hline 15 & -410 & -0.158 & -420 & -0.148 & 20 & 0.1105 \\
\hline 16 & -410 & -0.158 & -440 & -0.1485 & 20 & 0.1115 \\
\hline 17 & -420 & -0.1595 & -450 & -0.147 & 10 & 0.112 \\
\hline 18 & -420 & -0.159 & -460 & -0.1495 & 20 & 0.114 \\
\hline 19 & -430 & -0.161 & -460 & -0.1525 & 30 & 0.112 \\
\hline 20 & -450 & -0.16 & -490 & -0.151 & 30 & 0.1145 \\
\hline 21 & -470 & -0.1625 & -510 & -0.1535 & 20 & 0.115 \\
\hline 22 & -470 & -0.1605 & -510 & -0.152 & 30 & 0.1135 \\
\hline 23 & -460 & -0.161 & -510 & -0.152 & 30 & 0.1155 \\
\hline 24 & -460 & -0.161 & -510 & -0.1515 & 30 & 0.1115 \\
\hline 25 & -470 & -0.16 & -510 & -0.1505 & 30 & 0.118 \\
\hline 26 & -480 & -0.1605 & -530 & -0.1505 & 30 & 0.115 \\
\hline 27 & -500 & -0.1625 & -540 & -0.153 & 30 & 0.116 \\
\hline 28 & -500 & -0.164 & -550 & -0.1505 & 30 & 0.1185 \\
\hline
\end{tabular}


Table A.2, continued.

\begin{tabular}{|c|c|c|c|c|c|c|}
\hline \multirow[b]{3}{*}{$\begin{array}{c}\text { age } \\
\text { (days) }\end{array}$} & \multicolumn{6}{|c|}{ Virgin 2} \\
\hline & \multicolumn{2}{|c|}{ dry cure 1} & \multicolumn{2}{|c|}{ dry cure 2} & \multicolumn{2}{|c|}{ wet cure 1} \\
\hline & $\begin{array}{c}\text { strain } \\
(\mu \varepsilon)\end{array}$ & $\begin{array}{c}\text { weight change } \\
\text { (Ibs) }\end{array}$ & $\begin{array}{c}\text { strain } \\
(\mu \varepsilon)\end{array}$ & $\begin{array}{l}\text { weight change } \\
\text { (lbs) }\end{array}$ & $\begin{array}{c}\text { strain } \\
(\mu \varepsilon)\end{array}$ & $\begin{array}{c}\text { weight change } \\
\text { (lbs) }\end{array}$ \\
\hline 35 & -490 & -0.1565 & -560 & -0.148 & 30 & 0.1195 \\
\hline 42 & -550 & -0.1625 & -600 & -0.1525 & 40 & 0.123 \\
\hline 49 & -560 & -0.1645 & -610 & -0.1545 & 30 & 0.1265 \\
\hline 56 & -570 & -0.166 & -630 & -0.155 & 30 & 0.128 \\
\hline 63 & -590 & -0.169 & -650 & -0.1585 & 40 & 0.1265 \\
\hline 70 & -610 & -0.1705 & -680 & -0.16 & 20 & 0.1285 \\
\hline 77 & -620 & -0.169 & -690 & -0.1595 & 20 & 0.1315 \\
\hline 84 & -630 & -0.1685 & -700 & -0.158 & 20 & 0.133 \\
\hline 94 & -630 & -0.1695 & -710 & -0.1605 & 10 & 0.135 \\
\hline 104 & -630 & -0.17 & -710 & -0.16 & 10 & 0.139 \\
\hline 114 & -630 & -0.17 & -700 & -0.16 & 30 & 0.1385 \\
\hline 124 & -630 & -0.17 & -700 & -0.1605 & 20 & 0.1385 \\
\hline 134 & -630 & -0.1695 & -700 & -0.159 & 20 & 0.1355 \\
\hline 260 & -700 & -0.177 & -800 & -0.1665 & 10 & 0.1425 \\
\hline 358 & -710 & -0.1645 & -810 & -0.1515 & 100 & 0.1495 \\
\hline 397 & -710 & -0.1645 & -820 & -0.1525 & 130 & 0.1565 \\
\hline 398 & -400 & 0.1545 & -540 & 0.156 & 100 & 0.1395 \\
\hline 399 & -370 & 0.1565 & -490 & 0.1645 & 80 & 0.1315 \\
\hline 400 & -360 & 0.1625 & -490 & 0.162 & 60 & 0.1245 \\
\hline 402 & -440 & 0.169 & -560 & 0.174 & 0 & 0.1125 \\
\hline 404 & -360 & 0.17 & -490 & 0.1747 & -50 & 0.1025 \\
\hline 406 & -340 & 0.1795 & -500 & 0.1875 & -90 & 0.094 \\
\hline 409 & -320 & 0.1885 & -430 & 0.1905 & -140 & 0.0845 \\
\hline 411 & -330 & 0.191 & -430 & 0.192 & -160 & 0.08 \\
\hline 413 & -330 & 0.1945 & -440 & 0.1935 & -200 & 0.0745 \\
\hline 416 & -320 & 0.1965 & -420 & 0.1965 & -210 & 0.068 \\
\hline 418 & -320 & 0.196 & -420 & 0.2015 & -220 & 0.063 \\
\hline 420 & -320 & 0.1985 & -420 & 0.2005 & -250 & 0.0595 \\
\hline 426 & -310 & 0.202 & -410 & 0.2055 & -280 & 0.05 \\
\hline 433 & -300 & 0.2105 & -400 & 0.2155 & -330 & 0.0415 \\
\hline
\end{tabular}


Table A.2, continued.

\begin{tabular}{|c|c|c|c|c|c|c|}
\hline \multirow[b]{3}{*}{$\begin{array}{c}\text { age } \\
\text { (days) }\end{array}$} & \multicolumn{6}{|c|}{ Virgin 2} \\
\hline & \multicolumn{2}{|c|}{ dry cure 1} & \multicolumn{2}{|c|}{ dry cure 2} & \multicolumn{2}{|c|}{ wet cure 1} \\
\hline & $\begin{array}{c}\text { strain } \\
(\mu \varepsilon)\end{array}$ & $\begin{array}{l}\text { weight change } \\
\text { (Ibs) }\end{array}$ & $\begin{array}{c}\text { strain } \\
(\mu \varepsilon)\end{array}$ & $\begin{array}{l}\text { weight change } \\
\text { (Ibs) }\end{array}$ & $\begin{array}{c}\text { strain } \\
(\mu \varepsilon)\end{array}$ & $\begin{array}{c}\text { weight change } \\
\text { (lbs) }\end{array}$ \\
\hline 440 & -280 & 0.209 & -390 & 0.211 & -340 & 0.0335 \\
\hline 454 & -320 & 0.2195 & -400 & 0.22 & -380 & 0.021 \\
\hline 468 & -330 & 0.226 & -420 & 0.228 & -370 & 0.011 \\
\hline 482 & -300 & 0.223 & -400 & 0.226 & -380 & 0.0075 \\
\hline 497 & -300 & 0.228 & & 0.2325 & -450 & 0.0025 \\
\hline 572 & -280 & 0.2365 & -330 & 0.24 & & -0.022 \\
\hline 577 & & 0.228 & & 0.2315 & -420 & -0.023 \\
\hline 578 & -280 & 0.231 & -370 & 0.236 & -490 & -0.024 \\
\hline 579 & -270 & 0.236 & -380 & 0.239 & -500 & -0.024 \\
\hline 580 & -280 & & -370 & 0.1815 & -120 & 0.132 \\
\hline 581 & -320 & 0.161 & -410 & 0.162 & -90 & 0.1485 \\
\hline 582 & -310 & 0.2345 & -410 & 0.1445 & -60 & 0.152 \\
\hline 583 & -310 & 0.128 & -410 & 0.13 & -30 & 0.1525 \\
\hline 584 & -330 & 0.1145 & -430 & 0.118 & -20 & 0.155 \\
\hline 586 & & & & & 0 & 0.1595 \\
\hline 588 & & & & & 0 & 0.1605 \\
\hline 590 & -350 & 0.0675 & -450 & 0.0745 & 10 & 0.1635 \\
\hline 593 & & & & & 10 & 0.1655 \\
\hline 595 & -380 & 0.043 & -490 & 0.0505 & 10 & 0.1655 \\
\hline 597 & -390 & 0.035 & -480 & 0.042 & 10 & 0.16 \\
\hline 600 & & & & & 20 & 0.165 \\
\hline 602 & -390 & 0.019 & -500 & 0.027 & 10 & 0.1635 \\
\hline 604 & -430 & 0.0145 & -540 & 0.0225 & 20 & 0.1645 \\
\hline 611 & -460 & -0.0015 & -570 & 0.008 & 40 & 0.166 \\
\hline 612 & -470 & -0.003 & -580 & 0.0065 & 20 & 0.162 \\
\hline 613 & -460 & -0.0055 & -580 & 0.0045 & 30 & 0.1705 \\
\hline 614 & -340 & 0.143 & -430 & 0.156 & -10 & 0.143 \\
\hline 615 & -320 & 0.16 & -410 & 0.1745 & -40 & 0.129 \\
\hline 616 & -320 & 0.172 & -410 & 0.179 & -40 & 0.1195 \\
\hline 617 & -290 & 0.1715 & -430 & 0.185 & -60 & 0.109 \\
\hline
\end{tabular}


Table A.2, continued.

\begin{tabular}{|c|c|c|c|c|c|c|}
\hline \multirow[b]{3}{*}{$\begin{array}{c}\text { age } \\
\text { (days) }\end{array}$} & \multicolumn{6}{|c|}{ Virgin 2} \\
\hline & \multicolumn{2}{|c|}{ dry cure 1} & \multicolumn{2}{|c|}{ dry cure 2} & \multicolumn{2}{|c|}{ wet cure 1} \\
\hline & $\begin{array}{c}\text { strain } \\
(\mu \varepsilon)\end{array}$ & $\begin{array}{c}\text { weight change } \\
\text { (Ibs) }\end{array}$ & $\begin{array}{c}\text { strain } \\
(\mu \varepsilon)\end{array}$ & $\begin{array}{c}\text { weight change } \\
\text { (lbs) }\end{array}$ & $\begin{array}{c}\text { strain } \\
(\mu \varepsilon)\end{array}$ & $\begin{array}{c}\text { weight change } \\
\text { (lbs) }\end{array}$ \\
\hline 618 & -310 & 0.1795 & -440 & 0.1875 & -70 & 0.1005 \\
\hline 619 & -300 & 0.1835 & -410 & 0.189 & -110 & 0.0935 \\
\hline 621 & -300 & 0.1885 & -430 & 0.193 & -110 & 0.083 \\
\hline 623 & -310 & 0.1825 & -400 & 0.1895 & -120 & 0.075 \\
\hline 625 & -340 & 0.184 & -390 & 0.197 & -160 & 0.067 \\
\hline 628 & -330 & 0.1875 & -430 & 0.2 & -180 & 0.0565 \\
\hline 630 & -320 & 0.1915 & -430 & 0.197 & -200 & 0.051 \\
\hline 632 & -300 & 0.188 & -400 & 0.197 & -230 & 0.0465 \\
\hline 635 & -310 & 0.1895 & -390 & 0.1995 & -220 & 0.0415 \\
\hline 637 & -350 & 0.1885 & -390 & 0.199 & -280 & 0.038 \\
\hline 642 & -320 & 0.1915 & -380 & 0.199 & -290 & 0.0325 \\
\hline 644 & -300 & 0.189 & -380 & 0.197 & -310 & 0.0295 \\
\hline 646 & -340 & 0.191 & -390 & 0.199 & -330 & 0.027 \\
\hline 647 & -290 & 0.193 & -370 & 0.1995 & -330 & 0.026 \\
\hline 649 & -330 & 0.1935 & -400 & 0.2005 & -300 & 0.023 \\
\hline 651 & -320 & 0.1925 & -400 & 0.201 & -300 & 0.021 \\
\hline 653 & -280 & 0.1915 & -380 & 0.2015 & -320 & 0.0195 \\
\hline 655 & -290 & 0.192 & -390 & 0.1995 & -320 & 0.0175 \\
\hline 656 & -330 & 0.1505 & -420 & 0.155 & -60 & 0.138 \\
\hline 657 & -320 & 0.1365 & -410 & 0.1405 & -40 & 0.15 \\
\hline 658 & -340 & 0.1245 & -430 & 0.1295 & -50 & 0.155 \\
\hline 659 & -320 & 0.1145 & -410 & 0.119 & 20 & 0.161 \\
\hline 660 & -360 & 0.106 & -440 & 0.1105 & -40 & 0.1625 \\
\hline 661 & -420 & 0.098 & -510 & 0.1035 & 30 & 0.1615 \\
\hline 662 & -360 & 0.0915 & -460 & 0.0975 & -40 & 0.1675 \\
\hline 663 & -360 & 0.086 & -480 & 0.092 & -10 & 0.167 \\
\hline 664 & -400 & 0.081 & -600 & 0.087 & -10 & 0.164 \\
\hline 665 & -380 & 0.0765 & -500 & 0.083 & 30 & 0.1655 \\
\hline 667 & -490 & 0.0695 & -600 & 0.0765 & -30 & 0.168 \\
\hline 669 & -500 & 0.0625 & -520 & 0.07 & 0 & 0.166 \\
\hline
\end{tabular}


Table A.2, continued.

\begin{tabular}{|c|c|c|c|c|c|c|}
\hline \multirow[b]{3}{*}{$\begin{array}{c}\text { age } \\
\text { (days) }\end{array}$} & \multicolumn{6}{|c|}{ Virgin 2} \\
\hline & \multicolumn{2}{|c|}{ dry cure 1} & \multicolumn{2}{|c|}{ dry cure 2} & \multicolumn{2}{|c|}{ wet cure 1} \\
\hline & $\begin{array}{c}\text { strain } \\
(\mu \varepsilon)\end{array}$ & $\begin{array}{l}\text { weight change } \\
\text { (Ibs) }\end{array}$ & $\begin{array}{c}\text { strain } \\
(\mu \varepsilon)\end{array}$ & $\begin{array}{l}\text { weight change } \\
\text { (Ibs) }\end{array}$ & $\begin{array}{c}\text { strain } \\
(\mu \varepsilon)\end{array}$ & $\begin{array}{c}\text { weight change } \\
\text { (lbs) }\end{array}$ \\
\hline 671 & -430 & 0.0565 & -530 & 0.064 & 20 & 0.169 \\
\hline 673 & -420 & 0.0525 & -520 & 0.0605 & 30 & 0.169 \\
\hline 675 & -440 & 0.0475 & -530 & 0.0555 & 20 & 0.17 \\
\hline 677 & -460 & 0.0425 & -560 & 0.05 & 20 & 0.1685 \\
\hline 679 & -430 & 0.0375 & -540 & 0.046 & 60 & 0.171 \\
\hline 682 & -430 & 0.0345 & -540 & 0.0435 & 60 & 0.176 \\
\hline 684 & -450 & 0.032 & -560 & 0.041 & 40 & 0.1725 \\
\hline 686 & -470 & 0.029 & -580 & 0.038 & 40 & 0.174 \\
\hline 689 & -460 & 0.025 & -560 & 0.034 & 50 & 0.174 \\
\hline 692 & -490 & 0.022 & -590 & 0.032 & 60 & 0.176 \\
\hline 696 & -460 & 0.019 & -600 & 0.029 & 60 & 0.1795 \\
\hline 697 & -500 & 0.018 & -610 & 0.0285 & 40 & 0.1775 \\
\hline 698 & -510 & 0.0175 & -610 & 0.028 & 40 & 0.1765 \\
\hline 699 & -390 & 0.1175 & -490 & 0.136 & 20 & 0.1505 \\
\hline 700 & -350 & 0.1365 & -450 & 0.152 & -20 & 0.137 \\
\hline 701 & -340 & 0.147 & -430 & 0.1645 & -10 & 0.127 \\
\hline 702 & -310 & 0.157 & -430 & 0.1745 & -30 & 0.118 \\
\hline 703 & -310 & 0.1645 & -420 & 0.1815 & -30 & 0.1115 \\
\hline 706 & -320 & 0.1775 & -410 & 0.1955 & -50 & 0.0985 \\
\hline 708 & -330 & 0.1835 & -400 & 0.196 & -90 & 0.091 \\
\hline 710 & -310 & 0.1835 & -390 & 0.196 & -90 & 0.0835 \\
\hline 712 & -320 & 0.1855 & -400 & 0.1985 & -120 & 0.078 \\
\hline 714 & -290 & 0.1885 & -380 & 0.2 & -160 & 0.073 \\
\hline 717 & -290 & 0.19 & -380 & 0.2 & -120 & 0.0665 \\
\hline 719 & -300 & 0.1925 & -380 & 0.2055 & -130 & 0.0635 \\
\hline 722 & -270 & 0.1935 & -370 & 0.204 & -170 & 0.0585 \\
\hline 726 & -290 & 0.195 & -390 & 0.2065 & -190 & 0.0525 \\
\hline 734 & -290 & & -390 & 0.205 & -230 & 0.042 \\
\hline 737 & -290 & 0.198 & -380 & 0.2085 & -240 & 0.04 \\
\hline
\end{tabular}


Table A.3

Strain and weight change for Virgin 3.

\begin{tabular}{|c|c|c|c|c|c|c|}
\hline \multirow[b]{3}{*}{$\begin{array}{c}\text { age } \\
\text { (days) }\end{array}$} & \multicolumn{6}{|c|}{ Virgin 3} \\
\hline & \multicolumn{2}{|c|}{ dry cure 1} & \multicolumn{2}{|c|}{ dry cure 2} & \multicolumn{2}{|c|}{ wet cure 1} \\
\hline & $\begin{array}{l}\text { strain } \\
(\mu \varepsilon)\end{array}$ & $\begin{array}{l}\text { weight change } \\
\text { (lbs) }\end{array}$ & $\begin{array}{l}\text { strain } \\
(\mu \varepsilon)\end{array}$ & $\begin{array}{l}\text { weight change } \\
\text { (lbs) }\end{array}$ & $\begin{array}{c}\text { strain } \\
(\mu \varepsilon)\end{array}$ & $\begin{array}{l}\text { weight change } \\
\text { (lbs) }\end{array}$ \\
\hline 0.75 & 0 & 0 & 0 & 0 & 0 & 0 \\
\hline 1 & 0 & -0.0405 & 0 & -0.0355 & -40 & 0.089 \\
\hline 2 & -40 & -0.095 & 20 & -0.0895 & 30 & 0.103 \\
\hline 3 & -90 & -0.1075 & -30 & -0.1035 & 50 & 0.1105 \\
\hline 4 & -120 & -0.118 & -80 & -0.1125 & 70 & 0.112 \\
\hline 5 & -150 & -0.1145 & -100 & -0.1125 & 70 & 0.115 \\
\hline 6 & -170 & -0.127 & -110 & -0.1215 & 70 & 0.119 \\
\hline 7 & -200 & -0.1305 & -150 & -0.125 & 60 & 0.1185 \\
\hline 8 & -220 & -0.134 & -190 & -0.1285 & 60 & 0.119 \\
\hline 9 & -240 & -0.1365 & -220 & -0.131 & 60 & 0.125 \\
\hline 10 & -250 & -0.138 & -240 & -0.1315 & 60 & 0.1255 \\
\hline 11 & -270 & -0.1395 & -260 & -0.1345 & 60 & 0.126 \\
\hline 12 & -270 & -0.143 & -270 & -0.1375 & 70 & 0.124 \\
\hline 13 & -290 & -0.145 & -290 & -0.1395 & 70 & 0.1255 \\
\hline 14 & -300 & -0.147 & -300 & -0.141 & 70 & 0.1255 \\
\hline 15 & -320 & -0.1485 & -310 & -0.144 & 70 & 0.127 \\
\hline 16 & -340 & -0.1505 & -330 & -0.1445 & 80 & 0.125 \\
\hline 17 & -360 & -0.151 & -350 & -0.145 & 80 & 0.1285 \\
\hline 18 & -370 & -0.152 & -370 & -0.146 & 70 & 0.131 \\
\hline 19 & -370 & -0.155 & -380 & -0.1485 & 60 & 0.1285 \\
\hline 20 & -380 & -0.1555 & -390 & -0.1505 & 60 & 0.1265 \\
\hline 21 & -380 & -0.157 & -380 & -0.1505 & 60 & 0.1285 \\
\hline 22 & -390 & -0.1585 & -390 & -0.1525 & 50 & 0.13 \\
\hline 23 & -410 & -0.1605 & -410 & -0.1545 & 50 & 0.1375 \\
\hline 24 & -420 & -0.163 & -420 & -0.1555 & 50 & 0.1355 \\
\hline 25 & -430 & -0.1625 & -430 & -0.157 & 50 & 0.134 \\
\hline 26 & -430 & -0.164 & -440 & -0.158 & 50 & 0.132 \\
\hline 27 & -420 & -0.16 & -420 & -0.156 & 60 & 0.1305 \\
\hline 28 & -400 & -0.1585 & -400 & -0.1525 & 70 & 0.129 \\
\hline
\end{tabular}


Table A.3, continued.

\begin{tabular}{|c|c|c|c|c|c|c|}
\hline \multirow[b]{3}{*}{$\begin{array}{l}\text { age } \\
\text { (days) }\end{array}$} & \multicolumn{6}{|c|}{ Virgin 3} \\
\hline & \multicolumn{2}{|c|}{ dry cure 1} & \multicolumn{2}{|c|}{ dry cure 2} & \multicolumn{2}{|c|}{ wet cure 1} \\
\hline & $\begin{array}{c}\text { strain } \\
(\mu \varepsilon)\end{array}$ & $\begin{array}{c}\text { weight change } \\
\text { (lbs) }\end{array}$ & $\begin{array}{l}\text { strain } \\
(\mu \varepsilon)\end{array}$ & $\begin{array}{c}\text { weight change } \\
\text { (lbs) }\end{array}$ & $\begin{array}{l}\text { strain } \\
(\mu \varepsilon)\end{array}$ & $\begin{array}{c}\text { weight change } \\
\text { (lbs) }\end{array}$ \\
\hline 35 & -410 & -0.157 & -420 & -0.1485 & 70 & 0.1335 \\
\hline 42 & -420 & -0.155 & -430 & -0.1465 & 80 & 0.136 \\
\hline 49 & -430 & -0.154 & -440 & -0.146 & 70 & 0.1365 \\
\hline 56 & -440 & -0.153 & -450 & -0.143 & 60 & 0.139 \\
\hline 63 & -460 & -0.151 & -470 & -0.1435 & 100 & 0.144 \\
\hline 70 & -470 & -0.1515 & -480 & -0.1435 & 140 & 0.1415 \\
\hline 77 & -480 & -0.152 & -500 & -0.144 & 140 & 0.141 \\
\hline 87 & -490 & -0.1525 & -510 & -0.145 & 150 & 0.146 \\
\hline 97 & -520 & -0.1545 & -540 & -0.1465 & 150 & 0.1465 \\
\hline 107 & -510 & -0.1545 & -530 & -0.147 & 160 & 0.1465 \\
\hline 117 & -500 & -0.1525 & -510 & -0.1445 & 170 & 0.1475 \\
\hline 121 & -520 & -0.152 & -530 & -0.144 & 130 & 0.1535 \\
\hline 127 & -520 & -0.154 & -540 & -0.1465 & 160 & 0.15 \\
\hline 137 & -520 & -0.156 & -540 & -0.148 & 140 & 0.153 \\
\hline 335 & & -0.1665 & & -0.1585 & 200 & 0.151 \\
\hline 336 & -540 & -0.136 & -610 & -0.1895 & 170 & 0.152 \\
\hline 340 & -620 & -0.1665 & -610 & -0.159 & 180 & 0.158 \\
\hline 341 & -620 & -0.167 & -610 & -0.159 & 180 & 0.1575 \\
\hline 342 & -630 & -0.194 & -610 & -0.1595 & 180 & 0.152 \\
\hline 343 & -370 & 0.0415 & -450 & 0.007 & 150 & 0.1415 \\
\hline 344 & -310 & 0.135 & -310 & 0.1545 & 100 & 0.1365 \\
\hline 345 & -280 & 0.155 & -230 & 0.173 & 110 & 0.1305 \\
\hline 346 & -240 & 0.163 & -210 & 0.1745 & 80 & 0.124 \\
\hline 347 & -230 & 0.1665 & -210 & 0.179 & 40 & 0.12 \\
\hline 349 & -210 & 0.1715 & -190 & 0.18 & 10 & 0.1115 \\
\hline 351 & -200 & 0.1765 & -200 & 0.1845 & -30 & 0.1045 \\
\hline 353 & -200 & 0.1775 & -190 & 0.1855 & -60 & 0.098 \\
\hline 356 & -210 & 0.192 & & 0.199 & -110 & 0.0895 \\
\hline 358 & -190 & 0.1845 & -170 & 0.194 & -130 & 0.084 \\
\hline 360 & -180 & 0.183 & -170 & 0.1975 & -140 & 0.0795 \\
\hline
\end{tabular}


Table A.3, continued.

\begin{tabular}{|c|c|c|c|c|c|c|}
\hline \multirow[b]{3}{*}{$\begin{array}{c}\text { age } \\
\text { (days) }\end{array}$} & \multicolumn{6}{|c|}{ Virgin 3} \\
\hline & \multicolumn{2}{|c|}{ dry cure 1} & \multicolumn{2}{|c|}{ dry cure 2} & \multicolumn{2}{|c|}{ wet cure 1} \\
\hline & $\begin{array}{c}\text { strain } \\
(\mu \varepsilon)\end{array}$ & $\begin{array}{c}\text { weight change } \\
\text { (Ibs) }\end{array}$ & $\begin{array}{c}\text { strain } \\
(\mu \varepsilon)\end{array}$ & $\begin{array}{c}\text { weight change } \\
\text { (lbs) }\end{array}$ & $\begin{array}{c}\text { strain } \\
(\mu \varepsilon)\end{array}$ & $\begin{array}{c}\text { weight change } \\
\text { (lbs) }\end{array}$ \\
\hline 363 & -200 & 0.194 & -190 & 0.1975 & -180 & 0.0735 \\
\hline 365 & -180 & 0.1875 & -190 & 0.204 & -180 & 0.069 \\
\hline 367 & -160 & 0.195 & -170 & 0.209 & -220 & 0.067 \\
\hline 374 & -200 & 0.197 & -160 & 0.2145 & -250 & 0.0585 \\
\hline 375 & -170 & 0.195 & -190 & 0.211 & -240 & 0.0565 \\
\hline 376 & -180 & 0.204 & -180 & 0.219 & -260 & 0.0555 \\
\hline 377 & -220 & 0.1225 & -210 & 0.125 & -100 & 0.1025 \\
\hline 378 & -200 & 0.102 & -210 & 0.103 & -40 & 0.123 \\
\hline 379 & -210 & 0.091 & -210 & 0.092 & 10 & 0.127 \\
\hline 380 & -240 & 0.0805 & -230 & 0.081 & 20 & 0.133 \\
\hline 381 & -260 & 0.0725 & -250 & 0.0735 & 30 & 0.131 \\
\hline 382 & -270 & 0.066 & -270 & 0.067 & 50 & 0.1355 \\
\hline 384 & -260 & 0.0565 & -270 & 0.0565 & 60 & 0.135 \\
\hline 386 & -280 & 0.0485 & -290 & 0.0485 & 80 & 0.1425 \\
\hline 388 & -310 & 0.039 & -300 & 0.0405 & 70 & 0.1365 \\
\hline 391 & -320 & 0.028 & -330 & 0.029 & 80 & 0.143 \\
\hline 393 & -320 & 0.021 & -330 & 0.0225 & 80 & 0.1375 \\
\hline 395 & -350 & 0.0155 & -380 & 0.0165 & 80 & 0.139 \\
\hline 398 & -360 & 0.0085 & -350 & 0.01 & 80 & 0.1395 \\
\hline 400 & -370 & 0.005 & -390 & 0.006 & 100 & 0.1385 \\
\hline 405 & -400 & -0.0025 & -390 & -0.001 & 100 & 0.139 \\
\hline 407 & -410 & -0.0065 & -410 & -0.0045 & 90 & 0.1385 \\
\hline 409 & -430 & -0.0085 & -440 & -0.007 & 90 & 0.1405 \\
\hline 410 & -470 & -0.01 & -440 & -0.0085 & 110 & 0.1415 \\
\hline 412 & -470 & -0.014 & -440 & -0.011 & 100 & 0.142 \\
\hline 414 & -440 & -0.0155 & -420 & -0.014 & 120 & 0.1415 \\
\hline 416 & -450 & -0.0175 & -470 & -0.0155 & 90 & 0.142 \\
\hline 418 & -440 & -0.019 & -450 & -0.018 & 130 & 0.141 \\
\hline 419 & -310 & 0.1695 & -290 & 0.117 & 90 & 0.1305 \\
\hline 420 & -250 & 0.144 & -230 & 0.1595 & 80 & 0.126 \\
\hline
\end{tabular}


Table A.3, continued.

\begin{tabular}{|c|c|c|c|c|c|c|}
\hline \multirow[b]{3}{*}{$\begin{array}{c}\text { age } \\
\text { (days) }\end{array}$} & \multicolumn{6}{|c|}{ Virgin 3} \\
\hline & \multicolumn{2}{|c|}{ dry cure 1} & \multicolumn{2}{|c|}{ dry cure 2} & \multicolumn{2}{|c|}{ wet cure 1} \\
\hline & $\begin{array}{c}\text { strain } \\
(\mu \varepsilon)\end{array}$ & $\begin{array}{l}\text { weight change } \\
\text { (Ibs) }\end{array}$ & $\begin{array}{c}\text { strain } \\
(\mu \varepsilon)\end{array}$ & $\begin{array}{l}\text { weight change } \\
\text { (Ibs) }\end{array}$ & $\begin{array}{c}\text { strain } \\
(\mu \varepsilon)\end{array}$ & $\begin{array}{c}\text { weight change } \\
\text { (lbs) }\end{array}$ \\
\hline 421 & -210 & 0.169 & -170 & 0.1785 & 80 & 0.1215 \\
\hline 422 & -220 & 0.1785 & -130 & 0.1865 & 60 & 0.1165 \\
\hline 423 & -240 & 0.1875 & -200 & 0.194 & 30 & 0.1125 \\
\hline 424 & -210 & 0.1875 & -180 & 0.1935 & -40 & 0.109 \\
\hline 425 & -250 & 0.1985 & -210 & 0.2005 & 110 & 0.105 \\
\hline 426 & -180 & 0.197 & -180 & 0.203 & -70 & 0.1025 \\
\hline 427 & -340 & 0.195 & -320 & 0.202 & -30 & 0.0995 \\
\hline 428 & -220 & 0.194 & -200 & 0.199 & -80 & 0.0965 \\
\hline 430 & -190 & 0.196 & -140 & 0.2075 & -180 & 0.0925 \\
\hline 432 & -180 & 0.2 & -150 & 0.2045 & -70 & 0.089 \\
\hline 434 & -220 & 0.2005 & -200 & 0.2075 & -130 & 0.085 \\
\hline 436 & -130 & 0.201 & -220 & 0.207 & -130 & 0.0835 \\
\hline 438 & -200 & 0.2015 & -180 & 0.208 & -150 & 0.0805 \\
\hline 440 & -210 & 0.201 & -210 & 0.208 & -170 & 0.077 \\
\hline 442 & -160 & 0.2035 & -100 & 0.209 & -150 & 0.0745 \\
\hline 445 & -170 & 0.206 & -150 & 0.213 & -160 & 0.074 \\
\hline 447 & -180 & 0.207 & -170 & 0.211 & -170 & 0.073 \\
\hline 449 & -170 & 0.2065 & -160 & 0.2125 & -200 & 0.071 \\
\hline 452 & -170 & 0.203 & -110 & 0.216 & -190 & 0.0685 \\
\hline 455 & -180 & 0.207 & -170 & 0.2145 & -200 & 0.068 \\
\hline 459 & -160 & 0.212 & -110 & 0.216 & -200 & 0.066 \\
\hline 460 & -190 & 0.211 & $\begin{array}{l}-180 \\
\end{array}$ & 0.219 & -220 & 0.0655 \\
\hline 461 & -180 & 0.2125 & -160 & 0.218 & -210 & 0.066 \\
\hline 462 & -210 & 0.145 & -200 & 0.149 & -30 & 0.1145 \\
\hline 463 & -230 & 0.128 & -220 & 0.129 & 10 & 0.1235 \\
\hline 464 & -230 & 0.1175 & -230 & 0.1175 & 20 & 0.128 \\
\hline 465 & -220 & 0.111 & -210 & 0.11 & 50 & 0.129 \\
\hline 466 & -240 & 0.106 & -230 & 0.1045 & 70 & 0.132 \\
\hline 469 & -240 & 0.096 & -240 & 0.0945 & 70 & 0.1365 \\
\hline 471 & -270 & 0.0895 & -260 & 0.0885 & 90 & 0.1415 \\
\hline
\end{tabular}


Table A.3, continued.

\begin{tabular}{|c|c|c|c|c|c|c|}
\cline { 2 - 7 } \multicolumn{1}{c|}{} & \multicolumn{4}{c|}{ Virgin 3 } \\
\cline { 2 - 7 } \multicolumn{1}{c|}{} & \multicolumn{2}{c|}{ dry cure 1 } & \multicolumn{2}{c|}{ dry cure 2 } \\
$\begin{array}{c}\text { age } \\
(\text { days })\end{array}$ & $\begin{array}{c}\text { strain } \\
(\mu \varepsilon)\end{array}$ & $\begin{array}{c}\text { weight change } \\
(\mathrm{lbs})\end{array}$ & $\begin{array}{c}\text { strain } \\
(\mu \varepsilon)\end{array}$ & $\begin{array}{c}\text { weight change } \\
(\mathrm{lbs})\end{array}$ & $\begin{array}{c}\text { strain } \\
(\mu \varepsilon)\end{array}$ & $\begin{array}{c}\text { weight change } \\
(\mathrm{lbs})\end{array}$ \\
\hline 473 & -270 & 0.0825 & -210 & 0.081 & 90 & 0.1445 \\
\hline 475 & -310 & 0.077 & -300 & 0.0755 & 90 & 0.142 \\
\hline 477 & -340 & 0.0715 & -340 & 0.0705 & 130 & 0.1435 \\
\hline 480 & -300 & 0.065 & -300 & 0.064 & 120 & 0.145 \\
\hline 482 & -300 & 0.0615 & -290 & 0.0625 & 130 & 0.15 \\
\hline 485 & -340 & 0.0565 & -330 & 0.0565 & 140 & 0.149 \\
\hline 489 & -340 & 0.0495 & -340 & 0.0499 & 150 & 0.1475 \\
\hline 497 & -400 & 0.0385 & -390 & 0.0385 & 120 & 0.1507 \\
\hline 500 & -400 & 0.037 & -400 & 0.0375 & 130 & 0.1515 \\
\hline
\end{tabular}


Table A.4

Strain and weight change for Slag RCA.

\begin{tabular}{|c|c|c|c|c|c|c|}
\hline \multirow[b]{3}{*}{$\begin{array}{c}\text { age } \\
\text { (days) }\end{array}$} & \multicolumn{6}{|c|}{ Slag RCA } \\
\hline & \multicolumn{2}{|c|}{ dry cure 1} & \multicolumn{2}{|c|}{ dry cure 2} & \multicolumn{2}{|c|}{ wet cure 1} \\
\hline & $\begin{array}{l}\text { strain } \\
(\mu \varepsilon)\end{array}$ & $\begin{array}{l}\text { weight change } \\
\text { (Ibs) }\end{array}$ & $\begin{array}{l}\text { strain } \\
(\mu \varepsilon)\end{array}$ & $\begin{array}{l}\text { weight change } \\
\text { (Ibs) }\end{array}$ & $\begin{array}{c}\text { strain } \\
(\mu \varepsilon)\end{array}$ & $\begin{array}{l}\text { weight change } \\
\text { (Ibs) }\end{array}$ \\
\hline 0.75 & 0 & 0 & 0 & 0 & 0 & 0 \\
\hline 1 & -30 & -0.048 & 40 & -0.0435 & -130 & 0.057 \\
\hline 2 & 10 & -0.153 & 90 & -0.146 & -10 & 0.0735 \\
\hline 3 & -60 & -0.177 & -150 & -0.168 & 80 & 0.0815 \\
\hline 4 & -80 & -0.19 & -10 & -0.181 & 20 & 0.068 \\
\hline 5 & -90 & -0.2 & -240 & -0.19 & & \\
\hline 6 & -30 & -0.2065 & 50 & -0.1965 & 20 & 0.0665 \\
\hline 7 & -140 & -0.214 & -110 & -0.204 & -20 & 0.0675 \\
\hline 8 & -180 & -0.2195 & -80 & -0.209 & 20 & 0.0645 \\
\hline 9 & -170 & -0.2255 & -80 & -0.214 & 10 & 0.0745 \\
\hline 10 & -180 & -0.228 & -100 & -0.218 & 0 & 0.075 \\
\hline 11 & -220 & -0.232 & -220 & -0.221 & -20 & 0.075 \\
\hline 12 & -240 & -0.232 & -240 & -0.221 & 10 & 0.0755 \\
\hline 13 & -260 & -0.239 & -260 & -0.2275 & 60 & 0.0755 \\
\hline 14 & -280 & -0.2425 & -280 & -0.232 & 0 & 0.0755 \\
\hline 15 & -290 & -0.241 & -290 & -0.2285 & 10 & 0.0745 \\
\hline 16 & -290 & -0.2505 & -310 & -0.2395 & 20 & 0.085 \\
\hline 17 & -310 & -0.2525 & -320 & -0.242 & -10 & 0.0845 \\
\hline 18 & -310 & -0.255 & -310 & -0.244 & 60 & 0.087 \\
\hline 19 & -330 & -0.257 & -300 & -0.2465 & 70 & 0.086 \\
\hline 20 & -320 & -0.26 & -310 & -0.25 & 30 & 0.085 \\
\hline 21 & -370 & -0.2625 & -380 & -0.2525 & 40 & 0.0855 \\
\hline 22 & -360 & -0.265 & -340 & -0.2545 & 110 & 0.0885 \\
\hline 23 & -370 & -0.267 & -360 & -0.257 & 150 & 0.0885 \\
\hline 24 & -360 & -0.268 & -390 & -0.258 & 120 & 0.087 \\
\hline 25 & -350 & -0.27 & -410 & -0.2595 & 190 & 0.0905 \\
\hline 26 & -370 & -0.272 & -360 & -0.2615 & 150 & 0.092 \\
\hline 27 & -390 & -0.274 & -390 & -0.264 & 100 & 0.094 \\
\hline 28 & -370 & -0.2745 & -380 & -0.264 & 120 & 0.0935 \\
\hline
\end{tabular}


Table A.4, continued.

\begin{tabular}{|c|c|c|c|c|c|c|}
\hline \multirow[b]{3}{*}{$\begin{array}{c}\text { age } \\
\text { (days) }\end{array}$} & \multicolumn{6}{|c|}{ Slag RCA } \\
\hline & \multicolumn{2}{|c|}{ dry cure 1} & \multicolumn{2}{|c|}{ dry cure 2} & \multicolumn{2}{|c|}{ wet cure 1} \\
\hline & $\begin{array}{c}\text { strain } \\
(\mu \varepsilon)\end{array}$ & $\begin{array}{l}\text { weight change } \\
\text { (lbs) }\end{array}$ & $\begin{array}{c}\text { strain } \\
(\mu \varepsilon)\end{array}$ & $\begin{array}{l}\text { weight change } \\
\text { (lbs) }\end{array}$ & $\begin{array}{c}\text { strain } \\
(\mu \varepsilon)\end{array}$ & $\begin{array}{l}\text { weight change } \\
\text { (lbs) }\end{array}$ \\
\hline 29 & -390 & -0.277 & -390 & -0.2665 & 170 & 0.094 \\
\hline 30 & -420 & -0.278 & -440 & -0.2685 & 190 & 0.095 \\
\hline 31 & -390 & -0.28 & -400 & -0.2725 & 200 & 0.096 \\
\hline 32 & -390 & -0.2825 & -450 & -0.2765 & 200 & 0.101 \\
\hline 33 & -420 & -0.2855 & -450 & -0.2795 & 200 & 0.103 \\
\hline 34 & -430 & -0.288 & -430 & -0.2835 & 210 & 0.105 \\
\hline 39 & -450 & -0.291 & -480 & -0.2856 & 210 & 0.106 \\
\hline 40 & -450 & -0.2935 & -490 & -0.2858 & 210 & 0.108 \\
\hline 41 & -460 & -0.296 & -470 & -0.286 & 210 & 0.111 \\
\hline 42 & -460 & -0.2975 & -490 & -0.2868 & 210 & 0.111 \\
\hline 48 & -470 & -0.2995 & -490 & -0.2895 & 220 & 0.1235 \\
\hline 55 & -510 & -0.2965 & -520 & -0.3115 & 230 & 0.1265 \\
\hline 62 & -520 & -0.3025 & -520 & -0.292 & 240 & 0.139 \\
\hline 69 & -520 & -0.296 & -530 & -0.2945 & 260 & 0.141 \\
\hline 76 & -520 & -0.3 & -530 & -0.289 & 280 & 0.147 \\
\hline 86 & -520 & -0.2985 & -530 & -0.2905 & 270 & 0.1515 \\
\hline 96 & -520 & -0.2985 & -520 & -0.288 & 260 & 0.1545 \\
\hline 106 & -540 & -0.3005 & -560 & -0.2905 & 290 & 0.1485 \\
\hline 116 & -530 & -0.298 & -540 & -0.288 & 290 & 0.153 \\
\hline 126 & -530 & -0.2965 & -550 & -0.2865 & 300 & 0.159 \\
\hline 136 & -500 & -0.293 & -510 & -0.2815 & 350 & 0.162 \\
\hline 364 & -640 & -0.3085 & -620 & -0.297 & 350 & 0.179 \\
\hline 462 & -620 & -0.297 & -610 & -0.284 & & 0.199 \\
\hline 501 & -620 & -0.2995 & -610 & -0.2865 & 480 & 0.2015 \\
\hline 502 & -310 & 0.167 & -260 & 0.1675 & 460 & 0.179 \\
\hline 503 & -290 & 0.1725 & -270 & 0.1685 & 430 & 0.1705 \\
\hline 504 & -280 & 0.174 & -270 & 0.1705 & 410 & 0.162 \\
\hline 506 & -280 & 0.1825 & -340 & 0.1805 & 380 & 0.148 \\
\hline 508 & -260 & 0.187 & -250 & 0.1155 & 320 & 0.136 \\
\hline 510 & -260 & 0.191 & -280 & 0.19 & 290 & 0.124 \\
\hline
\end{tabular}


Table A.4, continued.

\begin{tabular}{|c|c|c|c|c|c|c|}
\hline \multirow[b]{3}{*}{$\begin{array}{l}\text { age } \\
\text { (days) }\end{array}$} & \multicolumn{6}{|c|}{ Slag RCA } \\
\hline & \multicolumn{2}{|c|}{ dry cure 1} & \multicolumn{2}{|c|}{ dry cure 2} & \multicolumn{2}{|c|}{ wet cure 1} \\
\hline & $\begin{array}{c}\text { strain } \\
(\mu \varepsilon)\end{array}$ & $\begin{array}{c}\text { weight change } \\
\text { (Ibs) }\end{array}$ & $\begin{array}{c}\text { strain } \\
(\mu \varepsilon)\end{array}$ & $\begin{array}{l}\text { weight change } \\
\text { (Ibs) }\end{array}$ & $\begin{array}{c}\text { strain } \\
(\mu \varepsilon)\end{array}$ & $\begin{array}{c}\text { weight change } \\
\text { (Ibs) }\end{array}$ \\
\hline 513 & -200 & 0.1975 & -180 & 0.2005 & 270 & 0.109 \\
\hline 515 & -200 & 0.2035 & -200 & 0.2005 & 220 & 0.099 \\
\hline 517 & -180 & 0.2075 & -180 & 0.2045 & 180 & 0.0865 \\
\hline 520 & -160 & 0.208 & -170 & 0.2125 & 130 & 0.0725 \\
\hline 522 & -160 & 0.214 & -170 & 0.205 & 130 & 0.0615 \\
\hline 524 & -140 & 0.211 & -170 & 0.208 & 90 & 0.0535 \\
\hline 530 & -120 & 0.215 & -160 & 0.219 & 50 & 0.033 \\
\hline 537 & -110 & 0.221 & -150 & 0.2175 & -20 & 0.0135 \\
\hline 544 & -110 & 0.221 & -140 & 0.2145 & -40 & -0.004 \\
\hline 558 & -60 & 0.2335 & -130 & 0.23 & -120 & -0.0305 \\
\hline 572 & -70 & 0.239 & -140 & 0.233 & -140 & -0.052 \\
\hline 586 & -100 & 0.237 & -120 & 0.239 & -160 & -0.063 \\
\hline 601 & 10 & 0.24 & -60 & 0.241 & -230 & -0.074 \\
\hline 676 & & 0.245 & & 0.251 & & -0.119 \\
\hline 681 & & 0.2465 & & 0.2475 & -260 & -0.1225 \\
\hline 682 & 0 & 0.2465 & -70 & 0.254 & -240 & -0.122 \\
\hline 683 & 10 & 0.248 & -70 & 0.2485 & -330 & -0.1225 \\
\hline 684 & -10 & 0.192 & -100 & 0.1945 & 90 & 0.1205 \\
\hline 685 & -30 & 0.1655 & -120 & 0.168 & 150 & 0.1505 \\
\hline 686 & -30 & 0.1405 & -110 & 0.1435 & 200 & 0.1725 \\
\hline 687 & -40 & 0.117 & -110 & 0.122 & 220 & 0.1765 \\
\hline 688 & -30 & 0.099 & -110 & 0.1035 & 250 & 0.176 \\
\hline 690 & & & & & 260 & 0.1845 \\
\hline 692 & & & & & 270 & 0.19 \\
\hline 694 & -90 & 0.03 & -130 & 0.0395 & 280 & 0.1875 \\
\hline 697 & & & & & 280 & 0.1885 \\
\hline 699 & -110 & -0.007 & -160 & 0.0055 & 290 & 0.1915 \\
\hline 701 & -120 & -0.0195 & -150 & -0.006 & 350 & 0.19 \\
\hline 704 & & & & & 310 & 0.1895 \\
\hline 706 & -130 & -0.0425 & -160 & -0.0285 & 290 & 0.192 \\
\hline
\end{tabular}


Table A.4, continued.

\begin{tabular}{|c|c|c|c|c|c|c|}
\hline \multirow[b]{3}{*}{$\begin{array}{c}\text { age } \\
\text { (days) }\end{array}$} & \multicolumn{6}{|c|}{ Slag RCA } \\
\hline & \multicolumn{2}{|c|}{ dry cure 1} & \multicolumn{2}{|c|}{ dry cure 2} & \multicolumn{2}{|c|}{ wet cure 1} \\
\hline & $\begin{array}{c}\text { strain } \\
(\mu \varepsilon)\end{array}$ & $\begin{array}{c}\text { weight change } \\
\text { (lbs) }\end{array}$ & $\begin{array}{c}\text { strain } \\
(\mu \varepsilon)\end{array}$ & $\begin{array}{c}\text { weight change } \\
\text { (lbs) }\end{array}$ & $\begin{array}{c}\text { strain } \\
(\mu \varepsilon)\end{array}$ & $\begin{array}{c}\text { weight change } \\
\text { (Ibs) }\end{array}$ \\
\hline 708 & & -0.0495 & -200 & -0.0355 & 300 & 0.1945 \\
\hline 715 & -180 & -0.074 & -230 & -0.059 & 300 & 0.1935 \\
\hline 716 & -180 & -0.0755 & -230 & -0.061 & 300 & 0.1955 \\
\hline 717 & -190 & -0.079 & -230 & -0.0645 & 300 & 0.2015 \\
\hline 718 & -100 & 0.1355 & -170 & 0.152 & 300 & 0.1725 \\
\hline 719 & -50 & 0.1595 & -120 & 0.1555 & 270 & 0.1595 \\
\hline 720 & -50 & 0.176 & -100 & 0.171 & 290 & 0.15 \\
\hline 721 & -50 & 0.1885 & -110 & 0.18 & 230 & 0.1385 \\
\hline 722 & -70 & 0.1935 & -100 & 0.1925 & 220 & 0.1285 \\
\hline 723 & -50 & 0.203 & -110 & 0.1965 & 190 & 0.1195 \\
\hline 725 & -20 & 0.2135 & -70 & 0.2075 & 180 & 0.102 \\
\hline 727 & -50 & 0.2145 & -100 & 0.211 & 180 & 0.087 \\
\hline 729 & -50 & 0.2125 & -90 & 0.21 & 150 & 0.072 \\
\hline 732 & -40 & 0.216 & -90 & 0.214 & 110 & 0.0525 \\
\hline 734 & -20 & 0.218 & -50 & 0.218 & 130 & 0.0405 \\
\hline 736 & -30 & 0.2205 & -90 & 0.2175 & 130 & 0.0295 \\
\hline 739 & -40 & 0.2205 & -70 & 0.2185 & 110 & 0.017 \\
\hline 741 & -30 & 0.218 & -70 & 0.2155 & 80 & 0.0095 \\
\hline 746 & -40 & & -60 & 0.2195 & 20 & -0.0065 \\
\hline 748 & -30 & 0.219 & -70 & 0.2165 & 40 & -0.0125 \\
\hline 750 & -30 & 0.221 & -110 & 0.2175 & 50 & -0.019 \\
\hline 751 & -30 & 0.2235 & -110 & 0.221 & 0 & -0.0215 \\
\hline 753 & -50 & 0.2215 & -90 & 0.2205 & 10 & -0.027 \\
\hline 755 & -20 & 0.2235 & -70 & 0.19405 & 0 & -0.032 \\
\hline 757 & -10 & 0.2245 & -50 & 0.2225 & -20 & -0.037 \\
\hline 759 & -30 & 0.2235 & -70 & 0.221 & -20 & -0.041 \\
\hline 760 & -50 & 0.169 & -100 & 0.1655 & 210 & 0.1305 \\
\hline 761 & -40 & 0.146 & -90 & 0.144 & 230 & 0.16 \\
\hline 762 & -50 & 0.1275 & -110 & 0.126 & 280 & 0.171 \\
\hline 763 & -40 & 0.111 & -110 & 0.1105 & 340 & 0.178 \\
\hline
\end{tabular}


Table A.4, continued.

\begin{tabular}{|c|c|c|c|c|c|c|}
\hline \multirow[b]{3}{*}{$\begin{array}{c}\text { age } \\
\text { (days) }\end{array}$} & \multicolumn{6}{|c|}{ Slag RCA } \\
\hline & \multicolumn{2}{|c|}{ dry cure 1} & \multicolumn{2}{|c|}{ dry cure 2} & \multicolumn{2}{|c|}{ wet cure 1} \\
\hline & $\begin{array}{c}\text { strain } \\
(\mu \varepsilon)\end{array}$ & $\begin{array}{l}\text { weight change } \\
\text { (Ibs) }\end{array}$ & $\begin{array}{l}\text { strain } \\
(\mu \varepsilon)\end{array}$ & $\begin{array}{l}\text { weight change } \\
\text { (Ibs) }\end{array}$ & $\begin{array}{c}\text { strain } \\
(\mu \varepsilon)\end{array}$ & $\begin{array}{l}\text { weight change } \\
\text { (Ibs) }\end{array}$ \\
\hline 764 & -70 & 0.0965 & -130 & 0.0965 & 300 & 0.184 \\
\hline 765 & -60 & 0.084 & -150 & 0.084 & 300 & 0.182 \\
\hline 766 & -90 & 0.0725 & -160 & 0.075 & 240 & 0.189 \\
\hline 767 & -100 & 0.063 & -150 & 0.066 & 250 & 0.19 \\
\hline 768 & -100 & 0.055 & -150 & 0.0585 & 260 & 0.1875 \\
\hline 769 & -110 & 0.047 & -150 & 0.0515 & 240 & 0.186 \\
\hline 771 & -130 & 0.0355 & -230 & 0.041 & 210 & 0.188 \\
\hline 773 & -130 & 0.024 & -150 & 0.031 & 220 & 0.1895 \\
\hline 775 & -130 & 0.013 & -210 & 0.0205 & 280 & 0.1915 \\
\hline 777 & -140 & 0.0065 & -190 & 0.0145 & 260 & 0.1925 \\
\hline 779 & -160 & -0.002 & -210 & 0.0065 & 290 & 0.192 \\
\hline 781 & -180 & -0.0105 & -220 & -0.002 & 290 & 0.1915 \\
\hline 783 & -140 & -0.018 & -190 & -0.009 & 320 & 0.193 \\
\hline 786 & -150 & -0.0235 & -200 & -0.015 & 330 & 0.193 \\
\hline 788 & -160 & -0.0275 & -210 & -0.0185 & 300 & 0.195 \\
\hline 790 & -180 & -0.0335 & -230 & -0.0245 & 310 & 0.195 \\
\hline 793 & -180 & -0.04 & -230 & -0.031 & 350 & 0.195 \\
\hline 796 & -190 & -0.045 & -250 & -0.0355 & 300 & 0.1705 \\
\hline 800 & -160 & -0.0515 & -220 & -0.042 & 330 & 0.1985 \\
\hline 801 & -210 & -0.0525 & -270 & -0.043 & 320 & 0.199 \\
\hline 802 & -210 & -0.0535 & -270 & -0.0435 & 310 & 0.1995 \\
\hline 803 & -130 & 0.108 & -170 & 0.15 & 280 & 0.1705 \\
\hline 804 & -110 & 0.124 & -150 & 0.1205 & 260 & 0.156 \\
\hline 805 & -90 & 0.1315 & -140 & 0.131 & 260 & 0.144 \\
\hline 806 & -80 & 0.1405 & -110 & 0.1365 & 270 & 0.135 \\
\hline 807 & -80 & 0.1485 & -110 & 0.1465 & 230 & 0.126 \\
\hline 810 & -80 & 0.1665 & -110 & 0.1605 & 250 & 0.1085 \\
\hline 812 & -80 & 0.1745 & -110 & 0.168 & 190 & 0.098 \\
\hline 814 & -40 & 0.1845 & -90 & 0.1725 & 200 & 0.0875 \\
\hline 816 & -40 & 0.1895 & -90 & 0.1815 & 170 & 0.0785 \\
\hline 818 & -30 & 0.1965 & -160 & 0.1885 & 190 & 0.07 \\
\hline
\end{tabular}


Table A.4, continued.

\begin{tabular}{|c|c|c|c|c|c|c|}
\cline { 2 - 7 } \multicolumn{1}{c|}{} & \multicolumn{3}{c|}{ Slag RCA } \\
\cline { 2 - 7 } \multicolumn{1}{c|}{} & \multicolumn{2}{c|}{ dry cure 1 } & \multicolumn{2}{c|}{ dry cure 2 } & \multicolumn{2}{c|}{ wet cure 1 } \\
\hline $\begin{array}{c}\text { age } \\
(\text { days })\end{array}$ & $\begin{array}{c}\text { strain } \\
(\mu \varepsilon)\end{array}$ & $\begin{array}{c}\text { weight change } \\
(\text { lbs })\end{array}$ & $\begin{array}{c}\text { strain } \\
(\mu \varepsilon)\end{array}$ & $\begin{array}{c}\text { weight change } \\
(\mathrm{lbs})\end{array}$ & $\begin{array}{c}\text { strain } \\
(\mu \varepsilon)\end{array}$ & $\begin{array}{c}\text { weight change } \\
(\mathrm{lbs})\end{array}$ \\
\hline 821 & -30 & 0.204 & -160 & 0.195 & 200 & 0.0585 \\
\hline 823 & -30 & 0.2085 & -70 & 0.2005 & 200 & 0.052 \\
\hline 826 & 0 & 0.2125 & -40 & 0.205 & 160 & 0.042 \\
\hline 830 & -10 & 0.2155 & -40 & 0.21 & 150 & 0.0285 \\
\hline 838 & -30 & 0.225 & -70 & 0.217 & 90 & 0.0065 \\
\hline 841 & -20 & 0.2275 & -70 & 0.221 & 80 & 0 \\
\hline
\end{tabular}


Table A.5

Strain and weight change for Limestone RCA.

\begin{tabular}{|c|c|c|c|c|c|c|}
\hline \multirow[b]{3}{*}{$\begin{array}{c}\text { age } \\
\text { (days) }\end{array}$} & \multicolumn{6}{|c|}{ Limestone RCA } \\
\hline & \multicolumn{2}{|c|}{ dry cure 1} & \multicolumn{2}{|c|}{ dry cure 2} & \multicolumn{2}{|c|}{ wet cure 1} \\
\hline & $\begin{array}{c}\text { strain } \\
(\mu \varepsilon)\end{array}$ & $\begin{array}{l}\text { weight change } \\
\text { (lbs) }\end{array}$ & $\begin{array}{c}\text { strain } \\
(\mu \varepsilon)\end{array}$ & $\begin{array}{l}\text { weight change } \\
\text { (Ibs) }\end{array}$ & $\begin{array}{c}\text { strain } \\
(\mu \varepsilon)\end{array}$ & $\begin{array}{c}\text { weight change } \\
\text { (Ibs) }\end{array}$ \\
\hline 0.75 & 0 & 0 & 0 & 0 & 0 & 0 \\
\hline 1 & -20 & -0.048 & -130 & -0.047 & -10 & 0.053 \\
\hline 2 & -90 & -0.166 & -140 & -0.1605 & 20 & 0.061 \\
\hline 3 & -130 & -0.187 & -280 & -0.181 & 0 & 0.0715 \\
\hline 4 & -210 & -0.1975 & -150 & -0.187 & 20 & 0.0655 \\
\hline 5 & -310 & -0.2045 & -230 & -0.199 & -180 & 0.062 \\
\hline 6 & 50 & -0.209 & -150 & -0.2035 & 50 & 0.058 \\
\hline 7 & -130 & -0.2145 & -190 & -0.209 & 30 & 0.058 \\
\hline 8 & -190 & -0.2175 & -260 & -0.2125 & 20 & 0.0565 \\
\hline 9 & -160 & -0.2215 & -260 & -0.216 & 30 & 0.0625 \\
\hline 10 & -180 & -0.2235 & -270 & -0.218 & 20 & 0.064 \\
\hline 11 & -200 & -0.2265 & -310 & -0.221 & 30 & 0.0635 \\
\hline 12 & -200 & -0.2265 & -310 & -0.221 & 30 & 0.0635 \\
\hline 13 & -250 & -0.2315 & -330 & -0.225 & 60 & 0.0715 \\
\hline 14 & -230 & -0.234 & -330 & -0.228 & 50 & 0.068 \\
\hline 15 & -290 & -0.233 & -350 & -0.226 & 40 & 0.069 \\
\hline 16 & -310 & -0.239 & -360 & -0.233 & 40 & 0.0795 \\
\hline 17 & -300 & -0.2405 & -360 & -0.2345 & 50 & 0.0765 \\
\hline 18 & -300 & -0.243 & -360 & -0.2365 & 60 & 0.08 \\
\hline 19 & -270 & -0.244 & -390 & -0.2375 & 60 & 0.0775 \\
\hline 20 & -290 & -0.2465 & -360 & -0.2405 & 70 & 0.078 \\
\hline 21 & -350 & -0.2485 & -440 & -0.242 & 30 & 0.0785 \\
\hline 22 & -330 & -0.2495 & -400 & -0.244 & 80 & 0.082 \\
\hline 23 & -350 & -0.251 & -410 & -0.245 & 90 & 0.081 \\
\hline 24 & -330 & -0.2515 & -410 & -0.246 & 70 & 0.0805 \\
\hline 25 & -350 & -0.253 & -390 & -0.247 & 90 & 0.0845 \\
\hline 26 & -340 & -0.254 & -370 & -0.248 & 100 & 0.085 \\
\hline 27 & -380 & -0.256 & -440 & -0.2505 & 100 & 0.0855 \\
\hline 28 & -360 & -0.256 & -420 & -0.2505 & 90 & 0.088 \\
\hline
\end{tabular}


Table A.5, continued.

\begin{tabular}{|c|c|c|c|c|c|c|}
\hline \multirow[b]{3}{*}{$\begin{array}{c}\text { age } \\
\text { (days) }\end{array}$} & \multicolumn{6}{|c|}{ Limestone RCA } \\
\hline & \multicolumn{2}{|c|}{ dry cure 1} & \multicolumn{2}{|c|}{ dry cure 2} & \multicolumn{2}{|c|}{ wet cure 1} \\
\hline & $\begin{array}{c}\text { strain } \\
(\mu \varepsilon)\end{array}$ & $\begin{array}{c}\text { weight change } \\
\text { (lbs) }\end{array}$ & $\begin{array}{c}\text { strain } \\
(\mu \varepsilon)\end{array}$ & $\begin{array}{c}\text { weight change } \\
\text { (lbs) }\end{array}$ & $\begin{array}{c}\text { strain } \\
(\mu \varepsilon)\end{array}$ & $\begin{array}{c}\text { weight change } \\
\text { (Ibs) }\end{array}$ \\
\hline 29 & -410 & -0.258 & -440 & -0.252 & 100 & 0.089 \\
\hline 30 & -400 & -0.2585 & -460 & -0.2525 & 100 & 0.093 \\
\hline 31 & -380 & -0.2589 & -430 & -0.253 & 110 & 0.094 \\
\hline 32 & -390 & -0.259 & -460 & -0.255 & 110 & 0.094 \\
\hline 33 & -410 & -0.2605 & -480 & -0.257 & 120 & 0.094 \\
\hline 34 & -430 & -0.2652 & -480 & -0.259 & 120 & 0.094 \\
\hline 39 & -460 & -0.279 & -500 & -0.262 & 130 & 0.094 \\
\hline 40 & -470 & -0.274 & -510 & -0.264 & 130 & 0.094 \\
\hline 41 & -470 & -0.289 & -510 & -0.266 & 140 & 0.1065 \\
\hline 42 & -490 & -0.309 & -520 & -0.267 & 140 & 0.1085 \\
\hline 48 & -460 & -0.272 & -520 & -0.266 & 160 & 0.119 \\
\hline 55 & -490 & -0.285 & -540 & -0.273 & 170 & 0.132 \\
\hline 62 & -500 & -0.2715 & -540 & -0.2645 & 220 & 0.132 \\
\hline 69 & -480 & -0.2695 & -560 & -0.266 & 220 & 0.136 \\
\hline 76 & -480 & -0.266 & -550 & -0.26 & 240 & 0.143 \\
\hline 86 & -490 & -0.263 & -550 & -0.2565 & 260 & 0.1435 \\
\hline 96 & -490 & -0.261 & -540 & -0.255 & 280 & 0.1495 \\
\hline 106 & -530 & -0.2515 & -570 & -0.255 & 280 & 0.1445 \\
\hline 116 & -520 & -0.2545 & -550 & -0.256 & 290 & 0.1535 \\
\hline 126 & -510 & -0.256 & -540 & -0.249 & 290 & 0.155 \\
\hline 136 & -480 & -0.25 & -510 & -0.243 & 290 & 0.164 \\
\hline 364 & -650 & -0.2515 & -650 & -0.245 & 270 & 0.1845 \\
\hline 462 & -620 & -0.236 & -650 & -0.229 & 410 & 0.1965 \\
\hline 501 & -630 & -0.2365 & -670 & -0.23 & 390 & 0.194 \\
\hline 502 & -310 & 0.171 & -270 & 0.182 & 380 & 0.172 \\
\hline 503 & -320 & 0.184 & -320 & 0.1885 & 380 & 0.165 \\
\hline 504 & -290 & 0.189 & -330 & 0.19 & 360 & 0.1565 \\
\hline 506 & -280 & 0.1985 & -430 & 0.202 & 340 & 0.143 \\
\hline 508 & -270 & 0.2 & -350 & -0.003 & 290 & 0.133 \\
\hline 510 & -300 & 0.2115 & -350 & 0.2135 & 270 & 0.1225 \\
\hline
\end{tabular}


Table A.5, continued.

\begin{tabular}{|c|c|c|c|c|c|c|}
\hline \multirow[b]{3}{*}{$\begin{array}{c}\text { age } \\
\text { (days) }\end{array}$} & \multicolumn{6}{|c|}{ Limestone RCA } \\
\hline & \multicolumn{2}{|c|}{ dry cure 1} & \multicolumn{2}{|c|}{ dry cure 2} & \multicolumn{2}{|c|}{ wet cure 1} \\
\hline & $\begin{array}{c}\text { strain } \\
(\mu \varepsilon)\end{array}$ & $\begin{array}{l}\text { weight change } \\
\text { (Ibs) }\end{array}$ & $\begin{array}{c}\text { strain } \\
(\mu \varepsilon)\end{array}$ & $\begin{array}{l}\text { weight change } \\
\text { (Ibs) }\end{array}$ & $\begin{array}{c}\text { strain } \\
(\mu \varepsilon)\end{array}$ & $\begin{array}{l}\text { weight change } \\
\text { (Ibs) }\end{array}$ \\
\hline 513 & -200 & 0.2215 & -270 & 0.2385 & 220 & 0.11 \\
\hline 515 & -190 & 0.219 & -270 & 0.2235 & 180 & 0.1025 \\
\hline 517 & -150 & 0.2255 & -230 & 0.2225 & 150 & 0.0935 \\
\hline 520 & -140 & 0.229 & -210 & 0.235 & 120 & 0.0815 \\
\hline 522 & -150 & 0.2315 & -200 & 0.235 & 110 & 0.0735 \\
\hline 524 & -150 & 0.231 & -190 & 0.2325 & 70 & 0.064 \\
\hline 530 & -130 & 0.239 & -190 & 0.2475 & 10 & 0.0465 \\
\hline 537 & -120 & 0.248 & -170 & 0.252 & -60 & 0.0295 \\
\hline 544 & -70 & 0.2475 & -140 & 0.2515 & -60 & 0.015 \\
\hline 558 & -90 & 0.255 & -150 & 0.259 & -130 & -0.0095 \\
\hline 572 & -90 & 0.264 & -170 & 0.2665 & -130 & -0.028 \\
\hline 586 & -60 & 0.2685 & -100 & 0.275 & & -0.037 \\
\hline 601 & -20 & 0.2765 & -90 & 0.2835 & -180 & -0.046 \\
\hline 676 & -20 & 0.283 & -80 & 0.289 & & -0.085 \\
\hline 681 & -20 & 0.2815 & -50 & 0.283 & -340 & -0.0875 \\
\hline 682 & -20 & 0.282 & -80 & 0.287 & -310 & -0.0875 \\
\hline 683 & 0 & 0.284 & -70 & 0.2875 & -290 & -0.088 \\
\hline 684 & -20 & 0.234 & -100 & 0.233 & 120 & 0.1295 \\
\hline 685 & -60 & 0.213 & -140 & 0.2105 & 170 & 0.1575 \\
\hline 686 & -50 & 0.193 & -120 & 0.1905 & 210 & 0.164 \\
\hline 687 & -50 & 0.1755 & -130 & 0.1735 & 240 & 0.171 \\
\hline 688 & -50 & 0.161 & -140 & 0.1585 & 250 & 0.1725 \\
\hline 690 & & & & & 280 & 0.182 \\
\hline 692 & & & & & 280 & 0.185 \\
\hline 694 & -100 & 0.0955 & -170 & 0.0905 & 280 & 0.1785 \\
\hline 697 & & & & & 300 & 0.185 \\
\hline 699 & -120 & 0.048 & -210 & 0.0455 & 300 & 0.185 \\
\hline 701 & -120 & 0.0335 & -210 & 0.0315 & 310 & 0.1815 \\
\hline 704 & & & & & 300 & 0.187 \\
\hline 706 & -150 & 0.0055 & -240 & 0.0045 & 300 & 0.1875 \\
\hline
\end{tabular}


Table A.5, continued.

\begin{tabular}{|c|c|c|c|c|c|c|}
\hline \multirow[b]{3}{*}{$\begin{array}{c}\text { age } \\
\text { (days) }\end{array}$} & \multicolumn{6}{|c|}{ Limestone RCA } \\
\hline & \multicolumn{2}{|c|}{ dry cure 1} & \multicolumn{2}{|c|}{ dry cure 2} & \multicolumn{2}{|c|}{ wet cure 1} \\
\hline & $\begin{array}{c}\text { strain } \\
(\mu \varepsilon)\end{array}$ & $\begin{array}{c}\text { weight change } \\
\text { (lbs) }\end{array}$ & $\begin{array}{c}\text { strain } \\
(\mu \varepsilon)\end{array}$ & $\begin{array}{c}\text { weight change } \\
\text { (lbs) }\end{array}$ & $\begin{array}{c}\text { strain } \\
(\mu \varepsilon)\end{array}$ & $\begin{array}{c}\text { weight change } \\
\text { (Ibs) }\end{array}$ \\
\hline 708 & -190 & -0.002 & -260 & -0.003 & 310 & 0.185 \\
\hline 715 & -210 & -0.027 & -280 & -0.028 & 330 & 0.1875 \\
\hline 716 & -210 & -0.028 & -280 & -0.029 & 310 & 0.1855 \\
\hline 717 & -220 & -0.0313 & -300 & -0.033 & 320 & 0.192 \\
\hline 718 & -100 & 0.156 & -170 & 0.1605 & 300 & 0.167 \\
\hline 719 & -170 & 0.1785 & -150 & 0.186 & 290 & 0.156 \\
\hline 720 & -50 & 0.189 & -130 & 0.191 & 270 & 0.1475 \\
\hline 721 & -40 & 0.197 & -140 & 0.21 & 260 & 0.138 \\
\hline 722 & -40 & 0.202 & -100 & 0.2085 & 240 & 0.131 \\
\hline 723 & -30 & 0.2125 & -130 & 0.219 & 230 & 0.123 \\
\hline 725 & -30 & 0.2205 & -100 & 0.2315 & 220 & 0.1095 \\
\hline 727 & -40 & 0.225 & -130 & 0.23 & 220 & 0.0985 \\
\hline 729 & -40 & 0.2225 & -110 & 0.2295 & 200 & 0.086 \\
\hline 732 & -40 & 0.226 & -120 & 0.2325 & 140 & 0.0675 \\
\hline 734 & -20 & 0.229 & -100 & 0.235 & 160 & 0.056 \\
\hline 736 & -20 & 0.23 & -90 & 0.235 & 140 & 0.046 \\
\hline 739 & -10 & 0.23 & -100 & 0.236 & 150 & 0.034 \\
\hline 741 & -40 & 0.228 & -140 & 0.2355 & 120 & 0.0265 \\
\hline 746 & -10 & 0.2305 & -80 & 0.2405 & 70 & 0.013 \\
\hline 748 & -50 & 0.229 & -90 & 0.2375 & 20 & 0.0075 \\
\hline 750 & -30 & 0.231 & -90 & 0.2375 & 40 & 0.0015 \\
\hline 751 & -20 & 0.2325 & -90 & 0.2405 & 20 & 0 \\
\hline 753 & -20 & 0.2305 & -120 & 0.2415 & 0 & -0.005 \\
\hline 755 & -30 & 0.232 & -110 & 0.241 & 10 & -0.0095 \\
\hline 757 & 0 & 0.232 & -90 & 0.239 & -10 & -0.013 \\
\hline 759 & 0 & 0.234 & -80 & 0.2445 & -10 & -0.017 \\
\hline 760 & -20 & 0.1895 & -110 & 0.2065 & 230 & 0.134 \\
\hline 761 & -30 & 0.1725 & -100 & 0.173 & 260 & 0.158 \\
\hline 762 & -50 & 0.1585 & -120 & 0.1585 & 260 & 0.1715 \\
\hline 763 & -40 & 0.146 & -120 & 0.1455 & 310 & 0.175 \\
\hline
\end{tabular}


Table A.5, continued.

\begin{tabular}{|c|c|c|c|c|c|c|}
\hline \multirow[b]{3}{*}{$\begin{array}{c}\text { age } \\
\text { (days) }\end{array}$} & \multicolumn{6}{|c|}{ Limestone RCA } \\
\hline & \multicolumn{2}{|c|}{ dry cure 1} & \multicolumn{2}{|c|}{ dry cure 2} & \multicolumn{2}{|c|}{ wet cure 1} \\
\hline & $\begin{array}{c}\text { strain } \\
(\mu \varepsilon)\end{array}$ & $\begin{array}{l}\text { weight change } \\
\text { (Ibs) }\end{array}$ & $\begin{array}{c}\text { strain } \\
(\mu \varepsilon)\end{array}$ & $\begin{array}{l}\text { weight change } \\
\text { (Ibs) }\end{array}$ & $\begin{array}{c}\text { strain } \\
(\mu \varepsilon)\end{array}$ & $\begin{array}{l}\text { weight change } \\
\text { (Ibs) }\end{array}$ \\
\hline 764 & -70 & 0.134 & -150 & 0.1335 & 290 & 0.1825 \\
\hline 765 & -110 & 0.1235 & -230 & 0.1235 & 300 & 0.1795 \\
\hline 766 & -110 & 0.1145 & -170 & 0.0495 & 290 & 0.1855 \\
\hline 767 & -120 & 0.106 & -180 & 0.1055 & 230 & 0.1885 \\
\hline 768 & -120 & 0.0985 & -200 & 0.0975 & 300 & 0.1835 \\
\hline 769 & -120 & 0.091 & -190 & 0.09 & 300 & 0.1835 \\
\hline 771 & -250 & 0.08 & -270 & 0.0795 & 230 & 0.1845 \\
\hline 773 & -120 & 0.0685 & -210 & 0.068 & 330 & 0.1865 \\
\hline 775 & -210 & 0.0585 & -240 & 0.058 & 280 & 0.187 \\
\hline 777 & -210 & 0.053 & -230 & 0.052 & 320 & 0.1885 \\
\hline 779 & -220 & 0.0445 & -250 & 0.043 & 310 & 0.186 \\
\hline 781 & -220 & 0.0365 & -270 & 0.035 & 300 & 0.19 \\
\hline 783 & -160 & 0.0295 & -230 & 0.0285 & 350 & 0.189 \\
\hline 786 & -170 & 0.024 & -240 & 0.023 & 350 & 0.1925 \\
\hline 788 & -180 & 0.0205 & -250 & 0.0195 & 330 & 0.191 \\
\hline 790 & -210 & 0.0155 & -280 & 0.0145 & 320 & 0.192 \\
\hline 793 & -200 & 0.01 & -270 & 0.0085 & 340 & 0.19 \\
\hline 796 & -220 & 0.0055 & -290 & 0.0045 & 330 & 0.1945 \\
\hline 800 & -180 & 0.0005 & -250 & -0.001 & 340 & 0.1935 \\
\hline 801 & -230 & -0.0005 & -300 & -0.002 & 330 & 0.1945 \\
\hline 802 & -240 & -0.001 & -310 & -0.0025 & 330 & 0.1955 \\
\hline 803 & -120 & 0.1325 & -190 & 0.1345 & 320 & 0.174 \\
\hline 804 & -90 & 0.1515 & -160 & 0.157 & 310 & 0.1635 \\
\hline 805 & -70 & 0.1625 & -140 & 0.1665 & 280 & 0.1545 \\
\hline 806 & -60 & 0.1695 & -130 & 0.1755 & 290 & 0.1475 \\
\hline 807 & -40 & 0.1765 & -130 & 0.184 & 270 & 0.141 \\
\hline 810 & -40 & 0.1905 & -130 & 0.2005 & 260 & 0.1265 \\
\hline 812 & -40 & 0.1985 & -130 & 0.2075 & 230 & 0.1175 \\
\hline 814 & -10 & 0.2005 & -90 & 0.2145 & 250 & 0.1075 \\
\hline
\end{tabular}


Table A.5, continued.

\begin{tabular}{|c|c|c|c|c|c|c|}
\cline { 2 - 7 } \multicolumn{1}{c|}{} & \multicolumn{3}{c|}{ Limestone RCA } \\
\cline { 2 - 7 } \multicolumn{1}{c|}{} & \multicolumn{2}{c|}{ dry cure 1 } & \multicolumn{2}{c|}{ dry cure 2 } & \multicolumn{2}{c|}{ wet cure 1 } \\
\hline $\begin{array}{c}\text { age } \\
\text { (days) }\end{array}$ & $\begin{array}{c}\text { strain } \\
(\mu \varepsilon)\end{array}$ & $\begin{array}{c}\text { weight change } \\
(\mathrm{lbs})\end{array}$ & $\begin{array}{c}\text { strain } \\
(\mu \varepsilon)\end{array}$ & $\begin{array}{c}\text { weight change } \\
(\mathrm{lbs})\end{array}$ & $\begin{array}{c}\text { strain } \\
(\mu \varepsilon)\end{array}$ & $\begin{array}{c}\text { weight change } \\
(\mathrm{lbs})\end{array}$ \\
\hline 816 & -20 & 0.2085 & -100 & 0.2225 & 230 & 0.0995 \\
\hline 818 & 0 & 0.2135 & -80 & 0.227 & 220 & 0.0915 \\
\hline 821 & -10 & 0.2195 & -80 & 0.2325 & 220 & 0.0815 \\
\hline 823 & 0 & 0.224 & -90 & 0.2375 & 210 & 0.075 \\
\hline 826 & 20 & 0.227 & -70 & 0.239 & 190 & 0.0665 \\
\hline 830 & 0 & 0.2295 & -70 & 0.2405 & 180 & 0.054 \\
\hline 838 & -10 & 0.2295 & -80 & 0.2465 & 140 & 0.032 \\
\hline 841 & 10 & 0.2375 & -60 & 0.246 & 110 & 0.027 \\
\hline
\end{tabular}


Table A.6

Strain and weight change for Gravel RCA 1.

\begin{tabular}{|c|c|c|c|c|c|c|}
\hline \multirow[b]{3}{*}{$\begin{array}{c}\text { age } \\
\text { (days) }\end{array}$} & \multicolumn{6}{|c|}{ Gravel RCA 1} \\
\hline & \multicolumn{2}{|c|}{ dry cure 1} & \multicolumn{2}{|c|}{ dry cure 2} & \multicolumn{2}{|c|}{ wet cure 1} \\
\hline & $\begin{array}{c}\text { strain } \\
(\mu \varepsilon)\end{array}$ & $\begin{array}{l}\text { weight change } \\
\text { (lbs) }\end{array}$ & $\begin{array}{c}\text { strain } \\
(\mu \varepsilon)\end{array}$ & $\begin{array}{c}\text { weight change } \\
\text { (lbs) }\end{array}$ & $\begin{array}{c}\text { strain } \\
(\mu \varepsilon)\end{array}$ & $\begin{array}{c}\text { weight change } \\
\text { (lbs) }\end{array}$ \\
\hline 0.75 & 0 & 0 & 0 & 0 & 0 & 0 \\
\hline 1 & -60 & -0.04 & -50 & -0.0465 & -10 & 0.061 \\
\hline 2 & -110 & -0.103 & -80 & -0.1075 & 50 & 0.0935 \\
\hline 3 & -160 & -0.1265 & -120 & -0.131 & 60 & 0.1045 \\
\hline 4 & -200 & -0.138 & -160 & -0.1425 & 80 & 0.112 \\
\hline 5 & -220 & -0.142 & -180 & -0.1475 & 90 & 0.1185 \\
\hline 6 & -280 & -0.1435 & -200 & -0.1485 & 100 & 0.1185 \\
\hline 7 & -320 & -0.15 & -240 & -0.155 & 100 & 0.121 \\
\hline 8 & -390 & -0.156 & -270 & -0.161 & 110 & 0.1235 \\
\hline 9 & -410 & -0.163 & -290 & -0.165 & 110 & 0.1315 \\
\hline 10 & -430 & -0.1635 & -310 & -0.1645 & 120 & 0.132 \\
\hline 11 & -450 & -0.165 & -330 & -0.1705 & 120 & 0.133 \\
\hline 12 & -470 & -0.171 & -350 & -0.176 & 130 & 0.1335 \\
\hline 13 & -530 & -0.1715 & -380 & -0.1775 & 130 & 0.1335 \\
\hline 14 & -580 & -0.176 & -410 & -0.178 & 130 & 0.139 \\
\hline 15 & -590 & -0.177 & -410 & -0.1805 & 130 & 0.142 \\
\hline 16 & -590 & -0.1765 & -410 & -0.18 & 140 & 0.1415 \\
\hline 17 & -610 & -0.177 & -420 & -0.181 & 150 & 0.144 \\
\hline 18 & -640 & -0.1765 & -430 & -0.1815 & 140 & 0.148 \\
\hline 19 & -640 & -0.179 & -460 & -0.1825 & 150 & 0.145 \\
\hline 20 & -730 & -0.182 & -470 & -0.187 & 150 & 0.149 \\
\hline 21 & -730 & -0.184 & -530 & -0.1905 & 160 & 0.147 \\
\hline 22 & -740 & -0.1855 & -540 & -0.1905 & 150 & 0.149 \\
\hline 23 & -760 & -0.1845 & -540 & -0.19 & 150 & 0.152 \\
\hline 24 & -770 & -0.183 & -540 & -0.1875 & 140 & 0.152 \\
\hline 25 & -760 & -0.1805 & -510 & -0.185 & 160 & 0.1545 \\
\hline 26 & -760 & -0.1705 & -510 & -0.1835 & 160 & 0.1605 \\
\hline 27 & -760 & -0.179 & -510 & -0.1855 & 150 & 0.153 \\
\hline 28 & -810 & -0.183 & -560 & -0.188 & 160 & 0.151 \\
\hline
\end{tabular}


Table A.6, continued.

\begin{tabular}{|c|c|c|c|c|c|c|}
\hline \multirow[b]{3}{*}{$\begin{array}{c}\text { age } \\
\text { (days) }\end{array}$} & \multicolumn{6}{|c|}{ Gravel RCA 1} \\
\hline & \multicolumn{2}{|c|}{ dry cure 1} & \multicolumn{2}{|c|}{ dry cure 2} & \multicolumn{2}{|c|}{ wet cure 1} \\
\hline & $\begin{array}{l}\text { strain } \\
(\mu \varepsilon)\end{array}$ & $\begin{array}{l}\text { weight change } \\
\text { (Ibs) }\end{array}$ & $\begin{array}{c}\text { strain } \\
(\mu \varepsilon)\end{array}$ & $\begin{array}{l}\text { weight change } \\
\text { (Ibs) }\end{array}$ & $\begin{array}{c}\text { strain } \\
(\mu \varepsilon)\end{array}$ & $\begin{array}{l}\text { weight change } \\
\text { (Ibs) }\end{array}$ \\
\hline 35 & -900 & -0.191 & -650 & -0.1975 & 180 & 0.159 \\
\hline 42 & -910 & -0.198 & -690 & -0.202 & 170 & 0.1615 \\
\hline 49 & -930 & -0.2035 & -730 & -0.208 & 180 & 0.1665 \\
\hline 56 & -950 & -0.2075 & -760 & -0.211 & 190 & 0.169 \\
\hline 63 & -990 & -0.2095 & -770 & -0.214 & 180 & 0.1695 \\
\hline 70 & -1000 & -0.209 & -790 & -0.2145 & 180 & 0.169 \\
\hline 77 & -1010 & -0.21 & -800 & -0.214 & 170 & 0.1695 \\
\hline 87 & -1020 & -0.2115 & -810 & -0.2165 & 190 & 0.174 \\
\hline 97 & -1020 & -0.213 & -820 & -0.218 & 180 & 0.1785 \\
\hline 107 & -1020 & -0.213 & -810 & -0.218 & 190 & 0.183 \\
\hline 117 & -1040 & -0.2145 & -820 & -0.219 & 190 & 0.183 \\
\hline 127 & -1050 & -0.2135 & -830 & -0.218 & 180 & 0.179 \\
\hline 136 & -1080 & -0.215 & -820 & -0.2195 & 180 & 0.1785 \\
\hline 253 & -1180 & -0.226 & -950 & -0.2305 & 170 & 0.19 \\
\hline 351 & -1200 & -0.2105 & -970 & -0.2155 & 260 & 0.208 \\
\hline 390 & -1220 & -0.213 & -1000 & -0.2195 & 300 & 0.21 \\
\hline 391 & -870 & 0.175 & -600 & 0.175 & 270 & 0.1875 \\
\hline 392 & -860 & 0.1805 & -600 & 0.181 & 270 & 0.1765 \\
\hline 393 & -880 & 0.1825 & -570 & 0.1855 & 230 & 0.1645 \\
\hline 395 & -850 & 0.1975 & -580 & 0.2075 & 200 & 0.141 \\
\hline 397 & -850 & 0.2145 & -550 & 0.213 & 130 & 0.122 \\
\hline 399 & -810 & 0.225 & -690 & 0.2285 & 50 & 0.104 \\
\hline 402 & -810 & 0.2285 & -530 & 0.235 & -40 & 0.083 \\
\hline 404 & -800 & 0.2295 & -520 & 0.243 & -90 & 0.071 \\
\hline 406 & -790 & 0.2395 & -530 & 0.244 & -130 & 0.0585 \\
\hline 409 & -760 & 0.2455 & & 0.2475 & -190 & 0.044 \\
\hline 411 & -760 & 0.254 & -490 & 0.2545 & -190 & 0.034 \\
\hline 413 & -750 & 0.25 & -480 & 0.2585 & -260 & 0.0255 \\
\hline 419 & -710 & 0.26 & -490 & 0.263 & -350 & 0.008 \\
\hline 426 & -720 & 0.265 & -450 & 0.2725 & -370 & -0.0085 \\
\hline
\end{tabular}


Table A.6, continued.

\begin{tabular}{|c|c|c|c|c|c|c|}
\hline \multirow[b]{3}{*}{$\begin{array}{l}\text { age } \\
\text { (days) }\end{array}$} & \multicolumn{6}{|c|}{ Gravel RCA 1} \\
\hline & \multicolumn{2}{|c|}{ dry cure 1} & \multicolumn{2}{|c|}{ dry cure 2} & \multicolumn{2}{|c|}{ wet cure 1} \\
\hline & $\begin{array}{c}\text { strain } \\
(\mu \varepsilon)\end{array}$ & $\begin{array}{c}\text { weight change } \\
\text { (Ibs) }\end{array}$ & $\begin{array}{c}\text { strain } \\
(\mu \varepsilon)\end{array}$ & $\begin{array}{l}\text { weight change } \\
\text { (lbs) }\end{array}$ & $\begin{array}{c}\text { strain } \\
(\mu \varepsilon)\end{array}$ & $\begin{array}{c}\text { weight change } \\
\text { (Ibs) }\end{array}$ \\
\hline 433 & -690 & 0.2655 & -430 & 0.2715 & -460 & -0.023 \\
\hline 447 & -690 & 0.2775 & -410 & 0.283 & -530 & -0.0435 \\
\hline 461 & -670 & 0.285 & -390 & 0.286 & -540 & -0.06 \\
\hline 475 & -670 & 0.286 & -430 & 0.2995 & -560 & -0.0645 \\
\hline 490 & -560 & 0.291 & -390 & 0.2965 & -620 & -0.0705 \\
\hline 565 & & 0.3025 & -390 & 0.3115 & & -0.1005 \\
\hline 570 & & 0.298 & -410 & 0.31 & -660 & -0.102 \\
\hline 571 & -610 & 0.309 & -370 & 0.3115 & -720 & -0.102 \\
\hline 572 & -610 & 0.308 & -380 & 0.312 & -670 & -0.103 \\
\hline 573 & -620 & 0.2395 & -380 & 0.219 & -80 & 0.172 \\
\hline 574 & -660 & 0.206 & -420 & 0.182 & -30 & 0.201 \\
\hline 575 & -640 & 0.1825 & -400 & 0.156 & 20 & 0.2055 \\
\hline 576 & -650 & 0.1625 & -400 & 0.1355 & 60 & 0.206 \\
\hline 577 & -660 & 0.1455 & -420 & 0.12 & 90 & 0.206 \\
\hline 579 & & & & & 100 & 0.213 \\
\hline 581 & & & & & 110 & 0.22 \\
\hline 583 & -710 & 0.0815 & -470 & 0.0565 & 140 & 0.217 \\
\hline 586 & & & & & 120 & 0.2225 \\
\hline 588 & -740 & 0.0445 & -510 & 0.021 & 140 & 0.2225 \\
\hline 590 & -750 & 0.032 & -540 & 0.0095 & 150 & 0.223 \\
\hline 593 & & & & & 150 & 0.2275 \\
\hline 595 & -790 & 0.0075 & -560 & -0.014 & 140 & 0.222 \\
\hline 597 & -830 & 0.0005 & -610 & -0.02 & 140 & 0.2285 \\
\hline 604 & -880 & -0.0235 & -660 & -0.0415 & 160 & 0.23 \\
\hline 605 & -870 & -0.026 & -650 & -0.043 & 170 & 0.23 \\
\hline 606 & -890 & -0.0285 & -670 & -0.045 & 160 & 0.234 \\
\hline 607 & -720 & 0.155 & -480 & 0.155 & 140 & 0.184 \\
\hline 608 & -690 & 0.175 & -460 & 0.1805 & 100 & 0.1555 \\
\hline 609 & -670 & 0.1905 & -430 & 0.189 & 60 & 0.1345 \\
\hline 610 & -660 & 0.204 & -420 & 0.2025 & 40 & 0.114 \\
\hline
\end{tabular}


Table A.6, continued.

\begin{tabular}{|c|c|c|c|c|c|c|}
\hline \multirow[b]{3}{*}{$\begin{array}{c}\text { age } \\
\text { (days) }\end{array}$} & \multicolumn{6}{|c|}{ Gravel RCA 1} \\
\hline & \multicolumn{2}{|c|}{ dry cure 1} & \multicolumn{2}{|c|}{ dry cure 2} & \multicolumn{2}{|c|}{ wet cure 1} \\
\hline & $\begin{array}{l}\text { strain } \\
(\mu \varepsilon)\end{array}$ & $\begin{array}{l}\text { weight change } \\
\text { (Ibs) }\end{array}$ & $\begin{array}{c}\text { strain } \\
(\mu \varepsilon)\end{array}$ & $\begin{array}{l}\text { weight change } \\
\text { (lbs) }\end{array}$ & $\begin{array}{c}\text { strain } \\
(\mu \varepsilon)\end{array}$ & $\begin{array}{l}\text { weight change } \\
\text { (Ibs) }\end{array}$ \\
\hline 611 & -650 & 0.2035 & -430 & 0.2085 & 10 & 0.098 \\
\hline 612 & -680 & 0.213 & -420 & 0.214 & -10 & 0.0835 \\
\hline 614 & -640 & 0.211 & -390 & 0.214 & -40 & 0.063 \\
\hline 616 & -640 & 0.218 & -420 & 0.2205 & -60 & 0.047 \\
\hline 618 & -660 & 0.2225 & -410 & 0.2185 & -90 & 0.0315 \\
\hline 621 & -630 & 0.2225 & -390 & 0.225 & -140 & 0.014 \\
\hline 623 & -630 & 0.219 & -400 & 0.2255 & -170 & 0.0045 \\
\hline 625 & -650 & 0.2205 & -390 & 0.225 & -200 & -0.0035 \\
\hline 628 & -630 & 0.222 & -390 & 0.226 & -220 & -0.0115 \\
\hline 630 & -640 & 0.216 & -390 & 0.22 & -280 & -0.017 \\
\hline 635 & -620 & 0.2245 & -380 & 0.2305 & -340 & -0.0265 \\
\hline 637 & -630 & 0.2235 & -380 & 0.2275 & -360 & -0.0305 \\
\hline 639 & -640 & 0.2245 & -430 & 0.2295 & -370 & -0.035 \\
\hline 640 & -620 & 0.2285 & -380 & 0.233 & -380 & -0.036 \\
\hline 642 & -680 & 0.2245 & -380 & 0.2335 & -400 & -0.04 \\
\hline 644 & -640 & & -380 & 0.2335 & -410 & -0.0435 \\
\hline 646 & -610 & 0.227 & -350 & 0.233 & -430 & -0.046 \\
\hline 648 & -630 & 0.2285 & -370 & 0.234 & -430 & -0.0485 \\
\hline 649 & -650 & 0.169 & -410 & 0.165 & 60 & 0.197 \\
\hline 650 & -650 & 0.149 & -400 & 0.1435 & 110 & 0.2145 \\
\hline 651 & -660 & 0.133 & -440 & 0.1265 & 120 & 0.223 \\
\hline 652 & -640 & 0.12 & -420 & 0.1125 & 180 & 0.226 \\
\hline 653 & -680 & 0.1085 & -440 & 0.1 & 140 & 0.2315 \\
\hline 654 & -760 & 0.098 & -540 & 0.089 & 140 & 0.2305 \\
\hline 655 & -720 & 0.0885 & -460 & 0.0795 & 120 & 0.2345 \\
\hline 656 & -710 & 0.081 & -480 & 0.071 & 140 & 0.241 \\
\hline 657 & -730 & 0.0735 & -520 & 0.0635 & 100 & 0.2385 \\
\hline 658 & -720 & 0.067 & -480 & 0.057 & 140 & 0.239 \\
\hline 660 & -810 & & -560 & 0.1595 & 170 & 0.2395 \\
\hline 662 & -780 & 0.0475 & -540 & 0.0375 & 150 & 0.2355 \\
\hline
\end{tabular}


Table A.6, continued.

\begin{tabular}{|c|c|c|c|c|c|c|}
\hline \multirow[b]{3}{*}{$\begin{array}{l}\text { age } \\
\text { (days) }\end{array}$} & \multicolumn{6}{|c|}{ Gravel RCA 1} \\
\hline & \multicolumn{2}{|c|}{ dry cure 1} & \multicolumn{2}{|c|}{ dry cure 2} & \multicolumn{2}{|c|}{ wet cure 1} \\
\hline & $\begin{array}{c}\text { strain } \\
(\mu \varepsilon)\end{array}$ & $\begin{array}{l}\text { weight change } \\
\text { (lbs) }\end{array}$ & $\begin{array}{c}\text { strain } \\
(\mu \varepsilon)\end{array}$ & $\begin{array}{l}\text { weight change } \\
\text { (lbs) }\end{array}$ & $\begin{array}{c}\text { strain } \\
(\mu \varepsilon)\end{array}$ & $\begin{array}{l}\text { weight change } \\
\text { (lbs) }\end{array}$ \\
\hline 664 & -800 & 0.039 & -550 & 0.0275 & 130 & 0.24 \\
\hline 666 & -800 & 0.033 & -560 & 0.022 & 170 & 0.2465 \\
\hline 668 & -820 & 0.026 & -580 & 0.015 & 180 & 0.246 \\
\hline 670 & -840 & 0.019 & -600 & 0.008 & 160 & 0.251 \\
\hline 672 & -840 & 0.013 & -590 & 0.0015 & 200 & 0.252 \\
\hline 675 & -840 & 0.0085 & -590 & -0.0025 & 210 & 0.2535 \\
\hline 677 & -870 & 0.0055 & -610 & -0.0055 & 210 & 0.251 \\
\hline 679 & -880 & 0.001 & -630 & -0.0095 & 190 & 0.2575 \\
\hline 682 & -880 & -0.0045 & -630 & -0.015 & 200 & 0.256 \\
\hline 685 & -920 & -0.0075 & -670 & -0.018 & 200 & 0.262 \\
\hline 689 & -920 & -0.012 & -660 & -0.022 & 210 & 0.261 \\
\hline 690 & -930 & -0.012 & -680 & -0.0225 & 200 & 0.264 \\
\hline 691 & -930 & -0.013 & -690 & -0.0235 & 210 & 0.2625 \\
\hline 692 & -770 & 0.133 & -520 & 0.1335 & 140 & 0.1845 \\
\hline 693 & -730 & 0.1495 & -460 & 0.1555 & 110 & 0.1525 \\
\hline 694 & -700 & 0.165 & -430 & 0.1705 & 90 & 0.13 \\
\hline 695 & -680 & 0.1765 & -420 & 0.18 & 80 & 0.114 \\
\hline 696 & -690 & 0.184 & -400 & 0.191 & 50 & 0.1015 \\
\hline 699 & -680 & 0.2025 & -410 & 0.2095 & 40 & 0.078 \\
\hline 701 & -690 & 0.206 & -410 & 0.214 & 10 & 0.0645 \\
\hline 703 & -660 & 0.207 & -390 & 0.215 & 10 & 0.051 \\
\hline 705 & -650 & 0.218 & -380 & 0.223 & -60 & 0.0405 \\
\hline 707 & -620 & 0.219 & -370 & 0.225 & -120 & 0.0325 \\
\hline 710 & & 0.2225 & -370 & 0.227 & -90 & 0.022 \\
\hline 712 & -620 & 0.2295 & -370 & 0.2305 & -110 & 0.016 \\
\hline 715 & -600 & 0.228 & -350 & 0.231 & -160 & 0.0095 \\
\hline 719 & -630 & 0.2285 & -360 & 0.2325 & -180 & -0.0005 \\
\hline 727 & -640 & & -380 & 0.235 & -270 & -0.0155 \\
\hline 730 & -620 & 0.234 & -360 & 0.2355 & -280 & -0.0175 \\
\hline
\end{tabular}


Table A.7

Strain and weight change for Gravel RCA 2.

\begin{tabular}{|c|c|c|c|c|c|c|}
\hline \multirow[b]{3}{*}{$\begin{array}{c}\text { age } \\
\text { (days) }\end{array}$} & \multicolumn{6}{|c|}{ Gravel RCA 2} \\
\hline & \multicolumn{2}{|c|}{ dry cure 1} & \multicolumn{2}{|c|}{ dry cure 2} & \multicolumn{2}{|c|}{ wet cure 1} \\
\hline & $\begin{array}{l}\text { strain } \\
(\mu \varepsilon)\end{array}$ & $\begin{array}{l}\text { weight change } \\
\text { (lbs) }\end{array}$ & $\begin{array}{l}\text { strain } \\
(\mu \varepsilon)\end{array}$ & $\begin{array}{l}\text { weight change } \\
\text { (lbs) }\end{array}$ & $\begin{array}{c}\text { strain } \\
(\mu \varepsilon)\end{array}$ & $\begin{array}{l}\text { weight change } \\
\text { (lbs) }\end{array}$ \\
\hline 0.75 & 0 & 0 & 0 & 0 & 0 & 0 \\
\hline 1 & -80 & -0.0145 & -60 & -0.037 & -110 & 0.054 \\
\hline 2 & -120 & -0.053 & -140 & -0.077 & -100 & 0.0755 \\
\hline 3 & -180 & -0.066 & -190 & -0.0905 & -90 & 0.082 \\
\hline 4 & -240 & -0.0725 & -240 & -0.0975 & -60 & 0.087 \\
\hline 5 & -250 & -0.075 & -250 & -0.1005 & -60 & 0.0915 \\
\hline 6 & -250 & -0.075 & -280 & -0.101 & -40 & 0.099 \\
\hline 7 & -290 & -0.0865 & -290 & -0.104 & -40 & 0.098 \\
\hline 8 & -320 & -0.0795 & -310 & -0.1045 & -30 & 0.1 \\
\hline 9 & -340 & -0.0875 & -360 & -0.114 & -30 & 0.1055 \\
\hline 10 & -360 & -0.097 & -380 & -0.123 & -20 & 0.107 \\
\hline 11 & -400 & -0.1015 & -410 & -0.1285 & -10 & 0.1095 \\
\hline 12 & -430 & -0.1 & -420 & -0.132 & -10 & 0.111 \\
\hline 13 & -460 & -0.1095 & -430 & -0.135 & 0 & 0.1115 \\
\hline 14 & -480 & -0.1065 & -460 & -0.137 & 10 & 0.114 \\
\hline 15 & -500 & -0.109 & -490 & -0.137 & 10 & 0.1115 \\
\hline 16 & -500 & -0.113 & -490 & -0.137 & 10 & 0.112 \\
\hline 17 & -520 & -0.1125 & -500 & -0.14 & 20 & 0.113 \\
\hline 18 & -530 & -0.114 & -520 & -0.1395 & 10 & 0.114 \\
\hline 19 & -530 & -0.1145 & -530 & -0.142 & 10 & 0.1145 \\
\hline 20 & -540 & -0.116 & -540 & -0.1435 & 10 & 0.116 \\
\hline 21 & -550 & -0.12 & -550 & -0.143 & 10 & 0.117 \\
\hline 22 & -570 & -0.1195 & -560 & -0.147 & 10 & 0.118 \\
\hline 23 & -580 & -0.1225 & -570 & -0.149 & 10 & 0.118 \\
\hline 24 & -590 & -0.121 & -570 & -0.1495 & 10 & 0.1195 \\
\hline 25 & -600 & -0.1255 & -580 & -0.1525 & 10 & 0.1185 \\
\hline 26 & -610 & -0.127 & -590 & -0.155 & 10 & 0.1185 \\
\hline 27 & -620 & -0.1275 & -600 & -0.156 & 10 & 0.119 \\
\hline 28 & -640 & -0.1295 & -630 & -0.1585 & 20 & 0.1235 \\
\hline
\end{tabular}


Table A.7, continued.

\begin{tabular}{|c|c|c|c|c|c|c|}
\hline \multirow[b]{3}{*}{$\begin{array}{c}\text { age } \\
\text { (days) }\end{array}$} & \multicolumn{6}{|c|}{ Gravel RCA 2} \\
\hline & \multicolumn{2}{|c|}{ dry cure 1} & \multicolumn{2}{|c|}{ dry cure 2} & \multicolumn{2}{|c|}{ wet cure 1} \\
\hline & $\begin{array}{c}\text { strain } \\
(\mu \varepsilon)\end{array}$ & $\begin{array}{l}\text { weight change } \\
\text { (Ibs) }\end{array}$ & $\begin{array}{c}\text { strain } \\
(\mu \varepsilon)\end{array}$ & $\begin{array}{l}\text { weight change } \\
\text { (Ibs) }\end{array}$ & $\begin{array}{c}\text { strain } \\
(\mu \varepsilon)\end{array}$ & $\begin{array}{c}\text { weight change } \\
\text { (lbs) }\end{array}$ \\
\hline 35 & -660 & -0.135 & -650 & -0.164 & 30 & 0.125 \\
\hline 42 & -690 & -0.141 & -680 & -0.1685 & 20 & 0.1275 \\
\hline 49 & -710 & -0.1415 & -700 & -0.1715 & 20 & 0.126 \\
\hline 56 & -740 & -0.143 & -720 & -0.1735 & 20 & 0.132 \\
\hline 63 & -750 & -0.147 & -740 & -0.1765 & 20 & 0.135 \\
\hline 70 & -760 & -0.1455 & -750 & -0.174 & 30 & 0.133 \\
\hline 77 & -760 & -0.15 & -750 & -0.181 & 30 & 0.138 \\
\hline 87 & -750 & -0.151 & -740 & -0.182 & 50 & 0.1435 \\
\hline 97 & -760 & -0.1545 & -760 & -0.185 & 50 & 0.1395 \\
\hline 107 & -770 & -0.1515 & -770 & -0.184 & 60 & 0.1415 \\
\hline 117 & -790 & -0.155 & -780 & -0.1875 & 50 & 0.1395 \\
\hline 127 & -820 & -0.1445 & -800 & -0.1815 & 50 & 0.133 \\
\hline 137 & -840 & -0.133 & -820 & -0.1755 & 50 & \\
\hline 234 & -880 & -0.1715 & -890 & -0.206 & 40 & 0.1605 \\
\hline 332 & -860 & -0.1615 & -860 & -0.1955 & 180 & 0.1715 \\
\hline 371 & -860 & -0.1645 & -850 & -0.199 & 210 & 0.183 \\
\hline 372 & -490 & 0.171 & -440 & 0.152 & 180 & 0.1535 \\
\hline 373 & -460 & 0.179 & -430 & 0.1615 & 170 & 0.1415 \\
\hline 374 & -440 & 0.182 & -410 & 0.165 & 120 & 0.1305 \\
\hline 376 & -460 & 0.1905 & -480 & 0.1715 & 90 & 0.112 \\
\hline 378 & -440 & 0.188 & -400 & 0.174 & 40 & 0.0985 \\
\hline 380 & -550 & 0.2025 & -410 & 0.1845 & -20 & 0.09 \\
\hline 383 & -400 & 0.207 & -340 & 0.187 & -50 & 0.081 \\
\hline 385 & -390 & 0.2125 & -340 & 0.193 & -90 & 0.076 \\
\hline 387 & -380 & 0.2085 & -340 & 0.1925 & -120 & 0.07 \\
\hline 390 & -370 & 0.213 & -320 & 0.1945 & -140 & 0.063 \\
\hline 392 & -370 & 0.2145 & -300 & 0.1945 & -160 & 0.0585 \\
\hline 394 & -360 & 0.213 & -320 & 0.195 & -180 & 0.0545 \\
\hline 400 & -350 & 0.222 & -320 & 0.204 & -230 & 0.044 \\
\hline 407 & -340 & 0.2335 & -300 & 0.2105 & -270 & 0.035 \\
\hline
\end{tabular}


Table A.7, continued.

\begin{tabular}{|c|c|c|c|c|c|c|}
\hline \multirow[b]{3}{*}{$\begin{array}{c}\text { age } \\
\text { (days) }\end{array}$} & \multicolumn{6}{|c|}{ Gravel RCA 2} \\
\hline & \multicolumn{2}{|c|}{ dry cure 1} & \multicolumn{2}{|c|}{ dry cure 2} & \multicolumn{2}{|c|}{ wet cure 1} \\
\hline & $\begin{array}{l}\text { strain } \\
(\mu \varepsilon)\end{array}$ & $\begin{array}{l}\text { weight change } \\
\text { (lbs) }\end{array}$ & $\begin{array}{l}\text { strain } \\
(\mu \varepsilon)\end{array}$ & $\begin{array}{l}\text { weight change } \\
\text { (lbs) }\end{array}$ & $\begin{array}{l}\text { strain } \\
(\mu \varepsilon)\end{array}$ & $\begin{array}{l}\text { weight change } \\
\text { (Ibs) }\end{array}$ \\
\hline 414 & -330 & 0.2285 & -270 & & -310 & 0.027 \\
\hline 428 & -350 & 0.2335 & -310 & 0.213 & -350 & 0.0125 \\
\hline 442 & -330 & 0.236 & -280 & 0.218 & -370 & 0 \\
\hline 456 & -310 & 0.2435 & -280 & 0.226 & & -0.005 \\
\hline 471 & -290 & 0.25 & -220 & 0.2296 & -400 & -0.0105 \\
\hline 546 & -210 & 0.2555 & -160 & 0.2375 & & -0.04 \\
\hline 551 & -260 & 0.252 & -210 & 0.2365 & -540 & -0.042 \\
\hline 552 & -280 & 0.2355 & -220 & 0.242 & -470 & -0.042 \\
\hline 553 & -270 & 0.26 & -230 & 0.2415 & -530 & -0.043 \\
\hline 554 & -280 & 0.1995 & -220 & 0.1815 & -70 & 0.1205 \\
\hline 555 & -320 & 0.176 & -270 & 0.1585 & -10 & 0.1355 \\
\hline 556 & -310 & 0.16 & -270 & 0.1425 & 30 & 0.149 \\
\hline 557 & -310 & 0.147 & -270 & 0.13 & 60 & 0.1575 \\
\hline 558 & -330 & 0.137 & -280 & 0.1195 & 80 & 0.1585 \\
\hline 560 & & & & & 100 & 0.167 \\
\hline 562 & & & & & 110 & 0.171 \\
\hline 564 & -380 & 0.0995 & -340 & 0.0815 & 120 & 0.168 \\
\hline 567 & & & & & 120 & 0.173 \\
\hline 569 & -430 & 0.0775 & -380 & 0.059 & 110 & 0.17 \\
\hline 571 & -430 & 0.07 & -370 & 0.051 & 150 & 0.1735 \\
\hline 574 & & & & & 140 & 0.174 \\
\hline 576 & -460 & 0.0545 & -490 & 0.035 & 130 & 0.177 \\
\hline 578 & -490 & 0.05 & -450 & 0.03 & 140 & 0.1785 \\
\hline 585 & -530 & 0.035 & -490 & 0.0145 & 160 & 0.172 \\
\hline 586 & -520 & 0.033 & -480 & 0.0135 & 160 & 0.1715 \\
\hline 587 & -530 & 0.031 & -500 & 0.01 & 160 & 0.182 \\
\hline 588 & -390 & 0.1655 & -360 & 0.148 & 110 & 0.1455 \\
\hline 589 & -360 & 0.1865 & -310 & 0.171 & 100 & 0.1275 \\
\hline 590 & -330 & 0.197 & -290 & 0.179 & 70 & 0.1165 \\
\hline 591 & -330 & 0.2085 & -280 & 0.1835 & 50 & 0.105 \\
\hline
\end{tabular}


Table A.7, continued.

\begin{tabular}{|c|c|c|c|c|c|c|}
\hline \multirow[b]{3}{*}{$\begin{array}{c}\text { age } \\
\text { (days) }\end{array}$} & \multicolumn{6}{|c|}{ Gravel RCA 2} \\
\hline & \multicolumn{2}{|c|}{ dry cure 1} & \multicolumn{2}{|c|}{ dry cure 2} & \multicolumn{2}{|c|}{ wet cure 1} \\
\hline & $\begin{array}{c}\text { strain } \\
(\mu \varepsilon)\end{array}$ & $\begin{array}{l}\text { weight change } \\
\text { (Ibs) }\end{array}$ & $\begin{array}{c}\text { strain } \\
(\mu \varepsilon)\end{array}$ & $\begin{array}{l}\text { weight change } \\
\text { (Ibs) }\end{array}$ & $\begin{array}{c}\text { strain } \\
(\mu \varepsilon)\end{array}$ & $\begin{array}{c}\text { weight change } \\
\text { (lbs) }\end{array}$ \\
\hline 592 & -340 & 0.2095 & -270 & 0.188 & 40 & 0.096 \\
\hline 593 & -330 & 0.2115 & -290 & 0.1965 & 10 & 0.089 \\
\hline 595 & -310 & 0.214 & -270 & 0.1965 & -10 & 0.079 \\
\hline 597 & -330 & 0.2175 & -270 & 0.203 & -30 & 0.071 \\
\hline 599 & -290 & 0.221 & -270 & 0.2065 & -60 & 0.062 \\
\hline 602 & -320 & 0.223 & -270 & 0.209 & -70 & 0.0525 \\
\hline 604 & -330 & 0.222 & -270 & 0.2075 & 110 & 0.046 \\
\hline 606 & -310 & 0.223 & -270 & 0.208 & -130 & 0.0415 \\
\hline 609 & -310 & 0.225 & -260 & 0.2115 & -140 & 0.0355 \\
\hline 611 & -360 & 0.2225 & -310 & 0.2085 & -140 & 0.032 \\
\hline 616 & -320 & 0.226 & -270 & 0.211 & -220 & 0.0255 \\
\hline 618 & -330 & 0.2265 & -280 & 0.2095 & -200 & 0.0225 \\
\hline 620 & -340 & 0.2255 & -280 & 0.2085 & -260 & 0.02 \\
\hline 621 & -330 & 0.2265 & -290 & 0.211 & -230 & 0.0185 \\
\hline 623 & -330 & 0.225 & -260 & 0.2125 & -250 & 0.016 \\
\hline 625 & -320 & 0.2275 & -260 & 0.2115 & -260 & 0.013 \\
\hline 627 & -290 & 0.2255 & -250 & 0.2125 & -290 & 0.011 \\
\hline 629 & -290 & 0.2275 & -250 & 0.2125 & -260 & 0.009 \\
\hline 630 & -350 & 0.183 & -270 & 0.165 & 60 & 0.1425 \\
\hline 631 & -330 & 0.1685 & -270 & 0.1505 & 100 & 0.157 \\
\hline 632 & -350 & 0.1565 & -280 & 0.139 & 130 & 0.161 \\
\hline 633 & -360 & 0.1465 & -280 & 0.1295 & 170 & 0.164 \\
\hline 634 & -360 & 0.138 & -320 & 0.1205 & 150 & 0.171 \\
\hline 635 & -390 & 0.1305 & -370 & 0.1135 & 130 & 0.173 \\
\hline 636 & -390 & 0.1245 & -260 & 0.107 & 130 & 0.176 \\
\hline 637 & -430 & 0.1185 & -350 & 0.101 & 150 & 0.176 \\
\hline 638 & -490 & 0.1135 & -440 & 0.096 & 100 & 0.1795 \\
\hline 639 & -400 & 0.109 & -380 & 0.091 & 150 & 0.1755 \\
\hline 641 & -530 & 0.102 & -470 & 0.0845 & 200 & 0.177 \\
\hline 643 & -570 & 0.0955 & -400 & 0.077 & 140 & 0.178 \\
\hline
\end{tabular}


Table A.7, continued.

\begin{tabular}{|c|c|c|c|c|c|c|}
\hline \multirow[b]{3}{*}{$\begin{array}{l}\text { age } \\
\text { (days) }\end{array}$} & \multicolumn{6}{|c|}{ Gravel RCA 2} \\
\hline & \multicolumn{2}{|c|}{ dry cure 1} & \multicolumn{2}{|c|}{ dry cure 2} & \multicolumn{2}{|c|}{ wet cure 1} \\
\hline & $\begin{array}{c}\text { strain } \\
(\mu \varepsilon)\end{array}$ & $\begin{array}{l}\text { weight change } \\
\text { (Ibs) }\end{array}$ & $\begin{array}{c}\text { strain } \\
(\mu \varepsilon)\end{array}$ & $\begin{array}{c}\text { weight change } \\
\text { (lbs) }\end{array}$ & $\begin{array}{c}\text { strain } \\
(\mu \varepsilon)\end{array}$ & $\begin{array}{c}\text { weight change } \\
\text { (Ibs) }\end{array}$ \\
\hline 645 & -450 & 0.0895 & -430 & 0.071 & 130 & 0.1815 \\
\hline 647 & -460 & 0.086 & -430 & 0.067 & 150 & 0.1805 \\
\hline 649 & -490 & 0.0805 & -450 & 0.0615 & 150 & 0.1845 \\
\hline 651 & -510 & 0.075 & -470 & 0.057 & 160 & 0.1825 \\
\hline 653 & -490 & 0.071 & -450 & 0.052 & 190 & 0.1835 \\
\hline 656 & -500 & 0.068 & -460 & 0.049 & 200 & 0.1845 \\
\hline 658 & -510 & 0.0655 & -470 & 0.0465 & 170 & 0.1855 \\
\hline 660 & -540 & 0.062 & -510 & 0.043 & 180 & 0.185 \\
\hline 663 & -530 & 0.059 & -490 & 0.0395 & 190 & 0.186 \\
\hline 666 & -560 & 0.056 & -520 & 0.037 & 190 & 0.186 \\
\hline 670 & -550 & 0.0525 & -520 & 0.0325 & 220 & 0.1875 \\
\hline 671 & -570 & 0.0515 & -530 & 0.032 & 170 & 0.1875 \\
\hline 672 & -550 & 0.0515 & -530 & 0.0315 & 180 & 0.1895 \\
\hline 673 & -420 & 0.1505 & -380 & 0.1305 & 140 & 0.159 \\
\hline 674 & -380 & 0.162 & -330 & 0.1475 & 110 & 0.1435 \\
\hline 675 & -380 & 0.1735 & -310 & 0.157 & 110 & 0.1315 \\
\hline 676 & -330 & 0.1835 & -270 & 0.165 & 110 & 0.122 \\
\hline 677 & -320 & 0.189 & -270 & 0.1775 & 70 & 0.115 \\
\hline 680 & -340 & 0.203 & -290 & 0.1895 & 80 & 0.0995 \\
\hline 682 & -310 & 0.206 & -270 & 0.1905 & 10 & 0.091 \\
\hline 684 & -300 & 0.209 & -250 & 0.1965 & 30 & 0.0825 \\
\hline 686 & -310 & 0.216 & -250 & 0.2025 & -30 & 0.0765 \\
\hline 688 & -280 & 0.22 & -230 & 0.209 & -60 & 0.0705 \\
\hline 691 & -280 & 0.2245 & -240 & 0.2065 & -30 & 0.0635 \\
\hline 693 & -280 & 0.229 & -220 & 0.2085 & -30 & 0.0595 \\
\hline 696 & -270 & 0.228 & -210 & 0.2105 & -80 & 0.055 \\
\hline 700 & -280 & 0.2275 & -230 & 0.214 & -90 & 0.0475 \\
\hline 708 & -290 & 0.231 & -230 & 0.214 & -160 & 0.037 \\
\hline 711 & -270 & 0.233 & -220 & 0.22 & -160 & 0.0345 \\
\hline
\end{tabular}


Table A.8

Strain and weight change for Twice Recycled RCA.

\begin{tabular}{|c|c|c|c|c|c|c|}
\hline \multirow[b]{3}{*}{$\begin{array}{c}\text { age } \\
\text { (days) }\end{array}$} & \multicolumn{6}{|c|}{ Twice Recycled } \\
\hline & \multicolumn{2}{|c|}{ dry cure 1} & \multicolumn{2}{|c|}{ dry cure 2} & \multicolumn{2}{|c|}{ wet cure 1} \\
\hline & $\begin{array}{l}\text { strain } \\
(\mu \varepsilon)\end{array}$ & $\begin{array}{l}\text { weight change } \\
\text { (lbs) }\end{array}$ & $\begin{array}{l}\text { strain } \\
(\mu \varepsilon)\end{array}$ & $\begin{array}{l}\text { weight change } \\
\text { (Ibs) }\end{array}$ & $\begin{array}{c}\text { strain } \\
(\mu \varepsilon)\end{array}$ & $\begin{array}{c}\text { weight change } \\
\text { (Ibs) }\end{array}$ \\
\hline 0.75 & 0 & 0 & 0 & 0 & 0 & 0 \\
\hline 1 & 20 & -0.063 & 20 & -0.066 & 0 & 0.075 \\
\hline 2 & 0 & -0.0995 & 0 & -0.104 & 30 & 0.0915 \\
\hline 3 & -30 & -0.1115 & -30 & -0.1165 & 40 & 0.097 \\
\hline 4 & -40 & -0.1205 & -50 & -0.1255 & 50 & 0.0965 \\
\hline 5 & -60 & -0.129 & -70 & -0.1345 & 70 & 0.101 \\
\hline 6 & -80 & -0.132 & -80 & -0.1385 & 90 & 0.106 \\
\hline 7 & -100 & -0.137 & -100 & -0.1415 & 90 & 0.1085 \\
\hline 8 & -110 & -0.14 & -120 & -0.1455 & 100 & 0.1125 \\
\hline 9 & -130 & -0.144 & -130 & -0.1515 & 100 & 0.1125 \\
\hline 10 & -150 & -0.146 & -150 & -0.1525 & 110 & 0.112 \\
\hline 11 & -160 & -0.1485 & -170 & -0.1555 & 110 & 0.116 \\
\hline 12 & -180 & -0.151 & -190 & -0.158 & 120 & 0.1155 \\
\hline 13 & -190 & -0.1525 & -200 & -0.1585 & 130 & 0.115 \\
\hline 14 & -190 & -0.1555 & -210 & -0.162 & 130 & 0.1175 \\
\hline 15 & -200 & -0.1575 & -220 & -0.1635 & 140 & 0.117 \\
\hline 16 & -220 & -0.159 & -240 & -0.1655 & 140 & 0.1175 \\
\hline 17 & -230 & -0.163 & -270 & -0.168 & 140 & 0.1165 \\
\hline 18 & -250 & -0.163 & -290 & -0.1685 & 170 & 0.118 \\
\hline 19 & -270 & -0.1655 & -300 & -0.1715 & 170 & 0.1185 \\
\hline 20 & -280 & -0.162 & -320 & -0.174 & 160 & 0.1195 \\
\hline 21 & -280 & -0.162 & -330 & -0.174 & 150 & 0.1205 \\
\hline 22 & -290 & -0.169 & -350 & -0.175 & 160 & 0.1205 \\
\hline 23 & -290 & -0.1705 & -340 & -0.1765 & 150 & 0.122 \\
\hline 24 & -320 & -0.173 & -360 & -0.179 & 150 & 0.126 \\
\hline 25 & -320 & -0.174 & -370 & -0.1805 & 150 & 0.1285 \\
\hline 26 & -330 & -0.1755 & -380 & -0.1815 & 150 & 0.1275 \\
\hline 27 & -330 & -0.177 & -380 & -0.183 & 150 & 0.127 \\
\hline 28 & -340 & -0.1775 & -390 & -0.184 & 150 & 0.1255 \\
\hline
\end{tabular}


Table A.8, continued.

\begin{tabular}{|c|c|c|c|c|c|c|}
\hline \multirow[b]{3}{*}{$\begin{array}{c}\text { age } \\
\text { (days) }\end{array}$} & \multicolumn{6}{|c|}{ Twice Recycled } \\
\hline & \multicolumn{2}{|c|}{ dry cure 1} & \multicolumn{2}{|c|}{ dry cure 2} & \multicolumn{2}{|c|}{ wet cure 1} \\
\hline & $\begin{array}{l}\text { strain } \\
(\mu \varepsilon)\end{array}$ & $\begin{array}{l}\text { weight change } \\
\text { (Ibs) }\end{array}$ & $\begin{array}{l}\text { strain } \\
(\mu \varepsilon)\end{array}$ & $\begin{array}{l}\text { weight change } \\
\text { (lbs) }\end{array}$ & $\begin{array}{c}\text { strain } \\
(\mu \varepsilon)\end{array}$ & $\begin{array}{l}\text { weight change } \\
\text { (Ibs) }\end{array}$ \\
\hline 35 & -300 & -0.171 & -360 & -0.178 & 180 & 0.127 \\
\hline 42 & -340 & -0.17 & -400 & -0.1765 & 170 & 0.13 \\
\hline 49 & -350 & -0.17 & -410 & -0.177 & 190 & 0.133 \\
\hline 56 & -350 & -0.1705 & -420 & -0.1775 & 220 & 0.136 \\
\hline 63 & -370 & -0.1695 & -430 & -0.177 & 280 & 0.1395 \\
\hline 77 & -390 & -0.1695 & -440 & -0.1775 & 360 & 0.142 \\
\hline 87 & -400 & -0.1725 & -450 & -0.1795 & 370 & 0.1435 \\
\hline 97 & -440 & -0.1755 & -500 & -0.1835 & 360 & 0.145 \\
\hline 107 & -450 & -0.1755 & -490 & -0.1835 & 360 & 0.148 \\
\hline 118 & -420 & -0.1735 & -470 & -0.1815 & 380 & 0.1485 \\
\hline 123 & -460 & -0.1745 & -500 & -0.1825 & 340 & 0.146 \\
\hline 127 & -420 & -0.1755 & -490 & -0.1835 & 390 & 0.151 \\
\hline 137 & -450 & -0.179 & -500 & -0.188 & 380 & 0.152 \\
\hline 337 & & -0.1975 & & -0.207 & 500 & 0.1585 \\
\hline 338 & -610 & -0.198 & -510 & -0.206 & 420 & 0.1625 \\
\hline 342 & -540 & -0.1985 & -660 & -0.206 & & \\
\hline 343 & -600 & -0.198 & -670 & -0.206 & & \\
\hline 344 & -600 & -0.199 & -660 & -0.207 & 420 & 0.161 \\
\hline 345 & -330 & 0.044 & -500 & -0.069 & 410 & 0.151 \\
\hline 346 & -270 & 0.112 & -380 & 0.067 & 360 & 0.145 \\
\hline 347 & -220 & 0.1485 & -300 & 0.132 & 370 & 0.138 \\
\hline 348 & -190 & 0.151 & -260 & 0.152 & 340 & \\
\hline 349 & -160 & 0.1545 & -250 & 0.1535 & 300 & 0.124 \\
\hline 351 & -160 & 0.16 & -230 & 0.162 & 300 & 0.114 \\
\hline 353 & -150 & 0.1695 & -220 & 0.1735 & 270 & 0.105 \\
\hline 355 & -140 & 0.177 & -180 & 0.1735 & 240 & 0.096 \\
\hline 358 & -140 & 0.179 & -220 & 0.178 & 210 & 0.0835 \\
\hline 360 & -130 & 0.1835 & -190 & 0.184 & 180 & 0.0765 \\
\hline 362 & -110 & 0.1835 & -170 & 0.188 & 160 & 0.0695 \\
\hline 365 & -120 & 0.197 & -190 & 0.1885 & 120 & 0.0615 \\
\hline
\end{tabular}


Table A.8, continued.

\begin{tabular}{|c|c|c|c|c|c|c|}
\hline \multirow[b]{3}{*}{$\begin{array}{l}\text { age } \\
\text { (days) }\end{array}$} & \multicolumn{6}{|c|}{ Twice Recycled } \\
\hline & \multicolumn{2}{|c|}{ dry cure 1} & \multicolumn{2}{|c|}{ dry cure 2} & \multicolumn{2}{|c|}{ wet cure 1} \\
\hline & $\begin{array}{c}\text { strain } \\
(\mu \varepsilon)\end{array}$ & $\begin{array}{c}\text { weight change } \\
\text { (Ibs) }\end{array}$ & $\begin{array}{c}\text { strain } \\
(\mu \varepsilon)\end{array}$ & $\begin{array}{l}\text { weight change } \\
\text { (Ibs) }\end{array}$ & $\begin{array}{c}\text { strain } \\
(\mu \varepsilon)\end{array}$ & $\begin{array}{c}\text { weight change } \\
\text { (Ibs) }\end{array}$ \\
\hline 367 & -110 & 0.1895 & -190 & 0.192 & 110 & 0.0565 \\
\hline 369 & -90 & 0.192 & & 0.1905 & 70 & 0.0525 \\
\hline 376 & -80 & 0.1925 & -150 & 0.1955 & 40 & 0.04 \\
\hline 377 & -80 & 0.1985 & -170 & 0.192 & 0 & 0.039 \\
\hline 378 & -90 & 0.202 & -180 & 0.2065 & 30 & 0.037 \\
\hline 379 & & 0.113 & -190 & 0.1115 & 170 & 0.0955 \\
\hline 380 & -120 & 0.0945 & -210 & 0.0935 & 220 & 0.1115 \\
\hline 381 & -120 & 0.0845 & -210 & 0.0845 & 290 & 0.117 \\
\hline 382 & -140 & 0.076 & -230 & 0.076 & 300 & 0.1305 \\
\hline 383 & -140 & 0.0685 & -230 & 0.069 & 290 & 0.1255 \\
\hline 384 & -160 & 0.0625 & -240 & 0.0635 & 240 & 0.1315 \\
\hline 386 & -160 & 0.0525 & -250 & 0.054 & 320 & 0.1315 \\
\hline 388 & -160 & 0.044 & -230 & 0.0465 & 310 & 0.143 \\
\hline 390 & -170 & 0.0355 & -260 & 0.038 & 320 & 0.133 \\
\hline 393 & -160 & 0.024 & -290 & 0.0265 & 330 & 0.1355 \\
\hline 395 & -180 & 0.0165 & -280 & 0.02 & 350 & 0.1395 \\
\hline 397 & -220 & 0.011 & -280 & 0.014 & 330 & 0.1405 \\
\hline 400 & -230 & 0.0035 & -290 & 0.0065 & 360 & 0.144 \\
\hline 402 & -300 & -0.0105 & -280 & 0.0015 & 340 & 0.145 \\
\hline 407 & -240 & -0.009 & -330 & -0.007 & 340 & 0.142 \\
\hline 409 & -270 & -0.014 & -360 & -0.011 & 360 & 0.1445 \\
\hline 411 & -280 & -0.017 & -380 & -0.015 & 360 & 0.146 \\
\hline 412 & -280 & -0.0185 & -390 & -0.017 & 350 & 0.147 \\
\hline 414 & -300 & -0.0215 & -380 & -0.021 & 360 & 0.147 \\
\hline 416 & -320 & -0.0255 & -350 & -0.0235 & 370 & 0.1485 \\
\hline 418 & -310 & -0.0275 & -360 & -0.026 & 390 & 0.148 \\
\hline 420 & -310 & -0.03 & -360 & -0.0285 & 390 & 0.1475 \\
\hline 421 & -190 & 0.106 & -270 & 0.0945 & 350 & 0.134 \\
\hline 422 & -170 & 0.1415 & -230 & 0.138 & 340 & 0.1285 \\
\hline 423 & -110 & 0.153 & -170 & 0.1565 & 320 & 0.123 \\
\hline
\end{tabular}


Table A.8, continued.

\begin{tabular}{|c|c|c|c|c|c|c|}
\hline \multirow[b]{3}{*}{$\begin{array}{c}\text { age } \\
\text { (days) }\end{array}$} & \multicolumn{6}{|c|}{ Twice Recycled } \\
\hline & \multicolumn{2}{|c|}{ dry cure 1} & \multicolumn{2}{|c|}{ dry cure 2} & \multicolumn{2}{|c|}{ wet cure 1} \\
\hline & $\begin{array}{l}\text { strain } \\
(\mu \varepsilon)\end{array}$ & $\begin{array}{l}\text { weight change } \\
\text { (Ibs) }\end{array}$ & $\begin{array}{c}\text { strain } \\
(\mu \varepsilon)\end{array}$ & $\begin{array}{l}\text { weight change } \\
\text { (Ibs) }\end{array}$ & $\begin{array}{c}\text { strain } \\
(\mu \varepsilon)\end{array}$ & $\begin{array}{l}\text { weight change } \\
\text { (Ibs) }\end{array}$ \\
\hline 424 & -50 & 0.1615 & -170 & 0.1675 & 330 & 0.117 \\
\hline 425 & -80 & 0.1705 & -250 & 0.1795 & 290 & 0.1115 \\
\hline 426 & -130 & 0.174 & -140 & 0.1775 & 270 & 0.1065 \\
\hline 427 & -120 & 0.182 & -180 & 0.186 & 320 & 0.102 \\
\hline 428 & -70 & 0.185 & -190 & 0.1905 & 250 & 0.0975 \\
\hline 429 & -100 & 0.1865 & -170 & 0.1915 & 210 & 0.094 \\
\hline 430 & -80 & 0.186 & -150 & 0.192 & 220 & 0.0905 \\
\hline 432 & -50 & 0.19 & -230 & 0.193 & 100 & 0.085 \\
\hline 434 & -90 & 0.1915 & -120 & 0.196 & 130 & 0.079 \\
\hline 436 & -90 & 0.189 & -160 & 0.197 & 170 & 0.0735 \\
\hline 438 & -100 & 0.198 & -170 & 0.202 & 140 & 0.0705 \\
\hline 440 & -80 & 0.198 & -160 & 0.2015 & 130 & 0.0665 \\
\hline 442 & -80 & 0.198 & -150 & 0.2065 & 100 & 0.062 \\
\hline 444 & -50 & 0.2015 & -110 & 0.205 & 120 & 0.0585 \\
\hline 447 & -40 & 0.202 & -110 & 0.21 & 110 & 0.0565 \\
\hline 449 & -60 & 0.2025 & -120 & 0.2125 & 90 & 0.0545 \\
\hline 451 & -70 & 0.2045 & -120 & 0.2105 & 60 & 0.052 \\
\hline 454 & -50 & 0.2075 & -120 & 0.211 & 70 & 0.0485 \\
\hline 457 & -50 & 0.206 & -110 & 0.211 & 60 & 0.047 \\
\hline 461 & -20 & 0.2085 & -80 & 0.213 & 40 & 0.045 \\
\hline 462 & -60 & 0.2085 & -130 & 0.215 & 30 & 0.0445 \\
\hline 463 & -50 & 0.211 & -130 & 0.215 & 30 & 0.044 \\
\hline 464 & -90 & 0.1405 & -160 & 0.141 & 190 & 0.104 \\
\hline 465 & -110 & 0.1205 & -160 & 0.121 & 270 & 0.114 \\
\hline 466 & -100 & 0.108 & -170 & 0.1095 & 270 & 0.1185 \\
\hline 467 & -100 & 0.0995 & -160 & 0.1015 & 340 & 0.1245 \\
\hline 468 & -110 & 0.0945 & -180 & 0.097 & 330 & 0.1295 \\
\hline 471 & -120 & 0.083 & -180 & 0.0865 & 340 & 0.135 \\
\hline 473 & -150 & 0.0755 & -200 & 0.08 & 350 & 0.137 \\
\hline 475 & -130 & 0.068 & -200 & 0.073 & 360 & 0.1435 \\
\hline
\end{tabular}


Table A.8, continued.

\begin{tabular}{|c|c|c|c|c|c|c|}
\cline { 2 - 7 } \multicolumn{1}{c|}{} & \multicolumn{3}{c|}{ Twice Recycled } \\
\cline { 2 - 7 } \multicolumn{1}{c|}{} & \multicolumn{2}{c|}{ dry cure 1 } & \multicolumn{2}{c|}{ dry cure 2 } & \multicolumn{2}{c|}{ wet cure 1 } \\
\hline $\begin{array}{c}\text { age } \\
\text { (days) }\end{array}$ & $\begin{array}{c}\text { strain } \\
(\mu \varepsilon)\end{array}$ & $\begin{array}{c}\text { weight change } \\
(\mathrm{lbs})\end{array}$ & $\begin{array}{c}\text { strain } \\
(\mu \varepsilon)\end{array}$ & $\begin{array}{c}\text { weight change } \\
(\mathrm{lbs})\end{array}$ & $\begin{array}{c}\text { strain } \\
(\mu \varepsilon)\end{array}$ & $\begin{array}{c}\text { weight change } \\
(\mathrm{lbs})\end{array}$ \\
\hline 477 & -170 & 0.0615 & -230 & 0.067 & 360 & 0.144 \\
\hline 479 & -200 & 0.0565 & -270 & 0.0615 & 390 & 0.1465 \\
\hline 482 & -160 & 0.05 & -230 & 0.055 & 390 & 0.1495 \\
\hline 484 & -170 & 0.0455 & -230 & 0.051 & 400 & 0.1535 \\
\hline 487 & -180 & 0.041 & -260 & 0.046 & 400 & 0.1535 \\
\hline 491 & -200 & 0.0335 & -250 & 0.038 & 410 & 0.153 \\
\hline 499 & -250 & 0.0215 & -310 & 0.026 & 400 & 0.157 \\
\hline 502 & -250 & 0.019 & -320 & 0.024 & 410 & 0.1585 \\
\hline
\end{tabular}


Table A.9

Strain and weight change for Lightweight 1.

\begin{tabular}{|c|c|c|c|c|c|c|}
\hline \multirow[b]{3}{*}{$\begin{array}{c}\text { age } \\
\text { (days) }\end{array}$} & \multicolumn{6}{|c|}{ Lightweight 1} \\
\hline & \multicolumn{2}{|c|}{ dry cure 1} & \multicolumn{2}{|c|}{ dry cure 2} & \multicolumn{2}{|c|}{ wet cure 1} \\
\hline & $\begin{array}{c}\text { strain } \\
(\mu \varepsilon)\end{array}$ & $\begin{array}{l}\text { weight change } \\
\text { (lbs) }\end{array}$ & $\begin{array}{c}\text { strain } \\
(\mu \varepsilon)\end{array}$ & $\begin{array}{l}\text { weight change } \\
\text { (Ibs) }\end{array}$ & $\begin{array}{c}\text { strain } \\
(\mu \varepsilon)\end{array}$ & $\begin{array}{c}\text { weight change } \\
\text { (Ibs) }\end{array}$ \\
\hline 0.75 & 0 & 0 & 0 & 0 & 0 & 0 \\
\hline 1 & -30 & -0.052 & -20 & -0.0525 & -50 & 0.073 \\
\hline 2 & -50 & -0.1225 & -80 & -0.1225 & 0 & 0.093 \\
\hline 3 & -100 & -0.13 & -120 & -0.1415 & 0 & 0.0955 \\
\hline 4 & -140 & -0.152 & -150 & -0.1505 & 0 & 0.099 \\
\hline 5 & -180 & -0.155 & -200 & -0.1615 & 0 & 0.1015 \\
\hline 6 & -210 & -0.111 & -240 & -0.1645 & -10 & 0.106 \\
\hline 7 & -240 & -0.171 & -280 & -0.1685 & -10 & 0.112 \\
\hline 8 & -270 & -0.174 & -310 & -0.177 & -10 & 0.1125 \\
\hline 9 & -290 & -0.1795 & -330 & -0.1775 & -10 & 0.1135 \\
\hline 10 & -330 & -0.1835 & -340 & -0.181 & 0 & 0.1145 \\
\hline 11 & -350 & -0.185 & -360 & -0.1835 & 0 & 0.116 \\
\hline 12 & -370 & -0.1865 & -380 & -0.1855 & 0 & 0.1185 \\
\hline 13 & -390 & -0.1895 & -400 & -0.1865 & 0 & 0.1205 \\
\hline 14 & -400 & -0.1895 & -400 & -0.1875 & 0 & 0.119 \\
\hline 15 & -430 & -0.1915 & -420 & -0.189 & 0 & 0.1185 \\
\hline 16 & -430 & -0.193 & -420 & -0.1905 & 0 & 0.1185 \\
\hline 17 & -470 & -0.198 & -470 & -0.1955 & 0 & 0.1205 \\
\hline 18 & -520 & -0.1995 & -510 & -0.197 & 0 & 0.1205 \\
\hline 19 & -530 & -0.2015 & -530 & -0.199 & 0 & 0.1235 \\
\hline 20 & -500 & -0.2005 & -500 & -0.195 & 0 & 0.122 \\
\hline 21 & -530 & -0.2015 & -530 & -0.199 & 0 & 0.1235 \\
\hline 22 & -540 & -0.203 & -550 & -0.2005 & 0 & 0.122 \\
\hline 23 & -550 & -0.204 & -570 & -0.2015 & 0 & 0.124 \\
\hline 24 & -540 & -0.207 & -580 & -0.2025 & 0 & 0.124 \\
\hline 25 & -550 & -0.2095 & -580 & -0.202 & 0 & 0.1235 \\
\hline 26 & -540 & -0.2065 & -580 & -0.204 & 0 & 0.123 \\
\hline 27 & -550 & -0.207 & -590 & -0.2045 & -10 & 0.124 \\
\hline 28 & -550 & -0.206 & -590 & -0.204 & -10 & 0.126 \\
\hline
\end{tabular}


Table A.9, continued.

\begin{tabular}{|c|c|c|c|c|c|c|}
\hline \multirow[b]{3}{*}{$\begin{array}{c}\text { age } \\
\text { (days) }\end{array}$} & \multicolumn{6}{|c|}{ Lightweight 1} \\
\hline & \multicolumn{2}{|c|}{ dry cure 1} & \multicolumn{2}{|c|}{ dry cure 2} & \multicolumn{2}{|c|}{ wet cure 1} \\
\hline & $\begin{array}{l}\text { strain } \\
(\mu \varepsilon)\end{array}$ & $\begin{array}{l}\text { weight change } \\
\text { (Ibs) }\end{array}$ & $\begin{array}{l}\text { strain } \\
(\mu \varepsilon)\end{array}$ & $\begin{array}{l}\text { weight change } \\
\text { (Ibs) }\end{array}$ & $\begin{array}{c}\text { strain } \\
(\mu \varepsilon)\end{array}$ & $\begin{array}{l}\text { weight change } \\
\text { (Ibs) }\end{array}$ \\
\hline 35 & -600 & -0.2035 & -630 & -0.2335 & -20 & 0.158 \\
\hline 42 & -640 & -0.1995 & -670 & -0.266 & -20 & 0.1865 \\
\hline 49 & -650 & -0.237 & -680 & -0.258 & -20 & 0.17 \\
\hline 56 & -660 & -0.2515 & -700 & -0.25 & -30 & 0.1435 \\
\hline 63 & -680 & -0.2365 & -720 & -0.231 & -50 & 0.1565 \\
\hline 70 & -700 & -0.2315 & -710 & -0.2495 & -40 & 0.149 \\
\hline 77 & -720 & -0.2275 & -710 & -0.2715 & -30 & 0.1335 \\
\hline 87 & -710 & -0.2315 & -740 & -0.23 & -20 & 0.1355 \\
\hline 97 & -730 & -0.232 & -760 & -0.2315 & -10 & 0.1355 \\
\hline 107 & -740 & -0.2335 & -770 & -0.2335 & 0 & 0.136 \\
\hline 117 & -730 & -0.2305 & -760 & -0.231 & -30 & 0.149 \\
\hline 127 & -730 & -0.231 & -750 & -0.2305 & -20 & 0.1435 \\
\hline 137 & -780 & -0.234 & -810 & -0.2335 & -10 & 0.141 \\
\hline 140 & -760 & -0.2355 & -790 & -0.2355 & -30 & 0.1545 \\
\hline 238 & -760 & -0.2195 & -810 & -0.218 & 50 & 0.1675 \\
\hline 452 & & -0.219 & & -0.2106 & 200 & 0.1885 \\
\hline 453 & -780 & -0.222 & -860 & -0.223 & 140 & 0.1715 \\
\hline 457 & -860 & -0.222 & -870 & -0.223 & 270 & 0.1785 \\
\hline 458 & -900 & -0.2215 & -930 & -0.223 & 80 & 0.1725 \\
\hline 459 & -880 & -0.2225 & -920 & -0.223 & 90 & 0.174 \\
\hline 460 & -670 & 0.0105 & -710 & 0.0125 & 70 & 0.156 \\
\hline 461 & -590 & 0.1125 & -630 & 0.1285 & 40 & 0.1455 \\
\hline 462 & -540 & 0.1495 & -590 & 0.1475 & 40 & 0.1355 \\
\hline 463 & -510 & 0.1585 & -570 & 0.158 & 10 & \\
\hline 464 & -510 & 0.155 & -560 & 0.159 & -10 & 0.116 \\
\hline 466 & -490 & 0.167 & -530 & 0.168 & -60 & 0.1005 \\
\hline 468 & -480 & 0.175 & -540 & 0.173 & -100 & 0.086 \\
\hline 470 & & 0.177 & -530 & 0.182 & -160 & 0.071 \\
\hline 473 & -470 & 0.1875 & -530 & 0.19 & -210 & 0.052 \\
\hline 475 & -460 & 0.1885 & -520 & 0.194 & -250 & 0.041 \\
\hline
\end{tabular}


Table A.9, continued.

\begin{tabular}{|c|c|c|c|c|c|c|}
\hline \multirow[b]{3}{*}{$\begin{array}{c}\text { age } \\
\text { (days) }\end{array}$} & \multicolumn{6}{|c|}{ Lightweight 1} \\
\hline & \multicolumn{2}{|c|}{ dry cure 1} & \multicolumn{2}{|c|}{ dry cure 2} & \multicolumn{2}{|c|}{ wet cure 1} \\
\hline & $\begin{array}{c}\text { strain } \\
(\mu \varepsilon)\end{array}$ & $\begin{array}{c}\text { weight change } \\
\text { (lbs) }\end{array}$ & $\begin{array}{c}\text { strain } \\
(\mu \varepsilon)\end{array}$ & $\begin{array}{c}\text { weight change } \\
\text { (lbs) }\end{array}$ & $\begin{array}{c}\text { strain } \\
(\mu \varepsilon)\end{array}$ & $\begin{array}{c}\text { weight change } \\
\text { (Ibs) }\end{array}$ \\
\hline 477 & -430 & 0.1955 & & 0.1985 & -270 & 0.031 \\
\hline 480 & -440 & 0.2 & -510 & 0.2035 & -300 & 0.0195 \\
\hline 482 & -450 & 0.2015 & -520 & 0.199 & -310 & 0.013 \\
\hline 484 & -430 & 0.212 & -490 & 0.2075 & -410 & 0.008 \\
\hline 491 & -430 & 0.2146 & -480 & 0.217 & -390 & -0.0095 \\
\hline 492 & -440 & 0.214 & -520 & 0.2145 & -380 & -0.011 \\
\hline 493 & -430 & 0.2195 & -520 & 0.222 & -390 & -0.013 \\
\hline 494 & -460 & 0.13 & -540 & 0.1255 & -200 & 0.0685 \\
\hline 495 & -460 & 0.097 & -550 & 0.092 & -110 & 0.0965 \\
\hline 496 & -470 & 0.0785 & -540 & 0.0735 & -70 & 0.0945 \\
\hline 497 & -500 & 0.063 & -570 & 0.0593 & -50 & 0.1005 \\
\hline 498 & -490 & 0.05 & -580 & 0.0445 & -40 & 0.1125 \\
\hline 499 & -510 & 0.0395 & -600 & 0.035 & -40 & 0.1135 \\
\hline 501 & -510 & 0.024 & -600 & 0.02 & -10 & 0.1255 \\
\hline 503 & -530 & 0.0125 & -600 & 0.009 & -20 & 0.123 \\
\hline 505 & -530 & 0.0005 & -620 & -0.003 & -10 & 0.13 \\
\hline 508 & -550 & -0.014 & -630 & -0.016 & -10 & 0.1315 \\
\hline 510 & -540 & -0.023 & -620 & -0.0245 & -10 & 0.129 \\
\hline 512 & -580 & -0.031 & -660 & -0.032 & 0 & 0.1295 \\
\hline 515 & -580 & -0.0405 & -670 & -0.0405 & 10 & 0.131 \\
\hline 517 & -590 & -0.0465 & -710 & -0.0465 & 20 & 0.13 \\
\hline 522 & -600 & -0.058 & -700 & -0.0575 & 0 & 0.1315 \\
\hline 524 & -630 & -0.0625 & -720 & -0.0625 & 20 & 0.131 \\
\hline 526 & -660 & -0.0675 & -750 & -0.067 & 20 & 0.1325 \\
\hline 527 & -700 & -0.069 & -760 & -0.069 & -10 & 0.134 \\
\hline 529 & -680 & -0.0735 & -740 & -0.0725 & 20 & 0.132 \\
\hline 531 & -670 & -0.077 & -740 & -0.076 & 0 & 0.133 \\
\hline 533 & -710 & -0.08 & -750 & -0.079 & 20 & 0.1345 \\
\hline 535 & -700 & -0.0825 & -750 & -0.082 & 20 & 0.1345 \\
\hline 536 & -580 & 0.053 & -620 & 0.0785 & 0 & 0.1165 \\
\hline
\end{tabular}


Table A.9, continued.

\begin{tabular}{|c|c|c|c|c|c|c|}
\hline \multirow[b]{3}{*}{$\begin{array}{c}\text { age } \\
\text { (days) }\end{array}$} & \multicolumn{6}{|c|}{ Lightweight 1} \\
\hline & \multicolumn{2}{|c|}{ dry cure 1} & \multicolumn{2}{|c|}{ dry cure 2} & \multicolumn{2}{|c|}{ wet cure 1} \\
\hline & $\begin{array}{c}\text { strain } \\
(\mu \varepsilon)\end{array}$ & $\begin{array}{l}\text { weight change } \\
\text { (Ibs) }\end{array}$ & $\begin{array}{c}\text { strain } \\
(\mu \varepsilon)\end{array}$ & $\begin{array}{c}\text { weight change } \\
\text { (Ibs) }\end{array}$ & $\begin{array}{c}\text { strain } \\
(\mu \varepsilon)\end{array}$ & $\begin{array}{c}\text { weight change } \\
\text { (lbs) }\end{array}$ \\
\hline 537 & -480 & 0.1255 & -570 & 0.127 & -20 & 0.108 \\
\hline 538 & -480 & 0.157 & -520 & 0.147 & -40 & 0.1005 \\
\hline 539 & -420 & 0.1685 & -500 & 0.16 & -80 & 0.0935 \\
\hline 540 & -500 & 0.1795 & -580 & 0.171 & -70 & 0.086 \\
\hline 541 & -450 & 0.1825 & -530 & 0.1725 & -100 & 0.079 \\
\hline 542 & -510 & 0.193 & -540 & 0.1845 & -30 & 0.072 \\
\hline 543 & -430 & 0.195 & -500 & 0.189 & -180 & 0.0665 \\
\hline 544 & -450 & 0.193 & -510 & 0.189 & -130 & 0.061 \\
\hline 545 & -470 & 0.189 & -530 & 0.192 & -150 & 0.0555 \\
\hline 547 & -460 & 0.1945 & -480 & 0.197 & -280 & 0.0475 \\
\hline 549 & -460 & 0.2 & -510 & 0.201 & -280 & 0.0395 \\
\hline 551 & -480 & 0.194 & -530 & 0.2025 & -210 & 0.032 \\
\hline 553 & -470 & 0.203 & -540 & 0.2035 & -250 & 0.0275 \\
\hline 555 & -440 & 0.2065 & -520 & 0.2055 & -280 & 0.0225 \\
\hline 557 & -450 & 0.2075 & -530 & 0.206 & -300 & 0.0165 \\
\hline 559 & -410 & 0.205 & -470 & 0.211 & -270 & 0.012 \\
\hline 562 & -400 & 0.212 & -480 & 0.2125 & -290 & 0.009 \\
\hline 564 & -440 & 0.2135 & -510 & 0.213 & -290 & 0.0065 \\
\hline 566 & -440 & 0.215 & -500 & 0.215 & -320 & 0.0035 \\
\hline 569 & -440 & 0.2115 & -490 & 0.219 & -320 & -0.0005 \\
\hline 572 & -420 & 0.2175 & -490 & 0.218 & -320 & -0.003 \\
\hline 576 & -400 & 0.2215 & -480 & 0.22 & -340 & -0.0055 \\
\hline 577 & -440 & 0.2075 & -510 & 0.225 & -350 & -0.006 \\
\hline 578 & -420 & 0.22 & -490 & 0.222 & -350 & -0.0065 \\
\hline 579 & -460 & 0.1335 & -530 & 0.135 & -180 & 0.059 \\
\hline 580 & -480 & 0.1105 & -540 & 0.111 & -100 & 0.08 \\
\hline 581 & -470 & 0.0965 & -540 & & -70 & 0.092 \\
\hline 582 & -470 & 0.087 & -540 & 0.087 & -30 & 0.1005 \\
\hline 583 & -470 & 0.08 & -550 & 0.08 & -50 & 0.108 \\
\hline 586 & -480 & 0.0655 & -560 & 0.0665 & -20 & 0.117 \\
\hline
\end{tabular}


Table A.9, continued.

\begin{tabular}{|c|c|c|c|c|c|c|}
\cline { 2 - 7 } \multicolumn{1}{c|}{} & \multicolumn{4}{c|}{ Lightweight 1 } \\
\cline { 2 - 7 } \multicolumn{1}{c|}{} & \multicolumn{2}{c|}{ dry cure 1 } & \multicolumn{2}{c|}{ dry cure 2 } & \multicolumn{2}{c|}{ wet cure 1 } \\
\hline $\begin{array}{c}\text { age } \\
\text { days })\end{array}$ & $\begin{array}{c}\text { strain } \\
(\mu \varepsilon)\end{array}$ & $\begin{array}{c}\text { weight } \\
\text { change }(\mathrm{lbs})\end{array}$ & $\begin{array}{c}\text { strain } \\
(\mu \varepsilon)\end{array}$ & $\begin{array}{c}\text { weight } \\
\text { change }(\mathrm{lbs})\end{array}$ & $\begin{array}{c}\text { strain } \\
(\mu \varepsilon)\end{array}$ & $\begin{array}{c}\text { weight } \\
\text { change }(\mathrm{lbs})\end{array}$ \\
\hline 588 & -510 & 0.057 & -590 & 0.058 & -30 & 0.126 \\
\hline 590 & -510 & 0.0475 & -570 & 0.0485 & -10 & 0.1325 \\
\hline 592 & -540 & 0.0395 & -610 & 0.041 & -30 & 0.1325 \\
\hline 594 & -580 & 0.032 & -640 & 0.034 & 20 & 0.1345 \\
\hline 597 & -540 & 0.0225 & -600 & 0.0245 & 20 & 0.137 \\
\hline 599 & -540 & 0.017 & -600 & 0.0205 & 20 & 0.14 \\
\hline 602 & -570 & 0.0095 & -640 & 0.0125 & 40 & 0.1415 \\
\hline 606 & -580 & -0.001 & -650 & 0.002 & 30 & 0.1425 \\
\hline 614 & -640 & -0.017 & -690 & -0.014 & 20 & 0.145 \\
\hline 617 & -640 & -0.02 & -710 & -0.0175 & 30 & 0.1475 \\
\hline
\end{tabular}


Table A.10

Strain and weight change for Lightweight 2.

\begin{tabular}{|c|c|c|c|c|c|c|c|c|}
\hline \multirow[b]{3}{*}{$\begin{array}{c}\text { age } \\
\text { (days } \\
\text { ) }\end{array}$} & \multicolumn{8}{|c|}{ Lightweight 2} \\
\hline & \multicolumn{2}{|c|}{ dry cure 1} & \multicolumn{2}{|c|}{ dry cure 2} & \multicolumn{2}{|c|}{ wet cure 1} & \multicolumn{2}{|c|}{ wet cure 2} \\
\hline & $\begin{array}{c}\text { strain } \\
(\mu \varepsilon)\end{array}$ & $\begin{array}{c}\text { weight } \\
\text { change } \\
\text { (lbs) }\end{array}$ & $\begin{array}{c}\text { strain } \\
(\mu \varepsilon)\end{array}$ & $\begin{array}{c}\text { weight } \\
\text { change } \\
\text { (lbs) }\end{array}$ & $\begin{array}{c}\text { strain } \\
(\mu \varepsilon)\end{array}$ & $\begin{array}{c}\text { weight } \\
\text { change } \\
\text { (lbs) }\end{array}$ & $\begin{array}{c}\text { strain } \\
(\mu \varepsilon)\end{array}$ & $\begin{array}{c}\text { weight } \\
\text { change } \\
\text { (lbs) }\end{array}$ \\
\hline 0.75 & 0 & 0 & 0 & 0 & 0 & 0 & 0 & 0 \\
\hline 1 & 20 & -0.091 & 10 & -0.082 & -30 & 0.0625 & -30 & 0.062 \\
\hline 2 & 0 & -0.1605 & -40 & -0.1395 & 10 & 0.0825 & 50 & 0.081 \\
\hline 3 & -50 & -0.181 & -120 & -0.1585 & 20 & 0.088 & 60 & 0.0875 \\
\hline 4 & -100 & -0.191 & -170 & -0.1675 & 20 & 0.0905 & 60 & 0.089 \\
\hline 5 & -140 & -0.199 & -200 & -0.1735 & 30 & 0.097 & 70 & 0.095 \\
\hline 6 & -170 & -0.2045 & -230 & -0.1795 & 30 & 0.098 & 70 & 0.0995 \\
\hline 7 & -190 & -0.21 & -260 & -0.184 & 30 & 0.0995 & 70 & 0.1025 \\
\hline 8 & -220 & -0.2145 & -280 & -0.1855 & 30 & 0.1015 & 60 & 0.103 \\
\hline 9 & -250 & -0.216 & -300 & -0.1885 & 20 & 0.103 & 60 & 0.104 \\
\hline 10 & -260 & -0.218 & -320 & -0.19 & 30 & 0.1065 & 70 & 0.1075 \\
\hline 11 & -290 & -0.221 & -350 & -0.1925 & 20 & 0.1085 & 60 & 0.108 \\
\hline 12 & -310 & -0.222 & -360 & -0.1935 & 20 & 0.1105 & 60 & 0.1085 \\
\hline 13 & -320 & -0.224 & -370 & -0.1955 & 20 & 0.1125 & 60 & 0.11 \\
\hline 14 & -340 & -0.2265 & -430 & -0.198 & 20 & 0.1115 & 60 & 0.112 \\
\hline 15 & -350 & -0.228 & -430 & -0.199 & 20 & 0.1135 & 60 & 0.1125 \\
\hline 16 & -350 & -0.226 & -470 & -0.1965 & 10 & 0.1215 & 50 & 0.1245 \\
\hline 17 & -370 & -0.2275 & -510 & -0.198 & 10 & 0.1145 & 60 & 0.114 \\
\hline 18 & -370 & -0.2285 & -540 & -0.198 & 20 & 0.1155 & 70 & 0.1165 \\
\hline 19 & -380 & -0.229 & -550 & -0.1985 & 30 & 0.1175 & 80 & 0.116 \\
\hline 20 & -390 & -0.2295 & -560 & -0.199 & 30 & 0.12 & 90 & 0.1165 \\
\hline 21 & -400 & -0.231 & -570 & -0.2005 & 30 & 0.119 & 80 & 0.117 \\
\hline 22 & -410 & -0.232 & -590 & -0.2015 & 30 & 0.1185 & 80 & 0.118 \\
\hline 23 & -410 & -0.233 & -600 & -0.203 & 30 & 0.119 & 80 & 0.1175 \\
\hline 24 & -420 & -0.2335 & -610 & -0.202 & 30 & 0.12 & 80 & 0.1165 \\
\hline 25 & -420 & -0.2335 & -630 & -0.2025 & 30 & 0.119 & 70 & 0.117 \\
\hline 26 & -430 & -0.234 & -630 & -0.203 & 40 & 0.1205 & 80 & 0.1175 \\
\hline 27 & -440 & -0.234 & -630 & -0.2025 & 40 & 0.121 & 80 & 0.1195 \\
\hline 28 & -450 & -0.2365 & -640 & -0.204 & 30 & 0.1195 & 80 & 0.1089 \\
\hline
\end{tabular}


Table A.10, continued.

\begin{tabular}{|c|c|c|c|c|c|c|c|c|}
\hline \multirow[b]{3}{*}{$\begin{array}{l}\text { age } \\
\text { (days) }\end{array}$} & \multicolumn{8}{|c|}{ Lightweight 2} \\
\hline & \multicolumn{2}{|c|}{ dry cure 1} & \multicolumn{2}{|c|}{ dry cure 2} & \multicolumn{2}{|c|}{ wet cure 1} & \multicolumn{2}{|c|}{ wet cure 2} \\
\hline & $\begin{array}{c}\text { strain } \\
(\mu \varepsilon)\end{array}$ & $\begin{array}{c}\text { weight } \\
\text { change } \\
\text { (Ibs) }\end{array}$ & $\begin{array}{c}\text { strain } \\
(\mu \varepsilon)\end{array}$ & $\begin{array}{l}\text { weight } \\
\text { change } \\
\text { (lbs) }\end{array}$ & $\begin{array}{c}\text { strain } \\
(\mu \varepsilon)\end{array}$ & $\begin{array}{c}\text { weight } \\
\text { change } \\
\text { (Ibs) }\end{array}$ & $\begin{array}{c}\text { strain } \\
(\mu \varepsilon)\end{array}$ & $\begin{array}{c}\text { weight } \\
\text { change } \\
\text { (lbs) }\end{array}$ \\
\hline 31 & -460 & -0.2385 & -680 & -0.2065 & 40 & 0.122 & 70 & 0.12 \\
\hline 32 & -470 & -0.238 & -700 & -0.205 & 50 & 0.1195 & 90 & 0.1185 \\
\hline 33 & -490 & -0.2405 & -710 & -0.2075 & 40 & 0.1205 & 90 & 0.1195 \\
\hline 34 & -510 & -0.2425 & -720 & -0.209 & 30 & 0.121 & 90 & 0.121 \\
\hline 35 & -500 & -0.2405 & -710 & -0.209 & 30 & 0.121 & 70 & 0.1185 \\
\hline 36 & -510 & -0.242 & -740 & -0.209 & 30 & 0.122 & 70 & 0.1245 \\
\hline 39 & -510 & -0.245 & -760 & -0.2115 & 20 & 0.126 & 60 & 0.129 \\
\hline 42 & -530 & -0.247 & -780 & -0.2135 & 20 & 0.124 & 60 & 0.123 \\
\hline 49 & -470 & -0.237 & -710 & -0.203 & 30 & 0.125 & 70 & 0.1235 \\
\hline 56 & & -0.235 & -740 & -0.201 & 10 & 0.1275 & 60 & 0.1245 \\
\hline 63 & & -0.2335 & -770 & -0.1995 & 20 & 0.13 & 70 & 0.1285 \\
\hline 70 & -510 & -0.2325 & -790 & -0.1975 & 40 & 0.134 & 80 & 0.128 \\
\hline 77 & & -0.2305 & -790 & -0.195 & 60 & 0.133 & 110 & 0.132 \\
\hline 87 & -540 & -0.229 & -810 & -0.194 & 120 & 0.1355 & & \\
\hline 97 & -550 & -0.2275 & -810 & -0.1915 & 130 & 0.138 & 170 & 0.1365 \\
\hline 107 & -560 & -0.2305 & -820 & -0.1945 & 132 & 0.141 & 170 & 0.1375 \\
\hline 117 & -590 & -0.229 & -860 & -0.193 & 130 & 0.1415 & 170 & 0.1385 \\
\hline 127 & -580 & -0.2275 & -860 & -0.191 & 150 & 0.145 & 190 & 0.145 \\
\hline 137 & -580 & -0.2255 & -820 & -0.189 & 90 & 0.148 & 140 & 0.1415 \\
\hline 351 & -480 & -0.229 & & -0.1917 & 180 & 0.1505 & 170 & 0.1445 \\
\hline 352 & -440 & -0.027 & -800 & -0.1905 & 140 & 0.1525 & 210 & 0.15 \\
\hline 356 & -640 & -0.027 & -950 & -0.1905 & 140 & 0.1525 & 210 & 0.15 \\
\hline 357 & -710 & -0.2275 & -980 & -0.191 & 130 & 0.151 & 180 & 0.153 \\
\hline 358 & -690 & -0.228 & -990 & -0.1915 & 140 & 0.1585 & 190 & 0.1555 \\
\hline 359 & -480 & 0.076 & -760 & 0.0205 & 120 & 0.135 & 170 & 0.134 \\
\hline 360 & -440 & 0.1345 & -700 & 0.1055 & 70 & 0.128 & 130 & 0.1275 \\
\hline 361 & -400 & 0.1415 & -680 & 0.1355 & 80 & 0.12 & 130 & 0.1215 \\
\hline 362 & -390 & 0.1805 & -660 & 0.1495 & 60 & 0.1115 & 100 & 0.114 \\
\hline 363 & -380 & 0.1475 & -660 & 0.1505 & 20 & 0.104 & 90 & 0.1075 \\
\hline 365 & -350 & 0.157 & -630 & 0.16 & -10 & 0.0915 & 40 & 0.097 \\
\hline
\end{tabular}


Table A.10, continued.

\begin{tabular}{|c|c|c|c|c|c|c|c|c|}
\hline \multirow[b]{3}{*}{$\begin{array}{c}\text { age } \\
\text { (days) }\end{array}$} & \multicolumn{8}{|c|}{ Lightweight 2} \\
\hline & \multicolumn{2}{|c|}{ dry cure 1} & \multicolumn{2}{|c|}{ dry cure 2} & \multicolumn{2}{|c|}{ wet cure 1} & \multicolumn{2}{|c|}{ wet cure 2} \\
\hline & $\begin{array}{c}\text { strain } \\
(\mu \varepsilon)\end{array}$ & $\begin{array}{c}\text { weight } \\
\text { change } \\
\text { (Ibs) }\end{array}$ & $\begin{array}{c}\text { strain } \\
(\mu \varepsilon)\end{array}$ & $\begin{array}{c}\text { weight } \\
\text { change } \\
\text { (lbs) }\end{array}$ & $\begin{array}{c}\text { strain } \\
(\mu \varepsilon)\end{array}$ & $\begin{array}{l}\text { weight } \\
\text { change } \\
\text { (Ibs) }\end{array}$ & $\begin{array}{c}\text { strain } \\
(\mu \varepsilon)\end{array}$ & $\begin{array}{c}\text { weight } \\
\text { change } \\
\text { (Ibs) }\end{array}$ \\
\hline 367 & -350 & 0.164 & -640 & 0.164 & -60 & 0.079 & 10 & 0.086 \\
\hline 369 & -340 & 0.168 & -630 & 0.169 & -100 & 0.0655 & -40 & 0.073 \\
\hline 372 & -350 & 0.177 & -640 & 0.1825 & -150 & 0.047 & -100 & 0.054 \\
\hline 374 & -340 & 0.1729 & -630 & 0.178 & -180 & 0.0375 & -110 & 0.044 \\
\hline 376 & -330 & 0.1815 & -620 & 0.184 & -210 & 0.028 & -160 & 0.0335 \\
\hline 379 & -350 & 0.185 & -640 & 0.1885 & -260 & 0.0165 & -210 & 0.0225 \\
\hline 381 & -350 & 0.1845 & -640 & 0.197 & -270 & 0.0105 & -200 & 0.0155 \\
\hline 383 & -330 & 0.1945 & -630 & 0.2015 & -360 & 0.0055 & -300 & 0.011 \\
\hline 390 & -320 & 0.2 & -620 & 0.2015 & -330 & -0.009 & -280 & -0.004 \\
\hline 391 & -330 & 0.191 & -620 & 0.1995 & -360 & -0.0105 & -290 & -0.005 \\
\hline 392 & -340 & 0.203 & -620 & 0.2105 & -350 & -0.0125 & -280 & -0.007 \\
\hline 393 & -370 & 0.087 & -670 & 0.0905 & -210 & & -130 & 0.046 \\
\hline 394 & -380 & 0.0505 & -680 & 0.0615 & -150 & 0.071 & -40 & 0.074 \\
\hline 395 & -390 & 0.033 & -690 & 0.047 & -70 & 0.0835 & 0 & 0.087 \\
\hline 396 & -410 & 0.019 & -710 & 0.0345 & -50 & 0.0865 & 30 & 0.09 \\
\hline 397 & -430 & 0.0085 & -730 & 0.025 & -50 & 0.095 & 20 & 0.0945 \\
\hline 398 & -450 & 0.0025 & -750 & 0.018 & -30 & 0.1015 & 40 & 0.103 \\
\hline 400 & -420 & -0.0095 & -740 & 0.0075 & 0 & 0.1065 & 80 & 0.11 \\
\hline 402 & -460 & -0.018 & -740 & -0.0005 & -10 & 0.109 & 60 & 0.114 \\
\hline 404 & -460 & -0.027 & -760 & -0.009 & -10 & 0.11 & 80 & 0.1135 \\
\hline 407 & -490 & -0.038 & -800 & -0.019 & 10 & 0.114 & 90 & 0.1185 \\
\hline 409 & -460 & -0.045 & -810 & -0.0255 & 10 & 0.1155 & 70 & 0.1215 \\
\hline 411 & -510 & -0.051 & -830 & -0.0305 & 20 & 0.123 & 170 & 0.124 \\
\hline 414 & -480 & -0.0575 & -790 & -0.0365 & 20 & 0.119 & 130 & 0.123 \\
\hline 416 & -500 & -0.0625 & -830 & -0.0405 & 10 & 0.1175 & 100 & 0.122 \\
\hline 421 & -510 & -0.0715 & -840 & -0.048 & 30 & 0.1185 & 140 & 0.1235 \\
\hline 423 & -550 & -0.076 & -880 & -0.0515 & 10 & 0.122 & & \\
\hline 425 & -580 & -0.079 & -920 & -0.0545 & 0 & 0.1235 & 120 & 0.1255 \\
\hline 426 & -570 & -0.081 & -920 & -0.056 & 50 & & 120 & 0.1255 \\
\hline 428 & -580 & -0.0845 & -920 & -0.059 & 0 & 0.1265 & 100 & 0.1245 \\
\hline
\end{tabular}


Table A.10, continued.

\begin{tabular}{|c|c|c|c|c|c|c|c|c|}
\hline \multirow[b]{3}{*}{$\begin{array}{l}\text { age } \\
\text { (days) }\end{array}$} & \multicolumn{8}{|c|}{ Lightweight 2} \\
\hline & \multicolumn{2}{|c|}{ dry cure 1} & \multicolumn{2}{|c|}{ dry cure 2} & \multicolumn{2}{|c|}{ wet cure 1} & \multicolumn{2}{|c|}{ wet cure 2} \\
\hline & $\begin{array}{c}\text { strain } \\
(\mu \varepsilon)\end{array}$ & $\begin{array}{c}\text { weight } \\
\text { change } \\
\text { (lbs) }\end{array}$ & $\begin{array}{c}\text { strain } \\
(\mu \varepsilon)\end{array}$ & $\begin{array}{c}\text { weight } \\
\text { change } \\
\text { (lbs) }\end{array}$ & $\begin{array}{c}\text { strain } \\
(\mu \varepsilon)\end{array}$ & $\begin{array}{c}\text { weight } \\
\text { change } \\
\text { (lbs) }\end{array}$ & $\begin{array}{c}\text { strain } \\
(\mu \varepsilon)\end{array}$ & $\begin{array}{c}\text { weight } \\
\text { change } \\
\text { (lbs) }\end{array}$ \\
\hline 430 & -580 & -0.087 & -910 & -0.0615 & 50 & 0.126 & 90 & 0.127 \\
\hline 432 & -620 & -0.0895 & -910 & -0.063 & 10 & 0.123 & 110 & 0.128 \\
\hline 434 & -590 & -0.092 & -900 & -0.0655 & 30 & 0.1275 & 120 & 0.127 \\
\hline 435 & -470 & 0.074 & -770 & 0.0795 & 40 & 0.1035 & 80 & 0.1075 \\
\hline 436 & -460 & 0.1135 & -720 & 0.125 & 20 & 0.0955 & 80 & 0.1015 \\
\hline 437 & -410 & 0.1285 & -690 & 0.1415 & -10 & 0.089 & 40 & 0.0945 \\
\hline 438 & -400 & 0.137 & -650 & 0.1535 & -10 & 0.082 & 50 & 0.0885 \\
\hline 439 & -480 & 0.1475 & -740 & 0.163 & -40 & 0.075 & 30 & 0.083 \\
\hline 440 & -420 & 0.151 & -720 & 0.1655 & -90 & 0.0685 & -30 & 0.077 \\
\hline 441 & -450 & 0.161 & -690 & 0.176 & 0 & 0.062 & 90 & 0.0715 \\
\hline 442 & -340 & 0.1645 & -630 & 0.1795 & -110 & 0.1265 & -20 & 0.067 \\
\hline 443 & -340 & 0.169 & -780 & 0.1825 & -90 & 0.0505 & -10 & 0.062 \\
\hline 444 & -460 & & -860 & 0.1785 & -160 & 0.0445 & -90 & 0.057 \\
\hline 446 & -370 & 0.1755 & -740 & 0.183 & -270 & 0.037 & -200 & 0.0495 \\
\hline 448 & -350 & 0.176 & -620 & 0.184 & -140 & 0.0305 & -90 & 0.042 \\
\hline 450 & -380 & 0.183 & -670 & 0.1945 & -180 & 0.0235 & -110 & 0.0345 \\
\hline 452 & -400 & 0.182 & -700 & 0.198 & -220 & 0.02 & -150 & 0.03 \\
\hline 454 & -390 & 0.183 & -660 & 0.1995 & -240 & 0.015 & -170 & 0.025 \\
\hline 456 & -390 & 0.184 & -680 & 0.2 & -260 & 0.0095 & -210 & 0.019 \\
\hline 458 & -350 & 0.188 & -630 & 0.202 & -250 & 0.0055 & -190 & 0.0145 \\
\hline 461 & -340 & 0.1895 & -640 & 0.2045 & -270 & 0.0035 & -200 & 0.012 \\
\hline 463 & -370 & 0.19 & -660 & 0.207 & -280 & 0.002 & -200 & 0.011 \\
\hline 465 & -380 & 0.1905 & -650 & 0.209 & -300 & -0.001 & -250 & 0.0075 \\
\hline 468 & -350 & 0.1995 & -640 & 0.2065 & -290 & -0.004 & -230 & 0.004 \\
\hline 471 & -350 & 0.195 & -630 & 0.2095 & -300 & -0.006 & -260 & 0.003 \\
\hline 475 & -340 & 0.197 & -620 & 0.2155 & -310 & -0.008 & -250 & 0.0005 \\
\hline 476 & -360 & 0.2025 & -660 & 0.211 & -310 & -0.0085 & -270 & 0 \\
\hline 477 & -340 & 0.1975 & -630 & 0.2135 & -310 & -0.0085 & -270 & \\
\hline 478 & -390 & 0.108 & -690 & 0.112 & -150 & 0.055 & -100 & 0.055 \\
\hline 479 & -410 & 0.081 & -710 & 0.091 & -70 & 0.0725 & -60 & 0.0725 \\
\hline
\end{tabular}


Table A.10, continued.

\begin{tabular}{|c|c|c|c|c|c|c|c|c|}
\cline { 2 - 9 } \multicolumn{1}{c|}{} & \multicolumn{9}{c|}{ dry cure 1 } & \multicolumn{2}{c|}{ dry cure 2 } & \multicolumn{2}{c|}{ wet cure 1 } & \multicolumn{2}{c|}{ wet cure 2 } \\
\cline { 2 - 9 } \multicolumn{1}{c|}{$\begin{array}{c}\text { age } \\
(\text { days })\end{array}$} & $\begin{array}{c}\text { strain } \\
(\mu \varepsilon)\end{array}$ & $\begin{array}{c}\text { weight } \\
\text { change } \\
(\mathrm{lbs})\end{array}$ & $\begin{array}{c}\text { strain } \\
(\mu \varepsilon)\end{array}$ & $\begin{array}{c}\text { weight } \\
\text { change } \\
(\mathrm{lbs})\end{array}$ & $\begin{array}{c}\text { strain } \\
(\mu \varepsilon)\end{array}$ & $\begin{array}{c}\text { weight } \\
\text { change } \\
(\mathrm{lbs})\end{array}$ & $\begin{array}{c}\text { strain } \\
(\mu \varepsilon)\end{array}$ & $\begin{array}{c}\text { weight } \\
\text { change } \\
(\mathrm{lbs})\end{array}$ \\
\hline 480 & -420 & 0.0645 & -700 & 0.078 & -50 & 0.085 & -20 & 0.087 \\
\hline 481 & -400 & 0.0554 & -710 & 0.0695 & -10 & 0.092 & 10 & 0.0955 \\
\hline 482 & -410 & 0.0825 & -710 & 0.065 & -20 & 0.099 & 30 & 0.1035 \\
\hline 485 & -420 & 0.0375 & -720 & 0.054 & 10 & 0.108 & 50 & 0.11 \\
\hline 487 & -460 & 0.03 & -760 & 0.0475 & -10 & 0.112 & 70 & 0.1125 \\
\hline 489 & -440 & 0.0225 & -740 & 0.04 & 40 & 0.1155 & 90 & 0.1155 \\
\hline 491 & -480 & 0.016 & -780 & 0.0345 & 20 & 0.1245 & 70 & 0.1245 \\
\hline 493 & -510 & 0.011 & -810 & 0.0295 & 70 & 0.1265 & 110 & 0.1285 \\
\hline 496 & -460 & 0.003 & -780 & 0.0235 & 70 & 0.1295 & 110 & 0.1295 \\
\hline 498 & -470 & -0.0015 & -780 & 0.0195 & 70 & 0.1325 & 120 & 0.1325 \\
\hline 501 & -500 & -0.006 & -810 & 0.0155 & 80 & 0.134 & & \\
\hline 505 & -500 & -0.0145 & -820 & 0.0085 & 70 & 0.133 & & 0.136 \\
\hline 513 & -560 & -0.0275 & -870 & -0.003 & 80 & 0.1365 & & 0.1365 \\
\hline 516 & -560 & -0.0295 & -870 & -0.0045 & 80 & 0.139 & 120 & 0.138 \\
\hline
\end{tabular}


Table A.11

Strain and weight change for Lightweight 3.

\begin{tabular}{|c|c|c|c|c|c|c|c|c|}
\hline \multirow[b]{3}{*}{$\begin{array}{c}\text { age } \\
\text { (days) }\end{array}$} & \multicolumn{8}{|c|}{ Lightweight 3} \\
\hline & \multicolumn{2}{|c|}{ dry cure 1} & \multicolumn{2}{|c|}{ dry cure 2} & \multicolumn{2}{|c|}{ wet cure 1} & \multicolumn{2}{|c|}{ wet cure 2} \\
\hline & $\begin{array}{c}\text { strain } \\
(\mu \varepsilon)\end{array}$ & $\begin{array}{l}\text { weight } \\
\text { change } \\
\text { (Ibs) }\end{array}$ & $\begin{array}{c}\text { strain } \\
(\mu \varepsilon)\end{array}$ & $\begin{array}{c}\text { weight } \\
\text { change } \\
\text { (lbs) }\end{array}$ & $\begin{array}{c}\text { strain } \\
(\mu \varepsilon)\end{array}$ & $\begin{array}{c}\text { weight } \\
\text { change } \\
\text { (lbs) }\end{array}$ & $\begin{array}{c}\text { strain } \\
(\mu \varepsilon)\end{array}$ & $\begin{array}{c}\text { weight } \\
\text { change } \\
\text { (lbs) }\end{array}$ \\
\hline 0.75 & 0 & 0 & 0 & 0 & 0 & 0 & 0 & 0 \\
\hline 1 & -90 & -0.0585 & 0 & -0.0635 & -10 & 0.0705 & -120 & 0.0675 \\
\hline 2 & -130 & -0.127 & -30 & -0.1725 & 40 & 0.08 & -90 & 0.081 \\
\hline 3 & -160 & -0.1745 & -70 & -0.1835 & 100 & 0.0905 & -60 & 0.088 \\
\hline 4 & -210 & -0.181 & -120 & -0.19 & 90 & 0.0955 & -70 & 0.0925 \\
\hline 5 & -270 & -0.1905 & -160 & -0.2 & 90 & 0.102 & -70 & 0.1 \\
\hline 6 & -290 & -0.197 & -190 & -0.2065 & 100 & 0.1025 & -70 & 0.1005 \\
\hline 7 & -320 & -0.204 & -210 & -0.213 & 110 & 0.103 & -60 & 0.1005 \\
\hline 8 & -350 & -0.2085 & -250 & -0.216 & 110 & 0.104 & -60 & 0.104 \\
\hline 9 & -390 & -0.2115 & -280 & -0.2165 & 100 & 0.106 & -60 & 0.103 \\
\hline 10 & -420 & -0.214 & -310 & -0.2235 & 100 & 0.109 & -60 & 0.107 \\
\hline 11 & -450 & -0.217 & -350 & -0.2265 & 120 & 0.111 & -110 & 0.1095 \\
\hline 12 & -470 & -0.219 & -370 & -0.229 & 100 & 0.1115 & -140 & 0.1105 \\
\hline 13 & -470 & -0.2205 & -380 & -0.2315 & 110 & 0.1155 & -130 & 0.109 \\
\hline 14 & -470 & -0.223 & -400 & -0.2325 & 110 & 0.1185 & -120 & 0.119 \\
\hline 15 & -490 & -0.2215 & -410 & -0.233 & 100 & 0.1155 & -130 & 0.1185 \\
\hline 16 & -510 & -0.224 & -420 & -0.234 & 100 & 0.115 & -140 & 0.121 \\
\hline 17 & -540 & -0.2255 & -440 & -0.236 & 100 & 0.1175 & -150 & 0.1155 \\
\hline 18 & -560 & -0.2275 & -460 & -0.237 & 90 & 0.1175 & -150 & 0.115 \\
\hline 19 & -560 & -0.228 & -470 & -0.238 & 90 & 0.1205 & -150 & 0.117 \\
\hline 20 & -570 & -0.229 & -480 & -0.239 & 100 & 0.1175 & -140 & 0.1155 \\
\hline 21 & -570 & -0.2305 & -490 & -0.24 & 110 & 0.1155 & -130 & 0.1135 \\
\hline 22 & -580 & -0.23 & -490 & -0.2395 & 100 & 0.118 & -150 & 0.1155 \\
\hline 23 & -610 & -0.2315 & -490 & -0.2405 & 90 & 0.123 & -140 & 0.122 \\
\hline 24 & -610 & -0.2315 & -500 & -0.241 & 100 & 0.1215 & -140 & 0.1225 \\
\hline 25 & -610 & -0.232 & -510 & -0.242 & 90 & 0.1205 & -140 & 0.1215 \\
\hline 26 & -620 & -0.2315 & -520 & -0.2415 & 90 & 0.124 & -140 & 0.122 \\
\hline 27 & -640 & -0.232 & -540 & -0.242 & 90 & 0.1265 & -150 & 0.1225 \\
\hline 28 & -660 & -0.232 & -550 & -0.242 & 90 & 0.127 & -150 & 0.1235 \\
\hline
\end{tabular}


Table A.11, continued.

\begin{tabular}{|c|c|c|c|c|c|c|c|c|}
\hline \multirow[b]{3}{*}{$\begin{array}{c}\text { age } \\
\text { (days) }\end{array}$} & \multicolumn{8}{|c|}{ Lightweight 3} \\
\hline & \multicolumn{2}{|c|}{ dry cure 1} & \multicolumn{2}{|c|}{ dry cure 2} & \multicolumn{2}{|c|}{ wet cure 1} & \multicolumn{2}{|c|}{ wet cure 2} \\
\hline & $\begin{array}{c}\text { strain } \\
(\mu \varepsilon)\end{array}$ & $\begin{array}{c}\text { weight } \\
\text { change } \\
\text { (Ibs) }\end{array}$ & $\begin{array}{c}\text { strain } \\
(\mu \varepsilon)\end{array}$ & $\begin{array}{c}\text { weight } \\
\text { change } \\
\text { (Ibs) }\end{array}$ & $\begin{array}{c}\text { strain } \\
(\mu \varepsilon)\end{array}$ & $\begin{array}{c}\text { weight } \\
\text { change } \\
\text { (lbs) }\end{array}$ & $\begin{array}{c}\text { strain } \\
(\mu \varepsilon)\end{array}$ & $\begin{array}{c}\text { weight } \\
\text { change } \\
\text { (lbs) }\end{array}$ \\
\hline 35 & -670 & -0.2345 & -570 & -0.2435 & 100 & 0.1275 & -140 & 0.1225 \\
\hline 42 & -690 & -0.2375 & -590 & -0.2475 & 100 & 0.1265 & -140 & 0.124 \\
\hline 49 & -710 & -0.227 & -610 & -0.213 & 100 & 0.166 & -140 & \\
\hline 56 & -720 & -0.2115 & -630 & -0.1965 & 90 & 0.1835 & -150 & 0.1515 \\
\hline 63 & -740 & -0.1965 & -650 & -0.1945 & 90 & 0.1925 & -150 & 0.152 \\
\hline 70 & -750 & -0.2125 & -660 & -0.223 & 80 & 0.1685 & -160 & 0.152 \\
\hline 77 & -750 & -0.2315 & -670 & -0.252 & 80 & 0.1445 & -160 & \\
\hline 87 & -760 & -0.266 & -670 & -0.26 & 80 & 0.141 & -170 & 0.1235 \\
\hline 97 & -760 & -0.3005 & -670 & -0.2675 & 80 & 0.134 & -170 & 0.1305 \\
\hline 107 & -790 & -0.247 & -680 & -0.258 & 80 & 0.1355 & -170 & 0.1325 \\
\hline 117 & -800 & -0.2475 & -700 & -0.2575 & 90 & 0.136 & -150 & 0.133 \\
\hline 128 & -810 & -0.247 & -710 & -0.258 & 100 & 0.1355 & -140 & 0.134 \\
\hline 137 & -810 & -0.2455 & -690 & -0.257 & 80 & 0.1395 & -160 & 0.1385 \\
\hline 161 & -830 & -0.246 & -730 & -0.257 & 70 & 0.146 & -190 & 0.144 \\
\hline 259 & -810 & -0.225 & -700 & -0.236 & 190 & 0.1625 & -70 & 0.1565 \\
\hline 473 & -880 & -0.2245 & & -0.2325 & & 0.1705 & 110 & 0.1605 \\
\hline 474 & & -0.2235 & & -0.2335 & 230 & 0.1705 & -60 & 0.1645 \\
\hline 478 & -890 & -0.224 & -750 & -0.2335 & 230 & 0.177 & 20 & 0.1635 \\
\hline 479 & -940 & -0.224 & -840 & -0.234 & 200 & 0.175 & -50 & 0.165 \\
\hline 480 & -950 & -0.2245 & -810 & -0.234 & 200 & 0.175 & -50 & 0.1705 \\
\hline 481 & -700 & 0.02 & -610 & -0.0275 & 180 & 0.1515 & -70 & 0.145 \\
\hline 482 & -660 & 0.13 & -490 & 0.1155 & 130 & 0.1425 & -120 & 0.133 \\
\hline 483 & -610 & 0.1555 & -510 & 0.1475 & 140 & 0.134 & -120 & 0.121 \\
\hline 484 & -590 & 0.154 & -490 & 0.1535 & 120 & 0.1255 & -130 & 0.11 \\
\hline 485 & -560 & 0.1655 & -490 & 0.1565 & 90 & 0.1475 & -170 & 0.0995 \\
\hline 487 & -540 & 0.176 & -450 & 0.171 & 50 & 0.1025 & -230 & 0.0795 \\
\hline 489 & -550 & 0.1785 & -420 & 0.1735 & 10 & 0.089 & -270 & 0.062 \\
\hline 491 & -540 & 0.1815 & -380 & 0.1805 & -40 & 0.0745 & -140 & 0.044 \\
\hline 494 & -550 & 0.2 & -390 & 0.1915 & -90 & 0.0525 & -370 & 0.0215 \\
\hline 496 & -520 & 0.1965 & -370 & 0.191 & -140 & 0.04 & -410 & 0.01 \\
\hline
\end{tabular}


Table A.11, continued.

\begin{tabular}{|c|c|c|c|c|c|c|c|c|}
\hline \multirow[b]{3}{*}{$\begin{array}{l}\text { age } \\
\text { (days) }\end{array}$} & \multicolumn{8}{|c|}{ Lightweight 3} \\
\hline & \multicolumn{2}{|c|}{ dry cure 1} & \multicolumn{2}{|c|}{ dry cure 2} & \multicolumn{2}{|c|}{ wet cure 1} & \multicolumn{2}{|c|}{ wet cure 2} \\
\hline & $\begin{array}{c}\text { strain } \\
(\mu \varepsilon)\end{array}$ & $\begin{array}{c}\text { weight } \\
\text { change } \\
\text { (Ibs) }\end{array}$ & $\begin{array}{c}\text { strain } \\
(\mu \varepsilon)\end{array}$ & $\begin{array}{c}\text { weight } \\
\text { change } \\
\text { (lbs) }\end{array}$ & $\begin{array}{c}\text { strain } \\
(\mu \varepsilon)\end{array}$ & $\begin{array}{c}\text { weight } \\
\text { change } \\
\text { (lbs) }\end{array}$ & $\begin{array}{c}\text { strain } \\
(\mu \varepsilon)\end{array}$ & $\begin{array}{c}\text { weight } \\
\text { change } \\
\text { (lbs) }\end{array}$ \\
\hline 498 & -500 & 0.2075 & -340 & 0.197 & & 0.027 & -440 & -0.001 \\
\hline 501 & -460 & & -340 & 0.206 & -220 & 0.015 & -470 & -0.014 \\
\hline 503 & -500 & 0.22 & -340 & 0.215 & -250 & 0.0025 & -500 & -0.021 \\
\hline 505 & -490 & 0.2175 & -320 & 0.216 & -350 & -0.0045 & -610 & -0.0265 \\
\hline 512 & -470 & 0.23 & -310 & 0.2245 & -350 & -0.0285 & -590 & -0.043 \\
\hline 513 & -470 & 0.2275 & -340 & 0.2255 & -340 & -0.03 & -580 & -0.045 \\
\hline 514 & -490 & 0.236 & -340 & 0.235 & -360 & -0.033 & -590 & -0.0465 \\
\hline 515 & -510 & 0.11 & -370 & 0.1055 & -170 & 0.0335 & -430 & 0.0325 \\
\hline 516 & -520 & 0.0675 & -390 & 0.063 & -70 & 0.0615 & -300 & 0.0755 \\
\hline 517 & -520 & 0.062 & -400 & 0.0405 & -20 & 0.079 & -260 & 0.095 \\
\hline 518 & -540 & 0.025 & -410 & 0.022 & 30 & 0.0885 & -210 & 0.1035 \\
\hline 519 & -590 & 0.011 & -450 & 0.0075 & 10 & 0.0945 & -200 & 0.106 \\
\hline 520 & -590 & -0.001 & -470 & -0.0045 & 0 & 0.103 & -190 & 0.112 \\
\hline 522 & -610 & -0.017 & -470 & -0.0195 & 60 & 0.102 & -140 & 0.156 \\
\hline 524 & -610 & -0.028 & -490 & -0.0315 & 40 & 0.1085 & -170 & 0.1165 \\
\hline 526 & -620 & -0.0405 & -480 & -0.044 & 40 & 0.1145 & -190 & 0.121 \\
\hline 529 & -640 & -0.0545 & -520 & -0.058 & 60 & 0.123 & -170 & 0.121 \\
\hline 531 & -640 & -0.062 & -540 & -0.0665 & 60 & 0.118 & -150 & 0.1305 \\
\hline 533 & -690 & -0.0685 & -550 & -0.073 & 80 & 0.121 & -150 & 0.133 \\
\hline 536 & -660 & -0.0765 & -550 & -0.0815 & 110 & 0.1245 & -130 & 0.137 \\
\hline 538 & -710 & -0.0815 & -560 & -0.0865 & 70 & 0.1215 & -180 & 0.13 \\
\hline 543 & -730 & -0.091 & -600 & -0.0965 & 80 & 0.128 & -160 & 0.131 \\
\hline 545 & -760 & -0.095 & -630 & -0.1005 & 70 & 0.124 & -160 & 0.13 \\
\hline 547 & -740 & -0.099 & -670 & -0.105 & 50 & 0.124 & -190 & 0.1305 \\
\hline 548 & -770 & -0.1005 & -650 & -0.1065 & 100 & 0.1265 & -150 & 0.1355 \\
\hline 550 & -790 & -0.1035 & -660 & -0.1105 & 80 & 0.1245 & -150 & 0.1325 \\
\hline 552 & -800 & -0.107 & -670 & -0.1135 & 90 & 0.1255 & -170 & 0.1345 \\
\hline 554 & -790 & -0.109 & -670 & -0.1155 & 100 & 0.1255 & -160 & 0.1325 \\
\hline 556 & -800 & -0.111 & -670 & -0.118 & 120 & 0.1265 & -110 & 0.1345 \\
\hline 557 & -630 & 0.0765 & -470 & 0.0895 & 70 & 0.1045 & -160 & 0.107 \\
\hline
\end{tabular}


Table A.11, continued.

\begin{tabular}{|c|c|c|c|c|c|c|c|c|}
\hline \multirow[b]{3}{*}{$\begin{array}{c}\text { age } \\
\text { (days) }\end{array}$} & \multicolumn{8}{|c|}{ Lightweight 3} \\
\hline & \multicolumn{2}{|c|}{ dry cure 1} & \multicolumn{2}{|c|}{ dry cure 2} & \multicolumn{2}{|c|}{ wet cure 1} & \multicolumn{2}{|c|}{ wet cure 2} \\
\hline & $\begin{array}{c}\text { strain } \\
(\mu \varepsilon)\end{array}$ & $\begin{array}{c}\text { weight } \\
\text { change } \\
\text { (Ibs) }\end{array}$ & $\begin{array}{c}\text { strain } \\
(\mu \varepsilon)\end{array}$ & $\begin{array}{c}\text { weight } \\
\text { change } \\
\text { (Ibs) }\end{array}$ & $\begin{array}{c}\text { strain } \\
(\mu \varepsilon)\end{array}$ & $\begin{array}{c}\text { weight } \\
\text { change } \\
\text { (lbs) }\end{array}$ & $\begin{array}{c}\text { strain } \\
(\mu \varepsilon)\end{array}$ & $\begin{array}{c}\text { weight } \\
\text { change } \\
\text { (lbs) }\end{array}$ \\
\hline 558 & -580 & 0.142 & -420 & 0.1395 & 50 & 0.0955 & -160 & 0.096 \\
\hline 559 & -530 & 0.164 & -380 & 0.1565 & 40 & 0.0875 & -170 & 0.0855 \\
\hline 560 & -500 & 0.175 & -330 & 0.1665 & 30 & 0.0795 & -140 & 0.0755 \\
\hline 561 & -570 & 0.181 & -410 & 0.175 & -50 & 0.072 & -230 & 0.066 \\
\hline 562 & -560 & 0.185 & -390 & & -70 & 0.064 & -340 & 0.0555 \\
\hline 563 & -570 & 0.1965 & -390 & 0.1875 & 50 & 0.058 & -160 & 0.048 \\
\hline 564 & -510 & 0.202 & -340 & 0.1935 & -70 & 0.0515 & -350 & 0.0385 \\
\hline 565 & -610 & 0.202 & -430 & 0.1935 & -50 & 0.0455 & -290 & 0.0315 \\
\hline 566 & -530 & 0.2 & -390 & 0.1925 & -120 & 0.039 & -350 & 0.0235 \\
\hline 568 & -600 & 0.2015 & -440 & 0.1895 & -250 & 0.0295 & -470 & 0.0135 \\
\hline 570 & -510 & 0.203 & -340 & 0.1985 & -270 & 0.02 & -410 & 0.003 \\
\hline 572 & -520 & 0.207 & -390 & 0.1995 & -220 & 0.0105 & -540 & -0.006 \\
\hline 574 & -550 & 0.214 & -390 & 0.205 & -210 & 0.005 & -430 & -0.0105 \\
\hline 576 & -520 & 0.2145 & -340 & 0.2065 & -250 & -0.0015 & -450 & -0.0165 \\
\hline 578 & -530 & 0.217 & -360 & 0.21 & -260 & -0.009 & -480 & -0.023 \\
\hline 580 & -460 & 0.218 & -320 & 0.2105 & -250 & -0.015 & -460 & -0.0275 \\
\hline 583 & -490 & 0.22 & -300 & 0.2145 & -260 & -0.018 & -470 & -0.0295 \\
\hline 585 & -500 & 0.222 & -340 & 0.217 & -270 & -0.0205 & -480 & -0.0315 \\
\hline 587 & -490 & 0.224 & -350 & 0.22 & -310 & -0.0245 & -510 & -0.035 \\
\hline 590 & -490 & 0.2255 & -310 & 0.22 & -300 & -0.029 & -500 & -0.039 \\
\hline 593 & -470 & 0.225 & -340 & 0.2205 & -320 & -0.031 & -510 & -0.0395 \\
\hline 597 & -460 & 0.229 & -290 & 0.2225 & -330 & -0.0345 & -510 & -0.042 \\
\hline 598 & -500 & 0.23 & -330 & 0.225 & -330 & -0.035 & -510 & -0.0425 \\
\hline 599 & -470 & 0.23 & -310 & 0.226 & -330 & -0.035 & -520 & -0.0425 \\
\hline 600 & -520 & 0.114 & -340 & 0.104 & -100 & 0.0505 & -300 & 0.068 \\
\hline 601 & -550 & 0.085 & -390 & 0.075 & -30 & 0.0745 & -210 & 0.102 \\
\hline 602 & -570 & 0.067 & -390 & 0.058 & 10 & 0.0875 & -180 & 0.1115 \\
\hline 603 & -550 & 0.055 & -390 & 0.047 & 30 & 0.097 & -160 & 0.122 \\
\hline 604 & -570 & 0.0475 & -410 & 0.04 & 40 & 0.1055 & -160 & 0.127 \\
\hline 607 & -560 & 0.032 & -410 & 0.0245 & 50 & 0.114 & -150 & 0.1325 \\
\hline
\end{tabular}


Table A.11, continued.

\begin{tabular}{|c|c|c|c|c|c|c|c|c|}
\cline { 2 - 9 } \multicolumn{1}{c|}{} & \multicolumn{9}{c|}{ dry cure 1 } & \multicolumn{2}{c|}{ dry cure 2 } & \multicolumn{2}{c|}{ wet cure 1 } & \multicolumn{2}{c|}{ wet cure 2 } \\
\cline { 2 - 10 } \multicolumn{1}{c|}{$\begin{array}{c}\text { age } \\
(\text { days })\end{array}$} & $\begin{array}{c}\text { strain } \\
(\mu \varepsilon)\end{array}$ & $\begin{array}{c}\text { weight } \\
\text { change } \\
(\mathrm{lbs})\end{array}$ & $\begin{array}{c}\text { strain } \\
(\mu \varepsilon)\end{array}$ & $\begin{array}{c}\text { weight } \\
\text { change } \\
(\mathrm{lbs})\end{array}$ & $\begin{array}{c}\text { strain } \\
(\mu \varepsilon)\end{array}$ & $\begin{array}{c}\text { weight } \\
\text { change } \\
(\mathrm{lbs})\end{array}$ & $\begin{array}{c}\text { strain } \\
(\mu \varepsilon)\end{array}$ & $\begin{array}{c}\text { weight } \\
\text { change } \\
(\mathrm{lbs})\end{array}$ \\
\hline 609 & -600 & 0.0225 & -460 & 0.015 & 50 & 0.117 & -140 & 0.132 \\
\hline 611 & -590 & 0.0125 & -460 & 0.005 & 80 & 0.1185 & -140 & 0.1355 \\
\hline 613 & -640 & 0.0045 & -490 & -0.003 & 90 & 0.127 & -150 & 0.136 \\
\hline 615 & -680 & -0.002 & -530 & -0.01 & 90 & 0.1461 & -120 & 0.142 \\
\hline 618 & -630 & -0.0105 & -490 & -0.0195 & 100 & 0.1325 & -130 & 0.143 \\
\hline 620 & -640 & -0.0165 & -490 & -0.0235 & 120 & 0.1365 & -110 & 0.1555 \\
\hline 623 & -680 & -0.024 & -520 & -0.031 & 120 & 0.136 & -100 & 0.1505 \\
\hline 627 & -680 & -0.0335 & -530 & -0.041 & 100 & 0.1365 & -120 & 0.1495 \\
\hline 635 & -740 & -0.048 & -590 & -0.0565 & 90 & 0.1385 & -120 & 0.152 \\
\hline 638 & -740 & -0.051 & -590 & -0.0585 & 110 & 0.142 & -110 & 0.154 \\
\hline
\end{tabular}




\section{Appendix B: Graphs of Strain versus Time}

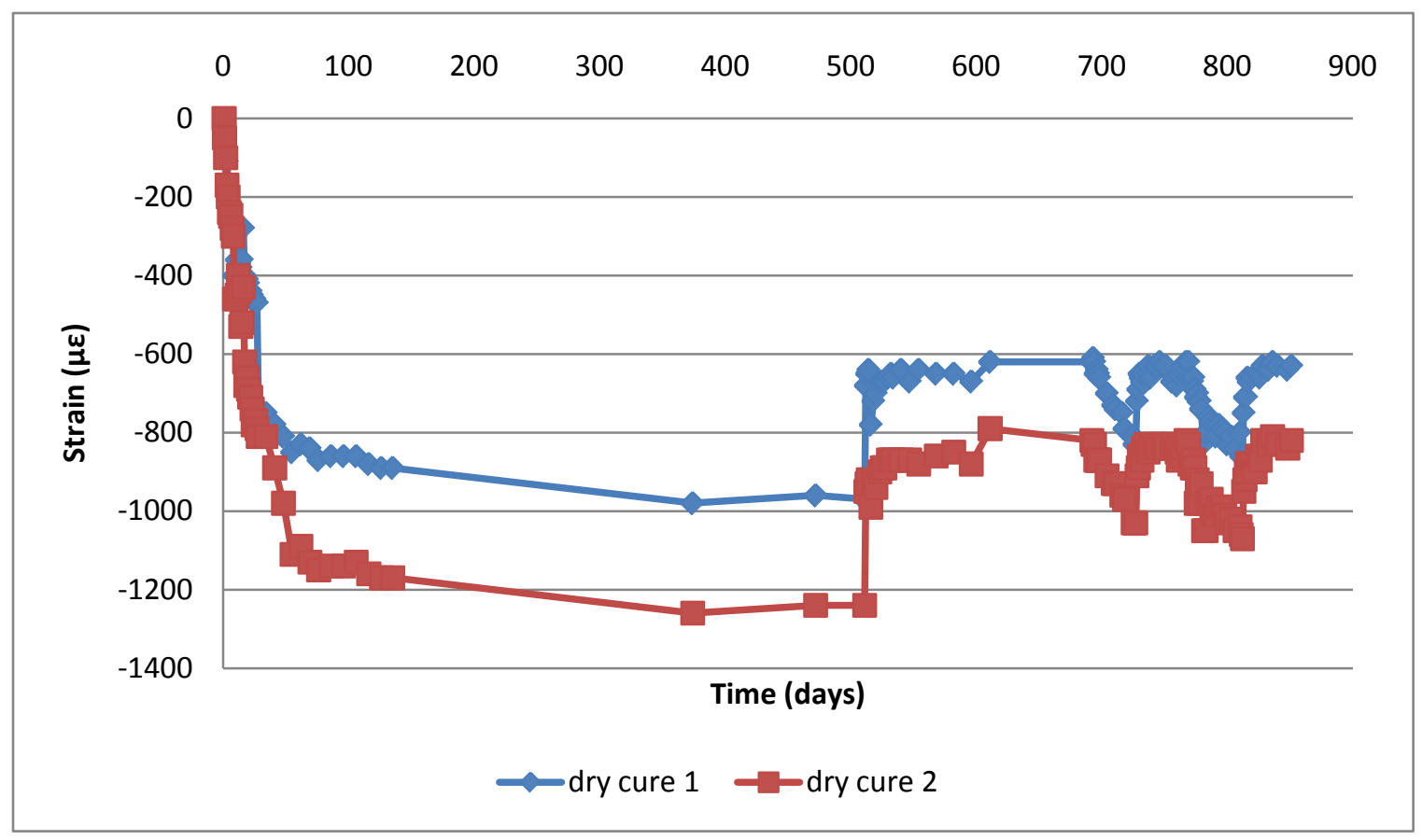

Figure B.1: Strain versus time for dry cured Virgin 1.

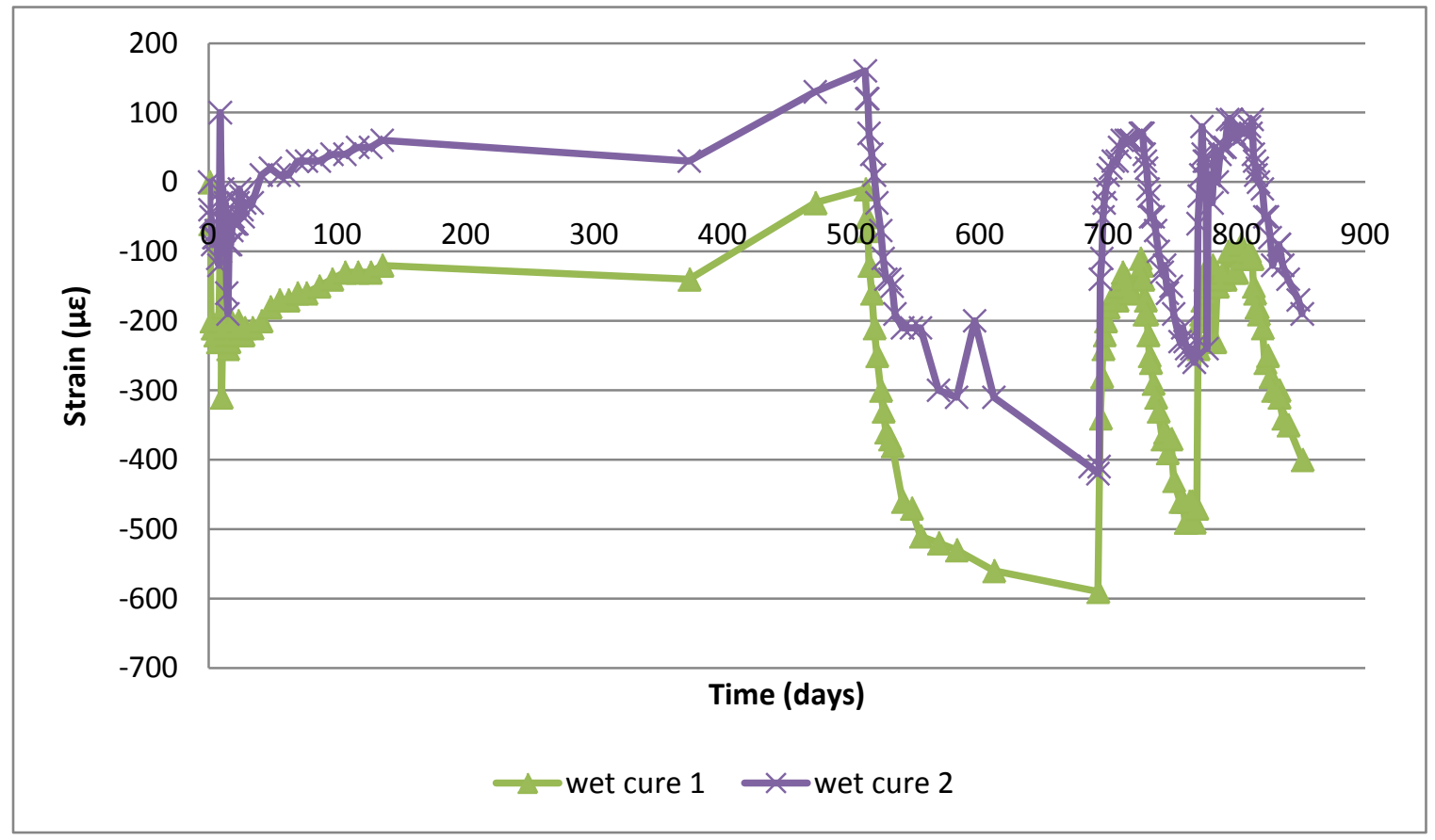

Figure B.2: Strain versus time for wet cured Virgin 1. 


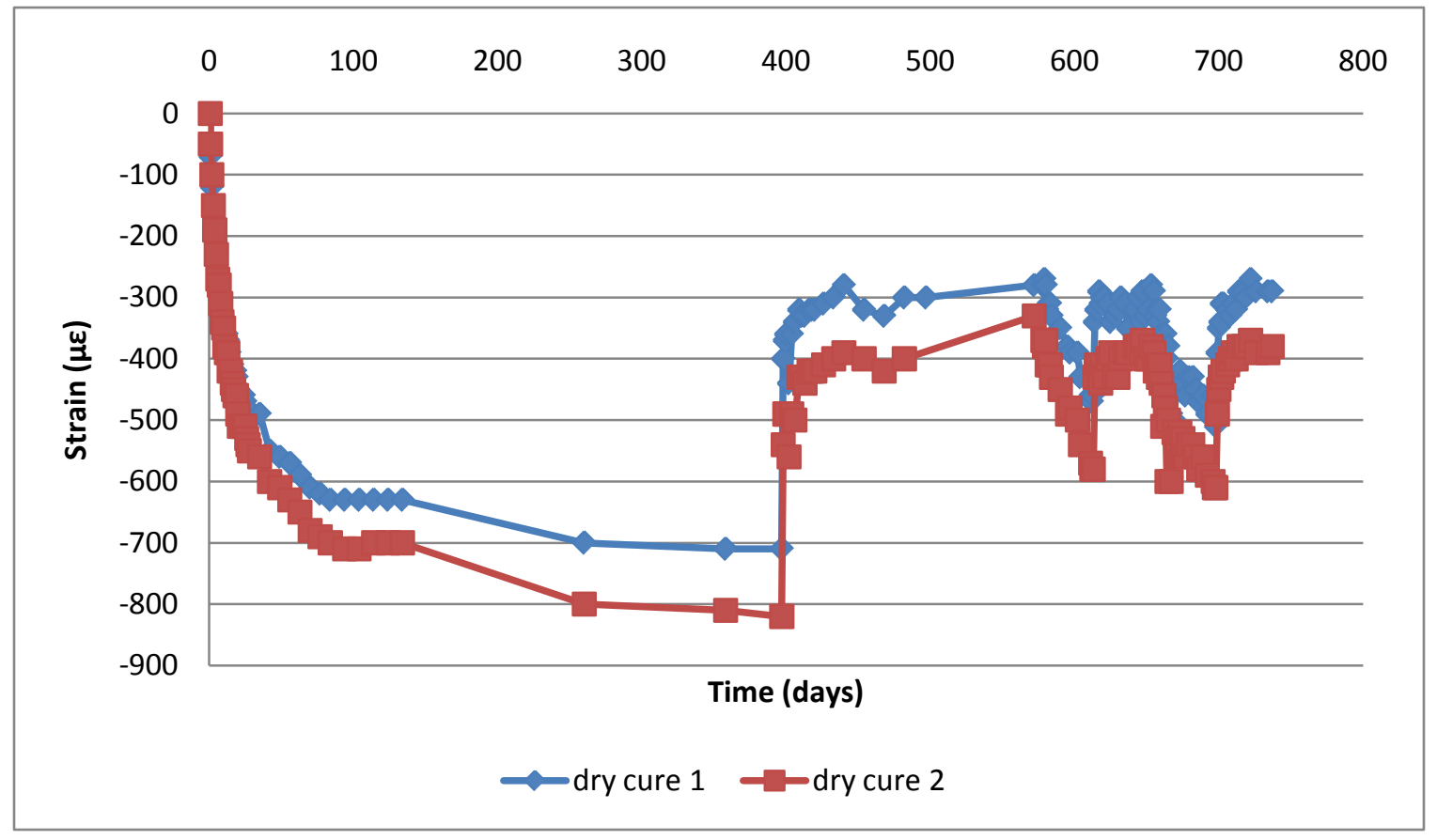

Figure B.3: Strain versus time for dry cured Virgin 2.

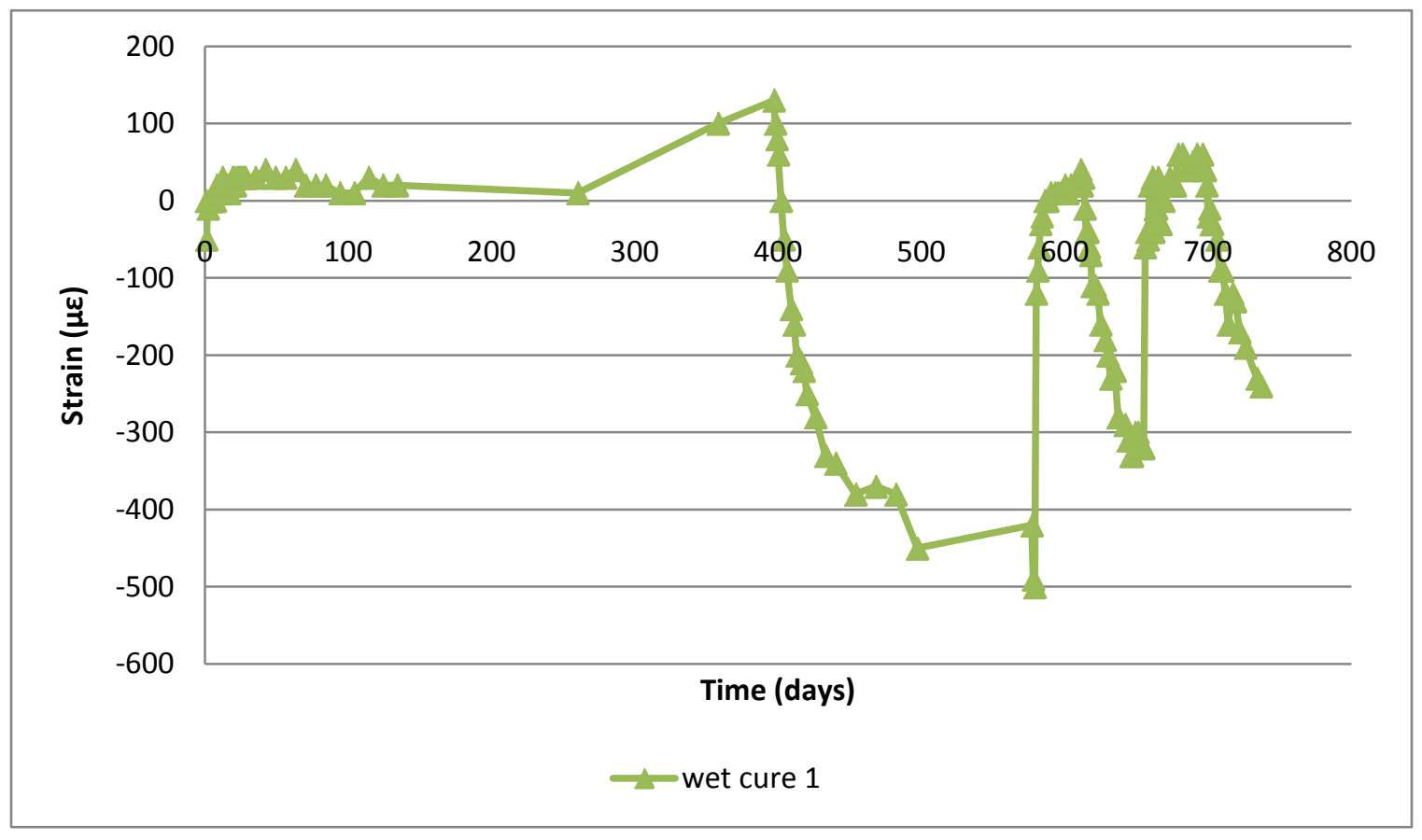

Figure B.4: Strain versus time for wet cured Virgin 2. 


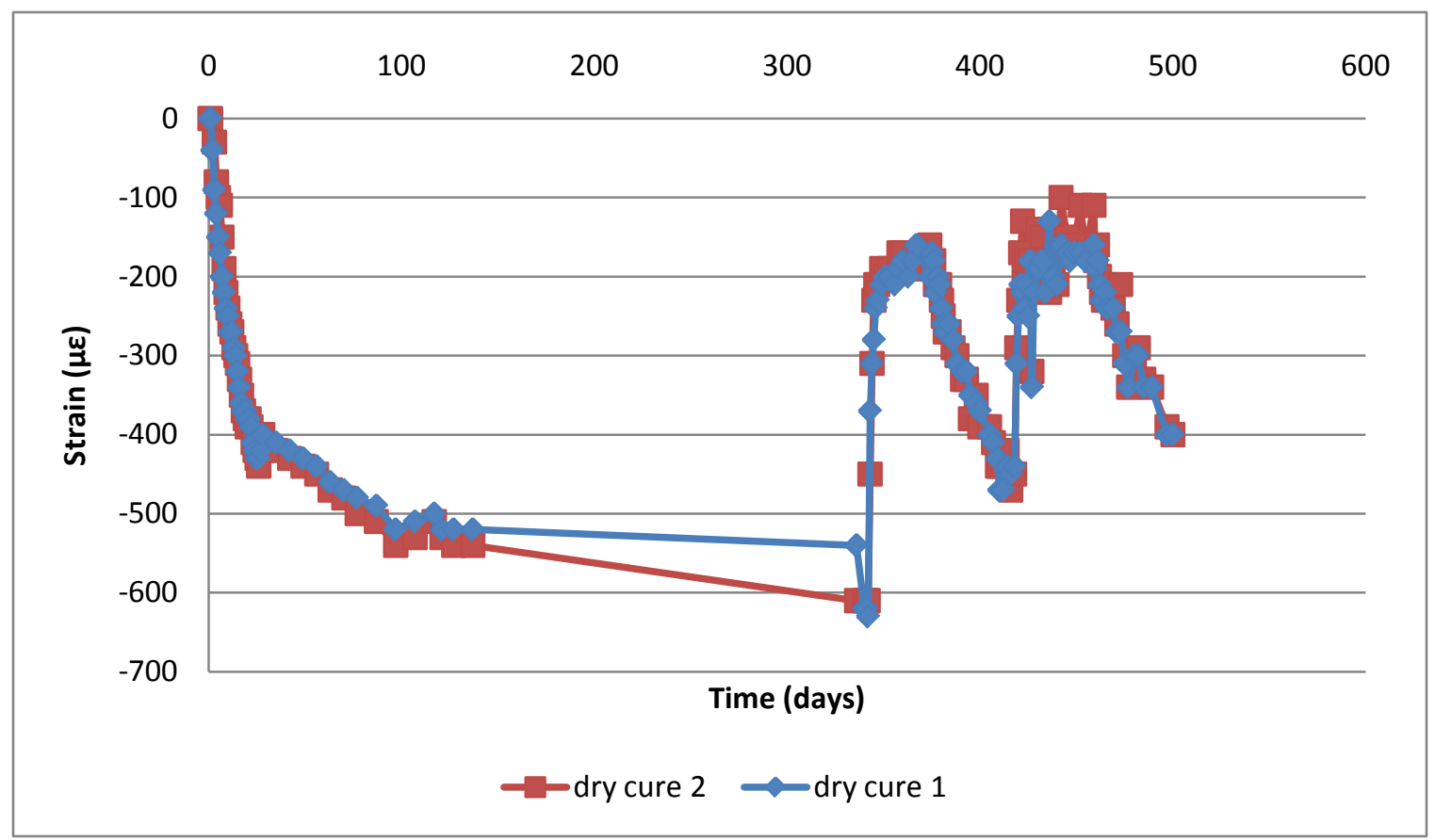

Figure B.5: Strain versus time for dry cured Virgin 3.

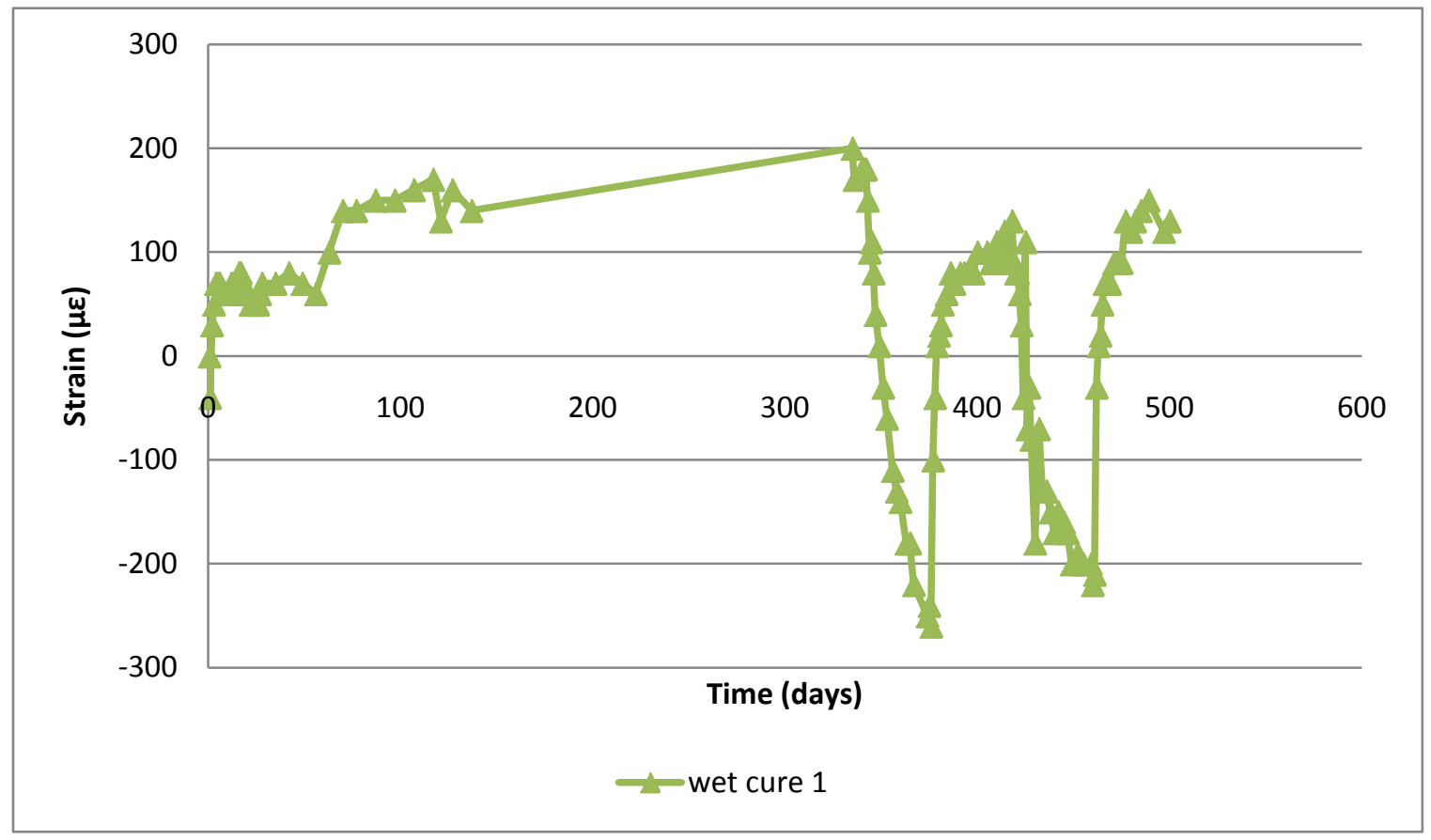

Figure B.6: Strain versus time for wet cured Virgin 3. 


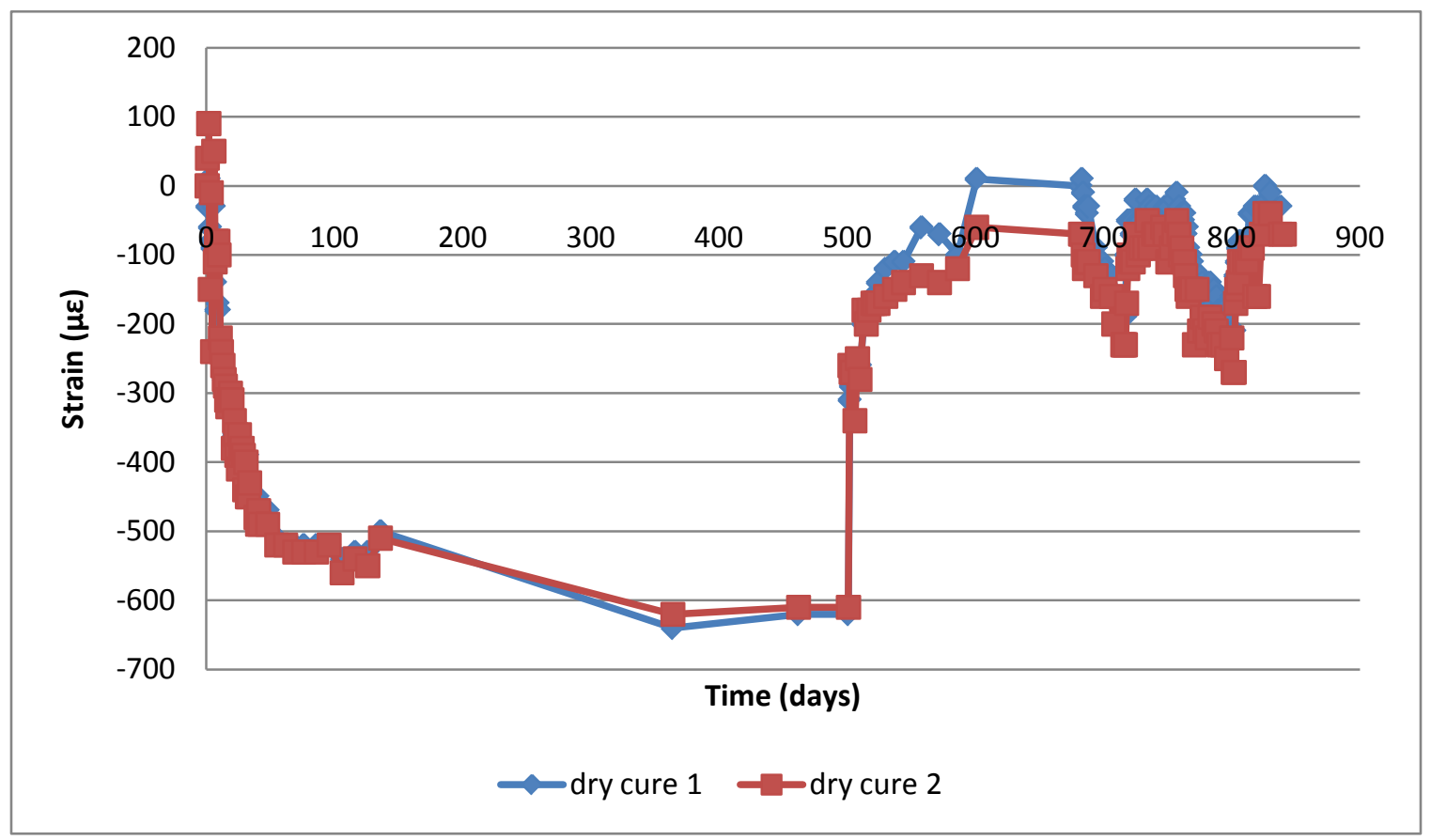

Figure B.7: Strain versus time for dry cured Slag RCA.

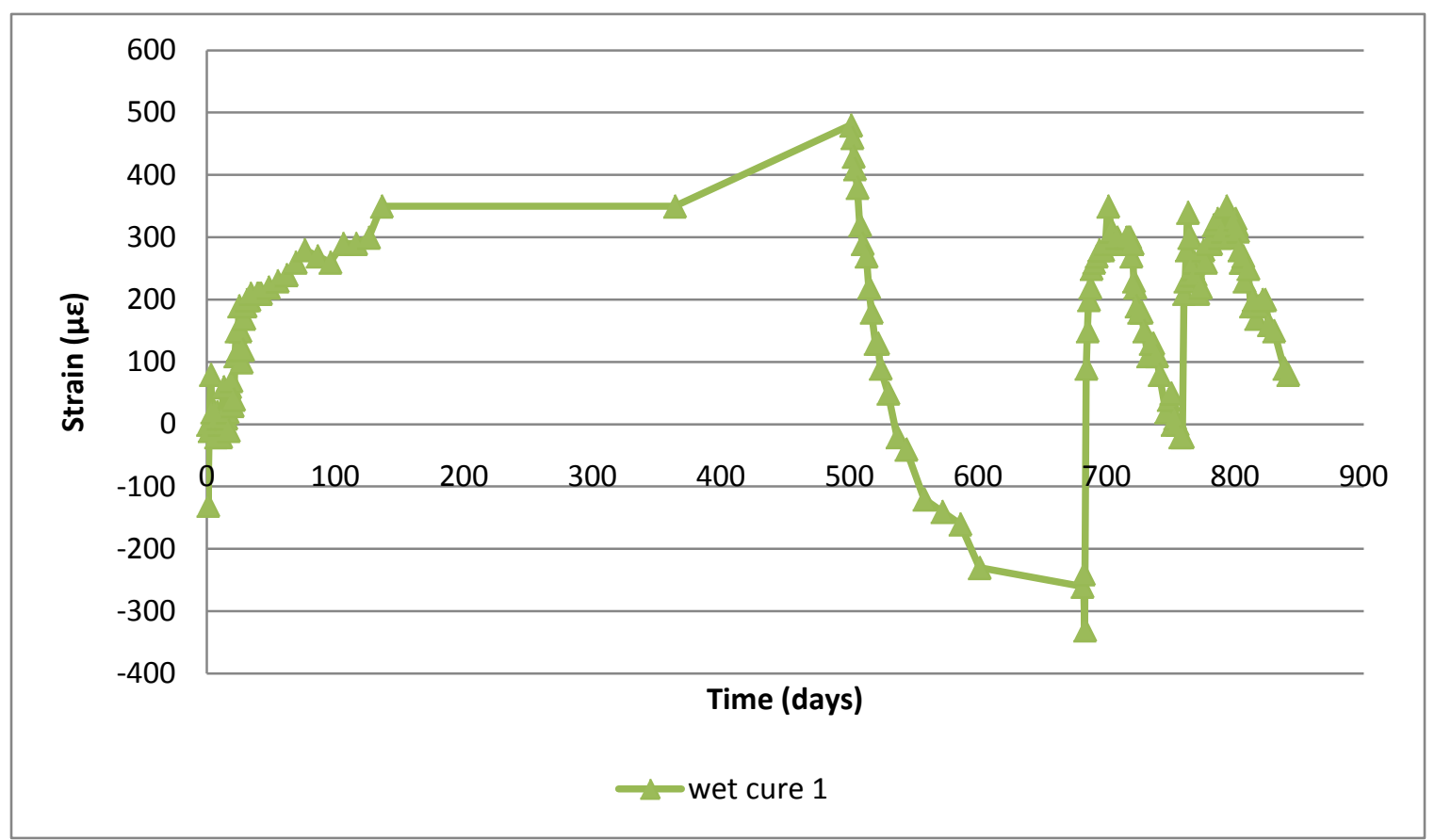

Figure B.8: Strain versus time for wet cured Slag RCA. 


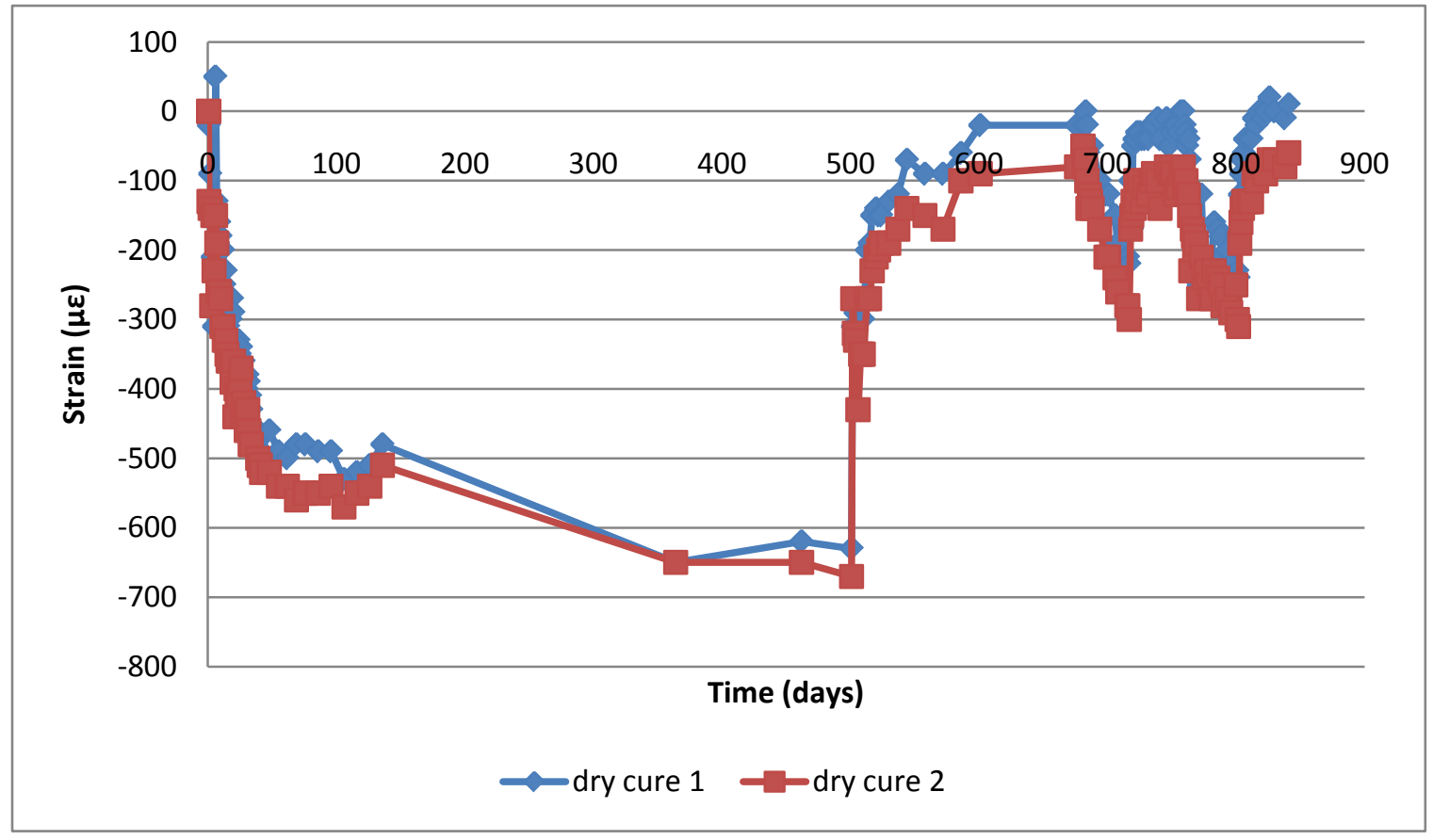

Figure B.9: Strain versus time for dry cured Limestone RCA.

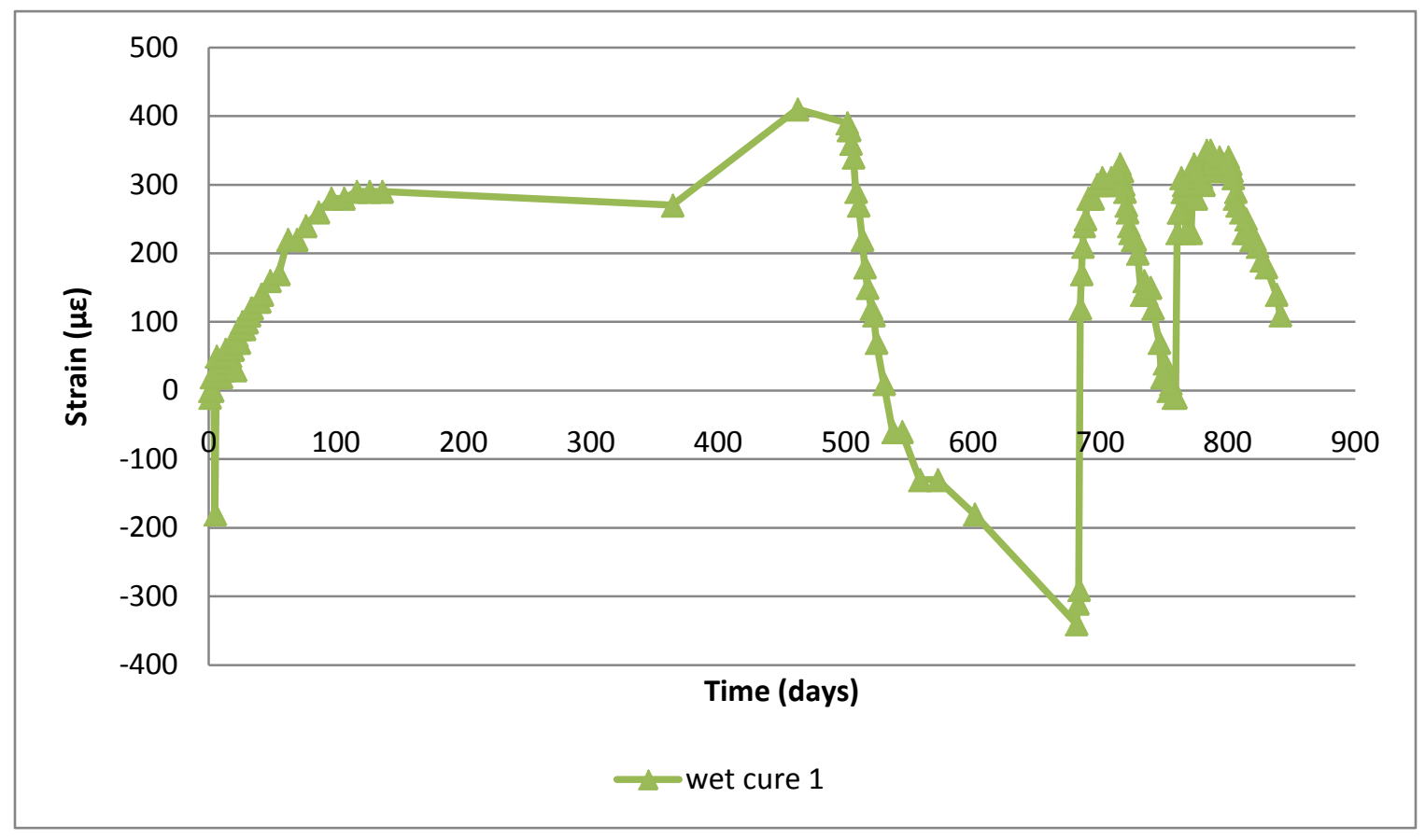

Figure B.10: Strain versus time for wet cured Limestone RCA. 


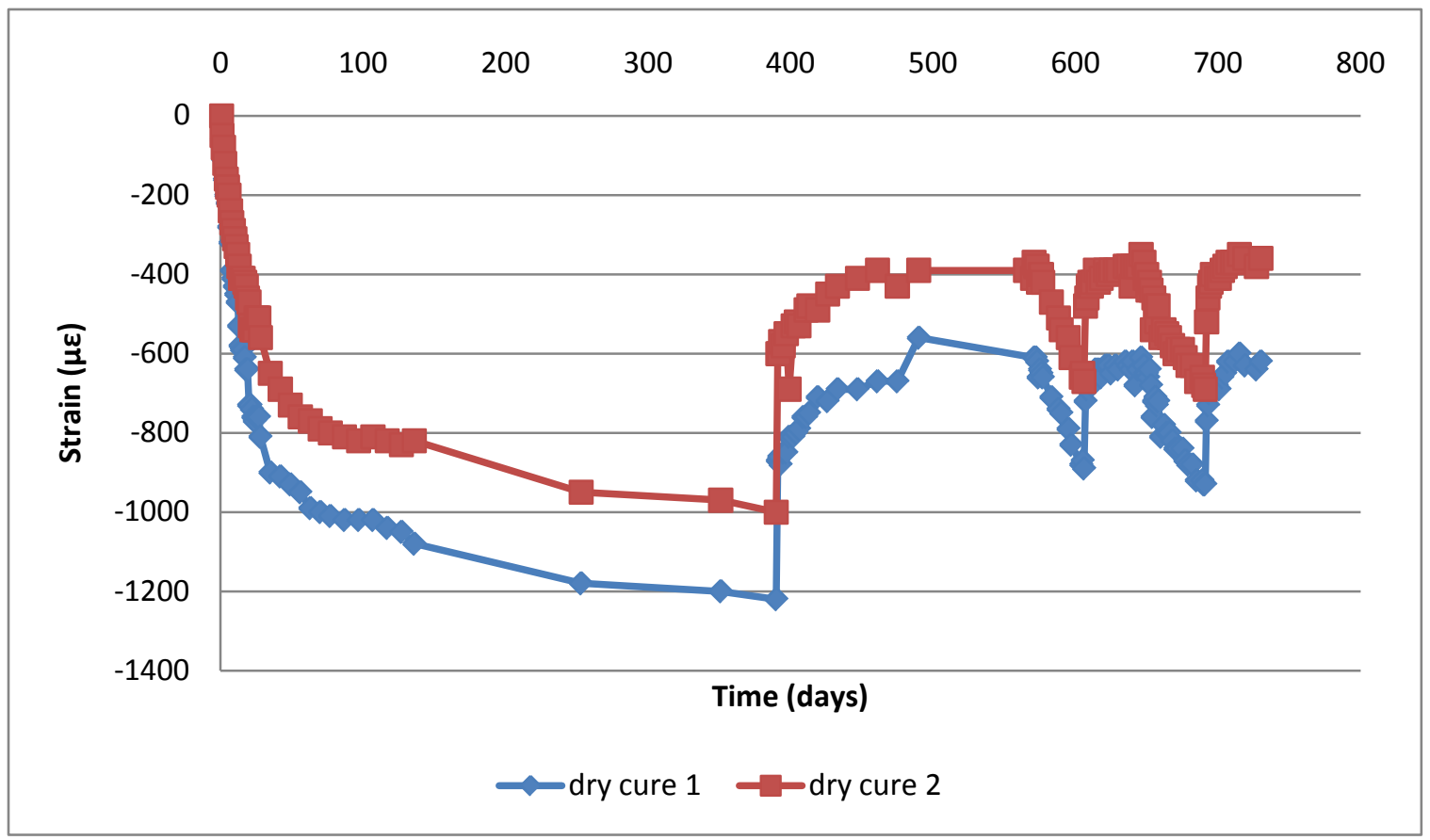

Figure B.11: Strain versus time for dry cured Gravel RCA 1.

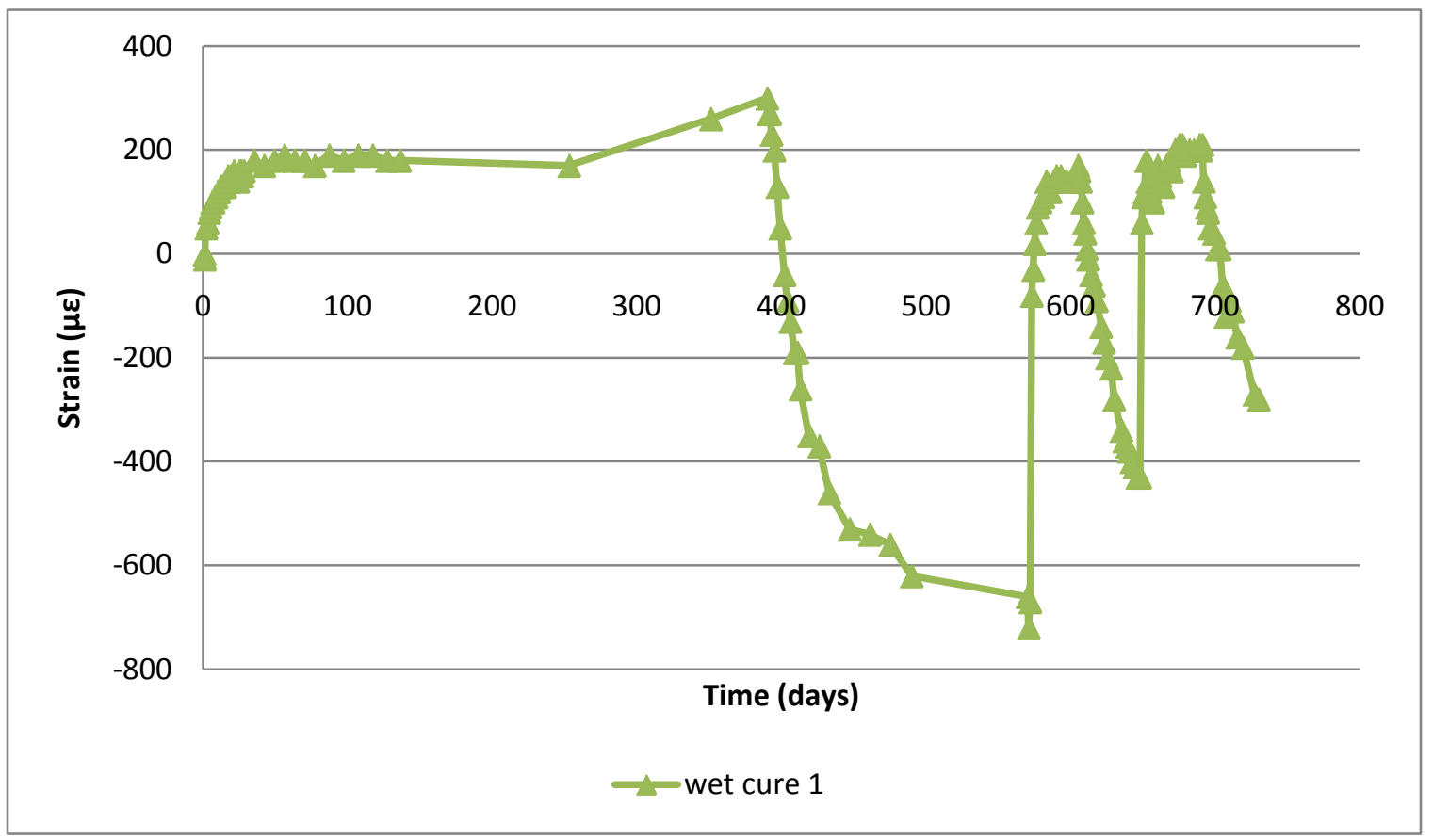

Figure B.12: Strain versus time for wet cured Gravel RCA 1. 


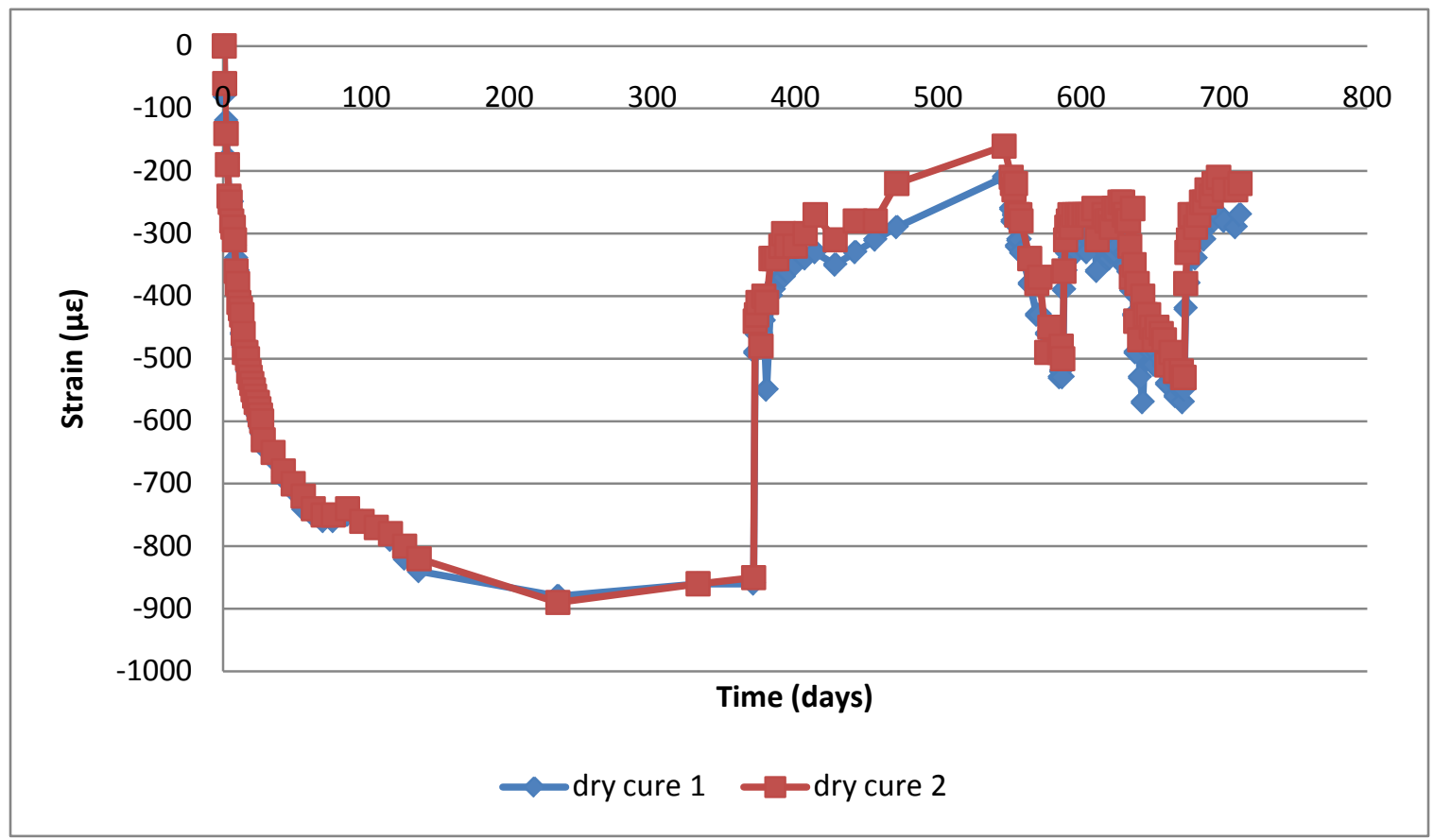

Figure B.13: Strain versus time for dry cured Gravel RCA 2.

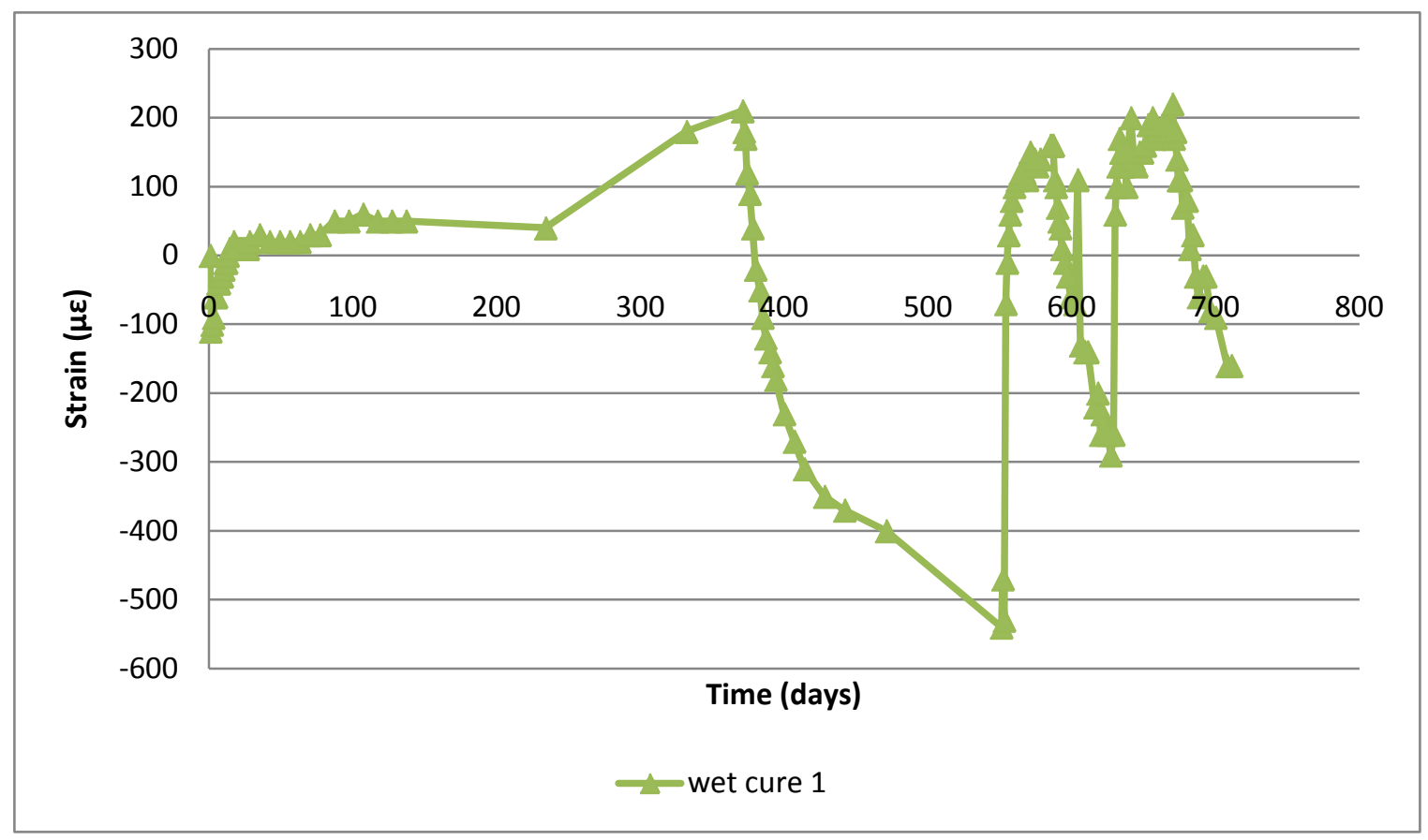

Figure B.14: Strain versus time for wet cured Gravel RCA 2. 


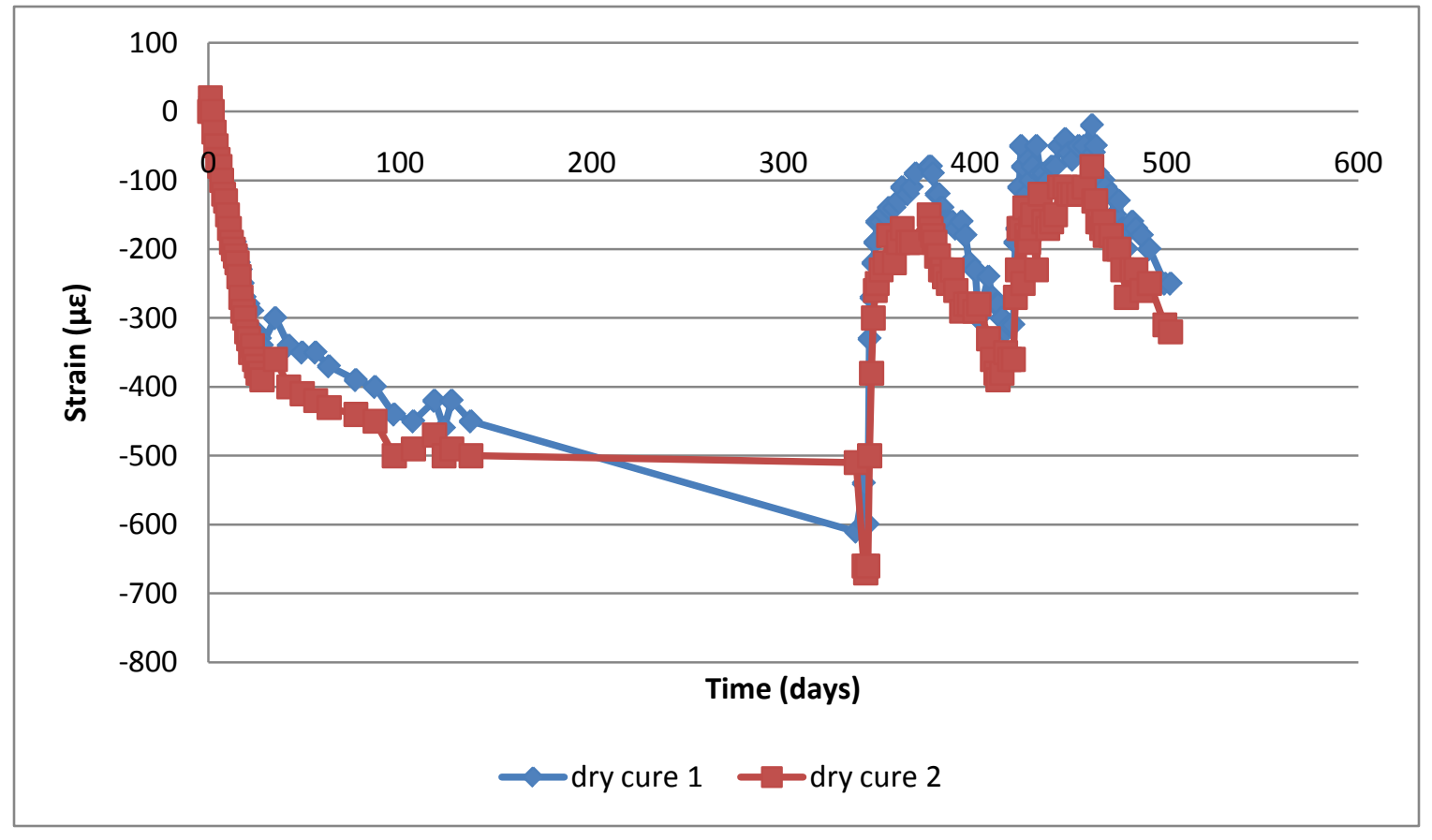

Figure B.15: Strain versus time for dry cured Twice Recycled RCA.

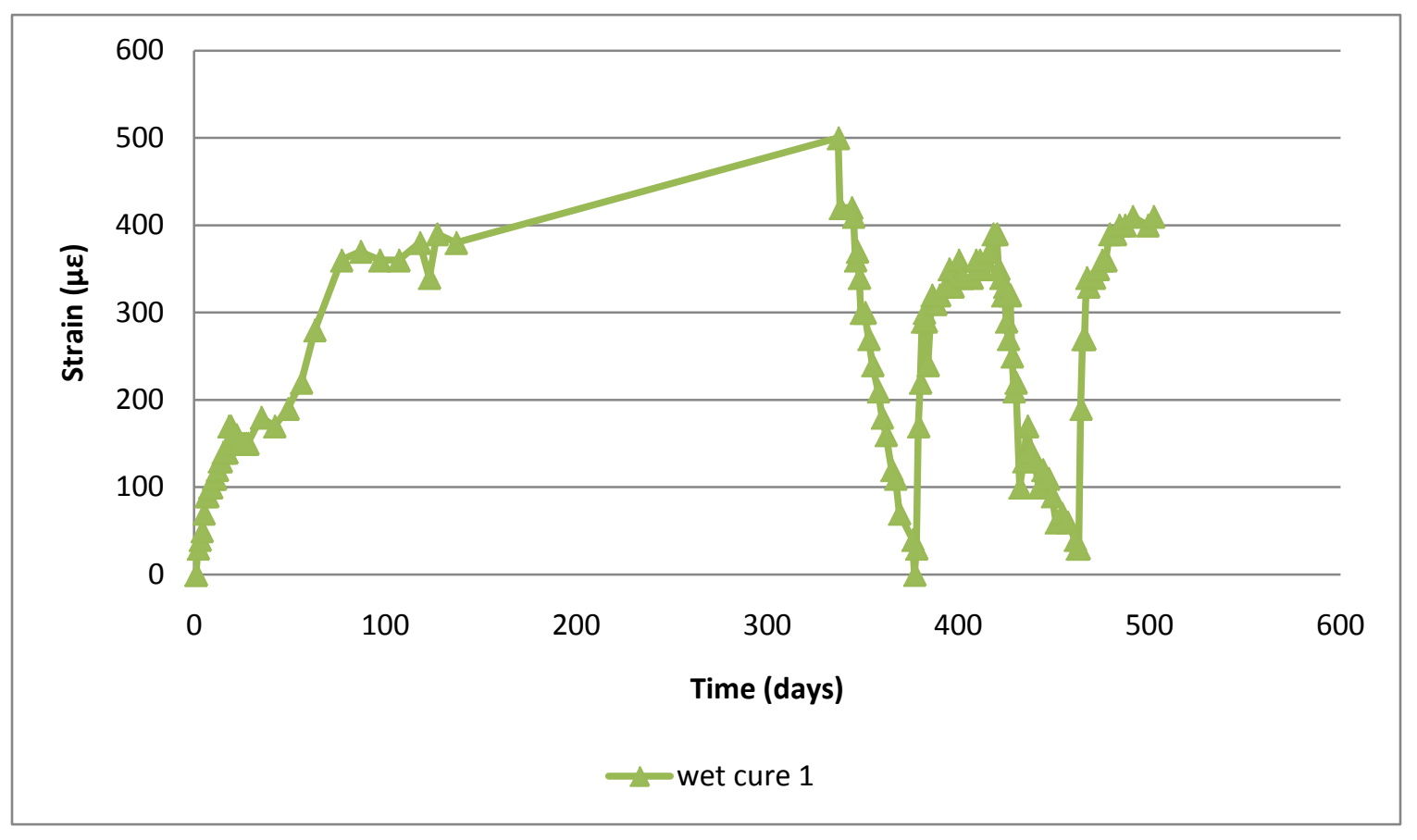

Figure B.16: Strain versus time for wet cured Twice Recycled RCA. 


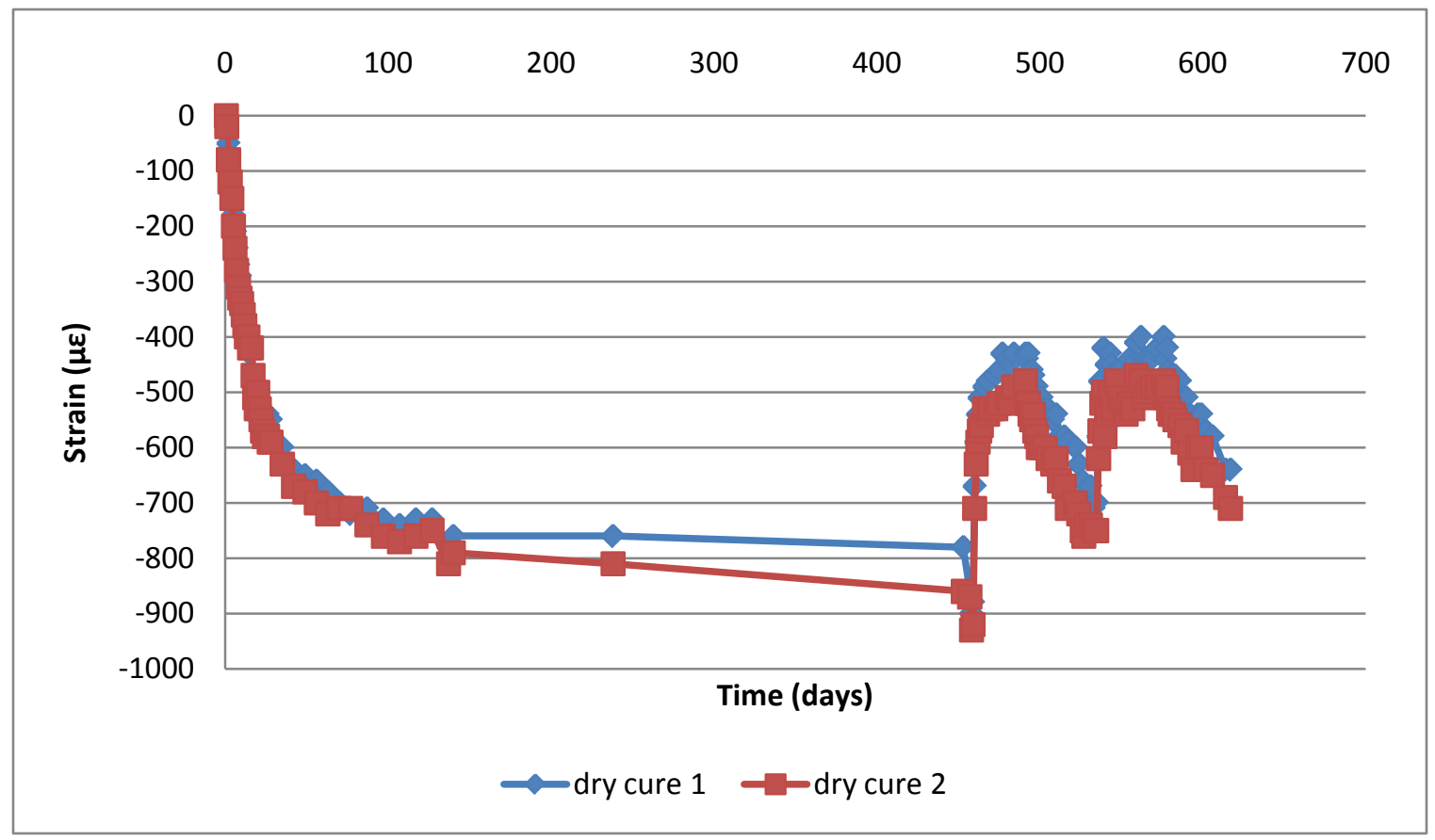

Figure B.17: Strain versus time for dry cured Lightweight 1.

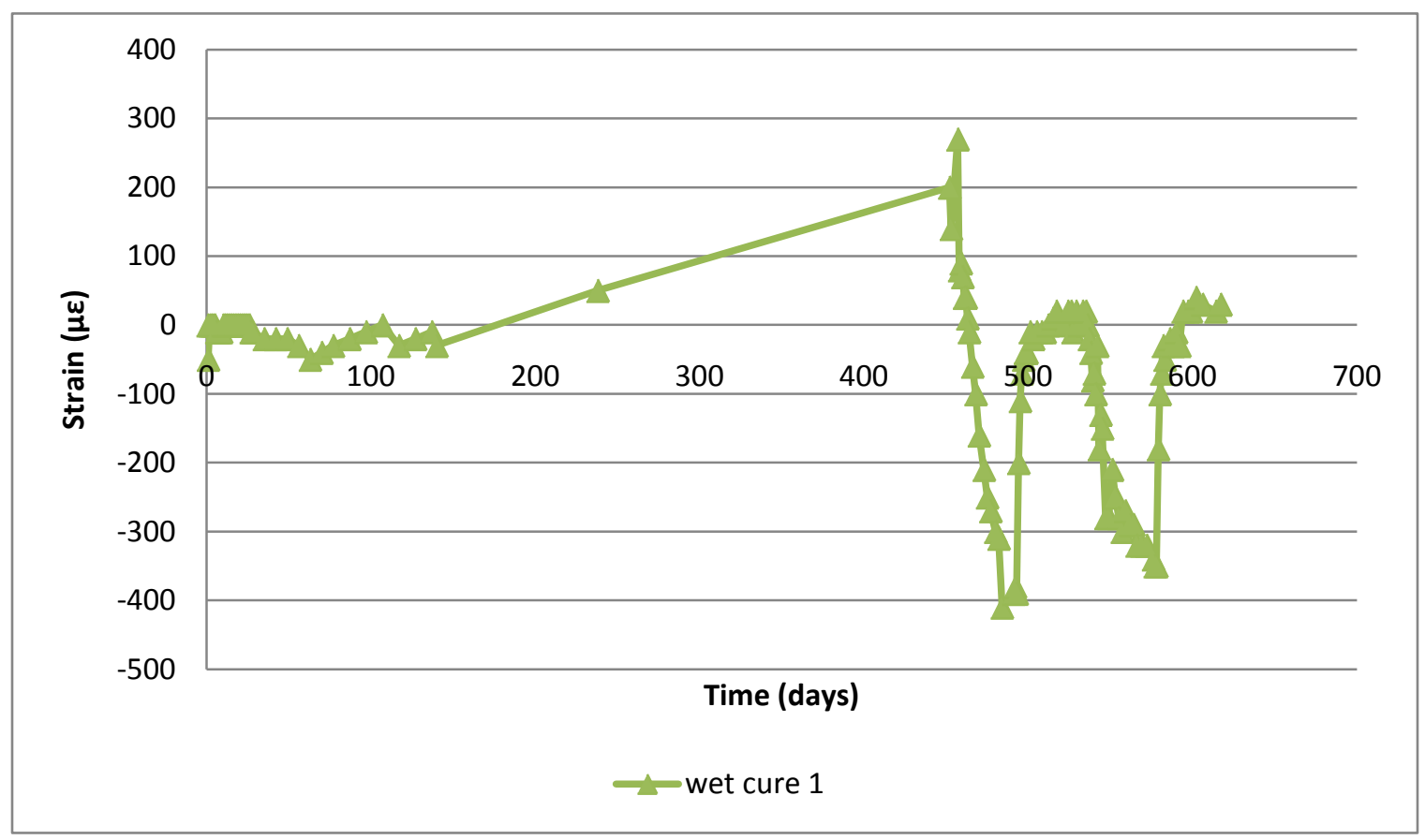

Figure B.18: Strain versus time for wet cured Lightweight 1. 


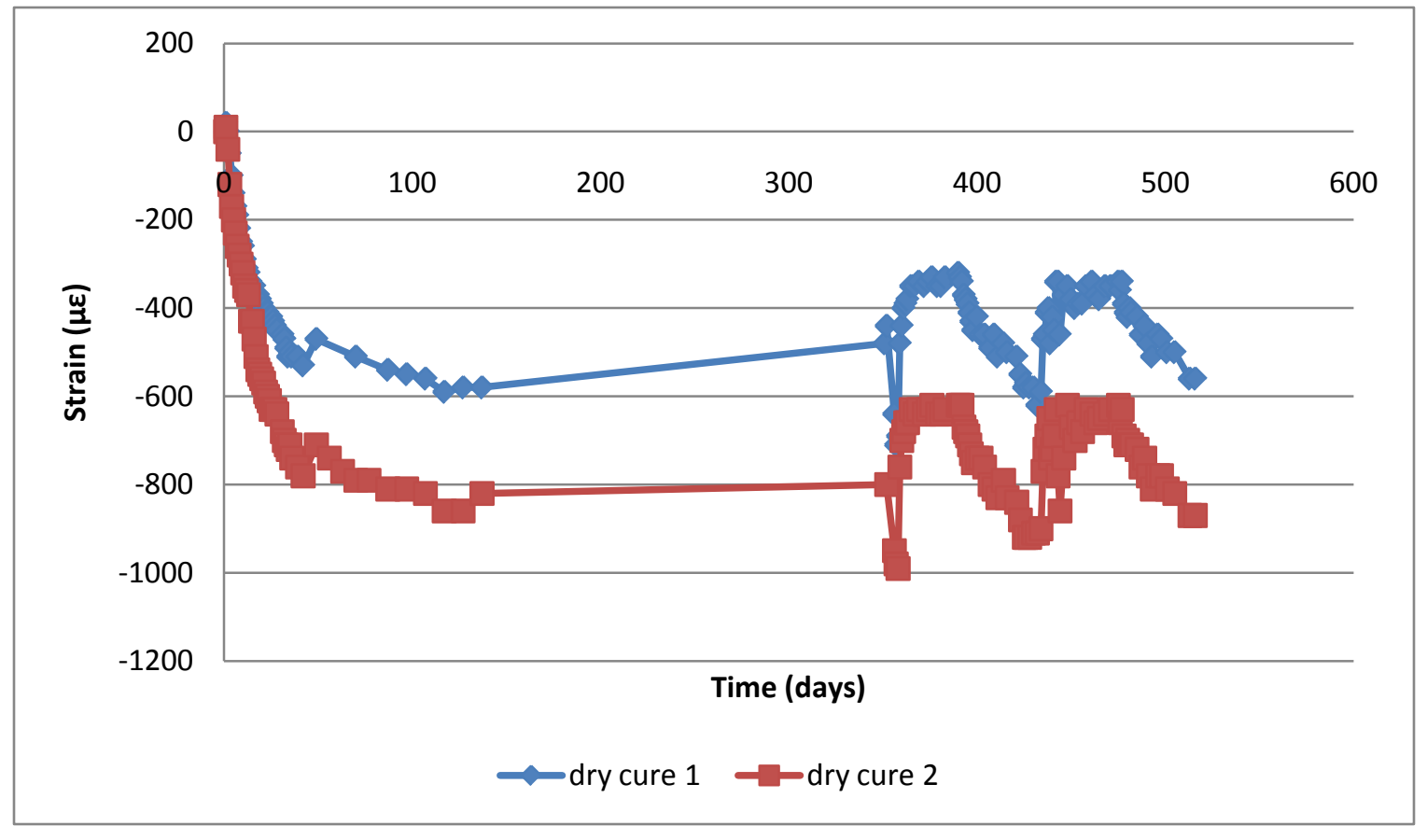

Figure B.19: Strain versus time for dry cured Lightweight 2.

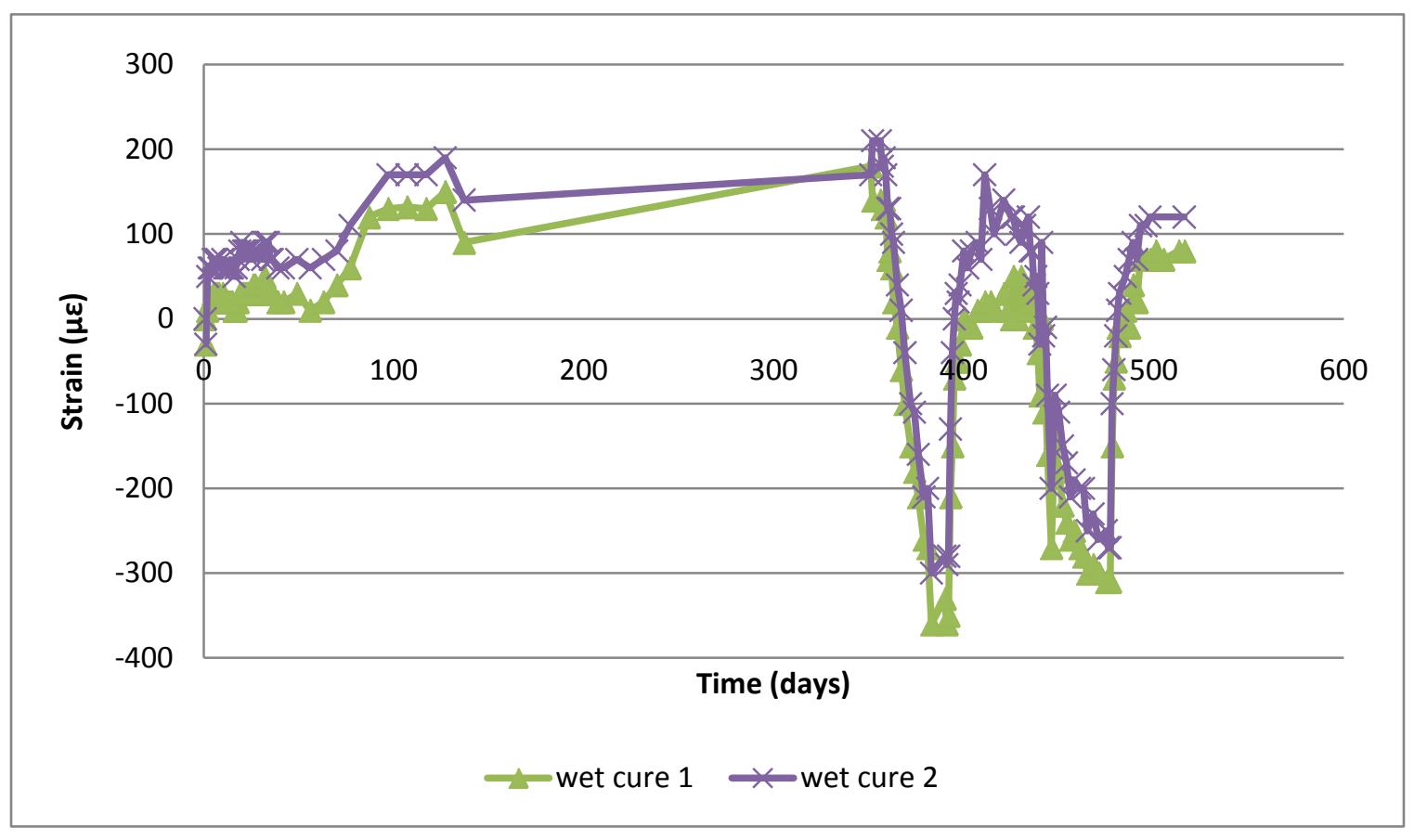

Figure B.20: Strain versus time for wet cured Lightweight 2. 


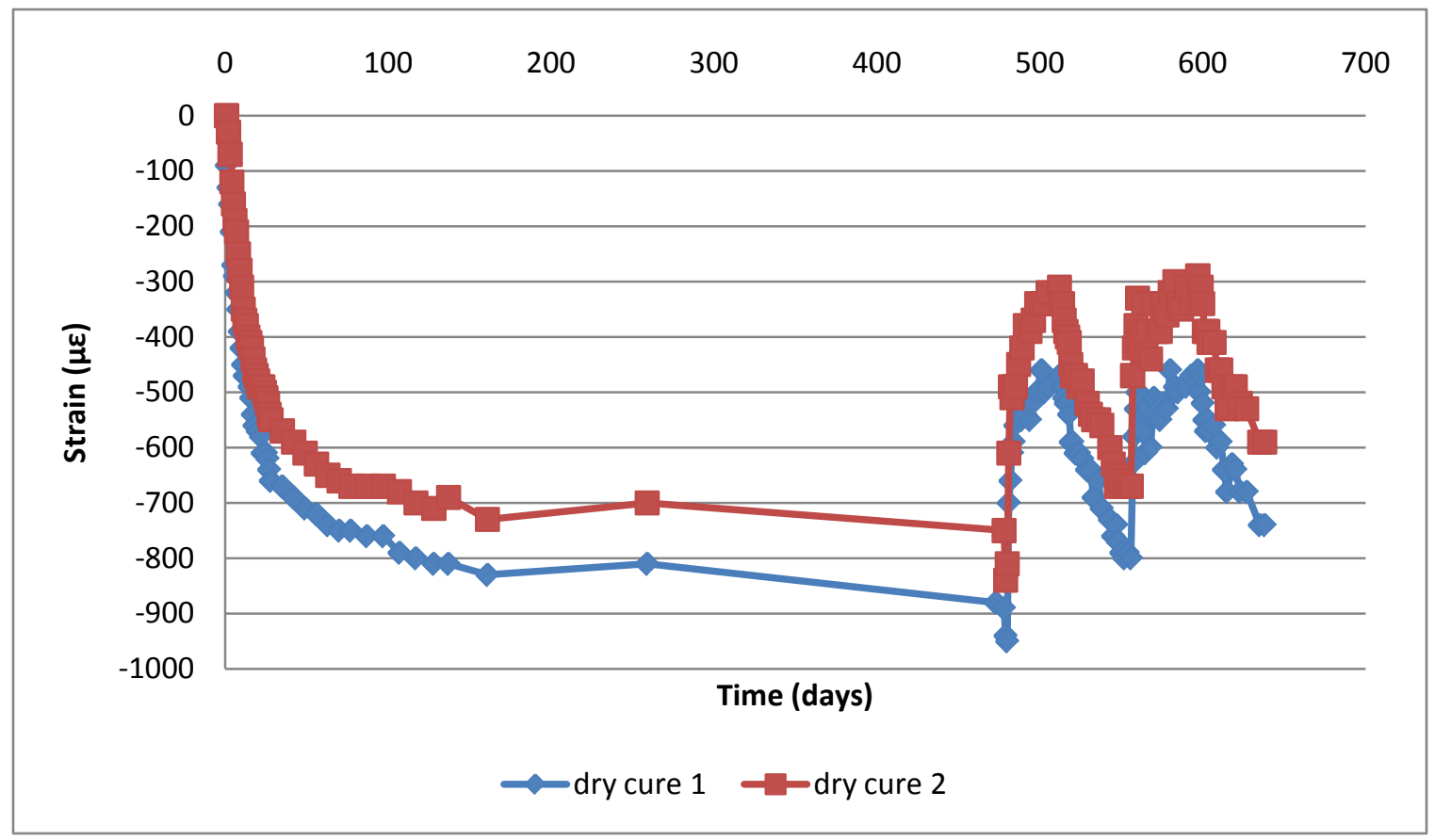

Figure B.21: Strain versus time for dry cured Lightweight 3.

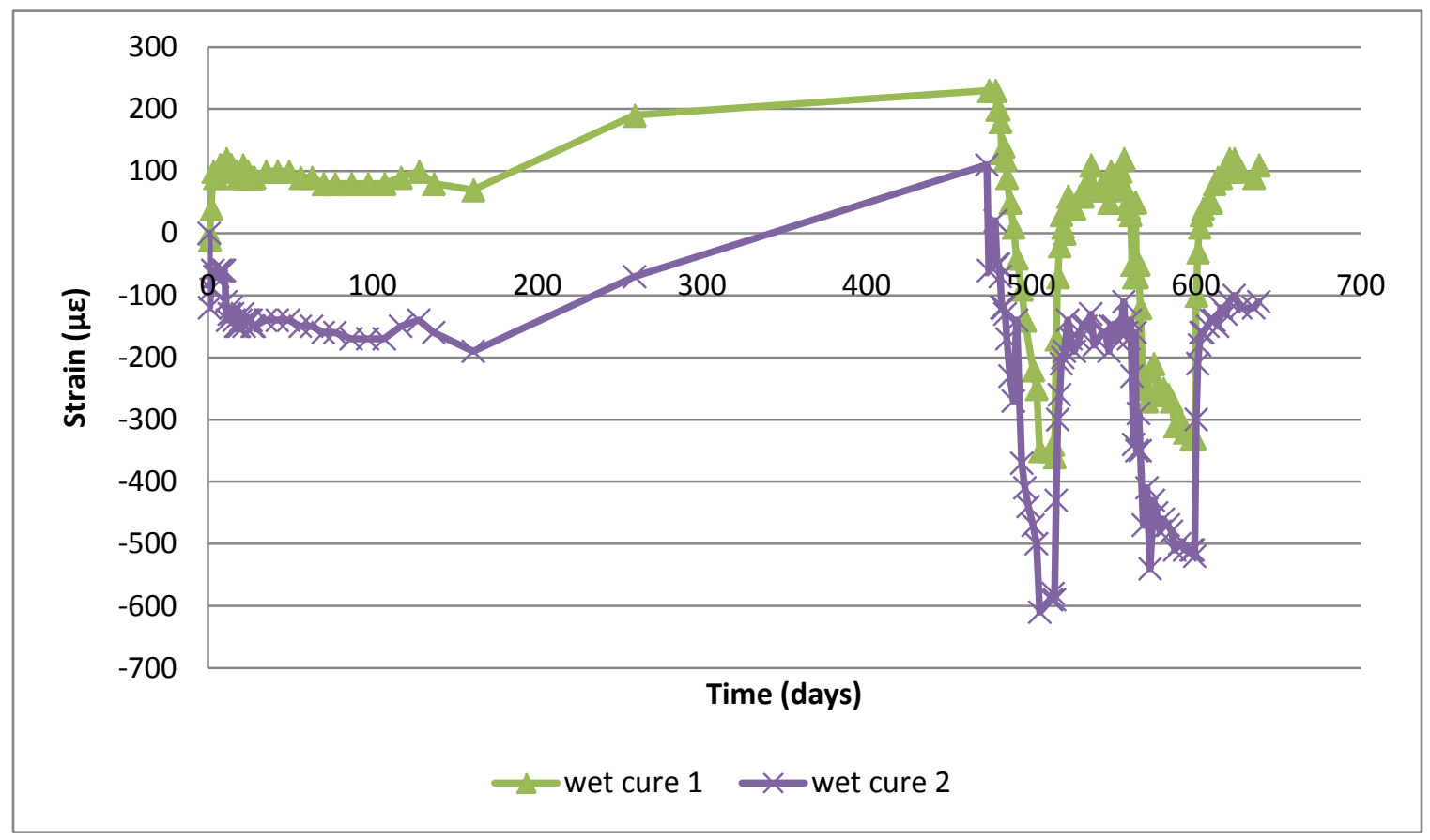

Figure B.22: Strain versus time for wet cured Lightweight 3. 


\section{Appendix C: Graphs of Weight Change versus Time}

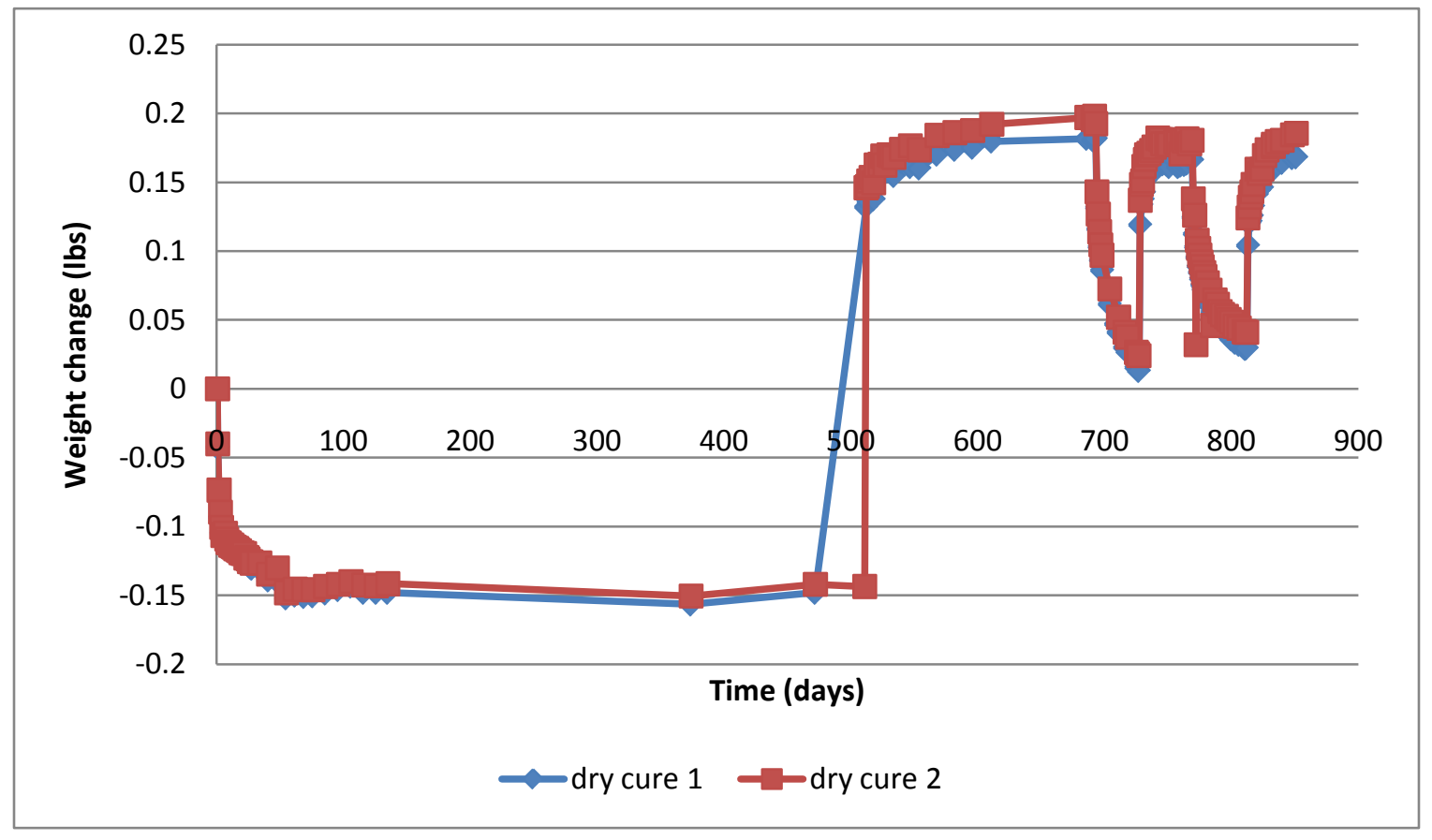

Figure C.1: Weight change versus time for dry cured Virgin 1.

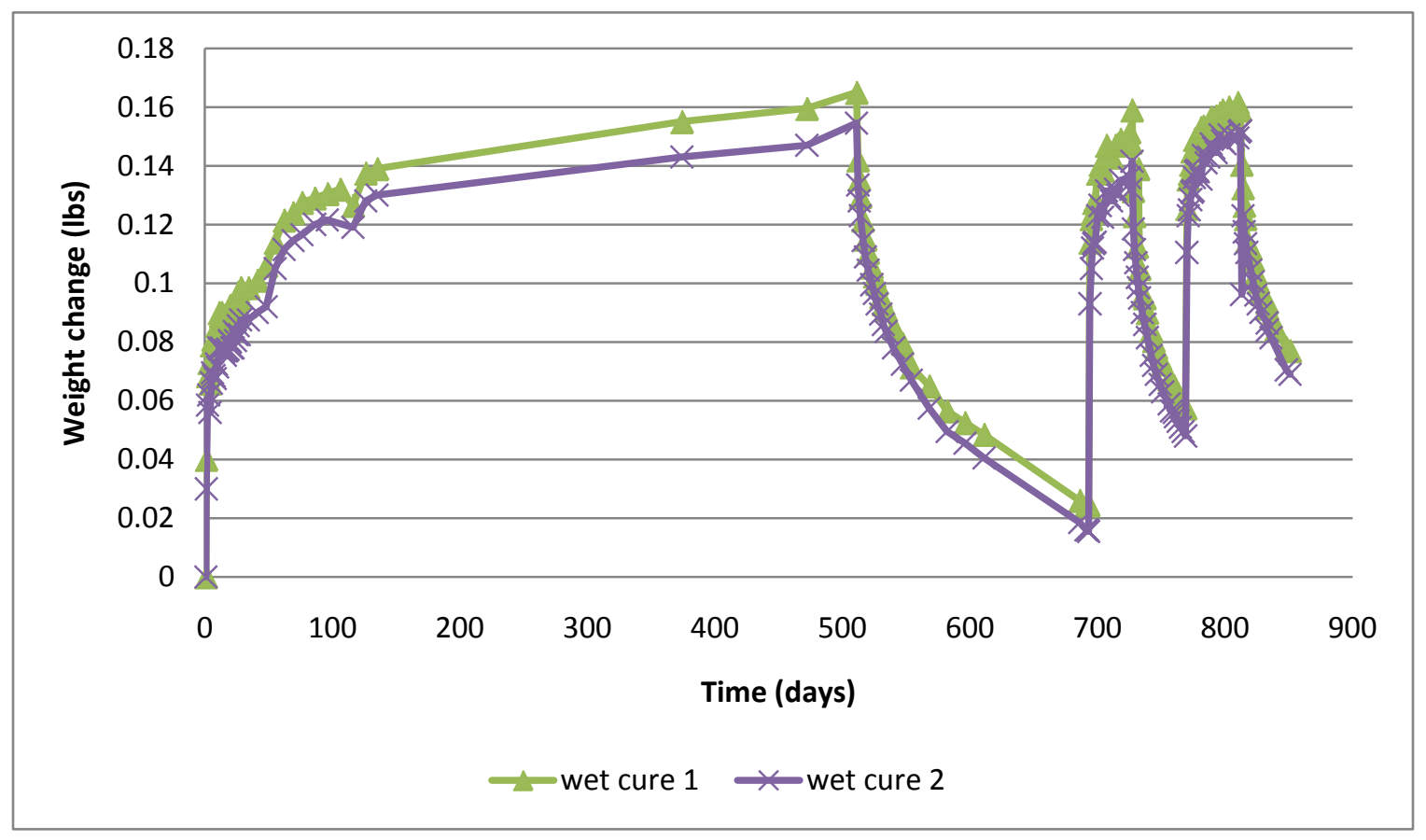

Figure C.2: Weight change versus time for wet cured Virgin 1. 


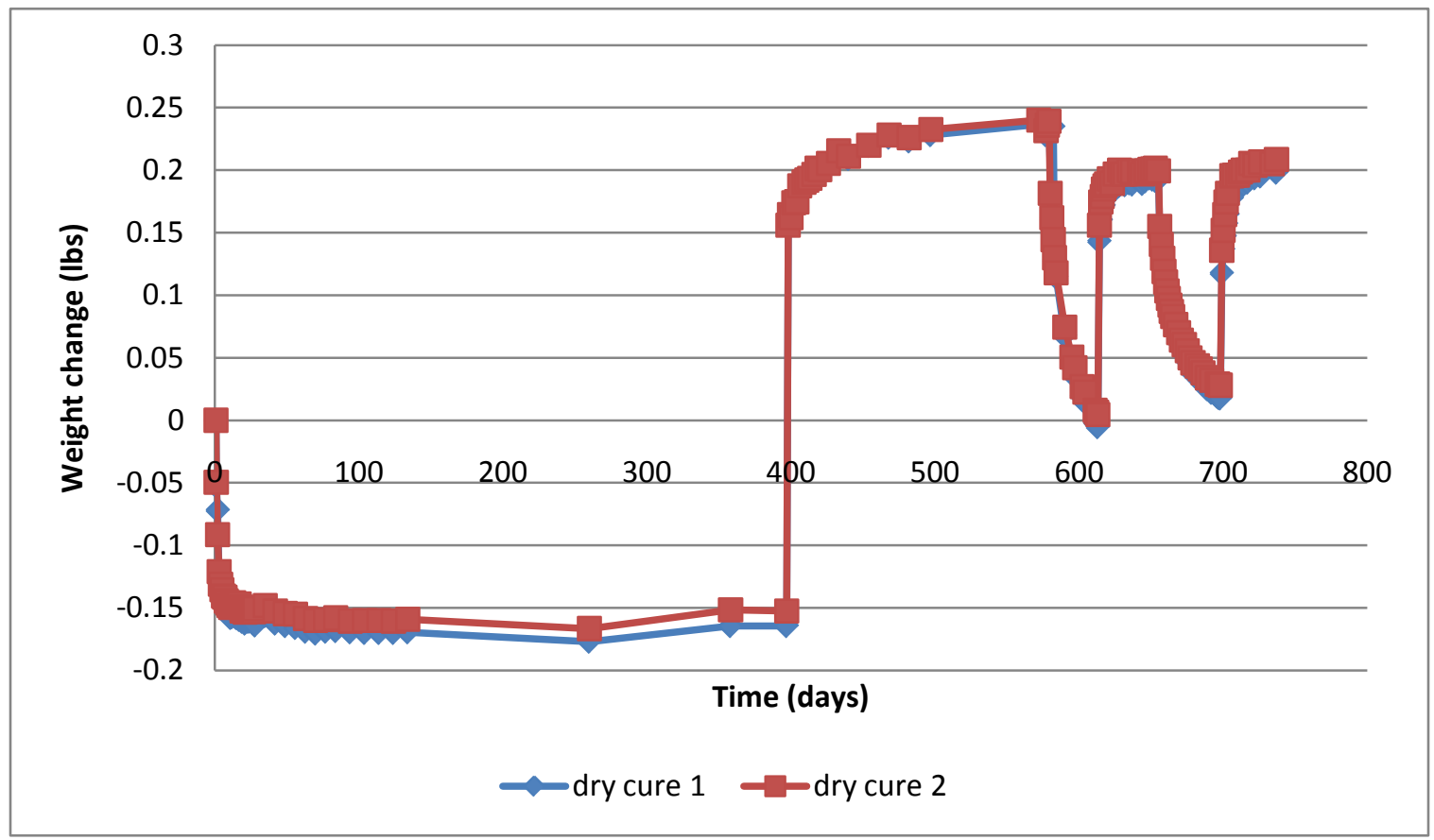

Figure C.3: Weight change versus time for dry cured Virgin 2.

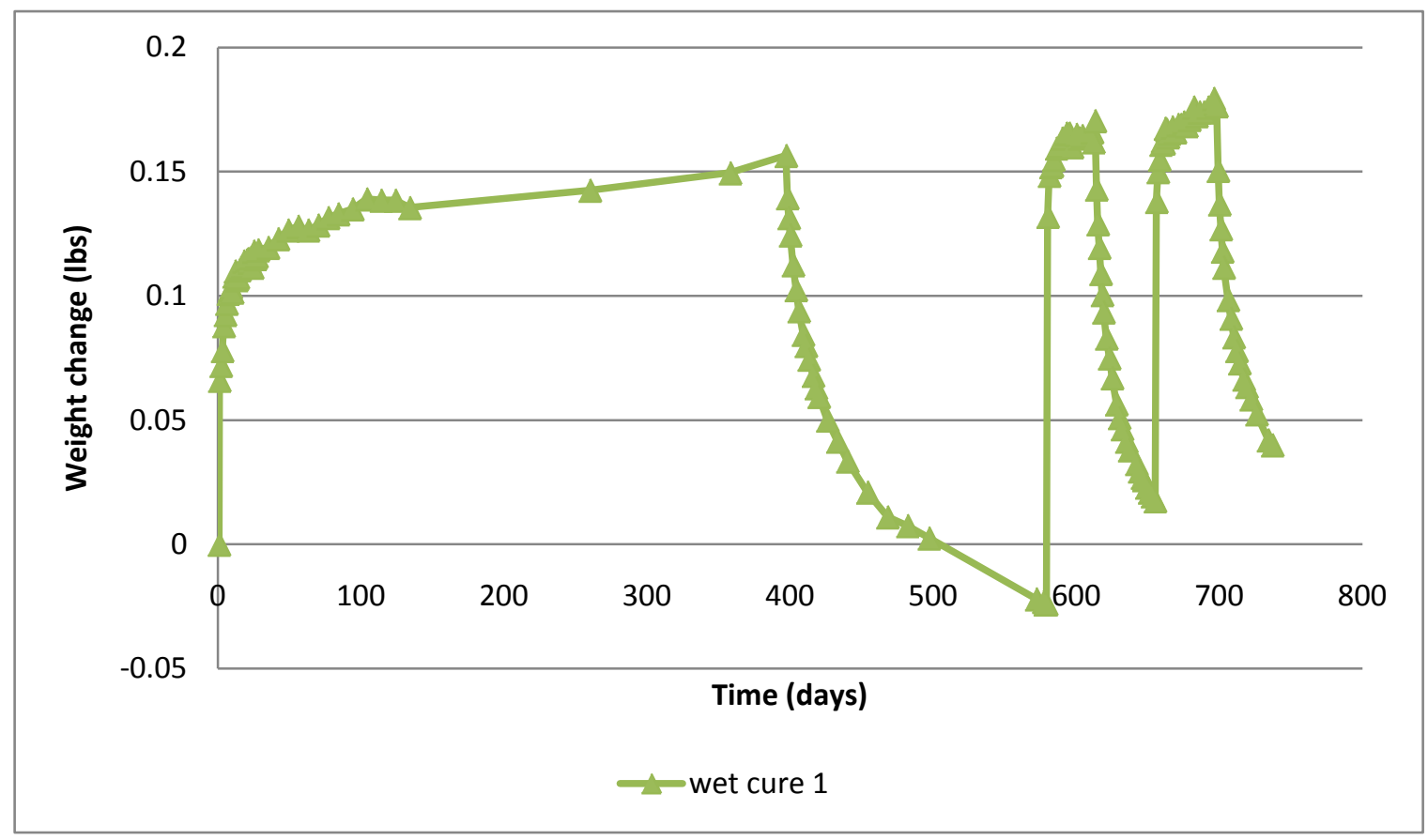

Figure C.4: Weight change versus time for wet cured Virgin 2. 


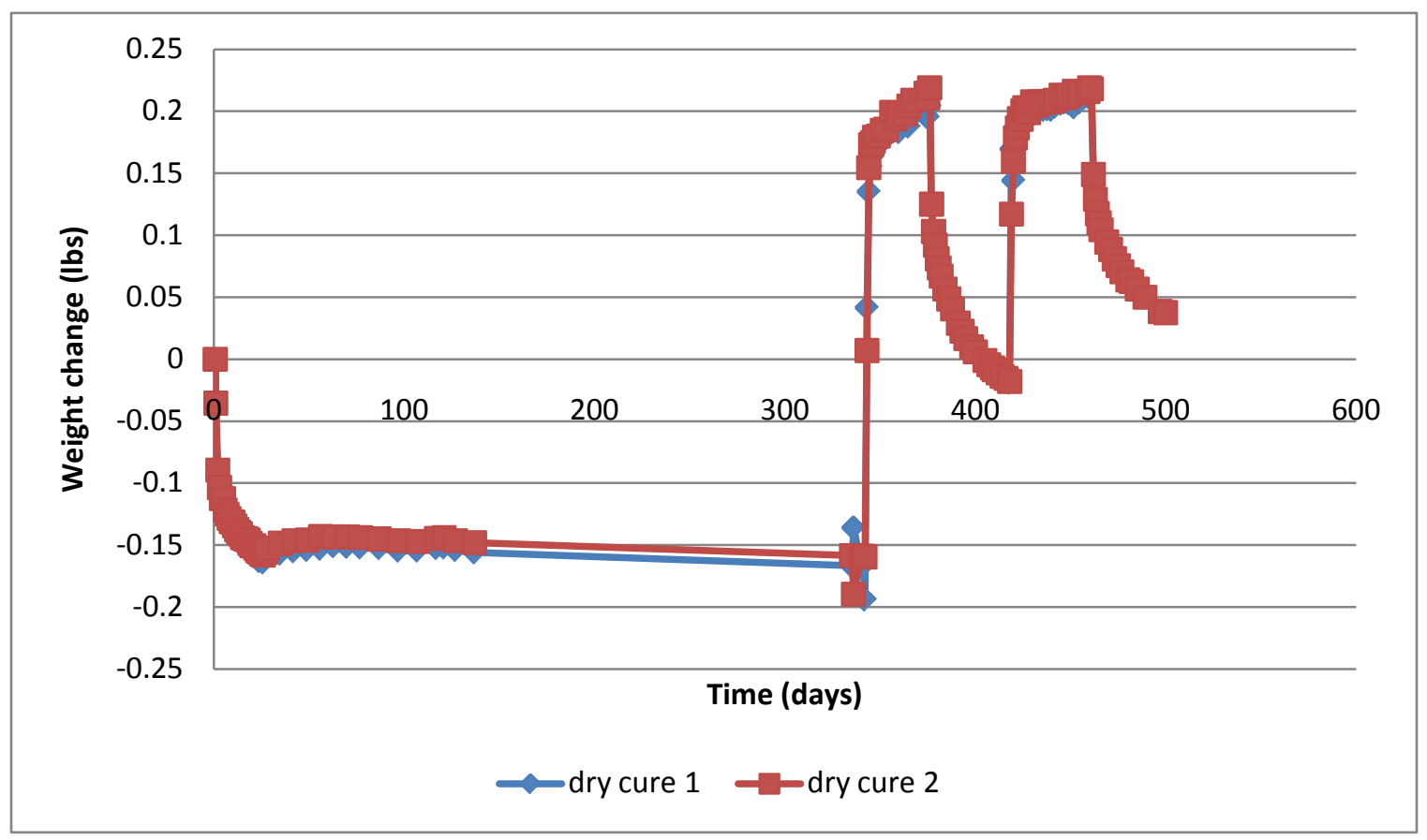

Figure C.5: Weight change versus time for dry cured Virgin 3.

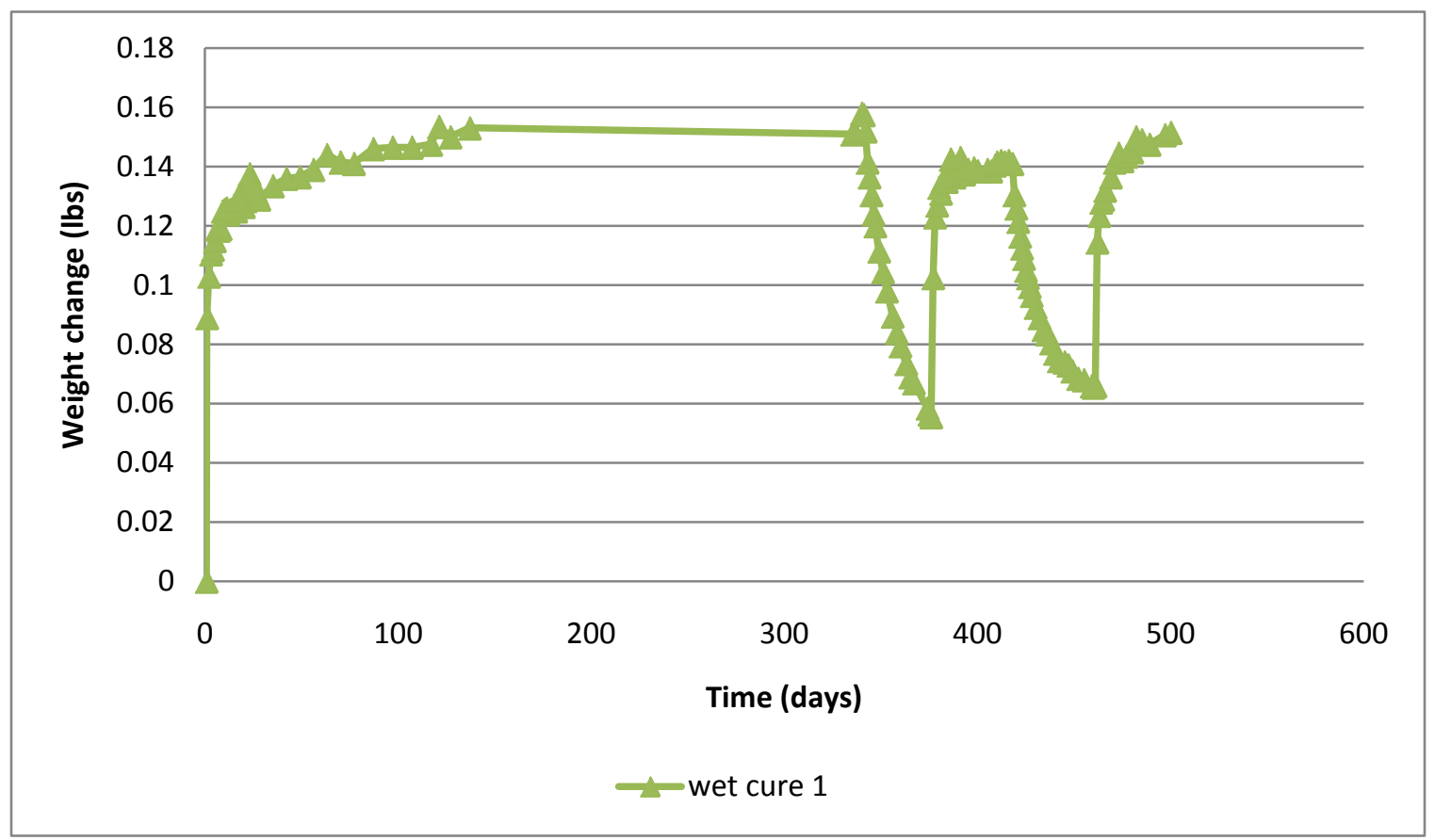

Figure C.6: Weight change versus time for wet cured Virgin 2. 


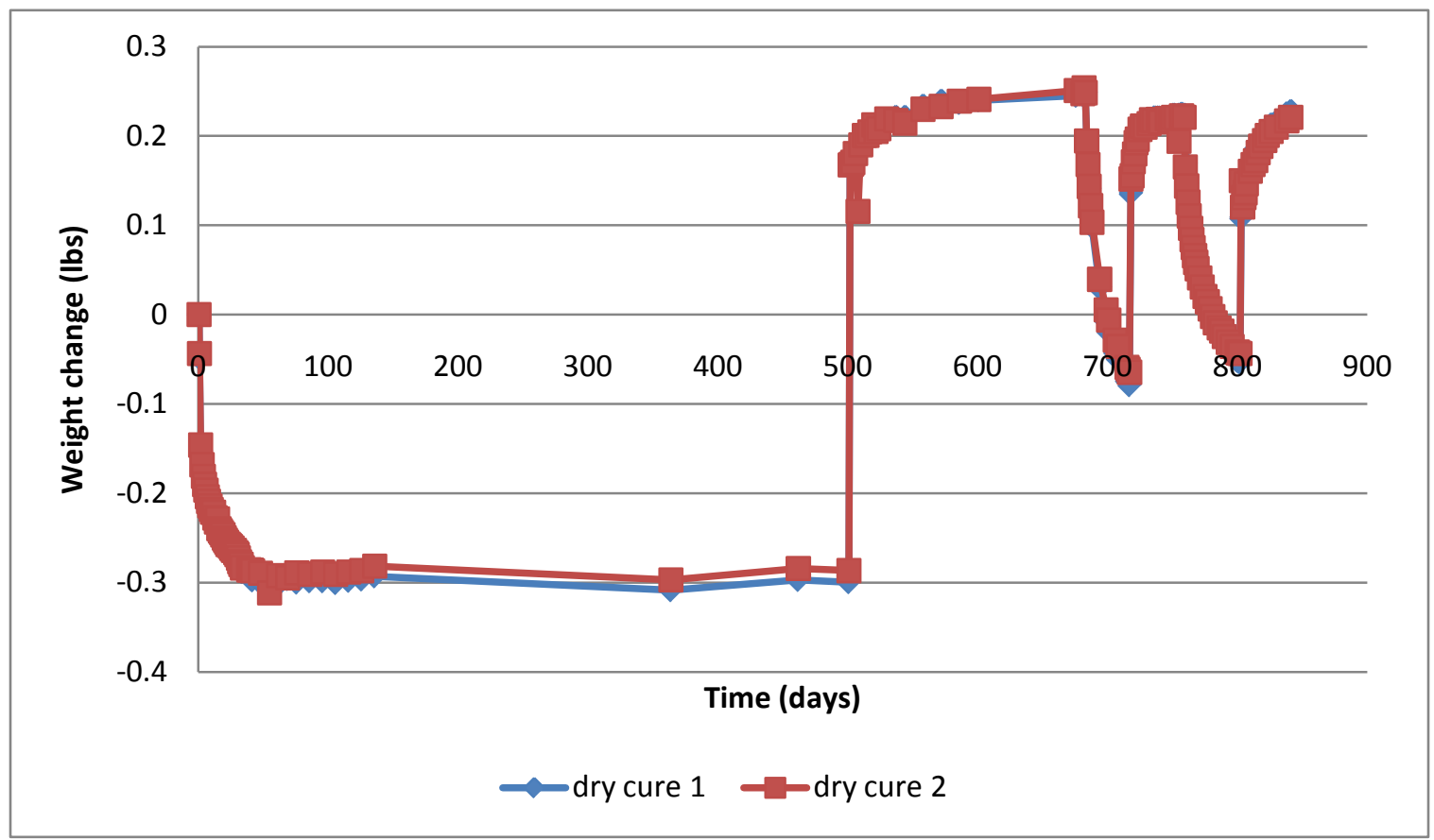

Figure C.7: Weight change versus time for dry cured Slag RCA.

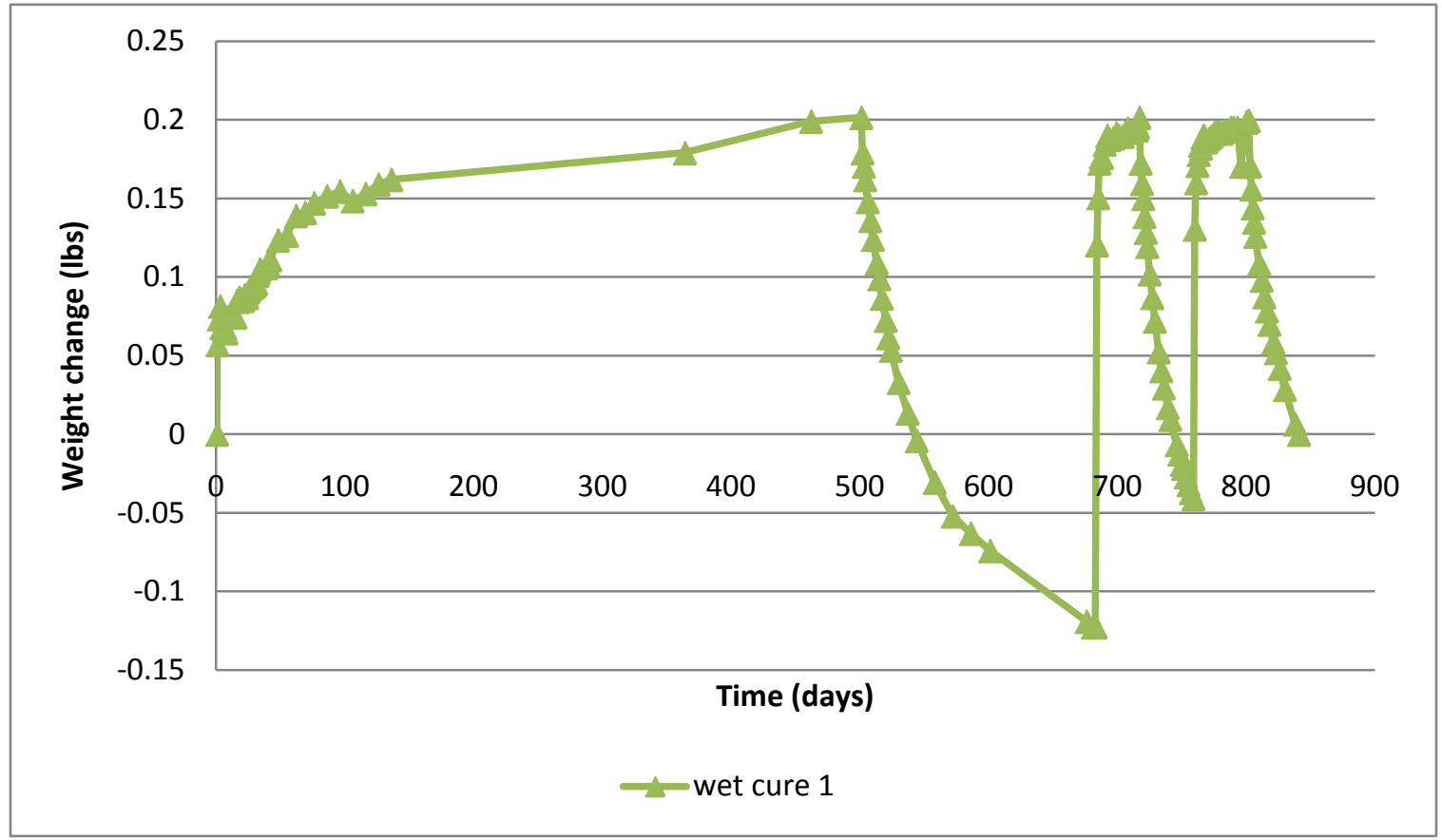

Figure C.8: Weight change versus time for wet cured Slag RCA. 


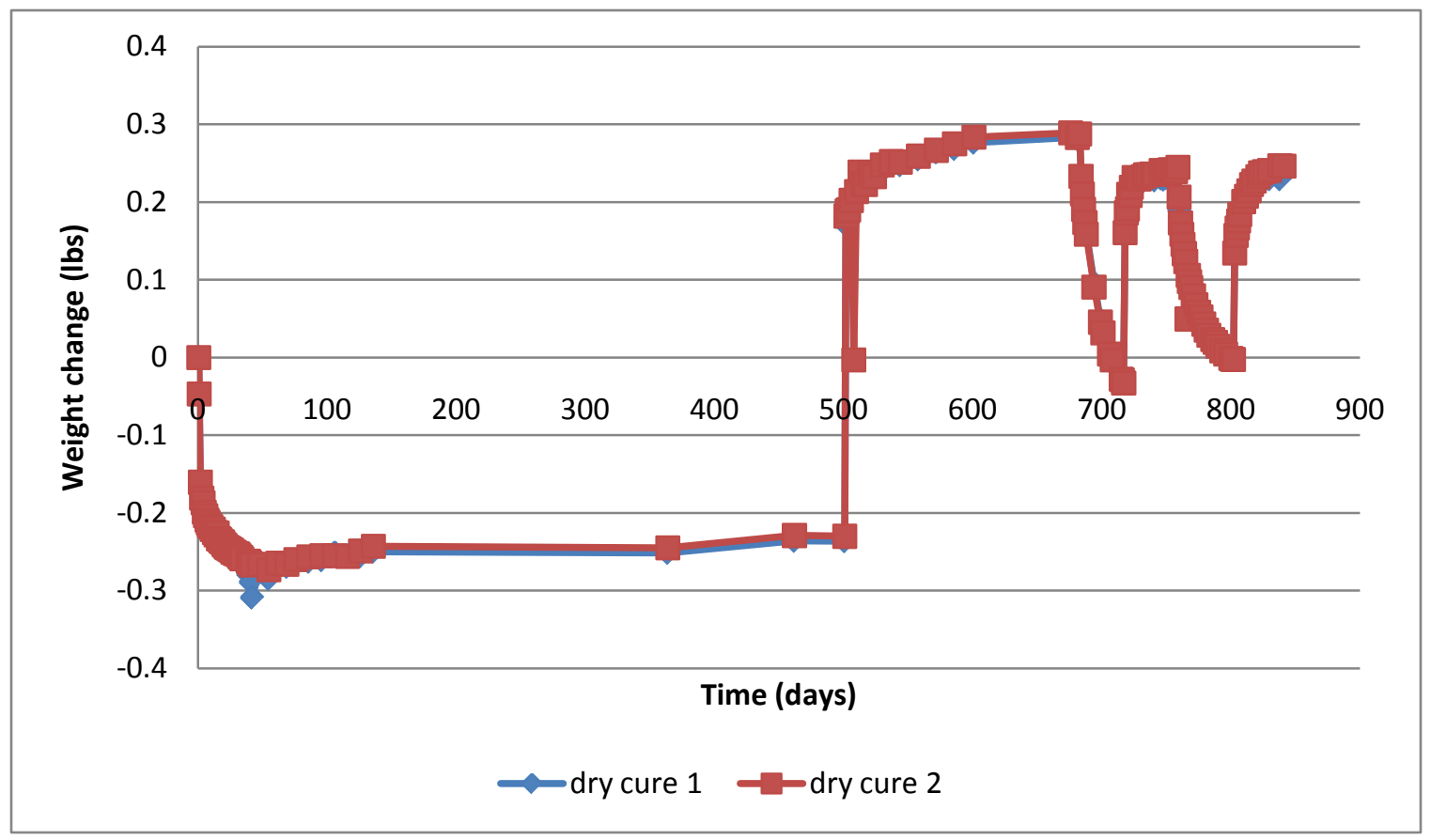

Figure C.9: Weight change versus time for dry cured Limestone RCA.

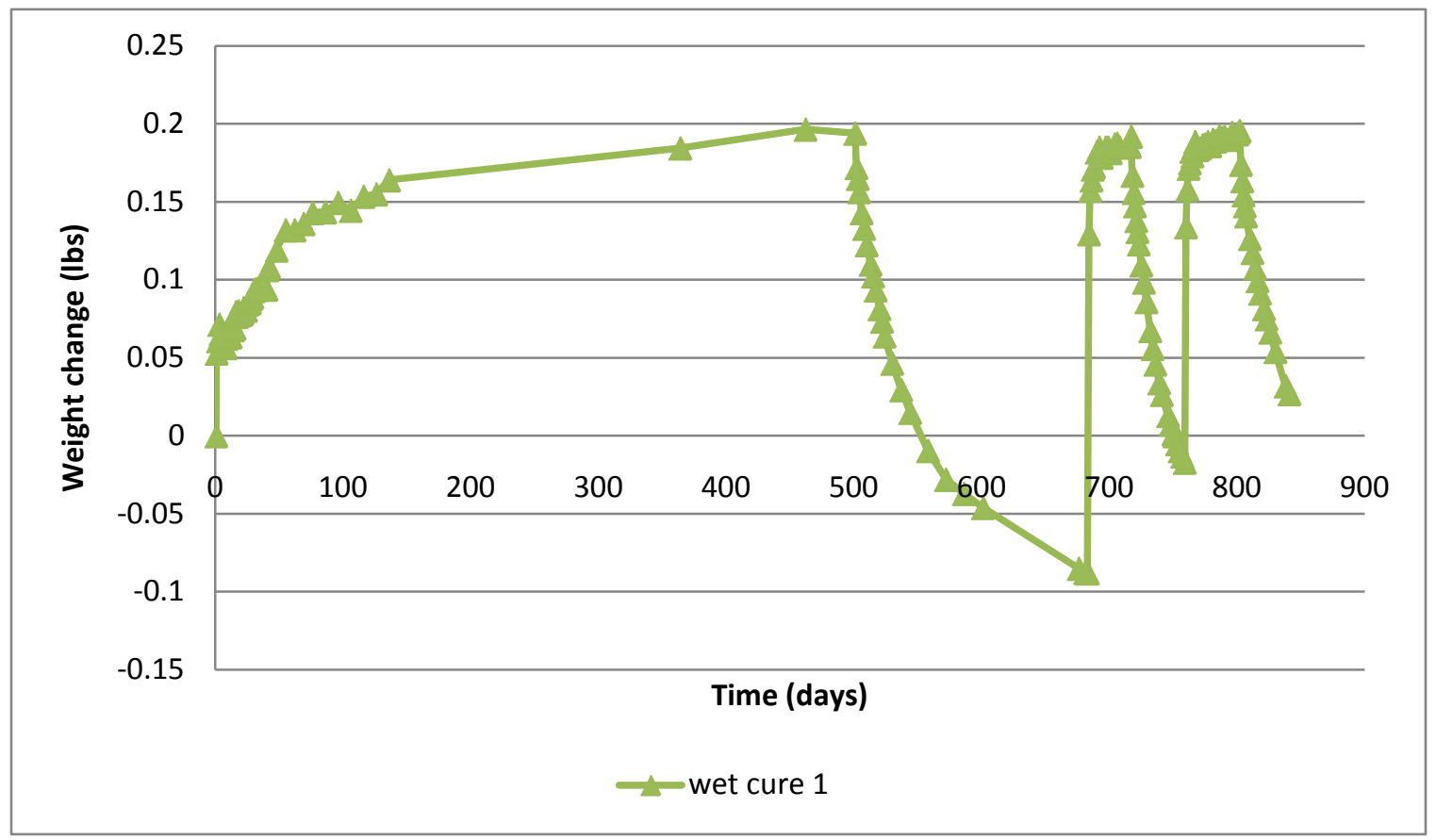

Figure C.10: Weight change versus time for wet cured Limestone RCA. 


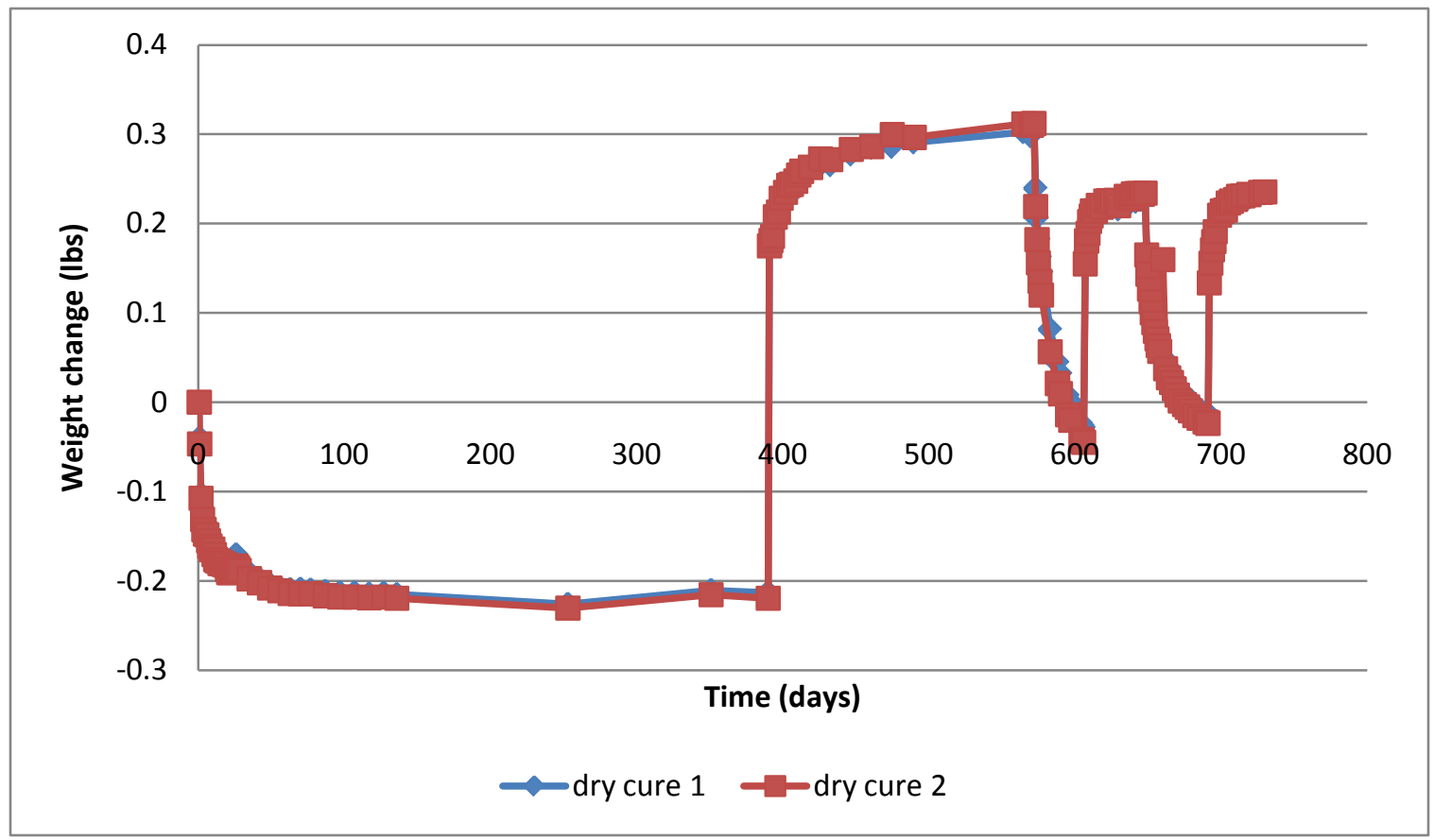

Figure C.11: Weight change versus time for dry cured Gravel RCA 1.

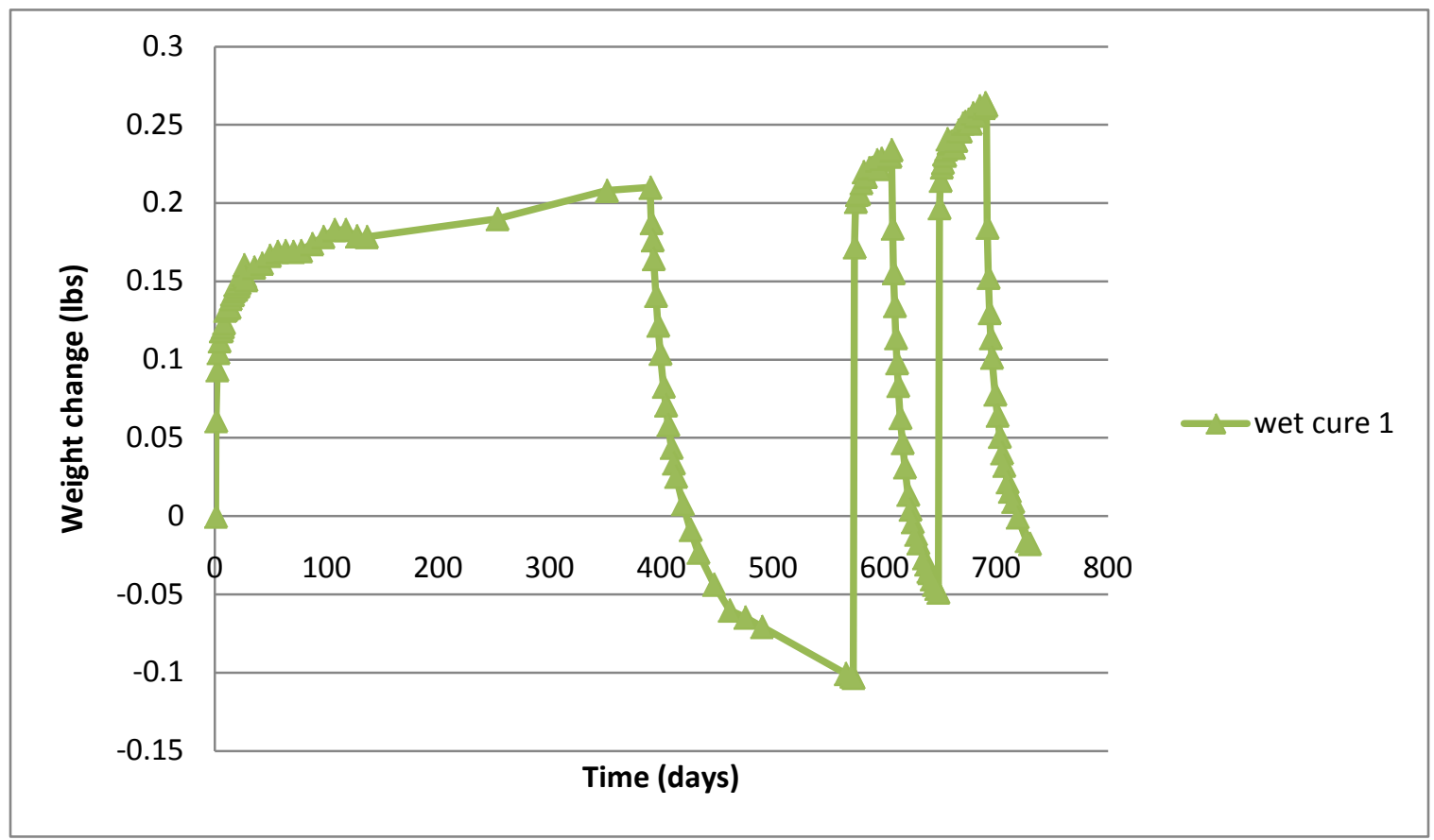

Figure C.12: Weight change versus time for wet cured Gravel RCA 1. 


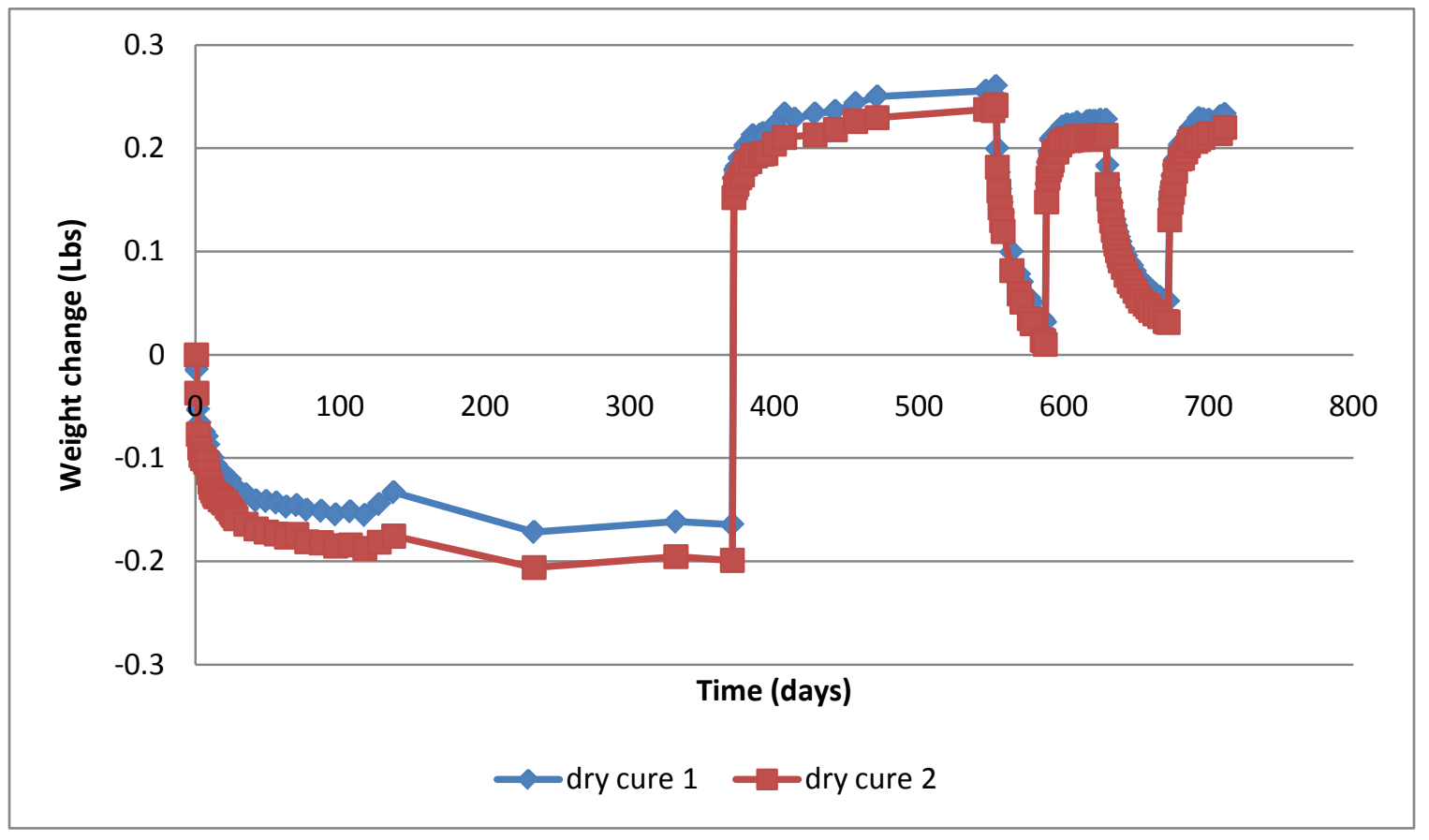

Figure C.13: Weight change versus time for dry cured Gravel RCA 2.

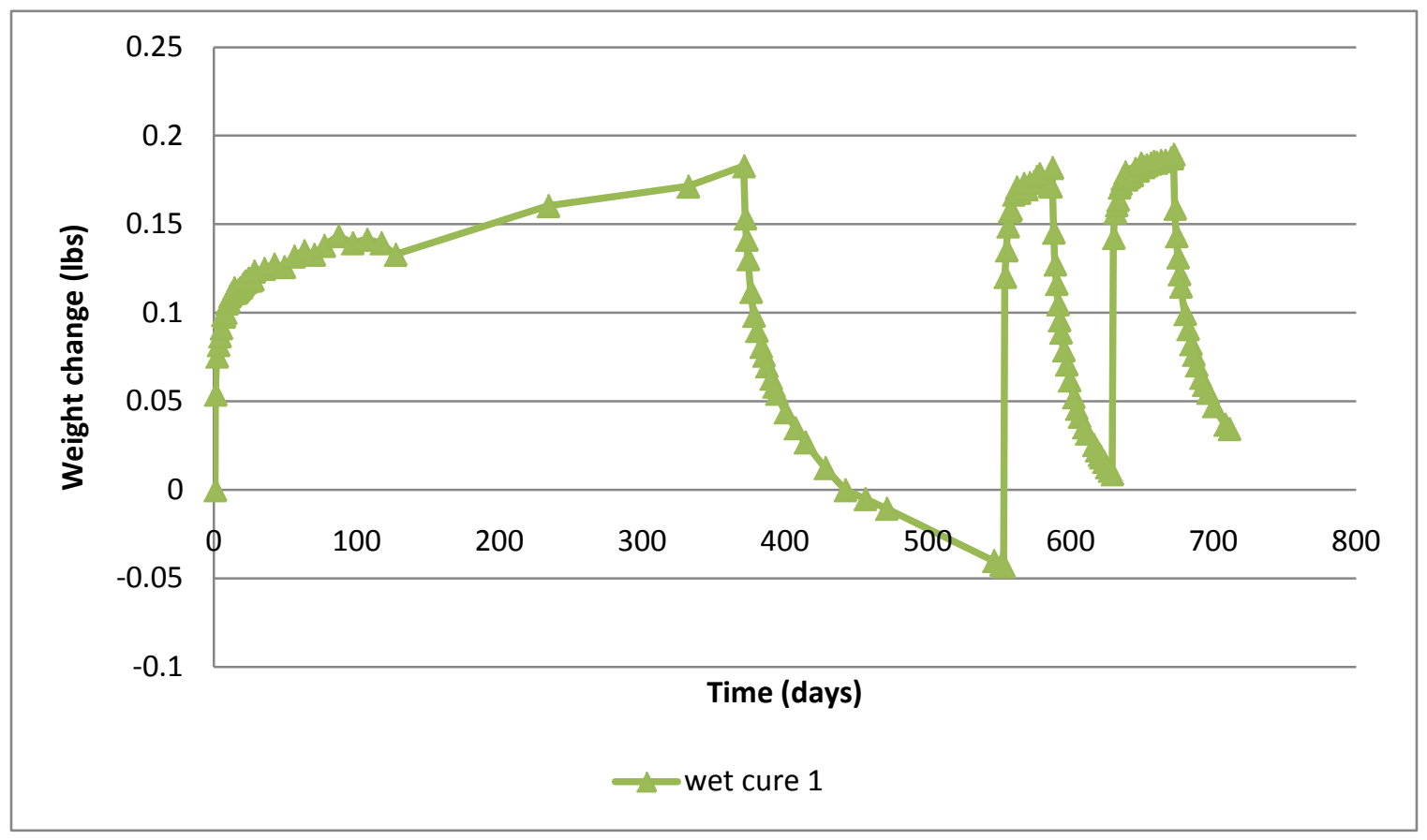

Figure C.14: Weight change versus time for wet cured Gravel RCA 1. 


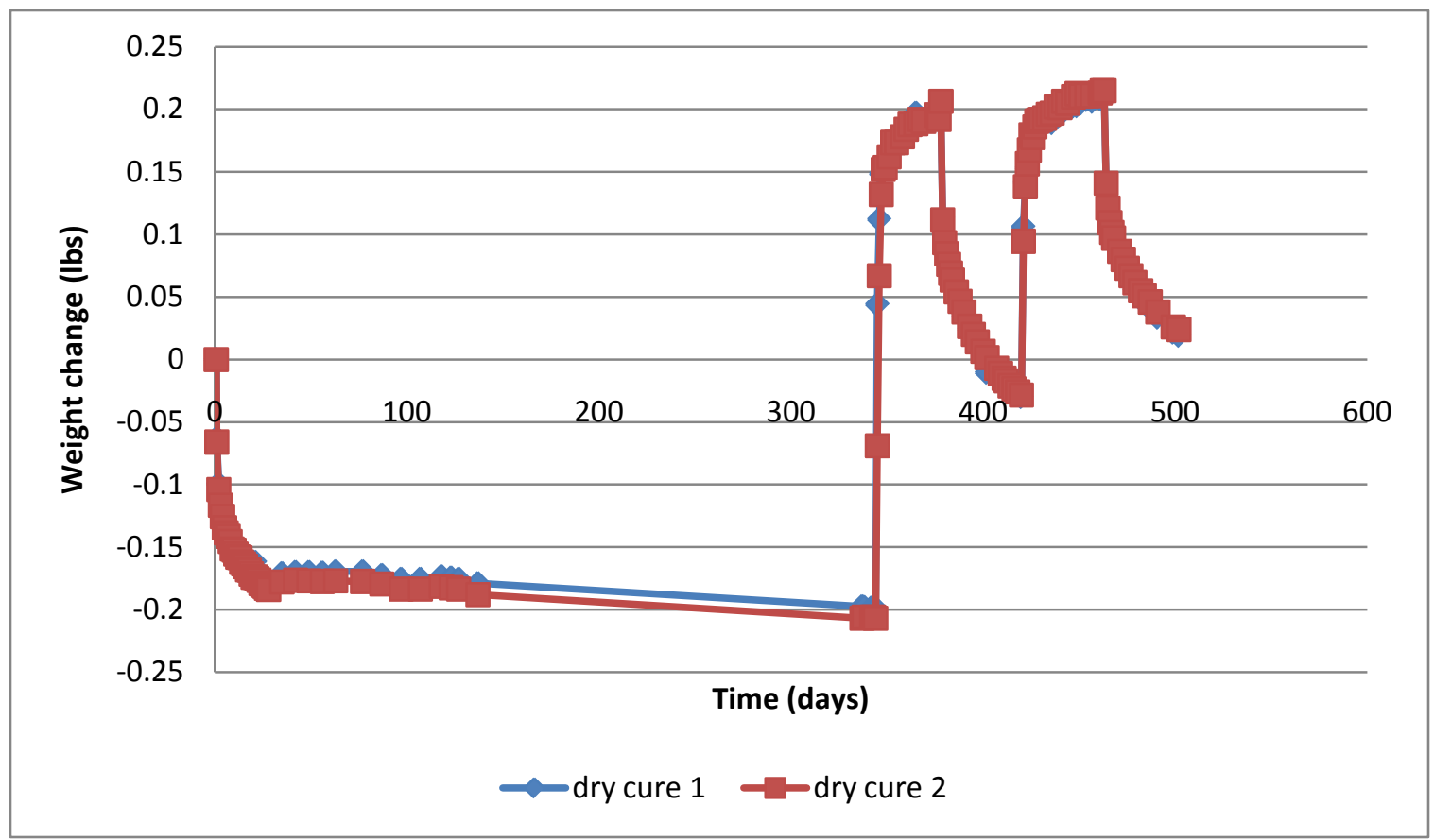

Figure C.15: Weight change versus time for dry cured Twice Recycled RCA.

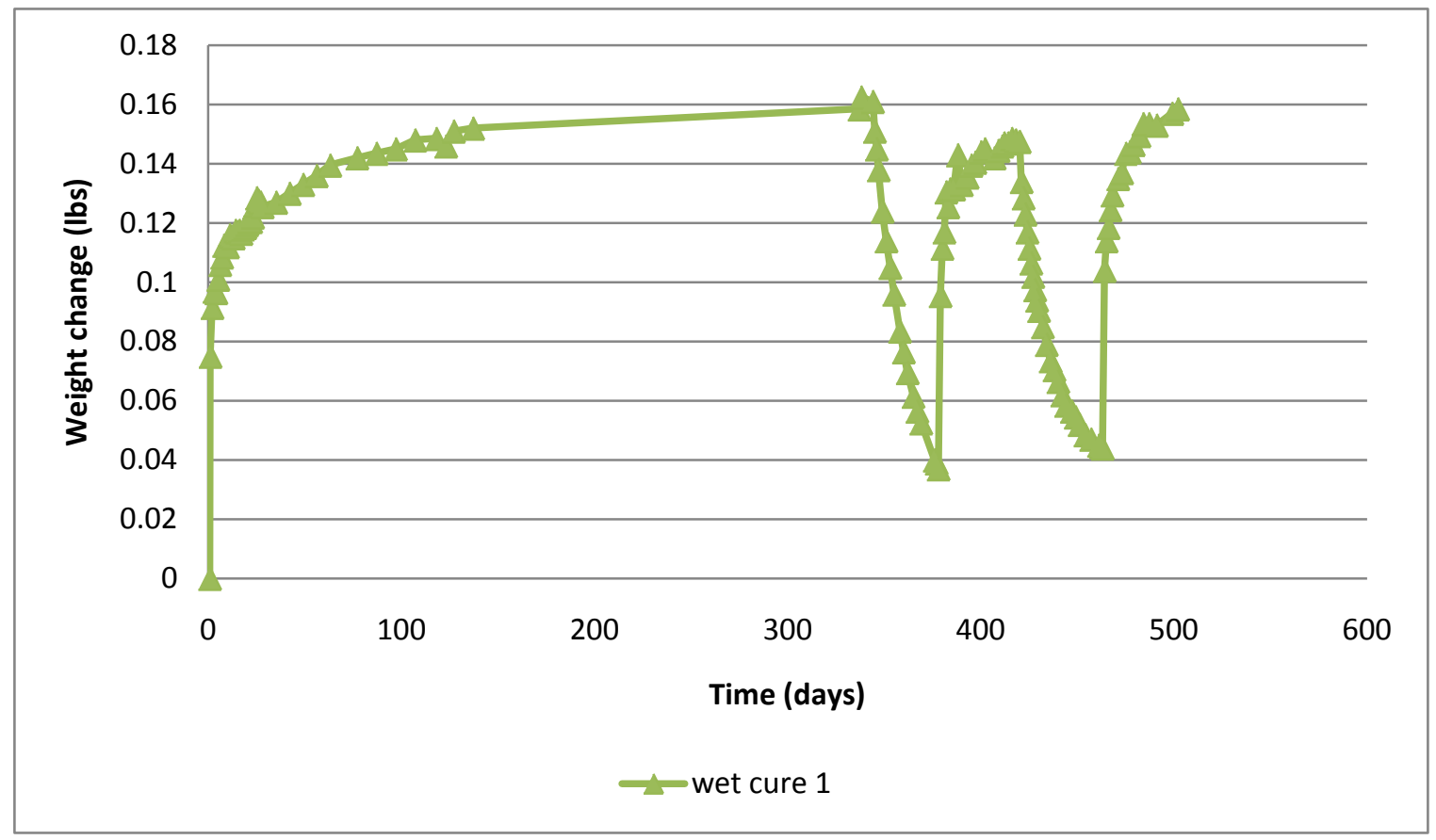

Figure C.16: Weight change versus time for wet cured Twice Recycled RCA. 


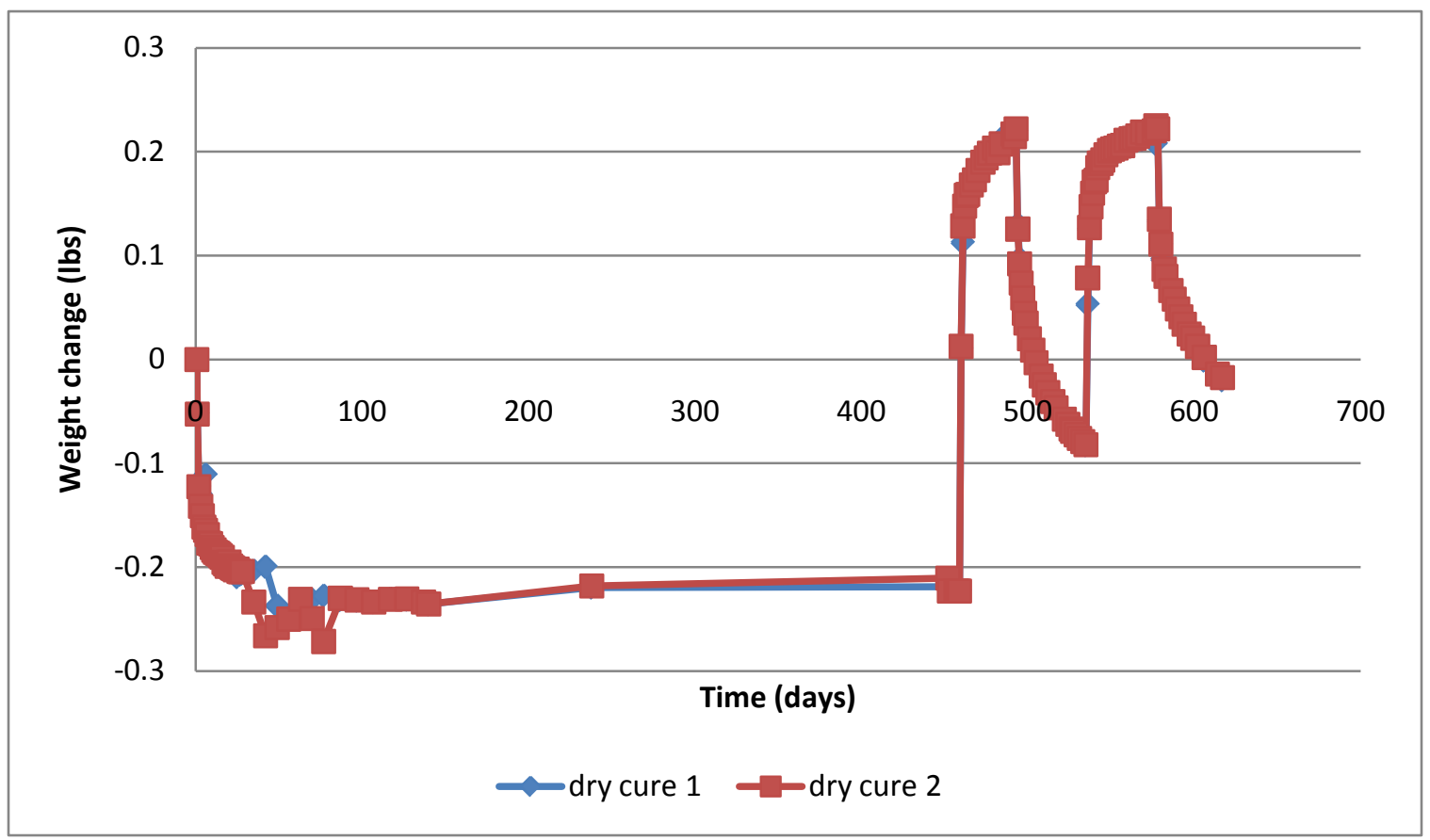

Figure C.17: Weight change versus time for dry cured Lightweight 1.

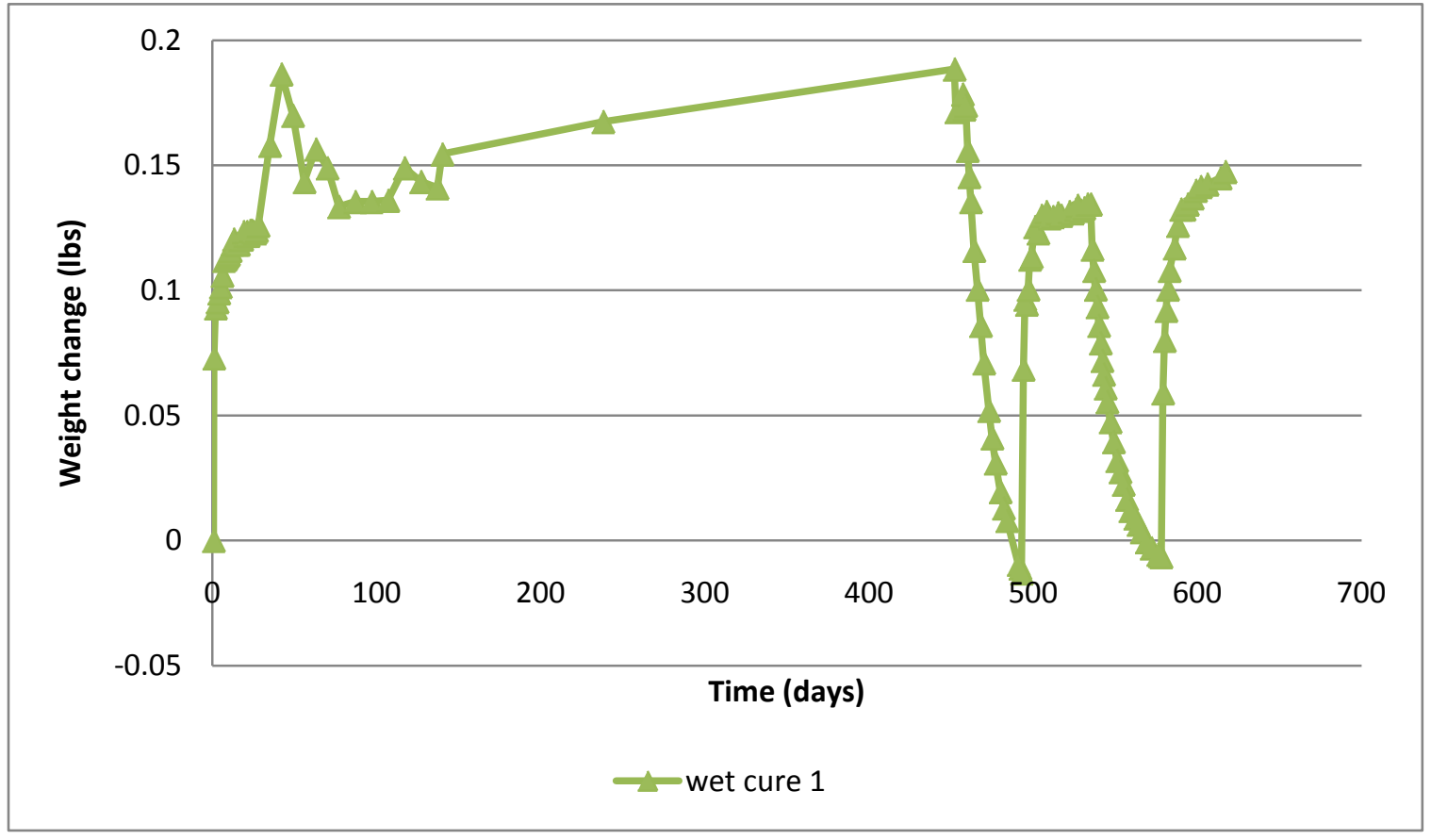

Figure C.18: Weight change versus time for wet cured Lightweight 1. 


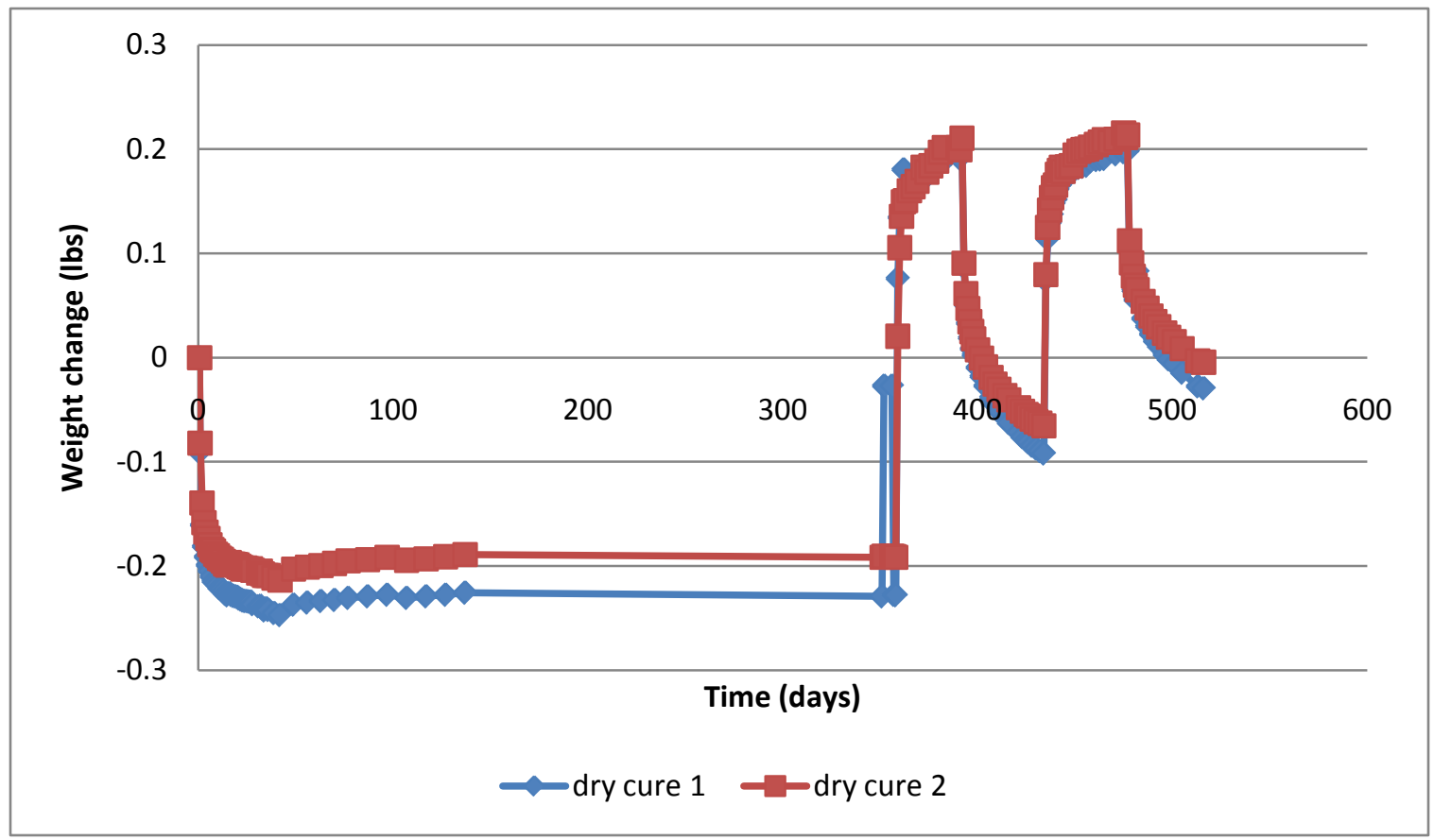

Figure C.19: Weight change versus time for dry cured Lightweight 2.

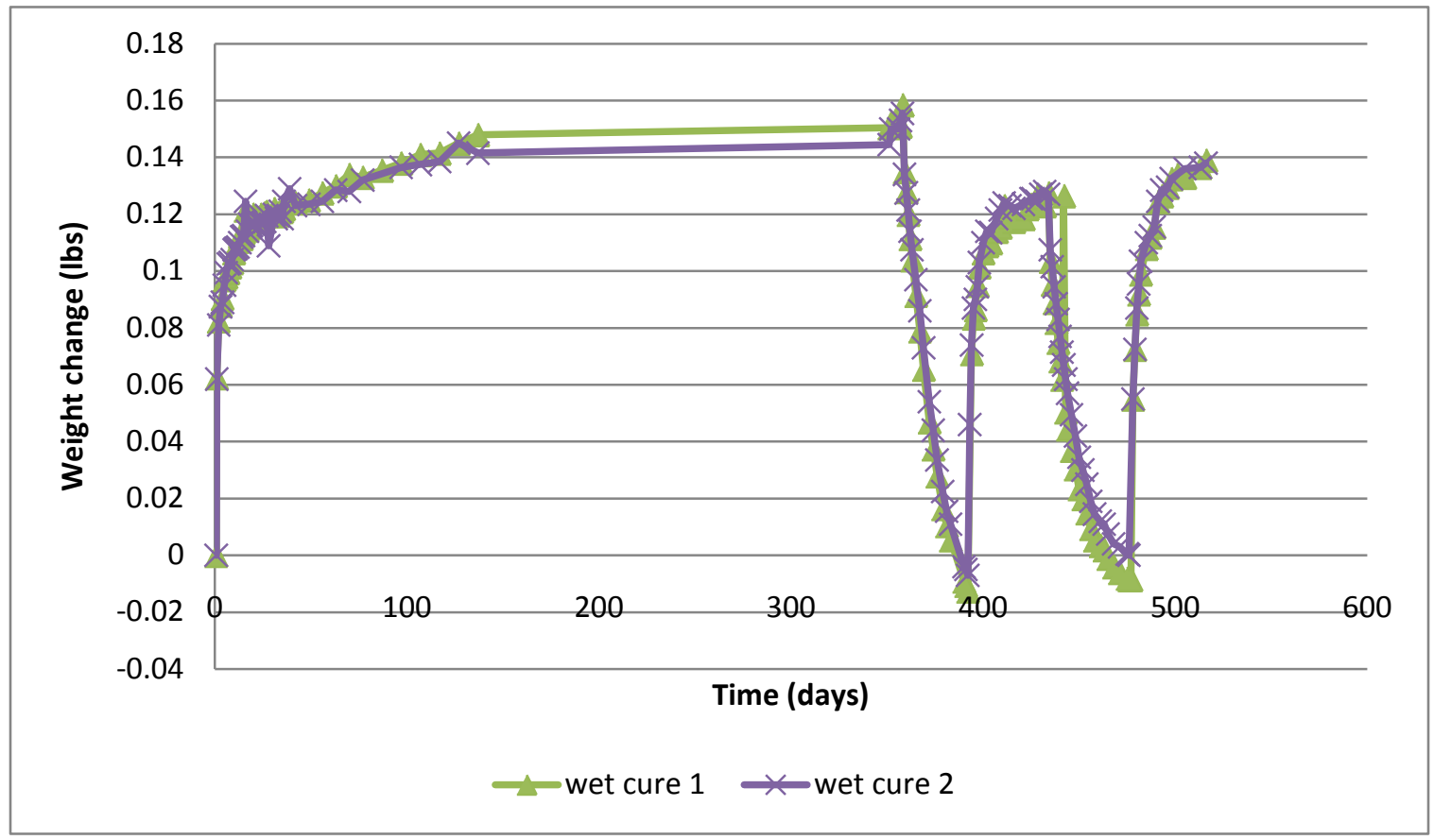

Figure C.20: Weight change versus time for wet cured Lightweight 2. 


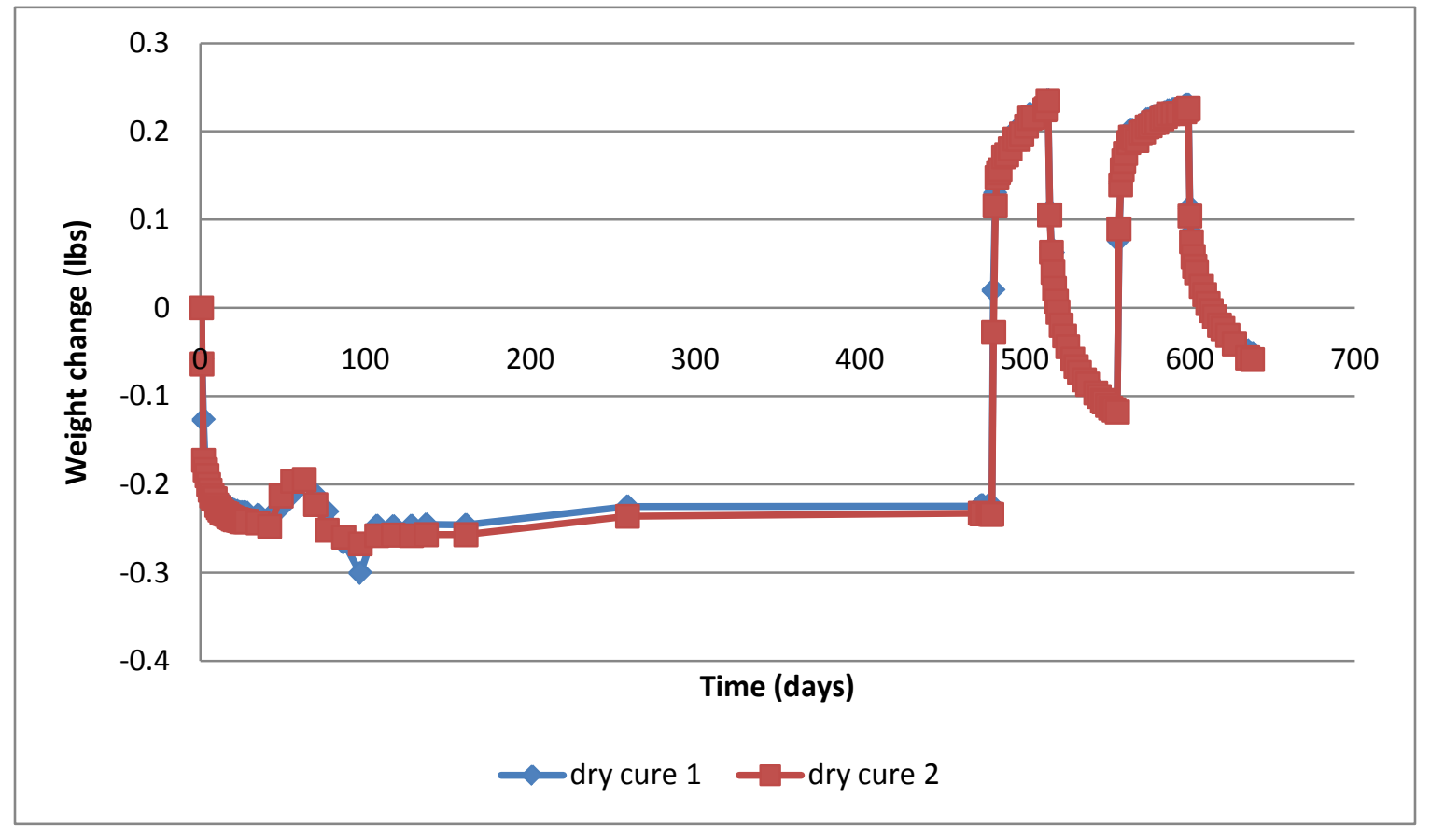

Figure C.21: Weight change versus time for dry cured Lightweight 3.

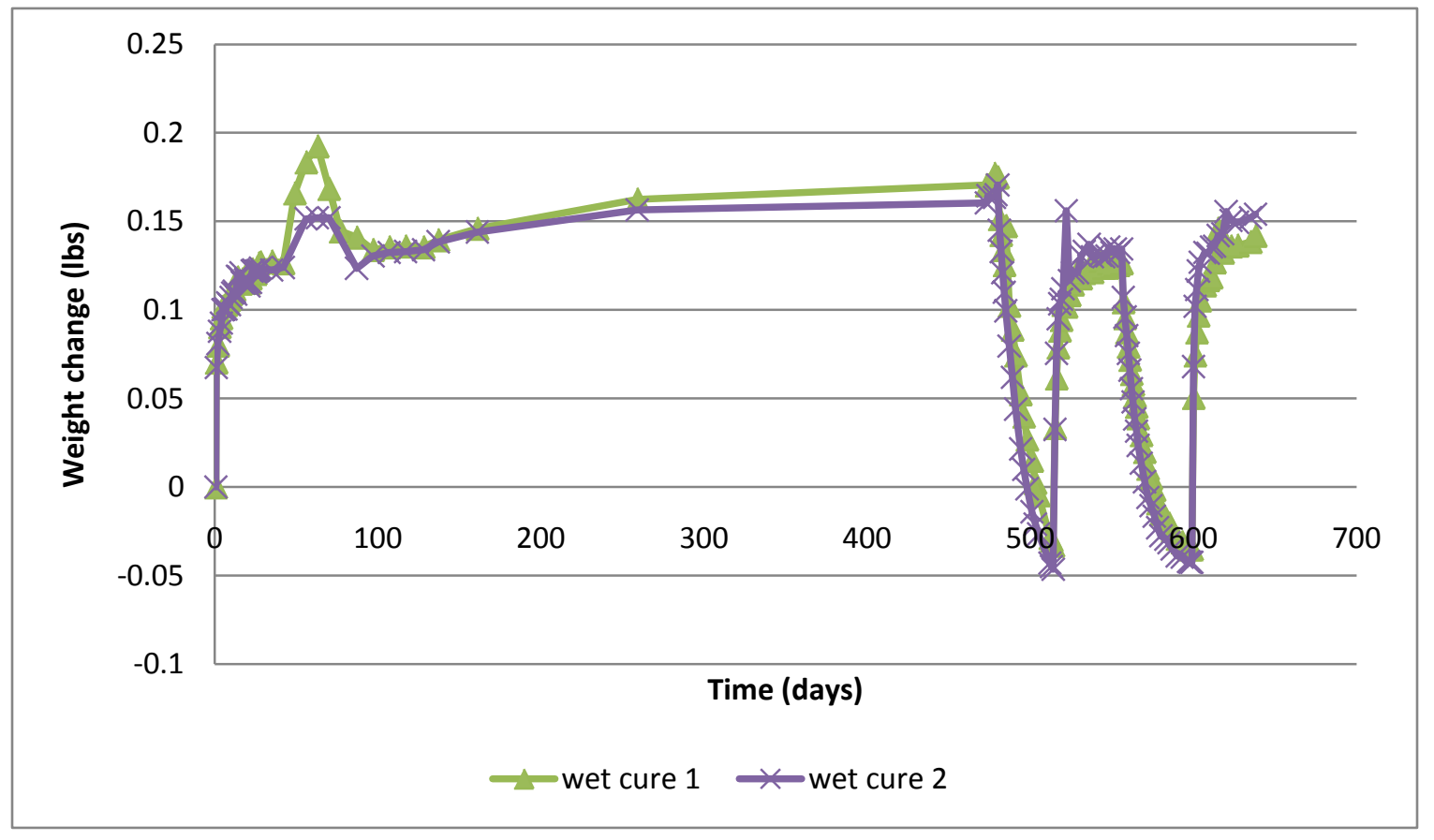

Figure C.22: Weight change versus time for wet cured Lightweight 3. 


\section{Appendix D: Graphs of Changes in Strain versus Changes in Weight}

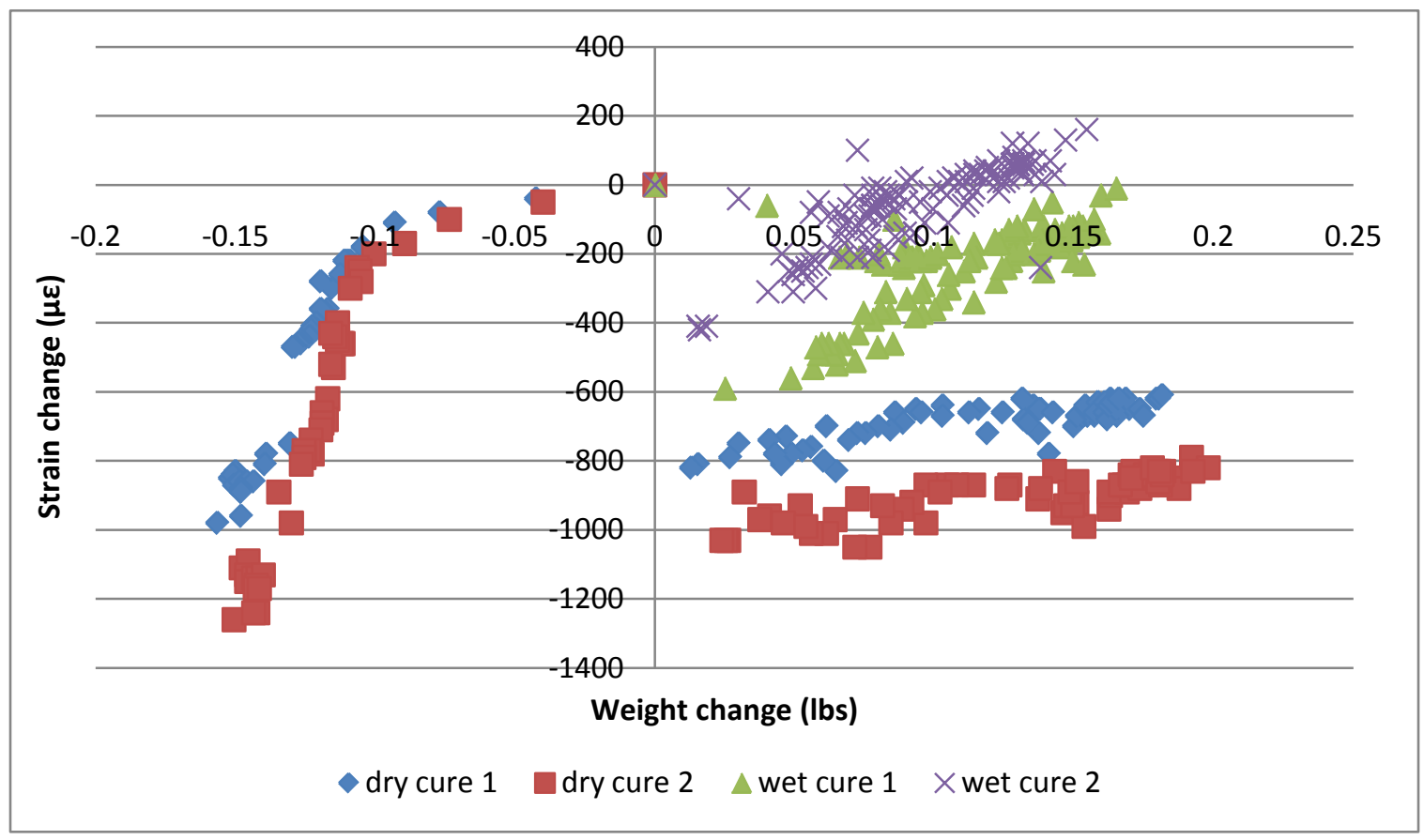

Figure D.1: Change in strain versus change in weight for Virgin 1.

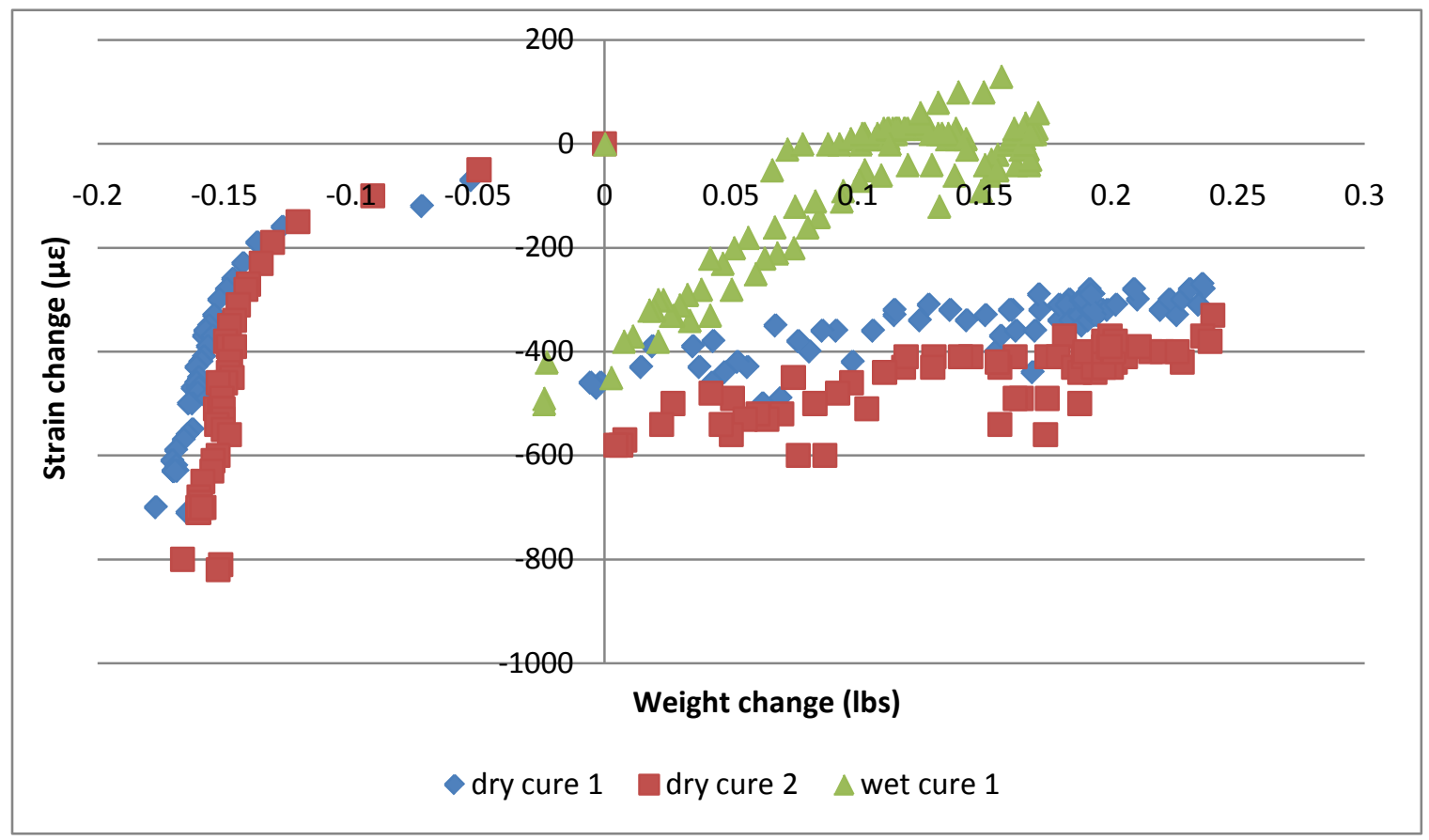

Figure D.2: Change in strain versus change in weight for Virgin 2. 


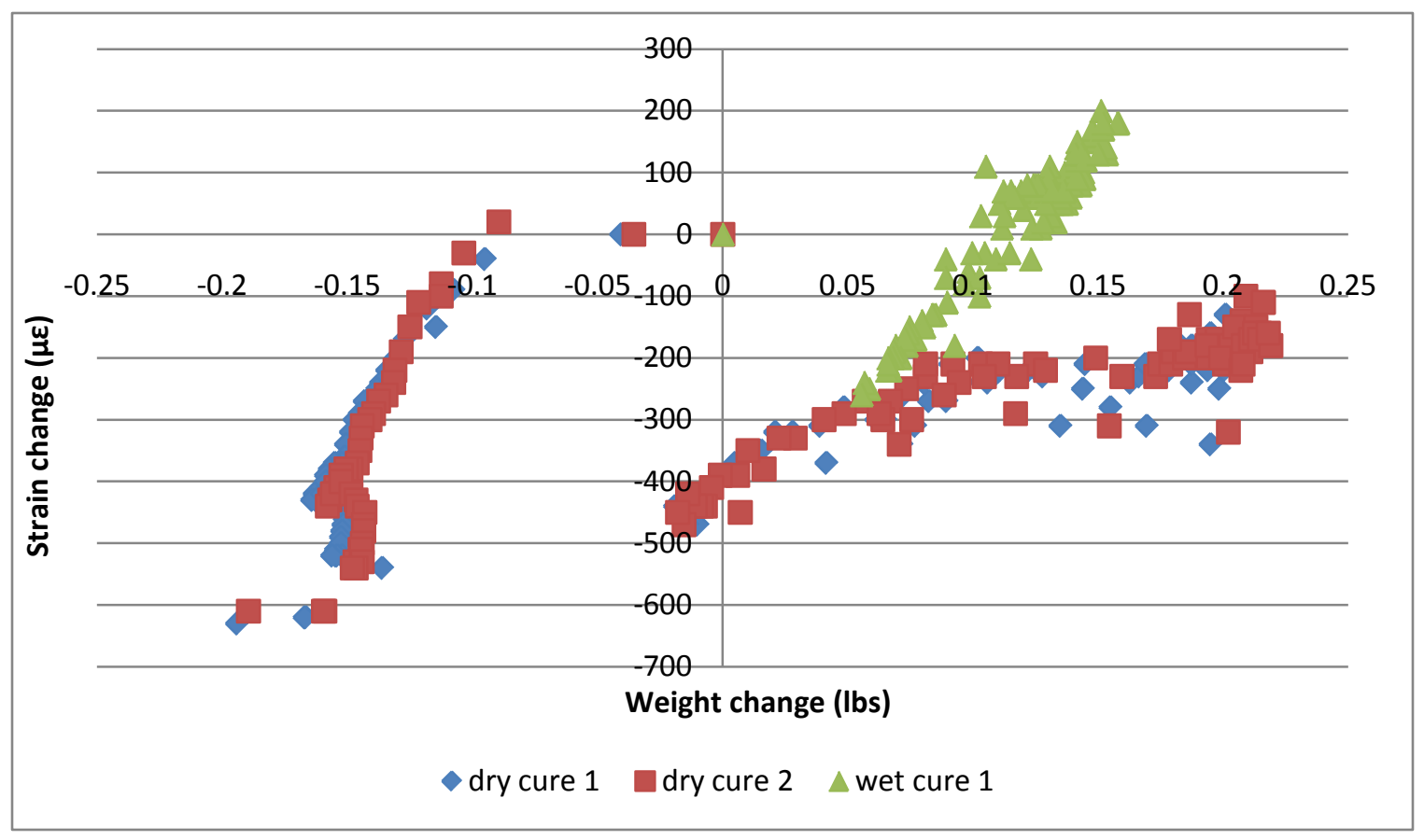

Figure D.3: Change in strain versus change in weight for Virgin 3.

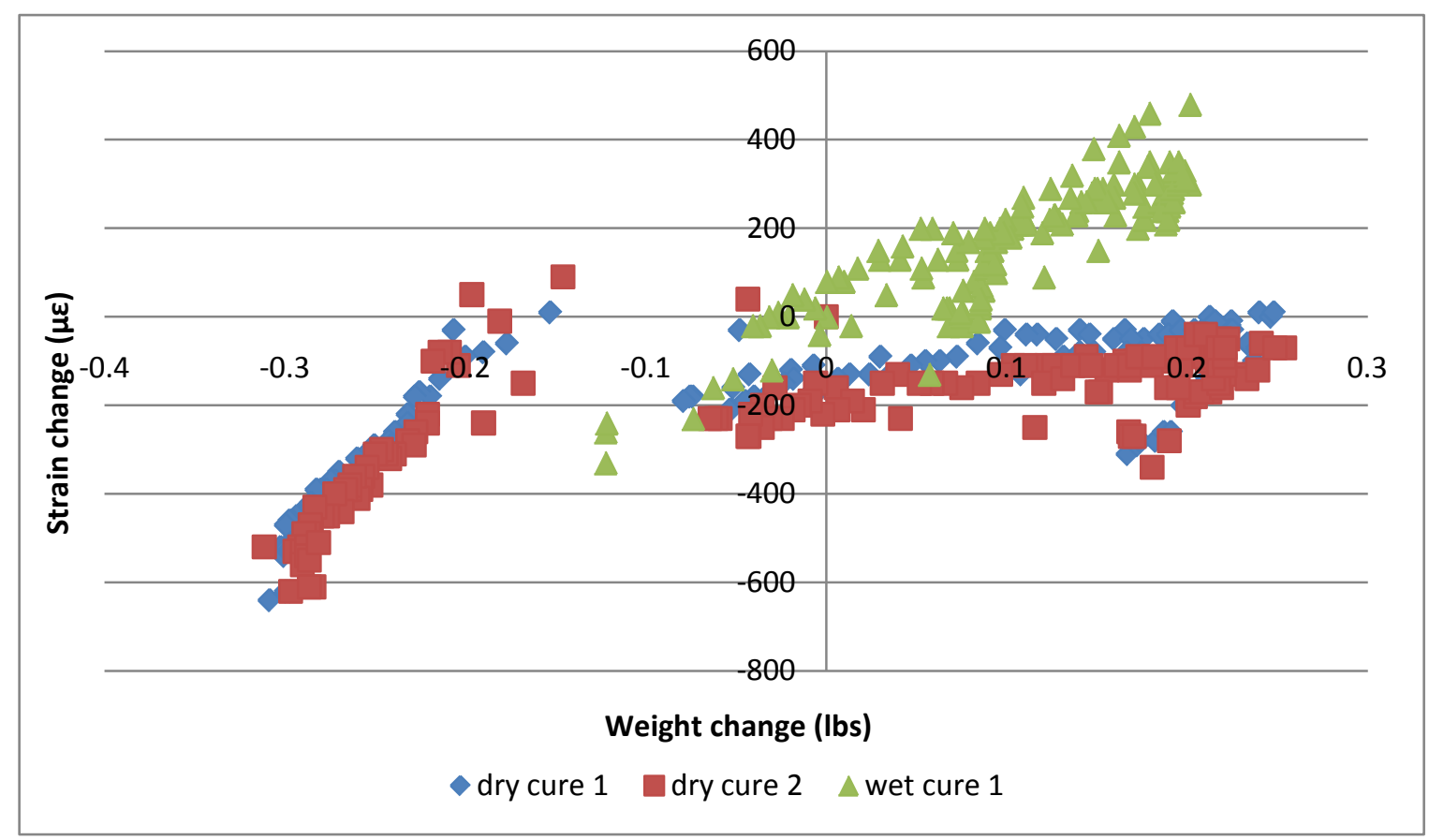

Figure D.4: Change in strain versus change in weight for Slag RCA. 


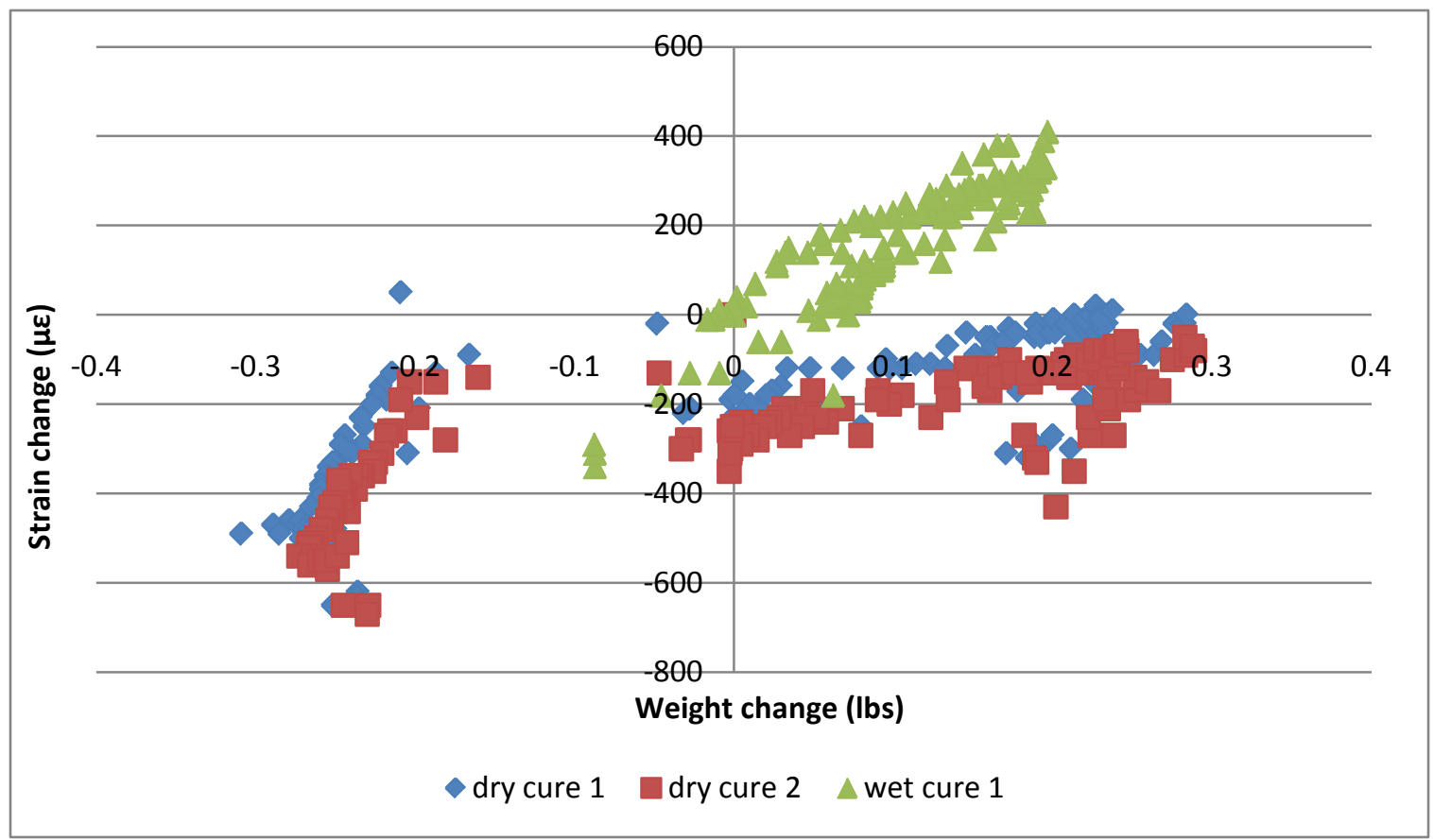

Figure D.5: Change in strain versus change in weight for Limestone RCA.

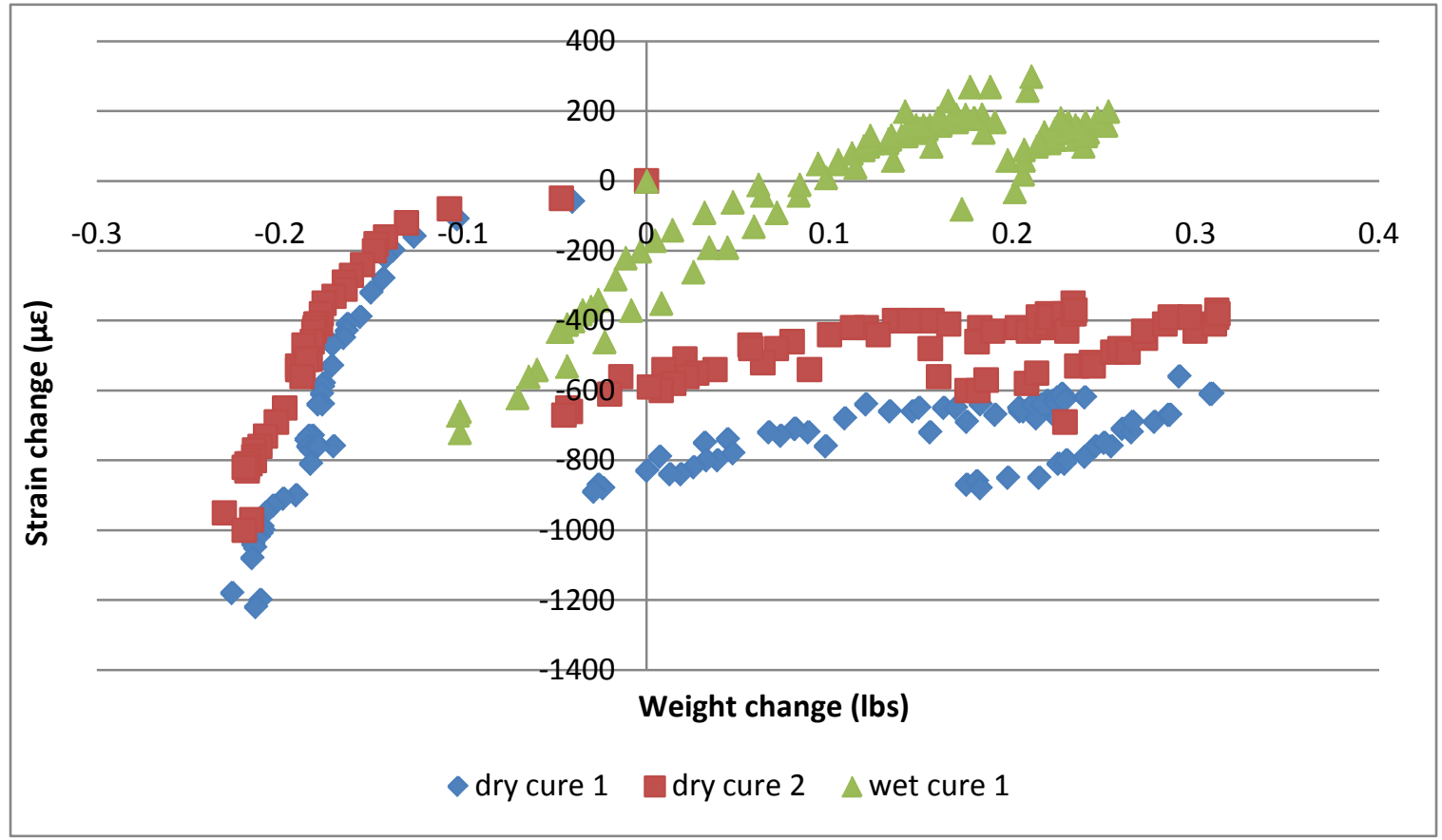

Figure D.6: Change in strain versus change in weight for Gravel RCA 1. 


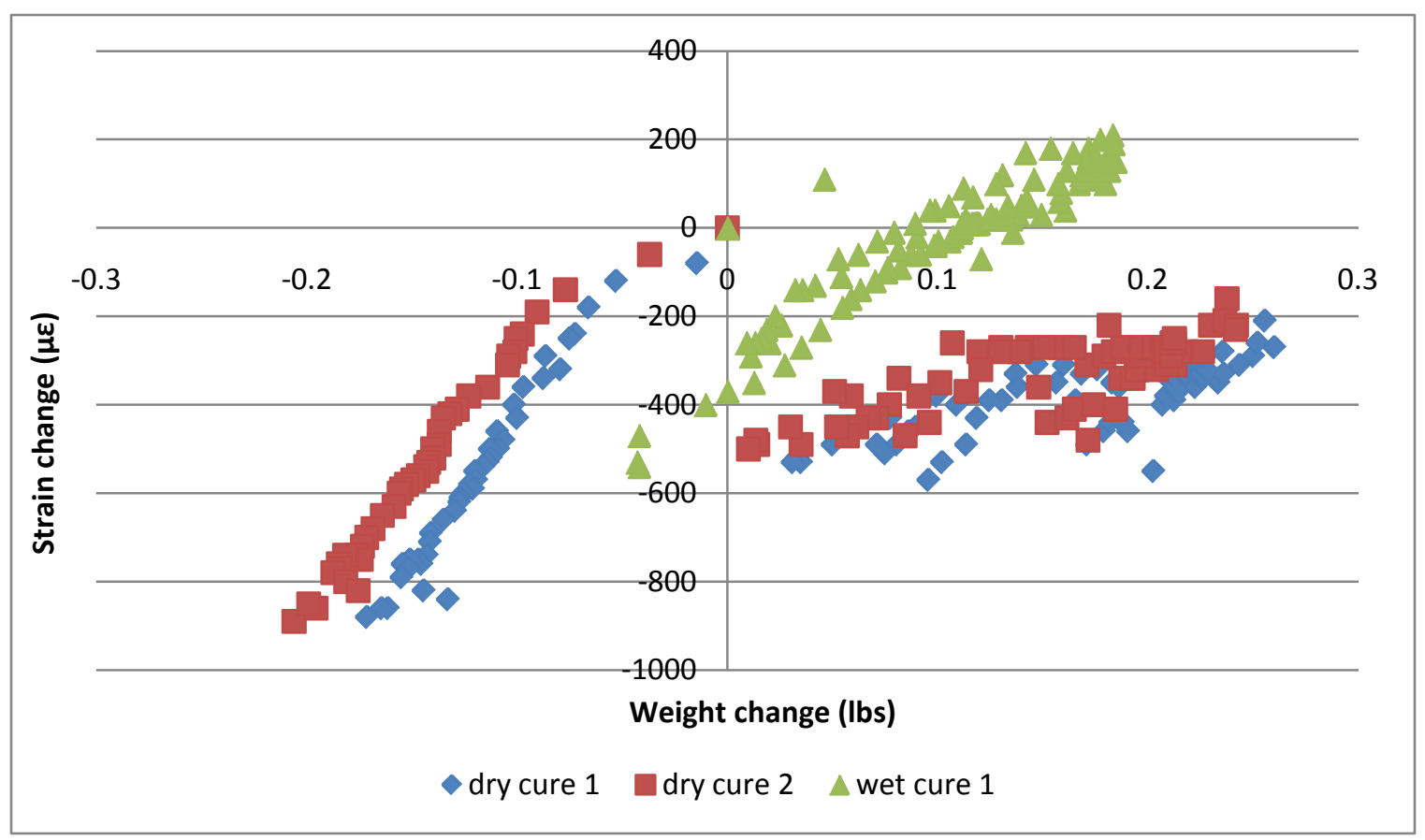

Figure D.7: Change in strain versus change in weight for Gravel RCA 2.

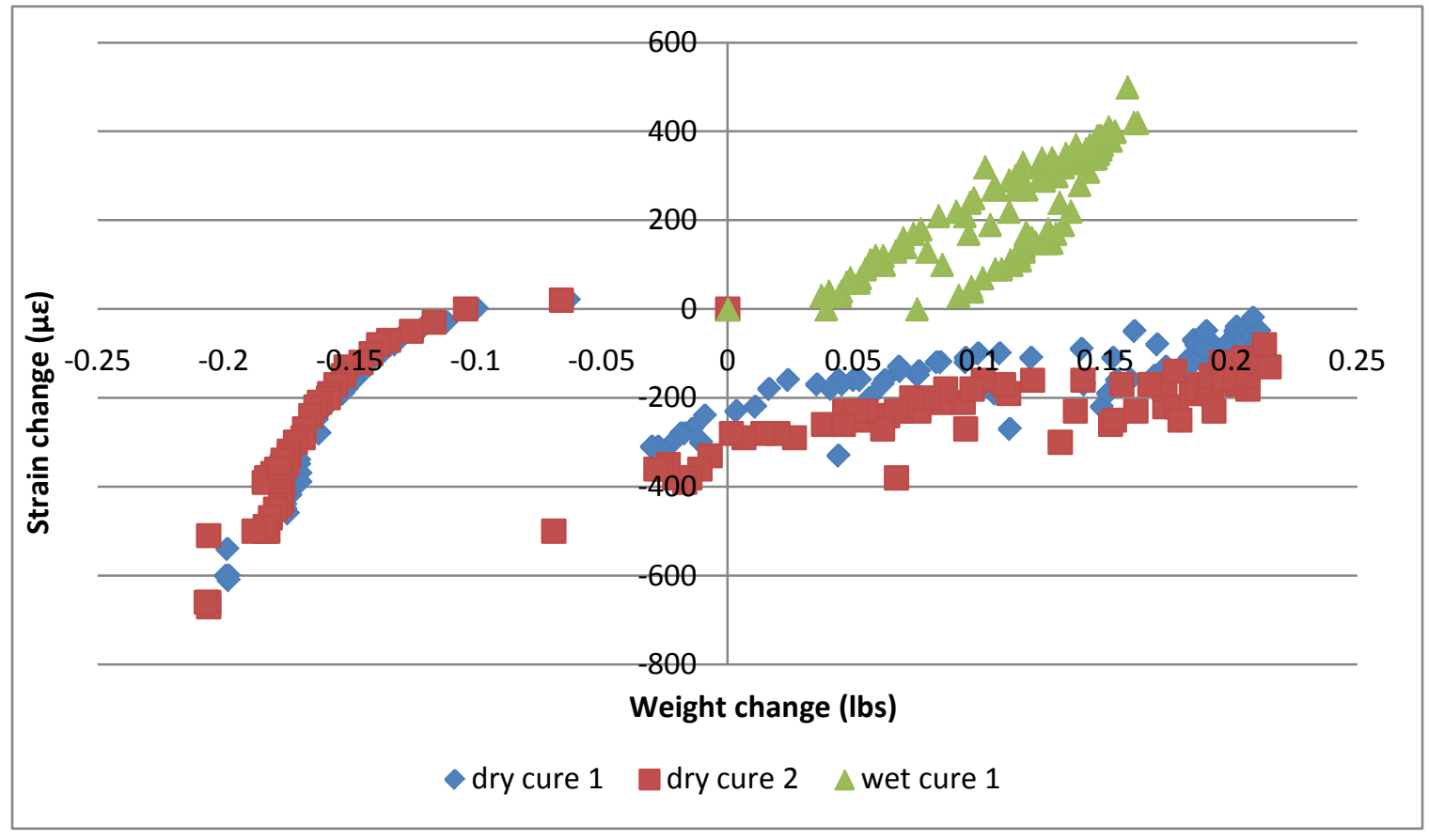

Figure D.8: Change in strain versus change in weight for Twice Recycled. 


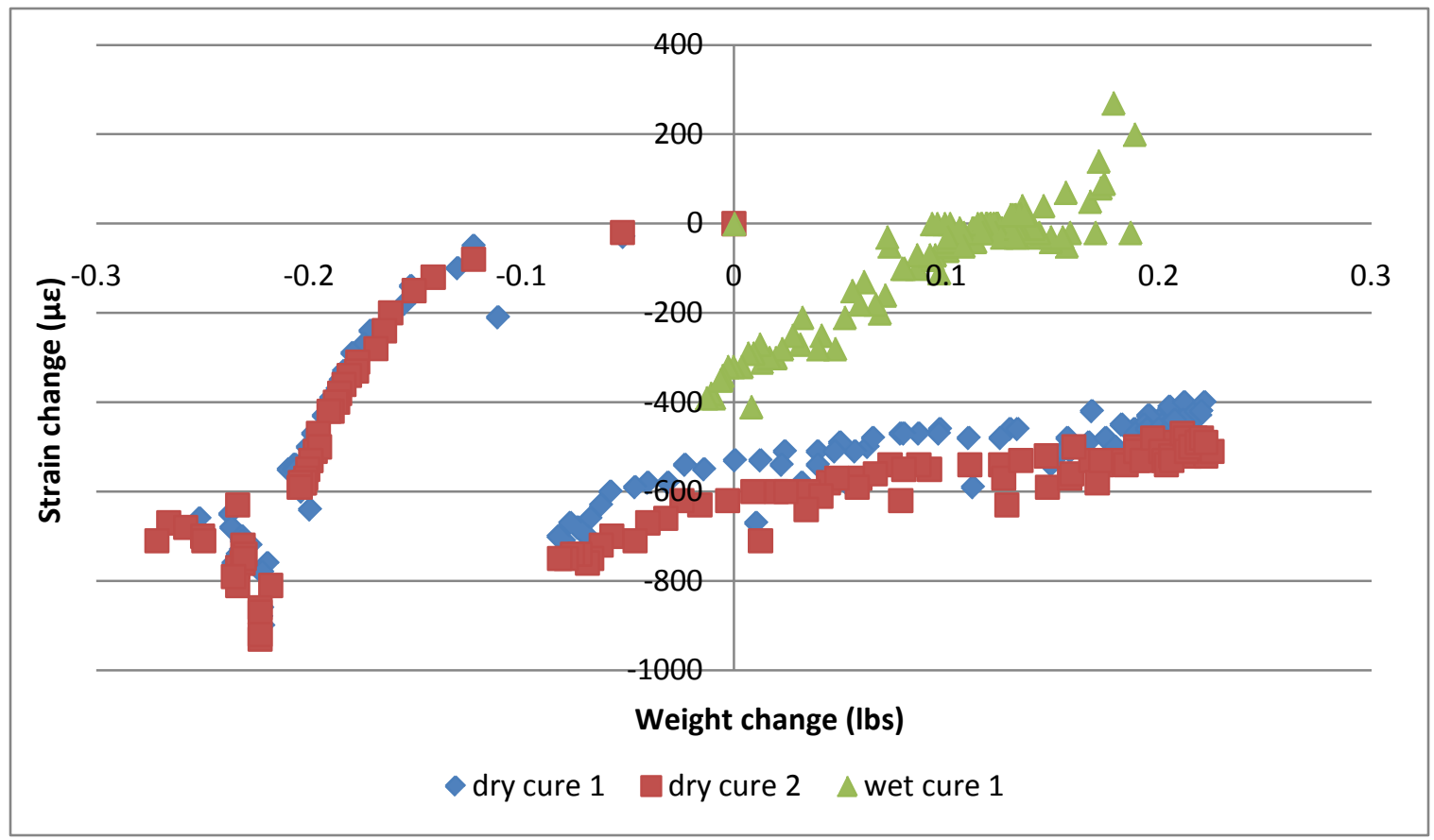

Figure D.9: Change in strain versus change in weight for Lightweight 1.

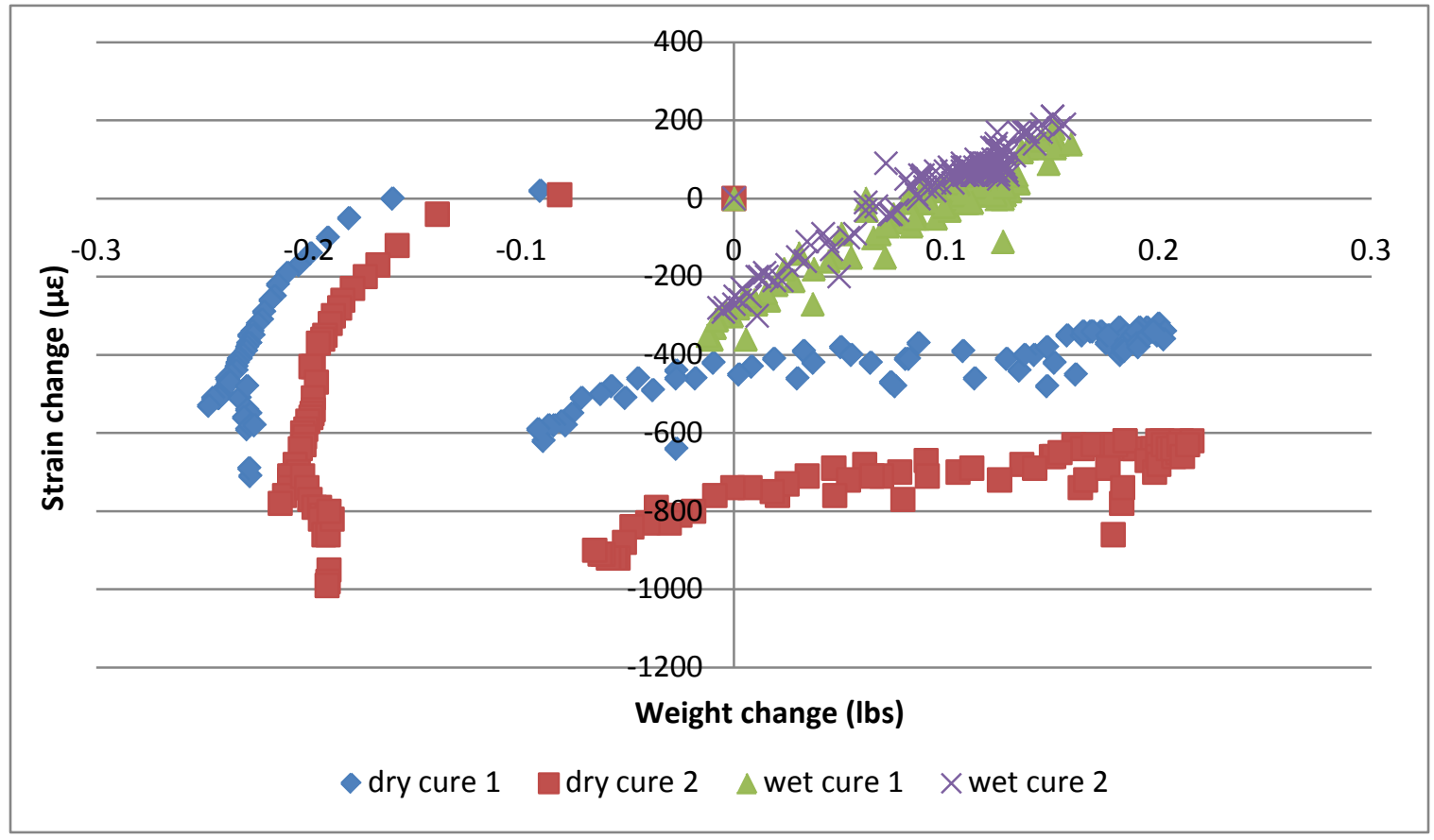

Figure D.10: Change in strain versus change in weight for Lightweight 2. 


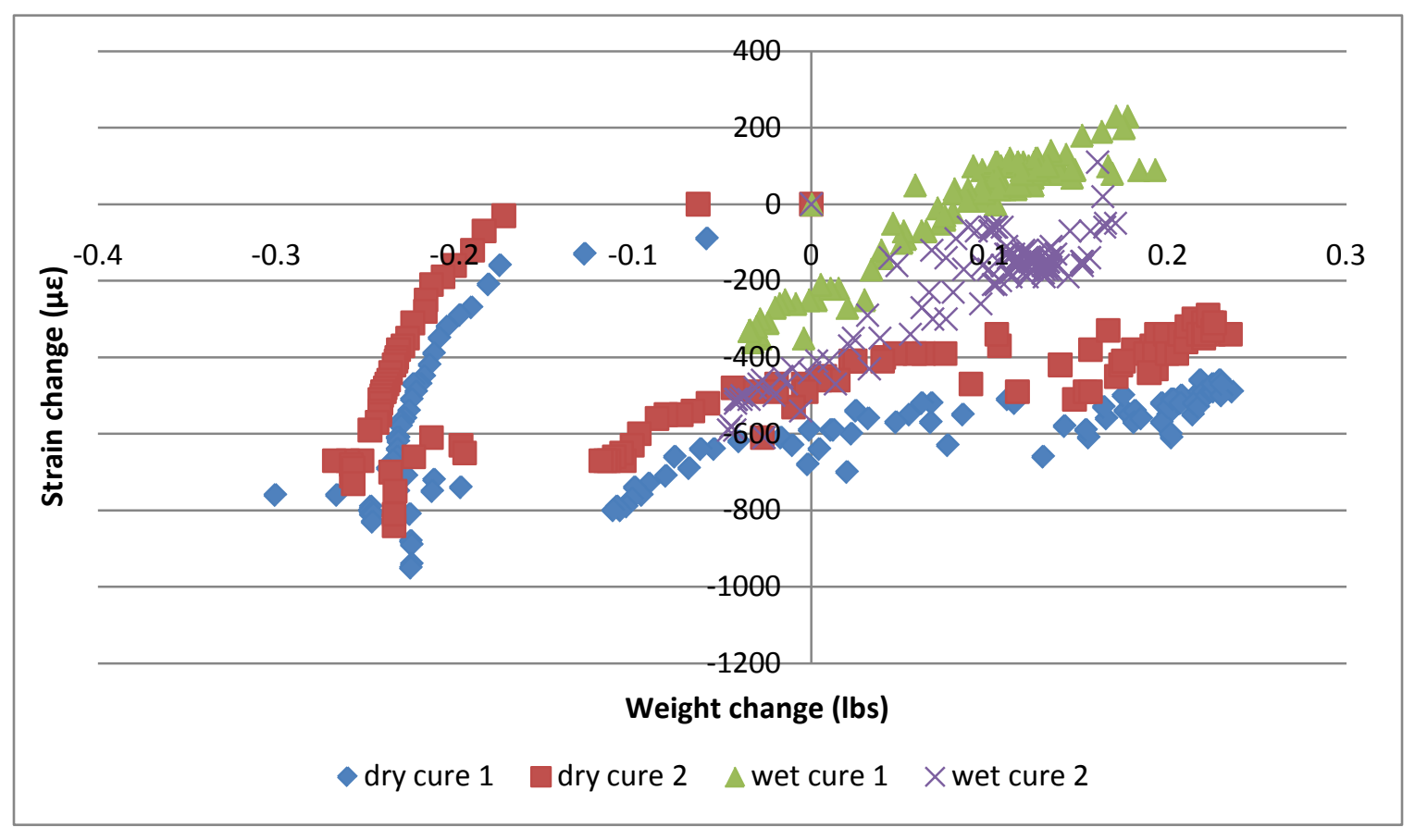

Figure D.11: Change in strain versus change in weight for Lightweight 3. 


\section{Appendix E: Validation of Elliptical Approximation for All Locations}

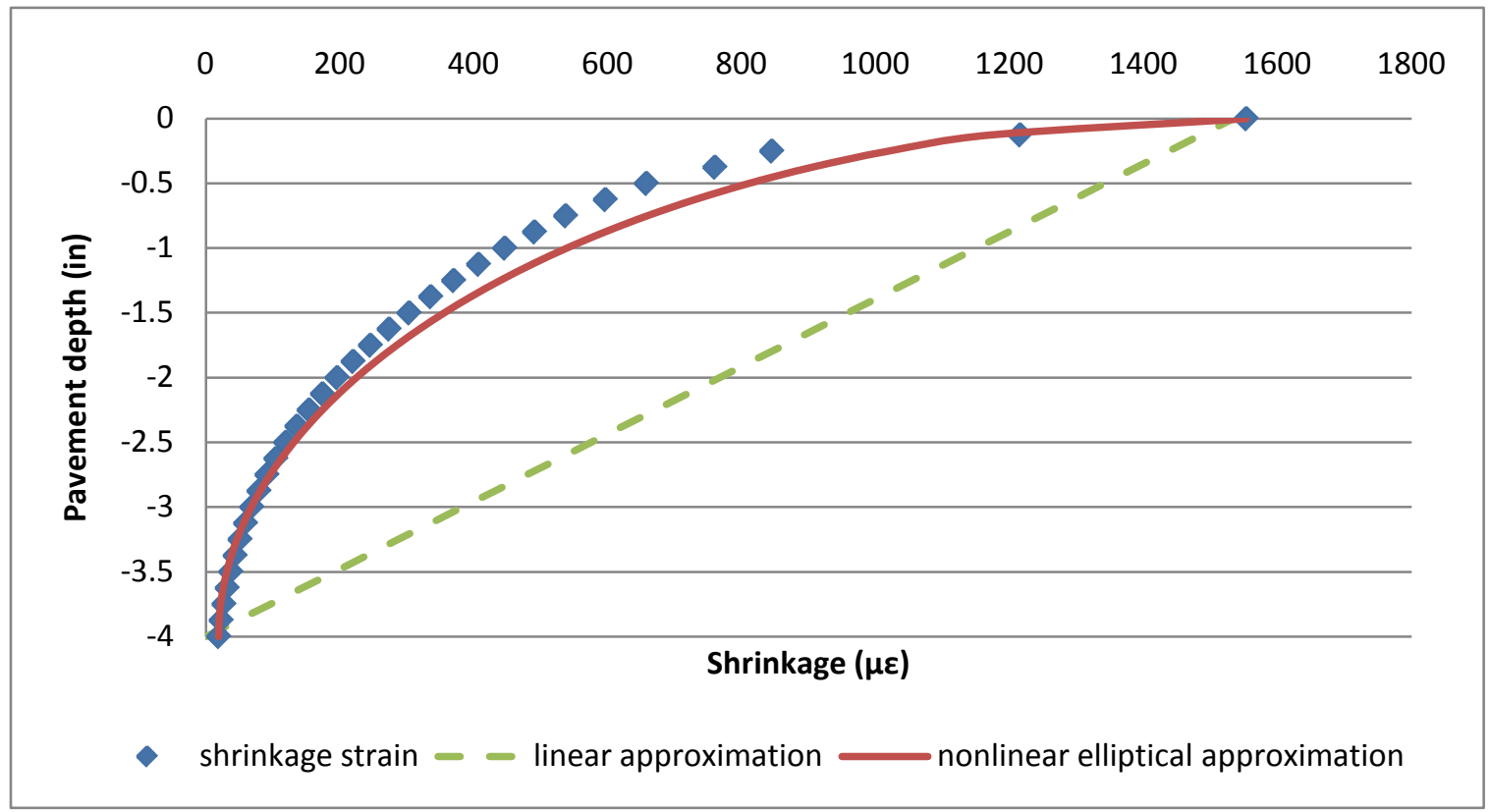

Figure E.1: Ultimate shrinkage through the depth of the pavement for Reno, Nevada 30 days after construction.

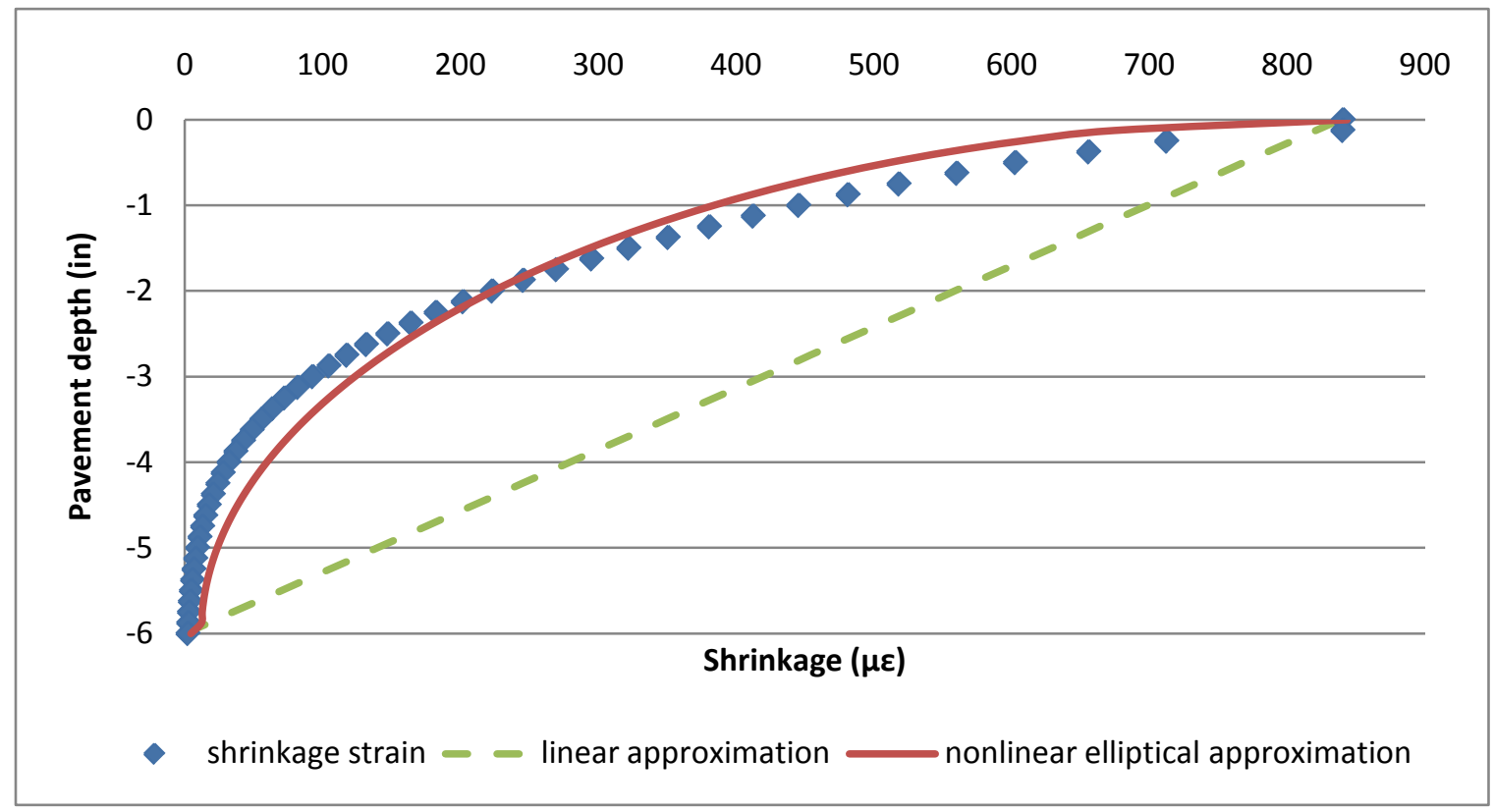

Figure E.2: Ultimate shrinkage through the depth of the pavement for Seattle, Washington 30 days after construction. 


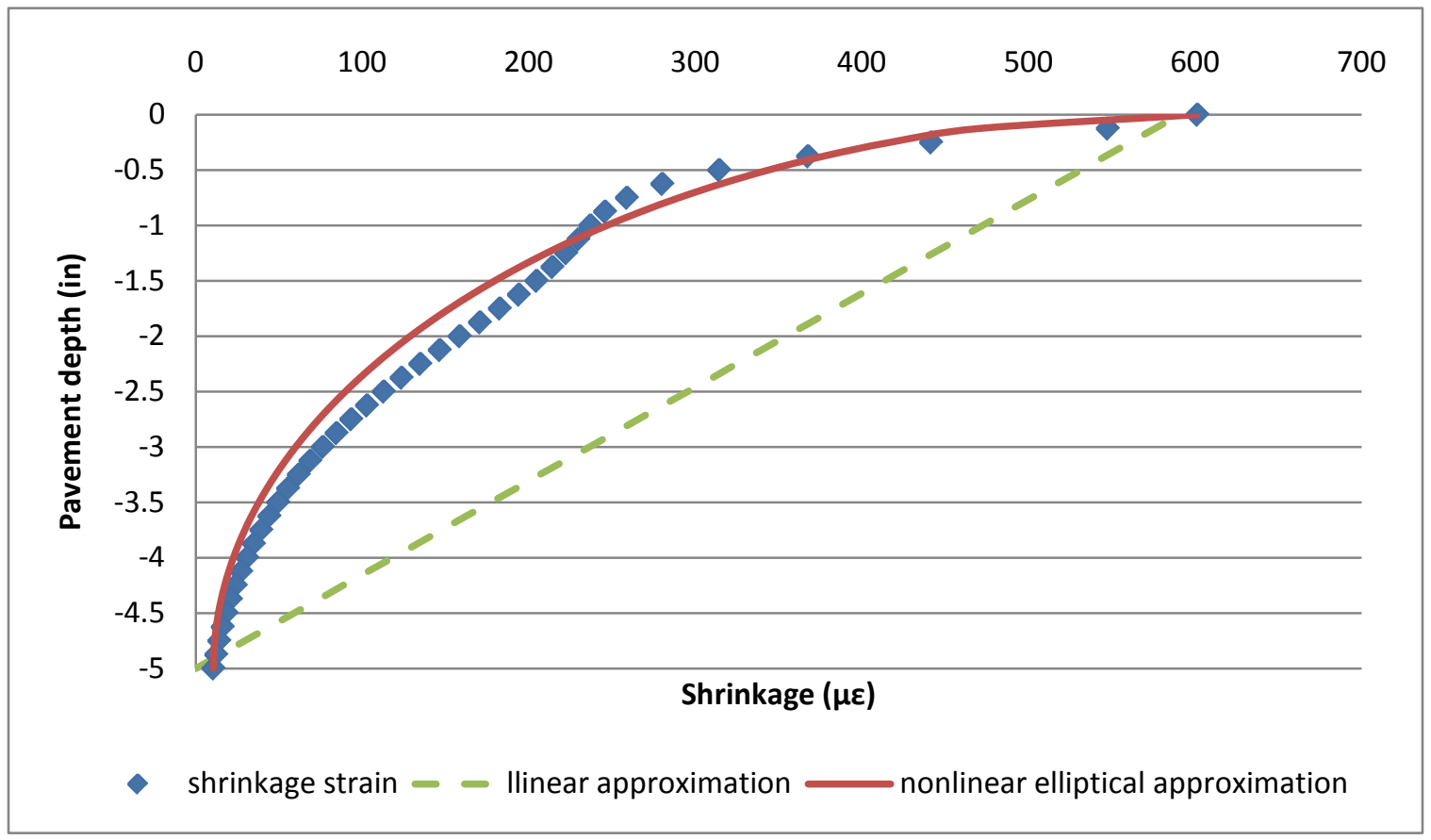

Figure E.3: Ultimate shrinkage through the depth of the pavement for Columbus, Ohio 30 days after construction.

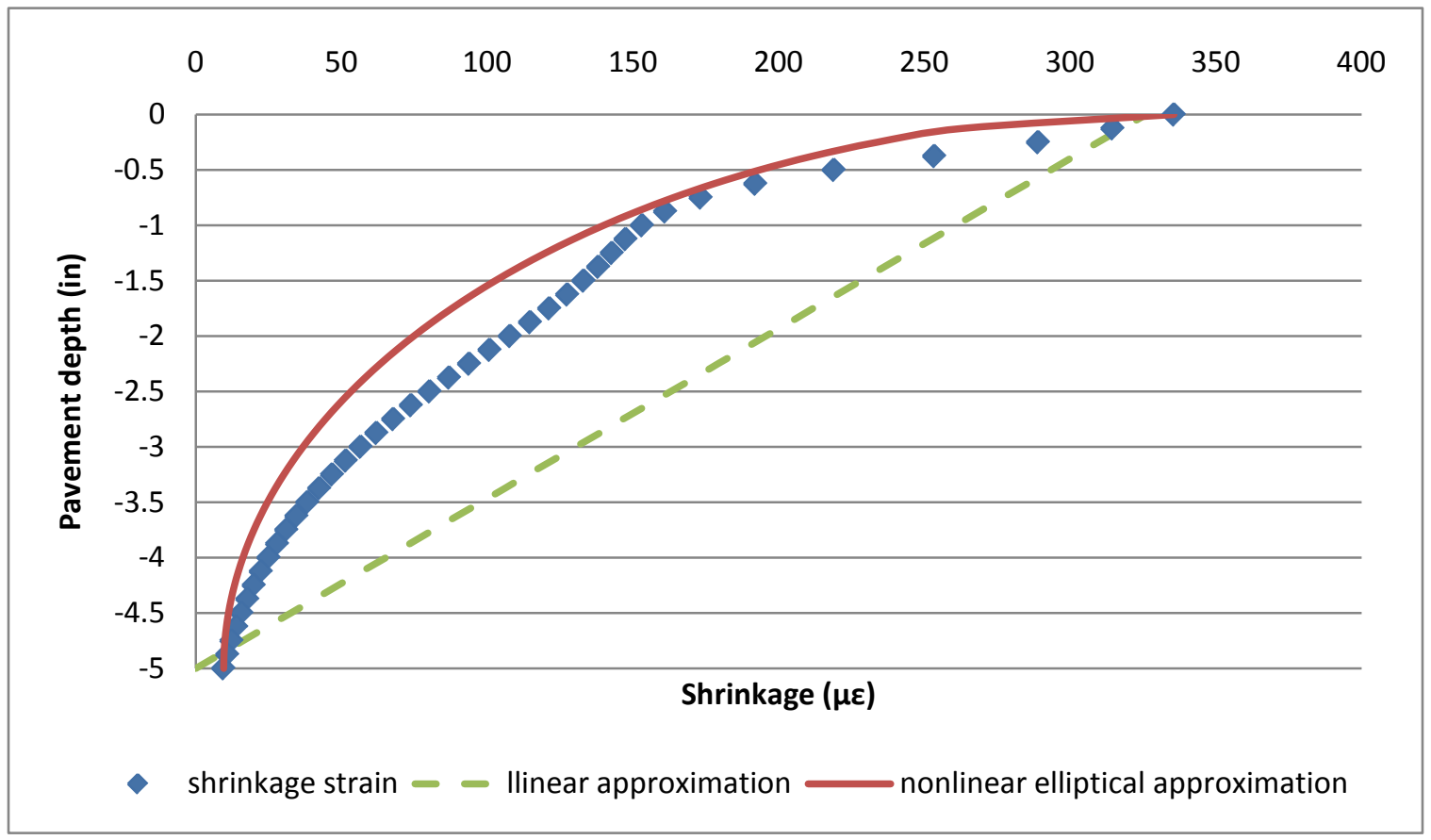

Figure E.4: Ultimate shrinkage through the depth of the pavement for New Orleans, Louisiana 30 days after construction. 


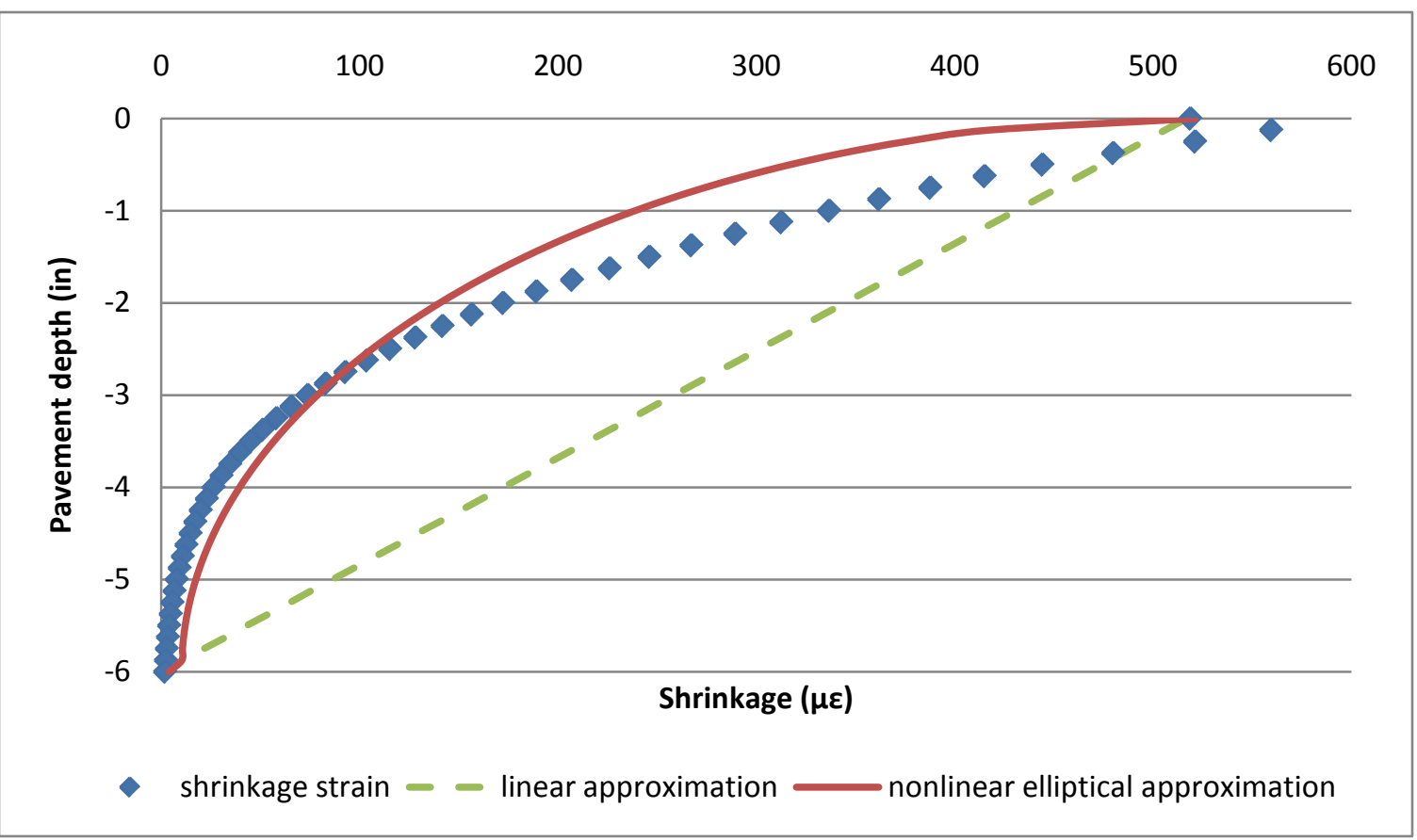

Figure E.5: Ultimate shrinkage through the depth of the pavement for Los Angeles, California 30 days after construction.

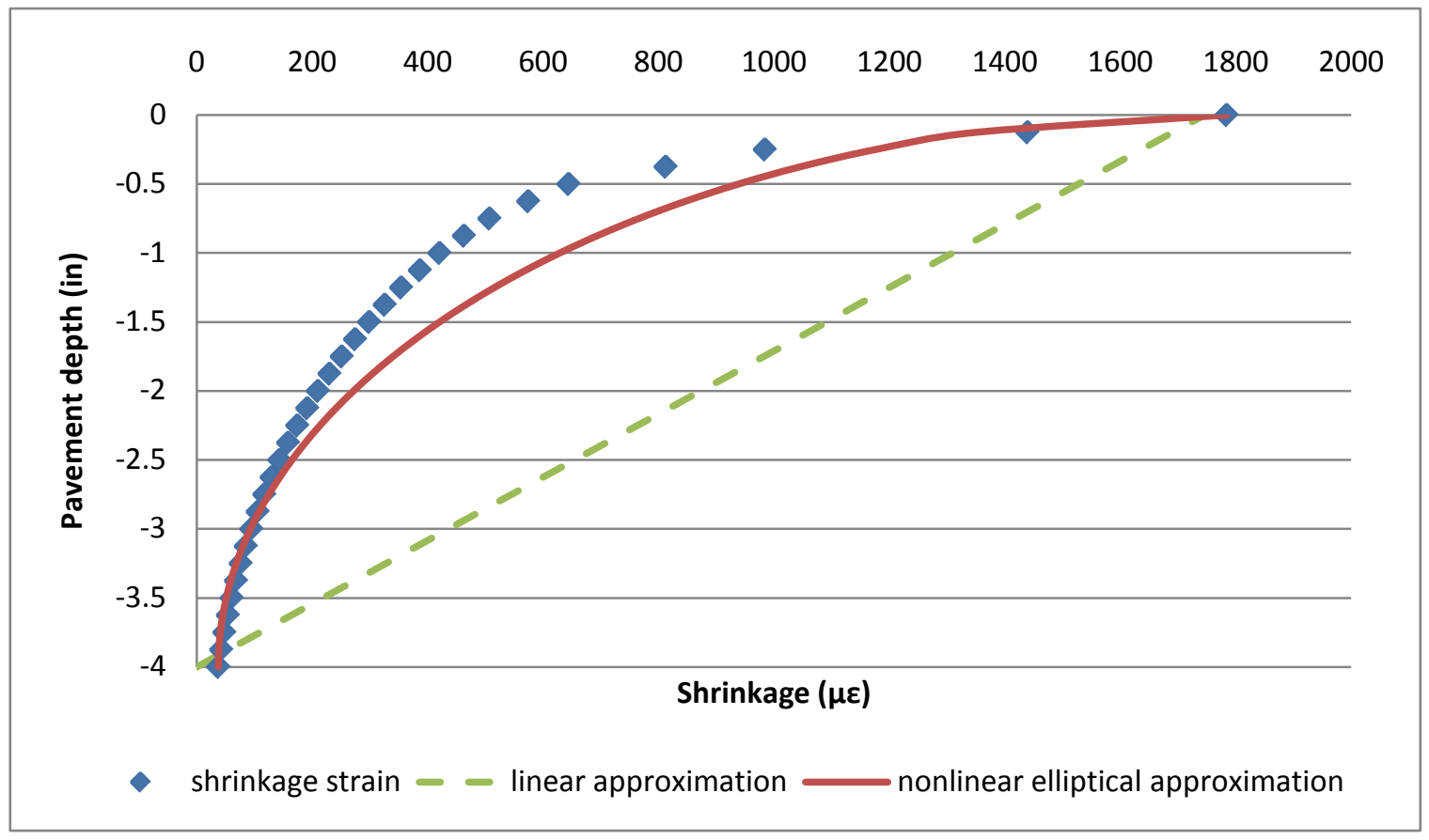

Figure E.6: Ultimate shrinkage through the depth of the pavement for Las Vegas, Nevada, 30 days after construction. 


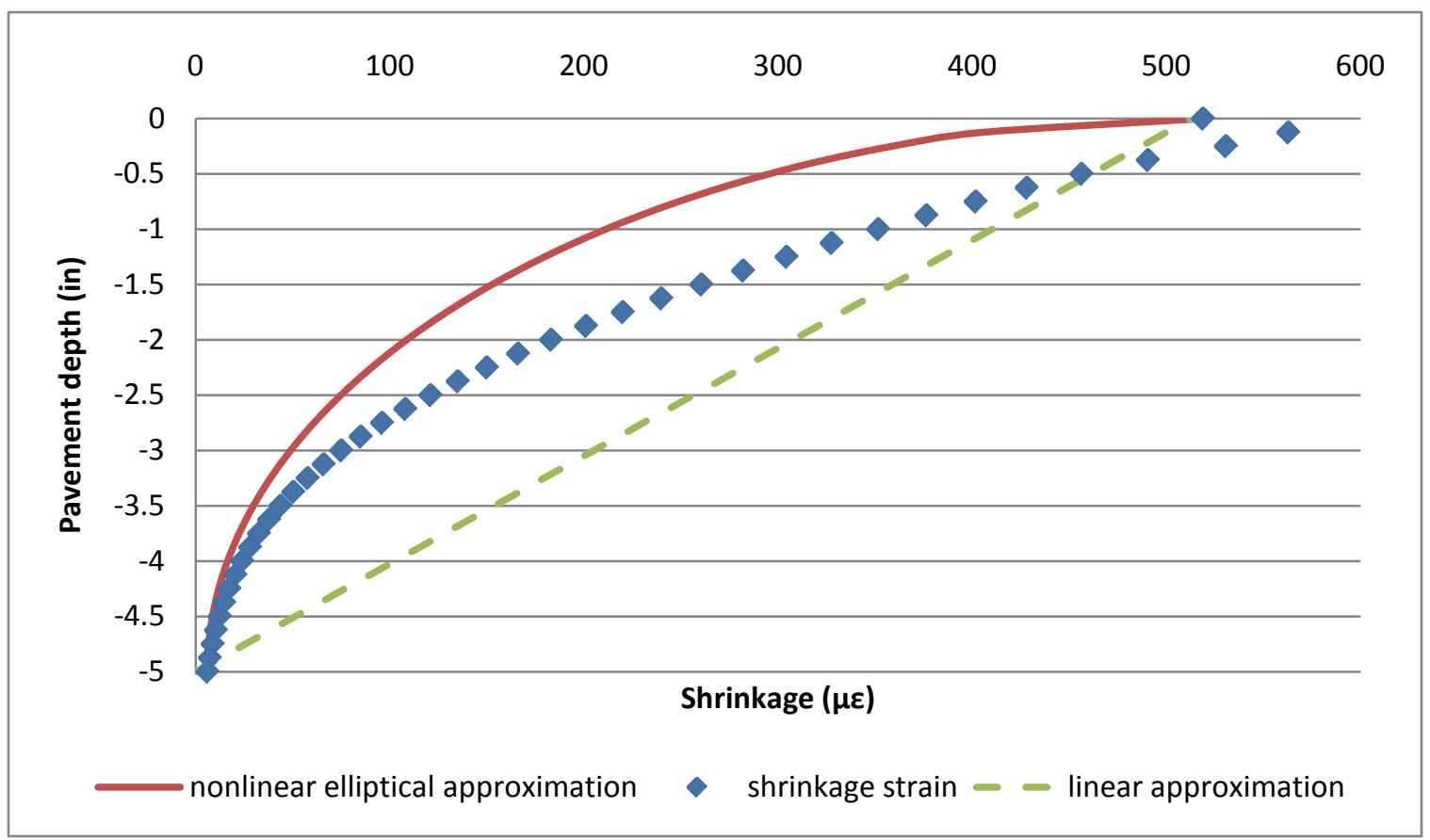

Figure E.7: Ultimate shrinkage through the depth of the pavement for Astoria, Oregon 30 days after construction.

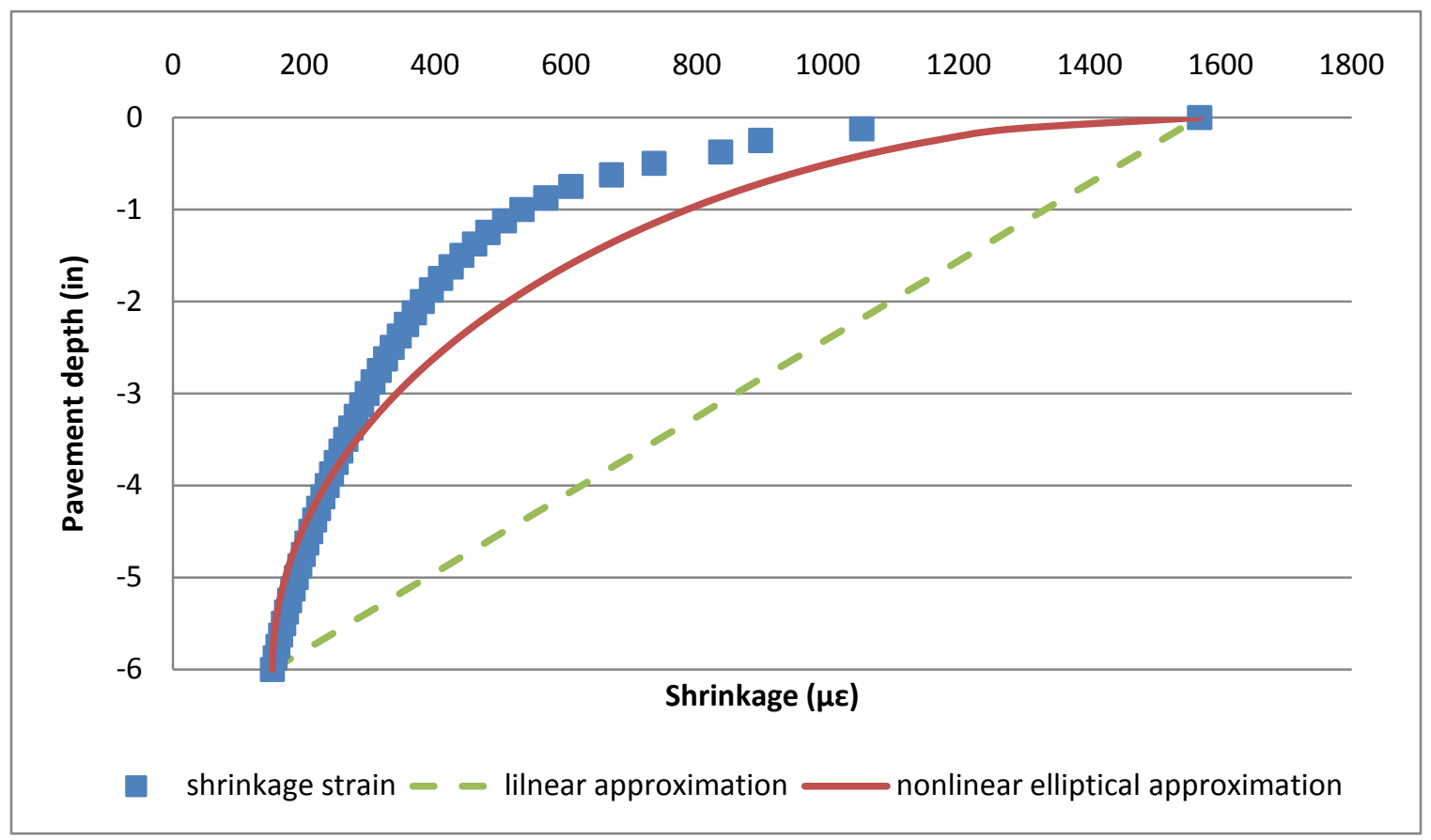

Figure E.8: Ultimate shrinkage through the depth of the pavement for Reno, Nevada 1 year after construction. 


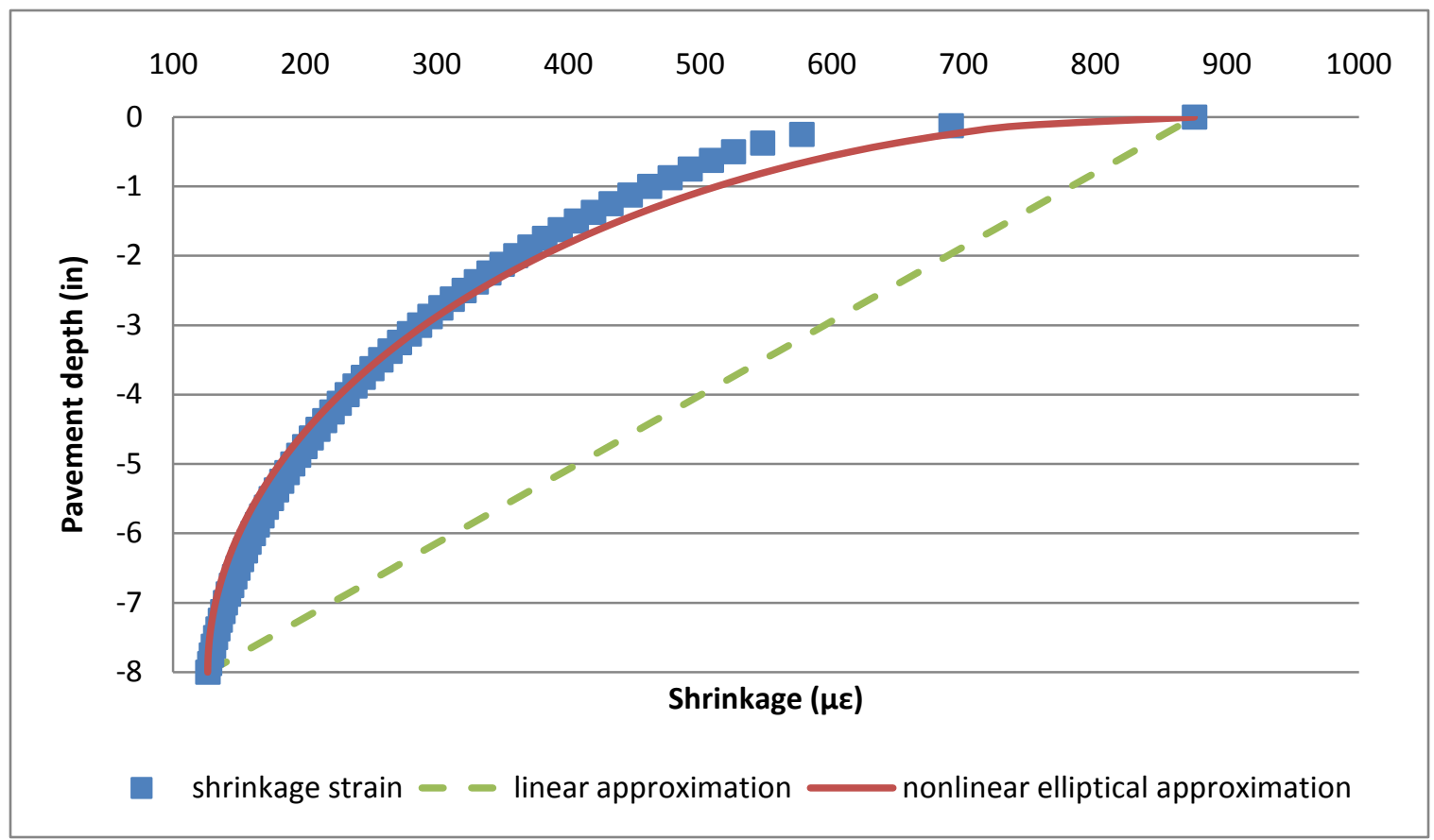

Figure E.9: Ultimate shrinkage through the depth of the pavement for Seattle, Washington 1 year after construction.

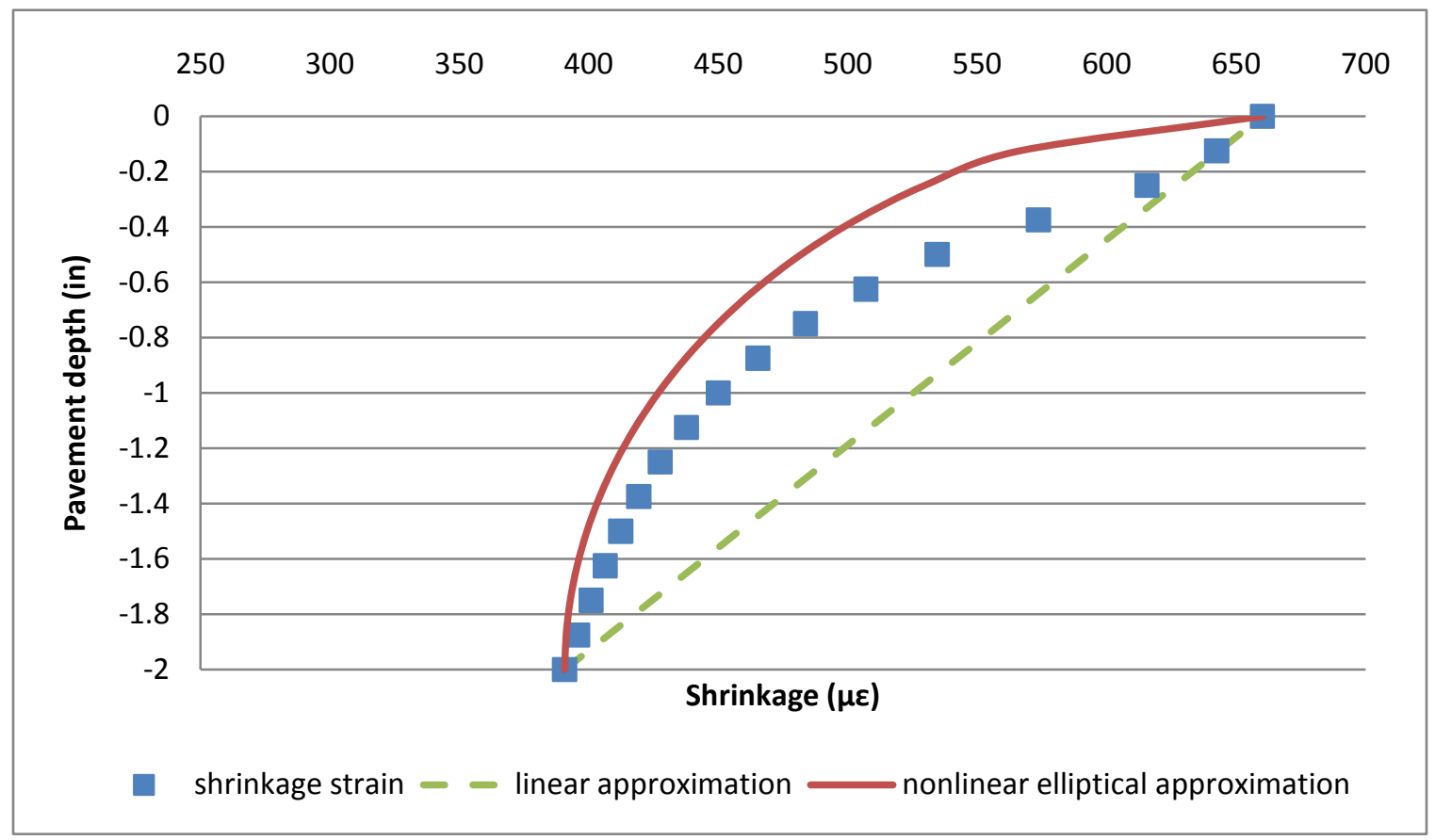

Figure E.10: Ultimate shrinkage through the depth of the pavement for Columbus, Ohio 1 year after construction. 


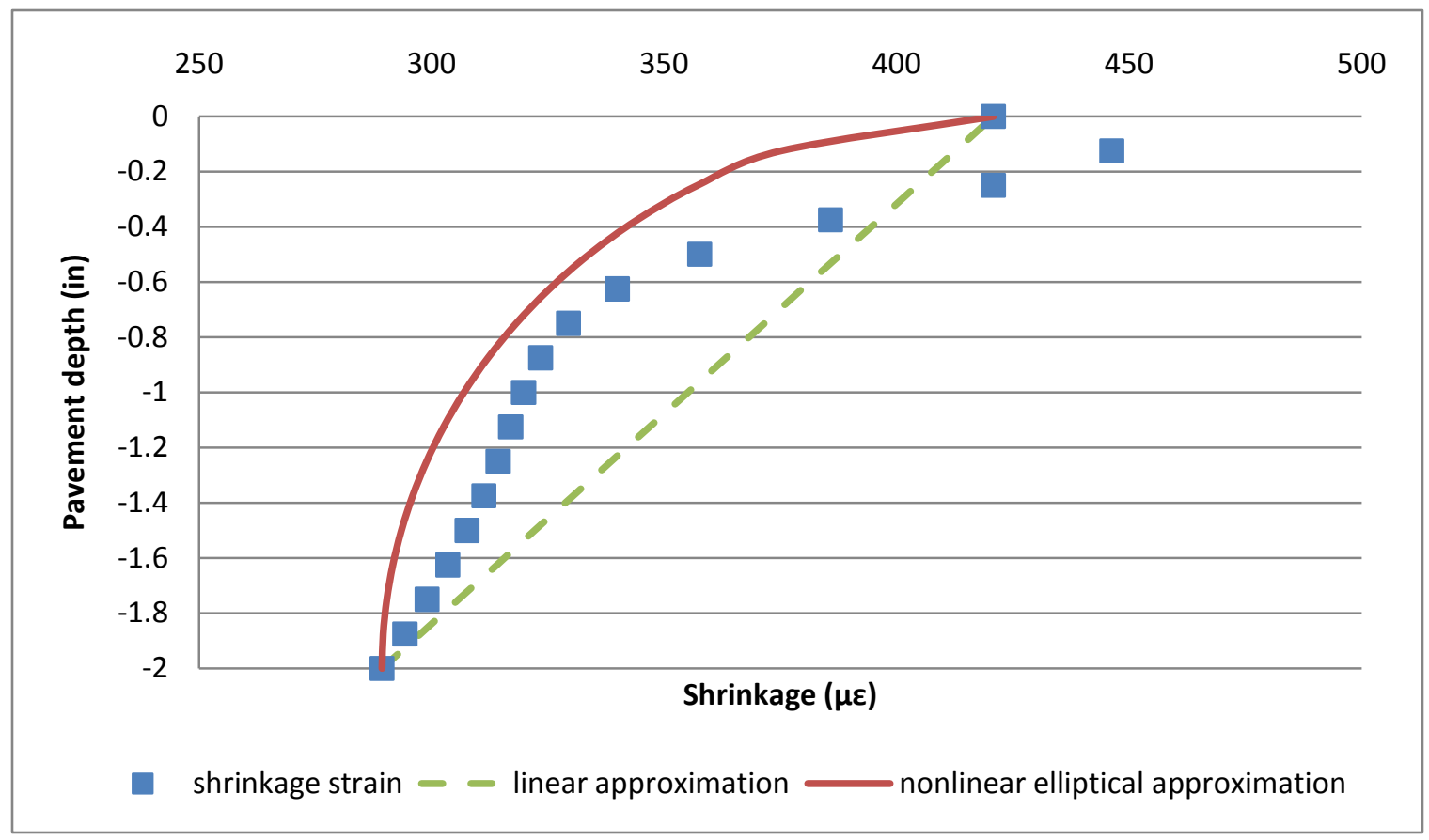

Figure E.11: Ultimate shrinkage through the depth of the pavement for New Orleans, Louisiana1 1 year after construction.

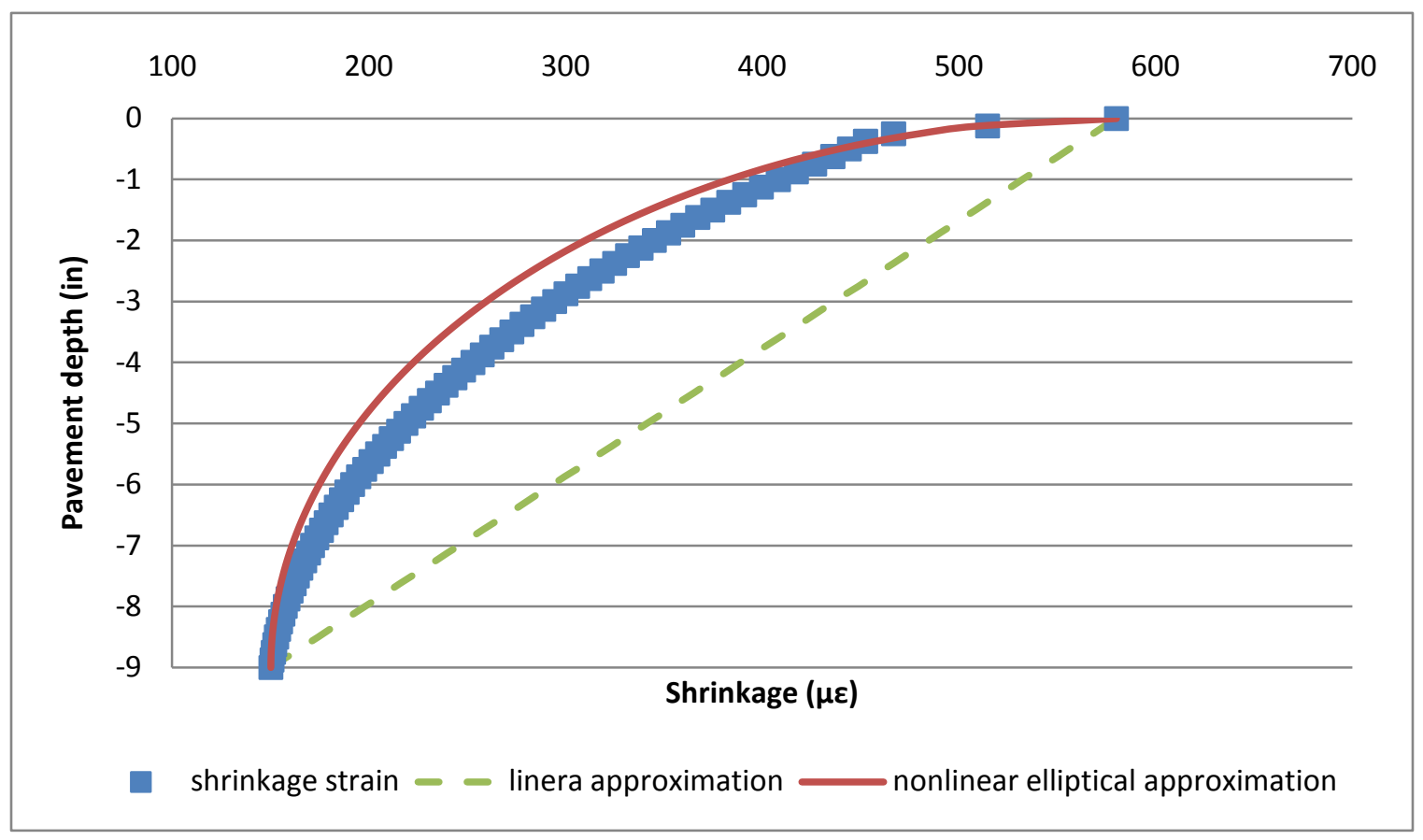

Figure E.12: Ultimate shrinkage through the depth of the pavement for Los Angeles, California 1 year after construction. 


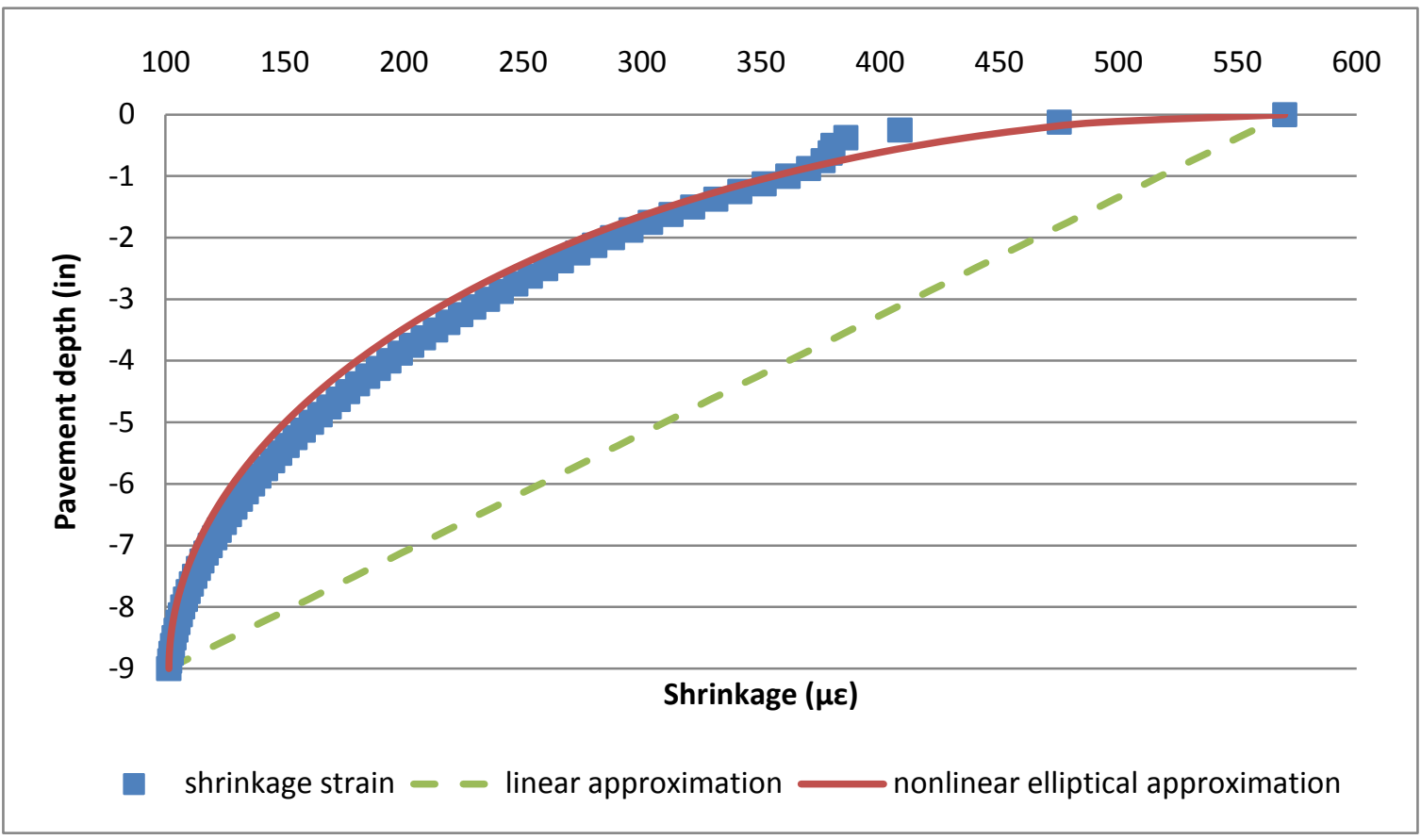

Figure E.13: Ultimate shrinkage through the depth of the pavement for Las Vegas, Nevada 1 year after construction.

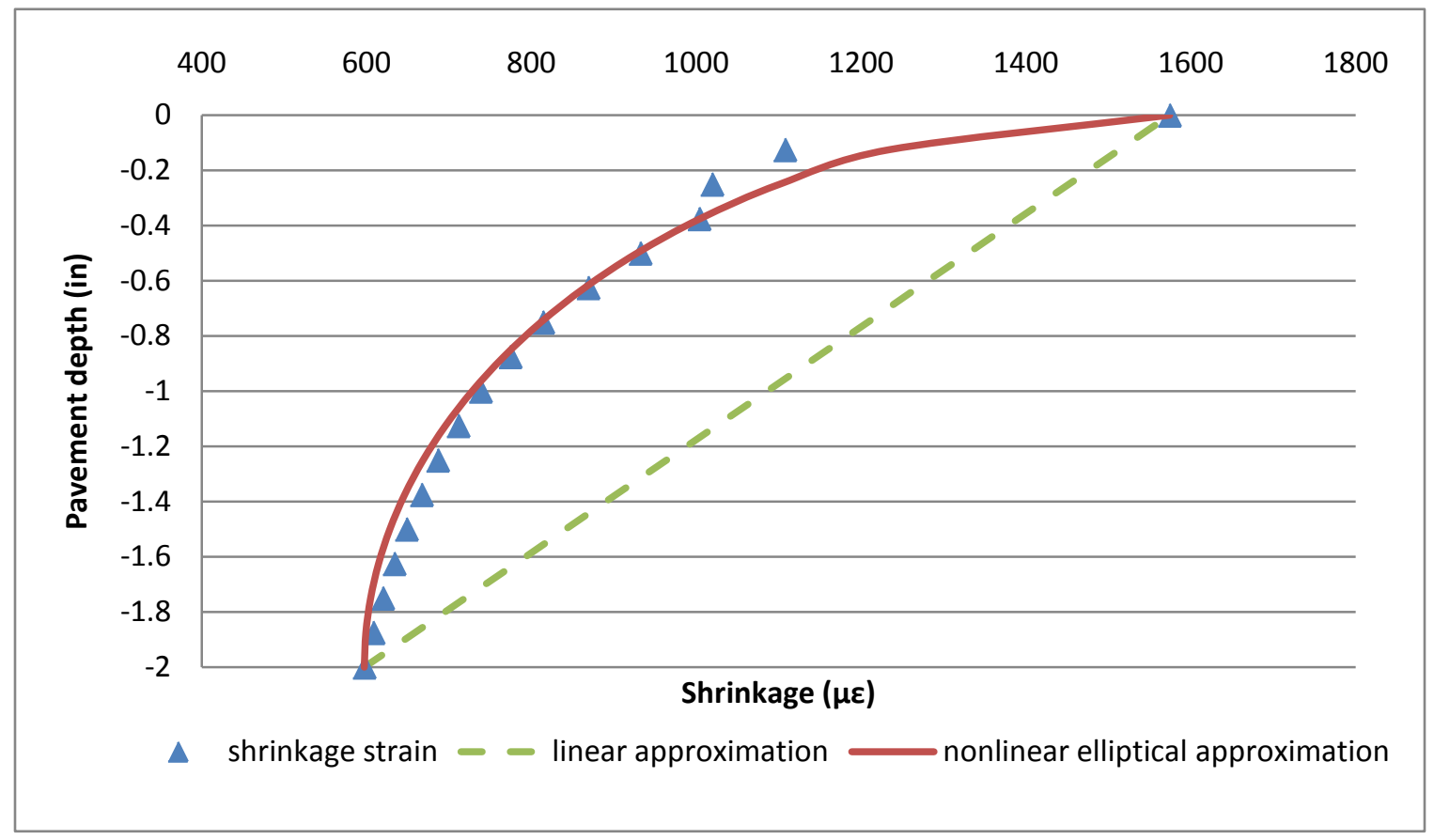

Figure E.14: Ultimate shrinkage through the depth of the pavement for Astoria, Oregon 1 year after construction. 


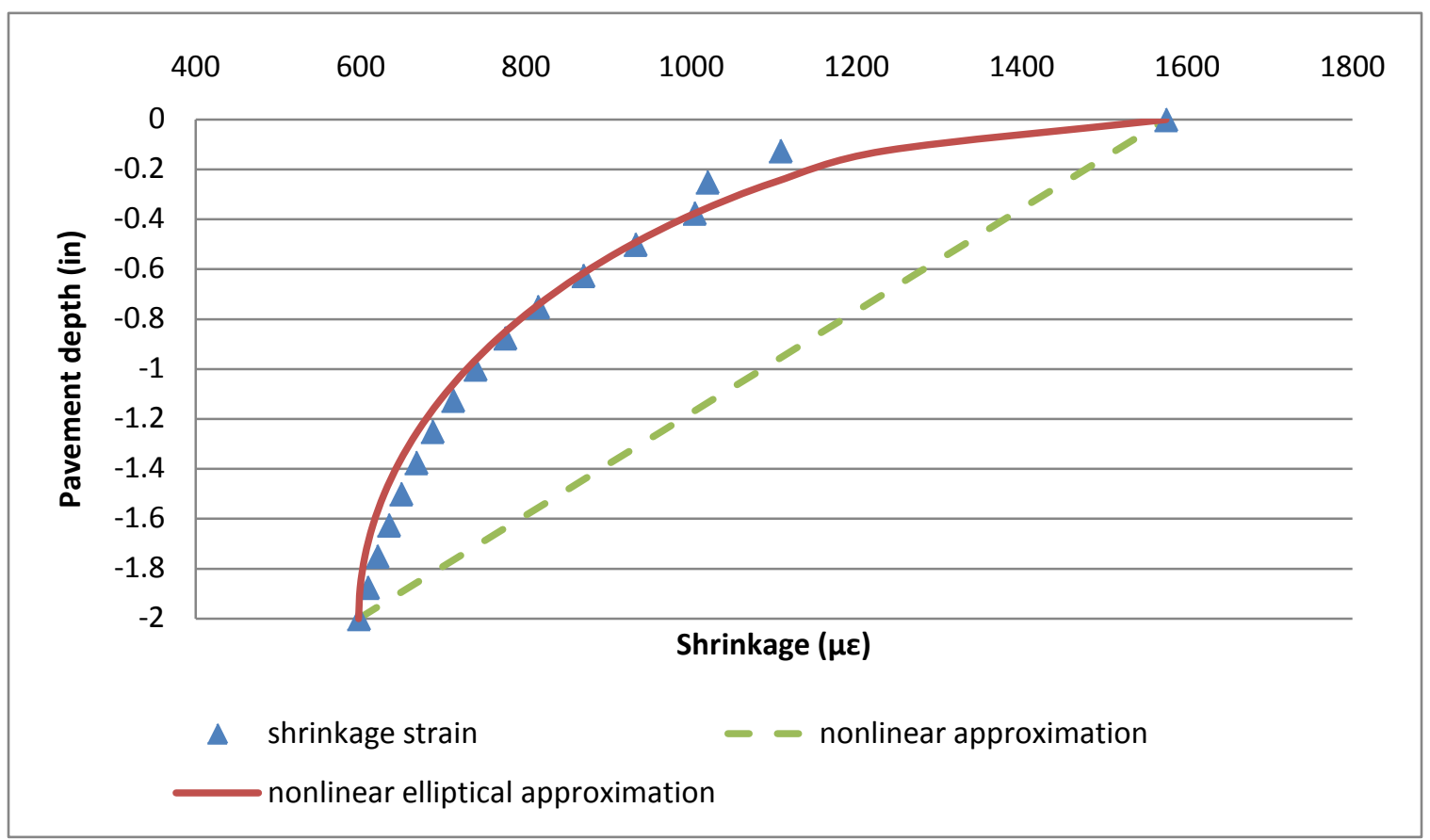

Figure E.15: Ultimate shrinkage through the depth of the pavement for Reno, Nevada 5 years after construction.

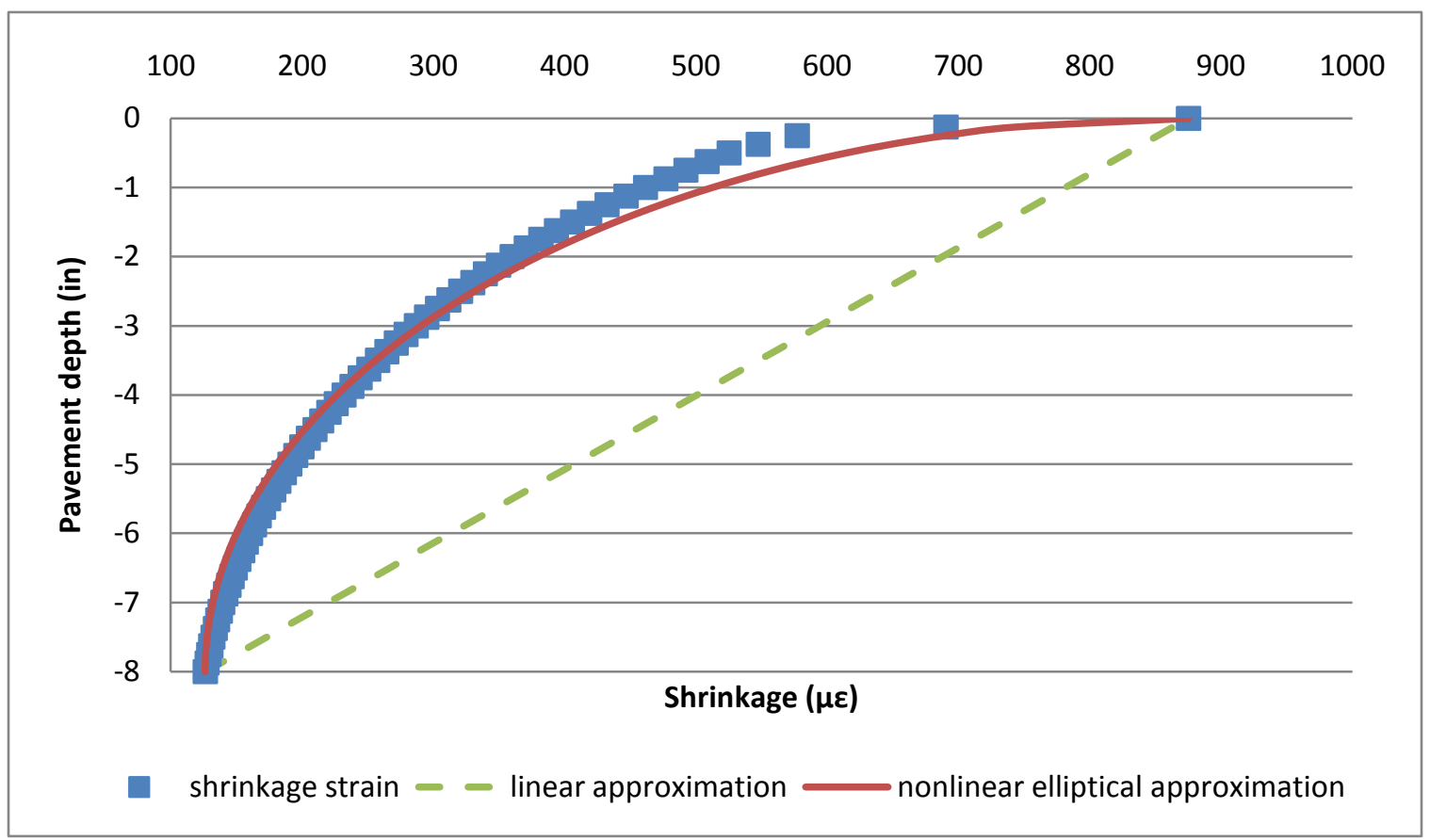

Figure E.16: Ultimate shrinkage through the depth of the pavement for Seattle, Washington 5 years after construction. 


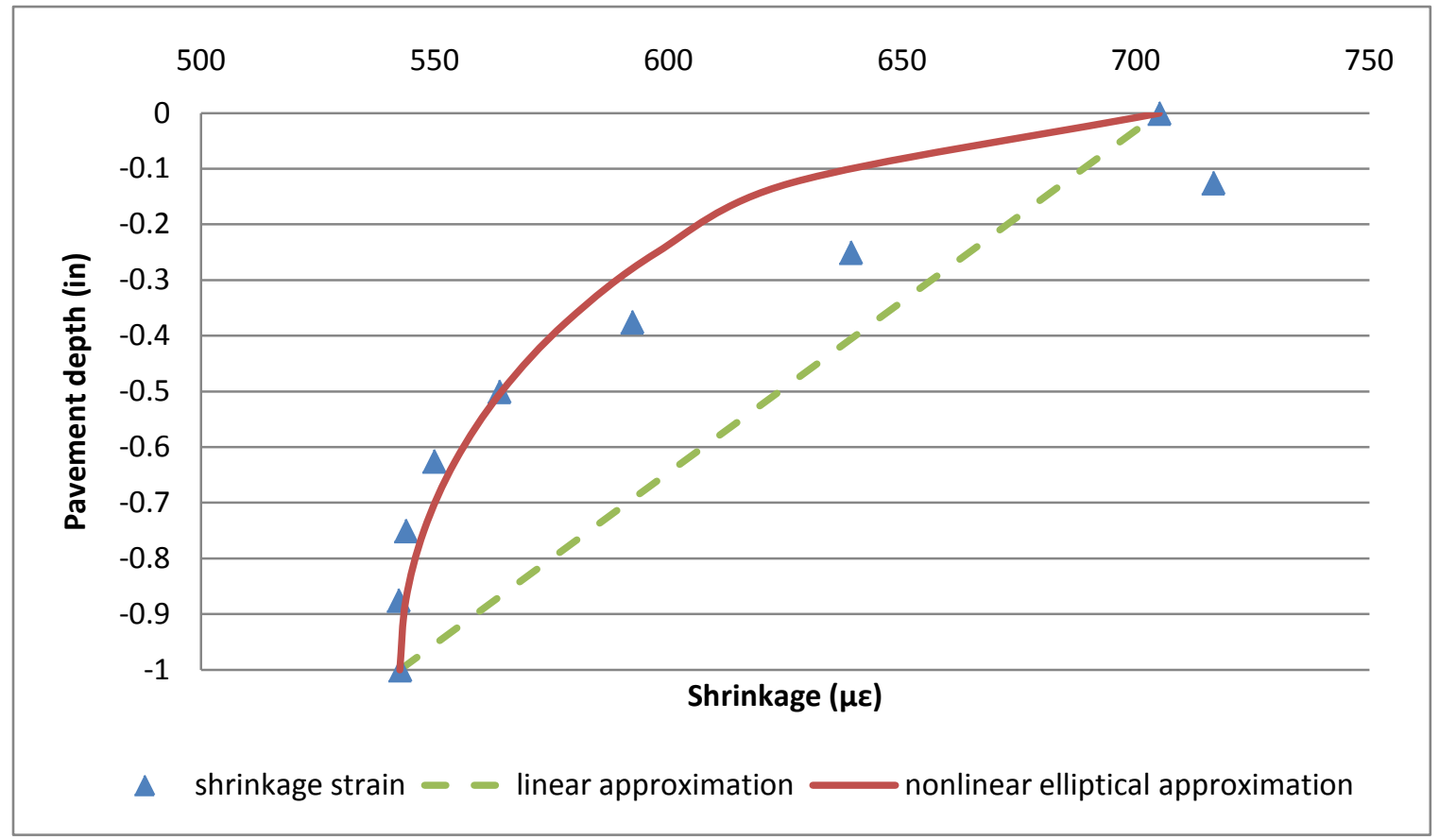

Figure E.17: Ultimate shrinkage through the depth of the pavement for Columbus, Ohio 5 years after construction.

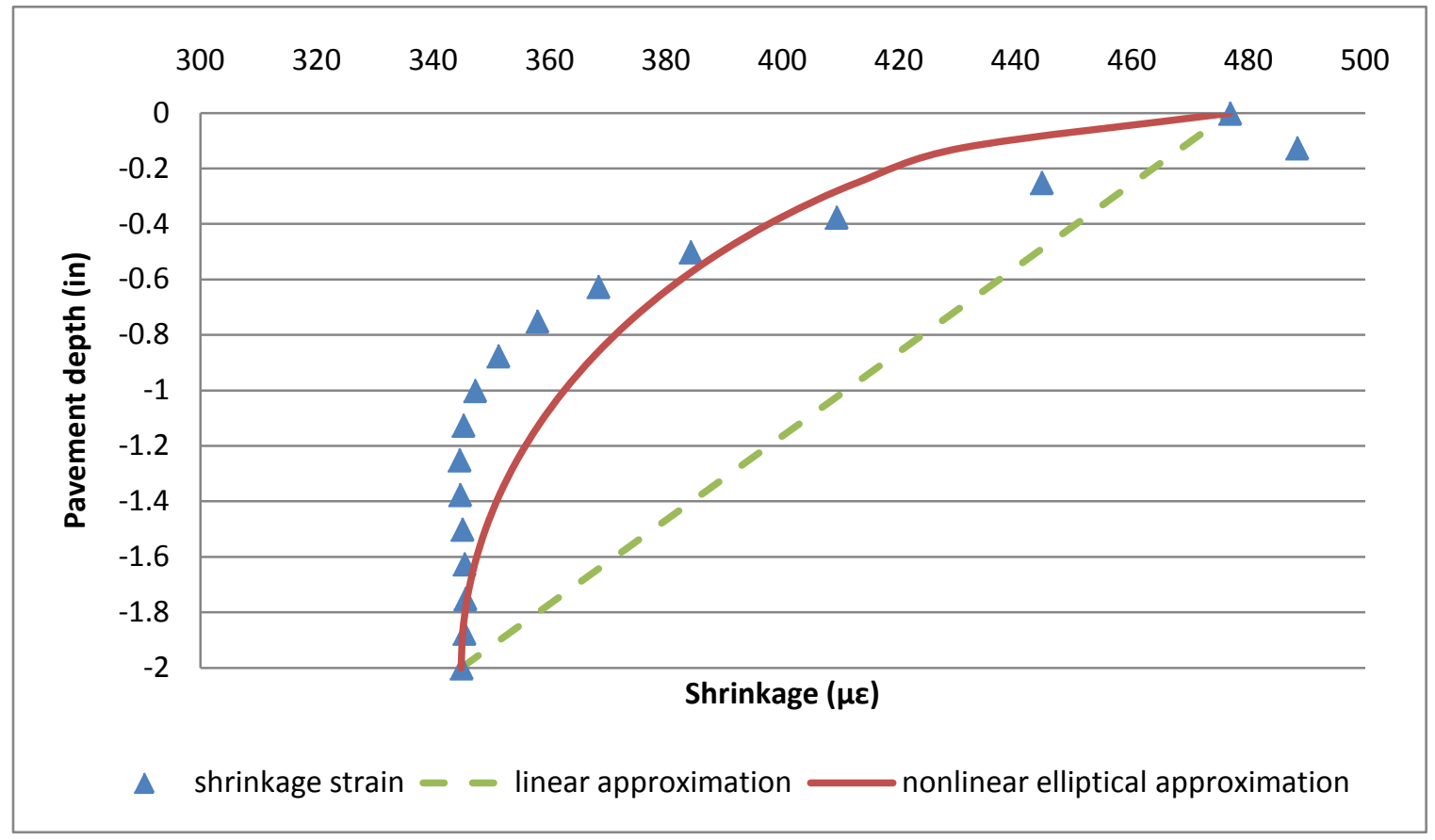

Figure E.18: Ultimate shrinkage through the depth of the pavement for New Orleans, Louisiana 5 years after construction. 


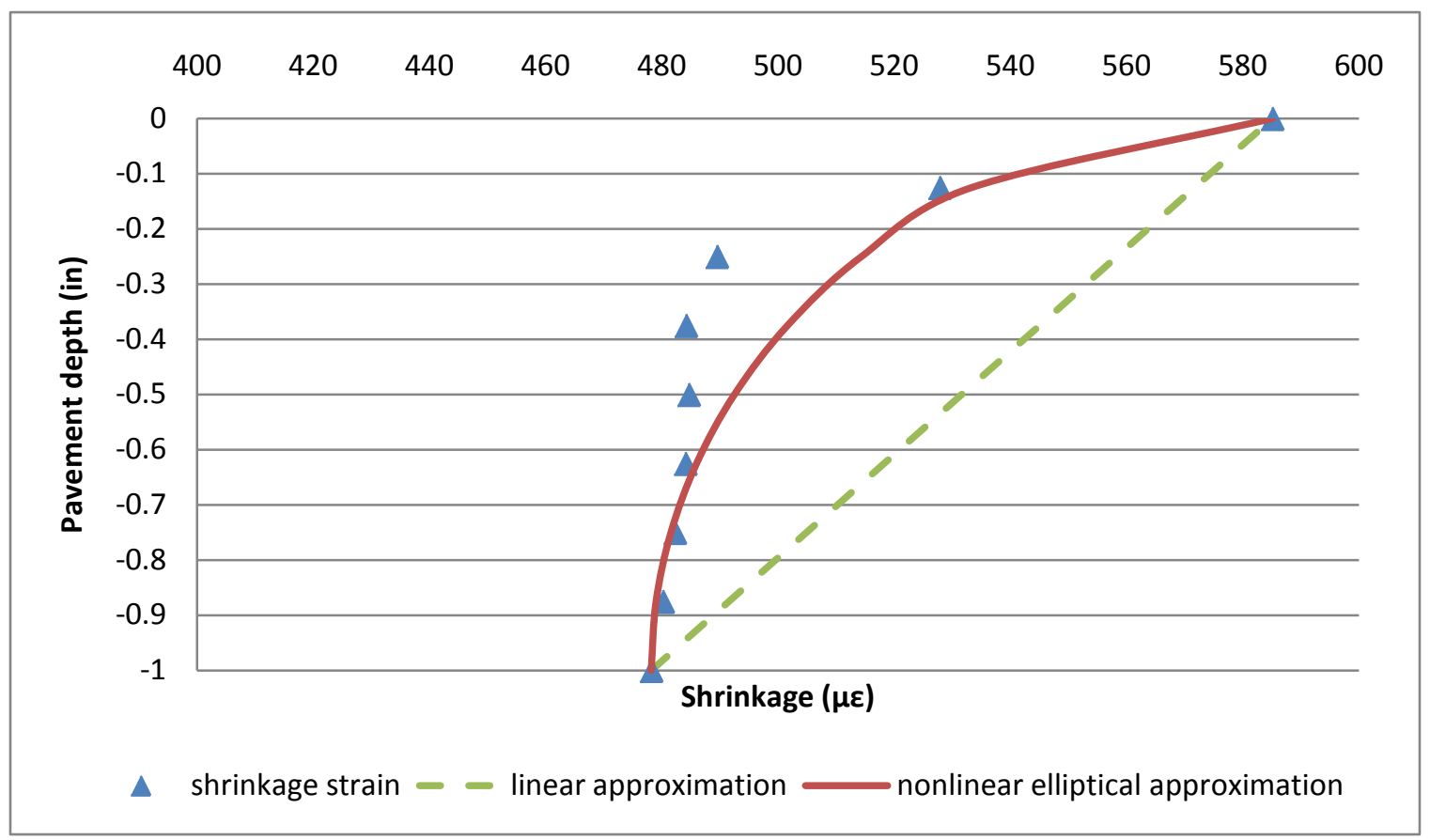

Figure E.19: Ultimate shrinkage through the depth of the pavement for Los Angeles, California 5 years after construction.

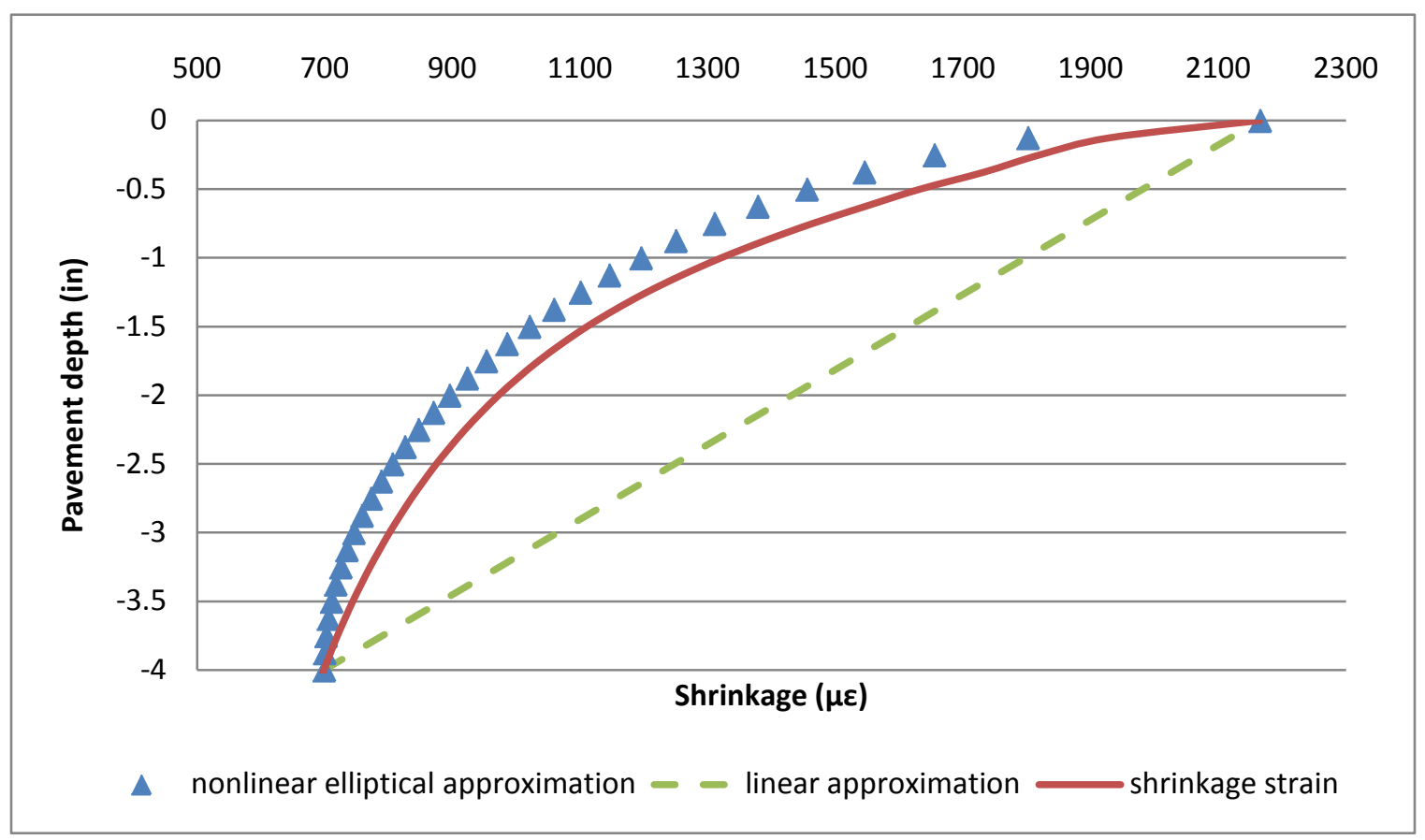

Figure E.20: Ultimate shrinkage through the depth of the pavement for Los Angeles, California 5 years after construction. 


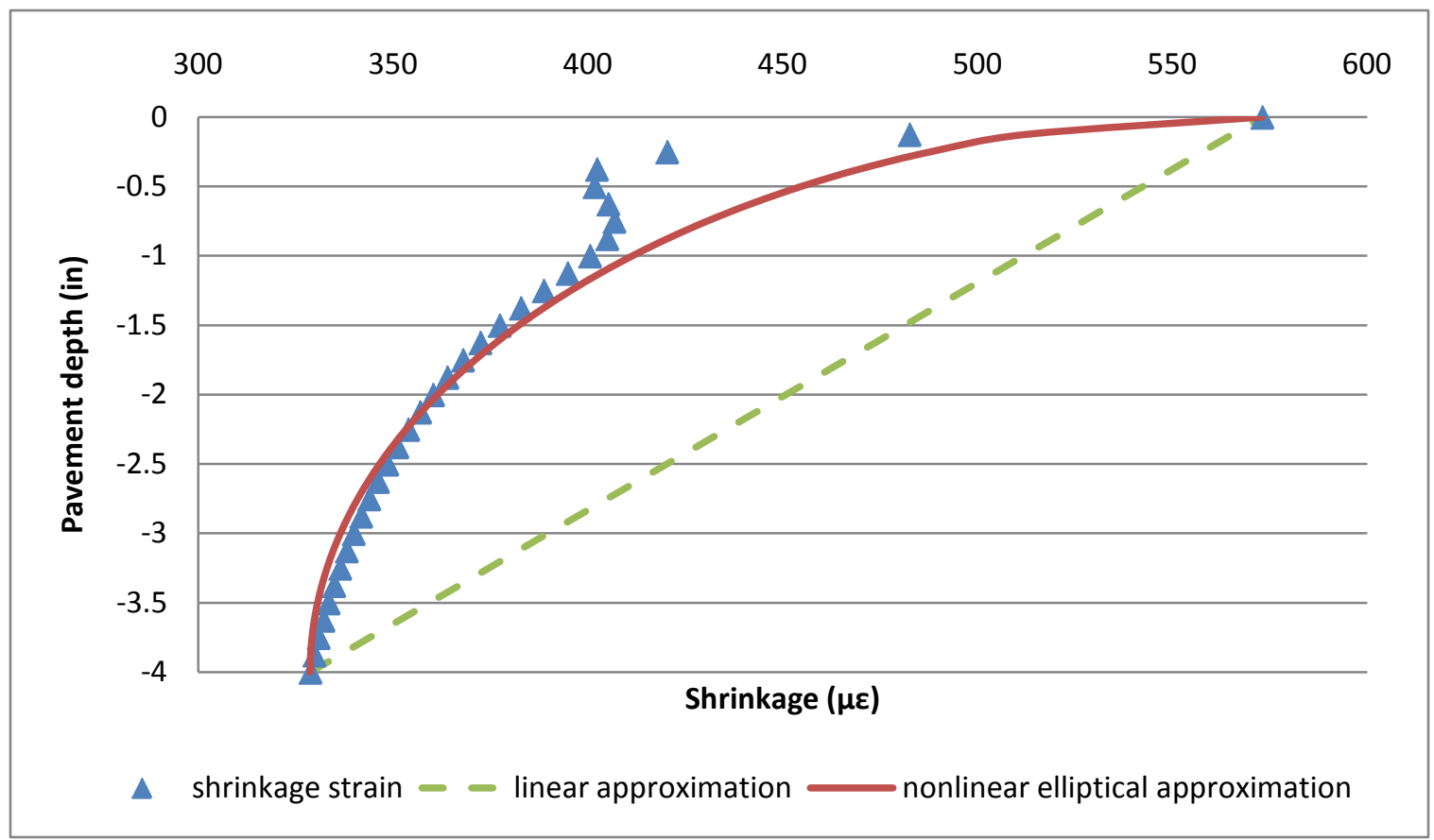

Figure E.21: Ultimate shrinkage through the depth of the pavement for Astoria, Oregon 5 years after construction. 


\section{Appendix F: Design Aids for the Prediction of Moisture Warping and Differential Drying}

Shrinkage

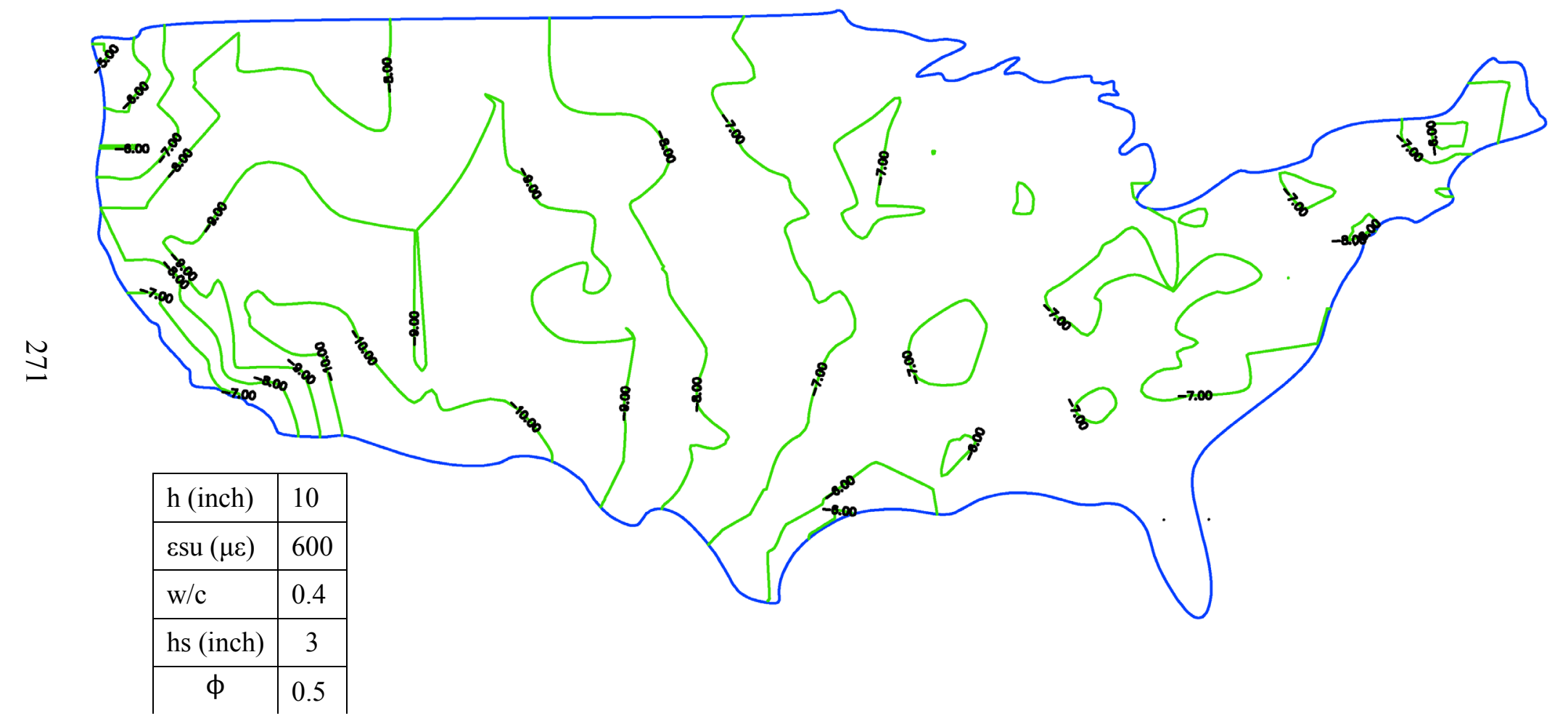

Figure F.1: Design Aid for a 10 inch thick slab with a w/c ratio of 0.4 , ultimate shrinkage of $600 \mu \varepsilon$, a reversible shrinkage factor of 0.5 and a depth of shrinkage zone of 3 inches. 


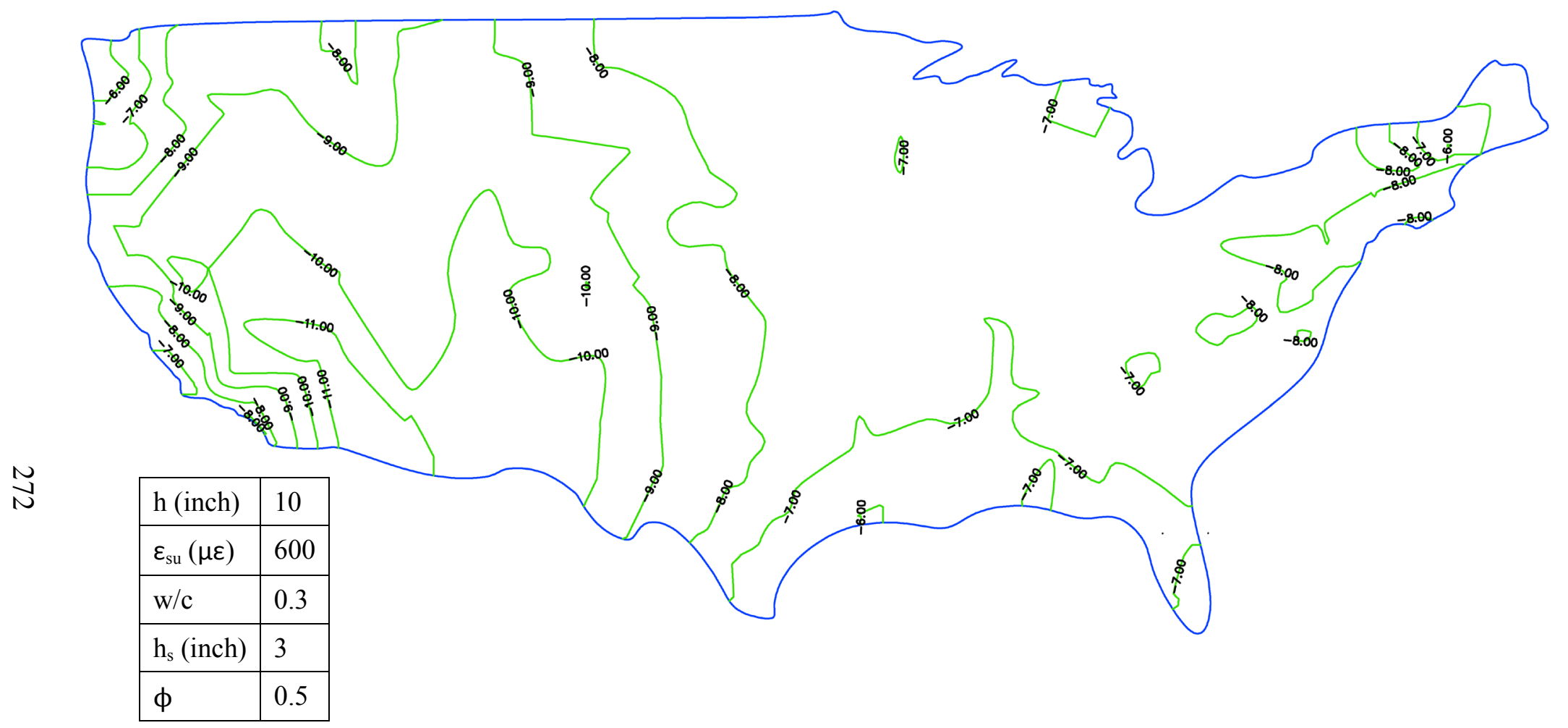

Figure F.2: Design Aid for a 10 inch thick slab with a w/c ratio of 0.3 , ultimate shrinkage of $600 \mu \varepsilon$, a reversible shrinkage factor of 0.5 and a depth of shrinkage zone of 3 inches. 


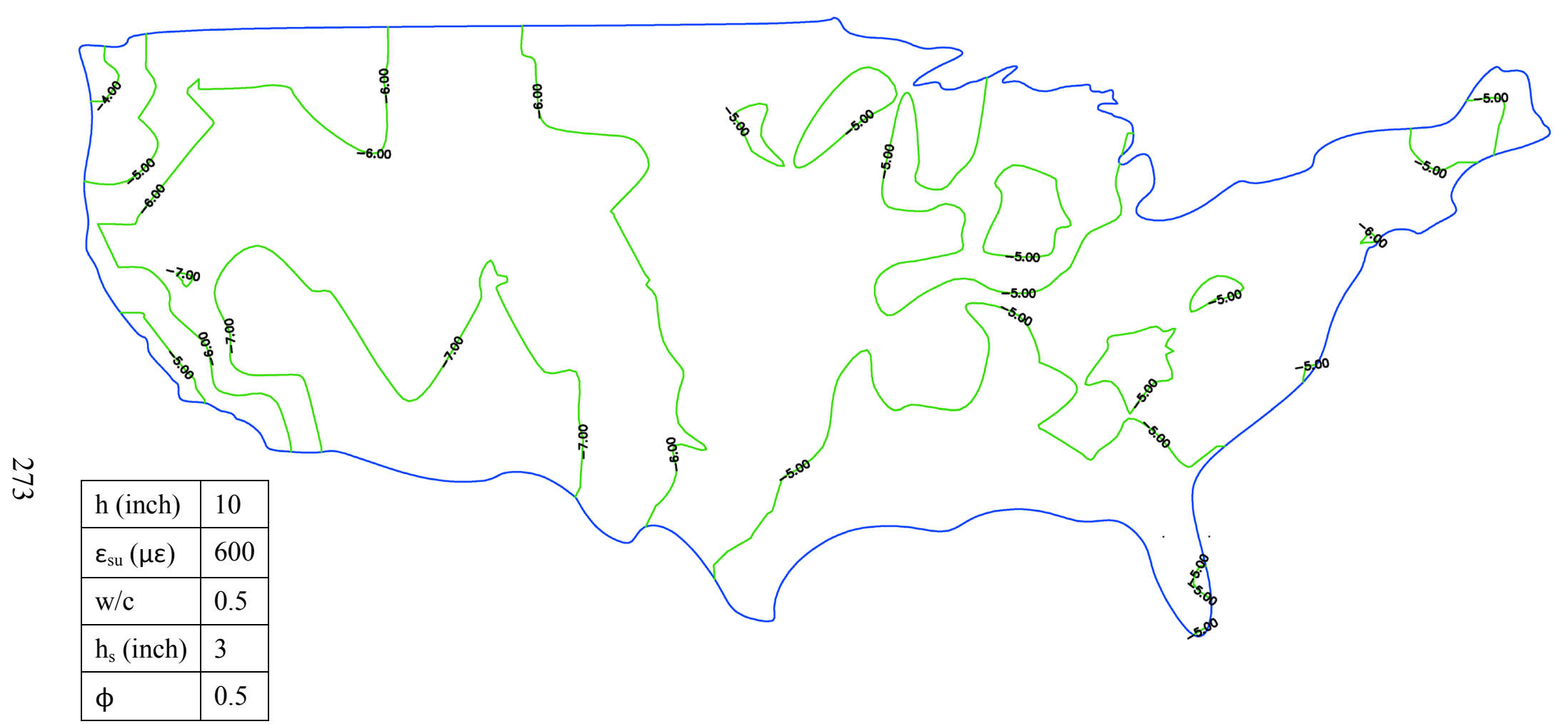

Figure F.3: Design Aid for a 10 inch thick slab with a w/c ratio of 0.5 , ultimate shrinkage of $600 \mu \varepsilon$, a reversible shrinkage factor of 0.5 and a depth of shrinkage zone of 3 inches. 


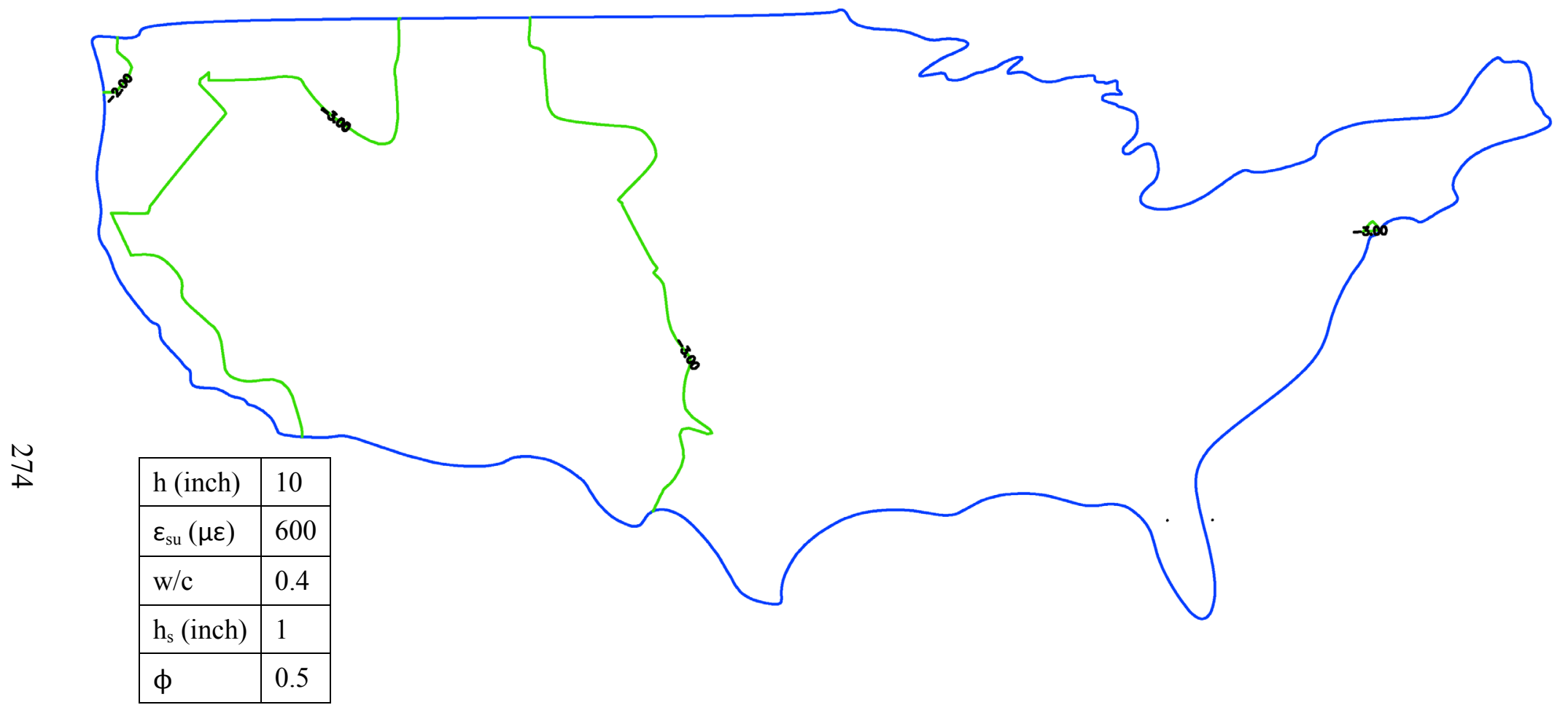

Figure F.4: Design Aid for a 10 inch thick slab with a w/c ratio of 0.4, ultimate shrinkage of $600 \mu \varepsilon$, a reversible shrinkage factor of 0.5 and a depth of shrinkage zone of 1 inch. 


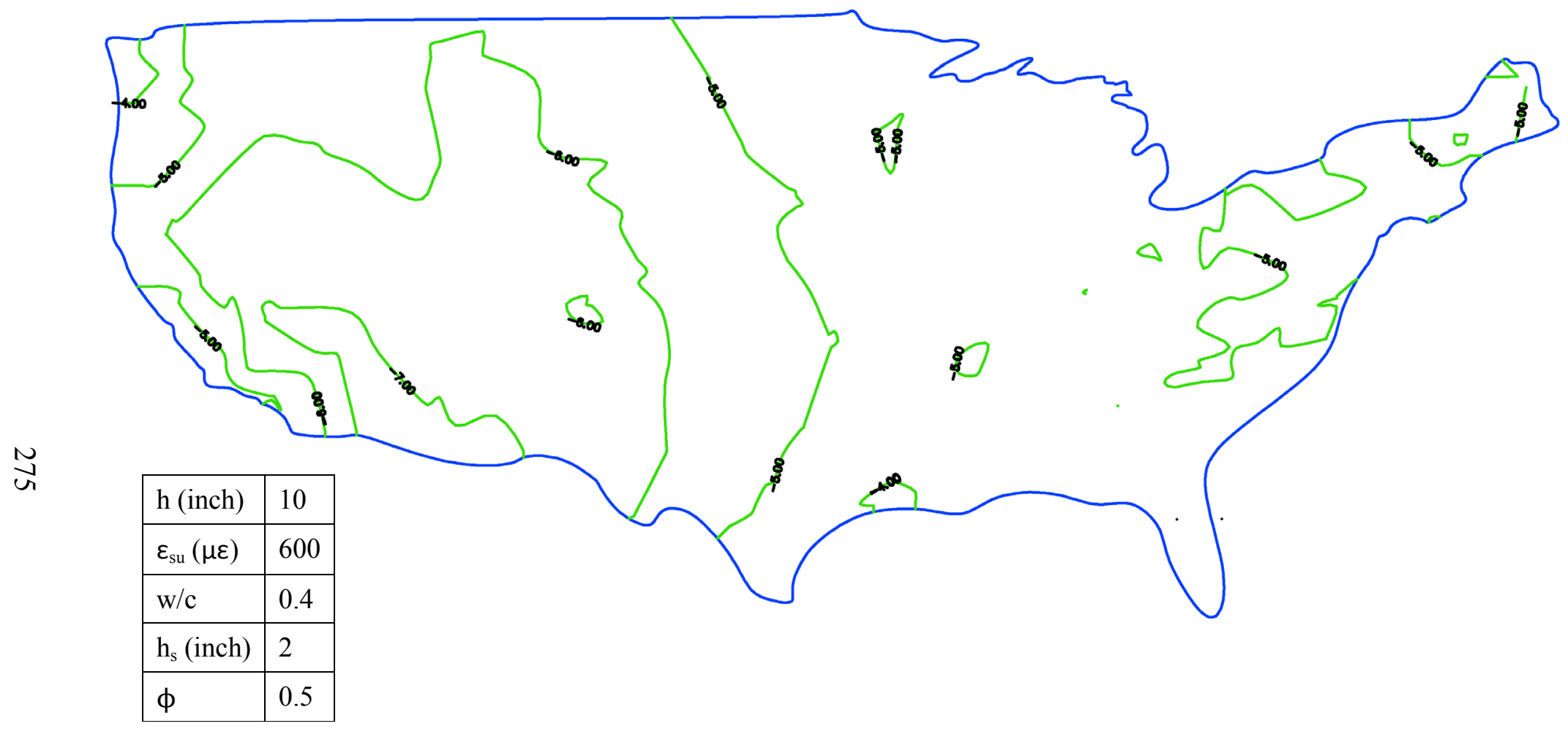

Figure F.5: Design Aid for a 10 inch thick slab with a w/c ratio of 0.4, ultimate shrinkage of $600 \mu \varepsilon$, a reversible shrinkage factor of 0.5 and a depth of shrinkage zone of 2 inches. 


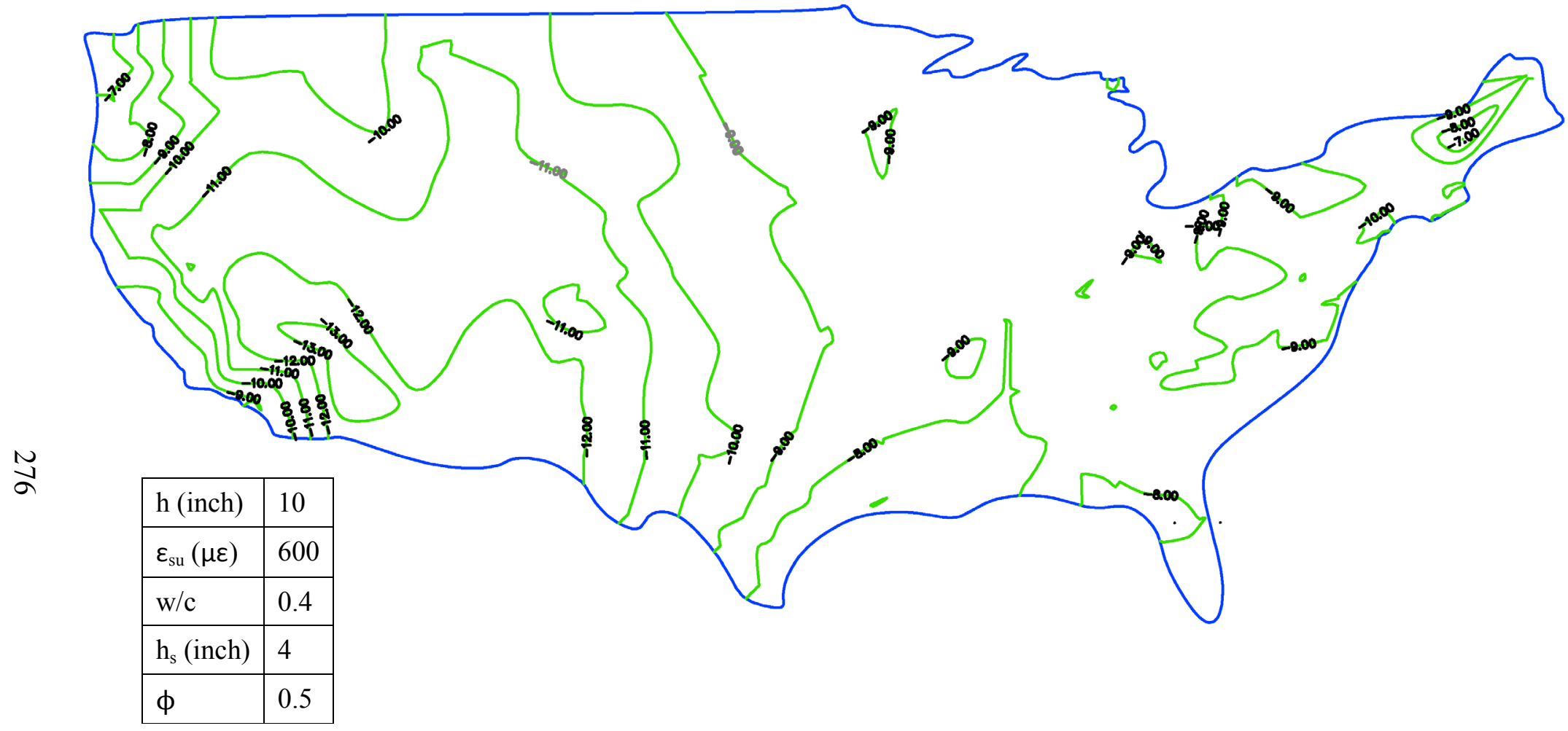

Figure F.6: Design Aid for a 10 inch thick slab with a w/c ratio of 0.4, ultimate shrinkage of $600 \mu \varepsilon$, a reversible shrinkage factor of 0.5 and a depth of shrinkage zone of 4 inches. 


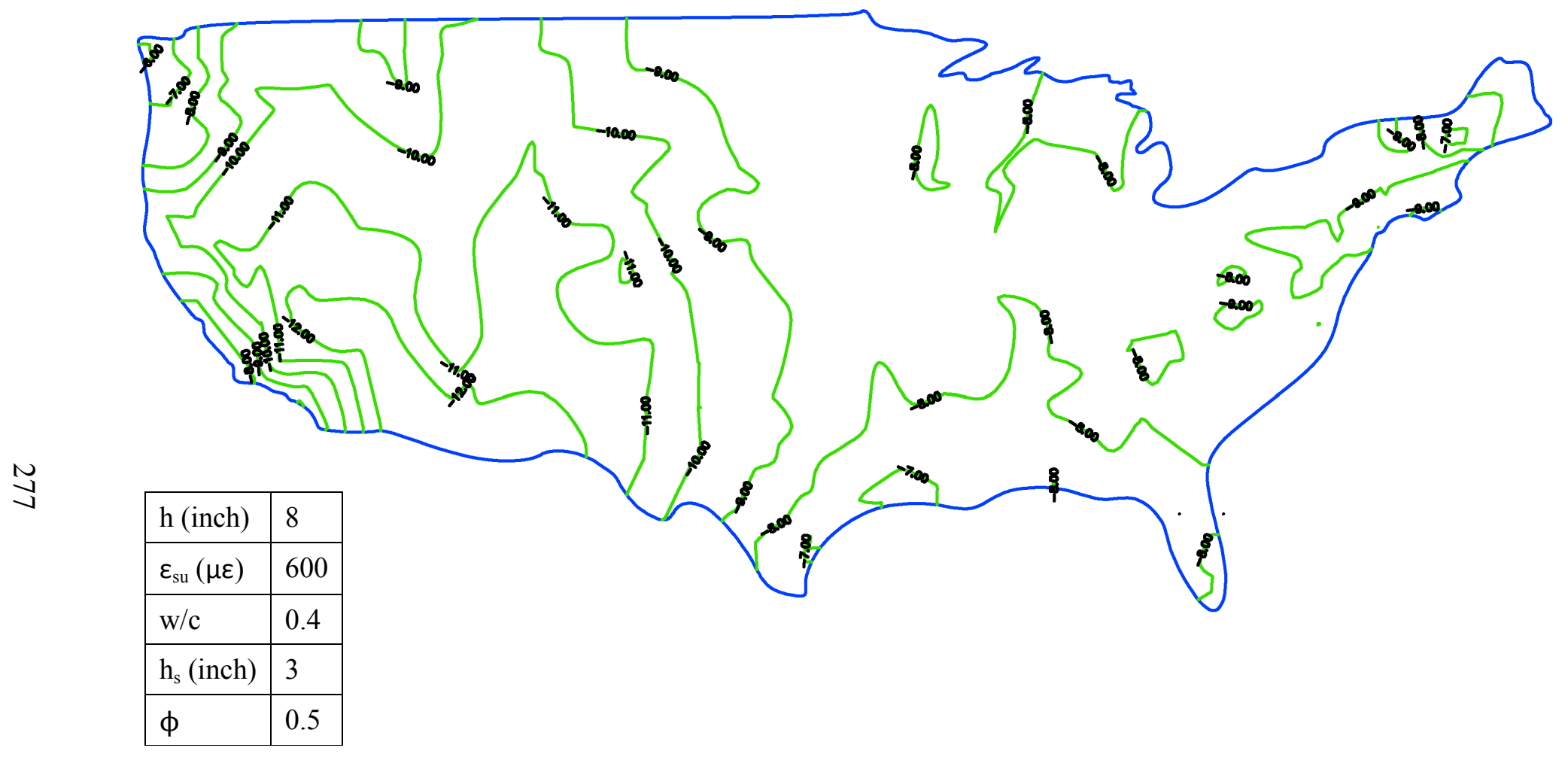

Figure F.7: Design Aid for an 8 inch thick slab with a w/c ratio of 0.4, ultimate shrinkage of $600 \mu \varepsilon$, a reversible shrinkage factor of 0.5 and a depth of shrinkage zone of 3 inches. 


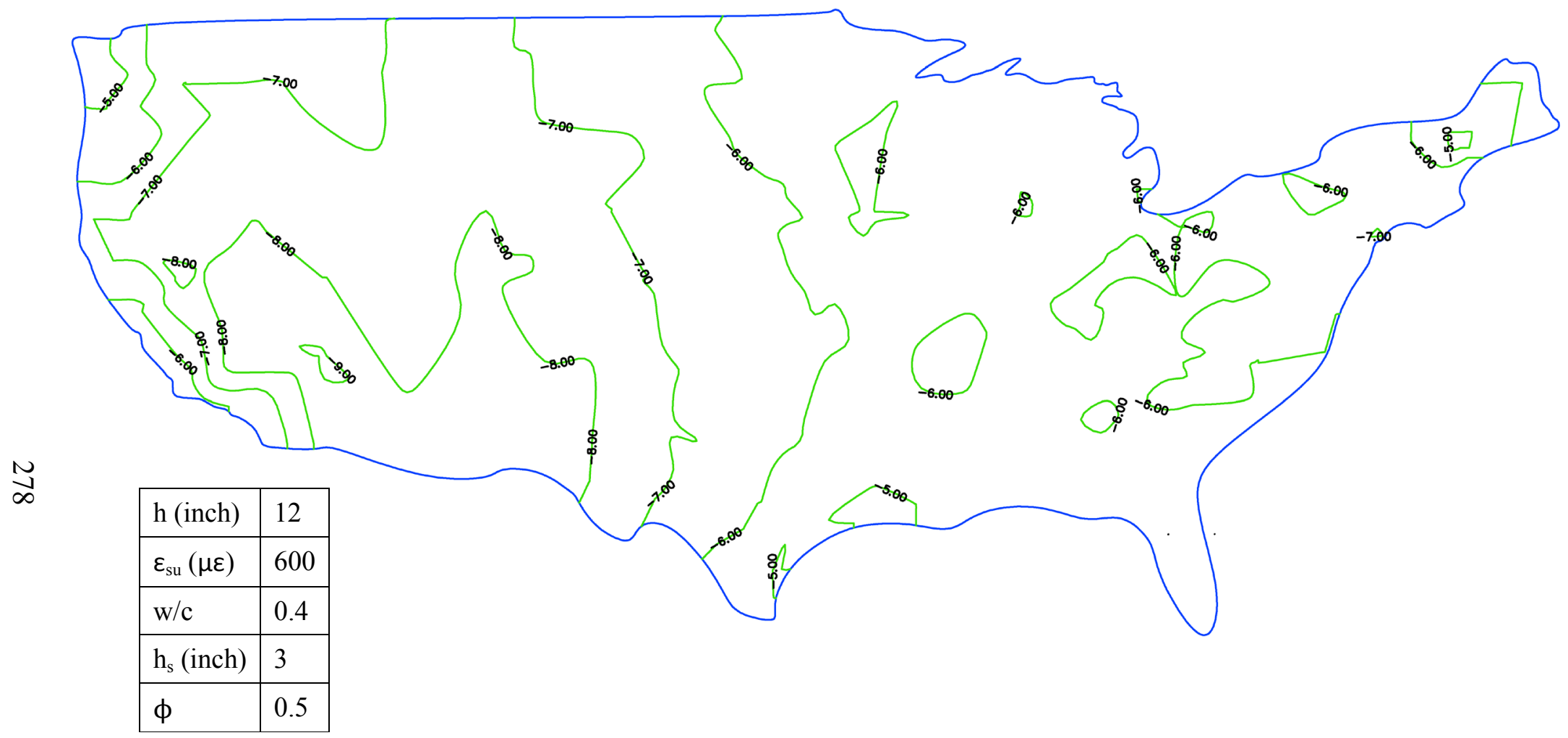

Figure F.8: Design Aid for a 12 inch thick slab with a w/c ratio of 0.4 , ultimate shrinkage of $600 \mu \varepsilon$, a reversible shrinkage factor of 0.5 and a depth of shrinkage zone of 3 inches. 


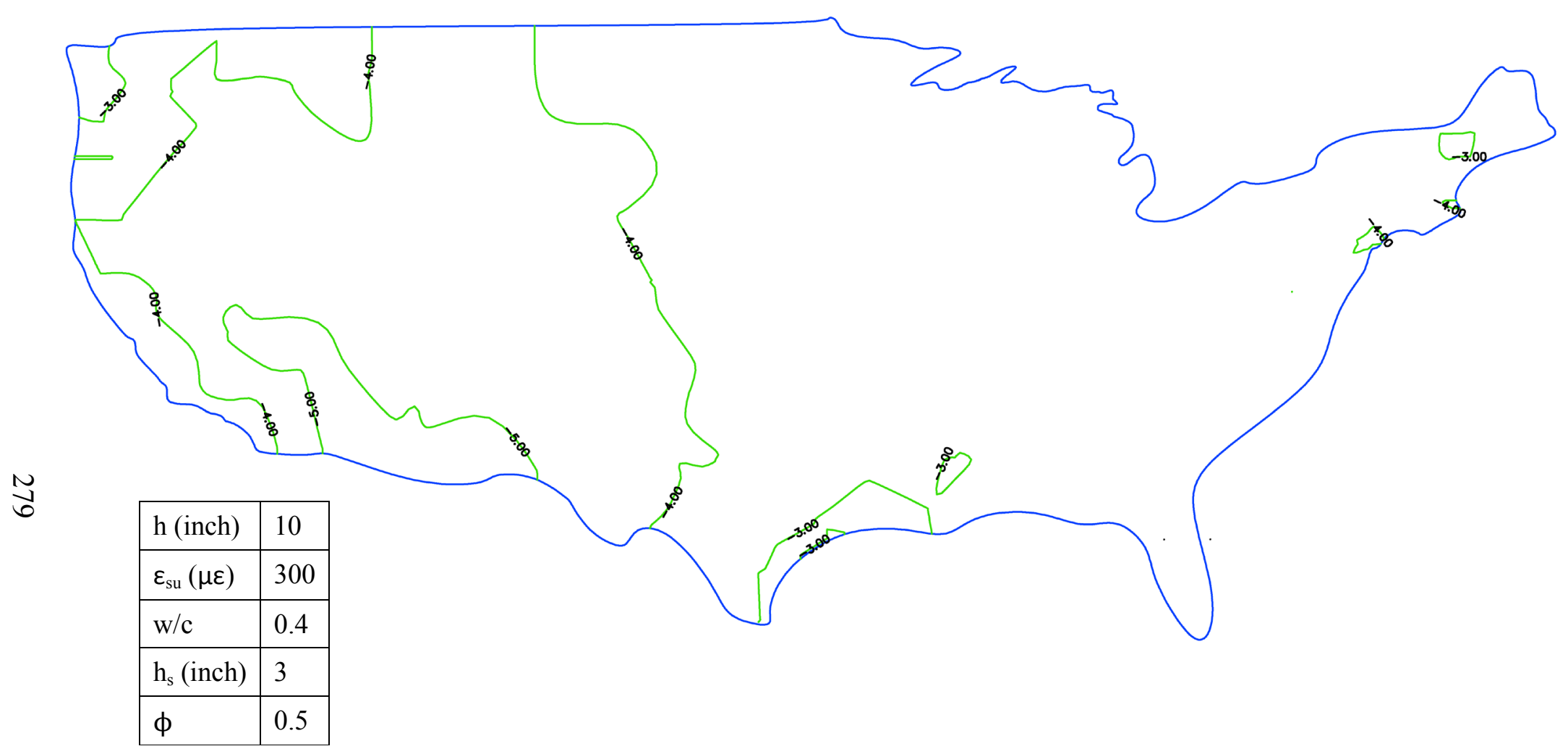

Figure F.9: Design Aid for a 10 inch thick slab with a w/c ratio of 0.4, ultimate shrinkage of $300 \mu \varepsilon$, a reversible shrinkage factor of 0.5 and a depth of shrinkage zone of 3 inches. 


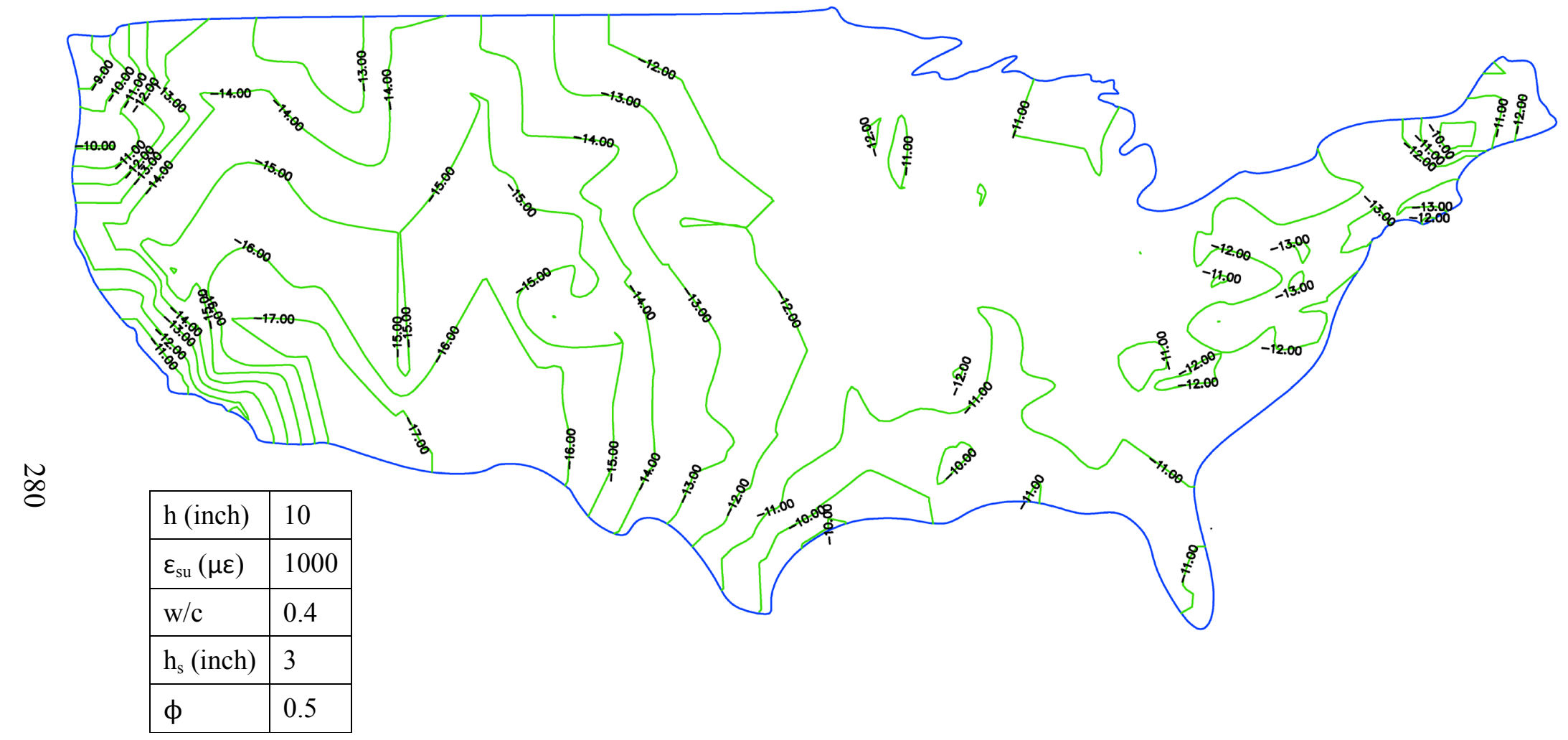

Figure F.10: Design Aid for a 10 inch thick slab with a w/c ratio of 0.4, ultimate shrinkage of $1000 \mu \varepsilon$, a reversible shrinkage factor of 0.5 and a depth of shrinkage zone of 3 inches. 


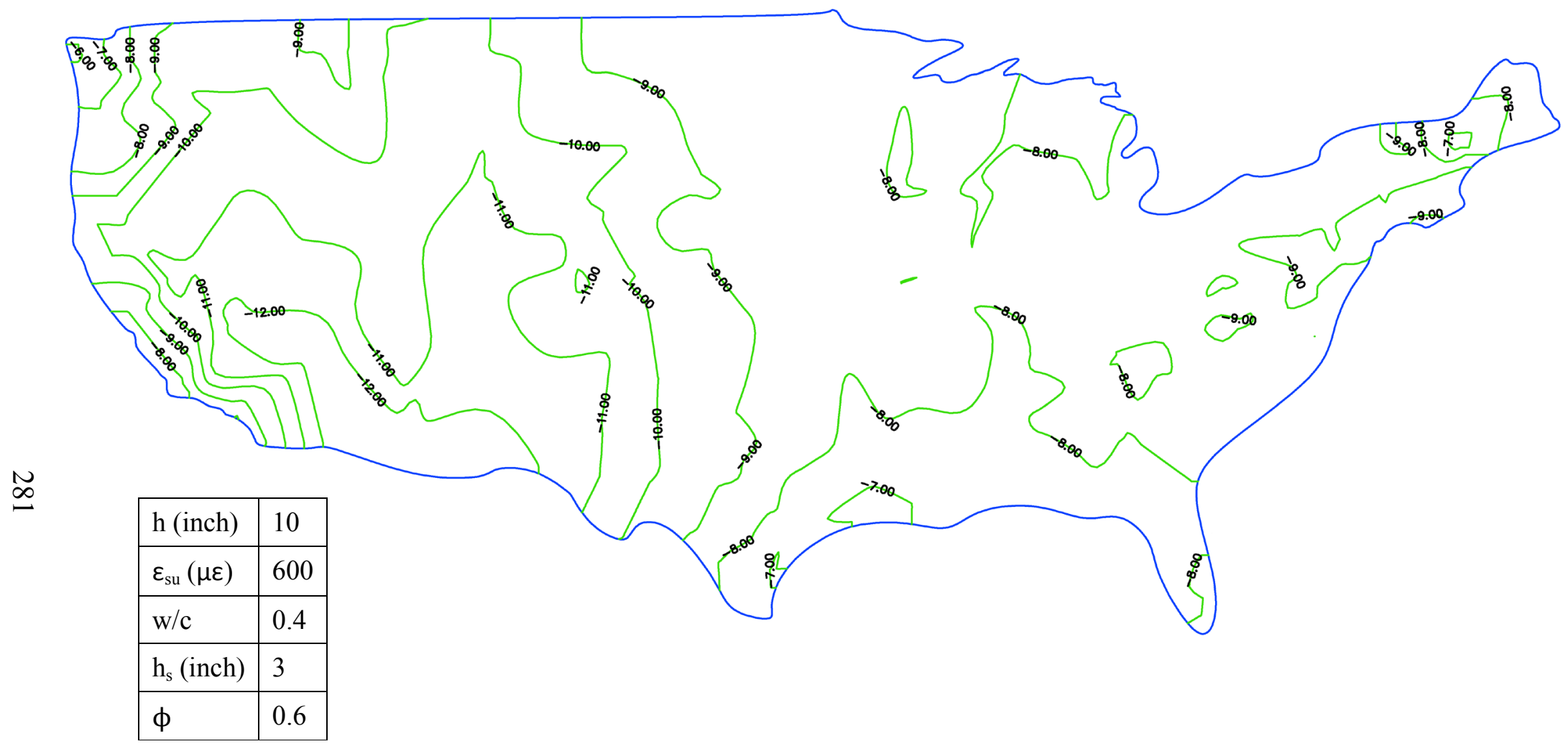

Figure F.11: Design Aid for a 10 inch thick slab with a w/c ratio of 0.4 , ultimate shrinkage of $600 \mu \varepsilon$, a reversible shrinkage factor of 0.6 and a depth of shrinkage zone of 3 inches. 


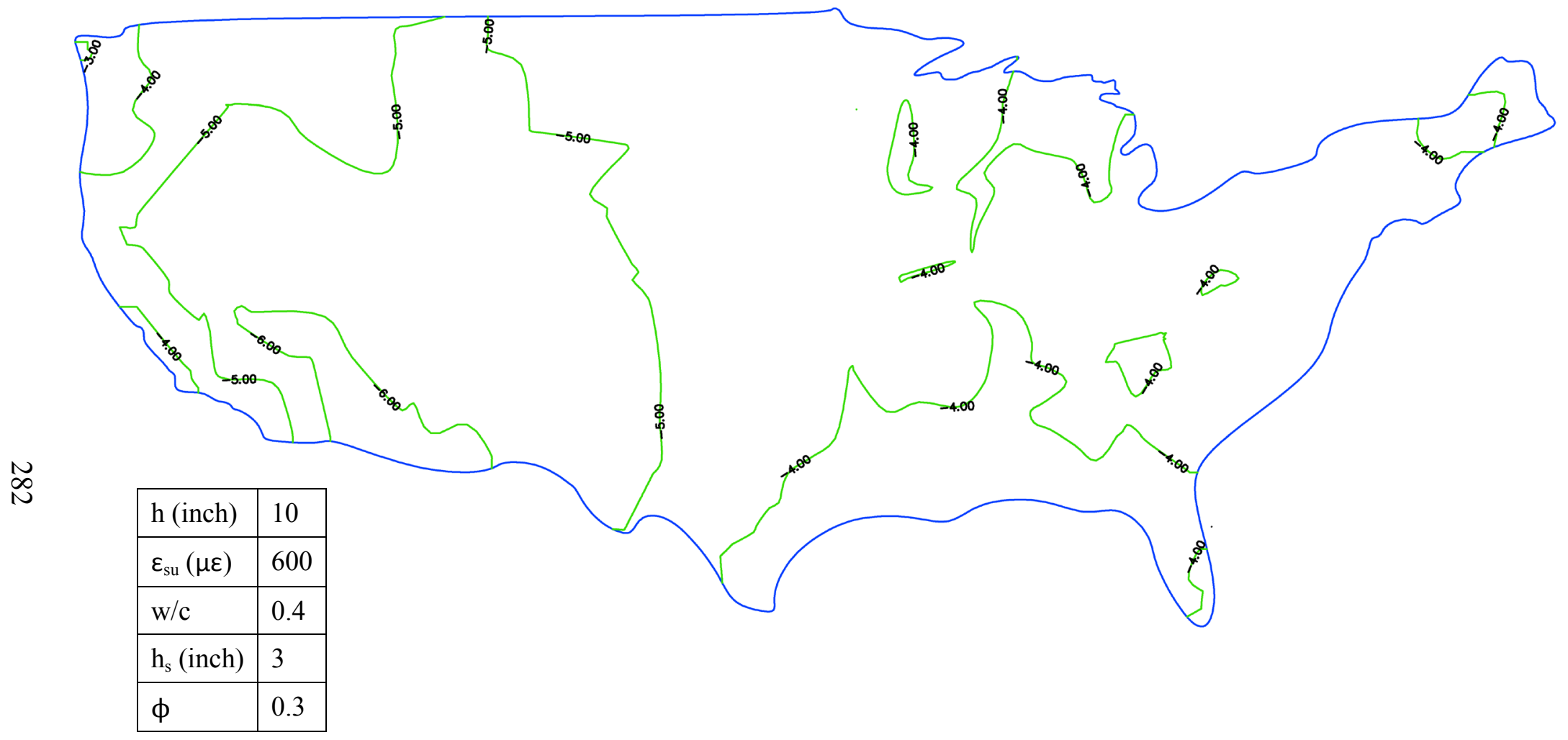

Figure F.12: Design Aid for a 10 inch thick slab with a w/c ratio of 0.4 , ultimate shrinkage of $600 \mu \varepsilon$, a reversible shrinkage factor of 0.3 and a depth of shrinkage zone of 3 inches. 


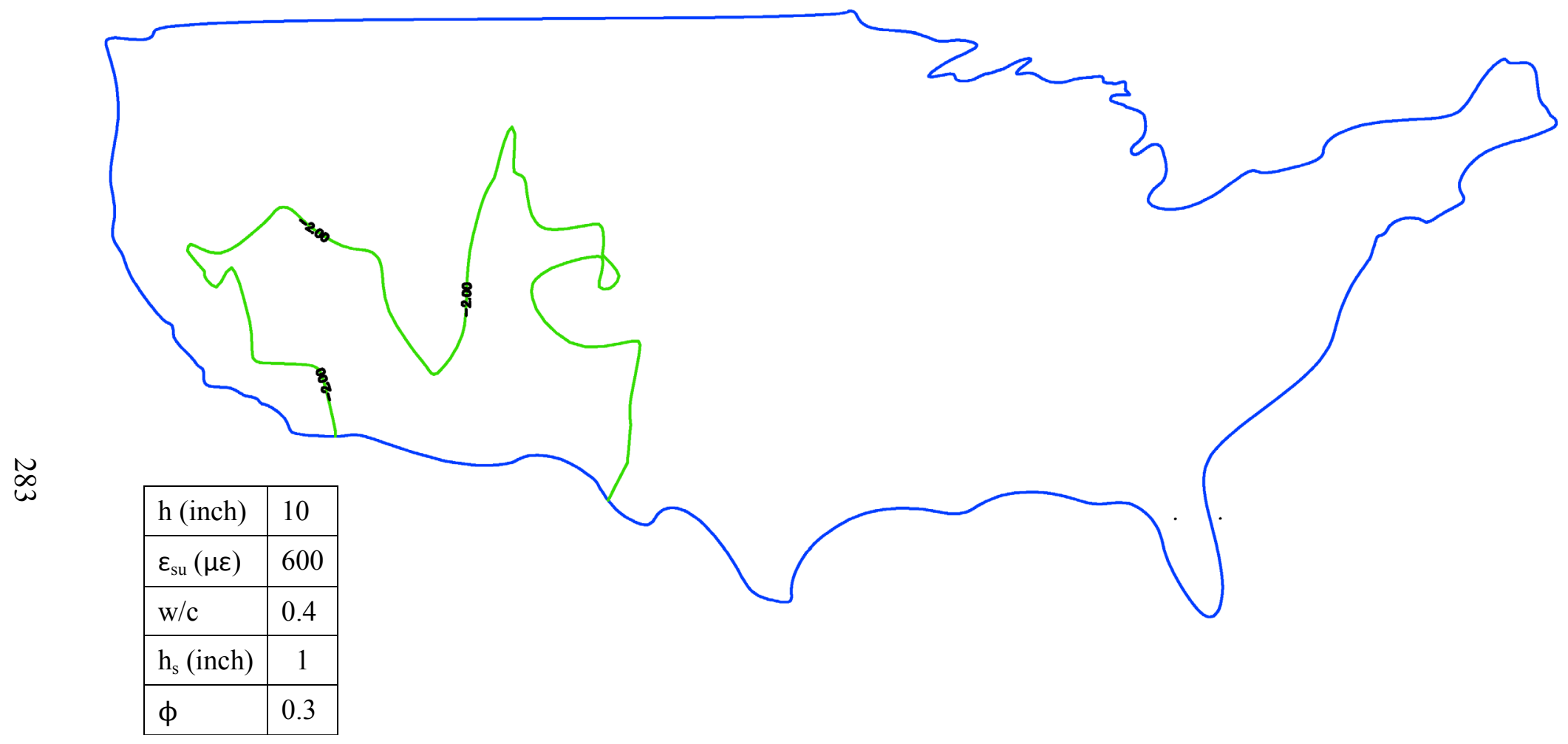

Figure F.13: Design Aid for a 10 inch thick slab with a w/c ratio of 0.4 , ultimate shrinkage of $600 \mu \varepsilon$, a reversible shrinkage factor of 0.3 and a depth of shrinkage zone of 1 inch. 


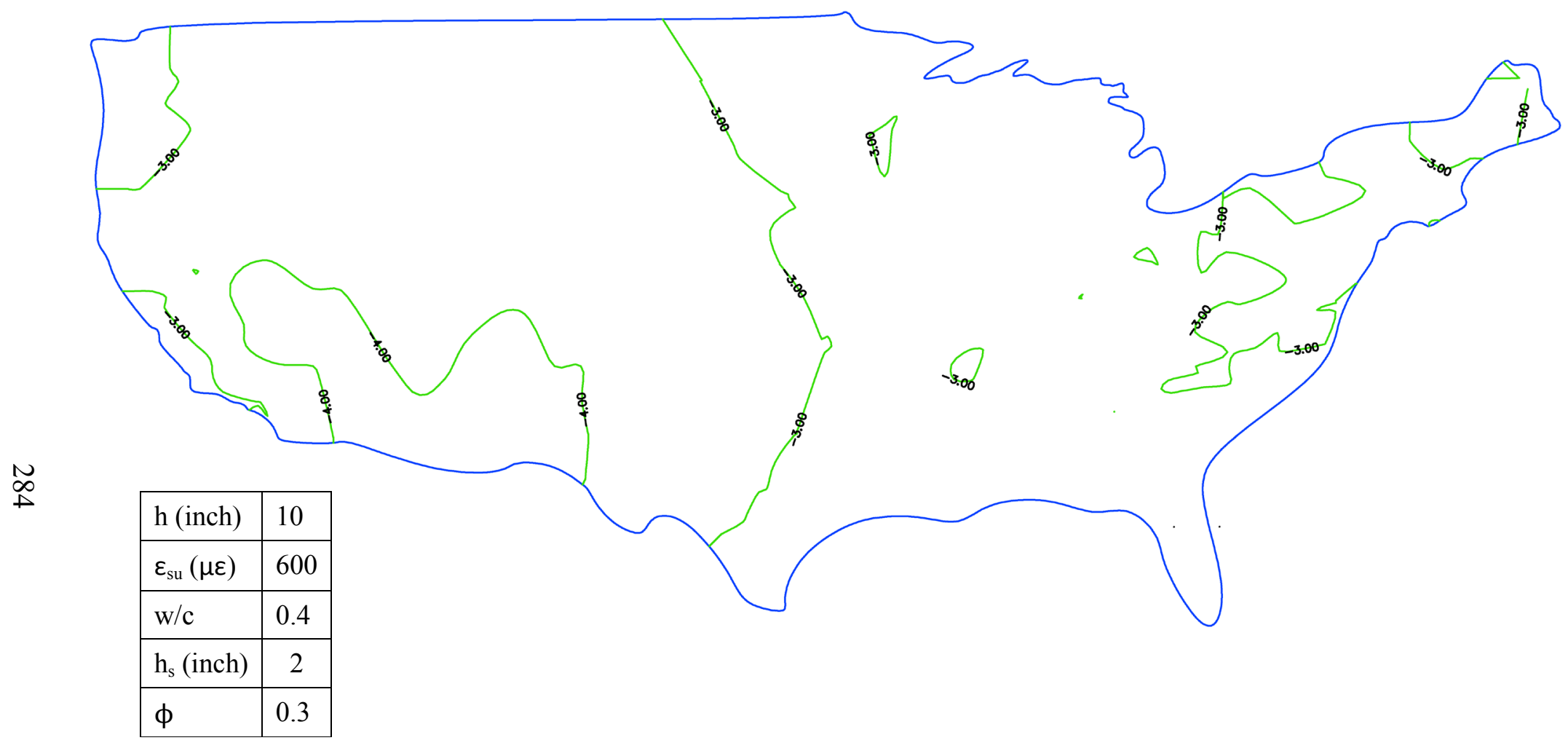

Figure F.14: Design Aid for a 10 inch thick slab with a w/c ratio of 0.4 , ultimate shrinkage of $600 \mu \varepsilon$, a reversible shrinkage factor of 0.3 and a depth of shrinkage zone of 2 inches. 


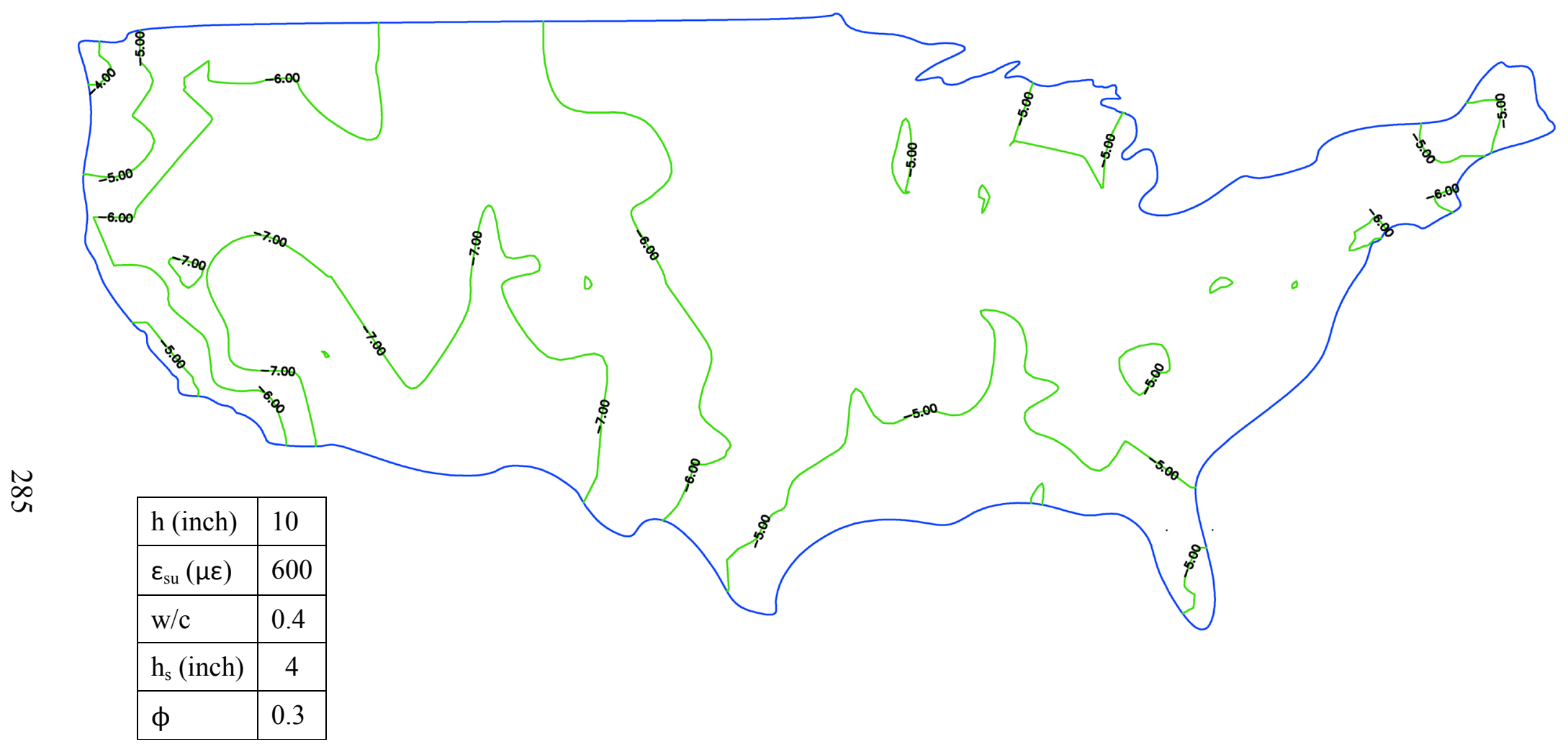

Figure F.15: Design Aid for a 10 inch thick slab with a w/c ratio of 0.4 , ultimate shrinkage of $600 \mu \varepsilon$, a reversible shrinkage factor of 0.3 and a depth of shrinkage zone of 4 inches. 


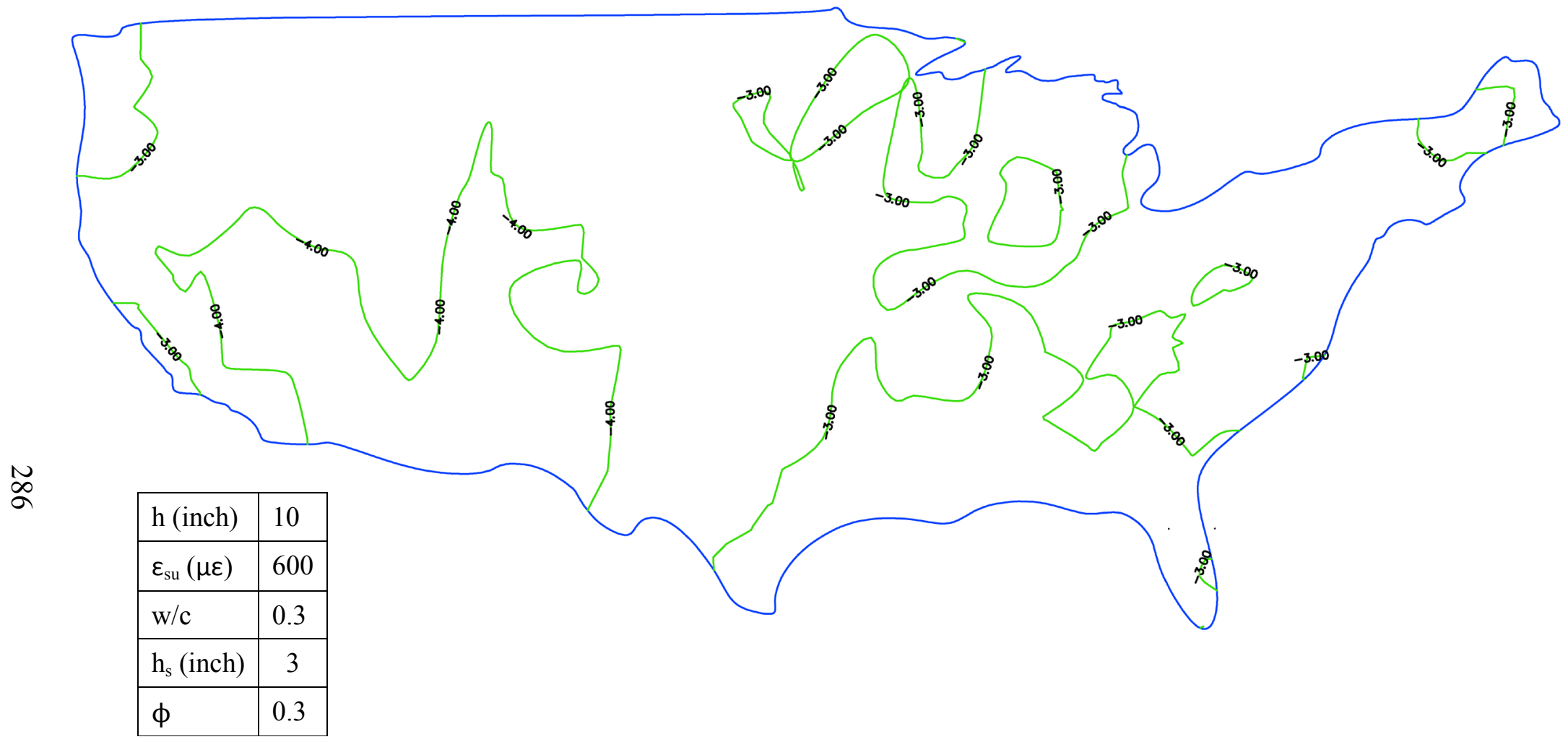

Figure F.16: Design Aid for a 10 inch thick slab with a w/c ratio of 0.3 , ultimate shrinkage of $600 \mu \varepsilon$, a reversible shrinkage factor of 0.3 and a depth of shrinkage zone of 3 inches. 


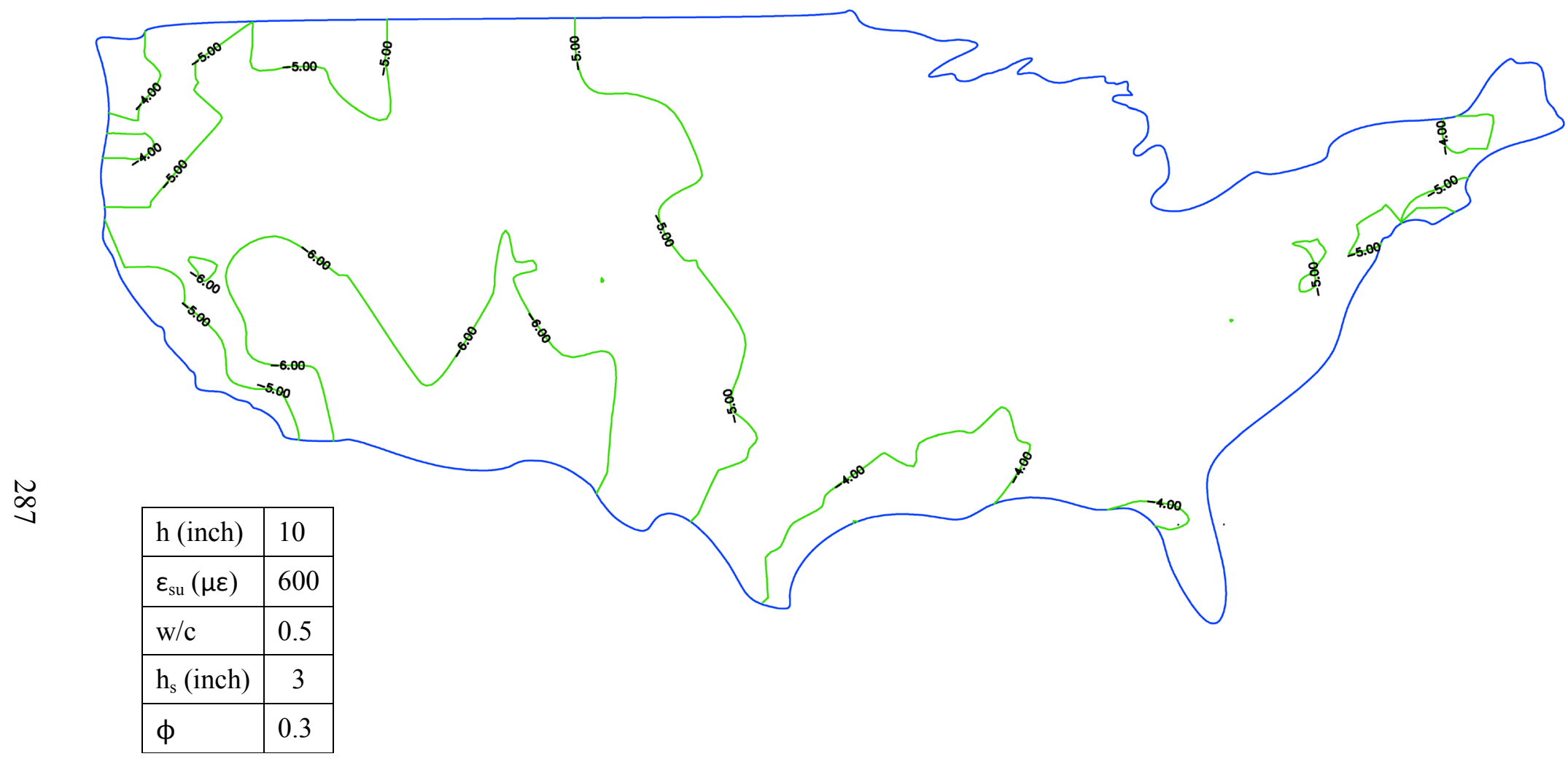

Figure F.17: Design Aid for a 10 inch thick slab with a w/c ratio of 0.5 , ultimate shrinkage of $600 \mu \varepsilon$, a reversible shrinkage factor of 0.3 and a depth of shrinkage zone of 3 inches. 


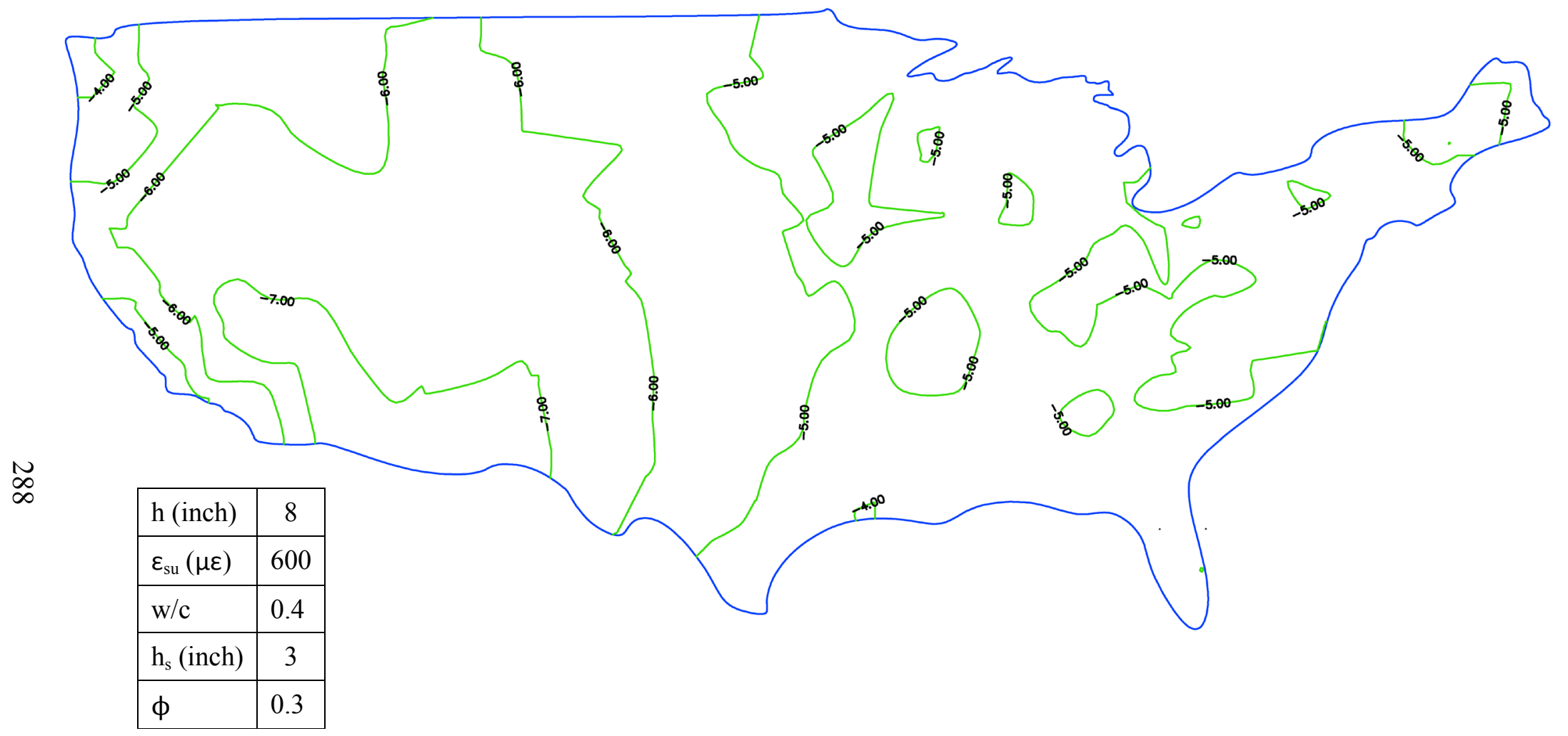

Figure F.18: Design Aid for an 8 inch thick slab with a w/c ratio of 0.4 , ultimate shrinkage of $600 \mu \varepsilon$, a reversible shrinkage factor of 0.3 and a depth of shrinkage zone of 3 inches. 


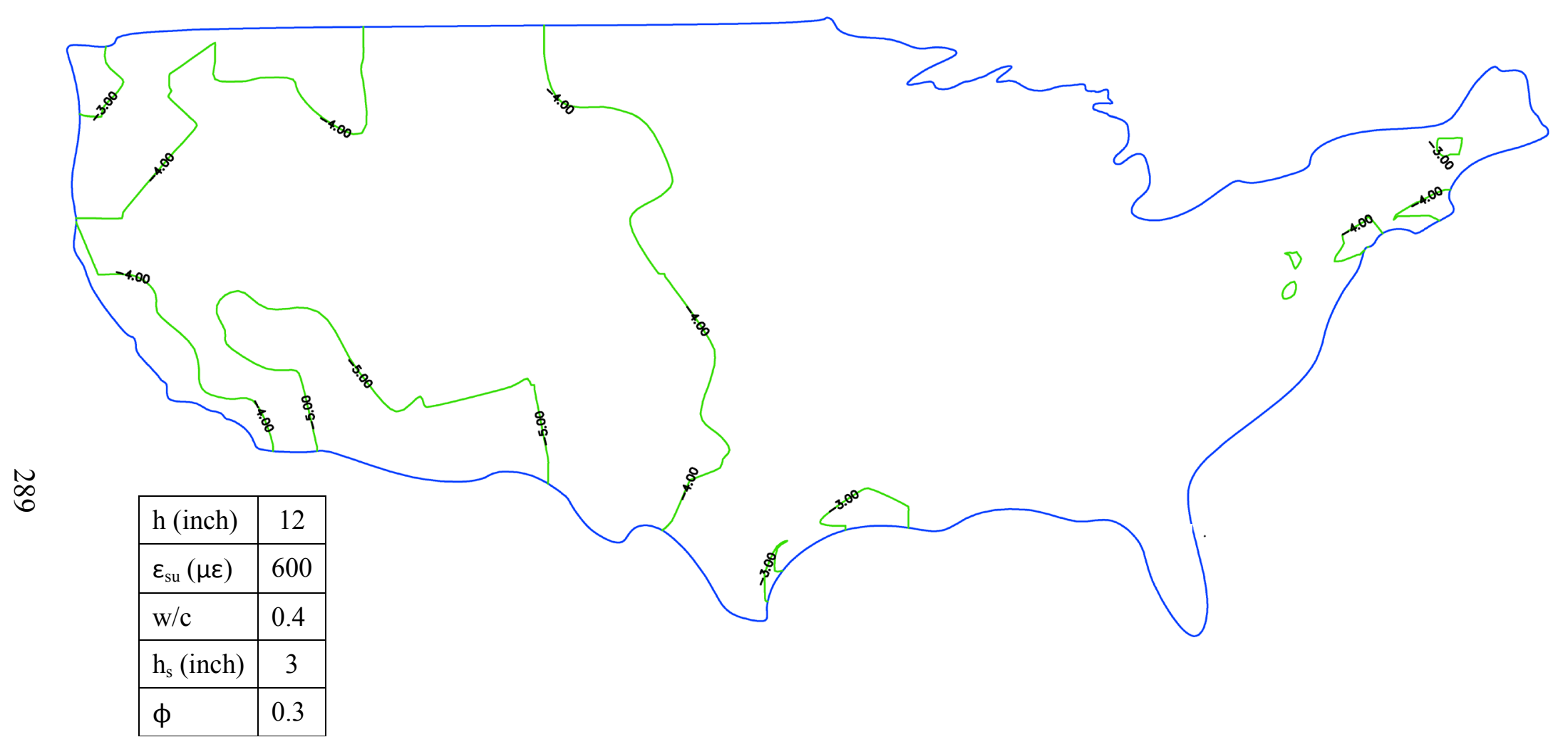

Figure F.19: Design Aid for a 12 inch thick slab with a w/c ratio of 0.4 , ultimate shrinkage of $600 \mu \varepsilon$, a reversible shrinkage factor of 0.3 and a depth of shrinkage zone of 3 inches. 


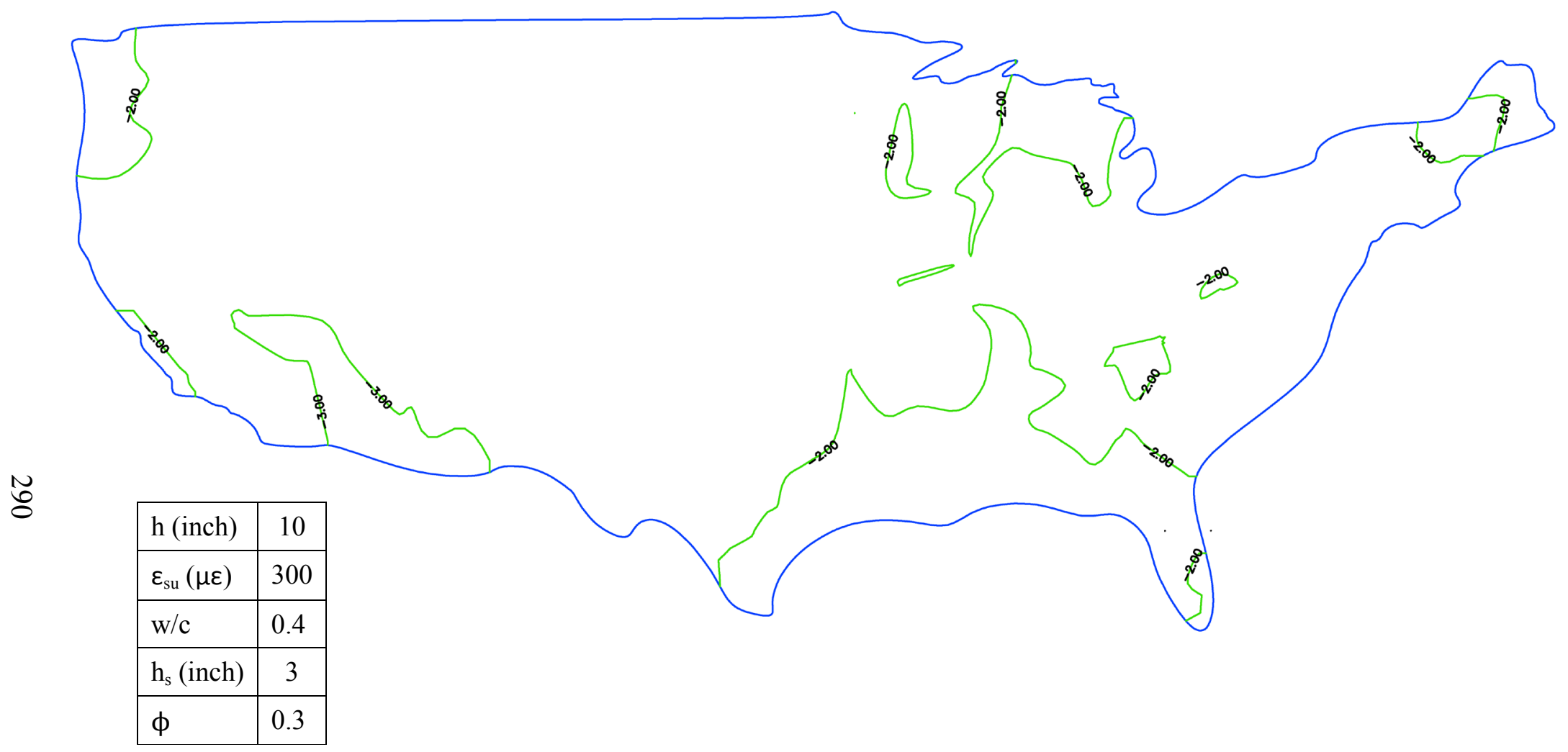

Figure F.20: Design Aid for a 10 inch thick slab with a w/c ratio of 0.4 , ultimate shrinkage of $300 \mu \varepsilon$, a reversible shrinkage factor of 0.3 and a depth of shrinkage zone of 3 inches. 


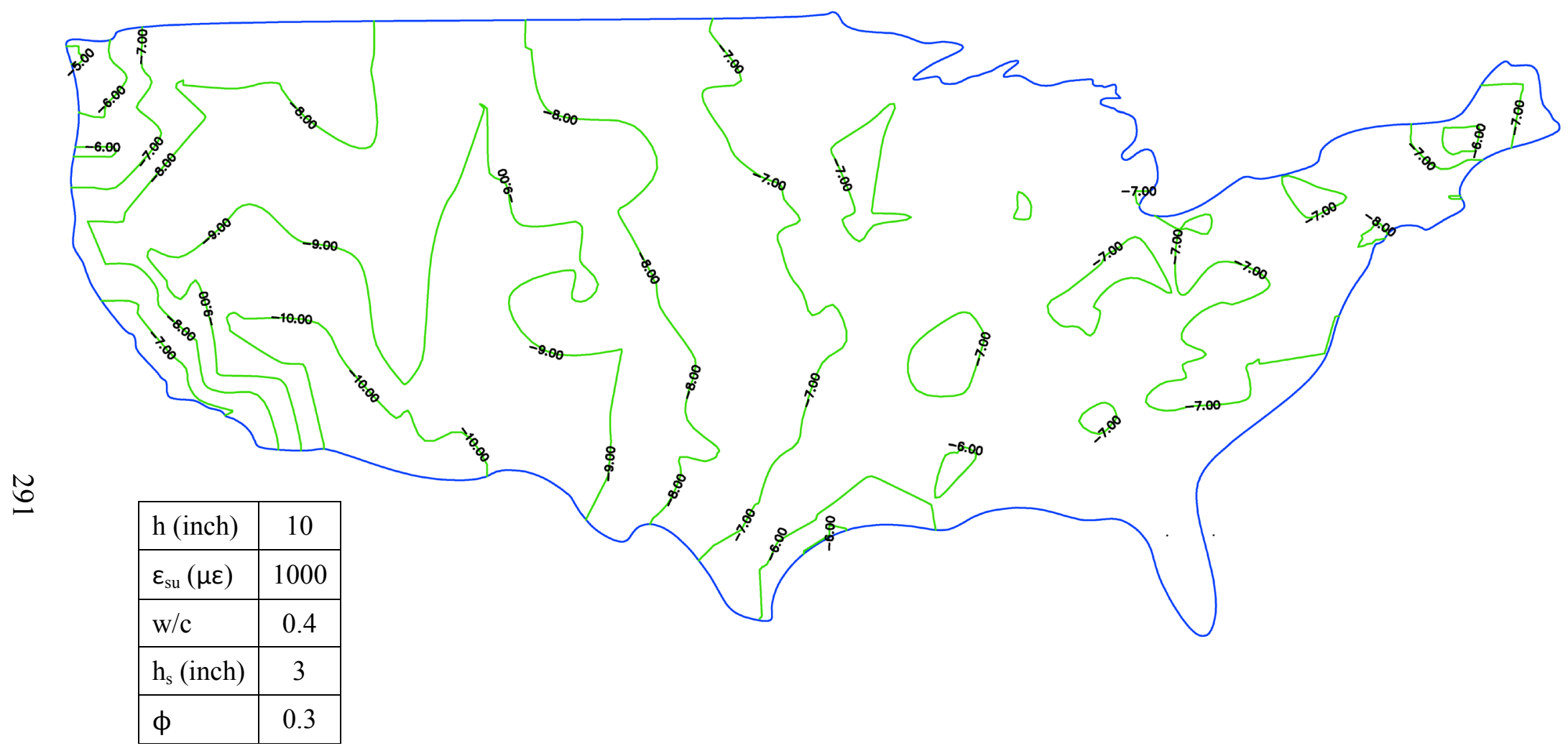

Figure F.21: Design Aid for a 10 inch thick slab with a w/c ratio of 0.4, ultimate shrinkage of $1000 \mu \varepsilon$, a reversible shrinkage factor of 0.3 and a depth of shrinkage zone of 3 inches. 


\section{Appendix G: Input Parameters for ISLAB 2000 Runs}

Table G.1

Input Prameters used in ISLAB 2000.

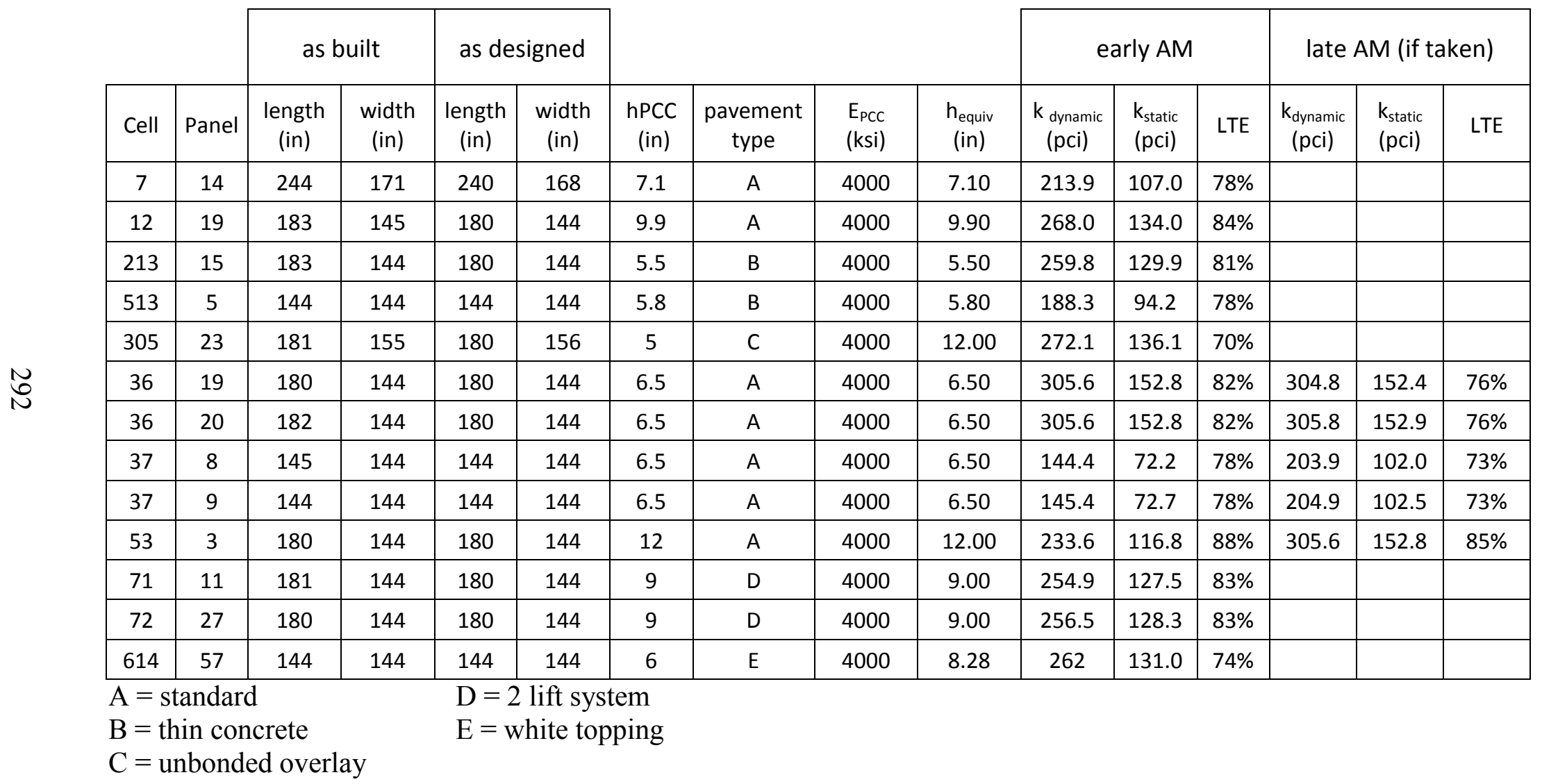

